
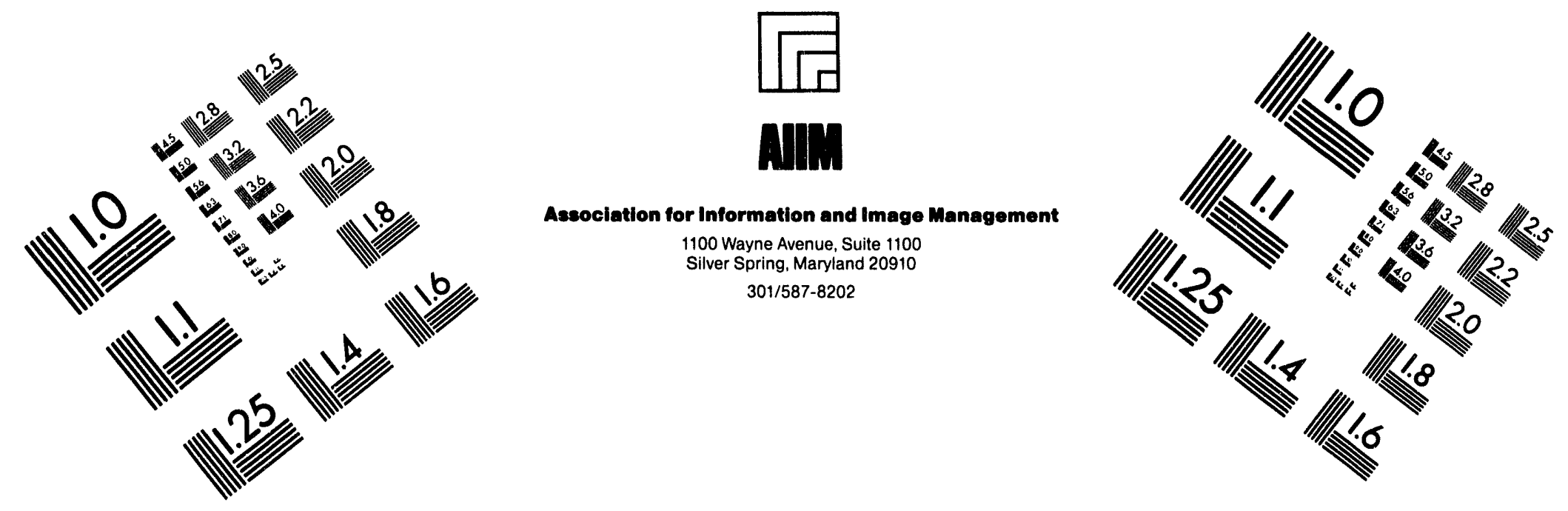

\title{
Centimeter
}

$\begin{array}{llllllllllllllll}1 & 2 & 3 & 4 & 5 & 6 & 7 & 8 & 9 & 10 & 11 & 12 & 13 & 14 & 15 & \mathrm{~mm}\end{array}$

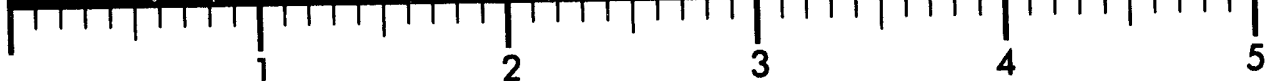
Inches
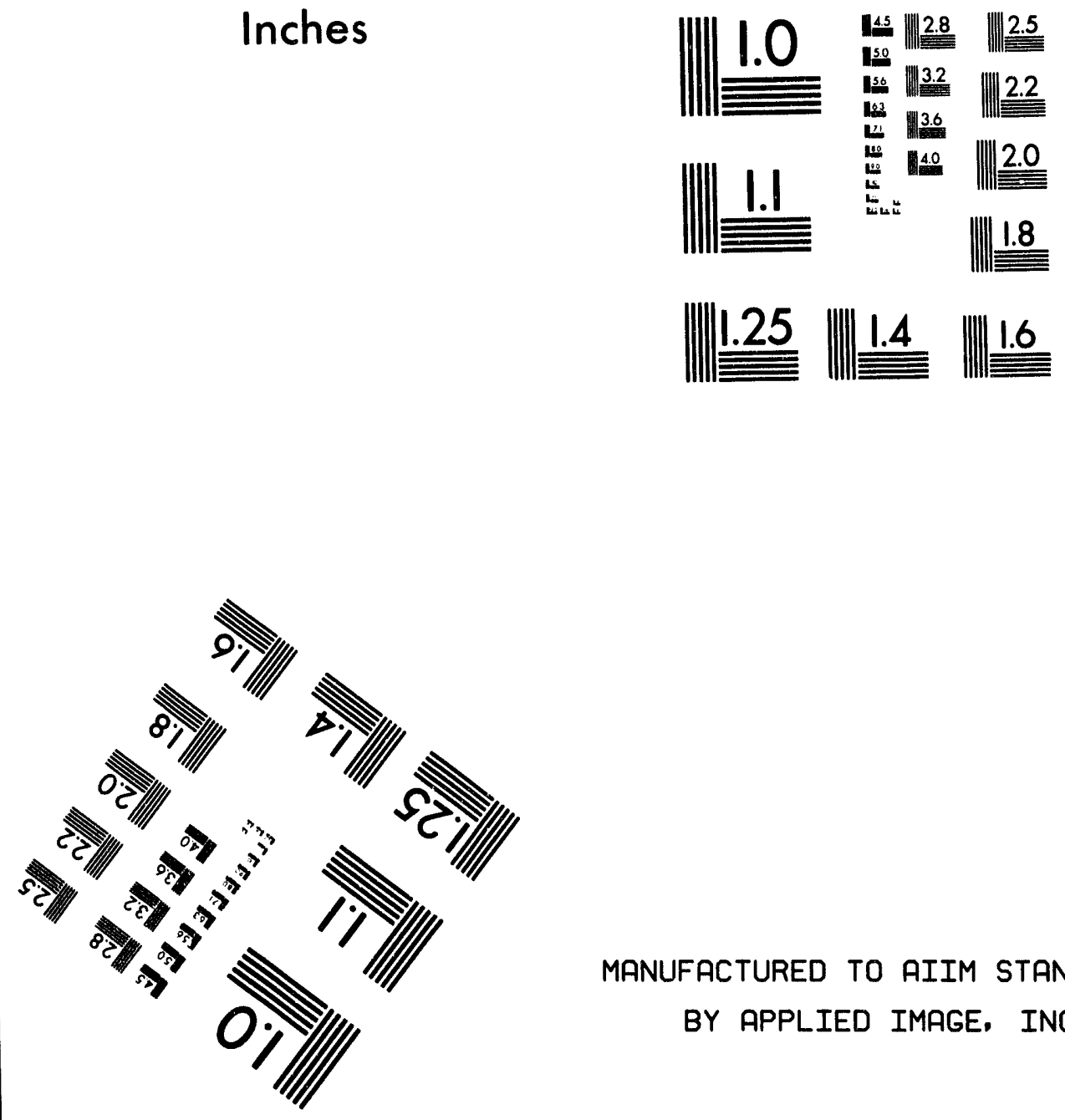

MANUFACTURED TO AIIM STANDARDS BY APPLIED IMAGE, INC.

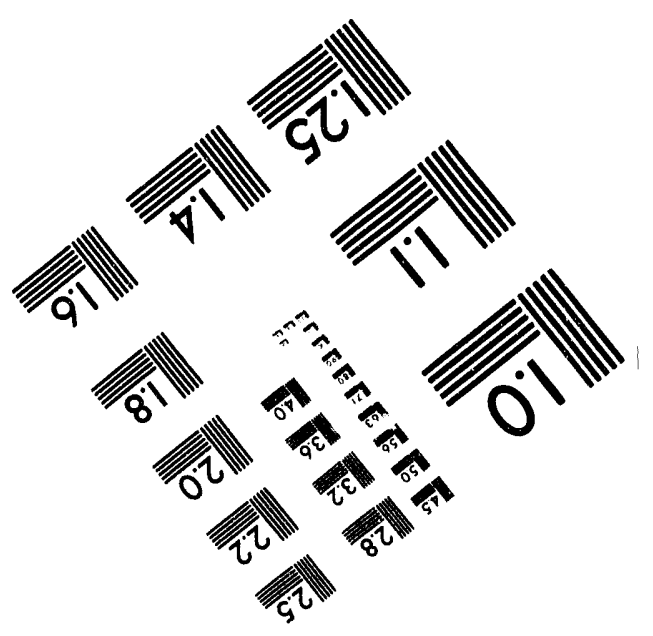



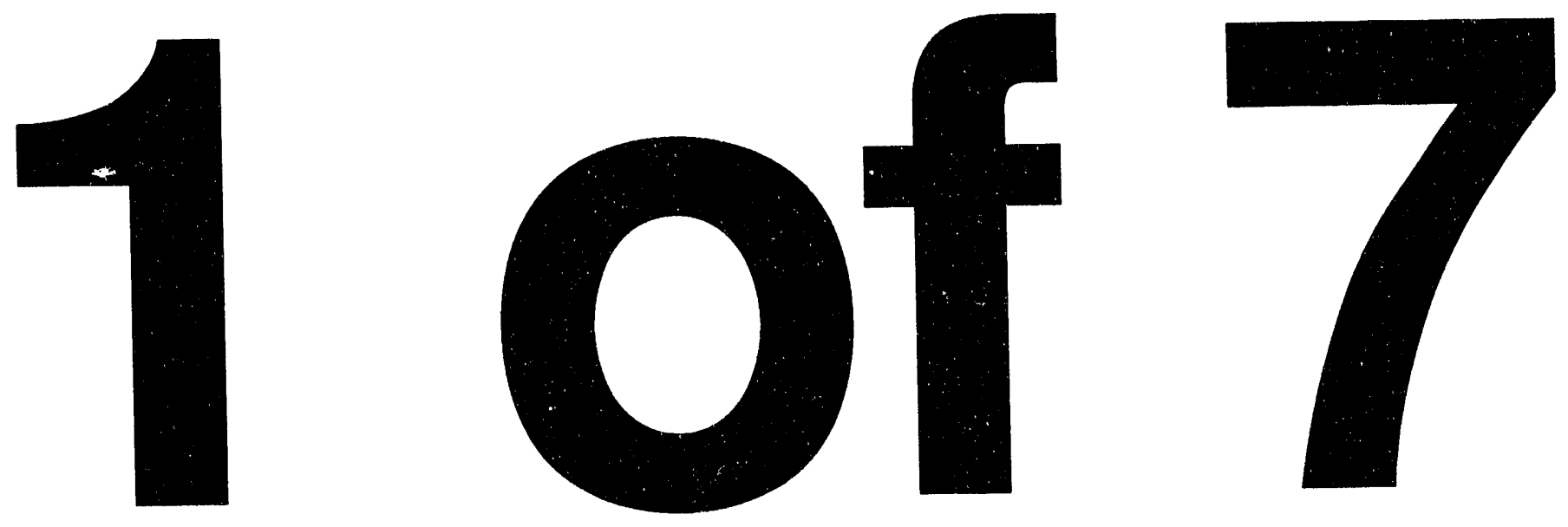
NEDO-32361

Contract DE-AC03-93SF19681

June 1, 1994

\section{Study of Plutonium Disposition Using Existing GE Advanced Boiling Water Reactors}

RECENEL

JUN 101994

OSTI

Evaluation of Pu Consumption in

Existing Light Water Reactors

Tprepared for the

United States Department of Energy

San Francisco, CA 


\section{Disclaimer}

This report was prepared as an account of work sponsored by an agency of the United States Government. Neither the United States Government nor any agency thereof, nor any of their employees, makes any warranty, express or implied, or assumes any legal liability or responsibility for the accuracy, completeness, or usefulness of any information, apparatus, product, or process disclosed, or represents that its use would not infringe privately owned rights. Reference herein to any specific commercial product, process, or service by trade name, trademark, manufacturer, or otherwise, does not necessarily constitute or imply its endorsement, recommendation, or favoring by the United States Government or any agency thereof. The views and opinions of authors expressed herein do not necessarily state or reflect those of the United States Government or any agency thereof.

\section{Disclaimer}

This document is prepared under a contract sponsored by an agency of the United States Government. Neither the contractor nor any subcontractor of any tier nor any employee of any of them makes any warranty, express or implied, or assumes any legal liability or responsibility for the accuracy, completeness, or usefulness of any information, apparatus, product, or process disclosed, or represents that its use would not infringe on any privately owned rights. Reference herein to any specific commercial product, process or service by trade name, trademark, manufacturer, or otherwise does not necessarily constitute or imply its endorsement, recommendation or favoring by any contractor of any tier. 


\section{Table of Contents}

$1.0 \quad$ Executive Summary ................................................................................. S-1

1.0 Introduction ......................................................................................

1.1 Technical Synopsis of Study From Introduction to Present ....................... 1.1-1

1.2 Definition of Commercial Power System Basis for Plutonium Disposition Study .................................................................................. 1.2-1

1.3 Licensing of Commercial System Basis ...................................................... 1.3-1

1.4 Reference Case and Alternatives ............................................................. 1.4-1

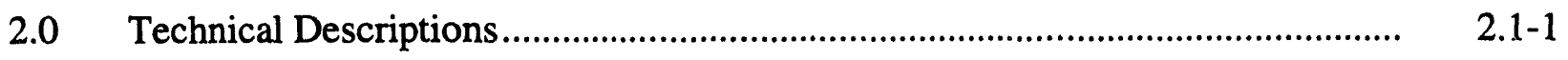

$2.1 \quad$ Reactor and Reactor Cooling Systems ..................................................... 2.1-1

2.2 Balance of Plant and Infrastructure ........................................................... 2.. 2-1

2.3 Reactor Safety Systems, Performance and Analysis ................................ 2.. 2.3-1

$2.4 \quad$ MOX Fuel Fabrication Facility ................................................................ 2.. 2.4

2.5 Ex-Reactor Storage for Fresh and Spent Fuel .......................................... 2..5-1

2.6 Fuel Management............................................................................. 2.6-1

$2.7 \quad$ Nuclear Material Transportation ............................................................. 2.. 2.7-1

$2.8 \quad$ References ...................................................................................... 2. $2.8-1$

3.0 Technology Needs....................................................................................

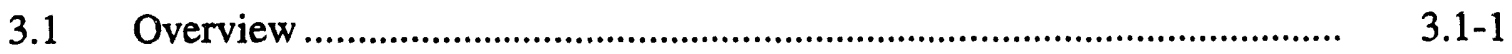

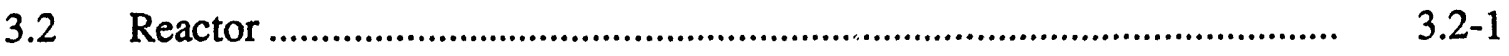

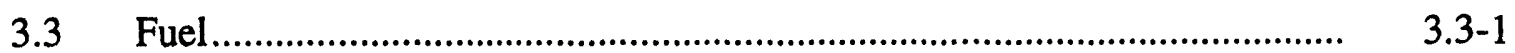

3.4 New Development and Test Facilities ...................................................... 3.. 3.4

3.5 References .....................................................................................

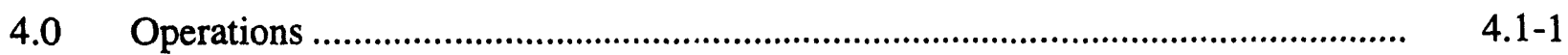

4.1 Government, Utility, and Private Industry Interfaces ................................ 4. 4.1-1

4.2 MOX Fuel Facility Preoperational Checkout and Startup Test Program ....................................................................................... 4. $4.2-1$

4.3 Initial Startup and Checkout ................................................................... 4. 4.3-1

4.4 Related Operating Experience on Availability and Capacity Factor ......... 4. 4.4-1 


\section{Table of Contents (Continued)}

5.0 BWR Reactor Complex Safety and Licensing

$5.1-1$

5.1 BWR Transient and Accident Analysis

5.2 Licensing the BWR Plant

5.3 Licensing the MOX Fuel Fabrication Facility

5.4 Environmental Impact Review Process

6.0 Estimated Cost and Schedule

$6.1-1$

6.1 Summary of Life Cycle Costs

6.2 Capital Cost Detail

6.3 Operations and Maintenance (O\&M) Cost Detail

6.4 Other Life Cycle Cost Detail

6.5 Anticipated Cost Risks

6.6 Plutonium Disposition Project Schedule

6.7 Anticipated Schedule Risks

6.8 References

7.0 Safeguards and Security

7.1 Overview

7.2 Fuel Fabrication Facility

7.3 New Fuel Receipt, Handling and Storage

7.4 Reactor Operations

7.5 Spent Fuel Handling and Storage..

7.6 Safeguards Risks in Shipping and Handling

7.7 Vulnerability Assessment

7.8 Description of Plutonium During Various Stages of Disposition

7.9 International Safeguards, Role of IAEA and IAEA Monitoring

7.10 Conclusions

7.11 References 
NEDO-32361

\section{Table of Contents (Continued)}

8.0 Environment, Safety and Health (ES\&H) ............................................... 8. 8-1

8.1 Transportation Safety ..................................................................... 8. 8.1-1

8.2 Environment, Safety, and Health Impacts of Fuel Activities .................. 8.2-1

$8.3 \quad$ Waste Streams ............................................................................... 8... 8.

8.4 Criticality Safety ........................................................................ 8.4-1

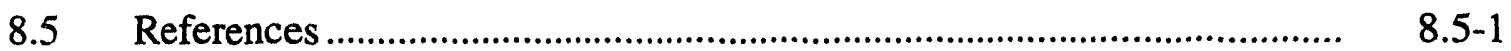

9.0 Non-Plutonium Dispositioning Operations.................................................. 9.1-1

9.1 Commercial Fuel Operation .................................................................. 9.1-1

9.2 Spent Fuel Storage (final ten years) .................................................. 9.2-1

9.3 Other Applications of this Technology ................................................ 9.3-1

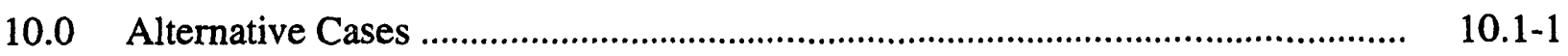

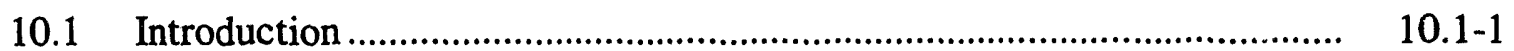

10.2 Technical Descriptions for Alternative Cases ........................................ 10.2-1

10.3 Estimated Incremental Cost and Schedule for Alternative Cases............. 10.3-1

10.4 Plutonium Disposition for the Alternative Cases................................... 10.4-1

10.5 Effect of Availability and Capacity Factor on the Alternative Cases ....... 10.5-1

10.6 Plant License Renewal Considerations ................................................ 10.6-1

10.7 Utilization of Existing DOE Facilities ............................................... 10.. 1-1

10.8 Disposition Options....................................................................... 10.8-1

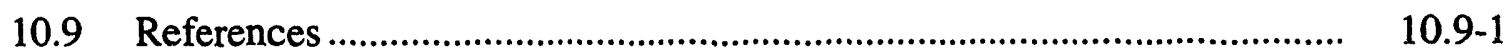

11.0 Public and Political Acceptance ............................................................... 11.1-1

11.1 Approach for Achieving Public Acceptance......................................... 11.1-1

11.2 Likelihood of National Political Acceptance ......................................... 11.2-1

11.3 Likelihood of Local or Regional Acceptance ..................................... 11.3-1 


\section{Table of Contents (Continued)}

12.0 Conclusions and Recommendations ...

12.1 Five Key Challenges

12.2 Five Key Advantages

12.3 Recommendations for Near-Term Implementation

Appendix A Uniform Descriptive Technical Data

A-1

Appendix B Nuclear Methods And Qualification

B-1

Appendix C Key BWR Operational Limits Definitions...

C-1

Appendix D Existing BWR Characteristics

D-1

Appendix E Feed Material Draft Specification

E-1

Appendix F Plutonium Disposition Complex Deployment Capabilities

F-1

Appendix G Comparison of Urania and MOX Fuel Properties

G-1

Appendix H Plan for Lead Fuel Testing for Verification of MOX Fuel Performance...

H-1

Appendix I Repository Considerations

I-1

Appendix J Adapting Commercial MOX Fuel Fabrication Experience

$\mathrm{J}-1$

Appendix K DOE Order Applicability Assessment for ABWR Complex

K-1 


\section{List of Figures}

Page

2.1-1 Generic BWR Fuel Bundle and Control Blade Configuration .................... 2.. 2.9

2.1-2 Full MOX Bundle Design for Generic BWR Core .................................. 2.. 2.1-10

2.1-3 Power Distribution for Full MOX Assembly ............................................ 2.1-11

2.1-4 Hot Uncontrolled K-Infinity for Full MOX Assembly .............................. 2.. 2.11

2.1-5 Island Bundle Design for Generic BWR Core ......................................... 2.1-13

2.1-6 Power Distribution for Island Design Assembly .................................... 2.1-14

2.1-7 Hot Uncontrolled K-Infinity for Island Design Assembly ........................... 2.1-14

2.1-8 Generic BWR-5 Core Configuration .................................................... 2.1-15

2.1-9 Equilibrium Cycle Loading Pattern ...................................................... 2.1-19

2.1-10 Equilibrium Cycle Maximum MAPRAT vs Exposure - Full MOX ............ 2.1-21

2.1-11 Equilibrium Cycle Maximum CPRRAT vs Exposure - Full MOX ............ 2.1-21

2.1-12 Equilibrium Cycle Maximum MAPRAT vs Exposure ............................... 2.. 2.22

2.1-13 Equilibrium Cycle Maximum CPRRAT vs Exposure - Island Design ........ 2.1-22

2.1-14 Equilibrium Cycle Hot Excess Reactivity - Full MOX ............................... 2.1-24

2.1-15 Equilibrium Cycle Minimum Cold Shutdown Margin - Full MOX ........... 2.1-24

2.1-16 Equilibrium Cycle Hot Excess Reactivity - Island Design ........................ 2.1-25

2.1-17 Equilibrium Cycle Minimum Cold Shutdown Margin - Island Design ....... 2.1-25

2.1-18 Equilibrium Cycle Dynamic Void Coefficient - Full MOX ........................ 2.. 2.1-27

2.1-19 Equilibrium Cycle Doppler Coefficient - Full MOX ............................... 2.1-27

2.1-20 Equilibrium Cycle Core Data Summary at $0 \mathrm{MWd}$ /st - Full MOX ............ 2.1-28

2.1-21 Equilibrium Cycle Core Data Summary at $2000 \mathrm{MWd}$ /st - Full MOX ...... 2.1-29

2.1-22 Equilibrium Cycle Core Data Summary at $4000 \mathrm{MWd} / \mathrm{st}$ - Full MOX ....... 2.1-30

2.1-23 Equilibrium Cycle Core Data Summary at $6000 \mathrm{MWd} / \mathrm{st}$ - Full MOX ....... 2.1-31

2.1-24 Equilibrium Cycle Core Data Summary at End of Cycle - Full MOX ....... 2.1-32

2.1-25 Equilibrium Cycle Core Data Summary at $0 \mathrm{MWd} / \mathrm{st}$ - Island Design ........ 2.1-33

2.1-26 Equilibrium Cycle Core Data Summary at $2000 \mathrm{MWd} / \mathrm{st}$ - Island Design .. 2.1-34

2.1-27 Equilibrium Cycle Core Data Summary at $4000 \mathrm{MWd} / \mathrm{st}$ - Island Design .. 2.1-35

2.1-28 Equilibrium Cycle Core Data Summary at $6000 \mathrm{MWd} / \mathrm{st}$ - Island Design .. 2.1-36

2.1-29 Equilibrium Cycle Core Data Summary at End of Cycle - Island Design ... 2.1-37

2.1-30 Spent Fuel Decay Heat for the Island Design ......................................... 2. 2.1-40

2.1-31 Spent Fuel Decay Heat for the Full MOX Design ...................................... 2.1-40 


\section{List of Figures (Continued)}

2.1-32

2.1-33

2.1-34

2.1-35

2.1-36

2.1-37

2.1-38

2.1-39

2.1-40

2.1-41

2.2-1

2.2-2

2.2-3

2.3-1

2.3-2

2.3-3

2.3-4

2.4-1

2.4-2

2.4-3

2.4-4

2.4-5

2.4-6

2.4-7

2.4-8

2.5-1

2.5-2

2.5-3

$2.5-4$

2.6-1
Full MOX and $\mathrm{UO}_{2}$, Core Decay Heat Difference

BWR Reactor Pressure Vessel.

BWR Control Rod Drive Unit

Control Rod Drive Hydraulic System

High Pressure Coolant Injection System

High Pressure Core Spray System

Reactor Core Isolation Cooling System.

Low Pressure Coolant Injection System and Containment Spray/Cooling ..

Core Spray System

Automatic Depressurization System Safety/Relief Valves.

Mark I Containment

Mark II Containment.

Mark III Containment

Generator Load Rejection without Bypass

Feedwater Controller Failure, Maximum Demand, HWL Trip

Typical Power/Flow Map.

MSIV Closure, Flux Scram

MOX Process Flow Sheet

Treatment of Manufacturing Waste

MOX Facility Arrangement

MOX Fuel Plant Organization Chart

Adaptation of FMEF 70 Foot Level Internal Area for MOX Fabrication ....

Upper Level of Fuel Processing Restoration Building .

Mezzanine Level of Fuel Processing Restoration Building.....

Adaptation of Waste Tank Exchange Gallery for MOX Fabrication

Fresh MOX Fuel Storage Vault

Ex-Reactor Fuel Storage

Typical Refueling Floor Layout.

Typical BWR Refueling Process

Composition of MOX Assemblies Charged to and Discharged from the

Generic BWR.
Page

$2.1-41$

$2.1-43$

2.1-46

2.1-47

2.1-50

2.1-51

2.1-52

2.1-54

2.1-55

2.1-56

2.2-18

2.2-19

2.2-20

2.3-7

2.3-8

2.3-11

2.3-14

2.4-3

2.4-15

2.4-18

2.4-20

2.4-23

2.4-24

2.4-25

2.4-28

2.5-3

2.5-4

2.5-5

2.5-8

2.6-2 


\section{List of Figures (Continued)}

2.6-2 Isotopic Composition of MOX Fuel Charged - Generic BWR-5 ................

Page

6.1-1 Break-Even Capital Value for Existing BWRs Compared to Building New ABWRs.

6.6-1 Overall Project Schedule for Dispositioning 50 MT Using Three

Existing BWRs.

Fuel Activities Schedule

Los Alamos National Laboratory Activities Schedule

MOX Facility Block Layout for 35,000 Rods/Year Reference Case

Showing Major Material Balance Areas

Period of Enhanced MOX Fuel Security

Plutonium Distribution During Reference Disposition Using

Three BWRs

7.8-2 Plutonium Distribution in Year 2012, Reference Option Using

Three BWRs

7.8-3 Plutonium Distribution in Year 2024, Reference Option Using

Three BWRs.

7.8-4 Plutonium Distribution for Alternate Case One.

8.4-3 Cross Sectional View of Proposed Spent Fuel Container in Repository ...... 


\section{List of Figures (Continued)}

Page

10.4-2 Plutonium Distribution During Alternate Case 1 Disposition Using 6 BWRs.

10.4-3

10.7-1 Adaptation of FMEF 70 Foot Level Internal Area for MOX Fabrication .... $10.7-3$

10.7-2 Upper Level of Fuel Processing Restoration Building

$10.7-4$

$10.7-3$ Mezzanine Level of Fuel Processing Restoration Building.

$10.7-5$

$10.7-4$ Adaptation of Waste Tank Exchange Gallery for MOX Fabrication

$10.7-8$ 


\section{List of Tables}

Fundamental Fuel Design Requirements

Low Pressure Coolant Systems

Power Production Data

Existing DOE Facilities Capabilities

Comparison of Isotopic Concentrations (Curies).

Nuclear Parameter Significance

Comparison of Key Parameters

Transient Results.

Decay Ratio at Natural Circulation and Rated Rod Line

Characteristics of Reactor Deployment and Fuel Fabrication Capacity ....

Reference Option Fuel Assembly Flow Summary 


\section{List of Tables (Continued)}

Evaluation of Amendment 22 Criteria in Regard to MOX Fuel

Potential Environmental Permit Requirements

BWR Plant Incremental O\&M Costs. 


\section{List of Tables (Continued)}

Page

8.2-1 BWR Occupational Dose Assessment ................................................ 8.2-6

8.2-2 BWR Offsite Radiological Impact (rem/yr ede - 1 BWR Unit)............... 8.. 8.2-7

8.2-3 MOX Fuel Facility Occupational Radiation Doses - Low Am Fuel ......... 8.2-8

8.2-4 MOX Fuel Facility Occupational Radiation Doses - High Am Fuel ........ 8.2-8

8.2-5 MOX Fuel Facility Offsite Radiological Impact .................................... 8.2-9

8.2-6 Impact Comparison of High- and Low-Americium Content Feed

Plutonium .................................................................................. 8...

8.3-1 BWR Radioactive Waste Generation (1 Unit) ........................................ 8.. 8.3-2

8.3-2 MOX Fuel Facility Radioactive Waste Generation Low and High Am

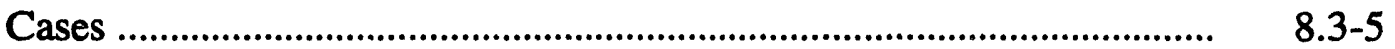

8.3-3 Hazardous and Non-Regulated Wastes Reference Case .......................... 8.. 8.3-5

8.3-4 Summary of Plutonium Carryover Quantities ....................................... 8.. 8.6

8.4-1 Hypothetical Accident Conditions ........................................................ 8.4-2

8.4-2 Fresh Fuel Bundle Neutron Multiplication Factors for Infinite Arrays

of RA-3D Containers in Accident Conditions ........................................ 8.4 8.8

8.4-3 Spent Fuel Waste Quantities ................................................................ 8.4.9

8.4-4 Neutron Multiplication Factors for Spent Fuel Bundles Infinite Arrays

of RA-3D Containers in Accident Conditions ........................................ 8.. 8.4-10

8.4-5 Neutron Multiplication Factors for Repository ....................................... 8.4-12

9.2-1 MOX Discharging Schedule and Spent Fuel Storage (Final Ten Years) .. $\quad$ 9.2-2

10.2-1 Characteristics of Reactor Deployment and Fuel Fabrication Capacity .... 10.2-1

10.3-1 Alternative Case Costs .................................................................... 10.3-2

10.3-2 Cash Flow - Incremental Cost ........................................................ 10.3-5

10.7-1 Potential Cost Savings from the Use of Existing DOE Facilities ............. 10.7-9

10.8-1 Browns Ferry Disposition Capability Based on 75\% C.F. ....................... 10.8-3

10.8-2 WNP-2, LaSalle 1/2 Disposition Capability Based on 75\% C.F. ............. 10.8-5

10.8-3 Grand Gulf, River Bend and Clinton Disposition Based on 75\% C.F...... $\quad 10.8-8$

10.8-4 Perry and Limerick Disposition Based on 75\% C.F. ............................. 10.8-9 


\section{List of Acronyms}

ABWR

ACS

AFUDC

ALARA

ARIES

ARMS

BAS

BAT

BCT

BNFP

BWR

BWROG

C/S

CAMS

COE

CRD

CRWMS

CWA

DCS

DE

DOE

DOT

DSC

E-MAD

EBS

EIA

EIS

EPA

FAA

FERC

FFTF

FMEF

FPR

FPS
Advanced Boiling Water Reactor

Atmospheric Control System

Allowance for Funds Used During Construction

As Low As Reasonably Achievable

Automated Retirement and Integrated Extraction System

Area Radiation Monitoring System

Breathing Air System

Best Available Technology

Best Conventional Technology

Barnwell Nuclear Fuel Plant

Boiling Water Reactor

BWR Owners Group

Containment and Surveillance

Containment Atmospheric Monitoring System

Corps of Engineers

Control Rod Drive

Civilian Radioactive Waste Management System

Clean Water Act

Drywell Cooling System

Dose Equivalent

Department of Energy

Department of Transportation

Dry Shielded Canister

Engine Maintenance, Assembly and Disassembly

Engineered Barrier System

Energy Information Agency

Environmental Impact Statement

Environmental Protection Agency

Federal Aviation Administration

Federal Energy Commission Reports

Fast Flux Test Reactor

Fuels Materials Examination Facility

Fuel Processing Restoration

Fire Protection System 
NEDO-32361

\section{List of Acronyms (Continued)}

FSAR

FWCF

GDC

GE

GENE

GSI

HPCI

HPCS

HPNSS

HSM

IAEA

IAS

ICA

ID

INEL

IPE

IRP

ISFSI

ISTM

KMP

LOCA

LD\&IS

LANL

LEID

LEU

LHGR

LLW

LPCI

LPCS

LRMS

LRNBP

LUA

MAPLHGR

MBA
Final Safety Analysis Report

Feedwater Controller Failure to Maximum Demand

General Design Criteria

General Electric Company (GE)

GE Nuclear Energy

Generic Safety Issues

High Pressure Coolant Injection

High Pressure Core Spray

High Pressure Nitrogen Gas Supply System

Horizontal Storage Module

International Atomic Energy Agency

Instrument Air System

Interim Correction Actions

Inventory Difference

Idaho National Engineering Laboratory

Individual Plant Evaluation

Integrated Resource Plan

Independent Spent Fuel Storage Installation

Inspection, Surveillance, Testing, and Monitoring

Key Measurement Points

Loss-of-Coolant Accident

Leak Detection and Isolation System

Los Alamos National Laboratory

Limit of Error for the Inventory Difference

Low Enriched Uranium

Linear Heat Generation Rate

Low-Level Radioactive Waste

Low Pressure Coolant Injection

Low Pressure Core Spray

Liquid Radwaste Management System

Load Rejection with No Bypass

Lead Use Assemblies

Maximum Average Planar Linear Heat Generation Rate

Material Balance Area 
NEDO-32361

\section{List of Acronyms (Continued)}

MCPR

MOX

MUF

MUWS

NAS

NEPA

NESHAP

NMIS

NMS

NPDES

NPT

NRDA

NRTA

NUPLEX

O\&M

OLMCPR

ORNL

ORR

OTA

PDC

PEIR

PIT

PIV

PRA

PRMS

PSD

PSO

PSS

PUC

$\mathrm{RBCW}$

RCIC

RCPB

RCRA

RHR
Minimum Critical Power Ratio

Mixed Oxide

Material Unaccounted For

Makeup Water System

National Academy of Sciences

National Environmental Policy Act

National Emission Standards for Hazardous Air Pollutants

New Material Isotopic Separation

Neutron Monitoring System

National Pollutant Discharge Elimination System

Treaty on the Non-Proliferation of Nuclear Weapons

Nevada Research \& Development Area

Near-Real-Time-Accountancy

Nuclear Plant Life Extension

Operations and Maintenance

Operating Limit MCPR

Oak Ridge National Laboratory

Operational Readiness Review

Office of Technology Assessment

Plutonium Disposition Complex

Programmatic Environmental Impact Review

Physical Inventory Taking

Physical Inventory Verification

Probabilistic Risk Assessment

Process Radiation Monitoring System

Prevention of Significant Deterioration

Program Secretarial Officer

Process Sampling System

Public Utility Commissions

Reactor Building Cooling Water

Reactor Core Isolation Cooling

Reactor Coolant Pressure Boundary

Resource Conservation and Recovery Act

Residual Heat Removal 
NEDO-32361

\section{List of Acronyms (Continued)}

\begin{tabular}{|c|c|}
\hline ROD & Record of Decision \\
\hline RPS & Reactor Protection Systems \\
\hline RRA & Regulatory Research Associates, Inc. \\
\hline RWCU & Reactor Water Cleanup \\
\hline SAF & Secure Automated Fabrication \\
\hline SER & Safety Evaluation Report \\
\hline SGTS & Standby Gas Treatment System \\
\hline SLMCPR & Safety Limit Minimum Critical Power Ratio \\
\hline SNM & Special Nuclear Material \\
\hline SPTM & Suppression Pool Temperature Monitoring \\
\hline SRL & Savannah River Laboratory \\
\hline SRMS & Solid Radwaste Management System \\
\hline SRP & Standard Review Plan \\
\hline SRV & Safety/Relief Valve \\
\hline SSAS & Station Service Air System \\
\hline SSSP & Site Safeguards and Security Plan \\
\hline SST & Safe Secure Transport \\
\hline TBCW & Turbine Building Cooling Water \\
\hline TEDE & Total Effective Dose Equivalent \\
\hline TID & Tamper Indicating Device \\
\hline TIG & Tungsten Inert Gas \\
\hline TRU & Transuranic \\
\hline TSR & Technical Safety Requirements \\
\hline TVA & Tennessee Valley Authority \\
\hline USI & Unresolved Safety Issues \\
\hline USQ & Unreviewed Safety Qualification \\
\hline UST & Underground Storage Tank \\
\hline VA & Vulnerability Assessment \\
\hline VAR & Vulnerability Analysis Report \\
\hline WIPP & Waste Isolation Pilot Project \\
\hline WTEG & Waste Tank Exchange Gallery \\
\hline ZPPR & Zero Power Physics Reactor \\
\hline
\end{tabular}


NEDO-32361

\section{Executive Summary}

GE SUPPORTS

THE FISSION DISPOSITION OPTION RECOMMENDED BY THE NATIONAL ACADENY OF SCIENCES

BWRS ARE WELL SUITED FOR THE PU DISPOSITION MISSION WTIH FULL MOX CORE CAPABILITY

NO REACTOR MODIFICATIONS ARE NEEDED FOR FULL MOX CAPABILITY

\section{Foreword}

The end of the cold war and the resulting dismantlement of nuclear weapons has resulted in the need for the U.S. to dispose of 50 to 100 metric tons of excess of plutonium in a safe and proliferation resistant manner. A number of studies, including the recently released National Academy of Sciences (NAS) study, have recommended conversion of plutonium into spent nuclear fuel with its high radiation barrier as the best means of providing permanent conversion and long-term diversion resistance to this material.

The NAS study "Management and Disposition of Excess Weapons Plutonium" identified light water reactor spent fuel as the most readily achievable and proven form for the disposition of excess weapons plutonium. The study also stressed the need for a U.S. disposition program which would enhance the prospects for a timely reciprocal program agreement with Russia.

\section{Technical Evaluation}

This summary provides the key findings of a GE study where plutonium is converted into Mixed Oxide (MOX) fuel and a typical 1155 MWe GE Boiling Water Reactor (BWR) is utilized to convert the plutonium to spent fuel. A companion study of the Advanced BWR has recently been submitted. The MOX core design work that was conducted for the ABWR enabled GE to apply comparable fuel design concepts and consequently achieve full MOX core loading which optimize plutonium throughput for existing BWRs.

BWRs are designed with core configurations that have readily permitted fuel design and technology improvements, including longer and more efficient fuel cycles and operation with MOX fuel. The boiling water reactor core and system performance under normal, transient and accident conditions have been evaluated with the conclusion that no modifications to the reactor systems are required for the use of MOX fuel over a range of plutonium enrichments. The core dynamics of the boiling water reactor allows full MOX core loading to be accomplished by fuel design alone. The full MOX core can be implemented in stages as the commercial urania fuel is discharged from the reactor.

Two fuel designs were developed for an 1155 MWe BWR-5 reactor which is representative of any of the later generation of reactors designed by General Electric. The two fuel design concepts are the "island" design 
ISLAND DESIGN FUEL ALLOW AN EARLY START OFPU DISPOSTTION

THREE BWRS CAN DISPOSITION 50 MT of Pu WITHIN 23 YEARS

MOX PLANTS ARE OPERATING IN EUROPE

U.S. HAS MOX CAPABILTY AT LOS ALAMOS and "full MOX" design. This island design is very similar to MOX fuel designs used in the Big Rock Point and Quad Cities BWRs in the 1960's and 1970's. The island design fuel bundle is very conservative from a design viewpoint, which minimizes potential licensing issues, and since only $20 \%$ of the fuel rods contain plutonium, the mission can be supported with existing plutonium fabrication capacity such as that available at Los Alamos National Laboratory (LANL). The island design fuel therefore provides an opportunity to commence a pilot demonstration program as early as 1997 with existing operating BWRs. The demonstration program would be used to validate and gain experience with various elements of the disposition program including transportation, storage, training, reactor licensing and operations, and safeguards while a MOX fuel plant with the capacity for a full MOX core program is being constructed. The full MOX design is based on maximizing the MOX throughput for the existing BWR cores. The design utilizes plutonium in all the fuel rods in the bundle and can be implemented in the currently operating BWRs as soon as the new MOX fabrication facility can be brought on line to provide the required fuel volume.

Using these bundle designs, any three of the most recent larger GE BWRs can disposition 50 MT of plutonium within 23 years of full MOX loading, assuming $75 \%$ capacity factor operation.

Commercial fabrication of MOX fuel is ongoing in the United Kingdom, Belgium and France with plans to do so in Japan. The fabrication process recommended by this study uses mechanical blending of urania and plutonia which is the most widely used process worldwide. Automated processing is used to lower worker exposure and improve waste management. A throughput of between 50 and 150 metric tons of MOX per year, comparable to plants already in operation in Europe, will be required. The plant will meet all environmental requirements and no technical developments are required for its design, licensing, permitting, construction and operation. The design and construction schedule for the MOX plant is compatible with the mission timeframe.

To support the earliest possible start of $\mathrm{Pu}$ disposition, the TA-55 facility at Los Alamos National Laboratory (LANL) was identified as being capable of fabricating the MOX fuel for "island" design reloads for up to three existing reactors. This facility could produce approximately $20 \mathrm{MT}$ of MOX fuel starting in 1996 and ending in 2000, at which time a large new MOX fabrication plant could be available. 
EXISTING BWRS ARE EASILY LICENSED FOR MOX

LICENSING ISSUES FOR MOX FACILITIES ARE WELL DEFINED

\section{Licensability}

The basis for the MOX fuel design was that the performance of the core would remain within the safety envelope currently approved for LEU fuel. The "island" and "full MOX" designs have been evaluated against these license requirements and were determined to comply with the necessary criteria. Certain physical and procedural changes will be required to implement the necessary security and safeguards requirements for handling MOX fuel, but these will not impact plant systems or the basis of the plart license.

The licensing plan for the MOX fuel would be to proceed under the provisions of GE's Standard Application for Reactor Fuel (GESTAR-II) Amendment 22. This provides a systematic NRC approved process that incorporates generic acceptance criteria with which GE can license new fuel designs.

Since there are no reactor system changes and the fuel has been designed within the envelope of existing criteria, there should be no schedule delays arising from licensing the existing BWRs to utilize MOX fuel.

MOX fuel fabrication facilities have been evaluated for safety and licensed in Europe. A hybrid approach with joint DOE-NRC safety reviews integrating both DOE Orders and NRC regulations could be adopted to license the MOX facility in the U.S.

\section{Schedule and Costs}

The use of existing reactors and existing MOX fabrication capability will provide the best assurance that plutonium can begin to be dispositioned at the earliest possible date (mid-1997). The schedule for the program shows that using three BWRs, and with a MOX plant available in 2001, $50 \mathrm{MT}$ can be converted to spent fuel by 2024 . The major uncertainties in this schedule are adequate political support with public acceptance and site selection.

The cost of the project was developed based on the facilities needed for MOX fabrication and use in the reference reactors. This cost estimate includes fabrication, transportation, storage, and use of the MOX fuel as well as storage of the spent fuel assemblies for 10 years at the completion of the mission. All of the aforementioned costs can be estimated reasonably well. A key cost uncertainty remains in determining what financial incentives will be required to obtain utility participation. These incentives may vary widely and can only be determined after detailed negotiations with the utilities. 
The estimated cost summary is presented in Table S-1. These estimates represent incremental costs and do not include utility incentives.

Table S-1: Cost Summary

\begin{tabular}{|c|c|c|c|}
\hline & Reference & Alternate 1 & Alternate 2 \\
\hline $\begin{array}{l}\text { Number of } \\
\text { Reactors }\end{array}$ & 3 & 6 & 3 \\
\hline $\begin{array}{l}\text { Disposition } \\
\text { Time, } \\
\text { Amount }\end{array}$ & $\begin{array}{l}23 \text { Reactor } \\
\text { Years, } \\
50 \mathrm{MT}\end{array}$ & $\begin{array}{l}24 \text { Reactor } \\
\text { Years, } \\
100 \mathrm{MT}\end{array}$ & $\begin{array}{l}46 \text { Reactor } \\
\text { Years, } \\
\text { 100MT }\end{array}$ \\
\hline $\begin{array}{l}\text { Reactor } \\
\text { Capital Cost }\end{array}$ & $\$ 167 \mathrm{M}$ & $\$ 306 \mathrm{M}$ & $\begin{array}{l}\text { Req's lic. } \\
\text { life ext. }\end{array}$ \\
\hline $\begin{array}{l}\text { MOX Plant } \\
\text { Capital Cost }\end{array}$ & $\$ 525 \mathrm{M}$ & $\$ 525 \mathrm{M}$ & $\$ 520 \mathrm{M}$ \\
\hline $\begin{array}{l}\text { Reactor } \\
\text { O\&M Cost }\end{array}$ & $\$ 6.4 \mathrm{M} / \mathrm{Yr}$ & $\$ 11.3 \mathrm{M} / \mathrm{Yr}$ & $\$ 6.4 \mathrm{M} / \mathrm{Yr}$ \\
\hline $\begin{array}{l}\text { MOX Plant } \\
\text { O\&M Cost }\end{array}$ & $\$ 52 \mathrm{M} / \mathrm{Yr}$ & $\$ 1049 \mathrm{M} / \mathrm{Yr}$ & $\$ 104 \mathrm{M} / \mathrm{Yr}$ \\
\hline
\end{tabular}

Use of existing infrastructure at DOE facilities, such as LANL, enables timely approvals for site-specific environmental impact reviews, cost savings, and schedule advantages. Other support activities are also in place at these DOE sites such as radwaste systems, safeguards, security infrastructure and site utility services. Participation agreements could also involve some form of sharing of the reactors' electric production revenues.

U.S. SAFEGUARDS REQUIREMENTS INCORPORATED INTO MOX AND REACTOR PLANT FACILITIES

\section{Safeguards}

All applicable DOE Orders and NRC Regulations for safeguarding Pu were evaluated for fuel fabrication, transportation, and in-reactor handling. The technical and administrative safeguards requirements are readily incorporated into both the MOX fuel fabrication and reactor plant facilities. Systems and practices equivalent to IAEA technical and inspection requirements for implementing safeguards - near real-time material accountability, and plutonium assay systems - are accommodated. 
FISSION OPTION WILL MAINTAIN U.S. LEADERSHIP IN SAFEGUARDS TECHNOLOGY AND NON-PROLIFERATION PRACTICES

BWR AND MOX FUEL FACILITIES PRODUCE NO UNACCEPTABLE ENVIRONMENTAL IMPACT

\section{DOE SITES}

ENVIRONMENTALLY

CHARACTERIZED, WOULD SUPPORT RAPID PERMITTING

OTHER NEEDED INFRASTRUCTURE IS READILY AVAILABLE
The BWR fission option facilitates bilateral agreements for transparency and reciprocity - exchange of observers, shared portal monitoring, access to accountability data, and offers the U.S. an opportunity to take a leadership role in instituting safeguards related technical improvements and innovations.

\section{Environment, Safety and Health}

The study assessments for waste streams, worker exposure and public exposure at site boundaries were found to meet all applicable standards and no conditions were identified that would preclude the environmental approval of the fabrication and use of MOX fuel. The evaluation encompassed transportation safety, reactor safety, and the safety of the MOX fabrication plant, as well as handling and disposition of transuranic and mixed wastes.

On-site discussions and evaluations of applicable DOE sites were conducted. All sites have been fully environmentally and geologically characterized. These DOE sites, including LANL, also have organizational, regulatory and technical infrastructures in place which are fully experienced in the preparation of NEPA, state and local documentation, and the permitting process.

\section{Other Infrastructure}

Other infrastructure needed to implement the disposition project (e.g., for conversion of metal to oxide feed material, transportation and storage) are available. The spent MOX fuel has been evaluated and was found to be acceptable in the permanent repository using the same criteria as for commercial spent fuel. Transportation systems and corresponding regulatory requirements are in place. While there are clear advantages to co-locate the MOX fabrication facility and all of the reactors used in the mission, the opportunity to do so is very limited. Two existing government owned BWR sites are adaptable for co-locating a MOX facility, the WNP-2 site located on the Hanford Reservation and TVA's Browns Ferry site.

\section{Reactor System Commercial Basis and Operational Interfaces}

This study assumes that the MOX fabrication plant will be owned by the U.S. Government and operated by private contractors. It is also assumed 
that the reactors will continue to be owned and operated by their current owners, although there are a number of possible alternative arrangements. The most likely commercial arrangement between the U.S. Government and the plant owner would involve the government providing the MOX fuel as well as other financial incentives and the coverage of certain risks. Since any three of the recent BWRs can be used for the disposition mission and several utilities have expressed interest in participating, the Government will have considerable flexibility in choosing the candidate reactors based on location and ownership.

\section{Public Acceptance}

PAOVEN TECHNOLOGY TO REMOVE THE CLEAR AND PRESENT DANGER OF WEAPONS PLUTONIUN
BWR FISSION OPTION, STRATEGICALLY AND TECHNICALLY THE MOST ATTRACTIVE
The strategic non-proliferation goals of the program should provide strong rationale and motivation for public acceptance. In addition, a dedicated MOX fuel plant and the integration of a commercial BWR for plutonium disposition would be major demonstrations of a "swords to plowshares" defense realignment program and a Government-Industry partnership.

\section{Technology Needs}

No new technology needs were identified for the reactors, MOX fabrication plants, or MOX fuel. Based on previous MOX fuel experience at Big Rock Point, Quad Cities, Garigliano, and elsewhere, as well as over 20 years experience with urania fuels which self-generate significant quantities of plutonium, GE is confident that its' fuel design methods are fully capable of supporting the full MOX fuel design.

An early start demonstration pilot program followed by the full MOX Pu Disposition program using the industrially mature, safe and reliable BWR technology will instill greater public confidence and trust as compared to lesser developed technology options. 


\section{NEDO-32361}

\subsection{Introduction}

\subsection{Technical Synopsis of Study From Introduction to Present}

\subsubsection{Core Design}

Detailed core design evaluations have shown that most existing BWRs, through appropriate core design, can utilize MOX fuel without any reactor modifications. Two core design concepts utilizing plutonium enriched uranium dioxide (referred to in this study as "mixed oxide" or "MOX") fuel were developed for the boiling water reactor (BWR) plutonium disposition mission. These core designs were developed for a 1155 MW BWR-5 reactor. This particular core is representative, although not identical, to all nuclear reactor cores designed by General Electric Company (GE). A description of the GE BWR core designs of interest for this mission is contained in Section 2. In the event that another BWR model is selected, any differences in MOX fuel design or mission capability will be minor.

The two bundle design concepts which are described in detail in Section 2 are the "island" design and "full MOX" design. This island design is very similar to MOX designs used in Big Rock Point, Quad Cities and Garigliano in the 1960's and 1970's. The island design bundle provides an opportunity to commence a pilot demonstration program in mid-1997 with existing operating BWRs . It is very conservative from a design viewpoint, which will minimize any potential licensing issues, and, since only $20 \%$ of the fuel rods contain plutonium, the mission can be supported with existing plutonium fabrication capacity such as that available at Los Alamos National Laboratory (LANL). The island design would be used to validate and gain experience with various elements of the disposition program including transportation, storage, training, reactor licensing, operations, and safeguards and also to provide an early demonstration of U.S. committment to the plutonium disposition program. The full MOX design is based on maximizing the MOX throughput for the existing BWR cores. The design utilizes plutonium in all the fuel rods in the bundle and can be implemented in the currently operating BWRs as soon as the new MOX fabrication facility can be brought on line to provide the required fuel volume.

Using these bundle designs, any three of the most recent larger GE BWRs can disposition 50 MT of plutonium within 23 years of full MOX loading, assuming $75 \%$ capacity factor operation.

\subsubsection{Reactor Plant Systems Evaluation}

The design basis for the MOX fuel was that the performance of an equilibrium core loading of that fuel would remain within the currently approved envelope for low enriched uranium (LEU) 
fuel. The resulting island and full MOX designs were therefore evaluated against the transient and accident criteria in the Safety Analysis Report for a reference plant (WNP-2) and were determined to comply with the necessary criteria. Accordingly, no system design changes will be required for the use of this fuel. Some plant procedural changes will be required, however, to implement the necessary safeguards and security requirements for handling MOX fuel. These changes, which are described in Section 2, would not be expected to impact plant systems.

\subsubsection{Reactor Plant and Fuel Licensing}

There are no licensing impacts on the design of the steam supply system of the plant; however, there will be some new issues arising from the need to handle and safeguard the fresh MOX fuel as well as to provide 10 years of onsite spent fuel storage at the end of the mission

A secured new fuel storage building is planned to accumulate and store incoming MOX fuel . This facility will avoid the need for additional Safe Secure Transports (SSTs) or long disruptions in operations around the refueling floor and the spent fuel pool. In addition, there is a DOE study requirement to store any remaining spent fuel for 10 years at the end of the mission. That spent fuel storage requirement is best met with a dry fuel storage complex outside the reactor building. The construction of the new fuel storage building and the spent fuel storage complex will require an amendment to the plant NRC license. Neither of these facilities, however, presents any technical issues which would be expected to preclude timely approval.

The licensing for the MOX fuel would proceed under the provisions of GE's existing Standard Application for Reactor Fuel (GESTAR-II) Amendment 22. GESTAR is a systematic NRC approved process that incorporates generic acceptance criteria with which GE licenses new BWR fuel designs.

While no delays to the reference schedule described in Section 6 are expected based on the technical issues involved in implementing the PDS at the operating reactor site and the MOX fabrication facility, there is always the potential for licensing delays due to the political process and the lack of public acceptance of the plutonium disposition program. The risk of any such delays will be minimized to the extent the public's concerns are satisfactorily addressed in the DOE's EIS and the Government is able to generate a broad political consensus in support of the mission. 


\subsubsection{MOX Fuel Fabrication}

In order to commence the disposition option in mid-1997, the TA-55 facility at Los Alamos National Laboratory (LANL) could begin fabrication of MOX fuel for the first one to three island reloads for each reactor. The facilities in the TA-55 area are the only ones currently existing in the United States with the capability to fabricate significant quantities of MOX fuel. This facility would produce approximately 20 MT of MOX fuel starting in 1996 and ending in early 2000 , at which time a large new MOX fabrication plant would be available. To minimize costs, some equipment used for the fabrication demonstration at LANL can be transferred and used in the new MOX facility.

After a careful evaluation of alternative processes, a powder blending process which has been successfully used both in the United States and Europe is recommended for the new MOX fabrication facility. The design basis of the facility was developed, including safety, waste minimization, ALARA, redundancy, throughput, and compatibility with processes at other commercial and DOE facilities. It was determined that the capital cost of the MOX facility is relatively insensitive to throughput in the range of 50 to $200 \mathrm{MT} / \mathrm{yr}$, due to the need to provide redundant capability as well as baseline capacity in key process steps. Plant layouts were completed based on experience with other currently operating fuel fabrication facilities. No technical, environmental or safety issues were identified which would preclude early use of the LANL facility or licensing of the new MOX fabrication plant.

\subsubsection{Safeguards}

All applicable DOE Orders and NRC Regulations for safeguarding plutonium were evaluated for fuel fabrication, transportation, and in-reactor handling. It was determined that no new technology is required to fully comply with existing safeguards procedures. Full compliance with International Atomic Energy Agency (IAEA) standards and procedures would not require new technology based on their similarity to current U.S. standards.

\subsubsection{Non-Plutonium Operations}

Any BWR selected would be capable of returning to LEU reload fuel at any time, again without plant modifications.

\subsubsection{Other Infrastructure}

Significant capability exists at DOE facilities such as Hanford, LANL, Savannah River Laboratory (SRL), Idaho National Engineering Laboratory (INEL), Oak Ridge National 
Laboratory (ORNL) which could be used to support the mission. These capabilities include process technology, buildings, equipment, safeguards and security infrastructure, waste management facilities, as well as a large and highly capable technical staff. While there are clear advantages to co-locate the MOX fabrication facility and all of the reactors used in the mission, the opportunity to do so is very limited. Two existing BWR sites are readily adaptable to a MOX fuel fabrication facility. WNP-2 is located on the Hanford Reservation, as is the FMEF facility. There may be an opportunity to utilize part of the existing SAF line and to retrofit the FMEF as a fuel fabrication facility to meet the mission objectives. The three Browns Ferry units are also on a Government site which could be suitable for a MOX facility. Both of these sites, as well as other possible DOE sites, are discussed in Sectior 10.

In order to meet current safeguards requirements, existing or additional DOE Safe Secure Transport (SST) vehicles will be used to transfer the fresh MOX fuel.

The discharged MOX fuel has been evaluated and found to comply with expected permanent repository criteria on spent fuel.

\subsubsection{Technology Needs}

No new technology needs were identified for the reactors, MOX fabrication plants, or MOX fuel. Based on previous experience at Big Rock Point, Quad Cities and Garigliano, as well as over 20 years experience with urania fuels which self-generate significant quantities of plutonium, GE's BWR design methods are capable of supporting the island or full MOX fuel design. Irradiation and analysis of MOX fuel rods and Lead Use Assemblies (LUAs) is planned to qualify the rod fabrication process and to further benchmark the nuclear design codes.

\subsubsection{Reactor Plant System Commercial Basis and Operational Interfaces}

This study assumes that the MOX fabrication plant will be owned by the U.S. Government and operated by private contractors, as is the case currently with a number of government-owned facilities. It is also assumed that the reactors will continue to be owned and operated by their current owners, although there are a number of possible alternative arrangements. The most likely commercial arrangement between the U.S. Government and the plant owner would involve the Government providing the MOX fuel as well as other financial incentives, including the coverage of certain risks. Additional discussion of the commercial issues associated with the use of existing BWRs is included in Section 4. 


\subsubsection{Safety and the Environment}

The impact of MOX fuel on environmental, health and safety factors in operating BWRs was evaluated. The evaluation encompassed transportation safety, reactor safety, and the safety of the MOX fabrication plant, as well as handling and disposition of transuranic and mixed waste. No technical issues related to worker safety, public health or the environment were identified which would impact the licensing, permitting, construction or operation of any facility needed for the project.

\subsubsection{Cost and Schedule}

The fundamental assumption in this study is that the use of existing reactors and an existing MOX fabrication facility will provide the best assurance that plutonium can begin to be dispositioned at the earliest possible date (i.e., mid-1997). The schedule for the completion of the mission shows that using three BWRs, and assuming the availability of the new MOX fabrication plant in early 2000,50 MT can be dispositioned by 2024 . The major uncertainties in this schedule are the timely issuance of the DOE Record of Decision, the availability date of the MOX plant and the actual size and capacity factors of the reactors chosen for the project.

The cost estimate for the project was developed based on the incremental facilities needed for MOX fabrication and use in the reference reactors. This cost estimate includes fabrication, transportation, storage, and use of the MOX fuel as well as storage of the spent fuel assemblies for 10 years at the completion of the mission. All of the aforementioned costs can be reasonably well estimated; however, a major uncertainty remains in determining what financial incentives will be required to obtain the utility's agreement to use their reactors. These incentives may vary widely and can only be fully determined after negotiations with the utility management.

\subsubsection{Public Acceptance}

The island design in an existing BWR provides an opportunity for a very early start to the disposition of weapons plutonium and therefore provides the U.S. with a visible demonstration of its committment to the success of the mission. In the longer term, the BWR fission option is consistent with the stated Russian position on plutonium disposition in a nuclear fuel cycle, and it positions the U.S. in a leadership role in safeguards for that technology.

These same benefits of early start, disposal effectiveness, revenue generation and safeguards leadership are valid arguments to support public acceptance in the United States. 


\subsection{Definition of Commercial Power System Basis for Plutonium Disposition Study}

The fleet of BWRs currently operating in the United States consists of 37 operating plants owned and operated by -22 utilities. The plant designs reflect the evolution of the BWR technology, with $2 / 3$ of the operating plants comprised of the later generation BWR-4,5\&6 designs. In the course of this study, GE has determined that there are various MOX fuel designs capable of operation in most of the existing reactors, each having unique benefits in speed of deployment (the island design) or plutonium metal throughput for the disposition mission (full MOX design). Thus, with most BWRs capable of contributing to the mission by utilizing a MOX fuel cycle, the selection of plants best suited for the mission will be a combination of other factors, including remaining license lifetime, plant capacity, location, and the costs and economic incentives necessary to gain utility involvement in the program.

\subsubsection{Plant Capability and Selection Criteria}

To evaluate the capability of the BWR fleet to perform the disposition mission, the total remaining plant capability for power production was estimated by multiplying the operating years of the plant, assuming first MOX fuel operation in 1998, by the annual plant capacity, assuming an average capacity factor for the mission of $75 \%$. This provided a relative plant capacity value, "Mission MW days", that was used to evaluate the individual plant ability to perform the disposition mission. These results are presented in Table 1.2-1.

This evaluation demonstrated the capacity for the plutonium disposition mission for any single unit of the later generation BWRs (the $\mathrm{C}$ core lattice designs) was almost twice that of any of the earlier plant designs. The primary contributor to this is the longer remaining lifetime of the current operating license of the later generation plants. This clear advantage in capacity, coupled with additional factors, suggested the following selection of plants/utilities to be evaluated for the mission. Each utility is listed after a description of the selection criteria it met for the $\operatorname{mission}(*)$.

NOTE $(*)$ Several utilities met more than one of the criteria. They are listed only once in the "primary" category for selection used for this study. 
Table 1.2-1 Evaluation of Existing Plant Capacity

\begin{tabular}{|c|c|c|c|c|c|c|}
\hline Utility & Plant Name & $\begin{array}{c}\text { Plant Size } \\
\text { (MWe) }\end{array}$ & Model & Lattice & $\begin{array}{c}\text { License } \\
\text { Expires } \\
\text { (yr) }\end{array}$ & $\begin{array}{l}\text { Mission } \\
\text { (MWd) }\end{array}$ \\
\hline Centerior Energy & Perry 1 & 1205 & 6 & C & 2026 & $9,236,325$ \\
\hline Entergy Operations & Grand Gulf & 1306 & 6 & C & 2023 & $8,937,938$ \\
\hline Entergy Operations & River Bend & 991 & 6 & C & 2025 & $7,324,729$ \\
\hline Illinois Power Co. & Clinton & 950 & 6 & C & 2026 & $7,281,750$ \\
\hline Commonwealth Edison & LaSalle 1 & 1144 & 5 & C & 2022 & $7,516,080$ \\
\hline Commonwealth Edison & LaSalle 2 & 1144 & 5 & C & 2023 & $7,829,250$ \\
\hline Niagara Mohawk Power Corporation & Nine Mile 2 & 1163 & 5 & C & 2026 & $8,914,395$ \\
\hline Washington Public Power Supply System & WNP-2 & 1155 & 5 & C & 2023 & $7,904,531$ \\
\hline Detroit Edison & Fermi 2 & 1154 & 4 & C & 2024 & $8,213,595$ \\
\hline PECO Energy Company & Limerick 1 & 1092 & 4 & C & 2024 & $7,772,310$ \\
\hline PECO Energy Company & Limerick 2 & 1092 & 4 & C & 2029 & $9,266,985$ \\
\hline Pennsylvania Power \& Light & Susquehanna 1 & 1085 & 4 & C & 2022 & $7,128,450$ \\
\hline Pennsylvania Power \& Light & Susquehanna 2 & 1085 & 4 & C & 2024 & $7,722,488$ \\
\hline Public Service Electric \& Gas & Hope Creek & 1118 & 4 & $\mathrm{C}$ & 2026 & $8,569,470$ \\
\hline Carolina Power \& Light & Brunswick 1 & 849 & 4 & D & 2016 & $4,183,448$ \\
\hline Carolina Power \& Light & Brunswick 2 & 849 & 4 & D & 2014 & $3,718,620$ \\
\hline Iowa Electric Light \& Power & Duane Amold & 565 & 4 & D & 2014 & $2,474,700$ \\
\hline Nebraska Public Power District & Cooper & 801 & 4 & D & 2014 & $3,508,380$ \\
\hline New York Power Authority & FitzPatrick & 850 & 4 & D & 2014 & $3,723,000$ \\
\hline PECO Energy Company & Peach Bottom 2 & 1098 & 4 & D & 2013 & $4,508,663$ \\
\hline PECO Energy Company & Peach Bottom 3 & 1098 & 4 & D & 2014 & $4,809,240$ \\
\hline Southern Nuclear Operating Company & Hatch 1 & 813 & 4 & D & 2014 & $3,560,940$ \\
\hline Southern Nuclear Operating Company & Hatch 2 & 822 & 4 & $\mathrm{D}$ & 2018 & $4,500,450$ \\
\hline Tennessee Valley Authority & Browns Ferry 1 & 1098 & 4 & D & 2013 & $4,508,663$ \\
\hline Tennessee Valley Authority & Browns Ferry 2 & 1098 & 4 & D & 2014 & $4,809,240$ \\
\hline Tennessee Valley Authority & Browns Ferry 3 & 1098 & 4 & D & 2016 & $5,410,395$ \\
\hline Vermont Yankee Nuclear Power Corp. & Vermont Yankee & 537 & 4 & D & 2013 & $3,087,079$ \\
\hline Boston Edison & Pilgrim & 678 & 3 & D & 2012 & $2,598,435$ \\
\hline Commonwealth Edison & Dresden 2 & 810 & 3 & D & 2010 & $2,660,850$ \\
\hline Commonwealth Edison & Dresden 3 & 810 & 3 & D & 2011 & $2,882,588$ \\
\hline Commonwealth Edison & Quad Cities 1 & 850 & 3 & D & 2011 & $3,024,938$ \\
\hline Commonwealth Edison & Quad Cities 2 & 850 & 3 & D & 2012 & $3,257,625$ \\
\hline Northeast Utilities & Millstone 1 & 650 & 3 & D & 2010 & $2,135,250$ \\
\hline Northern States Power & Monticello & 543 & 3 & D & 2010 & $1,783,755$ \\
\hline Niagara Mohawk Power Corp. & Nine Mile 1 & 620 & 2 & D & 2009 & $1,866,975$ \\
\hline Consumers Power & Big Rock Point & 75 & 1 & $\mathbf{K}$ & 2000 & 41,063 \\
\hline GPU Nuclear & Oyster Creek & 641 & 1 & D & 2009 & $1,930,211$ \\
\hline
\end{tabular}


- Remaining Plant "Mission MWd" for a Utility: This criterion is intended as an estimate of a utility's total potential mission capacity over the remaining plant life for the later generation plants it owns. The basis for this grouping was to identify individual utilities with the maximum capability for throughput in completing the mission:

Commonwealth Edison - LaSalle 1,2

Entergy - Grand Gulf, River Bend

PECO Energy - Limerick 1,2

Pennsylvania Power \& Light - Susquehanna 1,2

- Total BWR Capacity for a Given Utility: This criterion would maximize throughput given the commitment of a single utility to the disposition mission:

TVA - Browns Ferry 1,2 and 3 (NOTE: Table 1.2-1 assumed all plants restart before 1998). These plants employ a " $D$ " lattice core. Although a specific core design for a " $D$ " lattice core was not carried out due to the limited time for this study, the overall conclusions relative to full MOX utilization demonstrated for "C" lattice cores should apply equally well to " $D$ " lattice cores.

- Total Nuclear Capacity for a Utility that Owns a Single BWR: This criterion added those utilities with single unit, high capacity BWRs that had added capacity from PWRs and/or unfinished plants:

PSE\&G - Hope Creek \& Salem 1,2

Centerior - Perry $1 \& 2$ (unfinished plant)

Southern Nuclear - Hatch 1,2 and 4 PWRs

WPPSS - WNP-2 and WNP 1,3 (2 unfinished units)

- Demonstrated Interest in the Program with a High Capacity Plant:

WPPSS (met both criteria 3 and 4)

As the result of this selection process, the detailed technical studies to determine the plant capacity for the mission was focused on the later generation BWR-4,5\&6 plants. The resultant core design established that a combination of any three of the later generation plants could meet the mission goal to disposition $50 \mathrm{MT}$ of plutonium. It should be noted that similar core designs for the earlier plants can be generated if there are factors that make utilizing them preferable for 
NEDO-32361

the mission (e.g., use of the Browns Ferry units in conjunction with their license recovery program).

\subsubsection{Utility Interest and Commercial Involvement}

The key factors for utilization of commercial reactors to complete the disposition mission will be (1) interest in the utility sector to convert their plants to a MOX fuel cycle, and (2) the commercial incentives they will require to commit their plant(s) to the mission. Several utility contacts were made with utilities to quantify both their level of interest and the commercial incentives they might require to commit to the mission. The detailed results of these discussions are presented in Section 4.1.

As a result of these discussions, sufficient utility interest exists for the mission to be successfully completed within the remaining licensed lifetime of commercial BWRs. In general, most utilities are willing to commit to the disposition mission if the financial support from the program will significantly reduce their cost of power production from the plant. The total value of this support will be utility dependent, and will depend on several factors, including ownership structure of the plant, unamortized capital investment, costs for program implementation, operation and maintenance costs, the regulated rate structure currently in place for the facility, planned capital additions, and current competition in the local power sale market. The result of these factors will probably be a cost to the program approximately equal to the difference between the current total generation cost of the selected facility and the cost of alternate energy supplies, thus making the facility competitive in the utility integrated resource plan (IRP), as required under the Energy Policy Act of 1992. The total cost of this incentive, including fuel, could range as high as $\$ 0.06$ per kilowatt hour, depending on the plant and utility selected. It is expected that the exact value will be the result of detailed negotiations between the utility and the Government when the program goes forward.

Thus, the ability to complete the mission is not defined so much by the willingness of current plant operators to consider participation, but by the ability of the Government and the selected utilities to ultimately agree on the technical and commercial details of the program.

\subsubsection{Potential for Government Purchase of Existing Plants}

At the request of the DOE project staff, utility discussions included the subject of potential sale or transfer of units to the Government. GE's limited poll found that utilities do not have a significant interest in a sale or transfer arrangement if a commercial arrangement as outlined above can be negotiated. The primary influence is the utility interest in maintaining the plant as 
a power production asset, and meeting the existing grid demand. Transfer to the Government would necessitate contracts to repurchase the power to meet current demands, and several utilities indicated a desire to support the program while retaining the generation capacity (with improved economics). Transfer of the plant to the Government could also complicate the plant licensing for the mission, as it would require the license change to cover transfer of ownership as well as the change to a MOX fuel cycle.

Purchase of the plant capacity necessary for completion of the mission would not be an economic solution to the program. With current unamortized capital investments ranging from approximately $\$ 1$ Billion per unit to over $\$ 5.4$ Billion per unit, the cost to the Government for purchase of the capacity necessary to meet the mission would far exceed the cost of either new unit construction or the incentives and fuel costs necessary to involve commercial utilities in the program. This purchase would not include any operation costs, thus only worsening the cost benefit of the purchase option. For these reasons, with the level of utility interest demonstrated in the program, the purchase of the plants is not considered necessary for successful completion of the mission. 


\subsection{Licensing of Commercial System Basis}

The use of MOX fuel requires consideration of both the plant's and the fuel's safety and performance. The scope and timing of this project did not allow a complete safety analysis of the reference BWR-5 with both the island and the full MOX reload fuel to the level of detail that would be required for an NRC submittal. In addition, even if the analysis was complete, the NRC's decision on certain issues cannot be predetermined. Consequently, this report cannot be definitive on the course the actual licensing activity would take. Enough work was done, however, to provide reasonable assurance that there are no major obstacles to licensing both the fuel and the plant in accordance with the following considerations.

To burn MOX fuel in an existing BWR requires a complete evaluation of both the plant's licensing basis contained in the FSAR and the regulations and regulatory guidance of the NRC with respect to the impact of the new fuel on the reactor plant. If the introduction of MOX fuel results in changes to the plant's Technical Specifications or involves an unreviewed safety question, as defined in 10CFR50.59, then the plant's current operating license must be amended. The process for obtaining the amendment is contained in 10CFR50.90-50.92 and involves providing the NRC a thorough description and justification for the change. If the change does not result in a modification to the plant's Technical Specifications or involve an unreviewed safety question, the licensee simply follows the procedure in 10CFR50.59 and documents the change to the plant and the basis for determining that no unreviewed safety question is involved.

The process by which GE licenses new fuel designs with the NRC is in place and is described in Amendment 22 to the General Electric Standard Application for Reactor Fuel (GESTAR II) document. A new fuel design is considered to be licensed if all the fuel performance criteria listed in Amendment 22 are met.

The fundamental design criterion for both the island and the full MOX designs was that the fuel would perform within the envelope of licensed performance for LEU fuel. That design criterion was met for the most part, but there may be some minor performance differences relative to LEU fuel, particularly for the full MOX design, that will result in Technical Specification changes and which therefore result in plant license revisions.

The requirement to handle and store plutonium fuel will clearly result in the need for additional safeguards and security measures. In addition, the requirement to store 10 years worth of spent fuel will require the installation of additional spent fuel storage facilities. Implementing these 
NEDO-32361

new requirements will require review and approval by the NRC and a revision to the plant's license.

In summary, the mission will require amendments to the chosen plant's operating license, but the real effect of the MOX fuel and the additional safeguards and security requirements on plant operation and performance will be minor. 


\subsection{Reference Case and Alternatives}

The reference case in this study assumes the use of three 1155 MWe GE BWR-5 reactor units. These reactors are considered representative of the BWR reactor units most likely to be employed in the plutonium disposition mission. Although other candidate reactors may have slightly higher or lower ratings, or employ slightly different reactor and fuel designs, they are so similar in overall design and performance from the standpoint of the plutonium mission that they can be represented on a per megawatt basis by the reference case described. Section 10 discusses some specific deployment options for a number of actual reactor sites. The tables that follow in this section should be considered representative of most BWR-4,5\&6 plants, provided throughput adjustments are made for the actual reactor size and operating capacity factor.

The fuel cycles for these reactors employ two different MOX design concepts-the island and full MOX designs. The island design is used for the first three MOX reloads in Reactor 1 in order to achieve the earliest possible start to the mission and provide valuable lead experience in licensing, operations, and safeguards implementation. Similarly, the first two MOX reloads in Reactor 2 and the first MOX reload in Reactor 3 could be the island design. Following that, the new large MOX fabrication facility would be on-line to supply enough MOX fuel to support the full MOX fuel design in all three of the reactors. The characteristics of the island and full MOX bundle designs are shown in Table 1.4.2 and are described in detail in Section 2. Both the island and the full MOX designs have been evaluated and found to conform to the licensing basis for the reference BWR-5 reactor. While the performance of this fuel in BWR-4\&6 designs was not specifically evaluated, the performance of the reference MOX designs is expected to be fully within the licensing basis for those reactors as well, based on their similarity to the BWR-5.

Table 1.4-1 Key Reference Core Parameter Summary

\begin{tabular}{|l|c|}
\hline Reactor Type & BWR-5 \\
Reactor Power Level (MWt) & 3484 \\
Core Size (number of bundles) & 764 \\
Plant Capacity Factor (\%) & 75 \\
Reload Batch Size (bundles) & 176 \\
Bundle Parameters & \\
Bundle Type & GE9 \\
Number of Fuel Rods & 60 \\
Heavy Metal Weight (kg) & 179 \\
\hline
\end{tabular}


Table 1.4-2 Characteristics of the "Island" and "Full Core" MOX Bundle Designs

\begin{tabular}{|l|c|c|}
\hline Number of Fuel Rods & 1Gand & Full Core \\
Heavy Metal Mass (kg) & 60 & 60 \\
Number of MOX Rods & 179.0 & 179.0 \\
Average Pu Concentration (\%) & 12 & 60 \\
Plutonium Mass (kg) & 0.804 & 2.79 \\
Cycle Exposure (MWd/st) & 1.44 & 5.33 \\
Cycle Length (eff. full power days) & 8568 & 7857 \\
Ave Discharge Exposure (MWd/MT) & 371 & 340 \\
Total Assemblies per Batch & 39,205 & 37,609 \\
MOX Assemblies per Batch & 176 & 176 \\
Pu Disposition per Cycle (kg) & 58 & 176 \\
\hline
\end{tabular}

Table 1.4-3 summarizes the disposition performance and costs for the reference case and two alternates. The reference case assumes the use of three reactors to disposition $50 \mathrm{MT}$ of plutonium. Alternate 1 assumes the use of six reactors to disposition $100 \mathrm{MT}$ of plutonium. Alternate 2 assumes the use of the original three reactors for a total of 46 years to disposition 100 MT of plutonium. This option would require 20 years of plant life extension plus a few additional years for any plants selected.

The cost of the necessary incentives for utility participation cannot be quantified at the present time and is consequently not shown in Table 1.4-3; however, they are discussed further in Section 6. They are listed here as a line item primarily to identify them as a potential major cost element and to indicate that they are not included elsewhere in the table.

In summary, it is concluded that any three of the most recent GE domestic BWRs have the capacity to disposition $50 \mathrm{MT}$ of weapons plutonium within approximately 23 years after the availability of the first full MOX fuel. This time period is subject to minor adjustment upwards or downwards for reactor size and capacity factor. Similarly, six reactors could disposition 100 MT of plutonium in approximately the same time interval, while three reactors could disposition the $100 \mathrm{MT}$ in about 46 years. In this latter case, significant extension to any of the plant's current operating licenses would be required. 
NEDO-32361

Table 1.4-3 Disposition Performance Summary

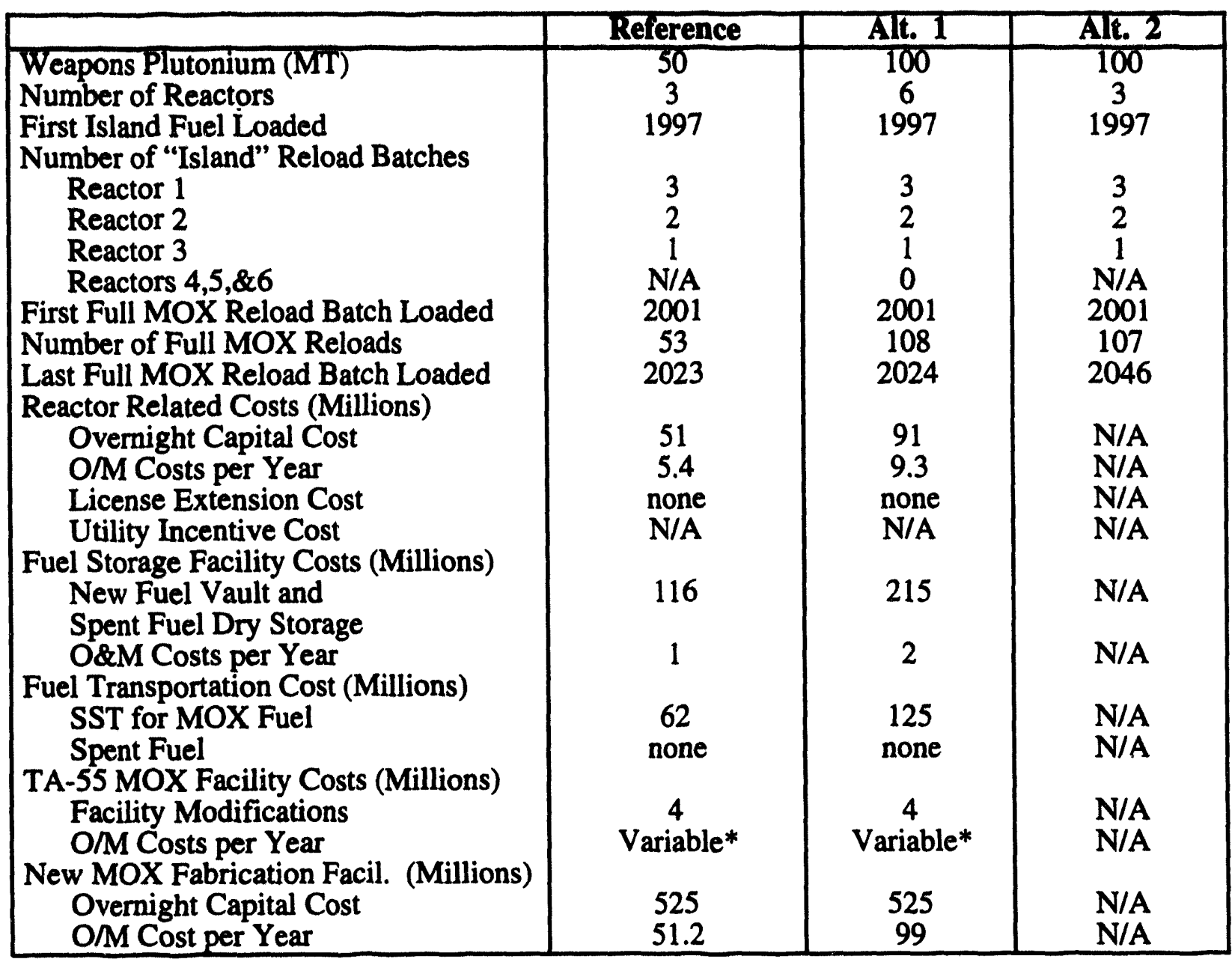

* See Reference and Alternate 1 cash flows: Table 6.4-3 and Table 10.3-2, respectively 


\subsection{Technical Descriptions}

BWRs have evolved over more than three decades of commercial operation to meet customer needs and regulatory requirements. They vary in a number of system respects; for example, the number of loops associated with the recirculation system, in the configuration of the containment system or in the core lattice design. These differences are described in more detail later in this section. The inherent flexibility of the BWR core enables full MOX core operation in most reactors by way of an appropriate currently licensed fuel design and does not require any modifications at the system level for the existing reactors discussed in this report.

This ability to accommodate a full MOX loading while fully meeting all safety requirements is discussed in this section. While the BWR-5 is used as a basis for this study, the conclusions are applicable to other BWR product lines. Plant-specific analyses will be initiated once specific plants are identified as candidates for disposition. Qualitative discussions are provided in this section where appropriate. As discussed in Section 1, two assembly designs have been developed for this study. The full MOX design is representative of a BWR core operating for the full plutonium disposition mission. The island design is a minor variation on existing $\mathrm{UO}_{2}$ bundle designs which contains limited plutonium. This design was developed to demonstrate the fission option at an early date and to utilize the early fabrication capability available at Los Alamos National Laboratory (LANL) for the production of MOX fuel rods. There would be minimal licensing impact for this design. Consequently, the island design could be implemented in BWRs as early as 1997.

After one or more island reloads, full MOX assemblies would be introduced. Although it is possible to discharge all the urania fuel from an existing core and start with a full MOX core, the preferable approach for MOX utilization is that MOX assemblies would be introduced into the core in normal reload batches. Use of the island design with a transition to full MOX eases the licensing, safeguards, and fuel storage issues, and provides ample testing opportunities both for plant operations and fuel design.

\subsection{Reactor and Reactor Cooling Systems}

Section 2.1.1 provides an assessment of a typical BWR reactor core and its performance. Section 2.1.2 describes the fluid systems which provide core cooling under normal and accident conditions. 
Previous studies of MOX utilization in BWRs have focused on one-third core loadings of MOX. This resulted in the paradigm that only one-third of a core may utilize MOX fuel. This basis has been re-evaluated as a result of the current project. Many changes in assembly designs have occurred since the early MOX calculations. In comparison with previous BWR MOX fuel designs, the lattice and control assembly designs for the latest BWRs discussed in this section have made it possible to achieve the near direct substitution of plutonium for U-235.

In the designs discussed in this section, the mechanical design assures that sufficient reactivity margin can be obtained in the hot, voided condition while still maintaining ample shutdown margin. A greater water gap between the assemblies is obtained by utilizing different assembly mechanical designs. The water gaps between assemblies also help to decouple the thermal neutron spectrum and minimize spectral interactions between fuel assemblies with large differences in fissile content and neutron absorption. High worth control blades also may be utilized if more control is necessary. The increased bypass gap width improves the void reactivity coefficient, which, in turn, results in acceptable pressurization transient and stability analysis.

Core characteristics have also evolved. Standard BWR uranium cores contain a certain amount of plutonium as a result of the neutron capture of fertile material. GE core management schemes have been developed to utilize this plutonium to improve fuel cycle efficiency. The principal difference between the mixed oxide (MOX) core described in this section and current uranium cores is the degree to which the composition of the fuel contains various plutonium isotopes.

A urania assembly contains about one percent plutonium at end of life, which is approximately the same value as a MOX assembly at end of life. In a standard urania core, about $40 \%$ of the power is produced by plutonium. These cores have more plutonium than earlier uranium cores, due to the higher fuel burnups in the more recent BWRs. Thus, more plutonium is created in recent cores and more power is produced by that plutonium. Current uranium cores have been moved closer to MOX cores, and plant systems analyses have improved in keeping with these changes.

In summary, full MOX cores have been evaluated and found to meet all current criteria. 


\subsubsection{Mechanical and Neutronic Design and Performance}

\subsubsection{Design of BWR Core and Fuel for Plutonium Utilization}

The core and fuel design of a generic BWR-5 utilizing plutonium fuel is not significantly different from that of a standard uranium fueled core. The generic BWR-5 discussed in this study can handle a full MOX core with some assembly mechanical changes and no system changes.

Standard BWR uranium cores, with the irradiated fuel, contain a certain amount of plutonium as a result of the neutron capture of fertile material. Core management schemes have been developed to utilize this plutonium to improve fuel cycle efficiency. The principal difference between the MOX core described in this section and current uranium cores is the degree to which the composition of the fuel contains various plutonium isotopes. A urania assembly contains about one percent plutonium at end of life, which is approximately the same value as a MOX assembly. In a standard urania core, about $40 \%$ of the power is produced by plutonium.

There are two parts to the design of a BWR for plutonium utilization: (1) lattice design, and (2) core configuration and management. Lattice design impacts spectrum changes, density changes, the resultant gadolinium loading and burnout rate of the fissile isotope, as well as the burn-in rate from fertile material.

The core configuration of the generic BWR-5 with plutonium MOX fuel is designed to be as interchangeable as possible with the standard uranium fuel it replaces. The key criteria in designing for optimal plutonium interchangeability with the standard designs are to:

- Minimize differences in reactivity versus burnup characteristics

- Maintain reasonable peak-to-average power factors

- Minimize effects on control blade reactivity worth

Experimental results from operating power reactors and test facilities, and detailed evaluations with design models that have been compared with experiments have verified that the above objectives are achievable. The MOX fuel for plutonium disposition is designed to the same thermal, mechanical and hydraulic conditions and limits as the uranium fuel. The nuclear 
characteristics are, however, affected by the plutonium. The MOX fuel designs have been developed with the following nuclear characteristics relative to uranium fuel:

- Improved local and global power distributions

- Adequate control rod worth and cold shutdown margin

- Increased cold-to-hot operating reactivity swing

- Acceptable dynamic and safety characteristics

Interchangeability and high plutonium loading has been achieved in previous BWR designs (see Section 5.3.2.1 for additional information) by use of an MOX fuel island design. In these designs, the plutonium-bearing rods were located in central regions of the lattice away from the control blades and the water gaps between assemblies. Location of MOX fuel in these lattice regions replaced the highest enrichment fuel rods in the BWR assembly, thereby maximizing uranium ore and separative work savings. The low uranium enrichment rods remained in the highest neutron importance regions at the edge of the assembly and effectively "shadow" the higher Pu-enriched MOX fuel rods with respect to neighboring assemblies.

This design also maximizes the plutonium concentration in each fuel rod to minimize the number of plutonium fuel rods and hence fabrication costs. Interchangeability was also considered for an all MOX fuel loading with a widespread use of gadolinium throughout the interior regions of the lattice. This allowed the burn rate of the interior plutonium bearing rods to be slowed so that the burnout rate of the fissile isotopes is more nearly that of the standard uranium fuel.

In comparison with previous BWR MOX fuel designs, the mechanical design of the commercial GE9 lattice and control assembly is one of the key improvements which makes it possible to achieve the near direct substitution of plutonium for U-235. One of the most important considerations in utilizing any appreciable amount of plutonium in the generic BWR core is maintenance of adequate control rod worth as plutonium is inserted into the lattice. The plutonium isotopes have an absorption cross section approximately three times greater than $\mathrm{U}-235$, and thus plutonium, when placed adjacent to a control blade, will compete with the control blade and reduce control rod effectiveness. Because of the mechanical design, sufficient reactivity margin can be obtained in the hot, voided condition while still maintaining ample shutdown margin. The water gaps between assemblies also help to decouple the thermal neutron spectrum and minimize spectral interactions between fuel assemblies with large differences in fissile content and neutron absorption. This is especially important to meet high exposure goals 
with MOX fuel. High worth control blades are standard commercial options and may also be utilized.

The interchangeability which has been designed into the lattices utilizing plutonium results in the ability to use core management techniques which have come into practice over years of operational experience. Fuel reliability and overall reactor safety response is equivalent to similar $\mathrm{UO}_{2}$ loaded BWR cores.

\subsubsection{Bundle Designs}

The nuclear fuel bundle contains fissionable material which produces thermal power while maintaining structural integrity. The basic configuration of the fuel design chosen for this application (Figure 2.1-1) consists of 60 fuel rods and one water rod in an 8x8 square array, spacers, water rods, upper and lower tie plates, channel and channel fastener, all fabricated into a transportable, interchangeable assembly. The outer envelope of the fuel assembly is square with distinguishing features which provide support, identification, orientation and handling capabilities. The rods, spacers, and tie rods constitute the fuel bundle. The fuel channel encloses the fuel bundle and provides: (1) a barrier between two parallel coolant flow paths, one for flow inside the bundle and the other for flow in the bypass region between channels; (2) a bearing surface for the control rod and, (3) rigidity for the fuel bundle. The channel fastener attaches the channel to the fuel bundle and, along with the channel spacer buttons, provides channel-tochannel spacing with resilient engagement. The bundle design codes are discussed in Appendix B.

\subsection{Bundle Design Limits and Considerations}

The generic BWR-5 reactor core analyzed for this report will accommodate any BWR fuel design, including a reload of full MOX assemblies. This ability derives from the advanced fuel technology that is constantly evolving for BWRs. In order to design a full MOX fuel assembly for a generic BWR, the limitations on full core MOX were studied. In general, necessary requirements were a large hot-to-cold swing and maximum bypass flow area. Increased hot-tocold swing eliminates any impact on the thermal margin or energy performance of the core design from cold shutdown margin considerations. An increased bypass flow area also reduces the magnitude of the void reactivity coefficient, which enhances the stability performance and reduces the impact of pressurization events.

Detailed BWR core designs for plutonium disposition have been developed utilizing GE's most advanced $8 \times 8$ fuel bundle, designated as GE9. The GE9 designs were chosen to fully meet the 
operating margin requirements typical of currently operating plants. GE9 has a large central water rod and improved hot-to-cold swing. The full MOX assembly design also utilizes a combination of licensed fuel mechanical components to maximize bypass flow area.

GE9 was developed primarily as a high exposure and high energy bundle design that is capable of batch discharge exposures up to $42,000 \mathrm{MWd} / \mathrm{st}$. The standard GE9 design has a peak pellet exposure limit of $60 \mathrm{GWd} / \mathrm{st}$. In order to take advantage of the high exposure mechanical design, GE9 has improved linear heat generation rate and reactivity margins (cold shutdown margin).

The bundle design integrates the lattice designs with the core loading design to permit all the design limits to be met in an optimized manner. The cycle energy and reactivity margin requirements determine the lattice enrichments and gadolinium loadings. These parameters control the hot and cold $k$-infinity characteristics and are selected such that cycle energy, hot excess reactivity and cold shutdown margins are met.

The thermal margin requirements are used to define the allowable local peaking and R-factor variations with exposure. The local peaking factor impacts the Linear Heat Generation Rate (LHGR), while the R-factor (a GE designation for the subchannel factor) has a direct influence on the Minimum Critical Power Ratio (MCPR) performance of the bundle. Key thermal limits used with BWRs are defined in Appendix C.

Axial enrichment zoning is typically used in BWRs, even if only to provide natural uranium sections at the top and bottom axial nodes. These short sections of natural uranium are generally provided to help reduce fluence on the core plates, as well as to provide some end-of-cycle power suppression in the upper end of the assembly. To improve plutonium disposition, however, these ends are not utilized. This results in slightly lower thermal margins at end-ofcycle, but since such margins are sizable in all these designs, the small decrease in the thermal margin is not considered to be of consequence. If it is necessary to reduce fluence above the core, these natural uranium end zones can be replaced.

The thermal-mechanical limits on peak pellet exposure and maximum LHGR versus exposure are considered during the lattice design process. These limits are utilized to derive the lattice dependent limits on the Maximum Average Planar Linear Heat Generation Rate (MAPLHGR). The resultant MAPLHGR limit provides confidence that neither the $\mathrm{UO}_{2}, \mathrm{MOX}$, or the $\mathrm{MOX} / \mathrm{Gd}_{2} \mathrm{O}_{3}$ rods will exceed their respective LHGR versus exposure limits. Thermalmechanical limits for the MOX fuel are assumed to be the same as for $\mathrm{UO}_{2}$ fuel. 
Although designs for a BWR are discussed in this section, this information generally applies to most plants. All BWR-5 plants, and many BWR-4 plants could directly use the same assemblies discussed here. BWR-6 plants use the same enrichment layout, but utilize a slightly different thermal-mechanical design. Some BWR-4 utilize an offset lattice design (or " $D$ " lattice). A new enrichment layout would be necessary for these plants, but a full MOX design would be possible.

A description of the physical configuration of the fuel lattice and the control blade configuration is given in Figure 2.1-1. The isotopic concentrations utilized in these designs are summarized in Table 2.1-1.

\subsection{Full MOX Bundle Design}

As shown in Figure 2.1-2, the full MOX bundle design for plutonium disposition contains a mixture of plutonium and uranium in all 60 power producing rods. This design also has a relatively large number of gadolinium-bearing burnable poison rods. This bundle design closely resembles the reactivity characteristics normally associated with enriched uranium fuel. The full MOX assembly design also utilizes a different combination of licensed fuel mechanical components to maximize bypass flow area. A smaller fuel assembly is combined with a $100 \mathrm{ml}$ thick channel (typical for BWR-5 assemblies) to increase bypass flow area. The increased bypass gap width reduces the void reactivity coefficient, which, in turn, reduces any impact from pressurization transient events. This combination does not introduce any new issues into the licensing or design effort.

The bundle axial and radial enrichment distribution is given in Figure 2.1-2 along with the gadolinium and plutonium concentration distribution. Values of enrichment for uranium are read in hundredths of a percent of total heavy metal. For instance, the "071" refers to $0.71 \% \mathrm{U}-235$ by weight, which is the fissile content of natural uranium. Natural uranium is used in the full MOX case since a slight contribution from the U-235 aids the reactivity coefficients. The island design uses depleted uranium to maximize the plutonium in the MOX rods. The plutonium concentrations are similarly given in hundredths of weight percent. The minimum plutonium concentration is $1.00 \mathrm{wt} \%$ and the maximum is $4.20 \mathrm{wt} \% \mathrm{PuO}_{2}$. Use is made of only one gadolinium concentration ( $1 \mathrm{wt} \%$ ). There are a total of seven pellet types required for this design, three of which are gadolinium rods. 
NEDO-32361

Table 2.1-1 Isotopic Concentrations of Actinides in Fresh Fuel

\begin{tabular}{|l|c|}
\hline Isotope & Weight Percent (\%) \\
\hline Pu-239 & 93.7 \\
\hline Pu-240 & 5.7 \\
\hline Pu-241 & 0.3 \\
\hline Am-241 & 0.3 \\
\hline Total & 100.0 \\
\hline & \\
\hline U-235 & 0.71 \\
\hline U-238 & 99.29 \\
\hline Total & 100.0 \\
\hline
\end{tabular}

The infinite lattice radial power peaking determines the peak power producing rod. The distribution of relative power peaking (normalized to unity across the lattice) is shown at beginning of life for the $40 \%$ void case in Figure 2.1-3. The exposure-dependent $\mathrm{k}_{\infty}$ are given in Figure 2.1-4 for the uncontrolled lattice for three void histories. This figure shows that this full MOX bundle design closely emulates a $\mathrm{UO}_{2}$ bundle. This is accomplished by means of the widely dispersed gadolinia in the interior rods.

\subsection{Island Bundle Design}

The island bundle design has 12 central MOX rods. These are clustered around the central water rod, thus the island appellation. These rods are located away from the outer water gaps so there is minimal effect on control rods. Also, these MOX rods have no gadolinia and are separated from the uranium-gadolinia rods, and so have a smaller effect on the gadolinia. This bundle design resembles LTA designs irradiated in various BWRs during concerted early MOX irradiation programs.

The island bundle design is a standard GE9, $8 \times 8$ bundle with a single lattice type. There are 12 rods containing $4 \%$ plutonium surrounding the central water rod. Figure 2.1-5 shows the rod enrichments and locations in the island design. Enrichments are given in hundredths of weight percent. The plutonium isotopic composition is the same as described before. The total bundle $\mathrm{Pu}$ enrichment is $0.804 \%$. This amounts to a total Pu weight per bundle of $1.439 \mathrm{~kg}$, of which $1.353 \mathrm{~kg}$ is fissile $\mathrm{Pu}$. The total uranium enrichment is $2.68 \%$, leading to a total heavy element weight for the bundle of $178.973 \mathrm{~kg}$. 


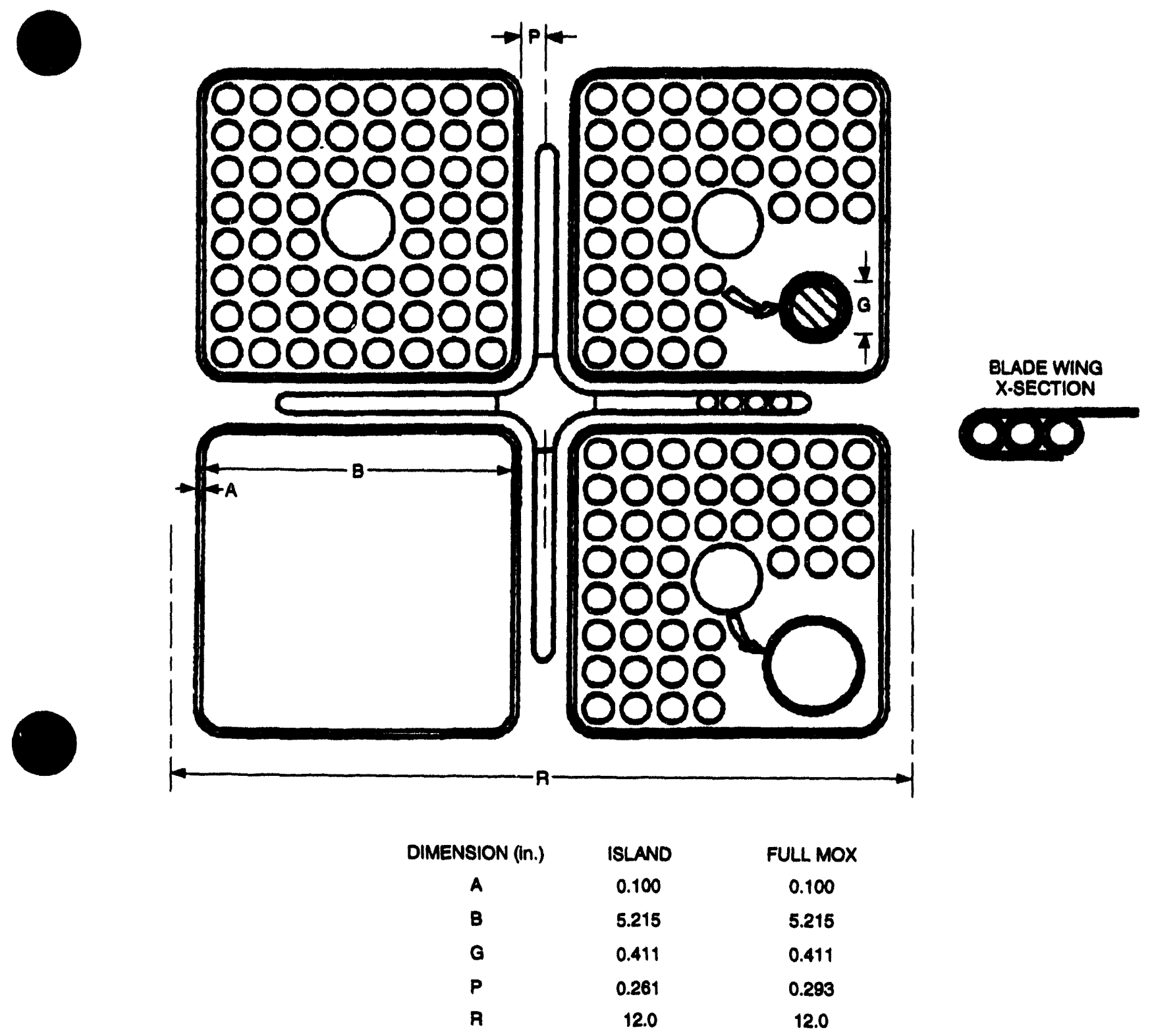

Figure 2.1-1 Generic BWR Fuel Bundle and Control Blade Configuration 

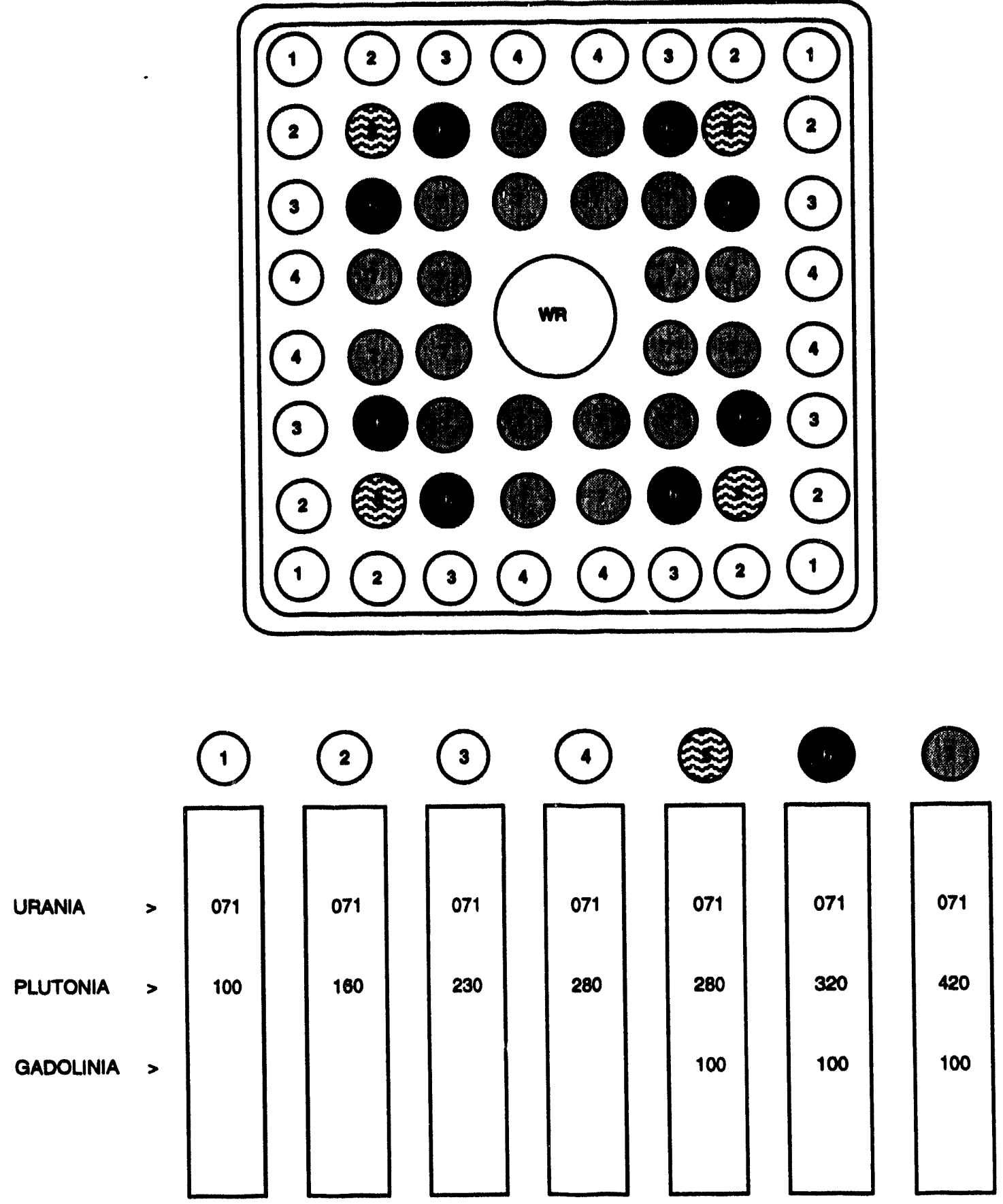

Figure 2.1-2 Full MOX Bundle Design for Generic BWR Core 
NEDO-32361

\begin{tabular}{|l|l|l|l|l|l|l|l|}
\hline 1.276 & 1.284 & 1.272 & 1.298 & 1.298 & 1.273 & 1.286 & 1.279 \\
\hline 1.284 & 0.797 & 0.717 & 0.776 & 0.776 & 0.718 & 0.788 & 1.257 \\
\hline 1.272 & 0.717 & 0.714 & 0.785 & 0.786 & 0.714 & 0.718 & 1.276 \\
\hline 1.296 & 0.776 & 0.785 & & & 0.785 & 0.776 & 1.298 \\
\hline 1.298 & 0.776 & 0.785 & & & 0.785 & 0.776 & 1.298 \\
\hline 1.273 & 0.718 & 0.714 & 0.785 & 0.785 & 0.714 & 0.719 & 1.276 \\
\hline 1.279 & 1.257 & 1.276 & 1.298 & 1.298 & 1.276 & 1.258 & 1.281 \\
\hline
\end{tabular}

Figure 2.1-3 Power Distribution for Full MOX Assembly (Beginning-of-Life Assembly)

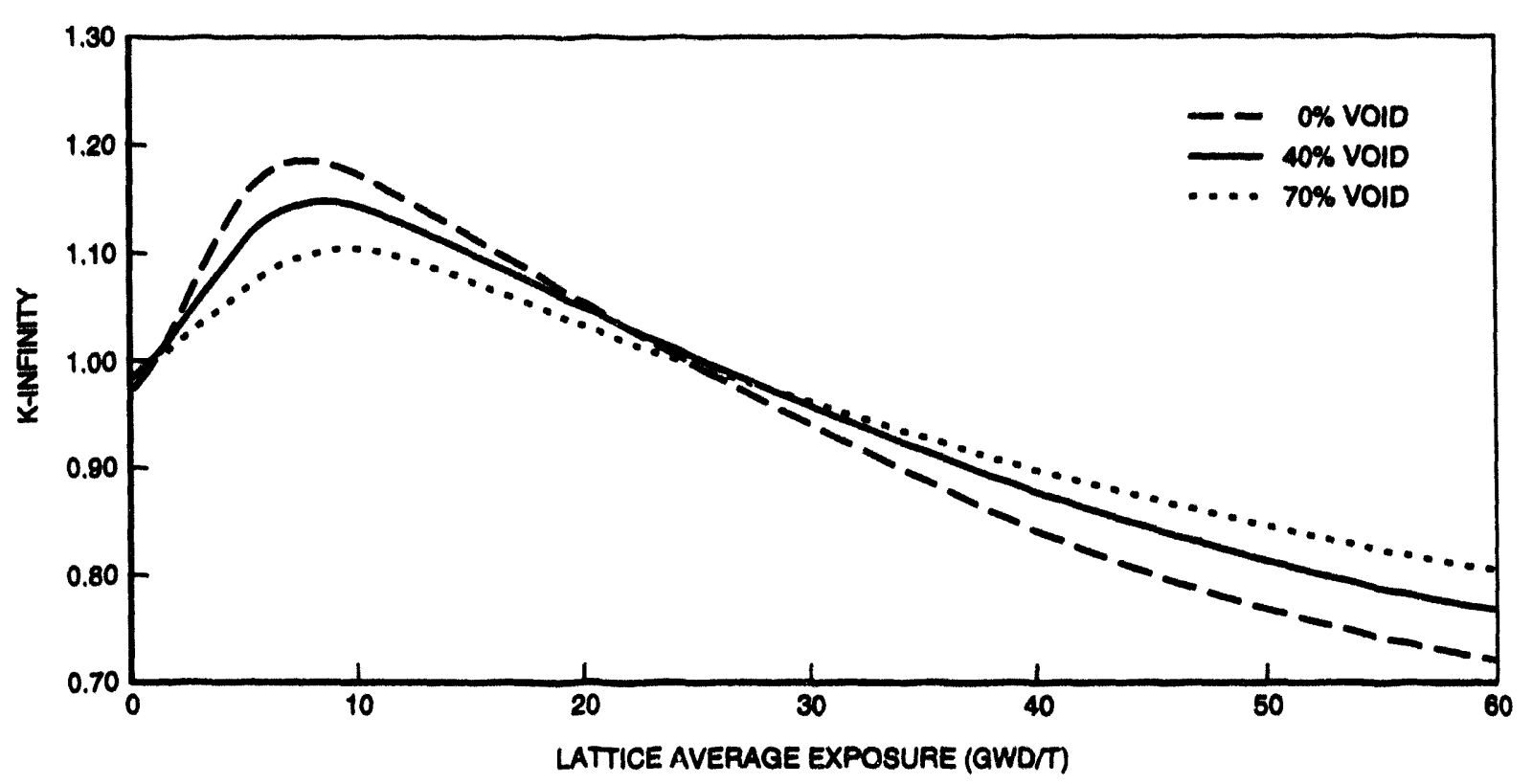

Figure 2.1-4 Hot Uncontrolled K-Infinity for Full MOX Assembly 
As can be seen in Figure 2.1-5, 10 rods contain 3\% gadolinia and there is no gadolinia in the rods which contain plutonium. Figure 2.1-6 shows the relative power peaking, normalized to unity, at the beginning of life for the $40 \%$ void case. The exposure-dependent $k_{\infty}$ for the uncontrolled lattice are shown in Figure 2.1-7 for 0,40 , and $70 \%$ voids.

\subsubsection{Equilibrium Core Dedgns}

The core designs utilized in this study were evaluated on the basis of equilibrium conditions. This is achieved by first developing a bundle load/shuffle and control rod pattern operational plan. This operational plan was then run in a multi-cycle analysis with the 3D simulator to achieve equilibrium cycle performance results. The target operational plan parameters are discussed in following sections. The loading was analyzed as a mirror and octant symmetric, control cell core.

\subsection{Loading Pattern Design}

The configuration of the generic BWR-5 core used in this study is presented in Figure 2.1-8. This core consists of 764 bundles operating at an average thermal power of $4.56 \mathrm{MW}$ for a total core output of $3484 \mathrm{MWth}$. The peripheral fuel locations are designed to preferentially force flow through the high power interior region. The specific design requirements are summarized in the following tables. Codes used in the core design are discussed in Appendix B.

The GE design of a reference loading pattern complies with several design criteria to demonstrate: (1) the Technical Specification license limits are met throughout the cycle; (2) full power operability; (3) operational flexibility; and (4) fuel bundle and channel reliability. The standard design criteria used by GE to establish a design reference loading pattern, and their bases, are described in Tables 2.1-2 and 2.1-3. The specific values used for these criteria are summarized in Table 2.1-2 through Table 2.1-4.

\subsection{Maximum Fuel Duty Versus Burnup}

A limit on fuel rod power as a function of fuel exposure is determined for each fuel design. This limit is specified in the Maximum Average Planar Linear Heat Generation Rate (MAPLHGR) limits for each fuel type. This provides confidence that fuel rod thermal mechanical design and licensing limits are not exceeded during normal operation, as well as in the event of an Anticipated Operational Occurrence (AOO). In the design of the reference loading pattern, margins to this limit are included to account for the known prediction uncertainties which are similar to the consideration for the LHGR. 
NEDO-32361

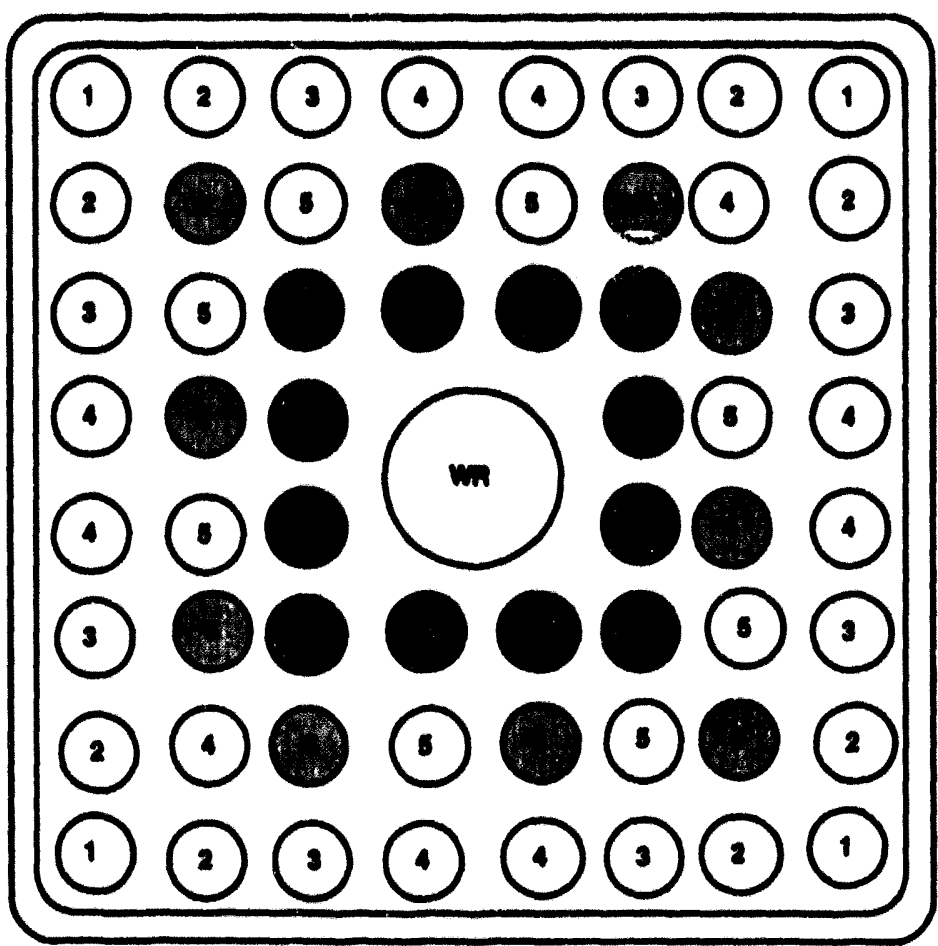

0

(1)
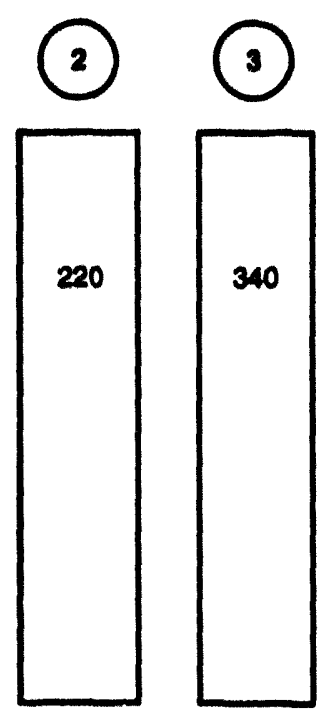

(4)
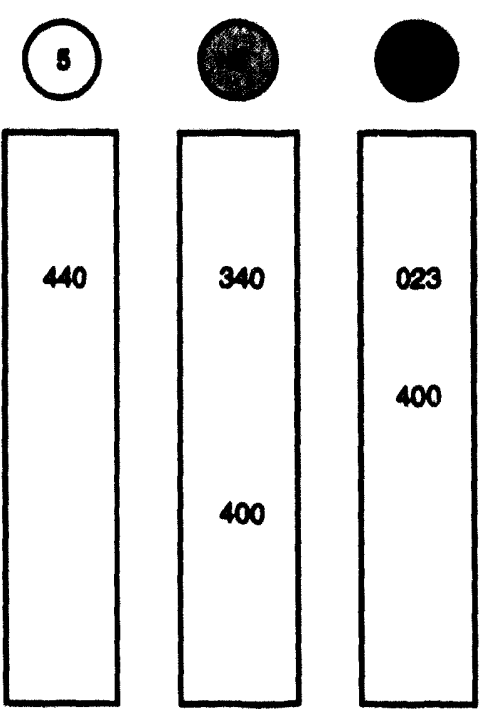

Figure 2.1-5 Island Bundle Design for Generic BWR Core 
NEDO-32361

\begin{tabular}{|l|l|l|l|l|l|l|l|}
\hline 0.861 & 0.977 & 1.187 & 1.210 & 1.236 & 1.196 & 1.031 & 0.893 \\
\hline 0.977 & 0.475 & 0.973 & 0.439 & 0.033 & 0.444 & 1.180 & 1.031 \\
\hline 1.187 & 0.973 & 1.208 & 1.248 & 1.279 & 1.122 & 0.444 & 1.195 \\
\hline 1.210 & 0.433 & 1.248 & & & 1.279 & 0.933 & 1.234 \\
\hline 1.236 & 0.939 & 1.279 & & & 1.248 & 0.433 & 1.209 \\
\hline 1.196 & 0.444 & 1.122 & 1.279 & 1.248 & 1.208 & 0.973 & 1.187 \\
\hline 1.031 & 1.150 & 0.444 & 0.933 & 0.433 & 0.973 & 0.476 & 0.977 \\
\hline 0.893 & 1.031 & 1.196 & 1.234 & 1.209 & 1.187 & 0.977 & 0.862 \\
\hline
\end{tabular}

Figure 2.1-6 Power Distribution for Island Design Assembly (Beginning-of-Life)

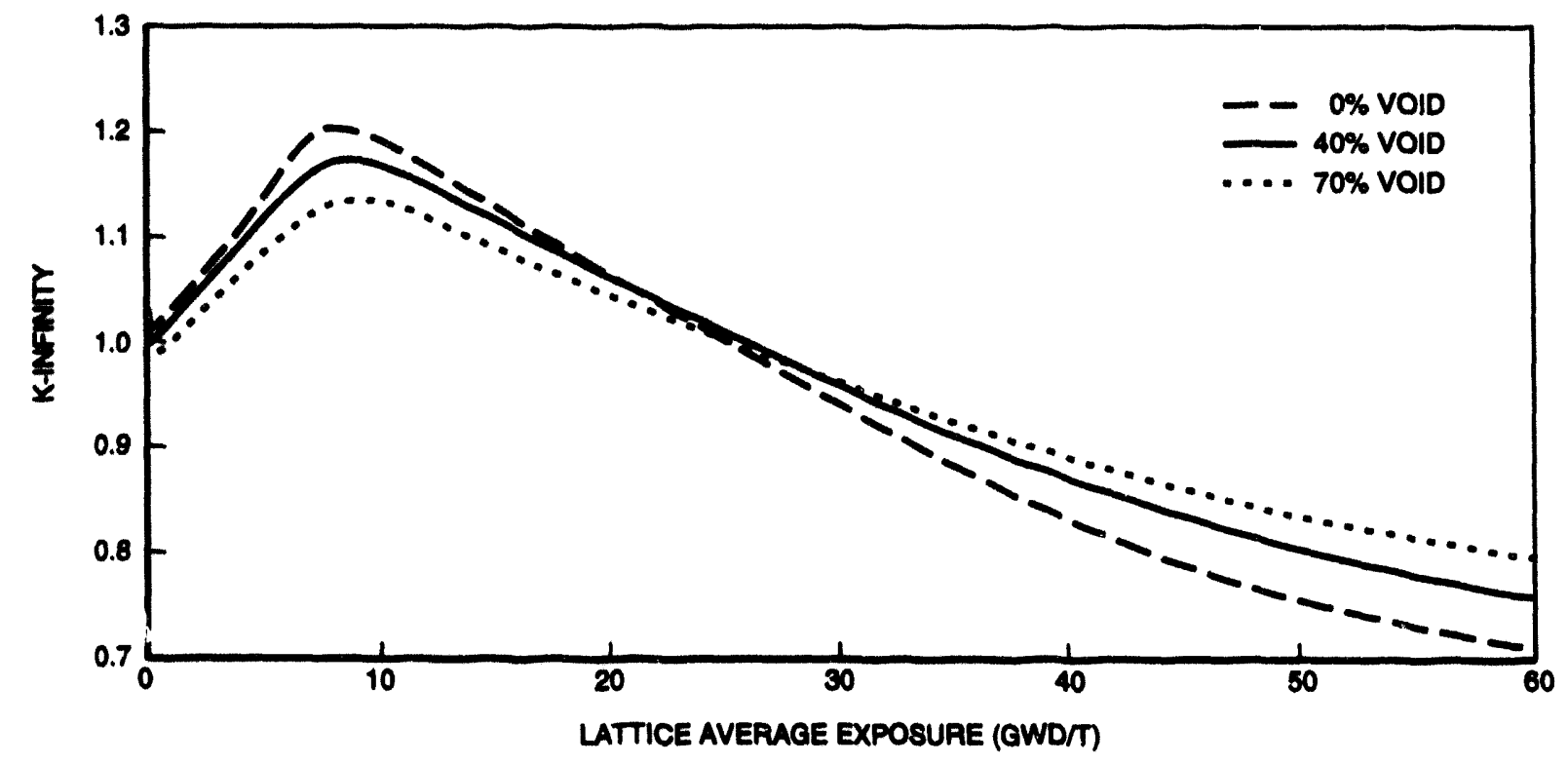

Figure 2.1-7 Hot Uncontrolled K-Infinity for Island Design Assembly 


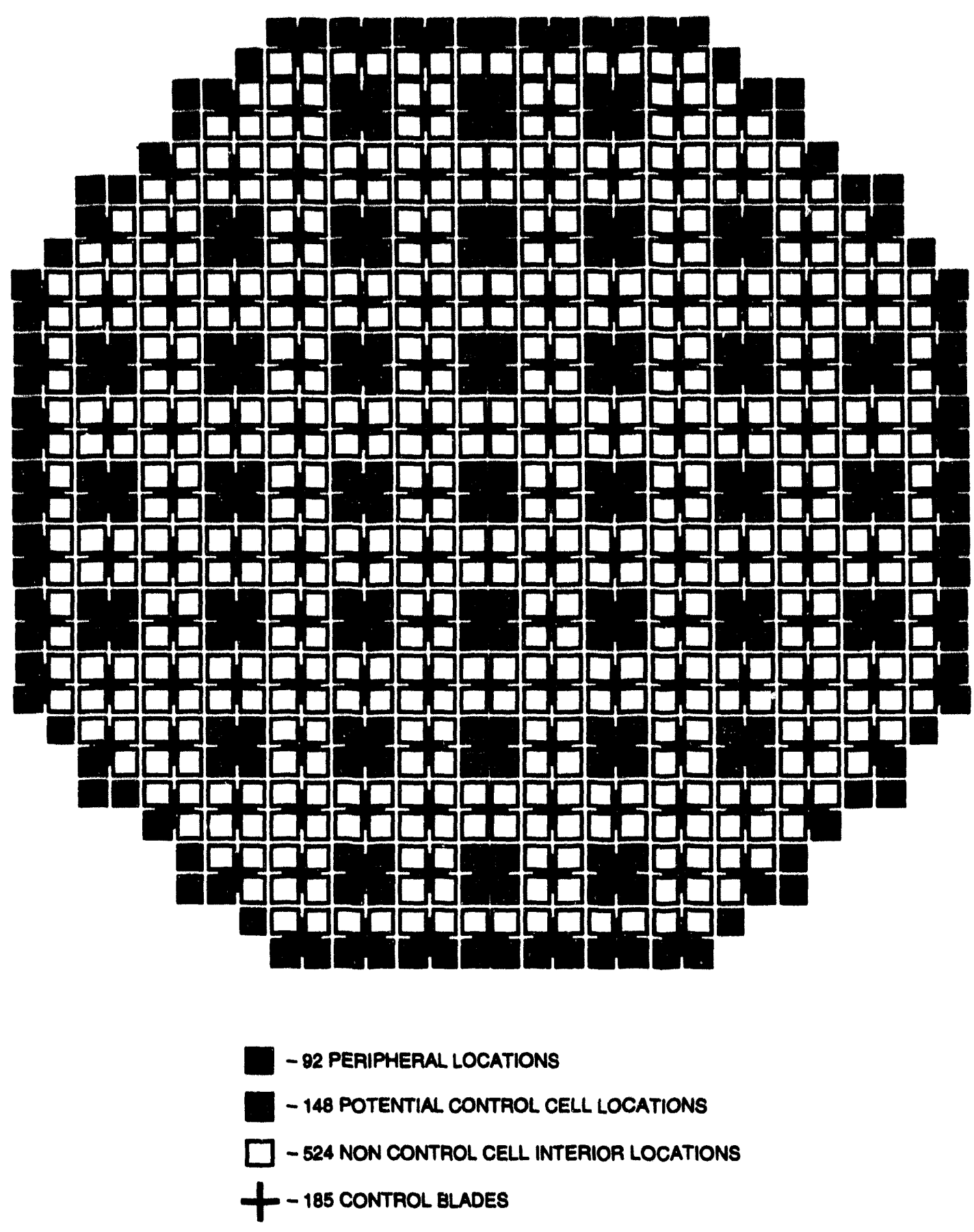

Figure 2.1-8 Generic BWR-5 Core Configuration 
Table 2.1-2 Performance Requirements

\begin{tabular}{|l|c|}
\hline Thermal Power Rating, MWth & 3484 \\
\hline Overall Capacity Factor, $\%$ & 75 \\
\hline
\end{tabular}

Table 2.1-3 Interface Requirements

\begin{tabular}{|l|c|}
\hline Number of Bundles & 764 \\
\hline Number of Control Blades & 185 \\
\hline Control Blade Pitch, in. & 12.0 \\
\hline Assembly Pitch, in. & 6.0 \\
\hline Power Density, kWh & 50 \\
\hline Rated Core Flow $(100 \%), \mathrm{Mlb} / \mathrm{hr}$ & 108.5 \\
\hline
\end{tabular}

Table 2.14 Fundamental Fuel Design Requirements

\begin{tabular}{|l|c|}
\hline Maximum Linear Heat Generation Rate, $\mathrm{kW} / \mathrm{ft}$ & 14.4 \\
\hline MCPR Operating Limit & 1.27 \\
\hline Minimum Cold Shutdown Margin, \%K & 1.0 \\
\hline Maximum Pellet Exposure, GWd/st & 60.0 \\
\hline Maximum Bundle Residence Time, years & 7.0 \\
\hline MLHGR Design Margin, $\%$ & 10 \\
\hline MCPR Design Margin, $\%$ & 15 \\
\hline Dynamic Void Coofficient, $\% / \%$ & 13 \\
\hline Doppler Coefficient $\% / \cdot \mathrm{K}$ & 0.5 \\
\hline
\end{tabular}

\subsection{Maximum Core Residence Time}

A maximum value for the fuel bundle core residence time is used as an integral part of the fuel bundle thermal-mechanical design basis. This maximum residence length is applied primarily in consideration of corrosion and hydriding of the fuel assembly component materials. Excessive corrosion and hydrogen embrittlement in the fuel assembly materials can lead to fuel assembly component failure. The projected cycle operating time is used with the bundle cumulative operating time in the process of selecting exposed bundles for use in the reference core loading.

\subsection{Peak Fuel Pellet Exposure Limit}

A maximum value for the individual fuel bundle peak pellet exposure is used as an integral part of the fuel rod thermal-mechanical design basis. This limit is selected to be compatible with the planned operating power history of that fuel design and will allow individual batches of reload fuel to achieve their design basis batch average exposure. 


\subsection{Special Bundle Shuffle Recommendations}

Longitudinal channel bowing results when channels are irradiated in a region of the core having a large gradient in fast neutron flux. This condition primarily exists in the core periphery, or in the nonperipheral control cells. The channel bowing, if large enough, may impact the control rod movement and reduce the expected channel lifetime.

To minimize the potential impact of channel bowing, the following bundle loading and shuffling recommendations are used in the reference core loading design for control cell cores:

- Those bundles which resided in the nonperipheral control cell locations during the previous cycle should not again be used in a nonperipheral control cell location.

- A fuel bundle should not be shuffled from a peripheral core location to a central core location after one or more cycles on the periphery.

- Channels that reside in the periphery for more than one cycle should be located so that the channel sides adjoining water will be different from the previous cycle (when possible, channels should reside on the periphery for only one cycle).

It should be noted that the above requirements usually will not have significant impact on core thermal performance or cycle energy. Should these recommendations become a significant impact on the core design, these restrictions can usually be relaxed to accommodate the core loading based on the balance of all design considerations or a different core loading can be developed.

\subsection{Core Design}

The general approach utilized in the core and fuel nuclear design is to establish the bundle average enrichment to enable the fuel to attain the required cycle length and capacity factor. The batch size utilized in this design is 176 bundles in equilibrium (a batch fraction of 23\%) which results in a batch average discharge exposure of approximately 38-40 GWd/MT. The resulting maximum residence time for these bundles is five years. This discharge exposure is consistent with the range normally associated with urania spent BWR fuel.

A summary of the important fuel cycle parameters and plutonium consumption rates is shown in Table 2.1-5. The island design analysis defined a core with all reload assemblies as island MOX assemblies. This case would be conservative. Any core with fewer island assemblies would have performance closer to a standard urania core. 
NEDO-32361

Table 2.1-5 Key Core Parameter Summary

\begin{tabular}{|l|c|c|}
\hline & ISland & FulIMOX \\
\hline Cycle Operating Period Length, EFPD & 370 & 340 \\
\hline Total Cycle Length, days & 493 & 453 \\
\hline Discharge Exposure, MWd/MT & 39,205 & 37,609 \\
\hline Reload Batch Size, bundles & 176 & 176 \\
\hline Plant capacity factor & $75 \%$ & $75 \%$ \\
\hline Pu Loading, kg/bundle & 1.44 & 5.33 \\
\hline Pu Usage, MT/yr & 0.19 & 0.75 \\
\hline MOX fuel rod usage, rods/yr & 1564 & 8509 \\
\hline
\end{tabular}

The equilibrium core design philosophy was to simulate the reactivity distribution of an equilibrium $\mathrm{UO}_{2}$ core in order provide simple operation. Also, the fuel fissile inventory was matched to the residence time of each batch in order to minimize any discharged plutonium inventory. A single fuel nuclear design was utilized in an equilibrium batch of 176 bundles.

The detailed core design layout for both cases is presented in Figure 2.1-9. The numbers shown in the beginning-of-equilibrium-cycle core map represent the relative number of cycles the fuel has been exposed. For instance, the number "1" refers to once burnt fuel (loaded this cycle) and the number " 4 " refers to bundles which have completed their fourth cycle.

For an equilibrium cycle, all fuel bundles loaded are of a common design. The important fuel bundle parameters were summarized previously. A control cell core loading strategy that contains 30 control cells was utilized. Due to the improved hot-to-cold reactivity swing characteristics of the GE9 bundle design, it was possible to design the fuel with a large cold shutdown margin and still maintain sufficient hot excess reactivity. The hot excess reactivity dictated the use of 30 control cells.

The important parameters of the equilibrium cycle design are summarized in Table 2.1-6. Examination of the results reveals that all thermal, reactivity and energy requirements are satisfied. The differences in thermal margins between the cases are due mainly to lack of optimization, rather than any inherent differences between the fuels. 


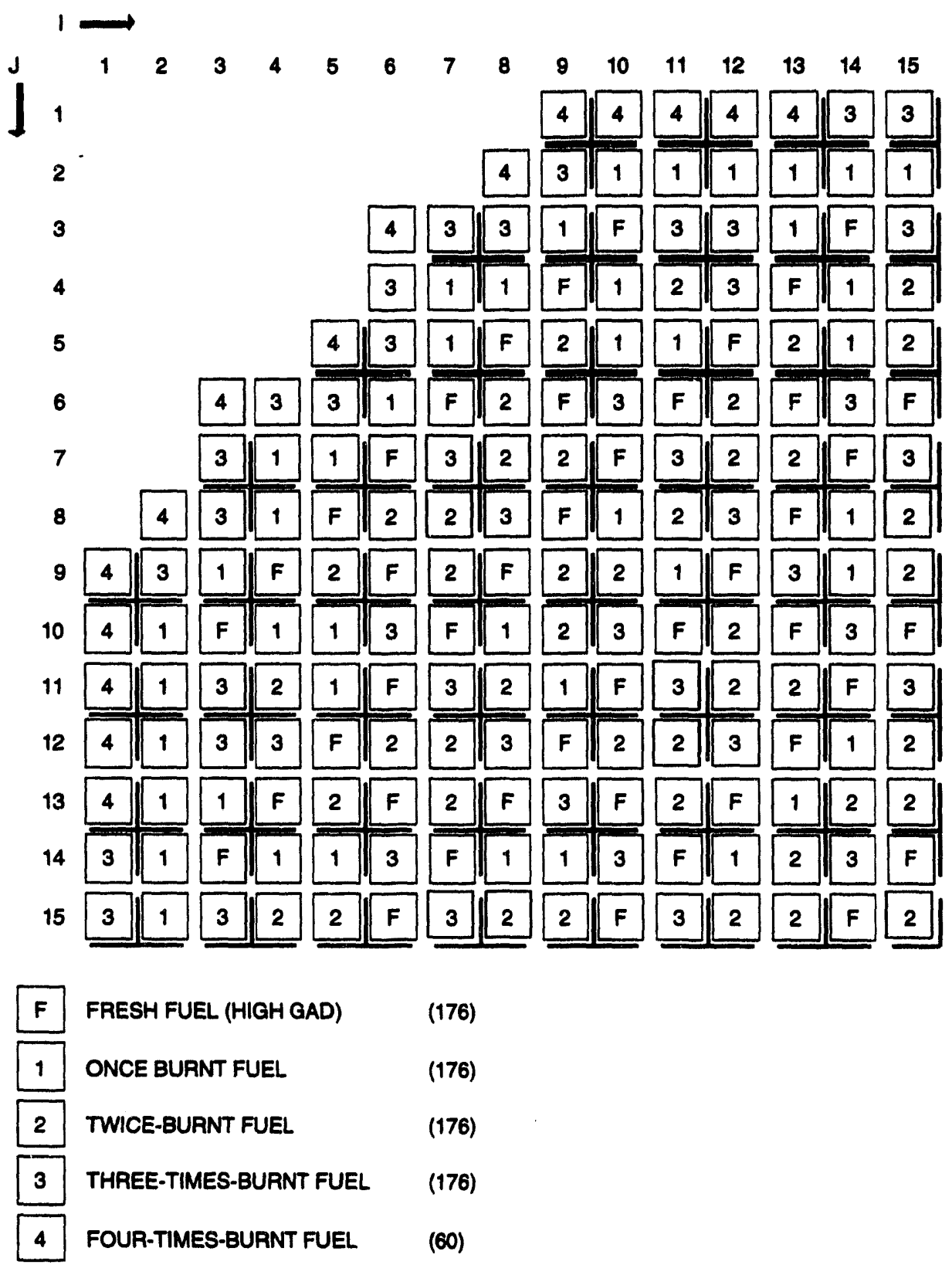

Figure 2.1-9 Equilibrium Cycle Loading Pattern 
Table 2.1-6 Equillbrium Cycle Key Parameter Summary

\begin{tabular}{|l|l|l|}
\hline & Island & Full MOX \\
\hline Cycle Length, EFPD & 370 & 340 \\
\hline Cycle Energy, GWd & 1229 & 1184 \\
\hline Cycle Exposure, MWdMT & 8992 & 8661 \\
\hline Core Mass, MT & 150.7 & 150.7 \\
\hline Reload Enrichment, w/o Pu & 0.79 & 2.79 \\
\hline Reload Batch Size, bundles & 176 & 176 \\
\hline Maximum MAPRAT & 0.88 & 0.98 \\
\hline Maximum CPRRAT & 0.83 & 0.79 \\
\hline Maximum LHGR, kW/ft (LHGR limit =14.4) & 12.7 & 12.8 \\
\hline MCPR (OLMCPR =1.25) & 1.51 & 1.58 \\
\hline Minimum Cold Shutdown Margin & 1.3 & 3.1 \\
\hline Hot Excess Reactivity at BOC & 1.4 & 1.2 \\
\hline
\end{tabular}

\subsubsection{Core Thermal Margins}

The reference loading pattern is designed such that the core performance maintains the MCPR equal to or above the Operating Limit MCPR throughout the cycle, without derating the thermal power. Additional margin is also included to account for the prediction uncertainty of the analytical calculation and possible differences between calculations and actual core operation. Transient analyses are discussed in Section 2.3.2.

The MCPR operating limit (1.25) shown on Table 2.1-6 was generated by performing detailed transient analyses for a range of core dynamic response factors. The operating limit is defined by the Load Rejection Without Bypass event and includes the effects of the turbine bypass capacity and the associated scram signals. As for a typical BWR with a uranium core, a $15 \%$ margin requirement was applied to MCPR.

The basis of linear heat generation rate (LHGR) margin is similar to the MCPR. The reference loading pattern design also provides confidence the LHGR margin limit is met with adequate margin to account for the prediction uncertainties. As for a typical BWR with a uranium core, a $10 \%$ margin requirement was applied to linear heat generation rate.

The critical power ratio and MAPLHGR thermal margin performance are plotted as a function of cycle exposure and ratios to limits (MAPRAT and CPRRAT). Figures 2.1-10 and 2.1-11 show limits for the full MOX case and Figures 2.1-12 and 2.1-13 show the island design. 
NEDO-32361

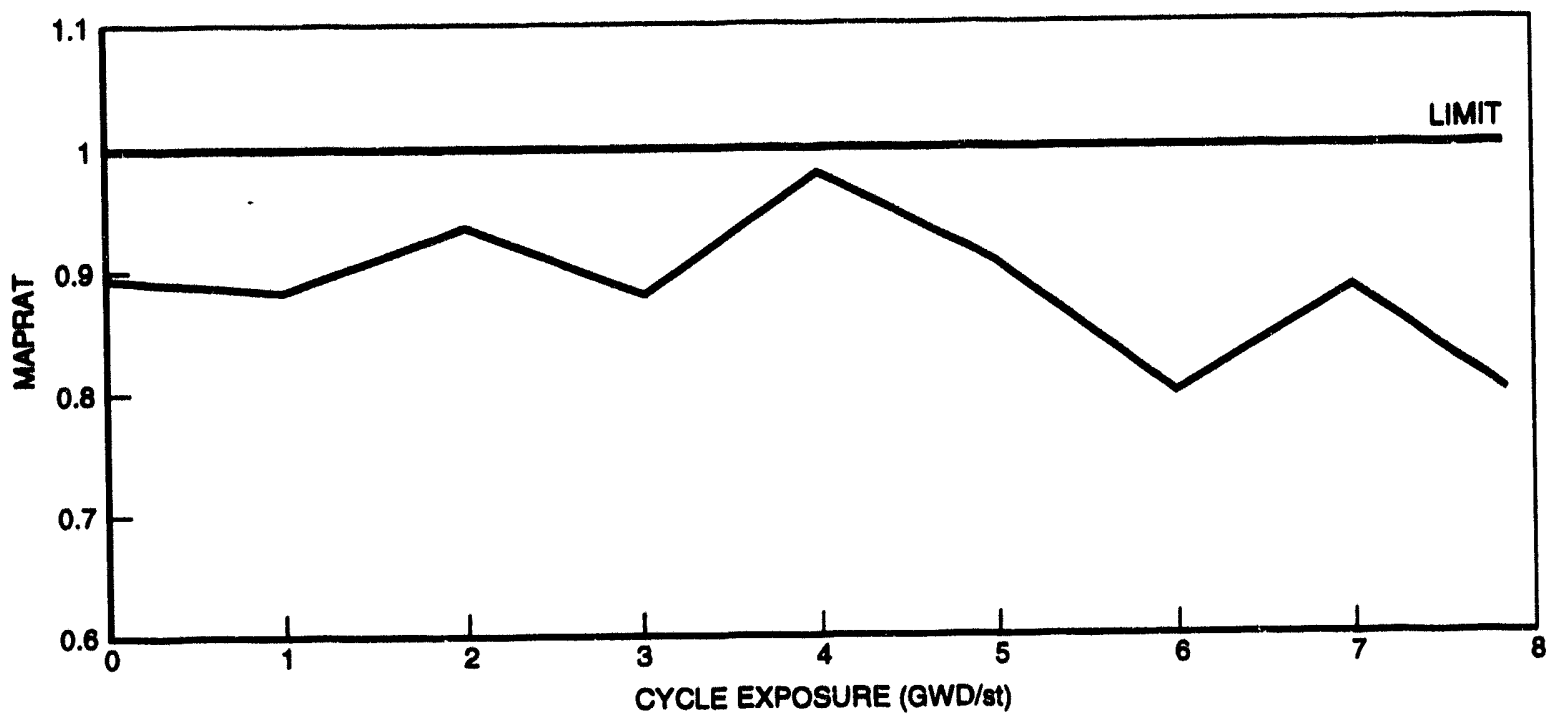

Figure 2.1-10 Equilibrium Cycle Maximum MAPRAT vs Exposure - Full MOX

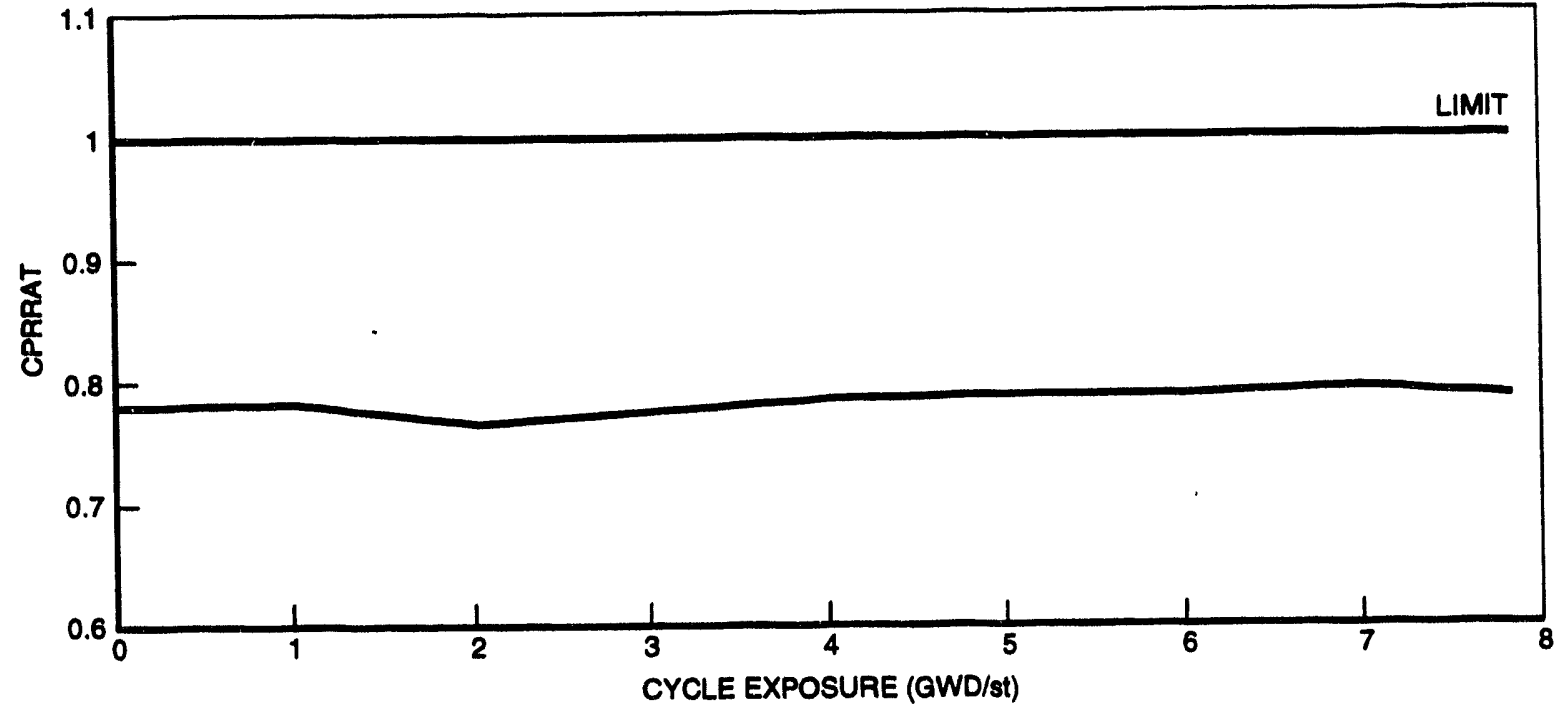

Figure 2.1-11 Equilibrium Cycle Maximum CPRRAT vs Exposure - Full MOX 
NEDO-32361

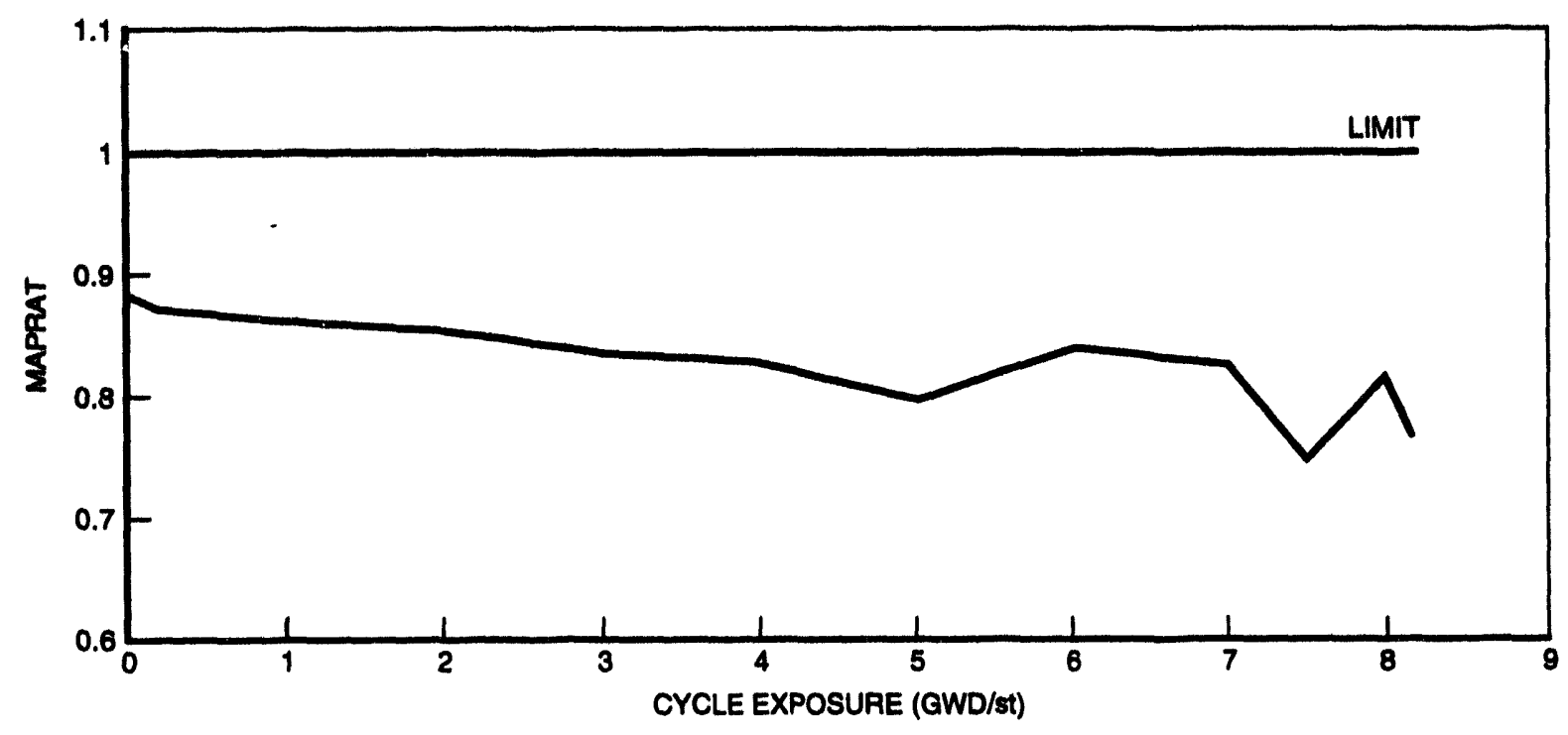

Figure 2.1-12 Equilibrium Cycle Maximum MAPRAT vs Exposure - Island Design

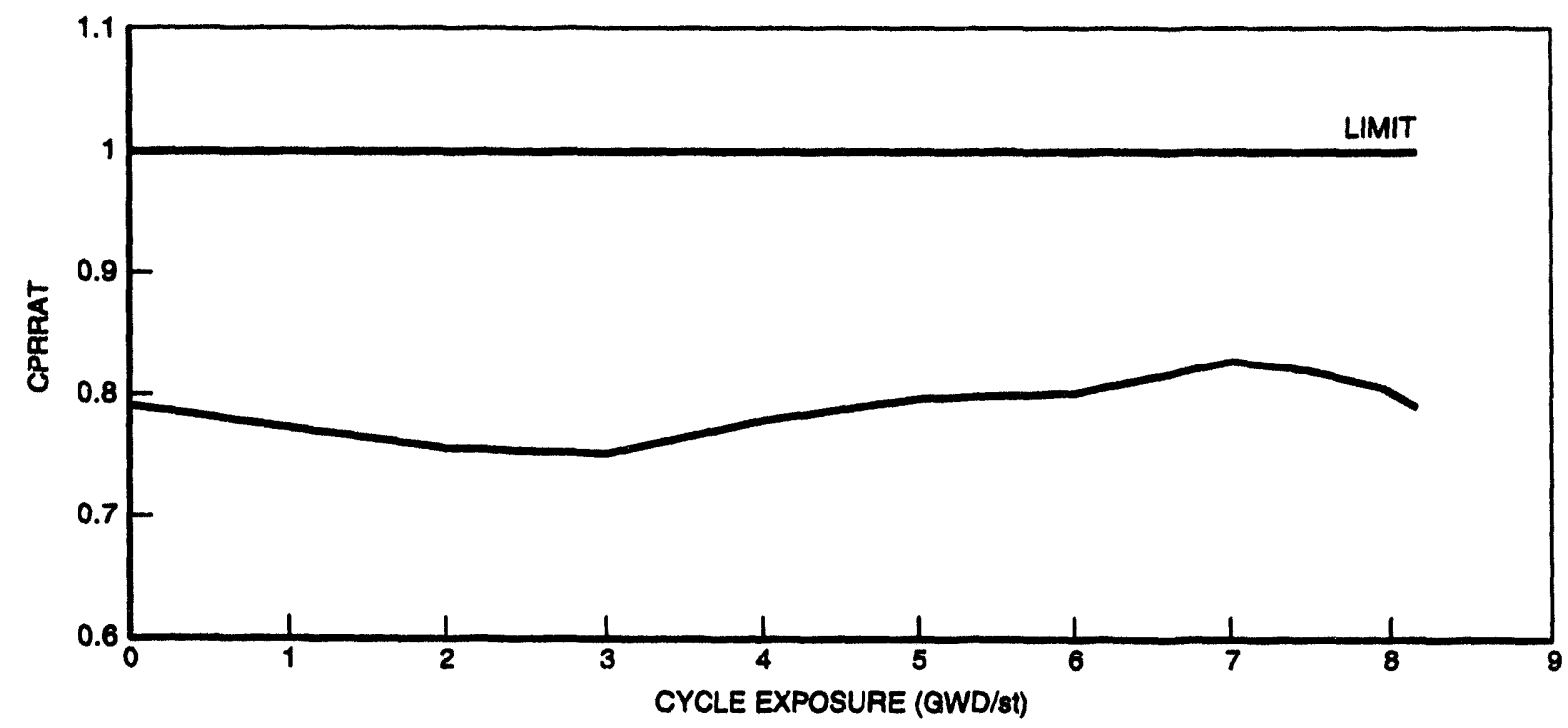

Figure 2.1-13 Equilibrium Cycle Maximum CPRRAT vs Exposure - Island Design 
Operation within MAPLHGR limit assures that the mechanical integrity of the fuel rods is maintained by limiting their power output in an appropriate manner throughout their lifetime. The MAPLHGR limits imposed on this cycle with a very low discharge exposure are the same as the fuel licensed for up to $42 \mathrm{GWd}$ /ton. These results demonstrate ample margin to core thermal limits.

\subsubsection{Reactivity Limit Summary}

A BWR core is designed such that it can be brought to cold shutdown condition at any point of the cycle, even with the strongest control rod withdrawn. In addition to meeting this Technical Specification requirement, the GE reference loading pattern design provides extra margin to cover the known prediction uncertainties, such as the projected critical eigenvalues for an equilibrium cycle and the projected end-of-cycle exposure and its distribution in the previous cycle (for reloads).

Hot excess reactivity is the margin to criticality for startup of the core design. Minimum hot excess reactivity margins are considered in the reference loading pattern design to demonstrate the full power capability.

The reactivity performance of the full MOX core design is summarized in Figure 2.1-14 and Figure 2.1-15. Figures 2.1-16 and 2.1-17 provide similar information for the island design. Due to the improved hot-to-cold reactivity swing of the GE9 bundle design, there is abundant cold shutdown margin; therefore, there is little or no impact of the MOX fuel utilization on core design from cold shutdown margin considerations. The gadolinium reduces peak hot excess and, in addition, maintains cold shutdown margins.

The minimum shutdown margin with the island design through the cycle is $1.266 \%$ at 8157 $\mathrm{MWd} / \mathrm{ST}$, which meets the design limit minimum of $1 \%$ with extra margin to cover prediction uncertainties such as the projected critical eigenvalues. The full MOX design has a minimum shutdown margin of $3.14 \%$. The hot excess reactivity at BOC for the island design is $1.4 \%$ and for the full MOX is $1.2 \%$. These are sufficient for full power capability while still allowing control of the core power shape with rod patterns. 
NEDO-32361

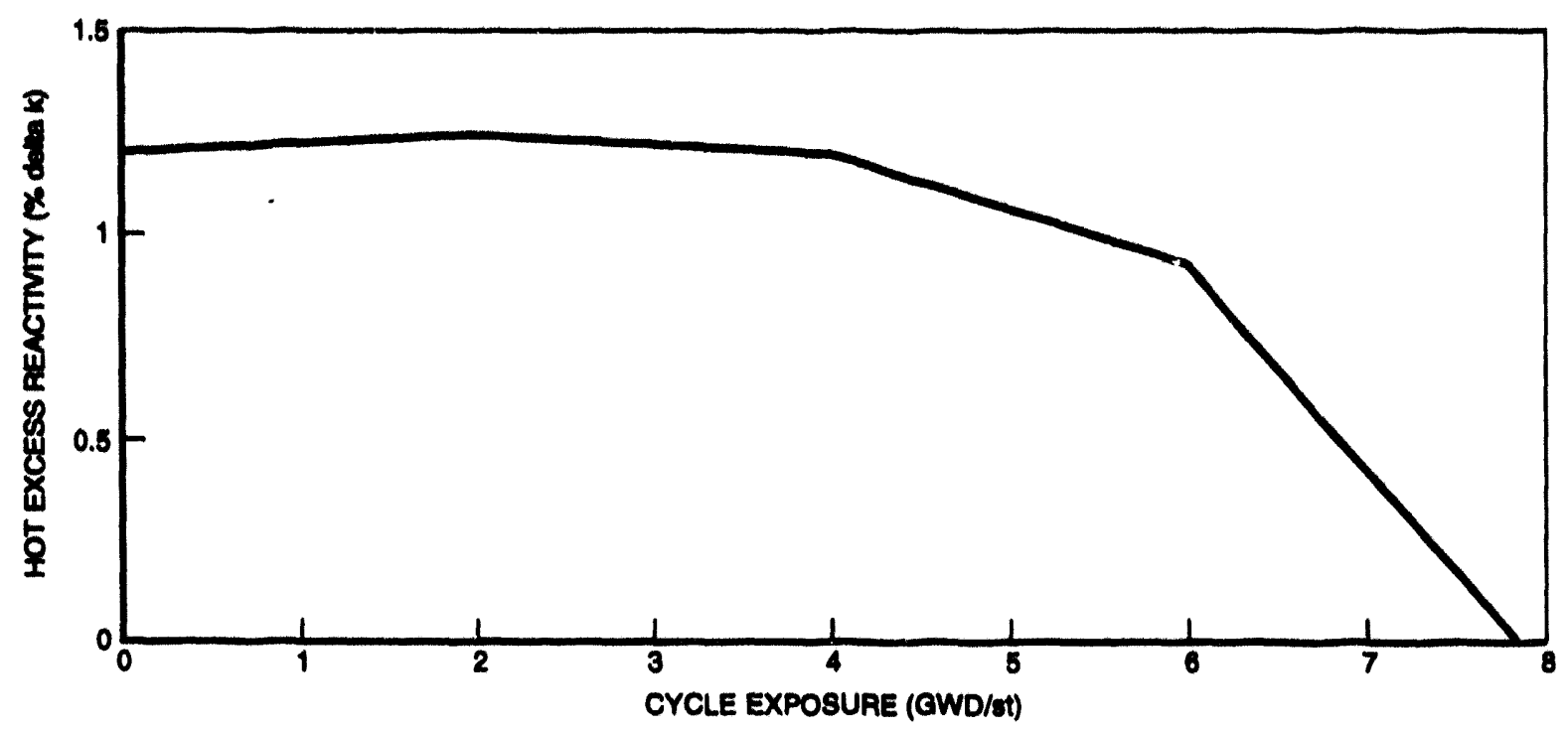

Figure 2.1-14 Equilibrium Cycle Hot Excess Reactivity - Full MOX

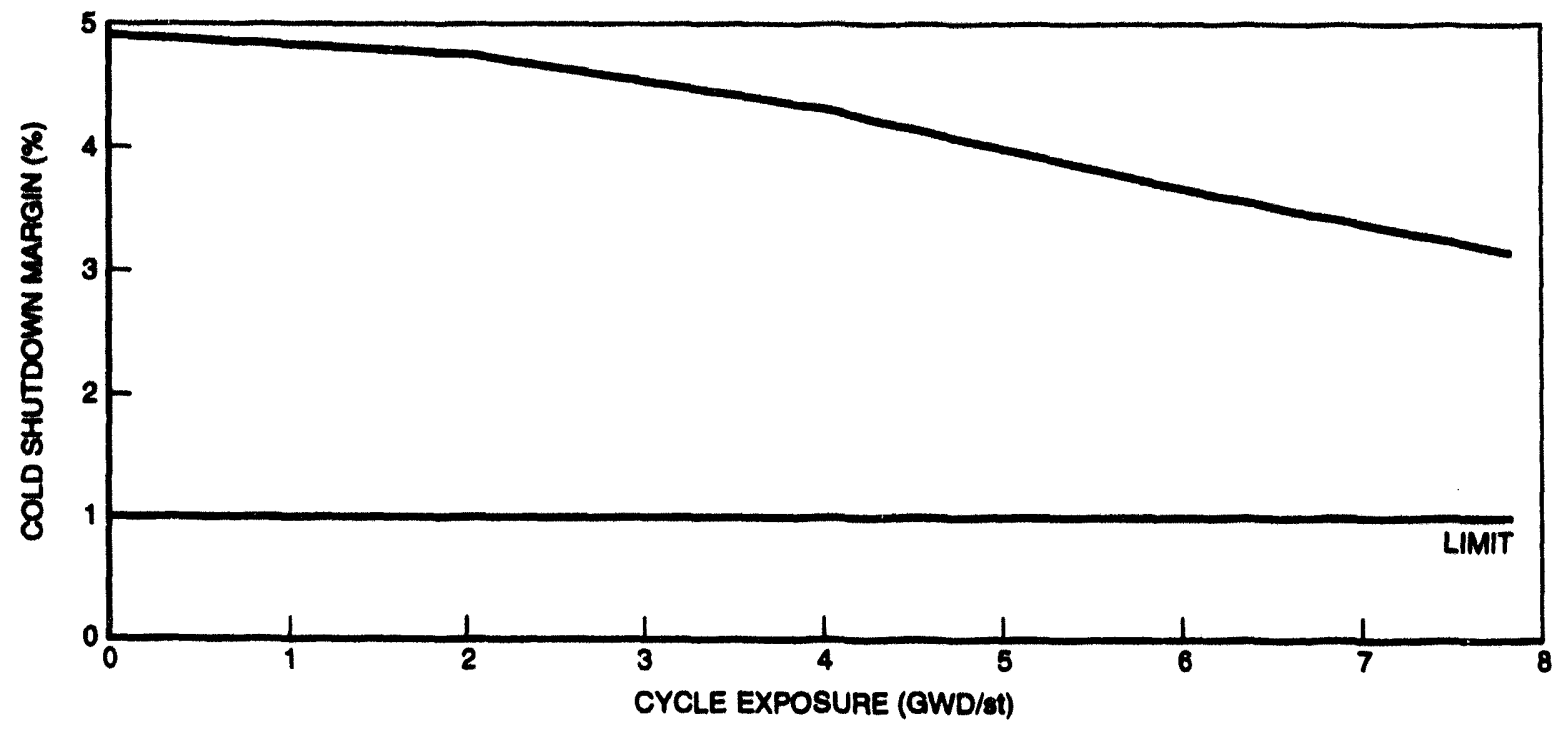

Figure 2.1-15 Equilibrium Cycle Minimum Cold Shutdown Margin - Full MOX 
NEDO-32361

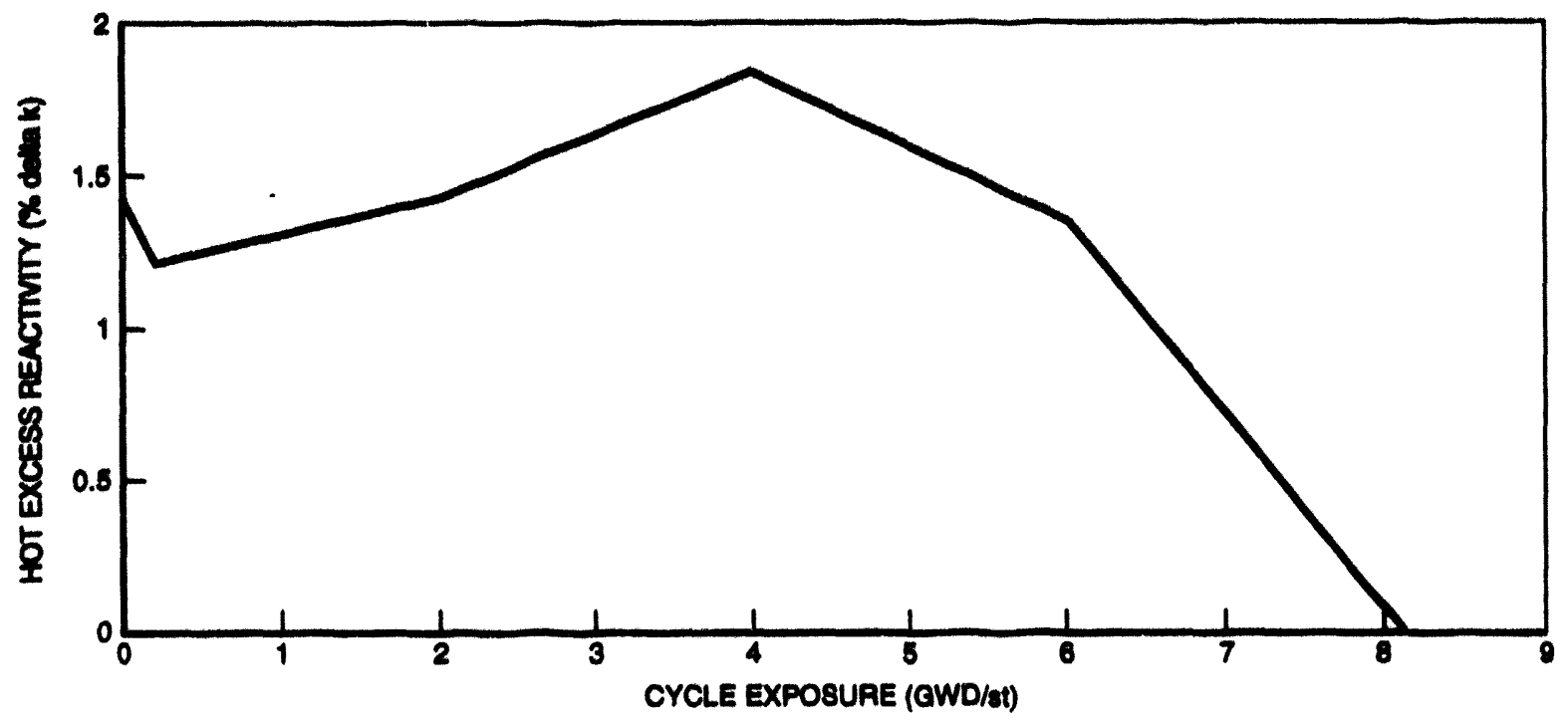

Figure 2.1-16 Equilibrium Cycle Hot Excess Reactivity - Island Design

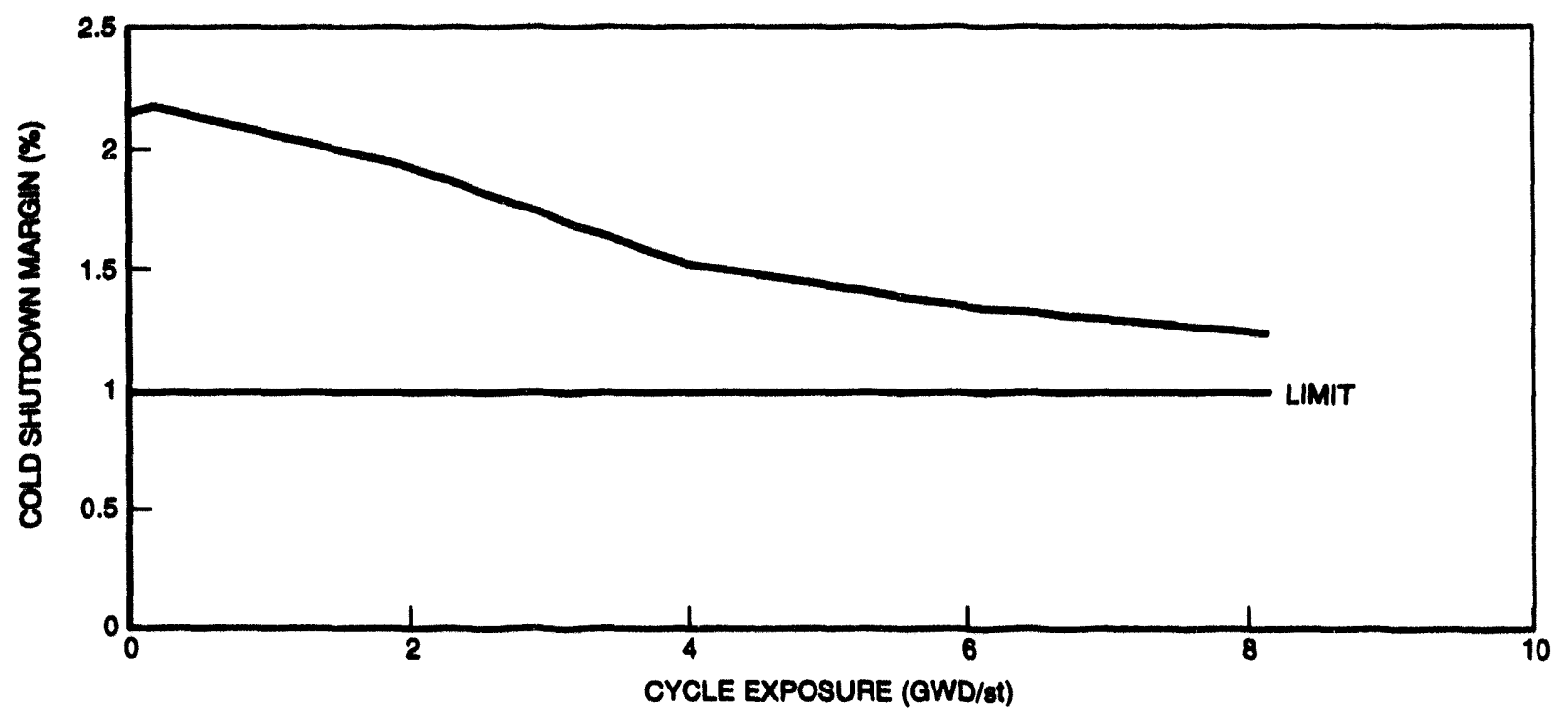

Figure 2.1-17 Equilibrium Cycle Minimum Shutdown Margin - Island Design 


\subsubsection{Reactivity Coemcients}

The dynamic void coefficient is a measure of the response of the reactor to changes in overall void fraction due to power, flow and pressure transients. This parameter is important in estimating system response to transient conditions and estimating reactor power/flow stability. BWR plants have been generically analyzed for the range of void coefficients from $-2.5 \% / \%$ to $-11.6 \notin / \%$ for transient response and up to $-9.0 \% / \%$ for stability. Values outside this range require special analysis to support establishment of Safety Limit CPR and power/flow operating window. Such analyses have been performed and are described in Section 2.3.

The fuel Doppler coefficient is a measure of the response of the reactor to changes in fuel temperature due to power transients. This parameter is important in estimating system response to transient conditions. BWR plants have been generically analyzed for the range of Doppler coefficients from -0.216 a/Kelvin to $-0.63 \mathrm{~d} / \mathrm{Kelvin}$ for transient response. Values outside this range require special analysis to support establishment of Safety Limit CPR.

The core dynamic void coefficient and Doppler coefficients of reactivity are plotted as a function of cycle exposure in Figure 2.1-18 and Figure 2.1-19 for the full MOX core. The void coefficient is somewhat larger than the upper generic limit for BWRs, while the Doppler coefficient is within the generic limits. The significance of the larger void coefficient for this core is discussed in Section 2.3.

The dynamic void and Doppler coefficients for the island design are similar to that of a typical uranium core. The transient responses of the island design are bounded by the full MOX design.

\subsubsection{Core Performance Description}

The core performance characteristics for the full MOX and island design cores as a function of exposure through the cycle are given in Figure 2.1-20 through Figure 2.1-29. These figures show the control rod pattern, core performance, axial power shape, and axial exposure shape for various exposure points through the cycle. The core maps in these figures show the control blade patterns in the core expressed in terms of notches (which are 3-inch sections of blade) withdrawn from the top of the core. Those cells which have no numbers represent cells in which there are no blades inserted. The thermal limits and reactivity margins associated with the given exposure are noted in the summary included with each figure. As seen from these figures, all thermal and reactivity margins are met. The resulting core average power and exposure profile are also 
NEDO-32361

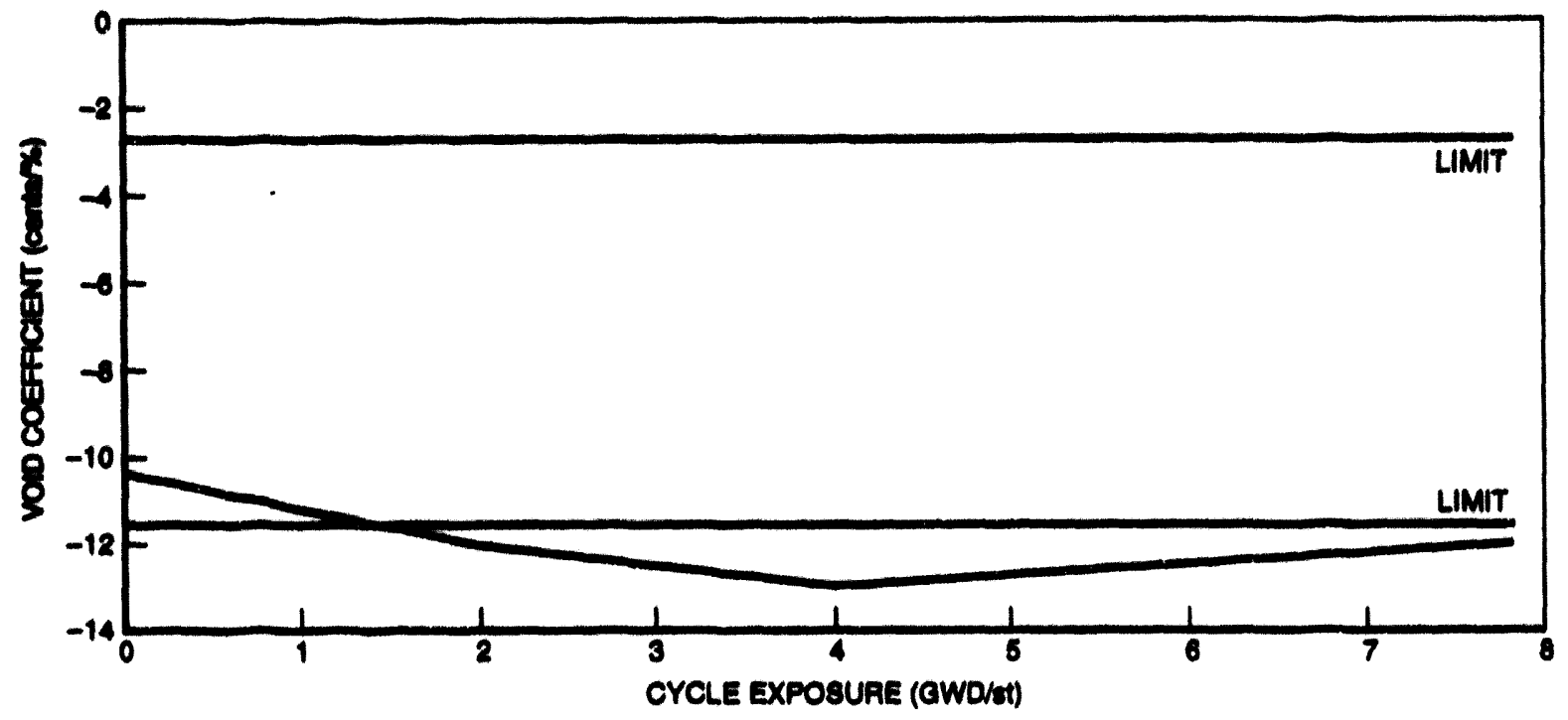

Figure 2.1-18 Equilibrium Cycle Dynamic Vold Coeficient - Full MOX

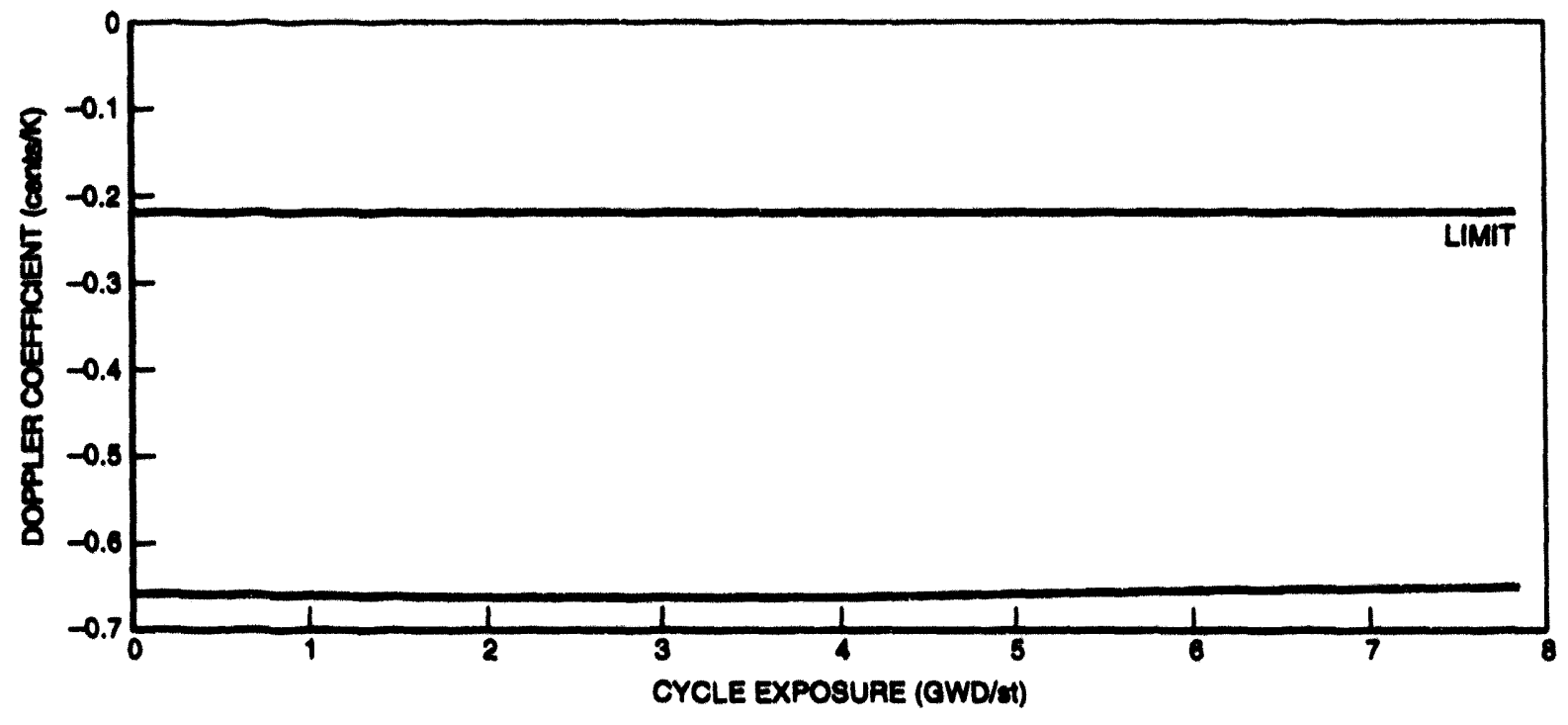

Figure 2.1-19 Equilibrium Cycle Doppler Coefficient - Full MOX 


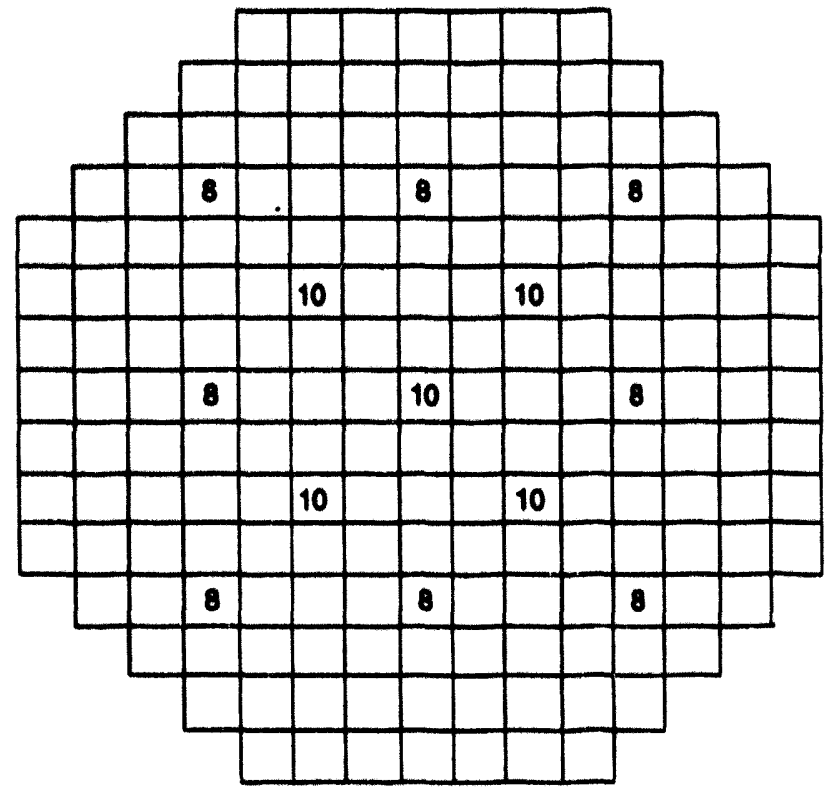

\begin{tabular}{|c|c|}
\hline CYCLE EXPOSUPE, MWd/St & 0 \\
\hline CYCLE ENEAOY, MWD & 0 \\
\hline NUMBEA OF FULL POWEA DAYS & 0 \\
\hline CORE AVERACE VOID FRACTION & .3905 \\
\hline COAE FLOW, Mb/hr & 1.086 \\
\hline MAXIMUM CHANNEL PEAKINO & 1.3628 \\
\hline COAE AXIAL POWEA PEAK & 1.181 \\
\hline MAXIMUM RAPLHOR & .847 \\
\hline MAXIMUM CPARAT & .77 \\
\hline HOT EXCESS REACTIVITY, \% & 1.20 \\
\hline COLD SHUTDOWN MARCIN & 4.91 \\
\hline
\end{tabular}

N $N$ - NUMBER OF 3 INCH INCREMENTS THAT THE CONTAOL BLADE IS WITHDAAWN FROM FULLY INSERTED
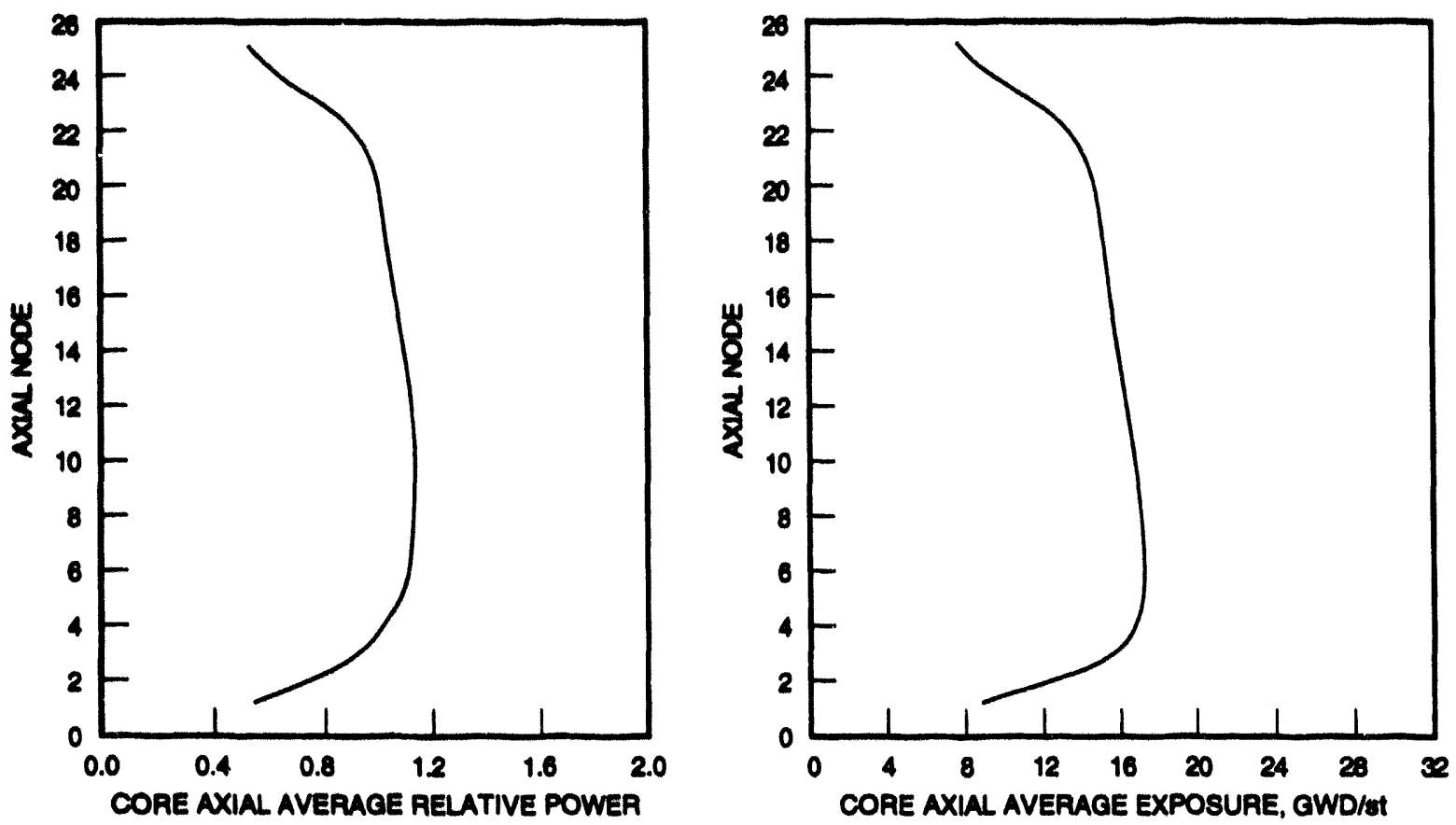

Figure 2.1-20 Equillbrium Cycle Core Data Summary at 0 MWd/st - Full MOX 


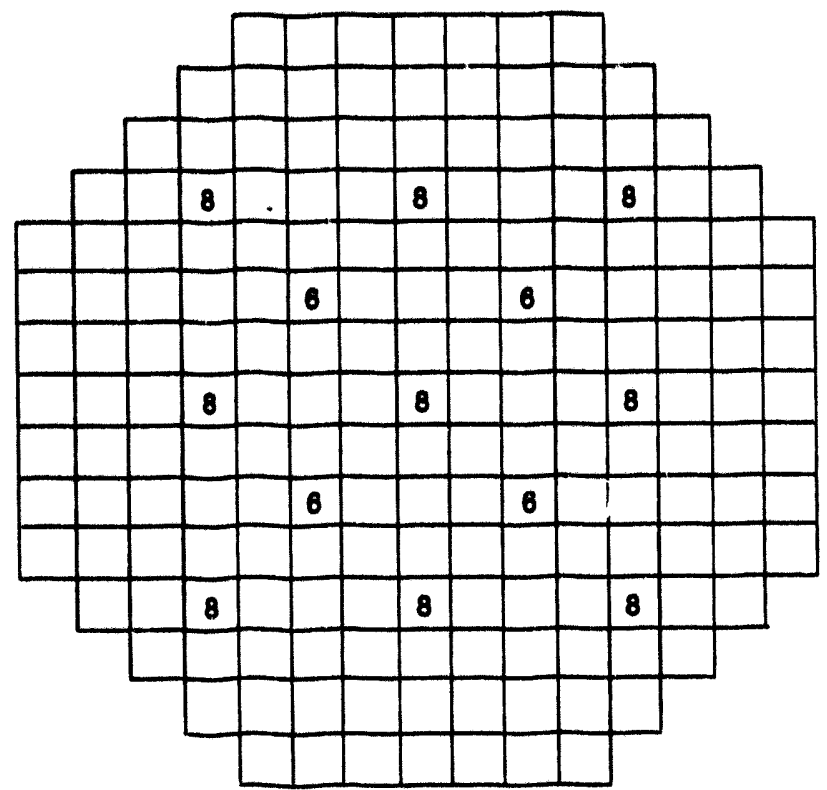

\begin{tabular}{|l|l|}
\hline CYCLE EXPOSURE, MWd/AT & 2000 \\
\hline CYCLE ENEAQY, MWD & 301469 \\
\hline NUMBEA OF FULL POWER DAYS & 87 \\
\hline CORE AVERAGE VOID FRACTION & .408 \\
\hline COAE FLOW, MIbMT & 1.085 \\
\hline MAXIMUM CHANNEL PEAKING & 1.322 \\
\hline CORE AXIAL POWER PEAK & 1.215 \\
\hline MAXIMUM RAPLHGR & .839 \\
\hline MAXIMUM CPARAT & .769 \\
\hline HOT EXCESS REACTIVITY, \% & 1.25 \\
\hline COLD SHUTDOWN MARGIN & 4.73 \\
\hline
\end{tabular}

$N$ N $N$ NUMBER OF 3 INCH INCREMENTS THAT THE CONTROL BLADE IS WITHDRAWN FROM FULLY INSERTED
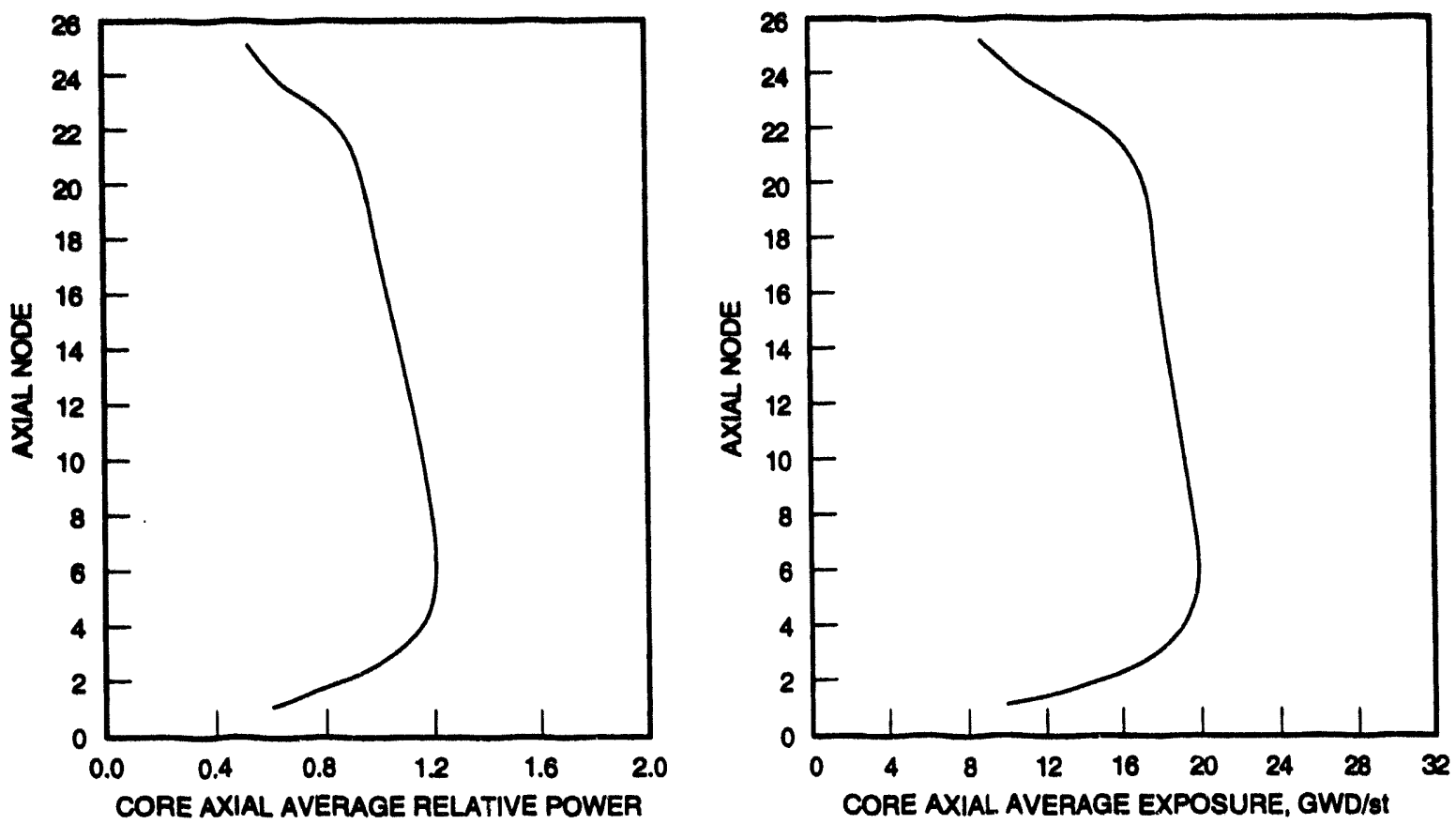

Figure 2.1-21 Equilibrium Cycle Core Data Summary at 2000 MWd/st - Full MOX 


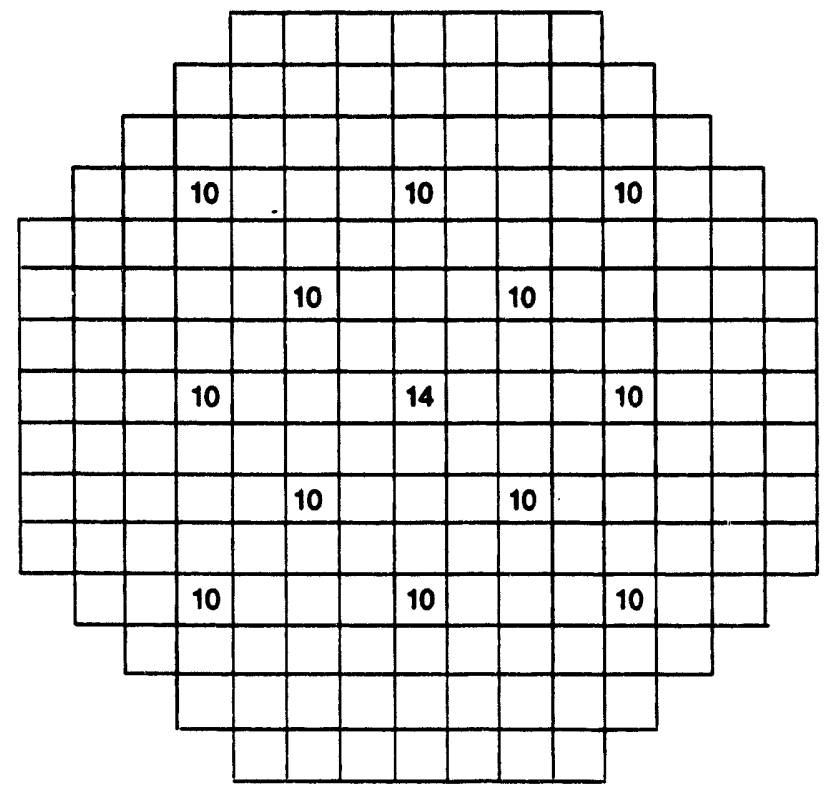

\begin{tabular}{|l|l|}
\hline CYCLE EXPOSURE, MWd/st & 4000 \\
\hline CYCLE ENERGY, MWD & 602937 \\
\hline NUMBER OF FULL POWER DAYS & 173 \\
\hline CORE AVERAGE VOID FRACTION & .4117 \\
\hline CORE FLOW, MIb/hr & 1.085 \\
\hline MAXIMUM CHANNEL PEAKING & 1.361 \\
\hline CORE AXIAL POWER PEAK & 1.262 \\
\hline MAXIMUM RAPLHGR & .942 \\
\hline MAXIMUM CPRRAT & .784 \\
\hline HOT EXCESS REACTIVITY, \% & 1.19 \\
\hline COLD SHUTDOWN MARGIN & 4.27 \\
\hline
\end{tabular}

N $N$ = NUMBER OF 3 INCH INCREMENTS THAT THE CONTROL BLADE IS WITHDRAWN FROM FULLY INSERTED
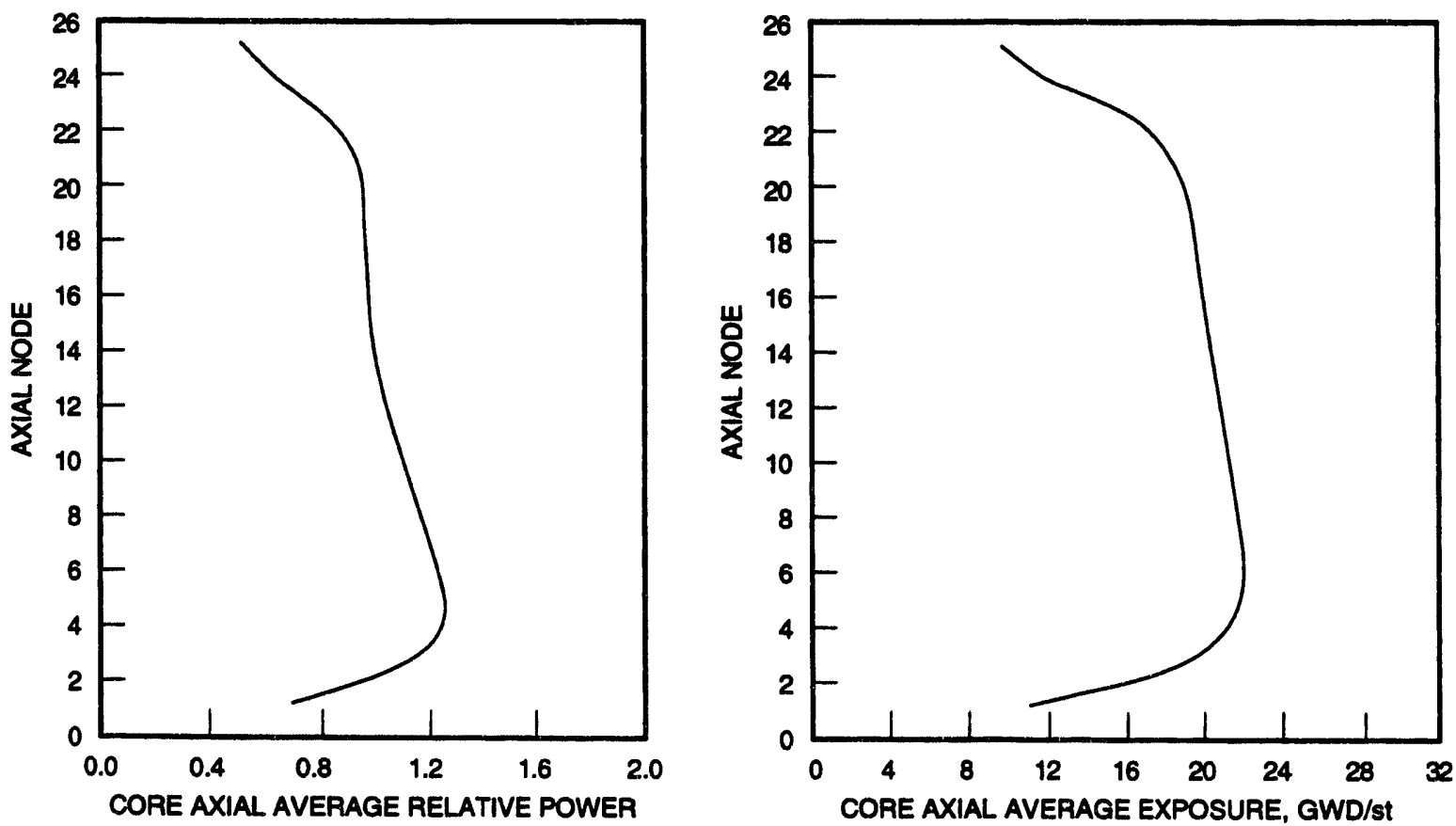

Figure 2.1-22 Equilibrium Cycle Core Data Summary at 4000 MWd/st - Full MOX 

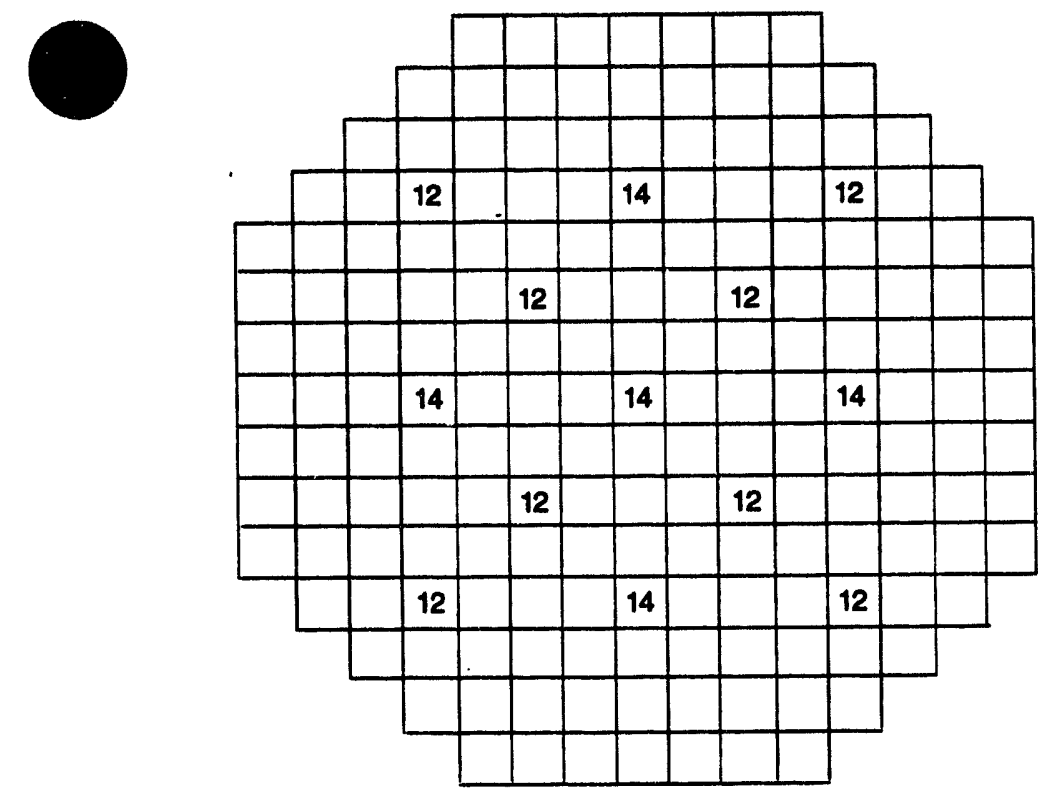

\begin{tabular}{|l|l|}
\hline CYCLE EXPOSURE, MWd/st & 6000 \\
\hline CYCLE ENERGY, MWD & 904406 \\
\hline NUMBER OF FULL POWER DAYS & 260 \\
\hline CORE AVERAGE VOID FRACTION & .3868 \\
\hline CORE FLOW, MIb/hr & 1.085 \\
\hline MAXIMUM CHANNEL PEAKING & 1.383 \\
\hline CORE AXIAL POWER PEAK & 1.109 \\
\hline MAXIMUM RAPLHGR & .77 \\
\hline MAXIMUM CPRRAT & .786 \\
\hline HOT EXCESS REACTIVITY, $\%$ & 0.93 \\
\hline COLD SHUTDOWN MARGIN & 3.65 \\
\hline
\end{tabular}

N $N=$ NUMBER OF 3 INCH INCREMENTS THAT THE CONTROL BLADE IS WITHDRAWN FROM FULLY INSERTED
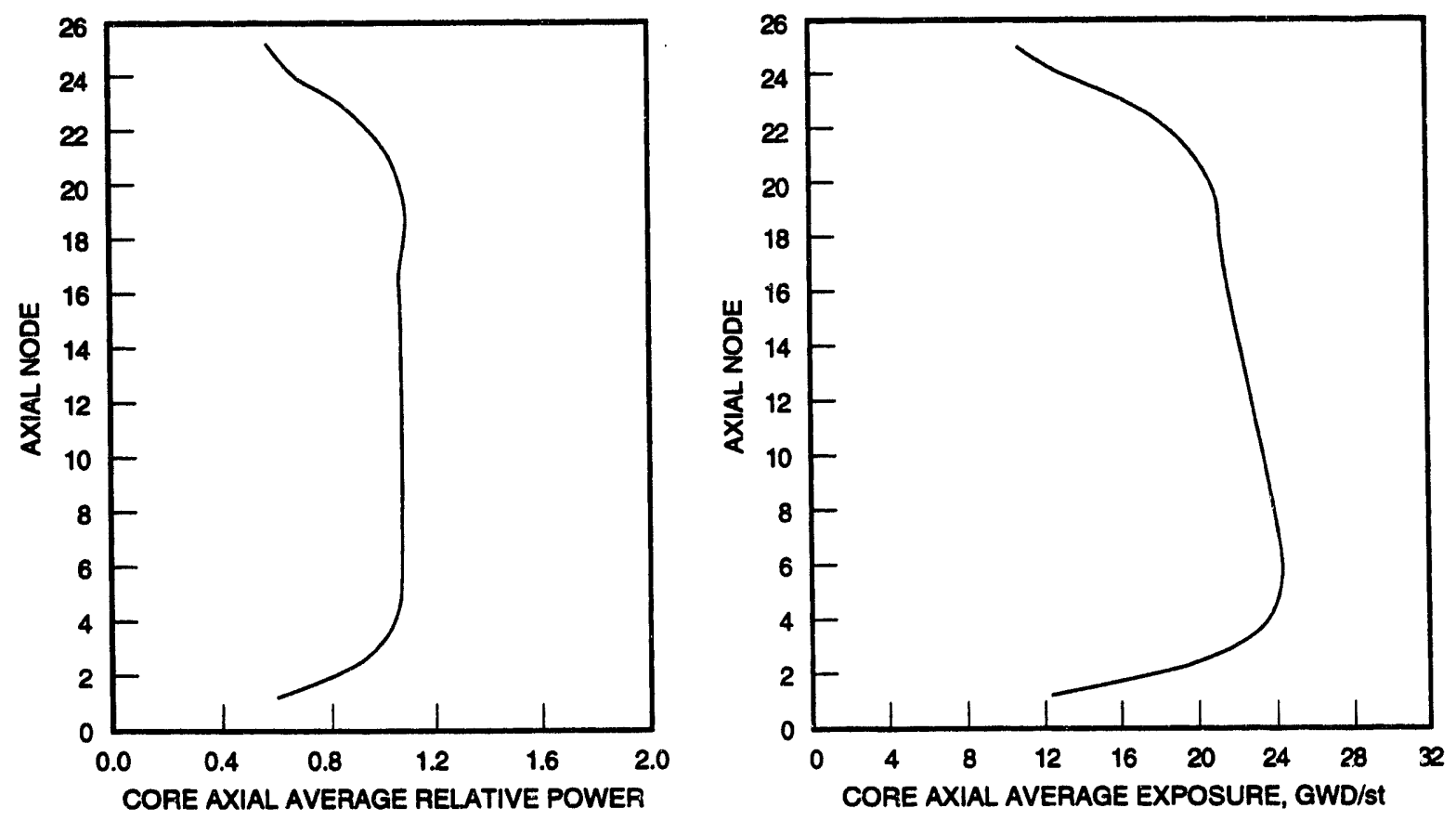

Figure 2.1-23 Equilibrium Cycle Core Data Summary at $6000 \mathrm{MWd}$ st - Full MOX 


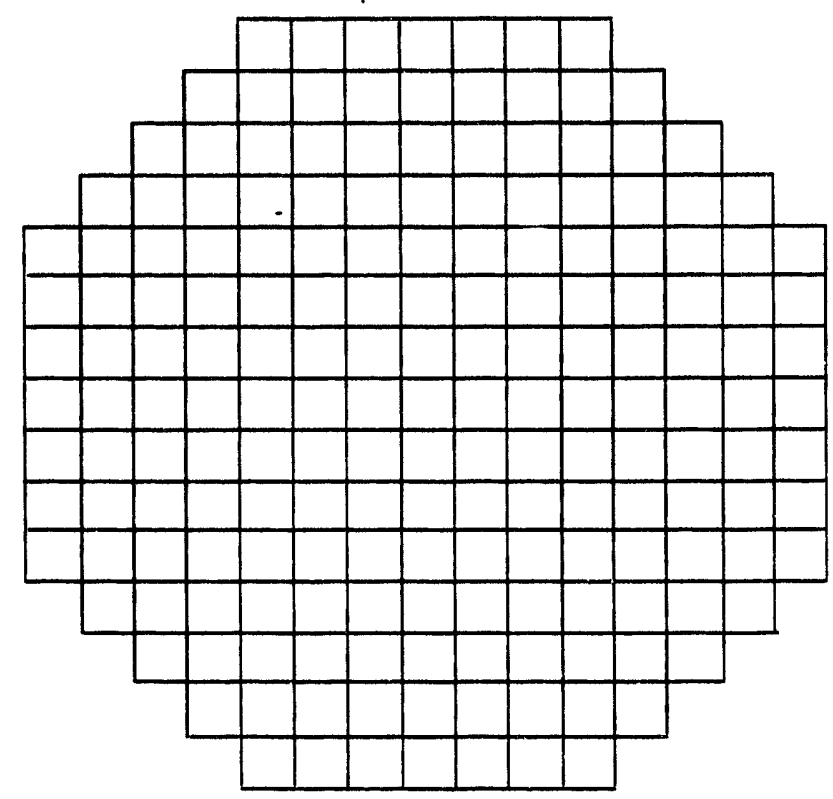

\begin{tabular}{|l|l|}
\hline CYCLE EXPOSURE, MWd/st & 7858 \\
\hline CYCLE ENERGY, MWD & 1184455 \\
\hline NUMBER OF FULL POWER DAYS & 340 \\
\hline CORE AVERAGE VOID FRACTION & .3845 \\
\hline CORE FLOW, MIb/hr & 1.085 \\
\hline MAXIMUM CHANNEL PEAKING & 1.364 \\
\hline CORE AXIAL POWER PEAK & 1.19 \\
\hline MAXIMUM RAPLHGR & .792 \\
\hline MAXIMUM CPRRAT & .786 \\
\hline HOT EXCESS REACTIVITY, \% & 0.0 \\
\hline COLD SHUTDOWN MARGIN & 3.1 \\
\hline
\end{tabular}

ALL RODS WITHDRAWN
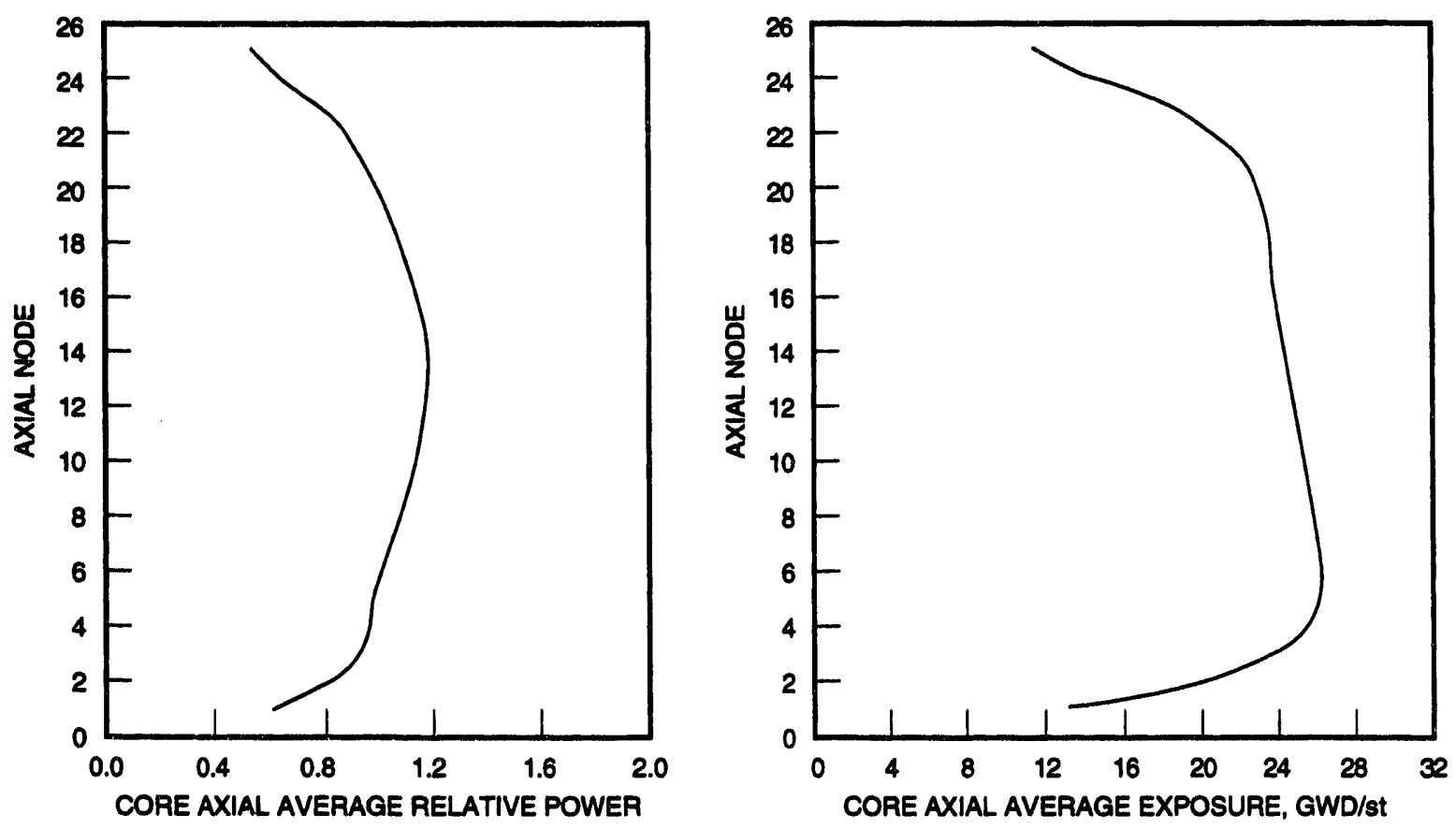

Figure 2.1-24 Equilibrium Cycle Core Data Summary at End of Cycle - Full MOX 


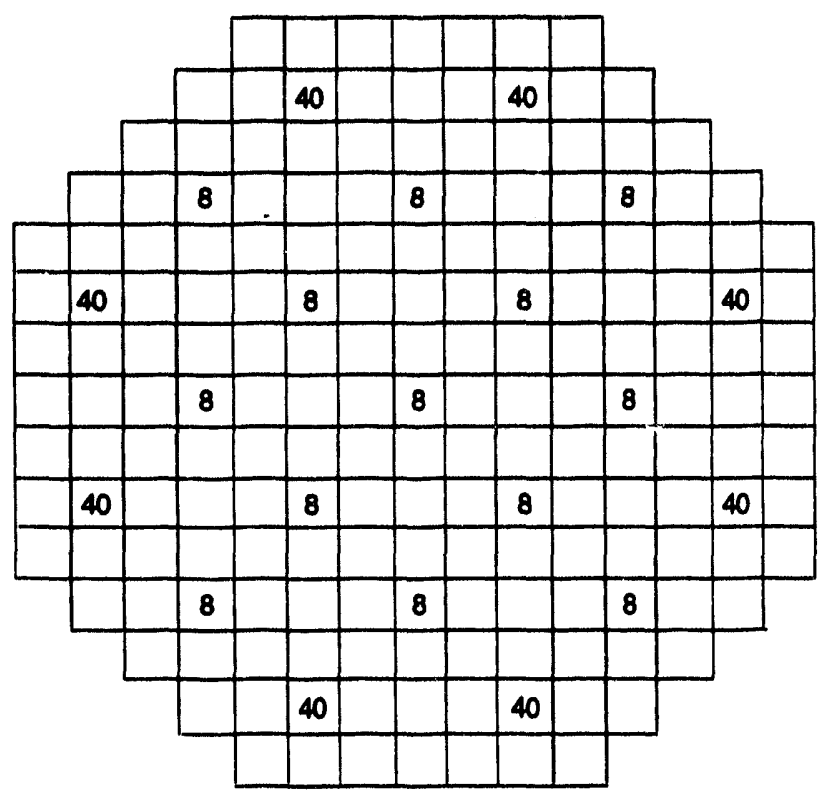

\begin{tabular}{|l|l|}
\hline CYCLE EXPOSURE, MWU/St & 0 \\
\hline CYCLE ENERGY, MWD & 0 \\
\hline NUMBER OF FULL POWER DAYS & 0 \\
\hline CORE AVERAGE VOID FRACTION & .4139 \\
\hline CORE FLOW, MIb/hr & 1.085 \\
\hline MAXIMUM CHANNEL PEAKING & 1.3743 \\
\hline CORE AXIAL POWER PEAK & 1.2695 \\
\hline MAXIMUM RAPLHGR & .883 \\
\hline MAXIMUM CPRRAT & .787 \\
\hline HOT EXCESS REACTIVITY, \% & 1.427 \\
\hline COLD SHUTDOWN MARGIN & 2.143 \\
\hline
\end{tabular}

$N$ N $=$ NUMBER OF 3 INCH INCREMENTS THAT THE CONTROL BLADE IS WITHDRAWN FROM FULL Y INSERTED
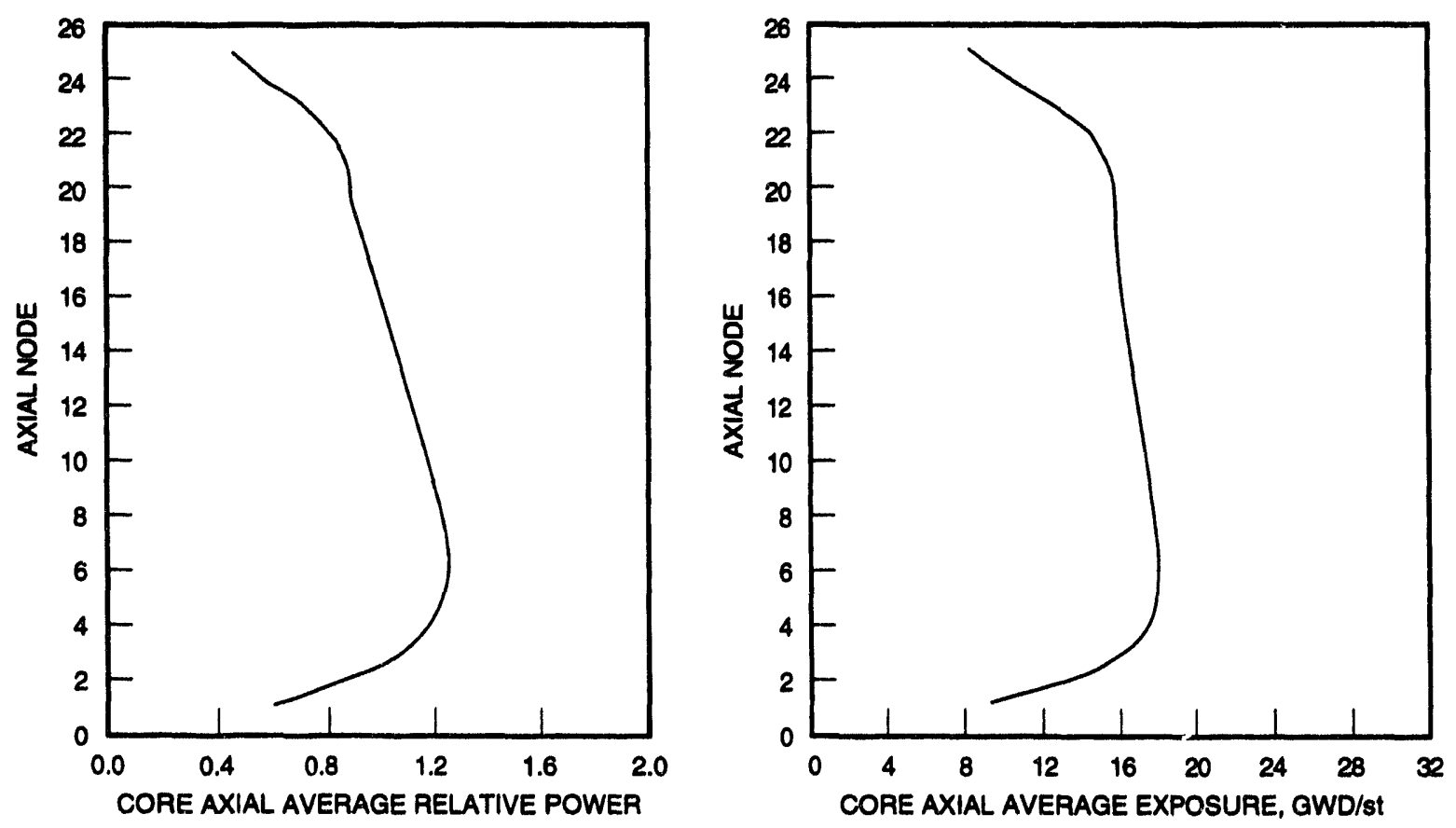

Figure 2.1-25 Equilibrium Cycle Core Data Summary at 0 MWd/st - Island Design 


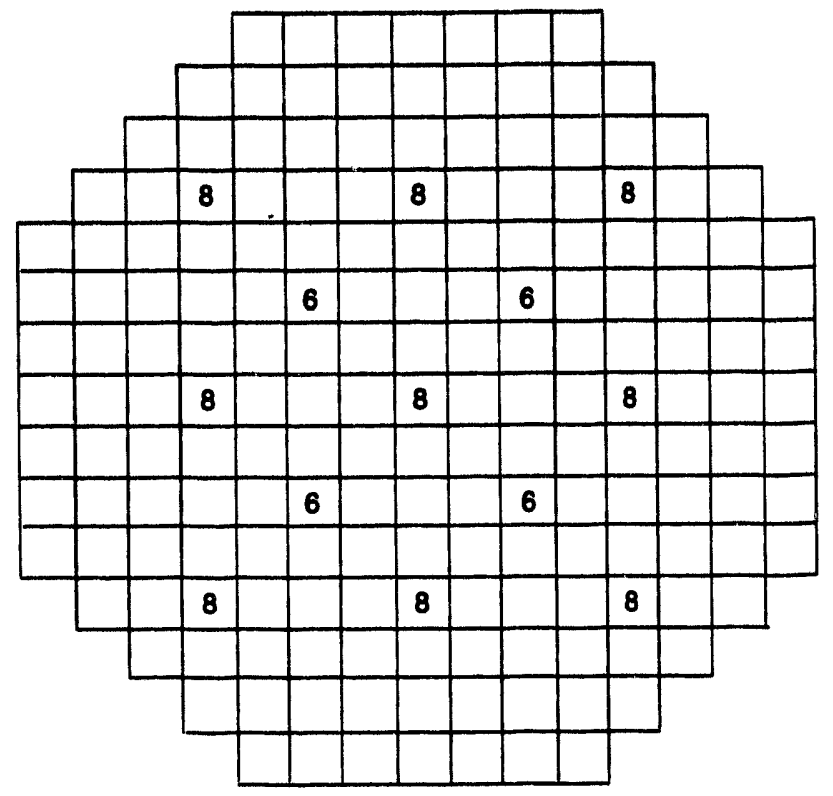

\begin{tabular}{|l|l|}
\hline CYCLE EXPOSURE, MWd/st & 2000 \\
\hline CYCLE ENERGY, MWD & 301447 \\
\hline NUMBER OF FULL POWER DAYS & 87 \\
\hline CORE AVERAGE VOID FRACTION & .407 \\
\hline CORE FLOW, MIb/hr & 1.085 \\
\hline MAXIMUM CHANNEL PEAKING & 1.330 \\
\hline CORE AXIAL POWER PEAK & 1.194 \\
\hline MAXIMUM RAPLHGR & .849 \\
\hline MAXIMUM CPRRAT & .759 \\
\hline HOT EXCESS REACTIVITY, \% & 1.426 \\
\hline COLD SHUTDOWN MARGIN & 1.913 \\
\hline
\end{tabular}

$N \quad N=$ NUMBER OF 3 INCH INCREMENTS THAT THE CONTROL BLADE IS WITHDRAWN FROM FULLY INSERTED
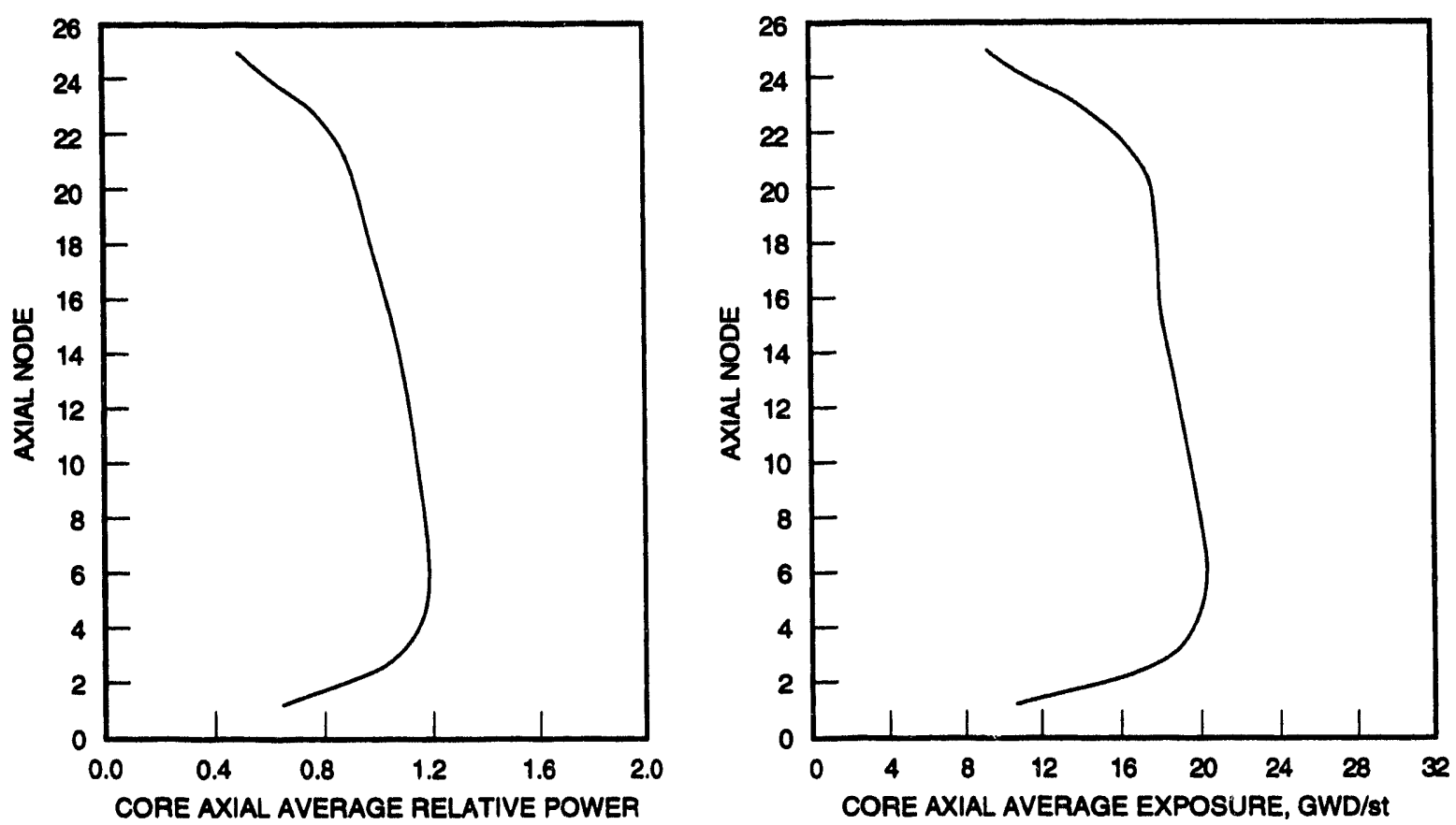

Figure 2.1-26 Equilibrium Cycle Core Data Summary at 2000 MWd/st - Island Design 


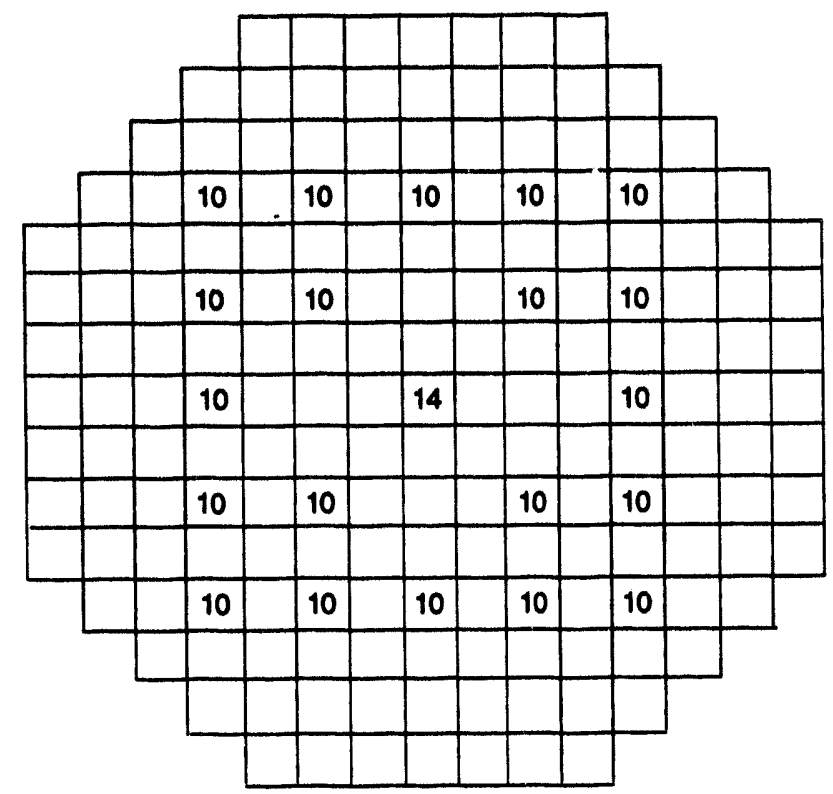

\begin{tabular}{|l|l|}
\hline CYCLE EXPOSURE, MWd/st & 4000 \\
\hline CYCLE ENERGY, MWD & 602894 \\
\hline NUMBER OF FULL POWER DAYS & 173 \\
\hline CORE AVERAGE VOID FRACTION & .3843 \\
\hline CORE FLOW, MIb/hr & 1.085 \\
\hline MAXIMUM CHANNEL PEAKING & 1.330 \\
\hline CORE AXIAL POWER PEAK & 1.138 \\
\hline MAXIMUM RAPLHGR & .824 \\
\hline MAXIMUM CPRRAT & .776 \\
\hline HOT EXCESS REACTIVITY, \% & 1.836 \\
\hline COLD SHUTDOWN MARGIN & 1.509 \\
\hline
\end{tabular}

$N \mathrm{~N}=$ NUMBER OF 3 INCH INCREMENTS THAT THE CONTROL BLADE IS WITHDRAWN FROM FULLY INSERTED
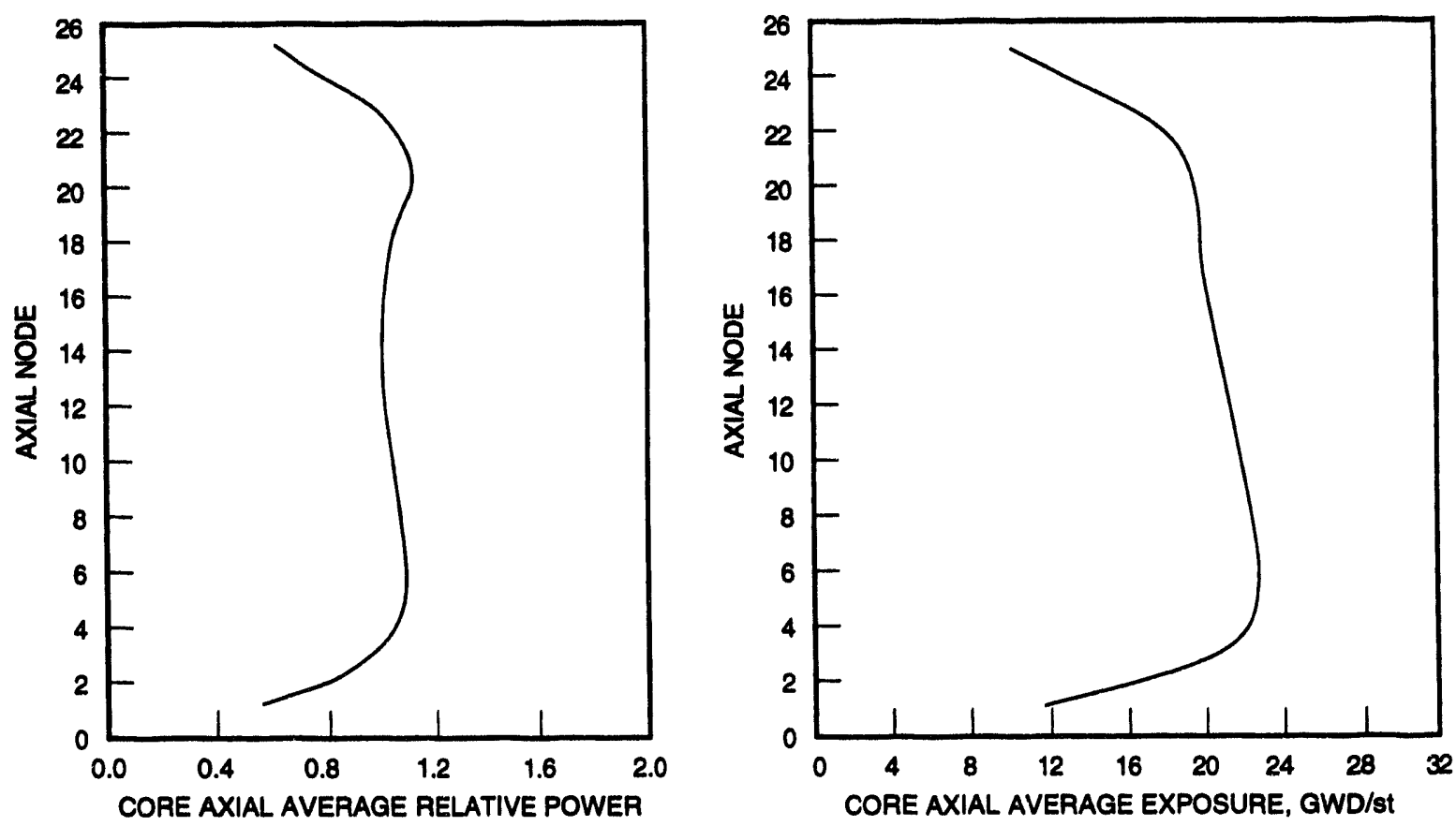

Figure 2.1-27 Equilibrium Cycle Core Data Summary at $4000 \mathrm{MWd}$ /st - Island Design 


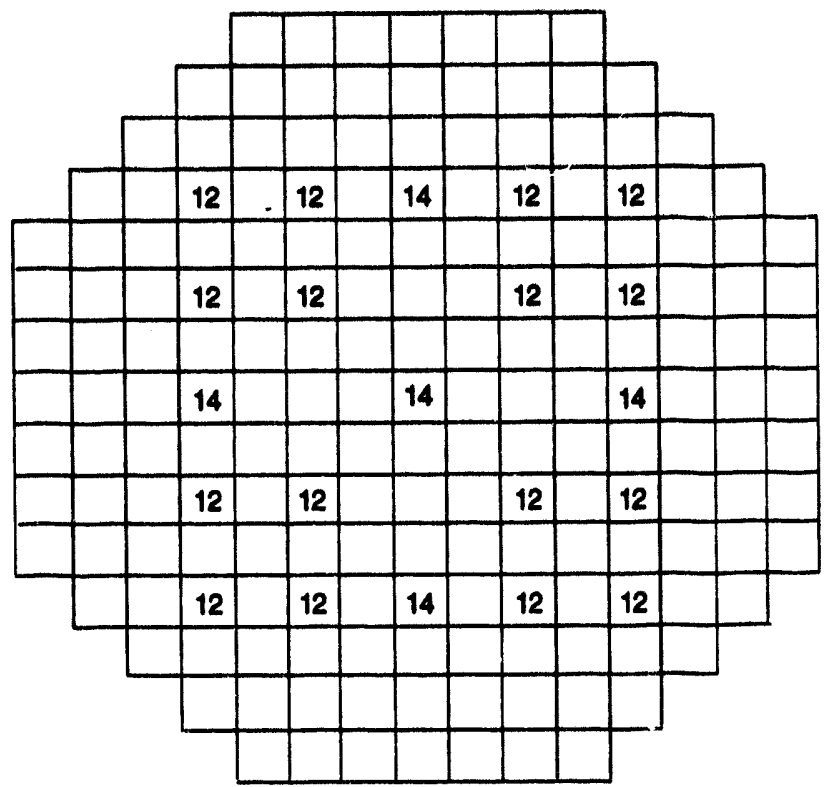

\begin{tabular}{|l|l|}
\hline CYCLE EXPOSURE, MWd/st & 6000 \\
\hline CYCLE ENERGY, MWD & 904341 \\
\hline NUMBER OF FULL POWER DAYS & 260 \\
\hline CORE AVERAGE VOID FRACTION & .3615 \\
\hline CORE FLOW, MIb/hr & 1.085 \\
\hline MAXIMUM CHANNEL PEAKING & 1.382 \\
\hline CORE AXIAL POWER PEAK & 1.244 \\
\hline MAXIMUM RAPLHGR & .799 \\
\hline MAXIMUM CPRRAT & .834 \\
\hline HOT EXCESS REACTIVITY, $\%$ & 1.356 \\
\hline COLD SHUTDOWN MARGIN & 1.332 \\
\hline
\end{tabular}

$N N=$ NUMBER OF 3 INCH INCREMENTS THAT THE CONTROL BLADE IS WITHDRAWN FROM FULLY INSERTED
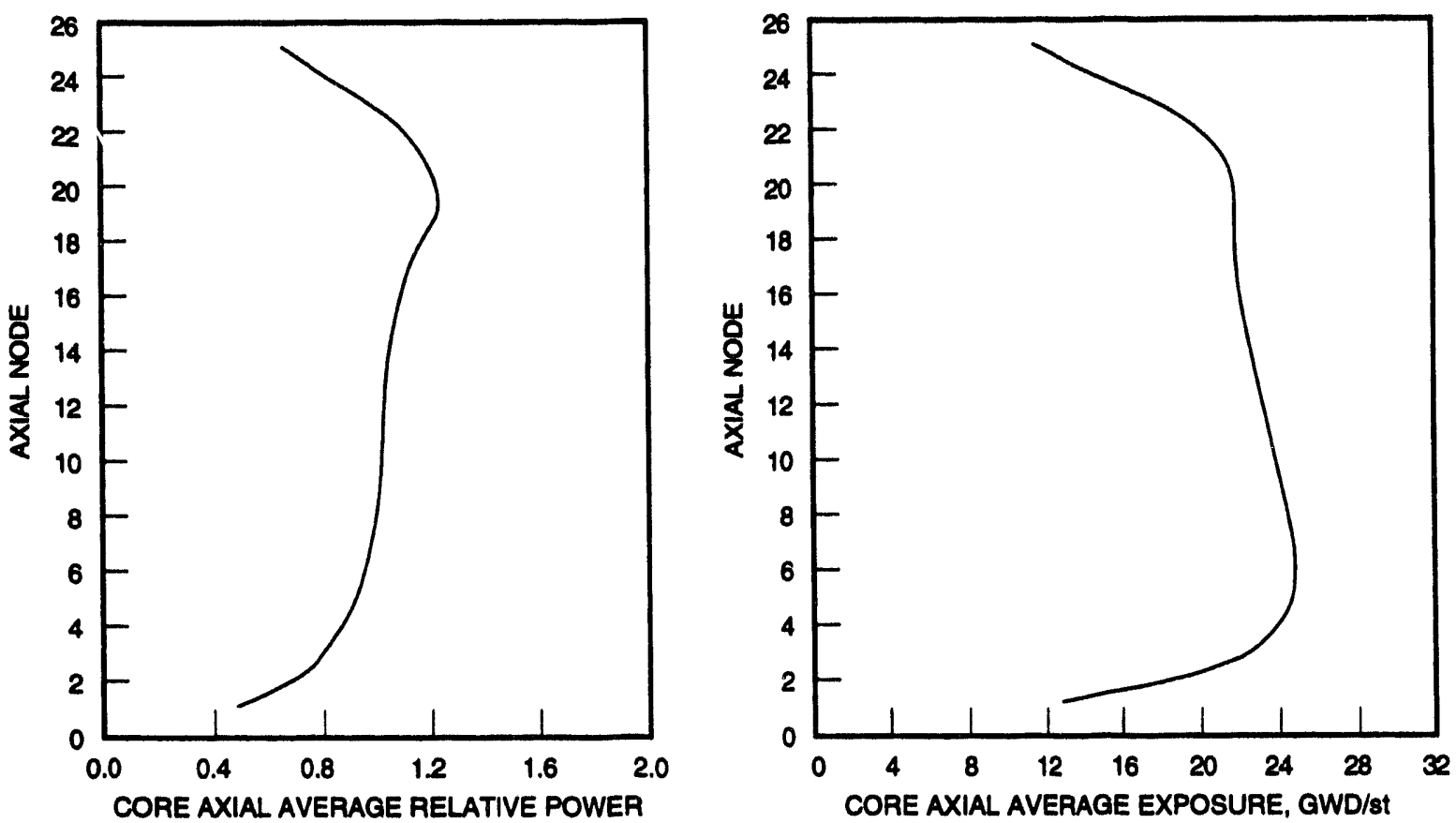

Figure 2.1-28 Equilibrium Cycle Core Data Summary at 6000 MWd/st - Island Design 


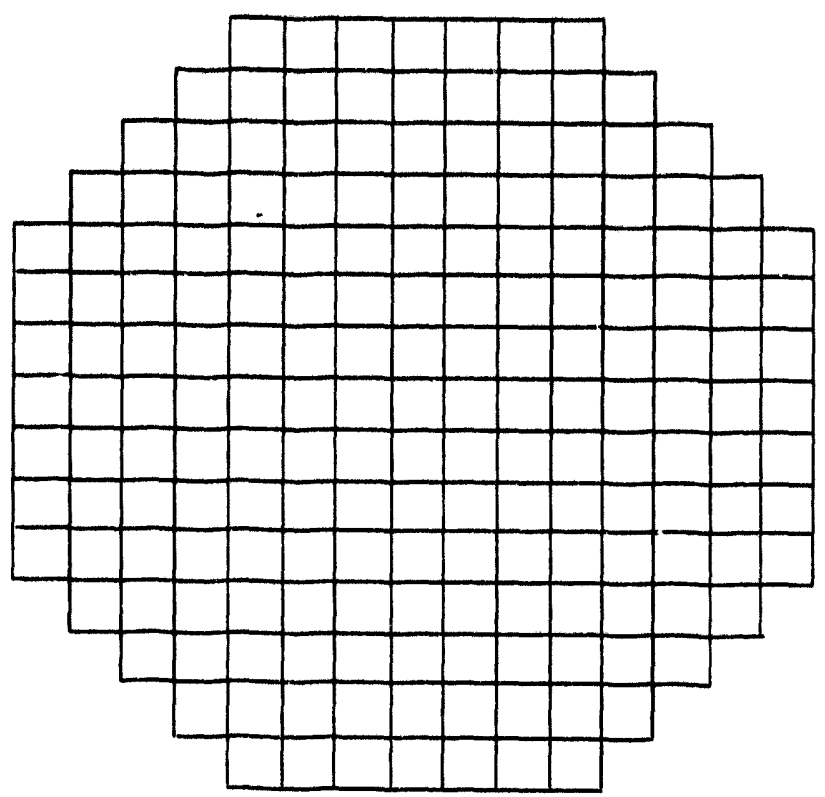

\begin{tabular}{|l|l|}
\hline CYCLE EXPOSURE, MWd/At & 8157 \\
\hline CYCLE ENERGY, MWD & 1236869 \\
\hline NUMBER OF FULL POWER DAYS & 365 \\
\hline CORE AVERAGE VOID FRACTION & .3756 \\
\hline CORE FLOW, MIb/hr & 1.085 \\
\hline MAXIMUM CHANNEL PEAKING & 1.383 \\
\hline CORE AXIAL POWER PEAK & 1.23 \\
\hline MAXIMUM RAPLHGR & .760 \\
\hline MAXIMUM CPRRAT & .792 \\
\hline HOT EXCESS REACTIVITY, $\%$ & 0.0 \\
\hline COLD SHUTDOWN MARGIN & 1.226 \\
\hline
\end{tabular}

ALL RODS WITHDRAWN
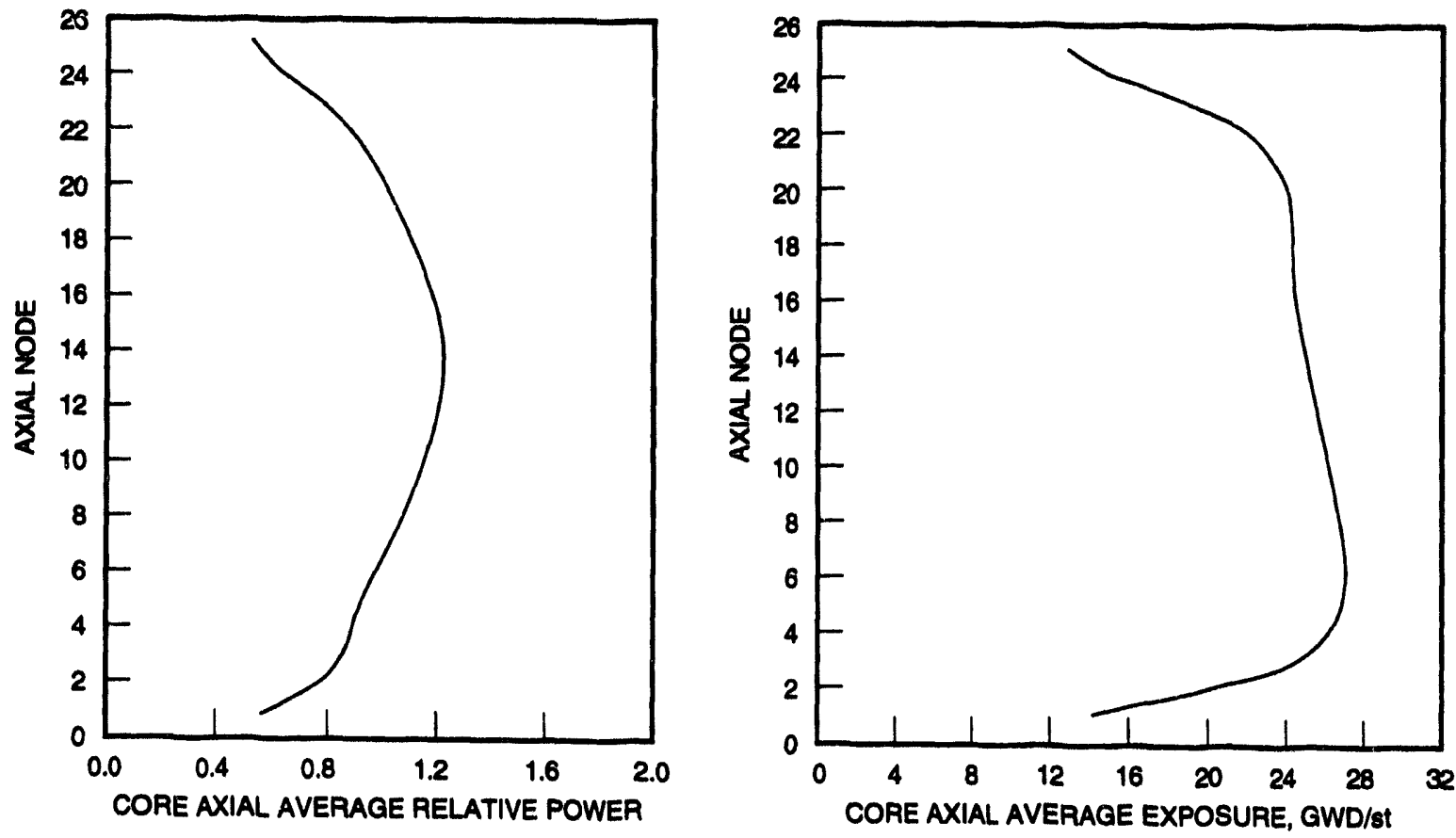

Figure 2.1-29 Equilibrium Cycle Core Data Summary at End of Cycle - Island Design 
given. Since the reactor core design itself provides sufficient margins, it is not necessary to axially grade the fuel assembly to accommodate the shift in power.

There is a larger-than-average control rod inventory when compared to the hot excess for the full MOX core. This result indicates that it might be necessary to use high worth control rods for cores with much higher hot excess. Types of cores in this category would be long cycle lengths or higher plutonium enrichment assemblies (i.e., "overloaded" assembly designs).

\subsubsection{Spent Fuel Characteristics}

The decay heat rate on a per bundle basis is shown in Table 2.1-7 and Figures 2.1-30 and 2.1-31. The decay power difference between island designs and the full MOX design also is shown in Table 2.1-7. The data show a slight increase in the full MOX bundle decay heat relative to the island bundles.

The average isotopic content of the discharged fuel bundles for the reference full MOX case is shown in Table 2.1-8. Th.s isotopics are calculated five days after reactor shutdown.

Table 2.1-7 Average Bundle Decay Power (watts) of SF 1 Fuel

\begin{tabular}{|c|r|r|r|}
\hline Time after Shutdown & \multicolumn{3}{|c|}{ Decay Power (watts) } \\
\hline & Full MOX & \multicolumn{1}{|c|}{ Island } & $\%$ Difr \\
\hline $0.00 \mathrm{sec}$ & 261,500 & 269,200 & 2.9 \\
\hline $10.00 \mathrm{sec}$ & 200,800 & 205,500 & 2.3 \\
\hline $10.00 \mathrm{~min}$ & 96,940 & 98,950 & 2.1 \\
\hline $30.00 \mathrm{~min}$ & 73,080 & 74,780 & 2.3 \\
\hline $10.00 \mathrm{hr}$ & 32,280 & 32,510 & 0.7 \\
\hline $50.00 \mathrm{hr}$ & 21,000 & 20,800 & -1.0 \\
\hline $100.00 \mathrm{hr}$ & 16,640 & 16,300 & -2.0 \\
\hline $0.50 \mathrm{yr}$ & 3171 & 2760 & -13.0 \\
\hline $1.00 \mathrm{yr}$ & 1898 & 1654 & -12.9 \\
\hline $1.50 \mathrm{yr}$ & 1324 & 1177 & -11.1 \\
\hline $5.00 \mathrm{yr}$ & 369 & 352 & -4.8 \\
\hline $10.00 \mathrm{yr}$ & 241 & 231 & -4.4 \\
\hline $30.00 \mathrm{yr}$ & 172 & 152 & -11.6 \\
\hline $50.00 \mathrm{yr}$ & 138 & 113 & -18.4 \\
\hline $100.01 \mathrm{yr}$ & 94 & 65 & -31.3 \\
\hline & & & \\
\hline
\end{tabular}


'ұеәч Кеәәр

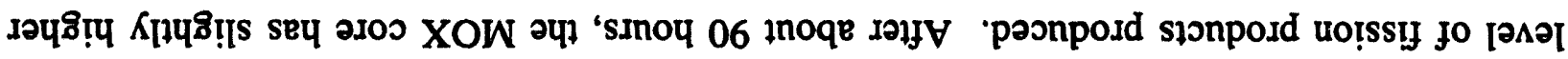

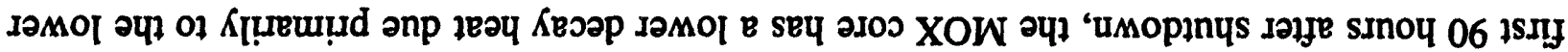

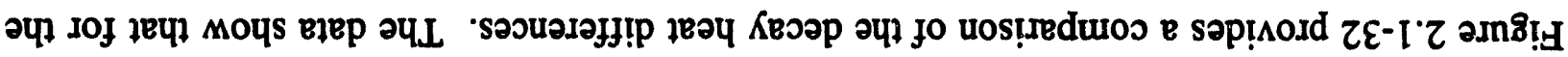

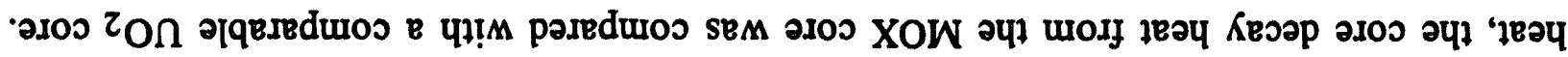

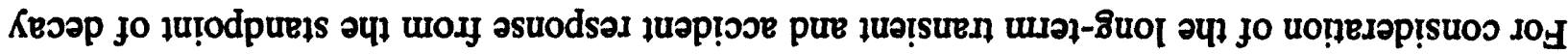

गpunq/sure18 S000 0 uRपp SSP - n

\begin{tabular}{|c|c|c|c|c|c|}
\hline$\varepsilon I^{\circ}+0 \triangleright \tau$ & $\begin{array}{c}\text { 20urereg } \\
\text { IonpoId } \\
\text { uo!ss! }\end{array}$ & $+00^{\circ} 0$ & ISI-wd & $28^{\circ} \mathrm{ZI}$ & $\downarrow t z-u \supset$ \\
\hline & & $0+0^{\circ} 0$ & $6 t I-u_{d}$ & $t S^{\circ} O I$ & 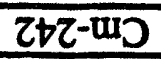 \\
\hline & & $\nabla 61^{\circ} 0$ & W8bI-Шd & & \\
\hline & & $69^{\circ} 81$ & $L \nabla I-w_{d}$ & I & $0 \varepsilon Z-4$. \\
\hline & & & & I & $8 z Z-4.1$ \\
\hline & & $6 L^{\circ} \nabla Z$ & ZSI-wS & & \\
\hline+0.0 & EII-PD & $\varepsilon I^{\prime} \varepsilon$ & ISI-WS & $\varepsilon \forall$ & $\varepsilon \forall z-u r \forall$ \\
\hline \multirow[t]{2}{*}{4} & $I \varepsilon Z^{-8} \mathrm{~d}$ & $80^{\circ} 25$ & OSI-שS & 4 & $z \nabla \tau-u r y$ \\
\hline & & $8 S t^{\circ} 0$ & $6 t[-w S$ & $\angle 6^{\circ} O E$ & Itz-ur \\
\hline $8 L 9$ & EOI-ny & $12.6 \varepsilon$ & $8 t I^{-w S}$ & & \\
\hline $9^{\circ} \varepsilon 9 I$ & I0I-ny & $\angle t^{\circ} D I$ & $\angle b I$ - ws & $56^{\circ} \mathrm{Z}$ & $6 \varepsilon z-d N$ \\
\hline & & & & S0.62 & $\angle E Z-d N$ \\
\hline tE0 0 & SOI-4d & E'SII & SDI-PN & & \\
\hline \multirow[t]{3}{*}{6.901} & $\varepsilon 0 I^{-4 d}$ & $6^{\circ} \varepsilon E I$ & $E t I-P N$ & $\varepsilon \mathcal{Z Z Z}$ & $z+z-n_{d}$ \\
\hline & & & & S'OIS & $I+z-n_{d}$ \\
\hline & & $\nabla I Z$ & $\varepsilon b I^{-I} \mathrm{~d}$ & S6II & $0 t z-n d$ \\
\hline$\triangle I^{\circ} \angle O Z$ & 09I-pD & 4 & $6 S I-9 L$ & EEDI & $6 \varepsilon Z-n_{d}$ \\
\hline S98.80t & 8SI-pD & $\forall \angle D I$ & 66-0I & $8^{\circ} \varepsilon \varepsilon$ & $8 E Z-n_{d}$ \\
\hline$\angle 6 I^{\circ} 0$ & $\angle S I-P D$ & $\varepsilon$ EIZ & EEI-\$D & & \\
\hline $28.81 \varepsilon$ & 9SI-PD & 4 & SEI-I & 001891 & $8 \varepsilon z-\Omega$ \\
\hline StI0 & SSI-PD & 4 & SEI-әX & L’9bI & $9 \varepsilon z-\Omega$ \\
\hline $89 \circ I$ & $\nabla S I-P D$ & $268^{\circ} 0$ & EEI-əXX & $S^{\circ} 18 \varepsilon$ & SEZ- $\Omega$ \\
\hline $910^{\circ} \mathrm{S}$ & SSI-ng & $0^{\circ} \varepsilon 8$ & IEI-0X & 9L'S & $\nabla \varepsilon q-\Omega$ \\
\hline $0 t^{\prime} Z I$ & $t S I-n g$ & & & म & $\varepsilon \varepsilon Z-\Omega$ \\
\hline $6 t^{\circ}$ IE & ESI-ng & $86 . S$ & $\varepsilon 8-\Gamma$ & 4 & ZEZ-4L \\
\hline 2uefu0D & adopos & 1"equos & 30101091 & mequos & Odopos \\
\hline
\end{tabular}

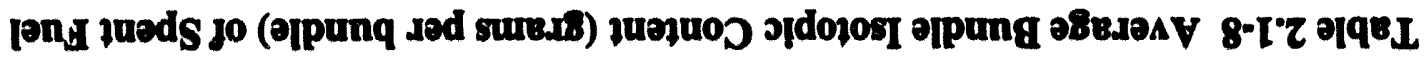


NEDO-32361

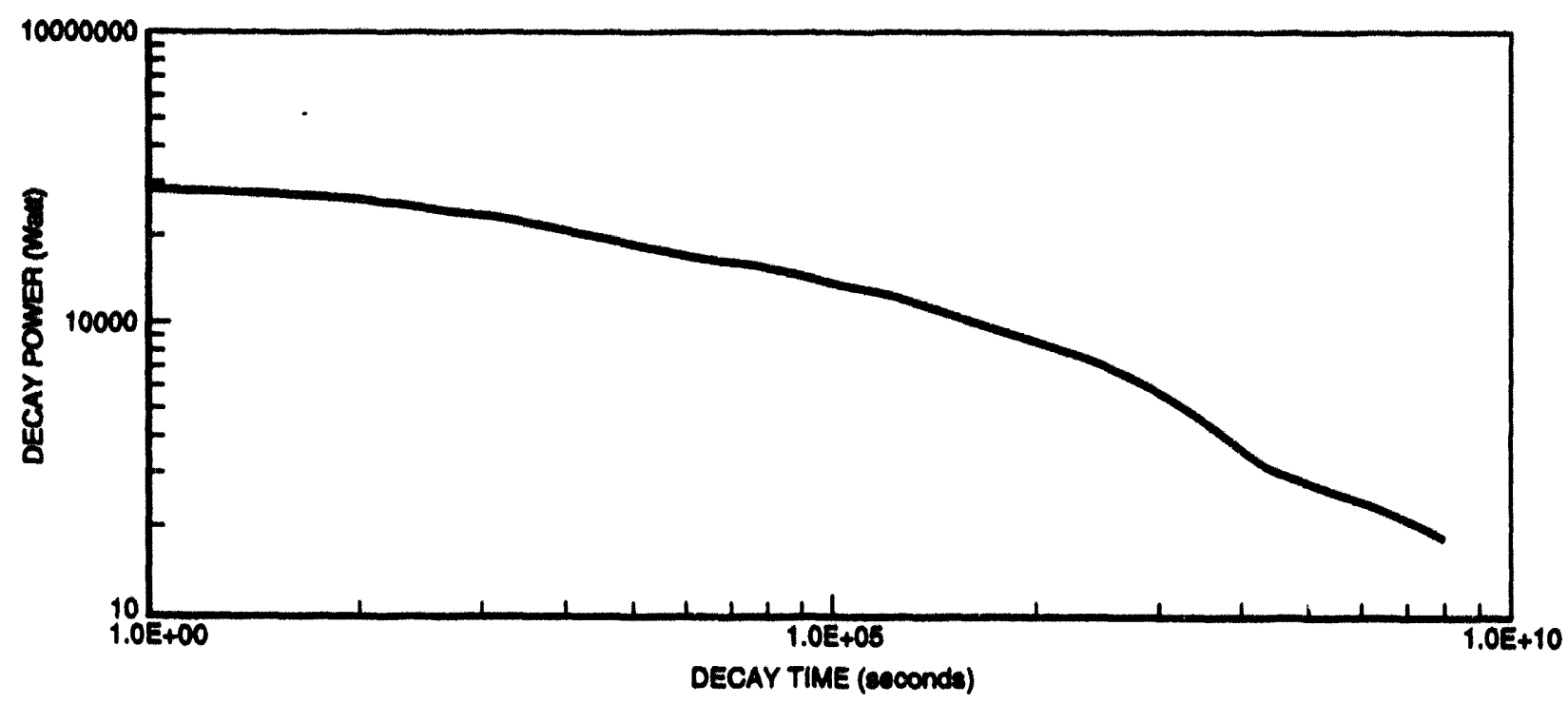

Figure 2.1-30 Spent Fuel Decay Heat for the Island Design

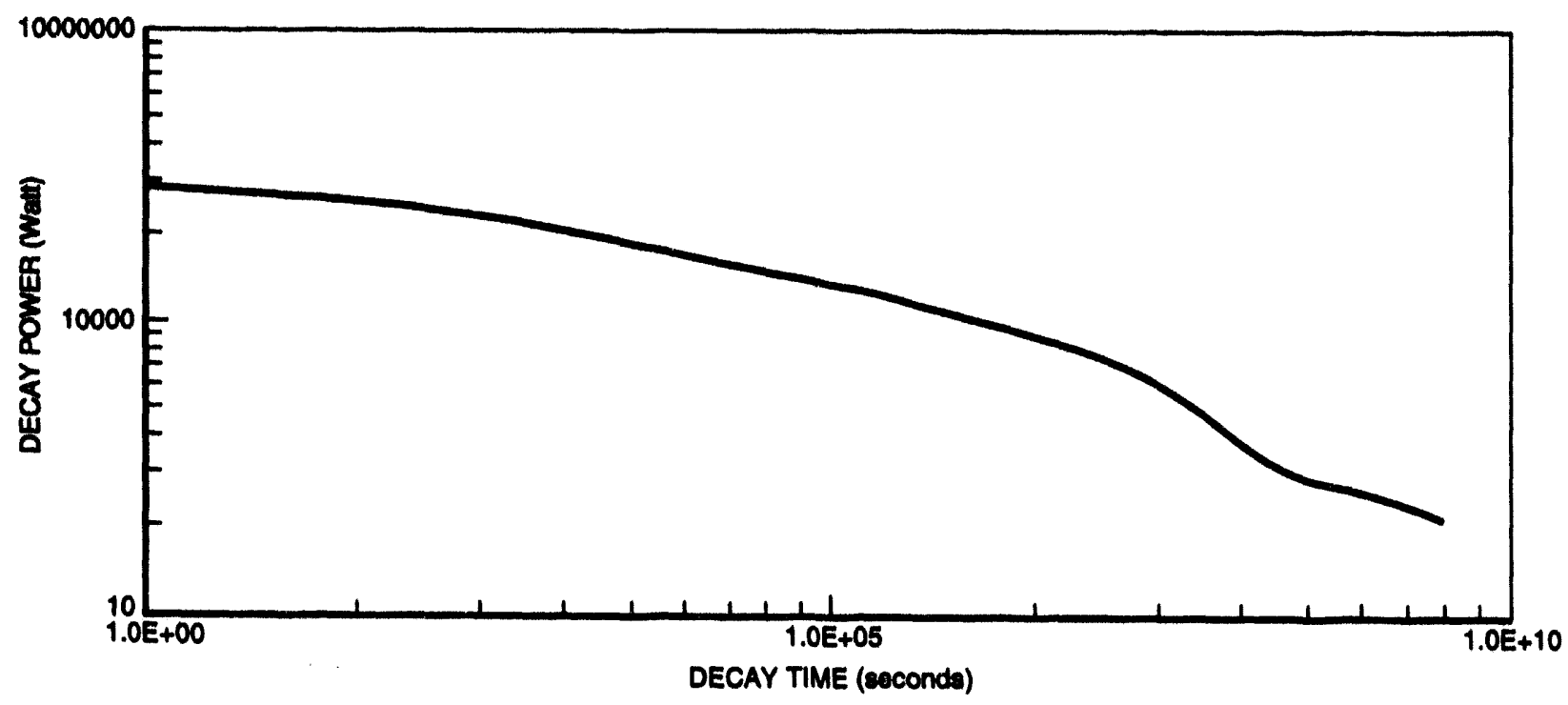

Figure 2.1-31 Spent Fuel Decay Heat for the Full MOX Design 


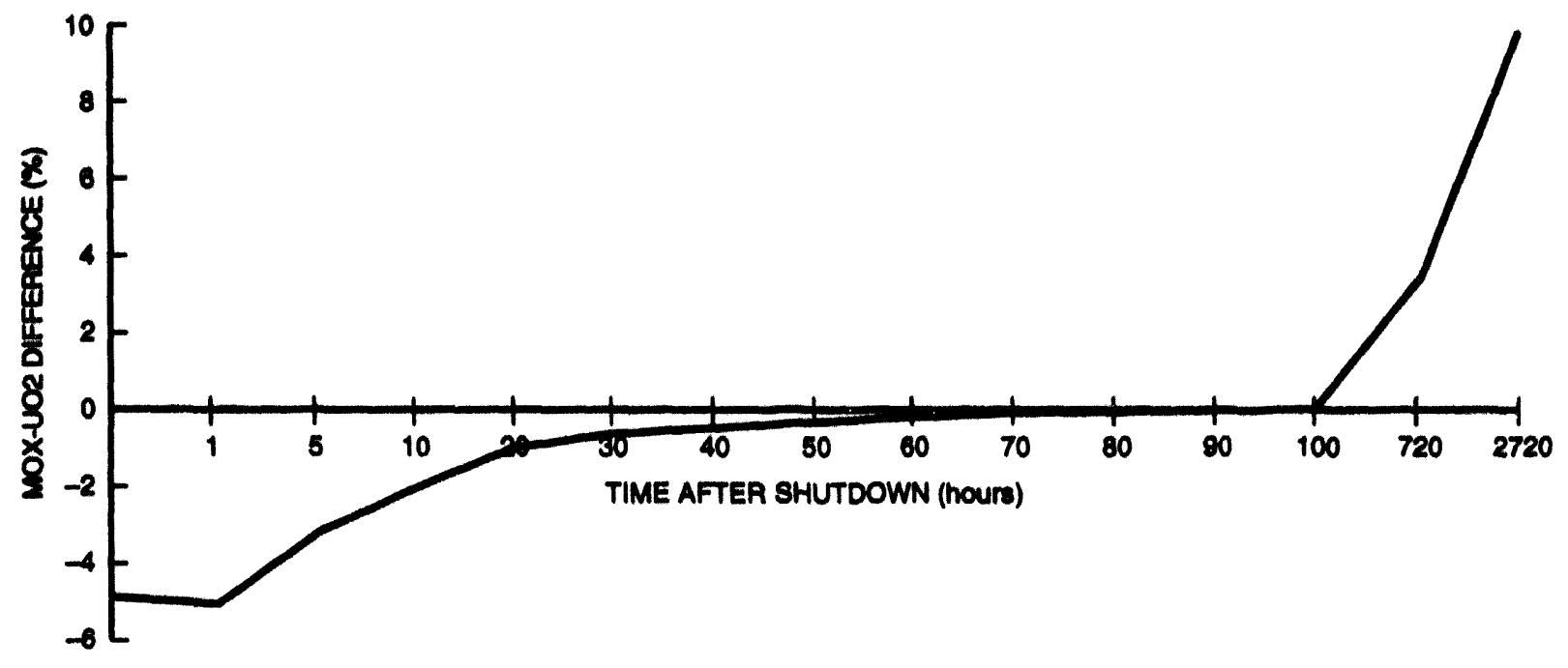

Figure 2.1-32 Full MOX and $\mathrm{UO}_{2}$ Core Decay Heat Difference 


\subsubsection{Cooling Under Normal Operations and Dealen Accidents}

\subsubsection{Nuclear Boller Systems}

The Nuclear Boiler System includes a direct-cycle forced-circulation boiling water reactor that produces steam for direct use in the steam turbine. A description of the subsystems and selected components is contained in the following paragraphs:

Reactor Pressure Vessel System: The BWR reactor pressure vessel (RPV) system (Figure 2.1-33) consists of the reactor pressure vessel with external reactor recirculation pumps, core and supporting structures, internal steam separators and dryers, control rod guide tubes, spargers for feedwater and low pressure emergency core cooling system (ECCS) injection, control rod drive penetrations, in-core nuclear instrumentation guide tubes, and other components. The reactor core is cooled by demineralized water that enters the lower portion of the core and boils as it flows upward around the fuel rods. The steam leaving the core is dried by steam separators and dryers located in the upper portion of the reactor vessel. The steam is then directed to the turbine through the four main steamlines. Detailed data for typical BWR-4, BWR-5 and BWR-6 plants are provided in Table 2.1-9.

The reactor vessel is subject to a rigid surveillance program during plant life to assure applicable codes and standards are maintained. Although no detailed fluence studies have been performed on a BWR plant to determine the impact of MOX fuel on the vessel and components, extensive study of the fluence conditions resulting from $\mathrm{UO}_{2}$ fuels have been made. $\mathrm{UO}_{2}$ core designs in BWRs produce half of their fission neutrons from plutonium at the end of their core life. Therefore, current fluence studies already account for a significant fraction of $\mathrm{Pu}$ fission neutrons and the impact that they have on the reactor vessel components.

The fluence conditions of the reactor vessel and components are affected primarily by the neutron production from the outer rows of fuel bundles. This is because the distance that a thermal neutron travels before it is absorbed or contributes to a fission (Mean Free Path) is relatively short - on the order of the magnitude of a fuel pin. Therefore, the fluence conditions on the RPV from MOX fuel is mainly affected by the fissions in the peripheral fuel bundles.

In a BWR plant, the peripheral fuel bundles of the $\mathrm{UO}_{2}$ core consist of higher exposure assemblies. These bundles contain approximately 0.8 to 1.3 weight $\% \mathrm{Pu}$ content at the end of core life. The MOX core considered in this study also contains about 1.0 to 1.6 weight \% Pu enrichment in the outer bundles. Therefore, even though a detailed fluence study has not yet 


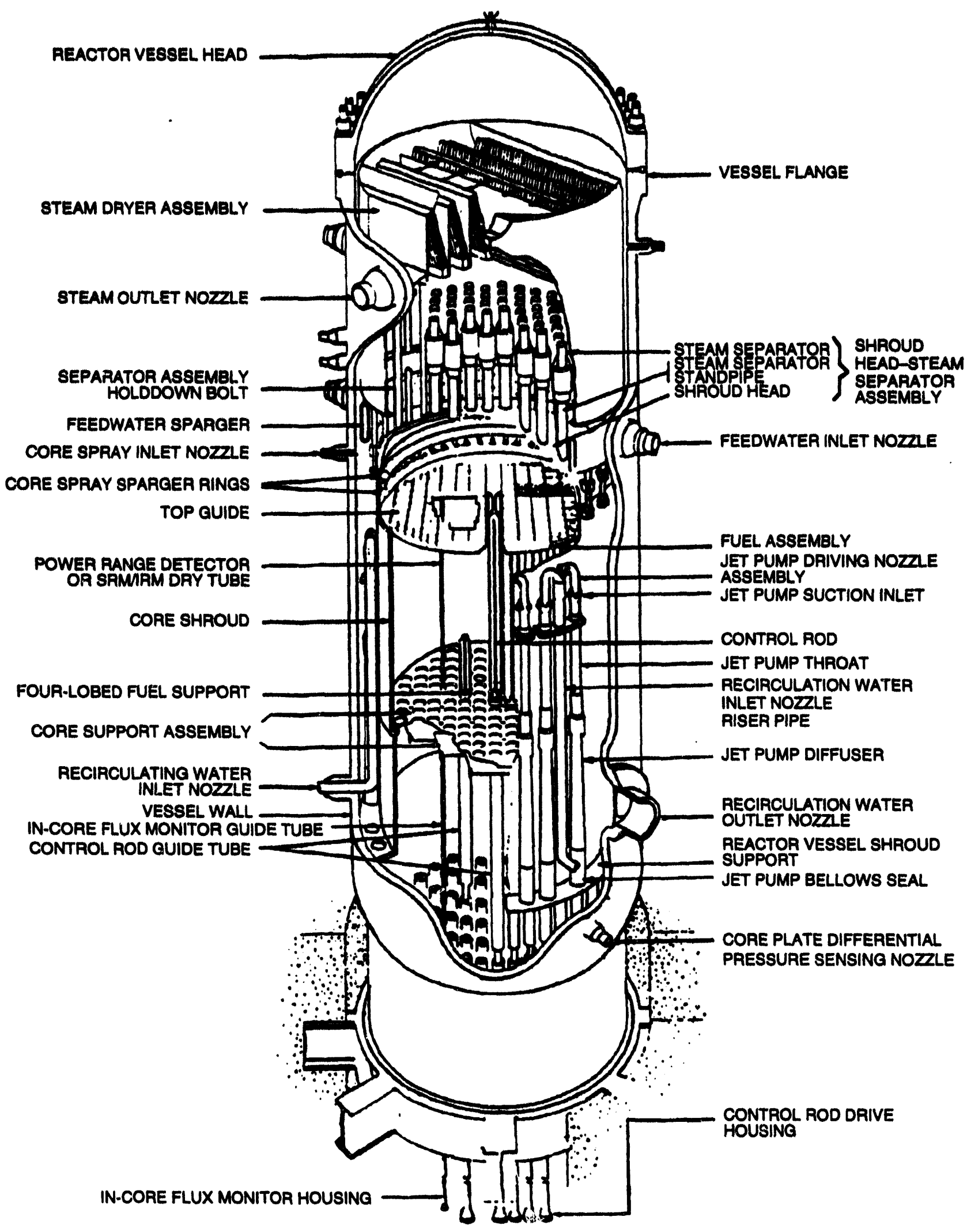

Figure 2.1-33 BWR Reactor Pressure Vessel 
Table 2.1-9 RPV Parameters

\begin{tabular}{|l|c|c|c|}
\hline & BWR/4 & BWR/5 & BWR/6 \\
\hline Design Pressure & $1250 \mathrm{psig}$ & $1250 \mathrm{psig}$ & $1250 \mathrm{psig}$ \\
\hline Design Temperature & $575^{\circ} \mathrm{F}$ & $575^{\circ} \mathrm{F}$ & $575^{\circ} \mathrm{F}$ \\
\hline Inside Diameter (in.) & 251 & 251 & 238 \\
\hline No. Fuel Bundles & 764 & 764 & 748 \\
\hline Jet Pumps & 20 & 20 & 20 \\
\hline Recirculation Flow/loop (Mlb/hr) & 17.1 & 17.85 & 15.88 \\
\hline Main Steam Lines & 4 & 4 & 4 \\
\hline Feedwater Nozzles & 2 & 2 & 2 \\
\hline Core Spray Nozzles & 2 & 2 & 2 \\
\hline Recirc Nozzles & 2 & 2 & 2 \\
\hline CRD Penetrations & 185 & 185 & 177 \\
\hline LPCI Injection vs Shroud & Outside & Outside & Inside \\
\hline LPCI Nozzles & 2 & 2 & 2 \\
\hline
\end{tabular}

been performed, it can be concluded that the fluence associated with the MOX design core would not be significantly different from the fluence conditions that have already been analyzed for $\mathrm{UO}_{2}$ cores of $\mathrm{BWR}$ plants.

It is concluded that no modifications to the RPV are needed to accommodate plutonium disposition.

\subsubsection{Reactor Coolant Systems}

Reactor Recirculation System: BWR/4, BWR/5 and BWR/6 plants contain 20 reactor jet pumps in the reactor vessel shroud which provide a forced circulation path for the core coolant flow (Figure 2.1-33). Ten jet pumps are driven by one of two recirculation loops each containing a single recirculation pump located in the drywell.

Although some minor operational changes are necessary to avoid operational regions which have a higher tendency for instabilities, no modifications to this system are required to accommodate plutonium disposition.

\subsubsection{Reactor Control and Backup Safety Systems}

\subsection{Reactor Control Systems}

Control Rod Drive System: The control rod design is a sheathed cruciform array of stainless steel tubes filled with Boron Carbide. When a scram is initiated by the Reactor Protection 
System, the control rod drive (Figure 2.1-34) inserts the rod to achieve sufficient negative reactivity to shut down the reactor.

Normal movement of control rods is provided by the CRD Hydraulic System (Figure 2.1-35). In addition to providing control rod movement, the CRD cooling water provides a constant flow of makeup water to the RPV. When a scram signal is received, high-pressure water stored in nitrogen charged accumulators forces the control rods into the core. BWR- 6 designs provide a higher accumulator pressure, which results in a control rod response time following scrams slightly faster than in BWR-4 and BWR-5 designs.

No modifications to the CRD System or control rods are needed for plutonium disposition. If extremely long operating cycles of high plutonium enrichments are desirable, it would be possible to replace the current control rods with commercially available currently licensed high worth rods. However, using the existing rods, the nuclear response of the reactor core to transients is verified by the analysis presented in Section 2.3. Therefore, the existing rods are considered adequate for the plutonium disposition mission. Depending on the specific plants chosen for implementation, a control rod interface study will be required to confirm dimensional acceptability of the control rod rollers and other dimensions. However, any inconsistencies can be accommodated.

Recirculation Flow Control System: Recirculation flow is varied in BWR-4 plants by variable speed motor generator (M-G) sets which adjust the recirculation pump motor operating frequency. In BWR-5 and BWR-6 plants, recirculation flow is controlled by a flow control valve. A low speed $(15 \mathrm{~Hz}) \mathrm{M}-\mathrm{G}$ set provides power to the recirculation pumps at minimum speed.

During normal power operation, the speed of the recirculation pumps (BWR-4) or a flow control valve (BWR-5 and BWR-6) is adjusted to control recirculation system flow. Adjusting recirculation system flow changes the coolant flow rate through the core, thereby changing the core power level. In response to certain transients, automatic runbacks or recirculation pump trips are initiated to reduce reactor power. The system can respond quickly to load transients and operator demands. The transient analysis confirms that no modifications for plutonium disposition are needed.

Standby Liquid Control System (SLCS): The Standby Liquid Control System provides a backup method to bring the nuclear reactor to subcriticality and to maintain subcriticality as the reactor cools. The system makes possible an orderly and safe shutdown in the event that not 
NEDO-32361

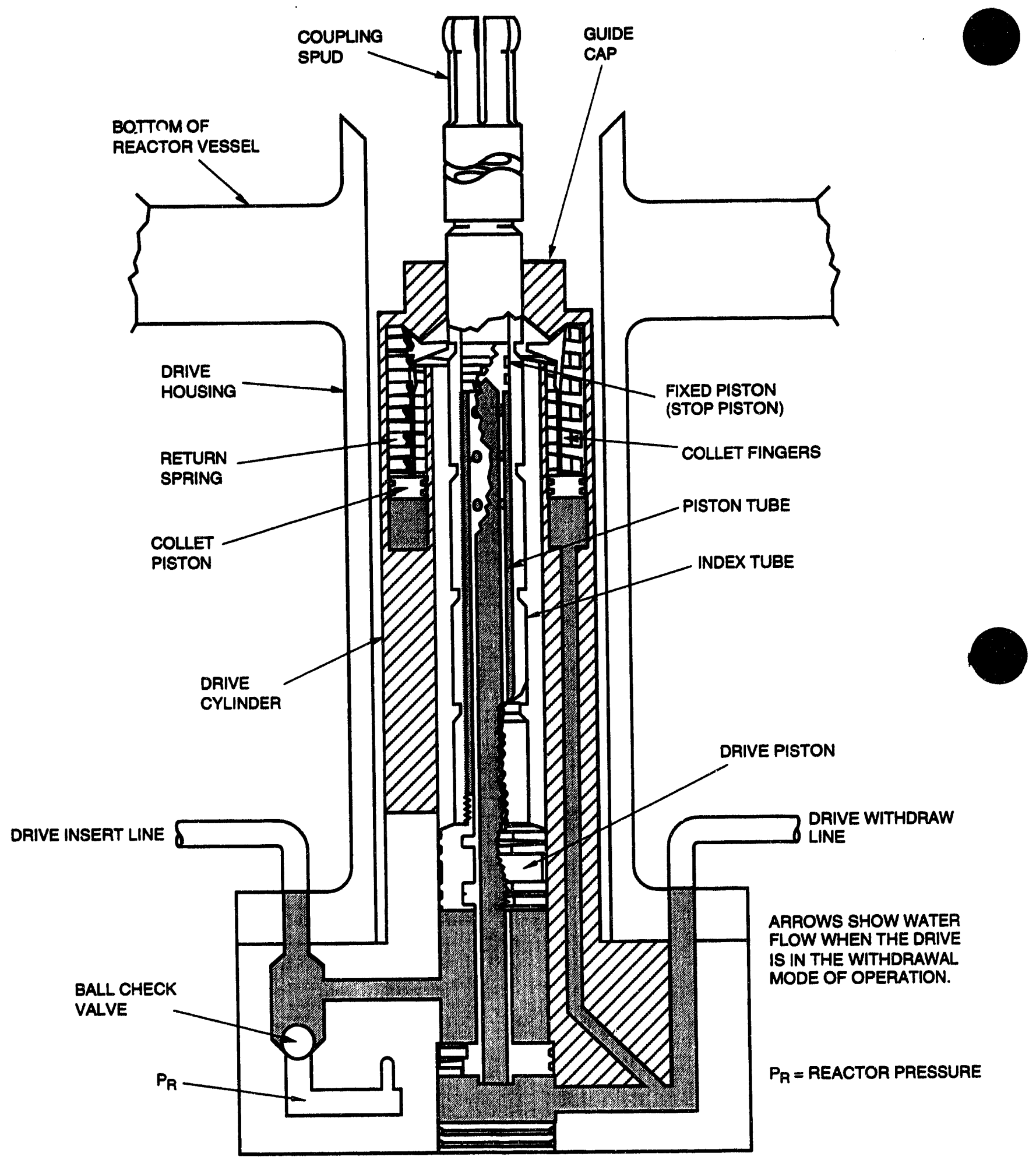

Figure 2.1-34 BWR Control Rod Drive Unit 


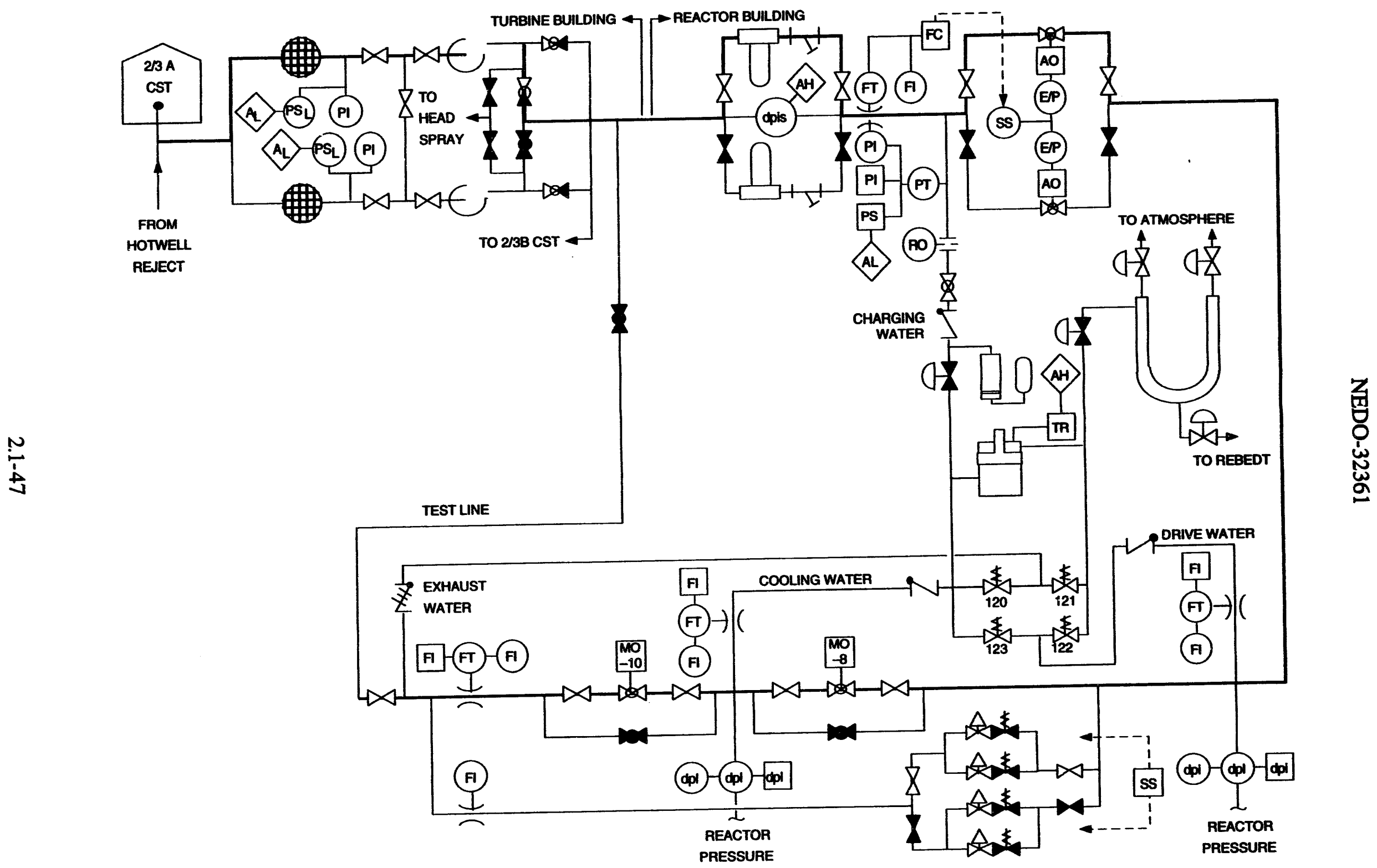

Figure 2.1-35 Control Rod Drive Hydraulic System 
enough control rods can be inserted into the reactor core to accomplish shutdown in the normal manner. The system is sized to counteract the positive reactivity effect of shutting down from rated power to cold shutdown condition. The SLCS is automatically initiated or can be manually initiated from the main control room to pump the neutron absorbing solution into the reactor.

The SLCS includes a boron solution tank, a test water tank, two positive displacement pumps, motor-operated injection and pump suction valves, and associated local piping, valves and controls in the Reactor Building outside the primary containment. The liquid is piped into the bottom of the reactor vessel through a dedicated penetration.

The boron absorbs thermal neutrons and thereby terminates the nuclear fission chain reaction in the fuel. The specified neutron absorber solution is sodium pentaborate. At all times, when it is possible to make the reactor critical, the SLCS will be able to deliver sufficient sodium pentaborate solution into the reactor to assure reactor shutdown.

No hardware upgrades or modifications are required to the current SLCS for use of MOX fuel. A comparison of the boron reactivity needed from the SLCS, as a function of exposure was reviewed for the reference full MOX core.

The boron worth is slightly lower in the MOX core in comparison with a $\mathrm{UO}_{2}$ core. This is due to the high absorption cross sections of plutonium relative to U-235, which results in a slight reduction in boron worth over the fuel lifetime. The analysis of the full MOX core shows that the core meets the SLCS requirement with a standard concentration of $660 \mathrm{ppm}$ boron.

If design uncertainties were to show that a change should be made, the enrichment of the sodium pentaborate solution can be increased slightly to account for the reduced worth. Increasing the enrichment of the sodium pentaborate solution in the storage tank is a routine operation and can be accomplished either by increasing the tank concentration or using a higher B-10 enrichment in the sodium pentaborate. A revision to the plant Technical Specifications, if warranted, would specify the new required concentration and would be determined during the fuel licensing process.

\subsection{Backup Safety Systems}

In the event of a breach in the reactor coolant pressure boundary (RCPB) that results in a loss of reactor coolant, independent divisions of the ECCS are provided to maintain fuel cladding below the temperature limit as defined by 10CFR50.46. The transient and accident analyses in 
Section 2.3 confirm that no modifications to the backup safety systems are needed for plutonium disposition.

High Pressure Core Cooling Systems: High pressure coolant makeup is provided by the High Pressure Coolant Injection (HPCI), High Pressure Core Spray (HPCS) and Reactor Core Isolation Cooling (RCIC) Systems (Table 2.1-10). Schematic diagrams of the systems are provided in Figures 2.1-36 through 38.

The high flow cooling systems (HPCI or HPCS) provide an adequate coolant inventory inside the reactor vessel to limit fuel cladding temperatures in the event of breaks in the reactor coolant boundary. The systems are initiated by either high pressure in the drywell or low water level in the vessel. The HPCS operates independently of all other systems over the entire range of system operating pressures; RCIC and HPCI System are steam turbine driven and have a minimum system operating pressure of about 150 psig.

The HPCS pump motors are powered by a dedicated emergency diesel generator if normal power is not available. The system may also be used as a backup for the reactor core isolation cooling system.

Low Pressure Core Cooling Systems: Low pressure coolant makeup is provided by the Low Pressure Coolant Injection (LPCI) or Low Pressure Core Spray (LPCS) Systems (Table 2.1-11). Schematic diagrams of the systems are provided in Figures 2.1-39 and 2.1-40. In order to utilize the low pressure systems in the event that high pressure systems are unavailable, an Automatic Depressurization System (ADS) is provided (Figure 2.1-41).

The ADS utilizes a subset of the safety/relief valves (SRVs) to depressurize the RPV. The SRVs used for ADS are set to open on detection of appropriate low reactor water level and high drywell pressure signals. To ensure that adequate coolant will be available to maintain water

Table 2.1-10 Typical High Pressure Coolant Makeup Systems

\begin{tabular}{|l|c|c|c|}
\cline { 2 - 4 } \multicolumn{1}{c|}{} & \multicolumn{3}{c|}{ Makeup Flow Rate/Pressure } \\
\hline System & BWR-4 & BWR-5 & BWR-6 \\
\hline High Pressure Core Spray & & $6250 \mathrm{gpm}$ & $6000 \mathrm{gpm}$ \\
(HPCS) & & $1000 \mathrm{psig}$ & $1000 \mathrm{psig}$ \\
\hline High Pressure Coolant Injection & $5600 \mathrm{gpm}$ & & \\
(HPCI) & $1120 \mathrm{psig}$ & & \\
\hline Reactor Core Isolation Cooling & $600 \mathrm{gpm}$ & $600 \mathrm{gpm}$ & $700 \mathrm{gpm}$ \\
(RCIC) & $1120 \mathrm{psig}$ & $1140 \mathrm{psig}$ & $1147 \mathrm{psig}$ \\
\hline
\end{tabular}



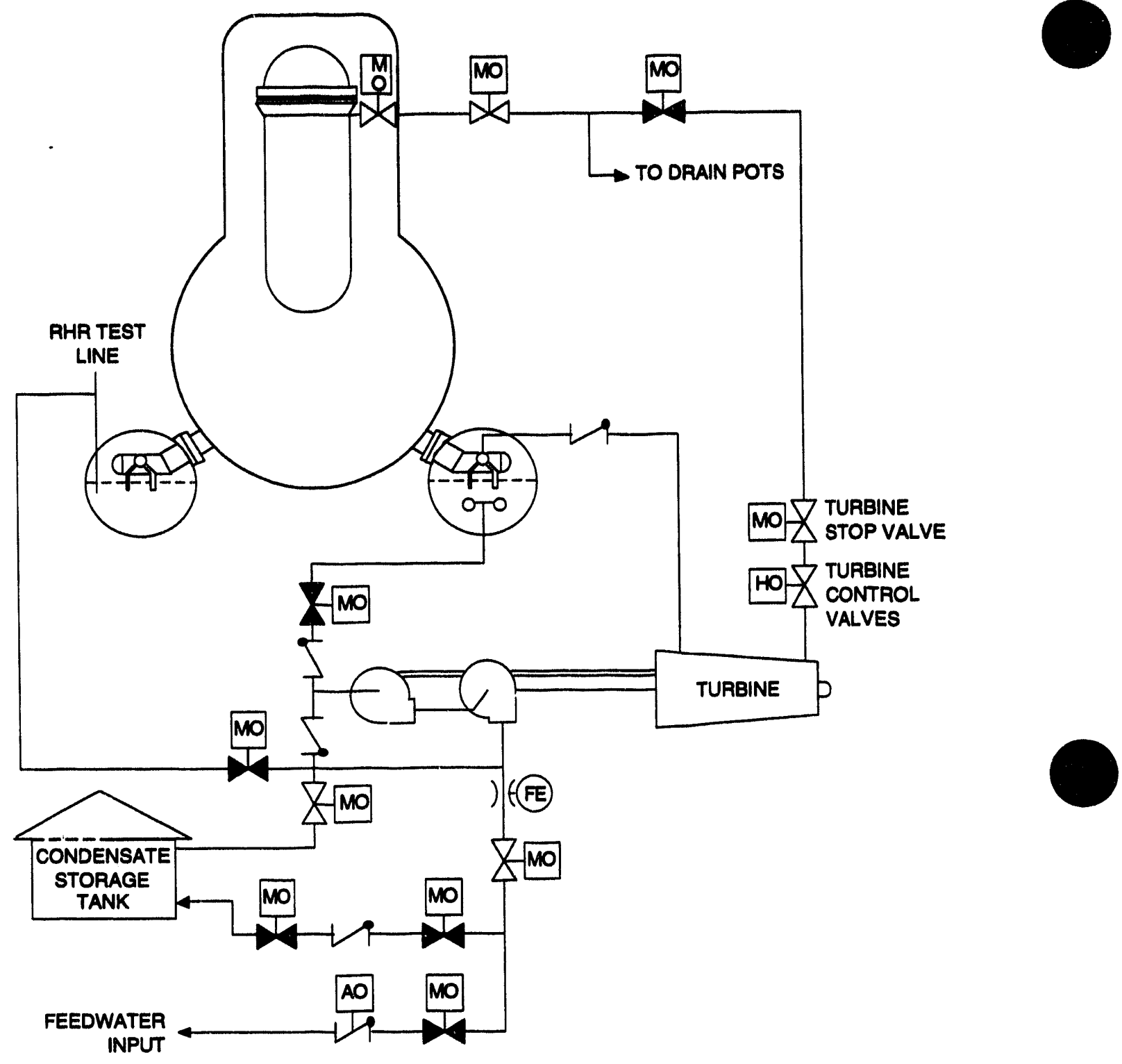

Figure 2.1-36 High Pressure Coolant Injection System 


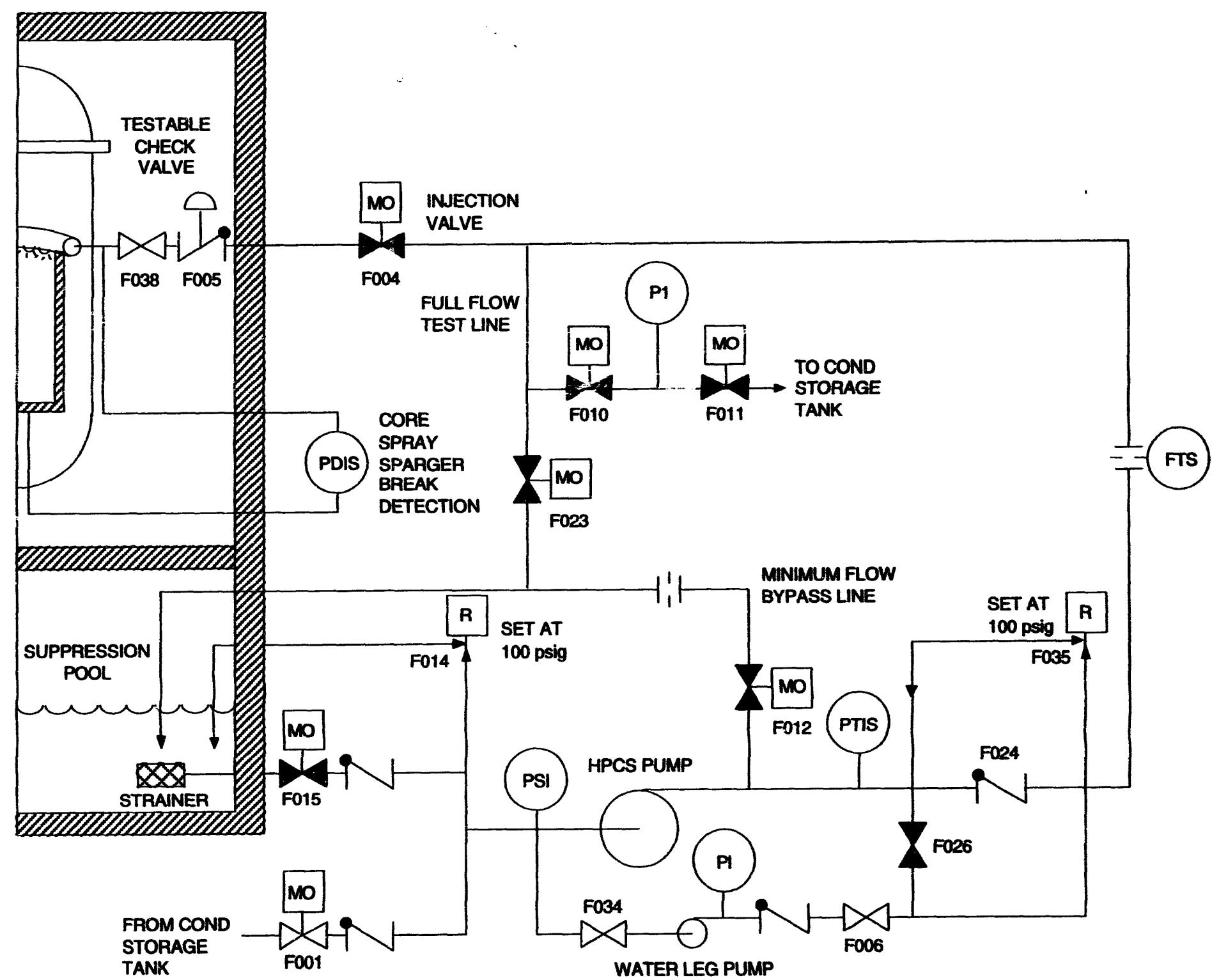

Figure 2.1-37 High Pressure Core Spray System 
NEDO-32361

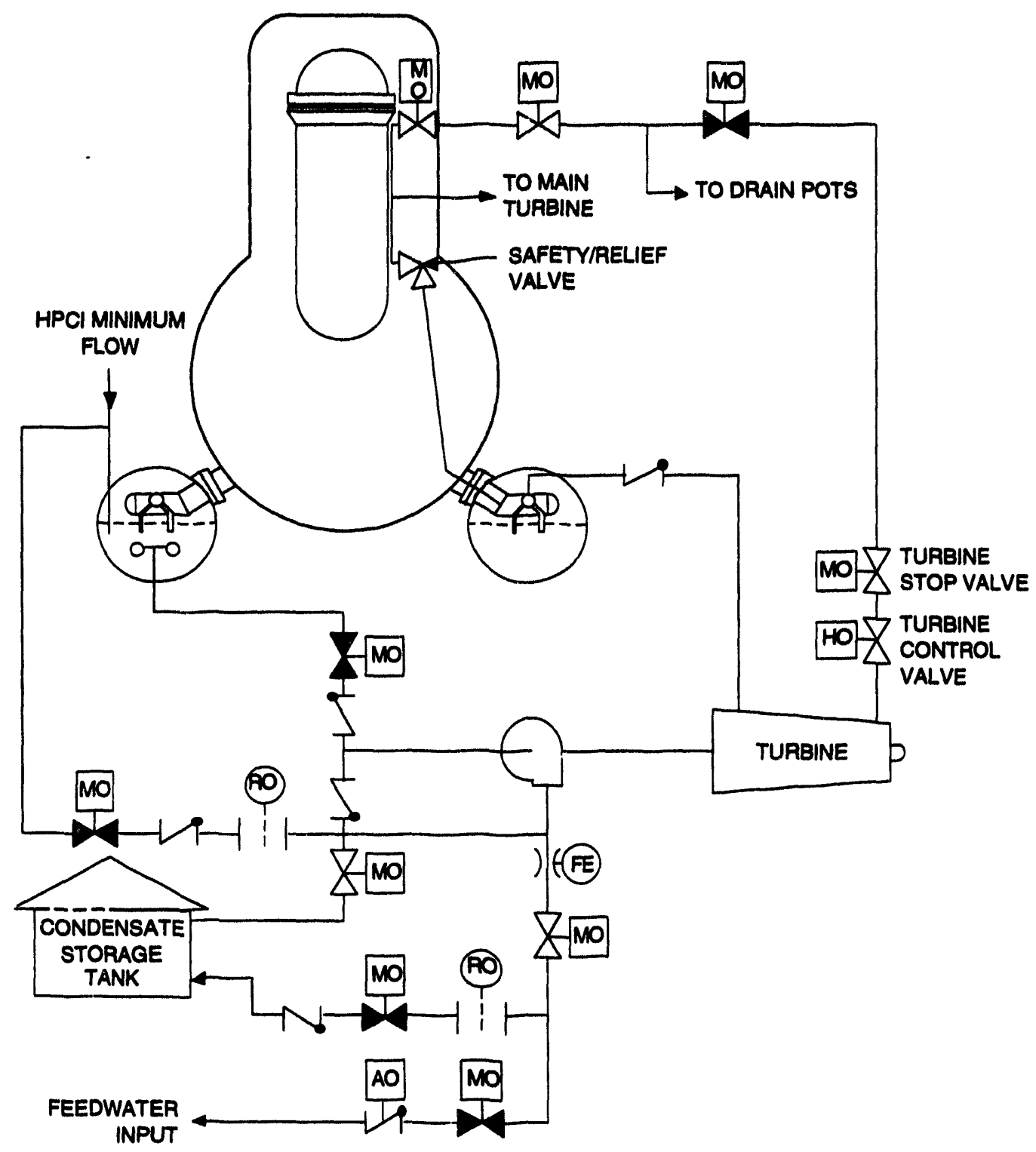

Figure 2.1-38 Reactor Core Isolation Cooling System 
NEDO-32361

Table 2.1-11 Low Pressure Coolant Systems

\begin{tabular}{|l|c|c|c|}
\cline { 2 - 4 } \multicolumn{1}{c|}{} & \multicolumn{3}{c|}{ Makeup Flow Rate/Pressure } \\
\hline System & BWR-4 & BWR-5 & BWR-6 \\
\hline Automatic Depressurization & 5 & 7 & 8 \\
System Valves & & & \\
\hline Low Pressure Coolant Injection & $10,000 \mathrm{gpm} / \mathrm{loop}$ & $10,600 \mathrm{gpm} / \mathrm{loop}$ & $6,500 \mathrm{gpm} / \mathrm{loop}$ \\
(LPCI) & $4 \mathrm{loops}$ & $2 \mathrm{loops}$ & $3 \mathrm{loops}$ \\
& $295 \mathrm{psig}$ & $225 \mathrm{psig}$ & $225 \mathrm{psig}$ \\
\hline Low Pressure Core Spray & $6250 \mathrm{gpm}$ & $6250 \mathrm{gpm}$ & $6000 \mathrm{gpm}$ \\
(LPCS) & $2 \mathrm{loops}$ & $1 \mathrm{loop}$ & $1 \mathrm{loop}$ \\
& $289 \mathrm{psig}$ & $289 \mathrm{psig}$ & $289 \mathrm{psig}$ \\
\hline
\end{tabular}

level after depressurization, the ADS will not be activated unless one or more low pressure cooling system is operating.

The Residual Heat Removal (RHR) System is a system of pumps, heat exchangers, and piping that fulfills the following functions: (1) Shutdown Cooling (SDC) - removes decay and sensible heat during and after plant shutdown; (2) LPCI - injects water into the reactor vessel following a loss-of-coolant accident to reflood the core in conjunction with other core cooling systems; and (3) Containment Spray - removes heat from the containment following a loss-of-coolant accident by cooling and recirculating the suppression pool water. The Core Spray System provides backup to the LPCI system, but does not provide the other functions. The RHR System pump motors are powered by the emergency diesel generators if normal power is not available.

Containment Cooling Systems: The Suppression Pool Cooling mode of RHR is placed in operation to: (1) limit the temperature of the water in the suppression pool following a design basis LOCA; (2) control the pool temperature during normal operation; and (3) reduce the pool temperature following a transient which results in operation of the safety/relief valves and/or the RCIC System. In this mode of operation, the RHR System pumps take suction from the suppression pool and pump the water through the RHR heat exchangers, where cooling takes place by transferring heat to the ser :ice water. The fluid is then discharged back to the suppression pool. In the Containment Spray mode, the RHR System is aligned to reduce high containment pressure and provide primary containment atmospheric cooling following a LOCA.

The Shutdown Cooling mode of RHR removes residual heat after reactor shutdown in preparation for refueling or nuclear system servicing. This mode is initiated and shut down manually. The flow path is from the vessel through the reactor shutdown cooling lines, through the RHR heat exchangers and back to the vessel. 


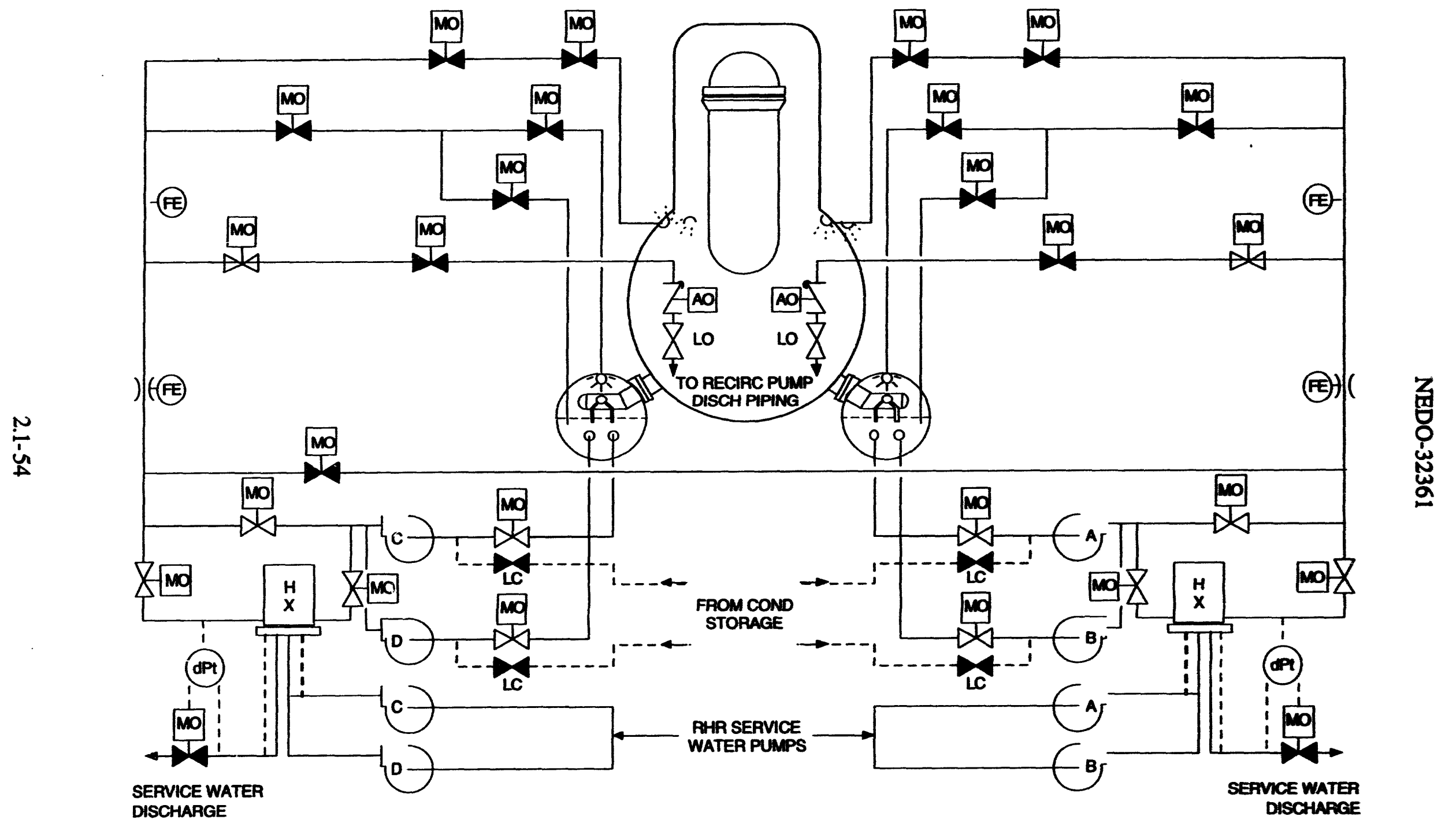

Figure 2-1-39 Low Pressure Coolant Injection System and Containment Spray/Cooling 


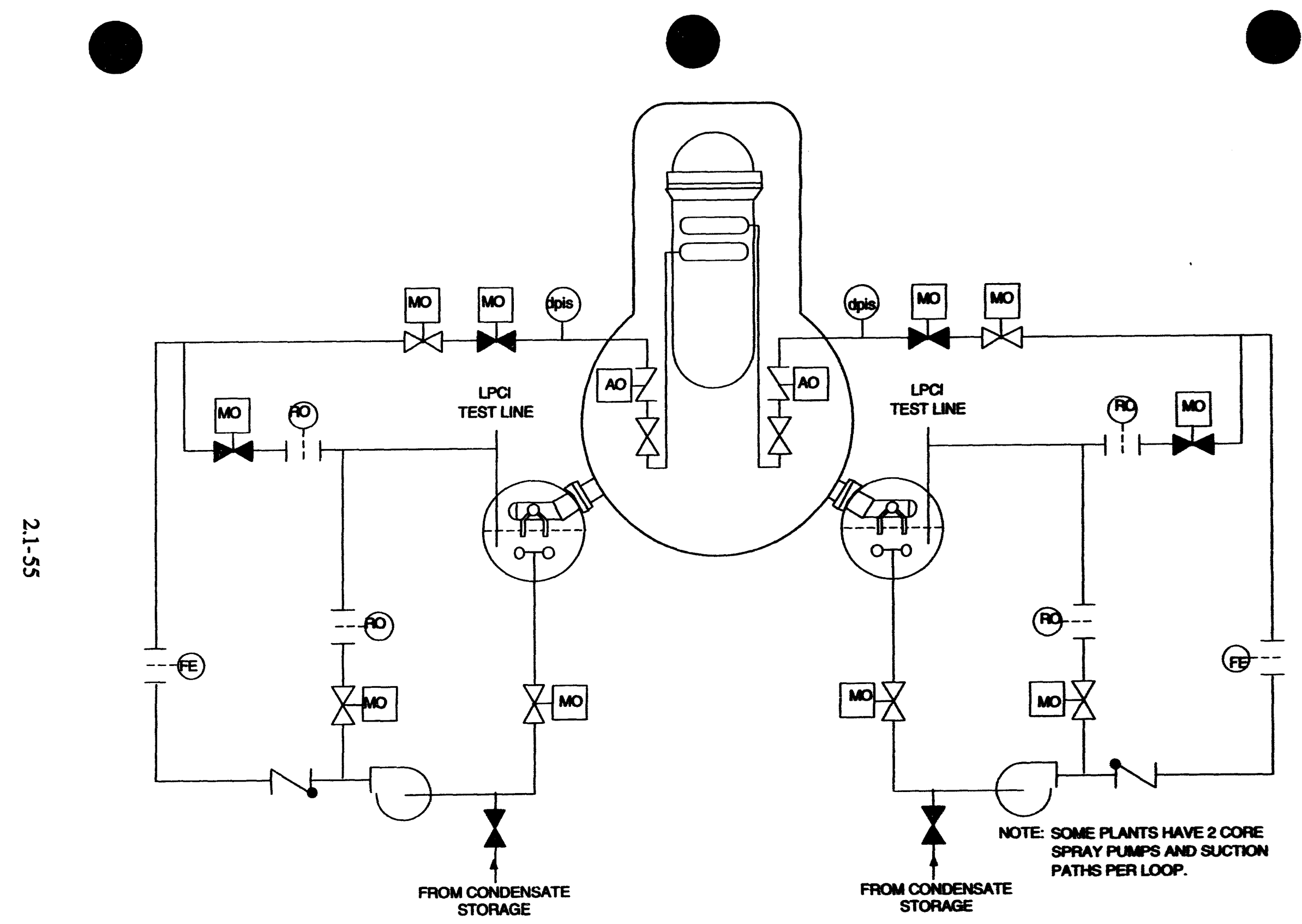

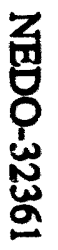

Figure 2-1-40 Core Spray System 
NEDO-32361

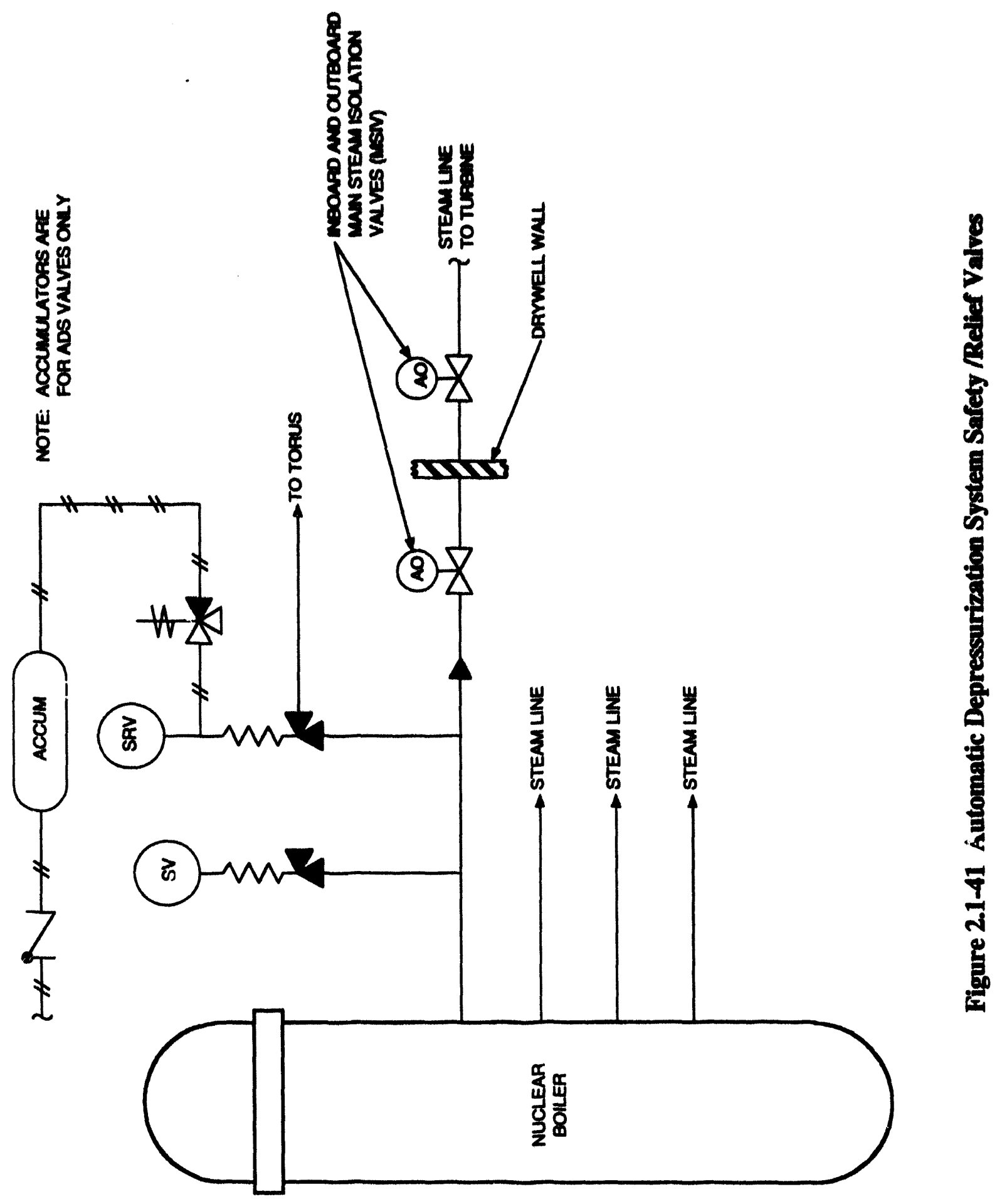




\subsection{Balance of Plant and Infrastructure}

\subsubsection{Balance of Plant}

\subsubsection{Power Production Systems}

Powe: production systems transfer steam from the reactor to the turbine generator for the production of electrical power. Data associated with power production in typical BWR-4, BWR-5 and BWR-6 plants are summarized in Table 2.2-1. Additional details on specific commercial BWRs are provided in Appendix D. The disposition of plutonium does not affect the rated power in commercial BWRs and no modifications are needed to power production systems for plutonium disposition.

The following systems constitute the power production systems common to commercial BWRs:

- Main Steamiline Systems: The main steamlines direct the flow of steam from the reactor vessel to the main turbine.

- Nuclear System Pressure Relief System: The pressure relief system consists of safety/relief valves mounted on the main steamlines and is provided to prevent excessive pressure inside the nuclear system as a result of operational transients or accidents. Typical numbers of relief valves are shown in Table 2.2-1.

- Main Steamline Isolation Valves (MSIVs): Each main steamline is provided with two isolation valves in series, one on each side of the primary containment barrier. All pipelines that penetrate the containment and offer a potential release path for radioactive material are provided with redundant isolation capabilities. Automatic isolation valves are provided in

Table 2.2-1 Power Production Data

\begin{tabular}{|l|c|c|c|}
\hline Parameter Description & BWR-4 & BWR-5 & BWR-6 \\
\hline Thermal Power Level (MWt) & 3293 & 3484 & 3579 \\
\hline Gross turbine Output (MWe) & 1092 & 1230 & 1200 \\
\hline Thermal Efficiency (\%) & 33.2 & 35.2 & 33.5 \\
\hline Steam Flow (Mlb/hr) & 14 & 15 & 15 \\
\hline Feedwater Temperature ( ${ }^{\circ}$ ) & 420 & 421 & 420 \\
\hline Vessel Dome Pressure (psig) & 1005 & 1020 & 1010 \\
\hline Mainsteam Relief Valves & 14 & 18 & 19 \\
\hline Turbine Bypass Capacity (\%) & 25 & 25 & 40 \\
\hline Feedwater Heater Drains & Cascade & Cascade & Pump Fwd \\
\hline
\end{tabular}



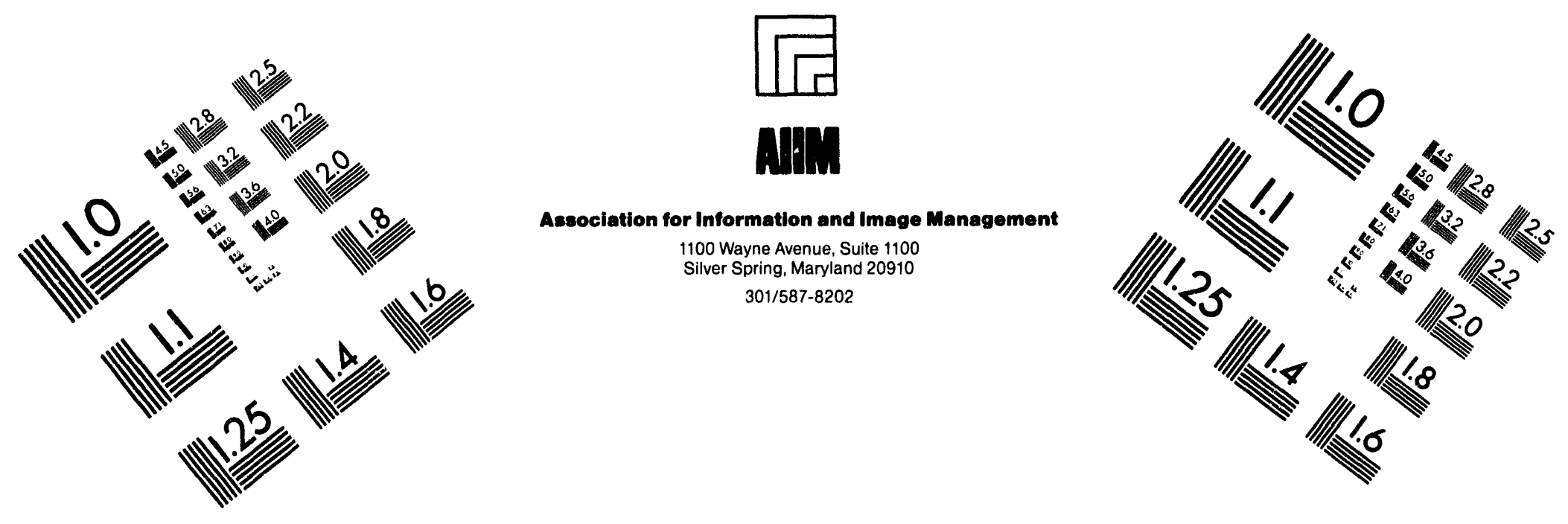

\section{Centimeter}

$\begin{array}{llllllllllllllll}1 & 2 & 3 & 4 & 5 & 6 & 7 & 8 & 9 & 10 & 11 & 12 & 13 & 14 & 15 & 15 m\end{array}$

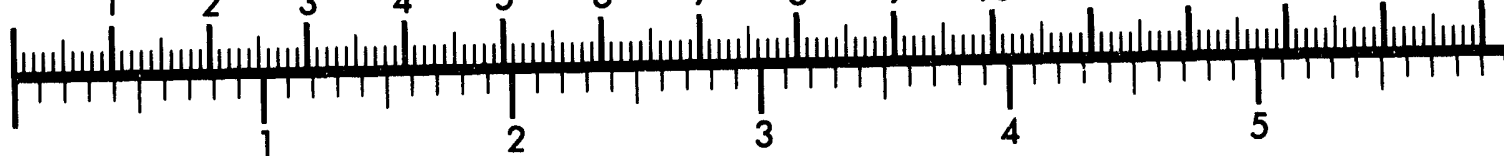
Inches
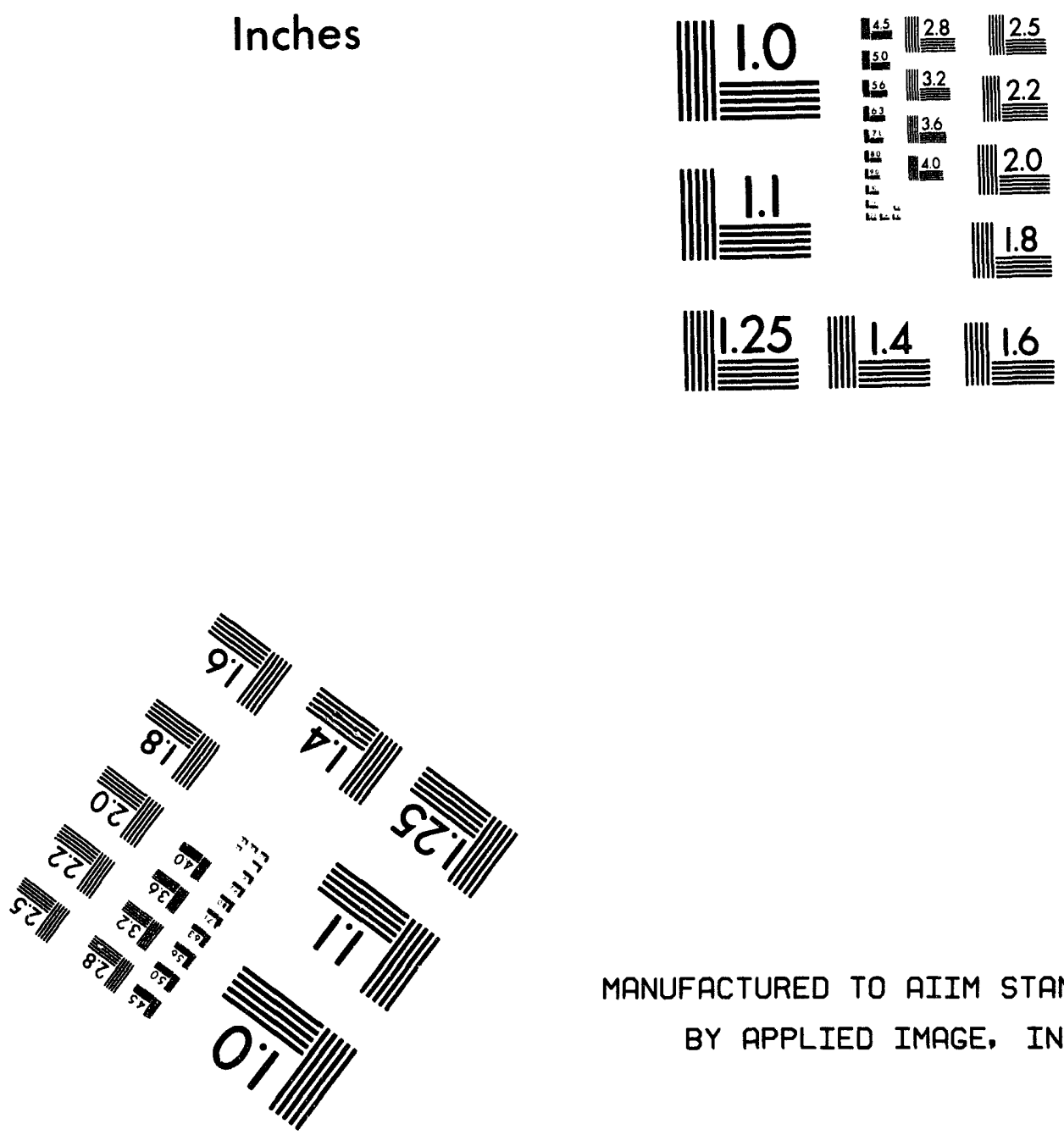

MANUFACTURED TO AIIM STANDARDS BY APPLIED IMAGE. INC.

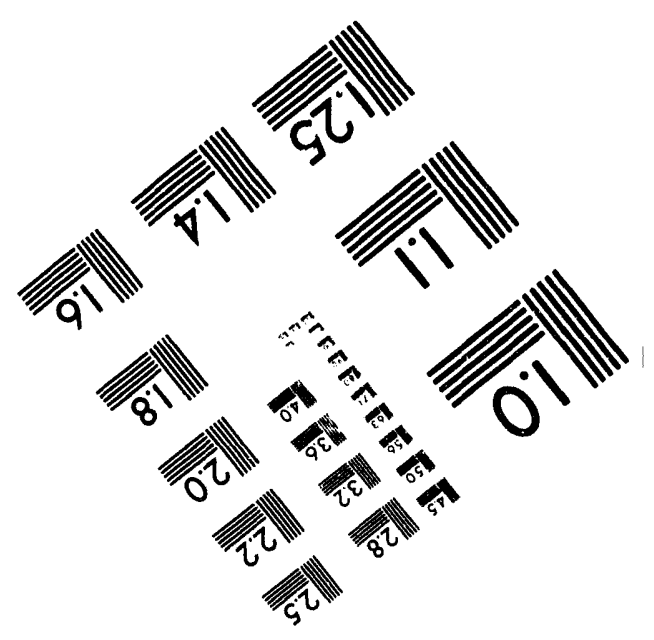



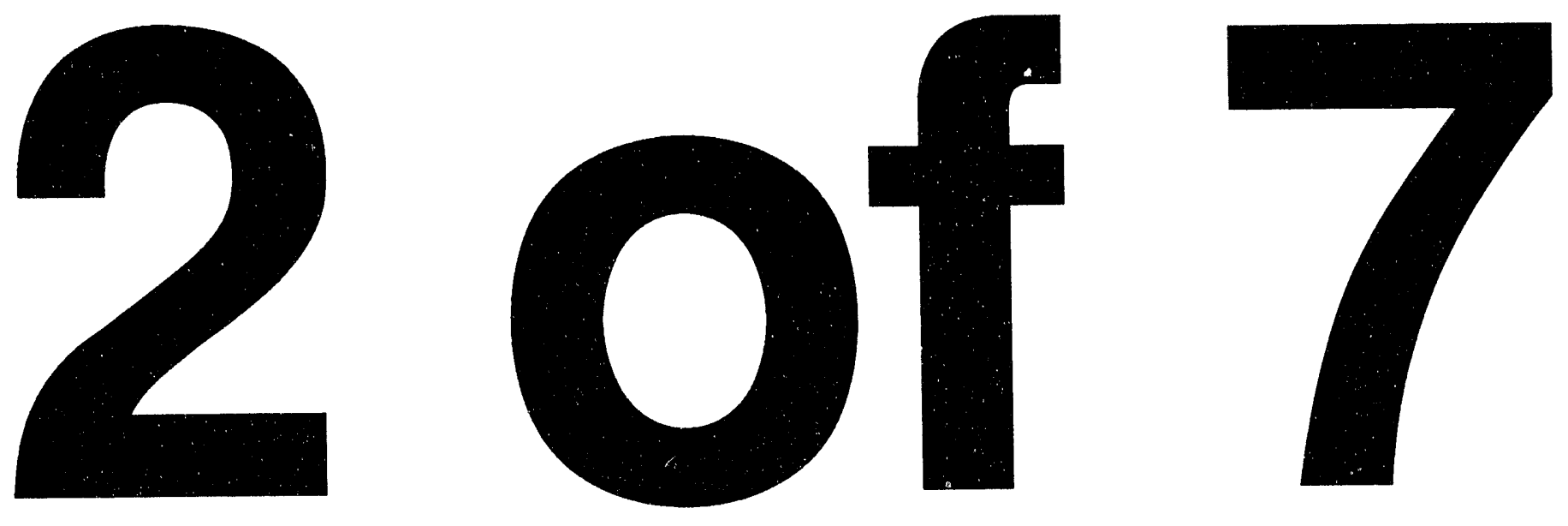
each main steamline. Each is powered by steam pressure, spring force and/or high pressure nitrogen gas. The typical closure period is three to five seconds. These valves fulfill the following objectives: (1) prevent excessive damage to the fuel barrier by limiting the loss of reactor coolant from the reactor vessel resulting from either a major leak from the steam piping outside the containment or a malfunction of the pressure control system resulting in excessive steam flow from the reactor vessel; and (2) limit the release of radioactive materials by isolating the reactor coolant pressure boundary in case of the detection of high steamline radiation.

- Turbine Main Steam System: The Main Steam System (MSS) delivers steam from the reactor to the curbine generator, the reheaters, the turbine bypass system, and the steam jet air ejectors from warmup to full-load operation. The MSS also supplies the steam seal system and the auxiliary steam system when other sources are not available.

- Main Turbine: The main turbines operate at $1800 \mathrm{rpm}$ and are equipped with an electrohydraulic control system and supervisory instruments to monitor performance. Both high pressure and low pressure turbines are provided to achieve the typical gross electrical output of the turbine generator is shown in Table 2.2-1.

- Turbine Control System: The turbine generators use an electro-hydraulic (EHC) control system which, in coordination with the turbine steam bypass and pressure control system, controls reactor pressure during startup, normal operation and operational transients. The EHC systems also operate the turbine stop valves, control valves, and combined intermediate valves. Turbine-generator supervisory instrumentation are provided for operational analysis and malfunction diagnosis. Automatic control functions are programmed to trip the turbinegenerator in response to turbine overspeed and excessive vibrations.

- Steam Bypass and Pressure Control System: A turbine bypass system is provided to pass steam directly to the main condenser under the control of the pressure regulator. Steam is bypassed to the condenser whenever the reactor steaming rate exceeds the flow permitted to pass to the turbine generator. Typical bypass capacities are shown in Table 2.2-1. These capacities are normally sufficient to prevent operation of the main steamline safety/relief valves. The pressure regulation systems provide main turbine control valve and bypass valve flow demands so as to maintain a nearly constant reactor pressure during normal operation. They also provide demands to the recirculation system to adjust power level by changing recirculation flow rate. 
- Moisture Separator Reheater: Four horizontal cylindrical-shell, combined moisture separator/reheaters are installed in the steam paths between the high and low pressure turbines. The moisture separator/reheaters serve to dry and reheat the high pressure turbine steam exhaust before it enters the low pressure turbines to improve cycle efficiency and reduces moisture-related erosion and corrosion in the low pressure turbines. Moisture is removed in chevron-type moisture separators, and is drained to the moisture separator drain tank and from there to the heater drain tank. The dry steam passes upward across there heater which is supplied with main steam. Finally, the reheated steam is routed to the combined intermediate valves which are located upstream of the low pressure turbines inlet nozzles.

- Turbine Gland Steam System: The Turbine Gland Steam System provides steam to the turbine glands and the turbine valve stems. The Turbine Gland Steam System prevents leakage of air into or radioactive steam out of the turbine shaft and turbine valves. The gland steam condenser collects air and steam mixture, condenses the steam, and discharges the air leakage to the atmosphere via the main vent by a motor-driven blower.

- Turbine Lubricating Oil System: The Turbine Lubricating Oil System supplies oil to turbine-generator bearing lubrication lines and mainly consists of a lube oil tank, oil pumps, oil coolers, and oil purifier equipment.

- Turbine Auxiliary Steam System: The Turbine Auxiliary Steam System supplies steam to the steam jet air ejectors for condenser deaeration and to the Turbine Gland Seal System, which prevents radioactive steam leakage out of the turbine casing and atmospheric air leakage into the casing during turbine operation. The house boiler steams provide a backup to the reactor generated steam during operation and would be used only when reactor steam is unavailable or too radioactive.

- Extraction System: Extraction steam from the high pressure turbine supplies the last stage (high pressure) feedwater heating and extraction steam from the low pressure turbines supplies the low pressure feedwater heaters.

- Generator: The generators are direct-driven, three-phase, $60 \mathrm{~Hz}, 27,000 \mathrm{~V}, 1800 \mathrm{rpm}$, conduction cooled, synchronous generators. At rated reactor power conditions, the gross electrical output and thermal efficiency from typical plants are shown in Table 2.2-1.

- Generator Cooling System: The Generator Cooling System includes the hydrogen-cooled rotor portion of the Hydrogen Gas Cooling System and the water-cooled stator portion of the Turbine Building Cooling Water System. 
- Generator Sealing Oil System: The Generator Sealing Oil System prevents hydrogen gas from leaking from the generator. The sealing oil is vacuum-treated to maintain the gas purity.

- Exciter: Main turbine-generator excitation power is provided by output of the alternatorexciter. This output is rectified by the stationary silicon-diode rectifiers. The direct current output of the rectifier banks then is applied to the main generator field through generator collectors.

- Heater, Drain and Vent System: The Heater, Drain and Vent System permits efficient and dependable operation of the heat cycle balance-of-plant equipment and, particularly, the condensate and feedwater heaters. All process equipment drains and vents are collected and routed to the appropriate points in the cycle and flows are controlled for equipment protection.

- Main Condenser: The main condensers are multipressure and multishell deaerating type condensers. During plant operation, steam expanding through the low pressure turbines is directed downward into the main condenser and is condensed. The main condenser also serves as a heat sink for the turbine bypass system, emergency and high level feedwater heater and drain tank dumps, and various other startup drains and relief valve discharges.

- Circulating Water System: The Circulating Water System provides a continuous supply of cooling water to the main condenser to remove the heat rejected by the steam cycle. The Circulating Water System consists of the following components: screen house and intake screens, condenser water boxes and water box fill and drain subsystem, and related support facilities such as for water treatment and general maintenance.

The Circulating Water System is normally designed to deliver water to the main condenser within a temperature range of 40 to $100^{\circ} \mathrm{F}$. The $40^{\circ} \mathrm{F}$ minimum temperature is maintained, when needed, by warm water recirculation. Heat is removed from the circulating water by means of a cooling tower or discharge to a lake or river.

The cooling water is normally circulated by fixed-speed motor-driven pumps. The pumps are arranged in parallel and discharged into a common header. The discharge of each pump is fitted with a butterfly valve. This arrangement permits isolation and maintenance of any one pump while the others remain in operation. The circulating water systems and condenser are designed to permit isolation of each set of the three series-connected tube-bundles to permit repair of leaks and cleaning of water boxes while operating at reduced power. 
- Condensate and Feedwater System: The Condensate and Feedwater System provides a dependable supply of high-quality feedwater to the reactor at the required flow, pressure, and temperature. The condensate pumps take the deaerated condensate from the main condenser hotwell and deliver it through the steam jet air ejector condenser, the gland steam condenser, the offgas condenser, the condensate demineralizer, and through parallel strings of low pressure feedwater heaters to the reactor feed pump suction.

The reactor feed pumps are either motor or steam turbine-driven and discharge through parallel strings of high-pressure heaters to the reactor. The drains from the high-pressure heaters are cascaded backward to the suction of the feed pumps or pumped forward to the reactor as indicated in Table 2.2-1.

The feedwater control system automatically controls the flow of feedwater into the reactor pressure vessel to maintain the water within the vessel at predetermined levels. A threeelement (steam flow, feedwater flow and RPV level) control scheme, which provides control signals to feedwater pumps, is used to accomplish this function.

- Condensate Purification System: BWRs are equipped with a $100 \%$ capacity condensate cleanup system, consisting of filter-demineralizers and, in some plants, deep bed demineralizers. The condensate cleanup system, with instrumentation and automatic controls, is designed to ensure a constant supply of high-quality water to the reactor.

\subsubsection{Instrumentation and Controls}

With the exception of the process computer and stability monitors, no significant instrumentation or control system changes are needed for the disposition of plutonium. The following sections describe commercial BWR instrumentation and controls. To address safeguards considerations, additional control systems for tracking of fuel assemblies also may be needed.

\subsection{Main Control Rooms}

Commercial BWR main control rooms have evolved in response to human factors considerations, especially following the accident at Three Mile Island (TMI). The primary controls are located on the reactor control, emergency core cooling, turbine control and balanceof-plant benchboards. Control parameter indications are located on vertical sections of the benchboards and alarms are provided above the vertical sections. Control panels and displays associated with automatic control functions are typically provided in other locations in the control room or the auxiliary relay rooms. 
The main control consoles provide the controls and displays required to operate the plant during normal plant operations, abnormal events and emergencies. These main control consoles provide controls and displays for both safety and non-safety system monitoring. The main control console is also equipped with dedicated displays for selected functions (e.g., the SLCS and the synchronization of the main generator to the electrical grid).

In addition to the above equipment, the main control consoles are equipped with both intra-plant and external communications equipment and laydown spaces for hard copies of procedures and other documents required by the operators during performance of their duties.

Critical plant parameter information necessary for emergency procedures and post-accident monitoring is also provided on separate computerized displays in the control room and in technical support centers.

\subsection{Reactor Vessel Instrumentation}

In addition to instrumentation for the nuclear safety systems and engineered safety features, instrumentation is provided to monitor and transmit information that can be used to assess conditions existing inside the reactor vessel and the physical condition of the vessel itself. This instrumentation monitors reactor vessel pressure, reactor water level, coolant temperature, reactor core differential pressure, coolant flow rates, and reactor vessel head seal leakage.

The steam flow instrumentation is connected to the venturi-type steam nozzle of the reactor pressure vessel. This instrumentation provides high nozzle flow isolation signals in case of a main steamline break.

\subsection{Leak Detection and Isolation System}

The Leak Detection and Isolation System (LD\&IS) consists of temperature, pressure, flow, and fission-product sensors with associated instrumentation, alarm, and isolation functions. The LD\&IS detects and annunciates leakage and provides signals to close containment isolation valves, as required, in (1) main steamlines, (2) the Reactor Water Cleanup System, (3) Residual Heat Removal System, (4) Reactor Core Isolation Cooling System, (5) Emergency Core Cooling Systems; and (6) other miscellaneous systems.

Small leaks in the containment or in the Reactor Building generally are detected by monitoring the air cooler condensate flow, radiation levels, equipment space temperature, and drain sump 
fill-up and pump-out rates. Large leaks are also detected ty changes in reactor water level, drywell pressure, and changes in flow rates in process lines.

\subsection{Suppression Pool Temperature Monitoring System}

The Suppression Pool Temperature Monitoring (SPTM) System is provided to monitor temperature at various quadrants and elevations in the suppression pools. Monitoring of suppression pool temperature is provided so that trends in suppression pool temperature can be established in sufficient time for proper cooling of the suppression pool water or for appropriate operator actions for suppression pool temperature control based upon symptom-based emergency operating procedures.

\subsection{Reactor Protection System}

The Reactor Protection System (RPS) consists of instrument channels, trip logics, trip actuators and scram logic circuitry that initiate rapid insertion of control rods (scram) to shut down the reactor. The RPS also establishes reactor operating modes and provides status and control signals to other systems and annunciators. To accomplish its overall function, the RPS interfaces with the Neutron Monitoring System, Process Radiation Monitoring System, Control Rod Drive System, Rod Control and Information System, Reactor Recirculation System, process computer, Nuclear Boiler System and other plant systems and equipment. The RPS acts in time to prevent fuel cladding damage and any nuclear system process barrier damage following abnormal operational transients. The RPS overrides all operator actions and process controls and is based on a fail-safe design philosophy that allows appropriate protective action even in the event of a single failure.

\subsection{Neutron Monitoring System}

- Power Monitoring: The Neutron Monitoring System (NMS) consists of in-core neutron detectors and out-of-core electronic monitoring equipment which provide indication of neutron flux for the entire range of flux conditions that can exist in the core.

The startup range (SRM) and intermediate range (IRM) neutron monitors consist of retractable in-core detectors which provide indications during reactor startup and low power operation. Local power range monitors (LPRMs) provide fixed indications of local core flux levels and are combined in the average power range monitors (APRMs) to indicate overall flux conditions during power range operation. The automatic traversing in-core probe system (TIP) provides a means to calibrate the power range monitors. 
The SRMs, IRMs and APRMs provide inputs to the RC\&IS to initiate rod blocks if preset flux limits or period limits for rod block are exceeded as well as inputs to the RPS if other limits for scram are exceeded.

Those portions of the NMS that input signals to the RPS qualify as a nuclear safety system. The APRMs also generates a simulated thermal power signal in some plants.

Detection and suppression of reactor instabilities are provided by one of two stability monitors which are acceptable to meet NRC requirements. At the time this report is written, commercial BWRs are selecting which of the two options they intend to implement. Implementation of a stability monitor is expected by 1997 . One approach (Option Ia) excludes a zone of operation in the power-flow map. During power ascension by pulling control rods, if the operator attempts to increase power above a specified point, an automatic rod block is issued by the control system, which prevents further rod withdrawal until the minimum pump speed is achieved. The other approach (Option III) uses LPRM signals to monitor the local power and initiate a scram if significant oscillations are detected. With the use of MOX fuel, option Ia designs will require a modification to its exclusion zone definition. Option III designs will not require modification. Section 2.3 discusses modifications to the exclusion zone associated with use of MOX fuel.

- Rod Worth Minimizer/Rod Sequence Control System: These systems electronically monitor control rod positions and initiate a control rod withdrawal block when the rod sequence and positions are outside of the bounds of the control rod drop safety analysis. No change to the system is required for the plutonium disposition mission. Evaluation of operational setpoints is evaluated as part of the fuel reload evaluation.

\subsection{Radiation Monitoring System}

- Process Radiation Monitoring System: The Process Radiation Monitoring System (PRMS) monitors radioacti ity in process and effluent streams and activate appropriate alarms and controls. The PRMS indicates and records radiation levels associated with selected plant process streams and effluent paths leading to the environment. All effluents from the plant which are potentially radioactive are monitored.

- Area Radiation Monitoring System: The Area Radiation Monitoring System (ARMS) alerts occupants and the control room personnel of excessive gamma radiation levels at selected locations within the plant. 
- Containment Atmospheric Monitoring: The Containment Atmospheric Monitoring System (CAMS) measures, alarms and records radiation levels and the oxygen concentration in the primary containment under post-accident conditions. It is automatically put in service upon detection of LOCA conditions.

\subsection{Plant Process Computer}

On-line process computers are provided to monitor and log process variables and make certain analytical computations. Modifications to the computer software associated with the MOX core are provided as part of the initial fuel load.

\subsection{Remote Shutdown System}

In the event that the control room becomes inaccessible, the reactor can be brought from power range operation to cold shutdown conditions by use of controls and equipment that are available outside the control room.

Manual transfer devices are provided which override controls from the main control room and transfer controls to remote shutdown control. Control and process sensor signals are interrupted by the transfer devices. All necessary power supply circuits are also transferred to other sources. Operation of the transfer devices causes an alarm in the main control room; access to the remote shutdown control panels (outside the main control room) is administratively and procedurally controlled. Instrumentation and controls on the remote shutdown panels include the following:

- Controls and indications for operation of high pressure and low pressure systems to control reactor water level.

- Controls and indications for operation of shutdown cooling once reactor pressure has been reduced, and suppression pool cooling to control suppression pool temperature which may rise due to SRV operation.

- Controls for operation of safety/relief valves sufficient for maintaining and reducing reactor pressure.

- Indications of reactor pressure vessel water level and pressure, and suppression pool temperature and level.

- Controls and indications for operation of the auxiliary systems. 
NEDO-32361

- Controls and indications for electrical power distribution.

- Controls for manually running and stopping emergency diesel generators.

\subsubsection{Electrical Distribution}

Onsite and offsite AC power distribution systems are provided to support normal and emergency operation. Although no modifications to the plant electrical distribution system are needed for plutonium disposition, there may be minor modifications to safeguards systems to assure reliable power.

- Offsite Electric Power System: The offsite electrical power systems are highly site specific, but have certain common characteristics in accordance with the General Design Criteria of 10CFR50. The systems include the switchyard, main power transformer from the main generator, the generator output breakers, and unit auxiliary transformers, connected to supply power to load groups of equipment including safety related buses. The "Normal Preferred" power feed is from the unit auxiliary transformers so that there normally are no bus transfers required when the unit is tripped off the line.

- Onsite Electric Power System: Safety-related equipment is powered from one of two independent electrical divisions. Turbine Building loads and one Reactor Building safetyrelated bus per electrical division receive power from a single auxiliary transformer. One winding of the reserve auxiliary transformer may also be utilized to supply reserve power to each of the non-safety-related buses either directly or indirectly through bus tie breakers. The safety-related buses also may be supplied power from the other winding of the reserve auxiliary transformer.

In general, motors larger than 300 kilowatts $(\mathrm{kW})$ are supplied from the 6.9 kilovolts $(\mathrm{kV})$ buses. Motors $300 \mathrm{~kW}$ or smaller but larger than $100 \mathrm{~kW}$ are supplied power from $480 \mathrm{~V}$ power center switchgear. Motors $100 \mathrm{~kW}$ or smaller are supplied power from $480 \mathrm{~V}$ motor control centers.

During normal plant operation, all of the non-safety buses and two of the safety-related buses are supplied with power from the turbine generator through the unit auxiliary transformers.

Diesel generators provide a separate onsite source of power for each safety-related bus when normal or alternate preferred power supplies are not available. The transfer from the normal preferred or alternate preferred power supplies to the diesel generator is automatic. The 
transfer back to the normal preferred or the alternate preferred power source is a manual transfer.

Any standby AC power system is capable of providing the required power to safely shutdown the reactor after loss of preferred power and/or loss-of-coolant accident or to maintain the safe shutdown condition and operate the safety related auxiliaries during and after shutdown.

The 125 VDC power distribution system provides independent and redundant on-site battery sources of power for operation of safety-related direct current loads. The 125 VDC nonsafety power is supplied from 125 VDC batteries.

The safety system and logic control for reactor protection system and MSIV isolation logic usually derives its power from uninterruptible $120 \mathrm{VAC}$ buses. The safety system and logic control for the ECCS derives its power from the safety related 125 VDC buses.

\subsubsection{Auxiliary Support Systems}

No modifications to the auxiliary support systems normally provided in BWRs are needed for plutonium disposition. The following brief descriptions summarize the system functions normally provided in BWRs.

- Reactor Water Cleanup System: The Reactor Water Cleanup (RWCU) System recirculates a portion of reactor coolant through a filter-demineralizer to remove particulate and dissolved impurities from the reactor coolant. It also removes excess coolant from the reactor system under controlled conditions during plant heatup and provides clean water for the head spray nozzle.

The RWCU System is a closed-loop system of piping, circulation pumps, a regenerative heat exchanger, non-regenerative heat exchangers, reactor water pressure boundary isolation valves, and two filter-demineralizers.

During normal plant operation, the RWCU System takes suction from the single bottom drain on the reactor pressure vessel and a recirculation system line, cools the water in the regenerative and non-regenerative heat exchangers, pumps it through the filter demineralizers, reheats it in the regenerative heat exchanger, and returns it to the reactor pressure vessel through the feedwater lines. During plant heatup, excess watei from reactor water swell can be directed to the main condenser or radwaste. 
- Process Sampling System: The Process Sampling System (PSS) provides process information that is required to monitor plant and equipment performance and changes to operating parameters. Representative liquid and gas samples are taken manually during plant operation for laboratory analyses.

- Atmospheric Control Systems: The Atmospheric Control System (ACS) is provided to establish and maintain an inert atmosphere within Mark I and Mark II containments during all plant operating modes except during shutdown for refueling or equipment maintenance, and during limited periods of time to permit access for inspection at low reactor power. A recombiner system is provided to control the concentration of hydrogen produced by radiolysis in the primary containment. The objective of the ACS is to preclude combustion of hydrogen and damage to essential equipment and structur ss.

- Drywell Cooling System: The Drywell Cooling System (DCS) is a recirculating system consisting of fan coil units. Cooling water comes from the Reactor Cooling Water System. Condensate that drips from the coils is routed to the drain system via the Leak Detection System. Instrumentation is installed in the drain line $t$. monitor cooler condensate flow and to provide input to the Leak Detection System.

The Drywell Cooling System supplies conditioned air to a common distribution header. The air/nitrogen is then ducted to areas within the drywell for equipment cooling. High drywell temperatures are alarmed in the main control room, alerting the operator to take appropriate corrective action.

- Standby Gas Treatment System: The Standby Gas Treatment System (SGTS) minimizes exfiltration of contaminated air from the secondary containment to the environment following an accident or abnormal condition which could result in abnormally high airborne radiation in the reactor building. In the event of an accident involving discharge of radioactive substances, the normal ventilation system will shutdown, and the radioactive substances which leaked in from the reactor containment vessel or elsewhere are passed through the filtering device and discharged through the plant stack.

- Makeup Water System: The Makeup Water System (MUWS) consists of a condensate storage tank, transfer pumps, and the necessary controls and instrumentation. The condensate storage tank receives demineralized water from the Condensate Water Makeup System and may also receive low conductivity water from the condensate return of the 
primary loop, from the radwaste disposal system and the condensate system in the Turbine Building.

- Service Water System: The Service Water System (SWS) provides cooling water during various operating modes, during shutdown and post-LOCA operations. The systems remove heat from the components cooled by the system and transfers it to the ultimate heat sink.

- HVAC Cooling Water Systems: The HVAC Normal Cooling Water Systems provide chilled water to the air supply cooling coils of the Reactor Building, to the heating/cooling coils in the drywell, and the Control Building electrical equipment room. The HVAC Emergency Cooling Water System provides chilled water to the cooling coils in the Control Building essential electrical equipment room, the main control room and the diesel generator electrical equipment areas.

- Reactor Building Cooling Water System: The Reactor Building Cooling Water (RBCW) System provides cooling water to certain designated equipment located in the Reactor Building, including the diesel generators, the RHR and fuel pool heat exchangers, and the high pressure and low pressure ECCS pump motors. Adequate capacity and redundancy is provided in heat exchangers and pumps to ensure performance of the cooling system. Under loss of offsite power, emergency power for the system is available from the onsite emergency diesel generators.

- Turbine Building Cooling Water System: The Turbine Building Cooling Water (TBCW) System cools non-safety-related loads, including the HVAC normal cooling water chillers, the feedwater, condensate, and heater drain pump motor coolers, and various turbine auxiliaries (generator stator coolers, hydrogen coolers, seal oil coolers, and exciter coolers).

- Station Heating, Ventilating and Air Conditioning: The plant environmental control systems control temperature, pressure, humidity, and airborne contamination to ensure the integrity of the plant equipment, provide acceptable working conditions for plani personnel, and limit offsite releases of airborne contaminants.

- Station Service Air System: The Station Service Air System (SSAS) provides a continuous supply of compressed air of suitable quality and pressure for general plant use. The service air compressor discharges into the air receivers and the air is then distributed throughout the plant. 
- Instrument Air System: The Instrument Air System (IAS) provides dry, oil-free, compressed air for valve actuators and for non-safety-related instrument control functions and for general instrumentation and valve services outside containment. All instrumentation and control systems located inside Mark I and Mark II containments are supplied with nitrogen gas during normal plant operation.

- High Pressure Nitrogen Gas Supply System: In Mark I and II containments, nitrogen gas is normally supplied to meet requirements on the main steam safety/relief valve automatic depressurization and relief function accumulators, the main steam isolation valves, and instruments and pneumatic valves using nitrogen in the Reactor Building. When this normal supply of pressurized nitrogen is not available, the High Pressure Nitrogen Gas Supply System (HPNSS) automatically maintains nitrogen pressure to this equipment. The system consists of high-pressure nitrogen storage bottles with piping, valves, instruments, controls, and control panel.

- Breathing Air System: The Breathing Air System (BAS) includes air compressors, dryers, purifiers and a distribution network. This network makes breathing air available in all plant areas where operations or maintenance must be performed and high radioactivity could occur in the ambient air. Special connections are provided to assure that this air is only used for breathing apparatus.

- Fire Protection System: The Fire Protection System (FPS) is designed to provide an adequate supply of water or chemicals to points throughout the plant where fire protection is required. Diversified fire-alarm and fire-suppression types are selected to suit the particular areas or hazards being protected. Chemical fire fighting systems are also provided as additions to or in lieu of the water fire-fighting systems. Appropriate instrumentation and controls are provided for the operation of the fire detection, annunciation and fire fighting systems.

\subsubsection{Waste Management Systems}

Waste management systems process radioactive waste for offsite disposal or recycling into the plant process streams. No modifications to these systems are needed for plutonium disposition.

- Liquid Radwaste Management System: The Liquid Radwaste Management System (LRMS) collects, monitors, and treats liquid radioactive wastes for return to the primary system whenever practicable. The BWR is designed to operate as a closed system with no routine discharges of liquid effluents from the reactor coolant and associated systems. The 
radwaste processing equipment is located in the Radwaste Building. Processed waste volumes discharged to the environs are expected to be small. Any discharge is such that concentrations and quantities of radioactive material and other contaminants are in accord with applicable local, state, and federal regulations.

All potentially radioactive liquid wastes are collected in sumps or drain tanks at various locations in the plant. These wastes are transferred to collection tanks in the radwaste facility.

Waste processing is normally done on a batch basis. Each batch is sampled as necessary in the collection tanks to determine concentrations of radioactivity and other contamination. Equipment drains and other low-conductivity wastes are treated by filtration and demineralization and are transferred to the condensate storage tank for reuse. Laundry drain wastes and other detergent wastes of low activity are treated by filtration, sampled and released via the liquid discharge pathway and may be released from the plant on a batch basis. Protection against inadvertent release of liquid radioactive waste is provided by design redundancy, instrumentation for the detection and alarm of abnormal conditions, automatic isolation, and administrative controls.

Equipment is selected, arranged, and shielded to permit operation, inspection, and maintenance with minimum radiation exposure to personnel.

- Solid Radwaste Management System: The Solid Radwaste Management System (SRMS) provides for the safe handling, packaging, and short-term storage of radioactive solid and concentrated liquid wastes that are produced. Wet waste produced by this system is transferred to the solidification system where it is solidified and loaded into containers. Dry active waste is surveyed and disposed of whenever possible. The remaining combustible waste is incinerated or compacted. Noncombustible dry waste is compacted and loaded into containers. Waste is shipped in containers for offsite disposal.

- Offgas System: The main condenser evacuation system removes the noncondensible gases from the main condenser and discharges them to the gaseous radwaste (offgas) system. The evacuation system consists of multiple-element, multi-stage steam jet air ejectors with intercondensers, for normal station operation, and mechanical vacuum pumps for use during startup.

The Offgas System receives air and noncondensible gases from the steam jet air ejectors and processes the effluent for the decay and/or removal of gaseous and particulate radioactive 
isotopes. The system processes the offgas and releases it to the environs through the plant stack, keeping the dose to the environment as low as practicable. The Offgas System also reduces the possibility of an explosion from the buildup of radiolytic hydrogen by the recombining -radiolytic hydrogen and oxygen under controlled conditions within a recombiner.

\subsubsection{Building Layout and Arrangement}

BWR plants include four main buildings: Reactor Building, Turbine Building, Control Building and Radwaste Building. In addition, Mark III plants (except Grand Gulf) contain a Fuel Building. The following paragraphs describe the contents of each building and the different containment types used in BWRs.

- Reactor Building: The Reactor Building houses the primary and secondary containments, and major portions of the Nuclear Steam Supply System, steam tunnel, refueling and fuel storage areas, diesel generators, essential power, nonessential power, emergency core cooling systems, HVAC System, and other supporting systems.

The secondary containment portion of the Reactor Building consists of the reactor area, which is exhausted by the Standby Gas Treatment System following accidents. The reactor area completely surrounds the primary containment. The interior is normally kept at subatmospheric pressure so that, even should there be leakage of radioactive substances from the reactor containment vessel, the direct discharge of radioactive material to the environs of the power station is prevented.

- Primary Containment: The primary containment system design for this plant incorporates the drywell/pressure suppression feature into a dry containment type structure. In fulfilling its design basis as a fission product barrier, the primary containment is a low leakage structure even at increased pressures that could follow a main steamline rupture or a fluid system line break. Penetrations through the liner for the drywell head, equipment hatches, personnel locks, piping, electrical and instrumentation lines are provided with seals and leak tight connections. The allowable leakage is about $0.5 \%$ per day from all sources.

- Mark I Containment: The Mark I containment is used on BWR-4 designs (some BWR-4s are surrounded by Mark II containments). The containment (Figure 2.2-1) consists of an inerted, steel construction drywell, downcomers and a torus-shaped suppression pool surrounding the drywell. 
In Mark I containments, new fuel is received through double secondary containment doors into an unloading area. A crane on the refueling floor lifts the new fuel through a vertical shaft to the refueling floor where it is inspected and placed in spent fuel storage pool awaiting loading into the reactor.

- Mark II Containment: The Mark II containment (Figure 2.2-2) differs from the Mark I containment in the arrangement of the suppression pool and its concrete construction. The torus is eliminated and replaced with a suppression pool under the drywell and vertical downcomers. Fuel handling in Mark II containments is similar to the Mark I containment designs.

- Mark III Containment: The Mark III containment (Figure 2.2-3) completely surrounds the reactor, refueling areas and several components contained in the Mark I and Mark II reactor buildings. Due to the need for maintenance of the equipment, the Mark III containment is not inerted during normal operation. Distributed igniters are provided for post accident hydrogen control. The drywell is vented to the suppression pool through a system of horizontal vents and a weir.

The Mark III containment contains limited new and spent fuel storage capacity. An inclined fuel transfer system is utilized to achieve an underwater transfer of fuel from the containment upper pools to the fuel building. New fuel is received in the Fuel Building in Mark III plants, inspected and stored in the fuel pools prior to transfer into the Mark III containment. Similarly, spent fuel is transferred to the fuel building for storage using the inclined fuel transfer system.

- Control Building: The Control Building houses the control room, the computer facility, the cable tunnels, some of the plant essential switchgear, the Reactor Building Cooling Water System, and the essential HVAC System.

- Turbine Building: The Turbine Building houses all equipment associated with the main turbine-generator and other auxiliary equipment. The turbine employs a conventional regenerative cycle with condenser deaeration and condensate demineralization. The turbinegenerator is equipped with an electrohydraulic control system and supervisory instruments to monitor performance. 
NEDO-32361

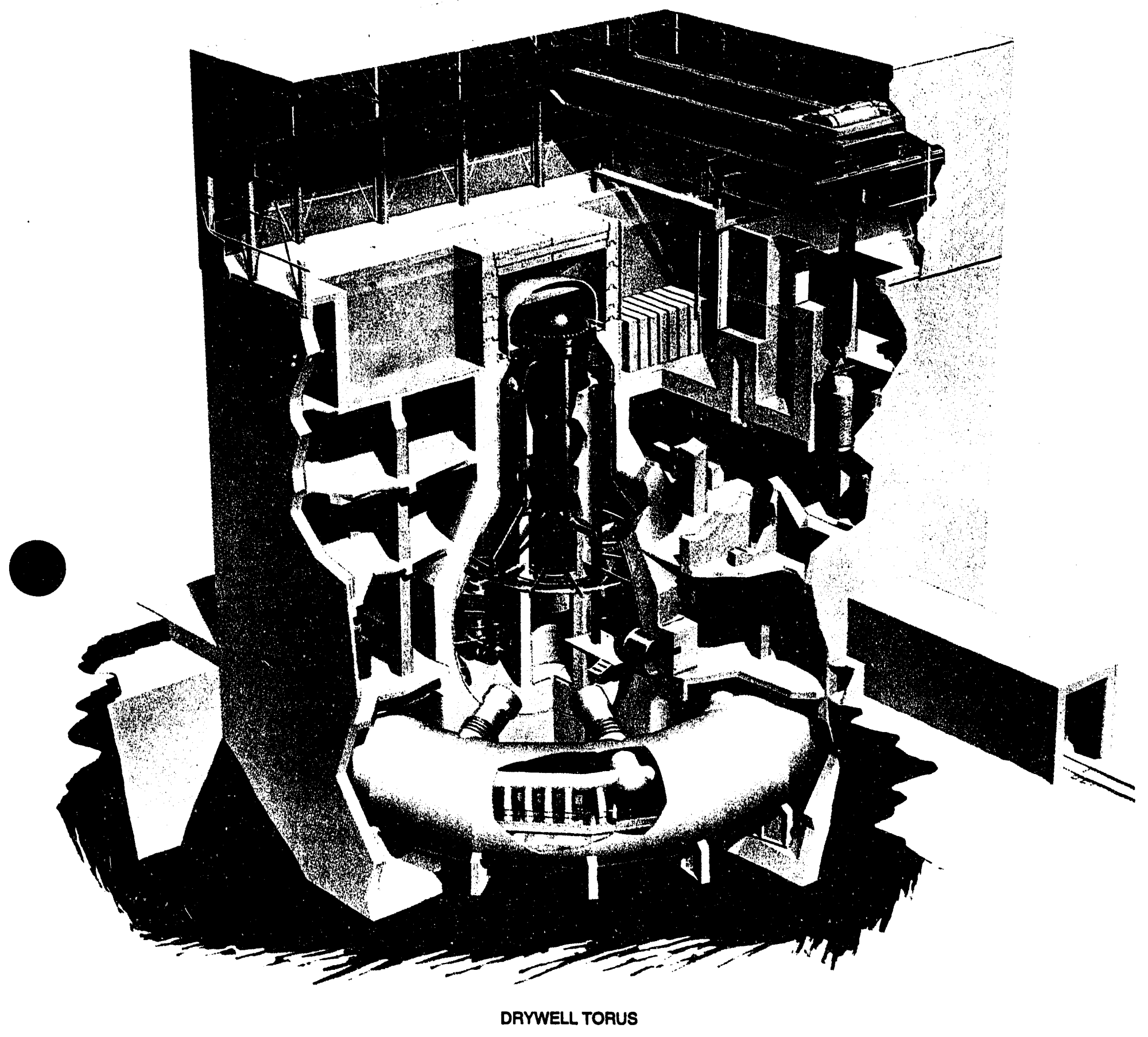

Figure 2.2-1 Mark I Containment 
NEDO-32361

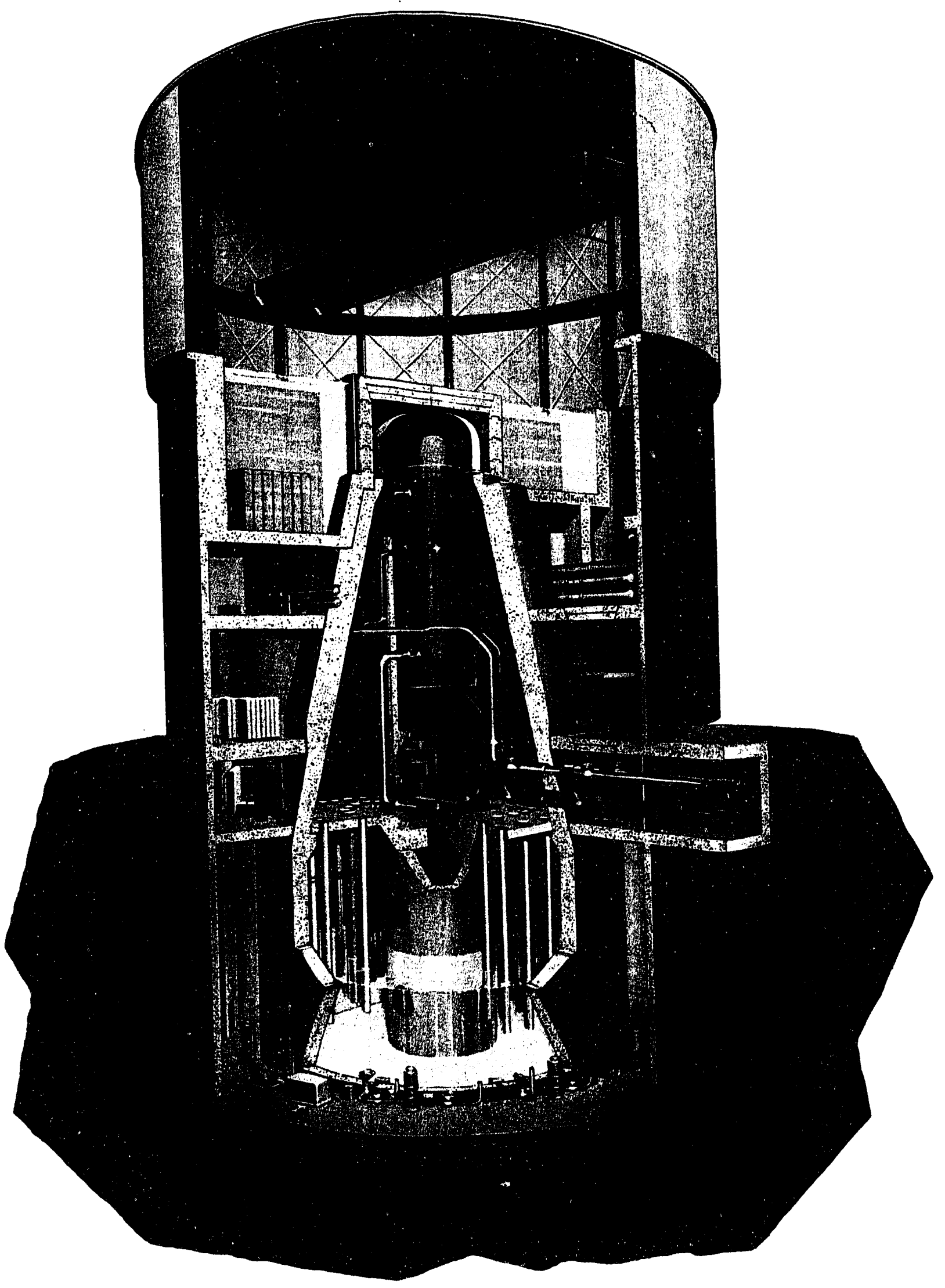

Figure 2.2-2 Mark II Containment 


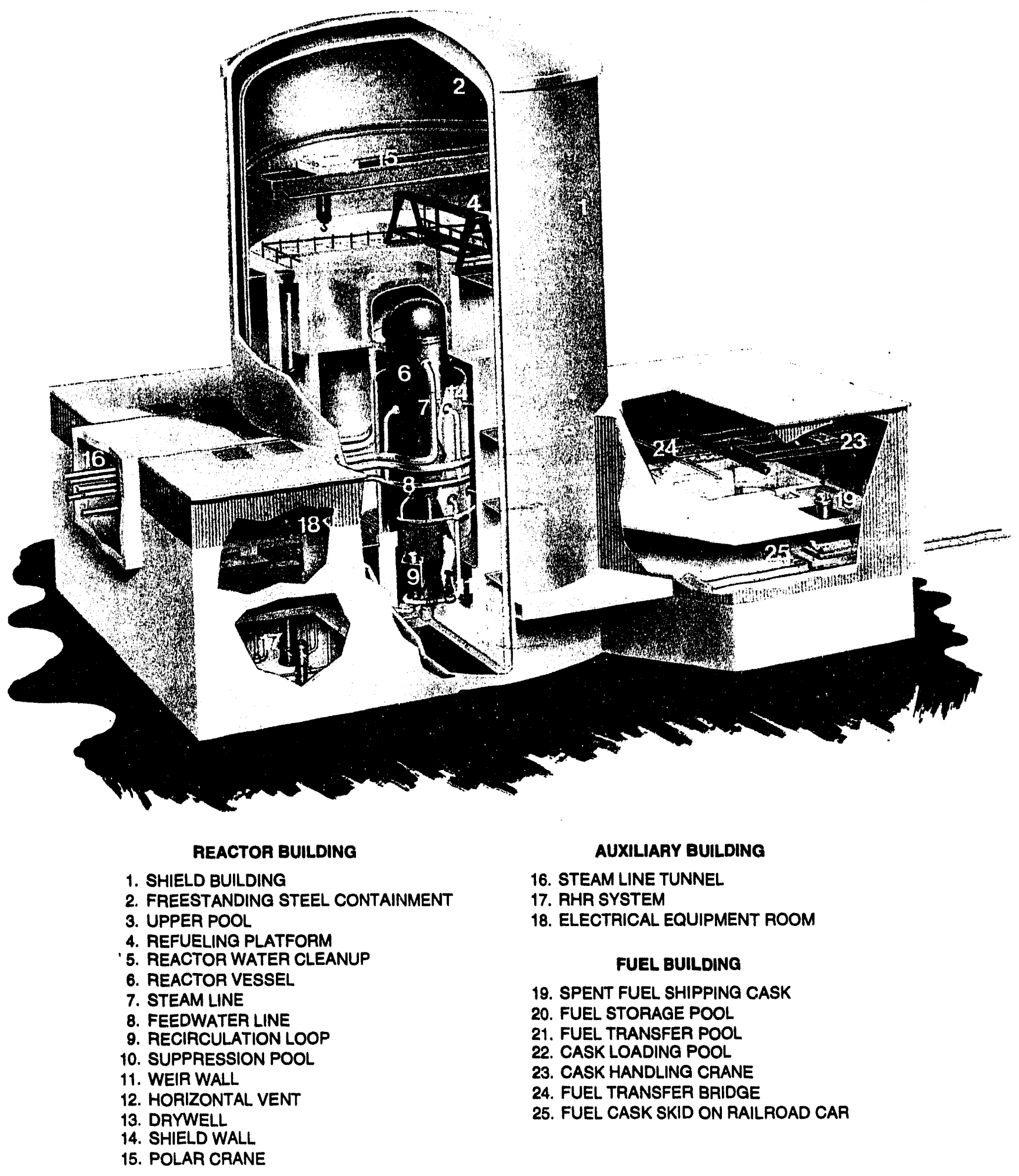

Figure 2.2-3 Mark III Containment 
- Radwaste Building: The Radwaste Building houses all equipment associated with the collection and processing of the liquid and solid radioactive waste generated by the plant. The Liquid Radwaste Management System collects, monitors, and treats liquid radioactive wastes for return to the primary system whenever practicable. The Solid Radwaste Management System provides for the safe handling, packaging, and short-term storage of radioactive solid and concentrated waste. The layout of the facility is designed so as to maximize the efficiency of the personnel, thereby minimizing the number of workers.

\subsubsection{Infrastructure for Plutonium Disposition}

This section discusses the infrastructure of organizations and site characteristics beyond the BWR and fuel fabrication facility which are necessary to support deployment of a BWR Plutonium Disposition Complex in the United States. This section also provides brief descriptions of DOE sites which could potentially form part of this needed infrastructure and the potential use of these facilities to support the plutonium disposition mission or supporting functions.

\subsubsection{Plutonium Disposition Site Requirements}

This section describes the capabilities and facilities needed by a commercial reactor site in order to be considered for location of some or all of the BWR plutonium disposition functions.

A selection criterion for commercial BWR compatibility with the plutonium disposition mission is provided in Section 1.2. All commercial BWRs are sufficiently large and have the physical resources and technical experience in nuclear programs to provide the space, utilities, and staff for the operation, safety, security, safeguards and maintenance of the plutonium disposition functions. However, the site selected for application of the plutonium disposition mission also may be influenced by other desirable features associated with existing sites within the DOE infrastructure. Some of the desirable features associated with BWRs used to support the plutonium disposition mission are:

- Reactor Plant Siting - Commercial BWRs are already sited and approved for operation. A site in close proximity to the MOX fuel fabrication facility presents the advantage of enhancing safeguards and minimizing the cost of transportation of the fresh MOX fuel assemblies.

- Power to the Grid - The operation of the BWR will provide the electric power generated to an existing utility grid. To achieve the planned capacity factors, the local demand or ability 
of the utility to sell the generated power should be sufficient that demand related power reductions are not experienced.

- Transportation - All BWR sites contain sufficient road or rail access to accommodate new fuel shipments and shipments of spent fuel and wastes from the site. Transportation safeguards considerations may make some sites more attractive than others.

- Site and Local Organizations - All BWR sites have a management organization experienced in large nuclear projects, a technical and production oriented work force, adequate utilities such as water and power, technology capabilities, and strict quality assurance requirements. A local community attitude that is favorable to plutonium transport and utilization would reduce any local opposition which may arise as a result of this program.

- New Fuel Storage - The weapons program is assumed to provide $\mathrm{PuO}_{2}$ feed material for plutonium disposition in accordance with the specifications for fabrication of MOX fuel for BWR fuel pins (see Section 2.4 for details of the MOX Fuel Fabrication Facility). The site must have the capability to receive the fresh MOX fuel and store it temporarily prior to transfer to the reactor refueling floor. A site with the capability for on-site temporary storage would have the advantage of reducing security provisions relative to another site in which remote temporary storage would need to be provided. It has been assumed that limited secure storage facilities will be required to be provided to accomplish the plutonium disposition mission.

- Spent Fuel Storage - A basis for the plant design is that it provide 10 years of storage at the reactor site for spent MOX fuel. It is assumed that the spent MOX fuel assemblies will be turned over to the U.S. Nuclear Waste Disposal Program after the 10-year cooling period. A plant with larger available capacity in its spent fuel pool has an advantage in that provisions for temporary storage would not need to be addressed in the early phases of the disposition mission.

It is anticipated that temporary storage of the fuel assemblies discharged from the in-reactor pool may be required at many operating BWRs pending development of the waste repository by DOE. A site with the capability for onsite temporary spent fuel storage presents the advantage of minimizing the complexity and cost of transportation of the spent MOX fuel assemblies to some other temporary storage location pending final disposal.

- Safety and Environmental Approvals - Commercial BWRs possess operating licenses issued by the NRC for a specific lifetime. Sufficient lifetime on the operating license to 
accomplish the plutonium disposition mission is one of the screening criteria used in selecting potential BWRs for the plutonium disposition mission.

Any request for amendment of an operating license requires the NRC to publish a notice of the proposed action in the Federal Register including a notice of opportunity for a public hearing. A change from $\mathrm{UO}_{2}$ to $\mathrm{MOX}$ fuel will require that the utility review the FSAR, the Bases to the Technical Specifications and all correspondence on the docket to determine if this change involves an Unreviewed Safety Question (USQ). From a utility perspective, this determination would likely be made in a conservative manner, since failure to identify a change as a USQ that is subsequently determined by the NRC to be a USQ could lead to escalated enforcement action, including a civil penalty.

Identification of a USQ requires a request for a license amendment in order to implement the change. A request for an amendment will also be required if it involves a change to the Technical Specifications (10CFR50.(a)(1)). It should be noted that "change" has frequently been interpreted by the NRC as any change, not just those that are in the less conservative direction. Since both changes to the Technical Specifications and USQ's are expected as a result of the change from $\mathrm{UO}_{2}$ to using a full $\mathrm{MOX}$ core, a license amendment will be required.

Whether a public hearing on the license amendment will be necessary depends to a large extent on the results of the Plutonium Disposition EIS process. If, during the EIS process, all the major issues were thoroughly discussed, then the likelihood of intervention in the license amendment process will be substantially reduced.

- Safeguards and Security - Operation of a BWR with MOX fuel will require safeguards and security for the storage, handling and transport of special nuclear materials (SNM) from the receipt of the MOX fuel through insertion of the MOX fuel assemblies into the reactor core and start of irradiation. After irradiation, the fuel assemblies will require safeguards and security equivalent to that for spent fuel in commercial reactors. A site with staffing and security fencing consistent with DOE requirements for safeguarding plutonium (see Section 7) has an advantage over a site that requires modification to achieve the needed capability.

\subsubsection{Capabilities at Potential Supporting Sites}

To establish the support capability of DOE and certain commercial facilities, a review of the sites in Table 2.2-2 was conducted. The goal of the review was to establish sites, facilities, resources 
Table 2.2-2 Existing DOE Facilities Capabilities

\begin{tabular}{|l|l|l|l|l|l|}
\hline \multicolumn{1}{|c|}{ Site } & $\begin{array}{l}\text { Pu Feed } \\
\text { Interface }\end{array}$ & $\begin{array}{l}\text { MOX } \\
\text { Fabrication }\end{array}$ & $\begin{array}{l}\text { Waste } \\
\text { Mgmnt }\end{array}$ & $\begin{array}{l}\text { Sfgrds } \\
\text { \& Sec. }\end{array}$ & \multicolumn{1}{|c|}{$\begin{array}{c}\text { Other } \\
\text { Comments }\end{array}$} \\
\hline $\begin{array}{l}\text { Idaho National } \\
\text { Engineering Lab. } \\
\text { (INEL) }\end{array}$ & Exists & $\begin{array}{l}\text { FPR can be } \\
\text { modified }\end{array}$ & Exists & Exists & $\begin{array}{l}\text { Experienced staff of } \\
12,500 \text { employees }\end{array}$ \\
\hline Hanford & Exists & $\begin{array}{l}\text { FMEF can be } \\
\text { modified }\end{array}$ & Exists & Exists & Shared site with WNP-2 \\
\hline $\begin{array}{l}\text { Savannah River } \\
\text { Site (SRS) }\end{array}$ & Exists & $\begin{array}{l}\text { Barnwell can } \\
\text { be modified }\end{array}$ & Exists & Exists & $\begin{array}{l}\text { Long-term Pu production } \\
\text { site }\end{array}$ \\
\hline $\begin{array}{l}\text { Oak Ridge } \\
\text { Reservation } \\
\text { (ORR) }\end{array}$ & Exists & $\begin{array}{l}\text { Y12 can be } \\
\text { modified }\end{array}$ & Exists & Exists & $\begin{array}{l}\text { DOE R\&D Center - close } \\
\text { to BFNP }\end{array}$ \\
\hline $\begin{array}{l}\text { Los Alamos } \\
\text { National } \\
\text { Laboratory }\end{array}$ & Exists & $\begin{array}{l}\text { Existing } \\
\text { Facilities can } \\
\text { be modified }\end{array}$ & Limited & Exists & $\begin{array}{l}\text { Island design could be } \\
\text { easily implemented }\end{array}$ \\
\hline $\begin{array}{l}\text { Lawrence } \\
\text { Livermore } \\
\text { National } \\
\text { Laboratory }\end{array}$ & Exists & $\begin{array}{l}\text { Some } \\
\text { fouldies } \\
\text { modified }\end{array}$ & None & Exists & $\begin{array}{l}\text { Pu process development } \\
\text { experience }\end{array}$ \\
\hline $\begin{array}{l}\text { Nevada Test } \\
\text { Site (NTS) }\end{array}$ & None & None & Exists & Exists & $\begin{array}{l}\text { Past experience with } \\
\text { weapons testing }\end{array}$ \\
\hline Pantex Plant & None & None & Limited & $\begin{array}{l}\text { Some } \\
\text { upgrade } \\
\text { needed }\end{array}$ & $\begin{array}{l}\text { Interim Pit Storage } \\
\text { Facility }\end{array}$ \\
\hline
\end{tabular}

and capabilities that already exist at DOE and commercial sites which could be used at a potential cost and schedule saving. A summary of findings is provided on Table 2.2-2.

It is clear that considerable cost effective, installed capability currently exists within the DOE community for meeting the plutonium disposition needs in the near term with an electric power producing, full MOX-fueled BWR plant.

Clear capability exists for early development of the island fuel design discussed in Section 2.1.1. These capabilities can be implemented in the short term with effort ranging from minor refurbishing to upgrading of existing facilities and only a few activities being "Greenfield" efforts. It is anticipated that a minimum cost deployment will be to locate the mission facilities in as close a proximity as possible.

Since it is not anticipated that any one BWR site (with the exception of Browns Ferry) would be sufficient to complete the entire disposition, multiple sites could be used to create a distributed "complex", with some additional cost for transportation between sites. 
NEDO-32361

\subsection{Reactor Safety Systems, Performance and Analysis}

\subsubsection{Reactor Safety Margins}

BWR reactor cores have operated for several years with MOX fuel both in test core loads in the U.S. and in foreign BWR cores. Therefore, the design and application of MOX fuel in BWRs does not present any new technological challenges. As with any core reload, GE has evaluated the entire spectrum of events in nuclear safety and operational analysis areas to establish the most limiting or design basis events. The scope of the situations analyzed includes anticipated operational occurrences, off-design abnormal (unexpected) transients that induce system operational disturbances, postulated accidents of low probability (e.g., the sudden loss of integrity of a major component), and finally, hypothetical events of extremely low probability (e.g., an anticipated transient without the operation of the entire Control Rod Drive System). In the event analysis, all essential protection sequences were evaluated until all required safety actions were successfully completed. The event analysis identified front line safety systems and their essential auxiliaries, operator actions, and limits to satisfy the required safety actions.

Defense-in-depth has been incorporated into the design of BWRs to provide margin in the design to assure safety. The traditional layers of defense have been reinforced by added redundancy or elimination of failure modes. The use of MOX fuel is not expected to reduce the margins of safety in the plant design. Potential impacts of MOX fuel on plant safety are discussed in the following paragraphs:

- Fresh Fuel: The radioactivity of plutonium $(\mathrm{Pu})$ and americium $(\mathrm{Am})$ in fresh fuel may affect handling. Potential fuel drop accidents could release a more hazardous source term than in previous designs.

Accidents involving fresh fuel are inherently less hazardous than spent fuel accidents because the radioactive materials are in the form of nonvolatile insoluble ceramics. There are no volatiles, as is the case with spent fuel fission gases, and there is no heat to provide a driving force. Consequently, fresh fuel drop accidents are not considered to be a safety consideration from the standpoint of public exposure.

- MOX Core Dynamics: The MOX cores potentially have a different response during Chapter 15 transients and accidents. The behavior of MOX cores during transients is analyzed and is addressed in Sections 2.3.2, 2.3.3 and 2.3.4. Substantial margin against limiting conditions is demonstrated. 
- Radiation Protection Source Terms: Source terms used to assess radiation protection in the BWR are slightly different when MOX fuel is used as compared with cases in which only $\mathrm{UO}_{2}$ fuel is evaluated. However, with the use of a MOX core, little difference in the isotopic content of the-core results. Table 2.3-1 compares the end-of-life inventory of isotopes in a comparable sized $\mathrm{UO}_{2}$ core and the reference MOX core. As shown in Table 2.3-1, the degree of difference is small.

With the normal release of isotopes from a BWR (including operation with small amounts of fuel failure), plutonium and other insoluble fuel isotopes released from the pins are normally not present. Therefore, on a realistic basis, the added increased amount of plutonium in the MOX core has no effect on the normal radiation release.

Chapter 12 of BWR safety analysis reports addresses conformance of normal operations to radiation protection limits on a conservative basis. As shown in Table 2.3-1, there is about three times as much Pu-239 in the reference MOX core as for a BWR core with $\mathrm{UO}_{2}$ at the end of cycle. However, plutonium and uranium are not considered in the Chapter 12 evaluation because they are not released to either air or liquid pathways. The impact of other isotopes can be seen in Table 2.3-1. Since the noble gas source term, which usually dominates the offsite exposure, is less than for the comparable $\mathrm{UO}_{2}$ core, the $\mathrm{MOX}$ core also is expected to result in lower exposure to the public.

- Severe Accident Source Terms: MOX core fission product source terms may differ slightly from those used in BWR responses to the NRC Severe Accident Policy Statement. These responses have taken several forms, but generally include an Individual Plant Evaluation (IPE) or a Probabilistic Risk Assessment (PRA). Since the IPE only involves core damage risk and not the source term, only the consequence portion of PRAs is affected by any differences in the MOX source term. Because no system design changes are required for the use of MOX fuel, IPE results remain valid. Regardless, the use of MOX fuel would not increase the evaluated risk because the fission product source terms are nearly identical and no uranium or plutonium is released in any of the accident source terms.

- Spent Fuel: Spent fuel characteristics could contain different isotopes (Table 2.3-1) and result in a more hazardous source term following bundle drop events than in previous cores. However, based on Table 2.3-1, any difference is not expected to be significant. 
NEDO-32361

Table 2.3-1 Comparison of Isotopic concentrations (Curies)

\begin{tabular}{|c|c|c|}
\hline Isotope/Group & Reference BWR & Reference MOX Core \\
\hline Group 1 & Noble Gases & \\
\hline KR-85. & $9.4 \mathrm{E5}$ & $5.8 \mathrm{E} 5$ \\
\hline KR-85M & $2.5 \mathrm{E7}$ & $1.7 \mathrm{E} 7$ \\
\hline KR-87 & 4.8E7 & 3.1E7 \\
\hline KR-88 & $6.8 \mathrm{E} 7$ & $4.3 \mathrm{E} 7$ \\
\hline XE-133 & $1.9 \mathrm{E} 8$ & $1.8 \mathrm{E} 8$ \\
\hline$\overline{X E-135}$ & 5.4E7 & $6.9 \mathrm{E} 7$ \\
\hline Group 3 & Halogens & \\
\hline $\mathrm{I}-131$ & $9.3 \mathrm{E} 7$ & $1.0 \mathrm{E} 8$ \\
\hline$\overline{\mathrm{I}-132}$ & $1.4 \mathrm{E} 8$ & $1.4 \mathrm{E} 8$ \\
\hline$\overline{I-133}$ & $1.9 \mathrm{E} 8$ & $1.9 \mathrm{E} 8$ \\
\hline I-134 & $2.1 \mathrm{E} 8$ & $2.6 \mathrm{E} 8$ \\
\hline $\mathrm{I}-135$ & $1.8 \mathrm{E} 8$ & $1.8 \mathrm{E} 8$ \\
\hline Group 4 & Alkali Metals & \\
\hline CS-134 & $1.3 \mathrm{E} 7$ & $1.4 \mathrm{E} 7$ \\
\hline CS-137 & $1.0 \mathrm{E} 7$ & $1.0 \mathrm{E} 7$ \\
\hline CS-138 & $1.8 \mathrm{E} 8$ & $1.6 \mathrm{E} 8$ \\
\hline Group 5 & Teleriums & \\
\hline TE-132 & $1.3 \mathrm{E} 8$ & $1.4 \mathrm{E} 8$ \\
\hline Group 6 & Alkali Earths & \\
\hline SR-91 & $1.1 \mathrm{E} 8$ & 7.7E7 \\
\hline SR-92 & $1.2 \mathrm{E} 8$ & $9.0 \mathrm{E} 7$ \\
\hline$\overline{B A-140}$ & $1.7 \mathrm{E} 8$ & $1.6 \mathrm{E} 8$ \\
\hline Group 7 & Noble Metals & \\
\hline RU-103 & $1.4 \mathrm{E} 8$ & $1.8 \mathrm{E} 8$ \\
\hline MO-99 & $1.7 \mathrm{E} 8$ & \\
\hline Group 8 & Rare Earths & \\
\hline Y-92 & $1.2 \mathrm{E} 8$ & 9.1E7 \\
\hline ZR-95 & $1.5 \mathrm{E} 8$ & $1.4 \mathrm{E} 8$ \\
\hline$\overline{\text { ZR-97 }}$ & $1.6 \mathrm{E} 8$ & $1.5 \mathrm{E} 8$ \\
\hline LA-140 & $1.7 \mathrm{E} 8$ & $1.6 \mathrm{E} 8$ \\
\hline CE-141 & $1.6 \mathrm{E} 8$ & $1.5 \mathrm{E} 8$ \\
\hline CE-144 & $1.0 \mathrm{E} 8$ & $8.5 \mathrm{E} 7$ \\
\hline & Transuranic & \\
\hline PU-238 & $2.2 \mathrm{E} 5$ & $2.0 \mathrm{E} 5$ \\
\hline PU-239 & $3.5 \mathrm{E} 4$ & $1.2 \mathrm{E} 5$ \\
\hline PU-240 & $3.9 \mathrm{E} 4$ & $2.1 \mathrm{E5}$ \\
\hline PU-241 & $1.4 \mathrm{E} 7$ & $3.2 \mathrm{E} 7$ \\
\hline AM-241 & $1.6 \mathrm{E} 4$ & $7.0 \mathrm{E} 4$ \\
\hline
\end{tabular}




\subsubsection{Transient Analysis}

GE uses a number of interrelated computer codes, correlations and analytical procedures to simulate the fuel, core, reactor and combined plant systems to predict the transient performance of BWRs for a variety of abnormal operating transients. These codes, correlations and procedures are used to provide a conservative prediction of the maximum changes in the safetyrelated parameters (e.g., $\triangle \mathrm{CPR}$ or reactor system pressure) that can occur and demonstrate that the applicable criteria are met for the most limiting transients.

BWR plant system transients have been assigned to one of eight separate categories:

- Decrease in reactor coolant temperature

- Increase in reactor pressure

- Decrease in reactor coolant system flow rate

- Reactivity increases and power distribution anomalies

- Increase in reactor coolant inventory

- Decrease in reactor coolant inventory

- Radioactive release from subsystems and components

- Anticipated transients without scram (ATWS).

The initial operating license of each BWR plant is based on the analyses of a spectrum of transient events in the above categories which are documented in each plant-specific FSAR. Each transient has been assigned to one of the above categories. In this manner, the most severe transient events relative to critical power ratio and reactor coolant system pressure have been identified.

The relative and absolute severity of the consequences of the transients analyzed are generally plant specific. It has been GE's experience that, regardless of the BWR plant considered, most of the events result in fairly mild plant disturbances. Thus, only a few events are severe enough to be potentially limiting such that operating limits are derived based on these results. 
Based on extensive experience with BWR transient behavior, representative limiting transients consistent with the licensing analyses of commercial BWR plants were chosen as the bases for this study. These transients are:

- Feedwater Controller Failure maximum demand (FWCF), representative of a decrease in core coolant temperature.

- Generator Load Rejection No Bypass (LRNBP), representative of an increase in reactor pressure.

The remaining transient categories are generally less limiting than the above two. These reactivity increases are spaced over a longer period of time, and the severity of the transient is dictated more by system characteristics rather than fuel design.

\subsubsection{Nuclear Parameters for Transient Analysis}

Three nuclear dynamic parameters are often used in qualitatively assessing the impact on $\triangle \mathrm{CPR}$. In order of importance, these are:

Table 2.3-2 Nuclear Parameter Significance

\begin{tabular}{|l|l|l|}
\hline Nuclear Parameter & MOX trend & \multicolumn{1}{|c|}{ Significance } \\
\hline Scram reactivity & lower & $\begin{array}{l}\text { Response dictated by the steady state } \\
\text { power shape }\end{array}$ \\
\hline Void reactivity & more negative & $\begin{array}{l}\text { Higher power increase in response to } \\
\text { void collapse }\end{array}$ \\
\hline Doppler reactivity & more negative & $\begin{array}{l}\text { More rapid response to power } \\
\text { increases }\end{array}$ \\
\hline
\end{tabular}

To provide a consistent comparison, a typical conservative top-peaked licensing basis power shape was used for both the reference $\mathrm{UO}_{2}$ core and the full MOX design. This eliminates the scram reactivity (i.e., power shape) as a factor in evaluating the core designs. Table 2.3-3 displays the key nuclear parameters affecting the transient analyses.

Table 2.3-3 Comparison of Key Parameters

\begin{tabular}{|l|c|c|c|}
\hline Parameter & $\begin{array}{c}\text { Reference UO } \\
\text { - EOC }\end{array}$ & $\begin{array}{c}\text { Island Design } \\
\text { - EOC }\end{array}$ & $\begin{array}{c}\text { Full MOX } \\
\text { - EOC }\end{array}$ \\
\hline Void coef (C/\%) & -7.46 & -9.46 & -12.96 \\
\hline Doppler (C/K) & -0.4590 & -0.5065 & -0.6580 \\
\hline
\end{tabular}


NEDO-32361

\subsubsection{Plant Operating Limit}

One purpose of the transient response analysis is to set plant operating limits to avoid the possibility of departure from nucleate boiling for events expected to occur during the plant lifetime. For BWRs this has been measured by the Minimum Critical Power Ratio (MCPR), which is the ratio of the bundle power at which departure from nucleate boiling is expected to occur on the hottest rod in a fuel bundle to the bundle's current power level. With departure from nucleate boiling MCPR at 1.0, statistical considerations are added which account for manufacturing and measurement tolerances, including uncertainties in the thermal-hydraulic correlations developed by test programs, to determine the Safety Limit MCPR (SLMCPR).

For BWRs, the SLMCPR has been established to be 1.07. It is defined such that if the most limiting fuel bundle in the core is calculated to have an MCPR of $1.07,99.9 \%$ of the fuel rods in the core will not experience departure from nucleate boiling. This is the acceptance criterion approved by the U.S. NRC.

Those transients identified which can occur due to a single equipment failure or single operator error are analyzed to determine the most limiting transient in terms of the change of critical power ratio which can occur. This $\triangle \mathrm{CPR}$ is added to the SLMCPR to determine the plant Operating Limit MCPR (OLMCPR). The core is designed and operated such that adequate MCPR operating margin exists.

One of the more limiting transient for BWR cores is the Load Rejection with No Bypass (LRNBP) event. Even though this event involves multiple failures, a long standing GE-USNRC agreement exists to consider this an initiating event as one of those in Regulatory Guide 1.70, Chapter 15. The primary nuclear dynamic parameter influencing this transient, other than scram reactivity, is the void coefficient. A collapse of voids due to primary system pressurization results in a power increase.

Since the MOX fuel design has a larger negative dynamic void coefficient than the reference design, a larger $\triangle C P R$ is predicted due to the void collapse associated with the transient. Figure 2.3-1 shows the progression of the LRNBP transient.

The Feedwater Controller Failure to Maximum Demand (FWCF) is a transient which can lead to excess coolant inventory. The supply of excess feedwater results in an increase in core subcooling which reduces void fraction and thus induces an increase in core power. Figure 2.3-2 shows the progression of the FWCF transient. 
NEDO-32361
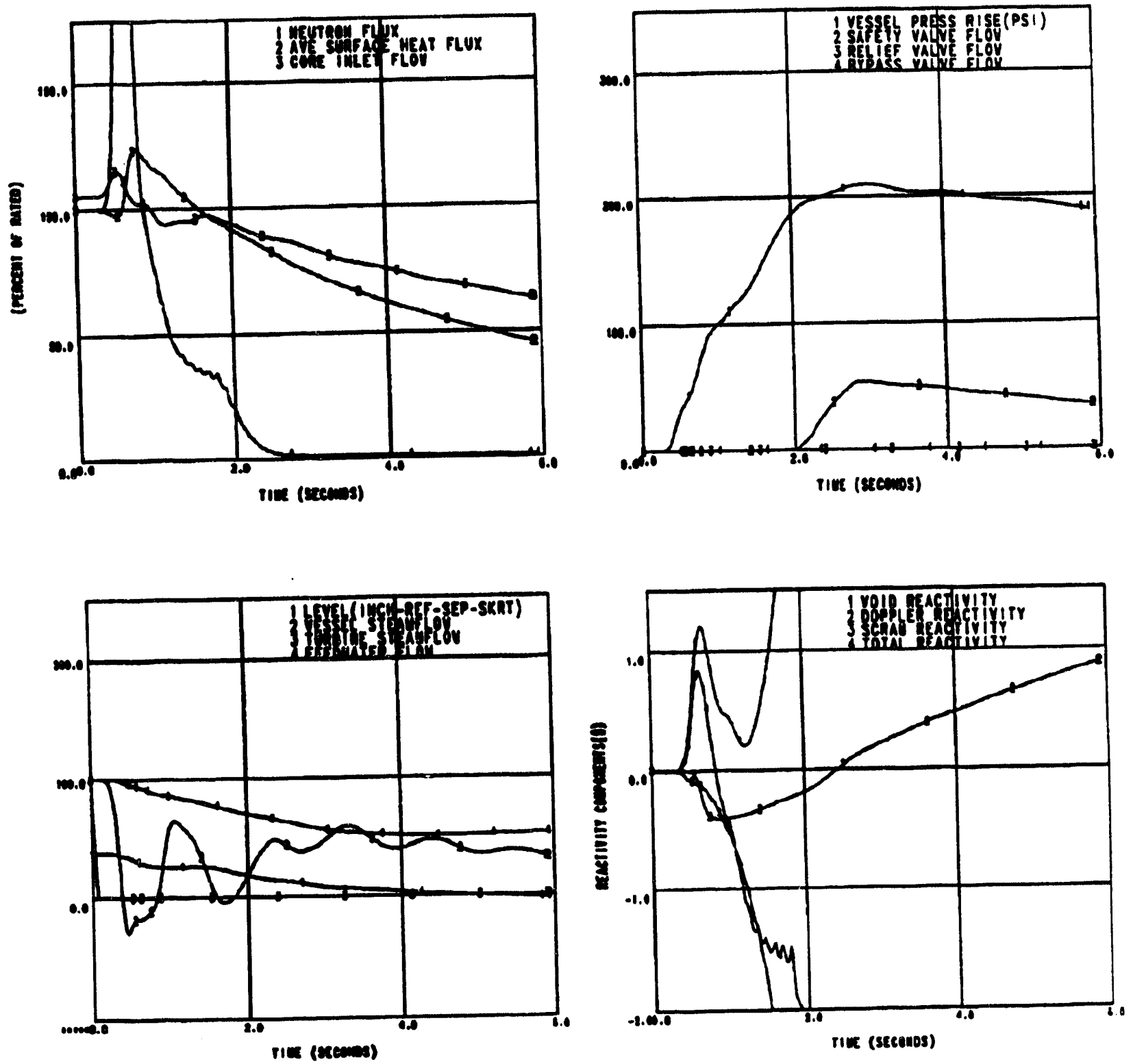

Figure 2.3-1 Generator Load Rejection without Bypass $100 \%$ Power, $105 \%$ Flow at EOC with ATWS, with RPT 

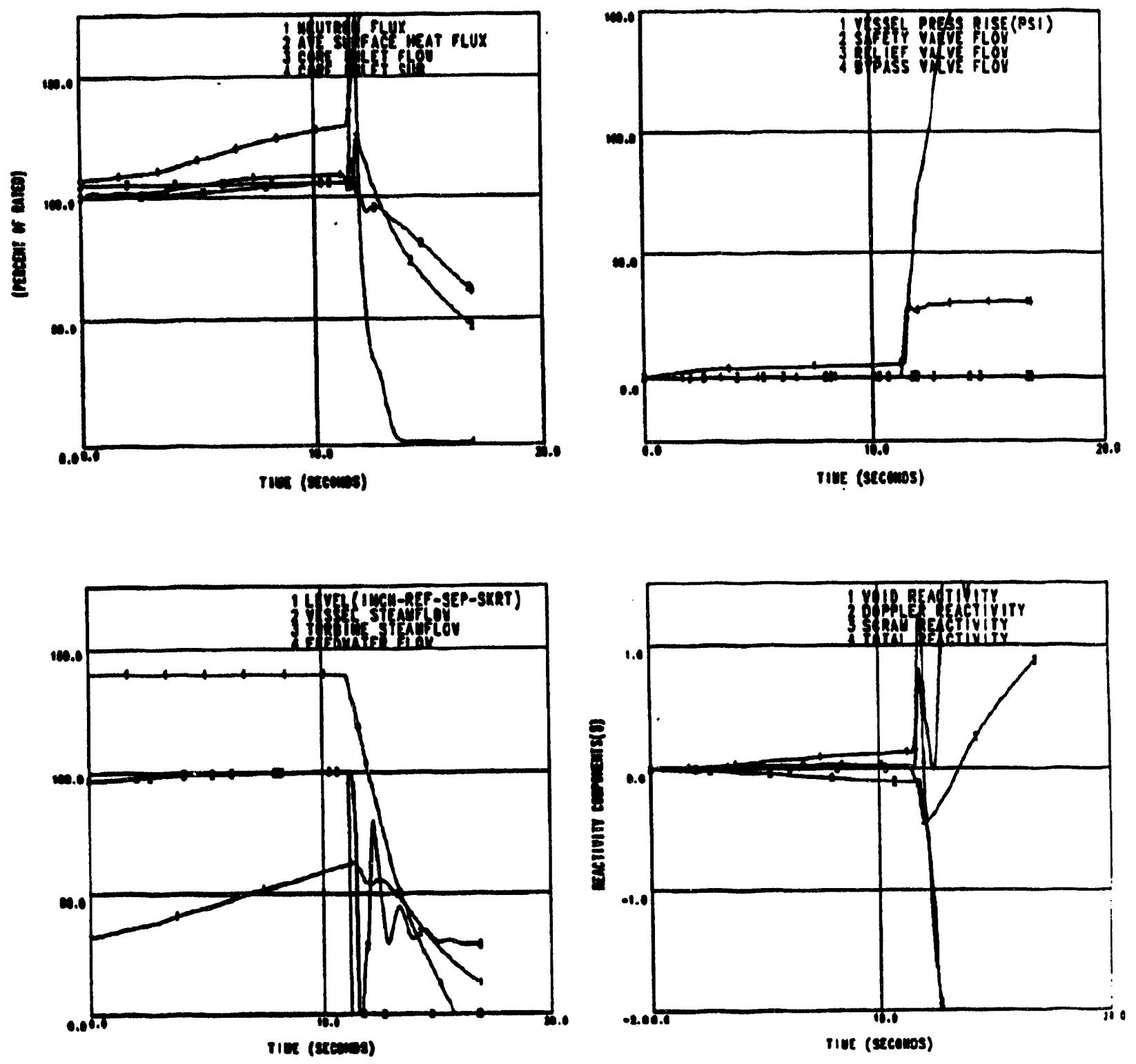

Figure 2.3-2 Feedwater Controller Failure, Maximum Demand, HWL Trip $100 \%$ Power, 105\% Flow at EOC with ATWS, with RPT 
The more negative void coefficient in the full MOX design bounds the transient results for the island design; hence, the island design $\triangle C P R$ results are not reported. Table 2.3-4 shows the comparison for the uncorrected $\triangle \mathrm{CPR}$ between the reference $\mathrm{UO}_{2}$ and full $\mathrm{MOX}$ designs.

These $\triangle C P R$ values would yield an OLCPR value of 1.27 (based on the LRNBP case), which allows a $24 \%$ margin based on a steady-state MCPR of 1.50 .

Table 2.3-4 Transient Results

\begin{tabular}{|l|c|c|}
\hline & Reference Core $\triangle$ CPR & Full MOX \\
\hline LRNBP & 0.18 & 0.20 \\
\hline FWCF & 0.16 & 0.19 \\
\hline
\end{tabular}

\subsubsection{Stability}

Utility requirements for interim correction actions (ICAs), to protect against reactor instabilities, is provided by NRC Bulletin 88-07 Supplement 1, which includes an exclusion zone bounded by the $45 \%$ flow and the $80 \%$ load line. Some plants have even more restrictive ICAs.

Protection from reactor instabilities is provided in the BWR by control which excludes a zone of pump speed/flow operation on the reactor power and flow map. For the MOX core evaluated, it is necessary to increase this zone slightly beyond that specified for the $\mathrm{UO}_{2}$ core. This modification is easily achieved and has minimal impact on the BWR plant operation.

From a safety point of view, the BWR has provided protection from reactor instabilities which might cause fuel damage. In BWRs density-wave oscillations have been observed under test and operating conditions as the reactor power and flow are lowered along the rated rod line. With this in mind, the BWR has provided administrative controls to exclude operation in a specified zone in the power-flow map (Figure 2.3-4). During power ascension by pulling control rods, as the operator increases power, recirculation pump speed is increased above a minimum speed to avoid the excluded region. During transients, if two recirculation pumps trip, an automatic scram is initiated or alarmed, depending on the specific plant design. In plants in which an alarm occurs, an automatic scram is initiated in response to LPRM signals if an instability occurs.

The basis of the minimum pump speed limit has been determined from stability analyses demonstrating a 95\% confidence that oscillations will not occur at or above this point. From many tests and analyses, GE has determined the primary drivers for instability and has determined that the void coefficient is the most significant parameter for the purpose of the 
mixed-oxide studies. Therefore, for the MOX core evaluation, stability limits were checked to see if further restrictions in the operating map would be necessary.

Stability is calculated by the use of frequency-domain codes which linearize the basic dynamic equations for small perturbations and report the behavior of the reactor in terms of a secondorder feedback system. Commonly, a decay ratio is reported; this is the ratio of the second overshoot to the first overshoot of the reactor response to a step change in input. Thus, a decay ratio $<1.0$ means the reactor is stable, and a decay ratio $>1.0$ means the reactor is unstable. In calibration of GE's methods to test data, a $20 \%$ conservatism is needed to achieve a $95 \%$ confidence of avoiding instability; thus, it is required to predict the decay ratio $<0.8$. The least stable point in the operating domain of Figure 2.3-3 is the intersection of the rated rod line with the natural circulation line.

Table 2.3-5 compares the decay ratios at the natural circulation flow and rated rod line for the reference $\mathrm{UO}_{2}$ design and the full MOX core design. Additional points on the rated rod line were evaluated for the decay ratio. For example, at $45 \%$ flow, the MOX decay ratio is 0.65 ; at $50 \%$ flow, the MOX decay ratio is $\mathbf{0 . 5 4}$. Administrative controls to avoid reactor instabilities are provided by NRC Bulletin 88-07, which specifies an exclusion zone bounded by the $45 \%$ flow and the $80 \%$ load line. On a generic basis, these boundaries have been judged to be sufficient to avoid regions of potential instabilities.

For the MOX design, the results indicate a higher potential for instabilities, relative to the reference core due to the more negative void coefficient. Relative to NRC Bulletin 88-07 Supplement 1, an expanded ICA zone is necessary as shown in Figure 2.3-3. The increased zone is nominally placed at $<50 \%$ flow based on estimates of the MOX decay ratios. The slightly greater exclusion zone should be readily achievable. This exclusion region will be precisely defined for the plants under consideration by analysis to support the MOX fuel licensing.

As discussed in Section 2.2.1, the stability long-term solutions to replace the ICAs are applicable on a plant-specinic basis and may include hardware/software devices such as local oscillation monitors or automatically enforced stability regions coupled with possible active components (e.g., Select Rod Insert, SRI). The impact of the MOX fuel design on the possible long-term solutions has not been evaluated, but is not considered to present more than a minor adjustment. A local oscillation based monitor should be unaffected. 
NEDO-32361

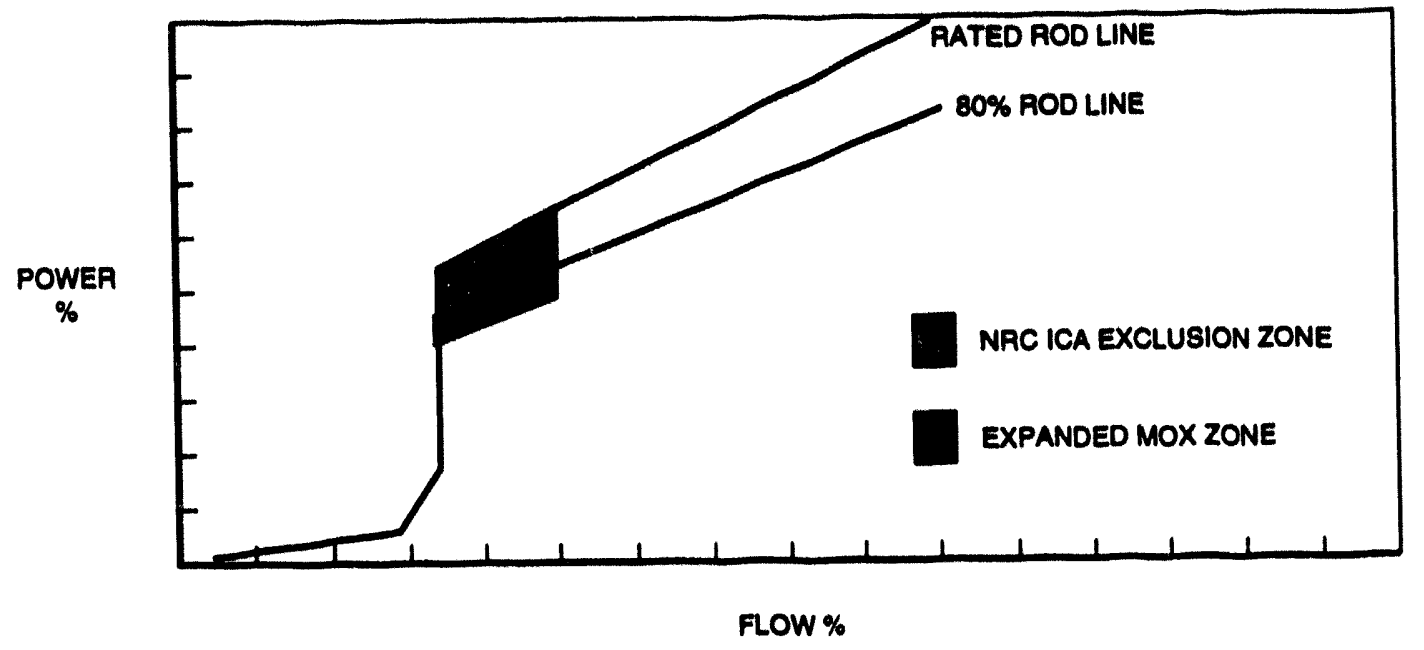

Figure 2.3-3 Typical Power/Flow Map 
NEDO-32361

Table 2.3-5 Decay Ratio at Natural Circulation and Rated Rod Line

\begin{tabular}{|c|c|}
\hline Reference $\mathrm{UO}_{2}$ & $\mathrm{MOX}$ \\
\hline 0.69 & 1.01 \\
\hline
\end{tabular}

\subsubsection{Anticipated Transient without Scram (ATWS)}

ATWS events are a special class of transients also analyzed in Chapter 15 of BWR safety analyses. Because of its low probability of occurrence, acceptance criteria for ATWS events are somewhat different than those for normal transients. These include:

- Core coolability, determined by demonstrating fuel temperatures $<2200^{\circ} \mathrm{F}$

- Peak nuclear boiler pressure below ASME service Level C (1500 psig)

- Containment parameters below limiting conditions

In the safety analyses, ATWS analyses are reported showing significant margins to the acceptance limits. In Mark I and Mark II plants, the containment pressure response is typically well below the design pressure; in Mark III plants, the containment temperature remains below the qualification temperature of equipment (especially SLC) inside containment.

The response of a BWR with MOX fuel to an ATWS event is not expected to change. If an isolation occurs, the increased void coefficient can lead to a slightly higher power spike, however the remaining heat discharged to the suppression pool is a function of the makeup injection rate and is unaffected by void coefficient. Therefore, the impact on the containment is not considered significant. Any potential for reactor instabilities would be addressed by boron injection in response to Emergency Procedure Guidelines.

As discussed in Section 2.1.2, if analysis indicates that additional enrichment of Boron is necessary, this modification can be easily accommodated with out a change in the plant design. Therefore, there is no significant impact is anticipated of using MOX fuel on the ATWS analysis. Detailed analysis would be performed as part of the fuel licensing activity.

\subsubsection{Overpressure Analysis}

In BWRs the number of safety/relief valves is normally set by requirements of the ASME Code for overpressure protection. This analysis is a special transient in which the MSIVs are suddenly closed, and the normal MSIV position scram is assumed to fail, which results in a reactor scram due to high neutron flux. In addition, no credit is given for safety/relief valves opening in the 
power actuated relief mode. Credit is given only for the spring mode. The acceptance criterion for this analysis is the Nuclear Boiler peak pressure less than Service Level B of the ASME Code (i.e., less than 1375 psig).

For the full MOX core design, the peak vessel pressure attained during the MSIVF transient is $1276 \mathrm{psig}$, which is comparable to the results from the reference $\mathrm{UO}_{2}$ core design.

Figure 2.3-4 shows the progression of the MSIVF transient.

\subsubsection{Accident Analysis}

Reactivity accidents, such as Rod Drop or Rod Ejection (dominated by the fuel Doppler Coefficient and control rod worth) are evaluated in BWRs safety analysis. Experience with these calculations has shown that they tend to be insignificant against the heat deposition limits associated with the event. Despite the smaller beta of MOX fuel, rod worth values are significantly lower than an equivalent uranium core. Thus, the severity of this event is reduced in comparison with $\mathrm{UO}_{2}$ based analyses contained in the safety analysis. Therefore, the rod drop accidents are not of concern.

For LOCAs, nuclear dynamic feedback plays an insignificant role, since the plant is presumed to scram at the start of the event. The only other core design dependent input is the decay heat level for the fuel after scram. As shown in Section 2.1.1.8, the decay heat level for the MOX core is expected to be slightly less than a $\mathrm{UO}_{2}$ core, because the fission product inventory immediately following a plant shutdown is lower than for a $\mathrm{UO}_{2}$ core. In any case, the BWR LOCA analysis is dominated by the inventory loss during the initial blowdown, and the results for a $\mathrm{UO}_{2}$ core show an acceptable response. Thus, it is concluded that MOX cores will not degrade the LOCA results.

\subsubsection{Radiological Impacts}

\subsubsection{Worker and Public Radiation Exposure (ALARA)}

BWR reactors, as discussed in the SSAR Chapters 11 and 12, have radiation protection features that comply with NRC requirements to maintain worker exposures "as low as reasonably achievable". Due to economic incentives, all BWRs have taken actions in the last several years to systematically reduce occupational exposures to workers. The median BWR exposure reported by INPO for 35 BWR units operating in 1993 shows 335 person-rem. Quantitative 

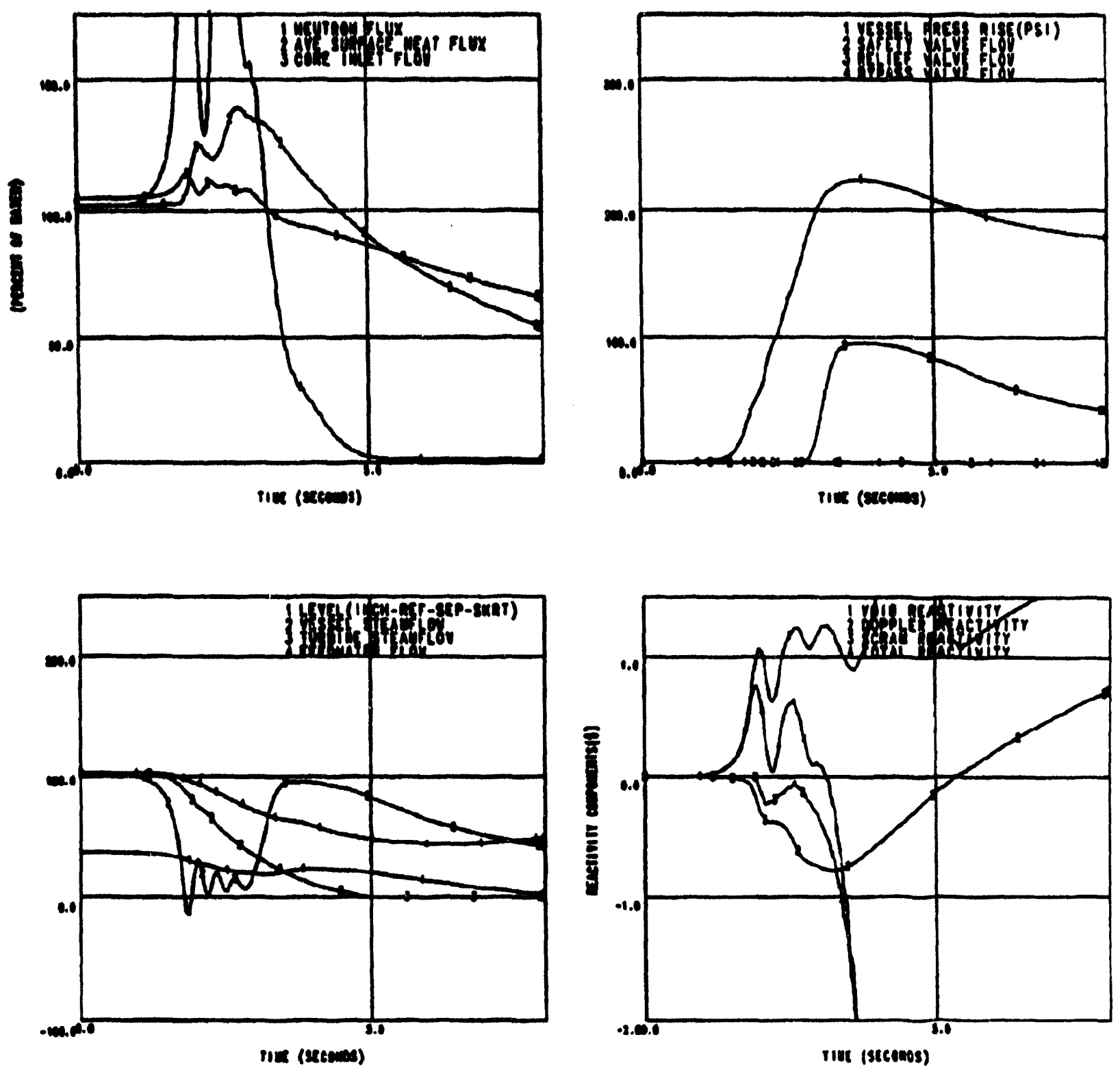

Figure 2.3-4 MSIV Closure, Flux Scram

$102 \%$ Power, $105 \%$ Flow at EOC with ATWS, without RPT 
estimates of the BWR reactor contribution to worker and public exposures are given in Section 8.2 .

\subsubsection{Offsite Radiological Impact from the Reactor Plant}

Offsite radiological impact from the BWR plants is derived from normal and accident releases. Accident releases do not significantly contribute to the offsite impact due to the low core damage frequency determined by PRA evaluations. Table 2.3-6 summarizes the estimated impacts. As can be seen from the table, the risk of offsite dose is extremely low and only a fraction of a percent of 10CFR50, Appendix I limits.

Table 2.3-6 Average BWR Offsite Radiological Impact

\begin{tabular}{|l|l|}
\hline Type of Exposure & \multicolumn{1}{|c|}{ Annual Dose Risk } \\
\hline Normal Operation - Gamma & $0.09 \mathrm{mrem} / \mathrm{yr}$ at site boundary \\
\hline Normal Operation - Beta & $0.05 \mathrm{mrem} / \mathrm{yr}$ at site boundary \\
\hline
\end{tabular}




\section{NEDO-32361}

\subsection{MOX Fuel Fabrication Facility}

This section describes the design requirements for the fabrication of either island or full MOX assemblies. The MOX facility arrangement is based on past industry experience and best practices. An initial set of derived requirements was adopted as follows:

- All materials required for fuel bundle fabrication shall be received in a form suitable for immediate use.

- All materials shall be made available as required to meet fabrication schedules.

- All process equipment shall be continuously monitored for acceptablr performance and, if necessary, adjusted on-line to maintain acceptable performance.

- Process equipment lifetimes shall be greater than MOX facility usage requirements, or their replacement impact on fabrication schedules shall be minimized.

- All glove box filter change operations shall be performed on-line.

- Fuel accountability procedures shall not have a significant impact on fabrication schedules.

- Fabrication process generation of fuel scrap material shall be minimized, and shall be better or equal to recent LWR fuel fabrication experience.

- The fuel scrap recovery process shall yield a minimum of $99.5 \%$ of plutonium back to the MOX facility.

- The storage area for completed fuel assemblies shall be sized to store all fabricated bundles consistent with the delivery schedule to the reactor site.

- Fuel fabrication facility generated waste shall be minimized.

- Worker exposure shall be maintained as low as practicable.

The selected BWR fuel design includes fuel assemblies which contain both MOX and MOX with gadolinia fuel rods. An extensive experience base exists for the fabrication process proposed for converting the weapons-grade plutonium into MOX fuel. This process has been utilized to fabricate both urania [Ref. 2-1] and MOX fuel [Ref. 2-2, 2-3, 2-4] for light water reactors, and MOX fuel [Ref. 2-5] for liquid metal reactors. A similar experience base for fabrication of MOX with gadolinia additions is not available, but urania fuel with gadolinia additions has been 
successfully fabricated for reloads in existing BWRs. Considerable material property work was performed on plutonia-rare earth combinations [Ref. 2-6, 2-7] which required high quality pellet samples. This material property sample information and the extensive fabrication experience with urania which contained gadolinia additions indicates that no significant problems should exist in the fabrication of MOX fuel with gadolinia additions. Since the urania concentration of the MOX fuel considered for BWRs is in the $90 \%$ range, the urania will tend to smooth out and mask most of the effects caused by plutonia and gadolinia additions. Although the fabrication of MOX with gadolinia additions is not expected to be a problem, testing will be required to verify the fabrication processes and benchmark the full MOX core.

The basic mill-prepress-granulate or powder blend process utilized to fabricate nuclear fuel is well developed. In this process, the feed powders are blended together in the desired concentration, milled to improve homogeneity, prepressed and granulated to form free flowing powder for pellet pressing. The resulting pellets are sintered in a reducing atmosphere, ground to size, inspected and loaded into fuel rods. The fuel rods are sealed by welding, inspected and assembled into fuel bundles. The completed fuel bundles are stored at the fabrication facility until they are needed to provide reload assemblies for a reactor. Several improvements in the fabrication process for urania fuels have been made in the past ten years. These process improvements have been incorporated in the adopted fuel fabrication process for MOX fuels and are detailed in Figure 2.4-1.

The relatively low radiation exposure levels associated with the weapons-grade r lutonium should allow the fuel fabrication activities to be performed in a traditional glove box arrangement. This concept has been demonstrated and tested extensively during previous MOX fabrication activities. Even though the anticipated worker exposure levels for this facility are lower than expected based on past experience, a reduction in worker exposure limits will be further assured by mechanizing the processing operations, which reduces the time the operator is in close proximity with the nuclear material. This process selection was based on the ease of operation and maintenance activities, as well as the lower capital costs associated with a glove box facility. Localized shielding will be installed on the equipment, and lead glass and sheet will be added to the exterior of the glove boxes to further reduce exposure levels. All maintenance activities will be accomplished by hands-on techniques only after all possible nuclear material has been removed to shielded storage areas. 


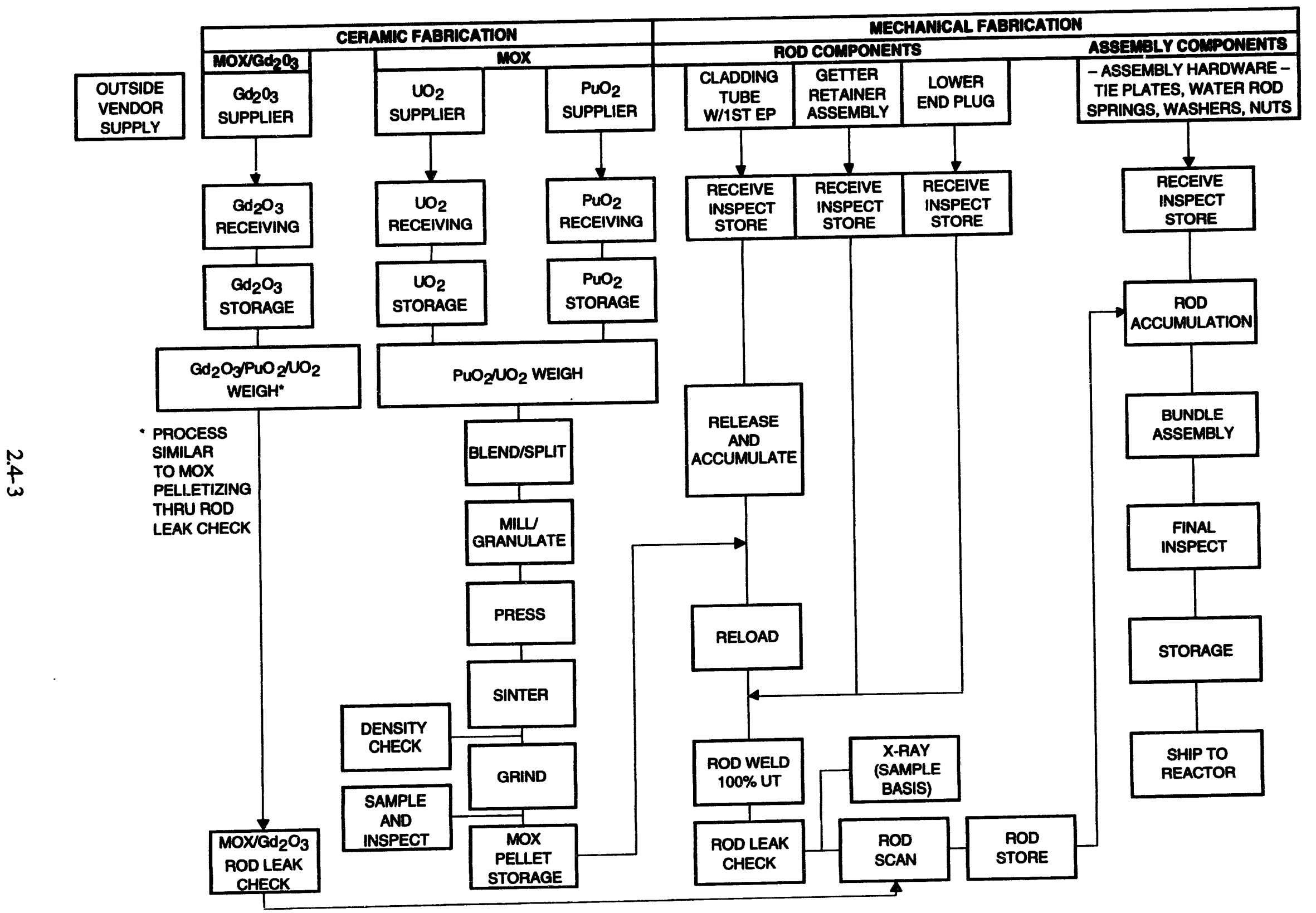

Figure 2.4-1 MOX Process Flow Sheet 


\subsubsection{Fuel Fabrication Throughputs}

The MOX facility capacity requirements are derived so that either the reference 50 or alternate $100 \mathrm{MT}$ of excess weapons plutonium can be dispositioned. The $50 \mathrm{MT}$ case requires 26.1 years of reactor operations, while the $100 \mathrm{MT}$ can be dispositioned in either 27.3 or 48.4 years. The selected bundle designs (Section 2.1.1) include both an island design to initiate disposition activities starting in 1997 and a full MOX design to maximize plutonium throughput beginning in 2001. Each core was designed so that the isotopics of the discharged fuel is similar to the isotopics of fuel discharged from typical operating commercial reactors utilizing urania fuel. The core fuel loading schemes uses a four batch reload with a $75 \%$ capacity factor. These factors result in a cycle time of 15 months, which yields an average discharge burnup, at equilibrium, of more than 37 GWd/MT.

The schedule for bringing the MOX facilities for fabrication of the island and full MOX assemblies to full operation are presented in Section 6.6. The following assumptions were made in establishing the MOX facility capacities at Los Alamos National Laboratory (LANL) for island fuel and at a new facility for full MOX fuel:

- One and one half years of fuel demonstration operation of the MOX facility at LANL is available to produce island assemblies prior to first fuel loading.

- Four and one half years until full production operation of the new MOX facility is available from the beginning of conceptual design.

- Island bundles can be substituted directly for one third of the standard urania fuel in a reload with no additional delays.

- At least one island reload is irradiated in each reactor prior to loading the first full MOX assemblies.

- Three months startup and testing required prior to full power operation for the first full MOX reload in each reactor.

- Reload fuel is delivered to the reactor site six months prior to core loading.

The dispositioning activities considered for processing either 50 or $100 \mathrm{MT}$ of plutonium are initiated with six island design reloads distributed among three reactors. These reloads provide adequate time for the construction of a facility for fabrication of full MOX assemblies necessary for completion of the disposition activities. Depending on the amount of plutonium to be 
dispositioned and the time frame required, either three or six reactors can be utilized. Each option requires slightly more plutonium ( 220 to $949 \mathrm{~kg}$ ) to fabricate the final core reload than the goal disposition amount. Two fabrication options to continue reactor operations after the disposition of the specified amount of plutonium have been defined should additional reactor life remain. The first is to continue fabricating MOX fuel using additional excess plutonium supplies while the second is to change the reactor core to urania fuel. This study assumes the usage of the urania fuel to discharge all MOX assemblies.

Table 2.4-1 provides a summary of the fuel fabrication throughput requirements for the reference $50 \mathrm{MT}$ and alternate $100 \mathrm{MT}$ disposition approaches, including key strategic material throughput needs. The MOX facility capacity is specified with a conservative $20 \%$ design margin to accommodate process losses, scrap and waste quantities.

\subsubsection{Reference Disposition Option Island Bundle Design}

Since the purpose of the island design is to begin irradiation of weapons-grade plutonium in 1997, existing facilities must be utilized to provide fabrication capability for the required fuel. The only operating facilities in the United States capable of producing MOX fuel are those located in the TA-55 area at LANL. Portions of these facilities were recently utilized to fabricate uranium nitride for space reactor applications [Ref. 2-8]. The process developed for these applications is similar to that required for fabrication of MOX fuel for a BWR. The scheduled activities for upgrading the LANL facility to the needs of this program, including equipment procurement, installation and testing, are summarized in Section 6.6. The facility for the fabrication of island design fuel would fabricate and insert $12 \mathrm{MOX}$ fuel rods into each of 116 urania bundles per year. This corresponds to a MOX fabrication rate of 6 MT per year. The details of the bundle fabrication and storage for island fuel are summarized in Table 2.4-2.

\subsubsection{Reference Disposition Full MOX Bundle Design}

The MOX facility is sized for the reference Pu disposition option with a core design that utilizes an average fuel bundle $\mathrm{Pu}$ enrichment of $2.79 \% \mathrm{Pu}$. This option requires three BWRs to disposition the specified $50 \mathrm{MT}$ of plutonium. Based on the construction and operational schedules detailed in Section 6.6, the MOX facility begins fabricating reactor fuel about one year prior to reactor fuel loading. The MOX facility design capacity for providing reload fuel for three reactors requires the fabrication of 400 bundles per year, which translates into a MOX capacity of $97 \mathrm{MT}$ per year. The resulting bundle fabrication rates and interim storage needs for 
Table 2.4-1 Characteristics of Reactor Deployment and Fuel Fabrication Capacity

\begin{tabular}{|l|c|c|c|}
\hline Disposition Option & Ref. & Alt. 1 & Alt. 2 \\
\hline Weapons Plutonium, MT & 50 & 100 & 100 \\
\hline Reactors, No. & 3 & 6 & 3 \\
\hline Defined Project Time, yr & 42.3 & 43.8 & 64.9 \\
\hline Reactor Disposition of Pu, yr & 26.1 & 27.3 & 48.4 \\
\hline BWR Fuel Bundle Design: & & & \\
Island Assembly: & 12 & 12 & 12 \\
$\quad$ Fuel Pins, no. & 1.434 & 1.434 & 1.434 \\
Plutonium, kg & 60 & 60 & 60 \\
Full MOX Assembly: & 5.334 & 5.334 & 5.334 \\
$\quad$ Fuel Pins, no. & & & \\
Plutonium, kg & 1,392 & 1,392 & 1,392 \\
\hline Fuel Fabrication Requirements: & $(1,400)^{1}$ & $(1,400)^{1}$ & $(1,400)^{1}$ \\
Island Assembly: & 116 & 116 & 116 \\
Fuel Pins, no./yr & 24,000 & 47,214 & 24,960 \\
Bundles, no./yr & $(24,000)^{1}$ & $(48,000)^{1}$ & $(25,000)^{1}$ \\
Full MOX Assembly: & 400 & 774 & \\
Fuel Pins, no./yr & & & \\
Bundles, no./yr & 6 & 6 & 6 \\
\hline Fuel Fabrication Design Rates: & 3.5 & 3.5 & 3.5 \\
Island Assembly: & & & \\
MOX Fab at 120\%, MT/yr & 97 & 189 & 101 \\
Fab Duration, yr & 23.5 & 24.5 & 45.5 \\
\hline Fuel MOX Assembly: & & & \\
MOX Fab at 120\%, MT/yr & & & \\
Fab Duration, yr & & & \\
\hline
\end{tabular}

(1) Rod totals utilized for capacity estimates

the full MOX reloads are shown in Table 2.4-2. Although the total fuel could be fabricated in a shorter period, the adopted approach was to keep the MOX facility operational for substantially the entire reactor Pu disposition interval. This approach maintains the availability of the trained MOX facility staff, equipment and facilities, should the overall goal of the program be modified or changed during the disposition time frame.

\subsubsection{Alternate Disposition Options with Full MOX Bundle Design}

Alternate-1 is the same as the reference option except that the disposition requirement is increased to $100 \mathrm{MT}$ of plutonium. Using the full MOX core design and the same schedule ground rules, the number of reactors required increases to six. The MOX facility fabrication capacity increases to 774 bundles per year to meet the core reloading schedules. The MOX 
NEDO-32361

Table 2.4-2 Reference Option Fuel Assembly Flow Summary

\begin{tabular}{|c|c|c|c|c|c|c|c|c|c|c|c|c|c|c|c|}
\hline \multirow{2}{*}{ Yerr } & \multirow{2}{*}{$\begin{array}{l}\text { Remeter } \\
O_{p \text { partion }} \\
\left(V^{\prime}(r)\right.\end{array}$} & \multicolumn{2}{|c|}{ Renctor 1} & \multicolumn{2}{|c|}{ Resetor 2} & \multicolumn{2}{|c|}{ Renetor 3} & Intand & Mox & $\begin{array}{c}\text { Tody } \\
\text { Mox } \\
\text { Any }\end{array}$ & Uranta & & & & Doll MOX \\
\hline & & $\pi m$ & Tod & $\pi m$ & Lond & $\operatorname{Tn}$ & Loed & & & & & (MT) & (MT) & & \\
\hline $19 \% .0$ & -1.5 & & & & & & & & & & & & & 29 & \\
\hline $19 \% 6.3$ & -1.0 & & & & & & & & & & & & & 29 & \\
\hline 19070 & $=0.3$ & & & & & & & & & & & & & 38 & \\
\hline 19925 & 0.0 & 0.00 & $5 B$ & & & & & 58 & & 58 & & 0.083 & 0.033 & 58 & \\
\hline 1908.0 & 0.5 & & & & & & & 0 & & 0 & & 0.000 & 0.0 .3 & 58 & \\
\hline 1995 & $\frac{1.0}{1.0}$ & 1.24 & 58 & & & & & 58 & & 58 & & 0.083 & 0.166 & 58 & \\
\hline 19920 & 1.5 & & & & & & & 0 & & 0 & & 0.000 & 0.166 & $\frac{18}{58}$ & \\
\hline 1985 & 2.0 & 2.48 & 58 & 2.24 & 58 & & & $\frac{116}{116}$ & & 116 & & 0.165 & 0.333 & & \\
\hline 2000.0 & 25 & & & & & & & 0 & & 0 & & 0000 & 0.333 & & 200 \\
\hline 2000.5 & 3.0 & & & 3.48 & 58 & & & 58 & & 51 & & 0.083 & 0,416 & & 200 \\
\hline 2001.0 & 3.5 & 3.72 & 176 & & & & & 0 & 176 & 125 & & 0.939 & 1,355 & & 200 \\
\hline 2001.5 & 4.0 & & & & & 4.48 & 58 & 58 & 0 & 5 & & 0.083 & 1.438 & & 200 \\
\hline 2002.0 & 4.5 & & & 4.72 & 176 & & & & 176 & 175 & & 0.939 & 2.377 & & 200 \\
\hline 2002.5 & 5.0 & 5.21 & 176 & & & & & & 176 & 176 & & 0.939 & 3.315 & & 200 \\
\hline 20030 & 5.5 & & & & & 5.72 & 176 & & 176 & 176 & & 0,939 & 4.254 & & 200 \\
\hline 2003.5 & 6.0 & 6.46 & 176 & 6.21 & 176 & & & & 352 & 352 & & $\frac{}{1.878}$ & 6.132 & & 200 \\
\hline 20040 & 6.5 & & & & & & & & 0 & 0 & & 0.000 & 6.132 & & 200 \\
\hline 2004.5 & 7.0 & & & 7.46 & 176 & 7.21 & 176 & & 352 & 352 & & $\frac{1.878}{1.87}$ & 8.009 & & 200 \\
\hline 2005.0 & 7.5 & 7.70 & 176 & & & & & & 176 & 176 & & 0.939 & 8.948 & & 200 \\
\hline 2005.5 & 8.0 & & & & & 8.46 & 176 & & 176 & 126 & & 0.939 & 9.887 & & 200 \\
\hline 2006.0 & 8.5 & 89 & 176 & 8.70 & 176 & & & & 352 & 352 & & 1.878 & 11.764 & & 200 \\
\hline 2006.5 & 9.0 & & & & & & & & 0 & 0 & & 0.000 & 11.79 & & 200 \\
\hline 2000 & 9.5 & & & 9.94 & 176 & 9.70 & 176 & & 352 & 352 & & 1.878 & 13.642 & & 200 \\
\hline 2007.5 & 10.0 & 10.18 & 176 & & & & & & 176 & 176 & & 0.939 & 14.581 & & 200 \\
\hline 20090 & 10.5 & & & & & 10.94 & 176 & & 176 & 176 & & 0.939 & 15.520 & & 200 \\
\hline 200.5 & 11.0 & 11.42 & 176 & 11.18 & 176 & & & & 352 & 352 & & 1.878 & 17.397 & & 200 \\
\hline 20090 & 11.5 & & & & & & & & 0 & 0 & & 0.000 & 17.397 & & 200 \\
\hline 2009.5 & 12.0 & & & 12.42 & 176 & 12.18 & 176 & & 352 & 352 & & 1.878 & 19.275 & & 200 \\
\hline 20100 & 12.5 & 12.66 & 176 & & & & & & 176 & 176 & & 0.939 & 20.213 & & 200 \\
\hline 2010.5 & 13.0 & & & & & 13,42 & 176 & & 176 & 176 & & 0.939 & 21.152 & & 200 \\
\hline 2011.0 & 13.5 & 13,90 & 176 & 13.66 & 176 & & & & 352 & 352 & & 1.878 & 23.030 & & 200 \\
\hline 2011.5 & 14.0 & & & & & & & & 0 & 0 & & 0.000 & 23.030 & & 200 \\
\hline 2012.0 & 14.5 & & & 14.90 & 176 & 14.66 & 176 & & 352 & 352 & & 1.878 & 24.907 & & 200 \\
\hline 2012.5 & 15.0 & 13.14 & 176 & & & & & & 176 & 176 & & 0.939 & 25,846 & & 200 \\
\hline 2013.0 & 15.5 & & & & & 15.90 & 176 & & 176 & 176 & & 0.939 & 26.785 & & 200 \\
\hline 2013.5 & 16.0 & 16.39 & 176 & 16.14 & 176 & & & & 352 & 352 & & 1.878 & 28.663 & & 200 \\
\hline 2014.0 & 16.5 & & & & & & & & 0 & 0 & & 0,000 & 28.663 & & 200 \\
\hline 2014.5 & 17.0 & & & 17.39 & 176 & 17.14 & 176 & & 352 & 352 & & 1.878 & 30.540 & & 200 \\
\hline 2015.0 & 17.5 & 17.63 & 176 & & & & & & 176 & 176 & & 0.939 & 31.479 & & 200 \\
\hline 2015.5 & 18.0 & & & & & 18.39 & 176 & & 176 & 176 & & 0.939 & 32.418 & & 200 \\
\hline 2016.0 & 18.5 & 18.87 & 176 & 18.63 & 176 & & & & 352 & 352 & & 1.878 & 34.295 & & 200 \\
\hline 2016.5 & 19.0 & & & & & & & & 0 & 0 & & 0.000 & 34.293 & & 200 \\
\hline 20170 & 19.5 & & & 19.87 & 176 & 19.63 & 176 & & 352 & 352 & & 1878 & 36.173 & & 200 \\
\hline 2017.5 & 20.0 & 20.11 & 176 & & & & & & 176 & 176 & & 0.939 & 37.112 & & 200 \\
\hline 2018.0 & 20.5 & & & & & 20.87 & 176 & & 176 & 175 & & 0.939 & 38.050 & & 200 \\
\hline 2018.5 & 21.0 & 21.35 & 176 & 21.11 & 176 & & & & 352 & 352 & & 1.878 & 39.928 & & 200 \\
\hline 20190 & 21.5 & & & & & & & & 0 & 0 & & 0.000 & 39,928 & & 200 \\
\hline 20195 & 22.0 & & & 22.35 & 176 & 22.11 & 176 & & 352 & 35 & & 1.878 & 41.806 & & 200 \\
\hline 20000 & 22.5 & 22.39 & 176 & & & & & & 176 & 175 & & 0.939 & 42.74 & & 200 \\
\hline 2020.5 & 23.0 & & & & & 23.35 & 176 & & 176 & 176 & & 0.939 & 43.683 & & 200 \\
\hline 2001.0 & 23.5 & 23.83 & 176 & 23.59 & 176 & & & & 352 & 35 & & 1.878 & $\frac{-5.561}{45.561}$ & & 200 \\
\hline 2001.5 & 24.0 & & & & & & & & 0 & 0 & & 0.000 & $\frac{15.561}{4.51}$ & & $2 m$ \\
\hline 20020 & 24.5 & & & 24.83 & 176 & 24.59 & 176 & & 352 & $35:$ & & 1.878 & 47.438 & & 200 \\
\hline 20225 & 25.0 & 25.07 & 176 & & & & & & 176 & 176 & & 0.939 & 4.37 & & 200 \\
\hline 20230 & 25.5 & & & & & 25.83 & 176 & & 176 & 172 & & 0.939 & 49.316 & & 128 \\
\hline 2003,5 & 26.0 & 26.31 & WF. & 26.07 & 176 & & & & 176 & $17 i$ & 176 & 0.939 & 50.255 & & \\
\hline 2024.0 & 26.5 & & & & & & & & & & 0 & 0.000 & 50.253 & & \\
\hline 20245 & 27.0 & & & 27,31 & 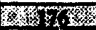 & 27.07 & Fink & & & & 352 & 0.000 & 50.255 & & \\
\hline 2025,0 & 27.5 & 27.56 & W & & & & & & & & 176 & 0.000 & 50.255 & & \\
\hline 2025.5 & 28.0 & & & & & 28.31 & 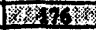 & & & & 176 & 0.000 & 50.255 & & \\
\hline 29060 & 28.5 & 28.80 & 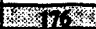 & 28.56 & SYX & & & & & & 352 & 0.000 & 50.255 & & \\
\hline 2006.5 & 29.0 & & & & & & & & & & 0 & 0.000 & 50.255 & & \\
\hline 2027.0 & 29.5 & & & 29.80 & \% & 29.56 & 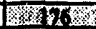 & & & & 352 & 0.000 & 50.255 & & \\
\hline 2007.5 & 30.0 & 30.04 & IVIV & & & & & & & & 176 & 0.000 & 50.255 & & \\
\hline 2009.0 & 30.5 & & & & & 30.80 & Kand & & & & 176 & 0.000 & 50.255 & & \\
\hline 2028.5 & 31.0 & & & 31.04 & 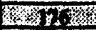 & & & & & & 176 & 0.000 & 50.255 & & \\
\hline & Totils & & & & & & & 348 & 9328 & 9576 & 2112 & 50.253 & & 348 & 9328 \\
\hline & MOX C194 & for wh & IDesirn & & $0.79 \mathrm{~K}$ & & or & Thtor & & & & & & & \\
\hline & MOX Capa & for Fu? & $\mathrm{CO}_{0} \mathrm{De}$ & & $13.34 \mathrm{~K}$ & & or & MT/NT & & & & & & & \\
\hline $\begin{array}{l}\text { 1. MOX } \\
\text { 2. U-23s } \\
\text { 3. Inidial } \\
\text { 4. No } \\
\text { 5. NII } \\
\text { 6. Minim } \\
\text { 7. Island } \\
\text { 8. Shnd }\end{array}$ & $\begin{array}{l}\text { Dad inclu } \\
\text { sembly. }\end{array}$ & & $x$ & & ope & cle. & & & & & & & & & \\
\hline
\end{tabular}


facility must be sized to process $189 \mathrm{MT}$ per year to produce the reload assemblies required for this alternative.

Alternate-2 dispositions $100 \mathrm{MT}$ of plutonium by extending the irradiation time for the three reactors in the reference option. Since the maximum existing operating license of any commercial BWR allows about 33 years of disposition, the existing BWRs chosen for this disposition alternative must be suitable for life extensions to satisfy the 48.4 year time frame. Should adequate life extensions not be available, the goal disposition quantity of $100 \mathrm{MT}$ plutonium must be reduced or additional reactors utilized. The MOX facility fabrication capacity remains nearly identical to the reference option with a capacity of 416 bundles per year, which requires a MOX processing capacity of $101 \mathrm{MT}$ per year to meet the core reloading schedules.

\subsubsection{Plutonium Feed Material Interface}

As weapons are retired from the existing nuclear arsenal, the warheads are removed and shipped to a disassembly location where the nuclear weapons components or "pits" are separated from the warheads and placed into storage. The plutonium in these weapons components is removed and either refabricated into new warheads, stored as part of the strategic reserve or identified as excess and placed into storage for non-military use. A plutonium hydride/dehydride process is being developed and demonstrated by Lawerence Livermor National Laboratory (LLNL) and LANL personnel for removing the plutonium from the "pits" in retired weapons. The four main advantages associated with this process are: (1) plutonium can be easily removed from the tight fitting "pit" configuration, (2) no additional waste is generated by the processing, (3) the plutonium configuration can be easily unclassified, and (4) consolidation into metal ingots provides a smaller, more compact form for storage. In this process, a "pit" is divided and each half is treated separately in a reaction vessel. A small amount of hydrogen is introduced into the reactor chamber at an elevated temperature and reacts with the plutonium, forming plutonium hydride (volume change during the reaction), which spalls from the "pit" and falls into a collection vessel. In the process under development by LLNL, hydrogen is added to the reactor until all of the plutonium has been converted to hydride and falls into the collection vessel. At that point, the hydride is heated to drive off the hydrogen (dehydrided), and the remaining plutonium is melted and cast into a metal ingot. In the LANL approach, uranium hydride is used to provide a small amount of hydrogen for reaction with the plutonium to form the hydride. The hydride falls into a heated collection vessel where the hydrogen is driven off and flows upward to the top of the reactor hydriding more plutonium. This cyclic process continues until all of the plutonium is converted to hydride and spalls from the "pit". The plutonium in the collection vessel is melted and cast to form a metal ingot. The reaction vessels required for the 
hydride/dehydride process are relatively small and can be readily adapted to glove box applications as well as automation.

The demonstration of the systems necessary for the disassembly of retired "pits" is underway in the Automated Retirement and Integrated Extraction System (ARIES) program at LANL [Ref. 2-9]. The conceptual design and prototype testing phases of this activity have been completed and the long lead time equipment items have been ordered. The need for the storage of the plutonium recovered from the retired "pits" was identified and LANL personnel are currently developing the requirements for this facility [Ref. 2-10]. This facility must be capable of handling the retired weapons plutonium as well as plutonium residues from various weapons sites located throughout the country. As a result, four different storage forms ("pits", oxide, metal and stabilized residues) are being considered for this facility. Ideally, "pit" storage would be eliminated from this facility because their classified nature requires additional security and control measures. The metal could be stored irrespective of impurity content, while the oxide would be accepted only after calcining so the loss on ignition at $1000^{\circ} \mathrm{C}$ would be less than $0.5 \%$. As in the case of metal, no impurity limits have been defined for the oxide form. The storage requirements for the stabilized residues have not been finalized.

Both LLNL and LANL have indicated there should be no problem converting either the hydride or metal form of plutonium to an acceptable oxide (see Appendix $\mathrm{E}$ for a draft specification). Each laboratory has some historical experience with converting metal to oxide, but additional testing will be required to evaluate the morphology of the resulting powder. Blending tests are also suggested to evaluate the plutonium homogenity of the material resulting from a physical blend process. Although the presence of gallium as an impurity does not appear to be a problem from the neutronics standpoint, the effect of gallium on the physical behavior (thermal conductivity, fission gas release, irradiation damage, cladding compatibility) of the fuel in a reactor has not been determined. Until testing verifies that gallium does not pose a problem to reactor operation, technology must be incorporated into the feed preparation processes to remove the gallium from either the oxide, hydride or metal form of plutonium.

The MOX fabrication process being considered for the MOX fabrication facility is based on proven glove box technology with automated operation and hands-on maintenance. In order to meet the requirement to minimize the worker radiation exposure during fabrication, the americium (Am) present in the plutonium feed material must be removed. The hydride/dehydride recovery process described above contains a step for Am removal just prior to the casting of plutonium ingots. Although this removal approach may be acceptable if the plutonium is fairly old because most of the $\mathrm{Pu}-241$ has decayed to Am-241, the lowest radiation 
exposure plutonium dioxide feed for MOX processing would result if the americium were removed just prior to oxide conversion. In this scenario, the plutonium is removed from the "pits" by the hydride/dehydride process, cast into ingsts and placed in storage. The plutonium identified for MOX fabrication is obtained from storage, heated to melting in small lots and the americium removed by chlorination. During subsequent steps, any undesirable impurities can be removed from the plutonium by either electro-refining or vacuum distillation. The resulting plutonium could be converted to oxide by an oxidation technique and shipped to the MOX fabrication facility.

Discussions with personnel at LLNL indicate that the feed material supplier would incur significantly lower costs if the americium was not removed from the feed material. In addition to the savings associated with the elimination of processing steps, the problems associated with handling and storing the americium could also be avoided. Installation of additional shielding in the MOX processing area, particularly the feed handling and blending areas, could reduce the worker exposure to acceptable levels. After the blending operations, the shielding provided by the urania should significantly reduce the exposure and the amount of additional shielding required. These process modifications to the MOX facility to accommodate additional exposure only transfer the location of the americium challenge. During the sintering operation, the americium tends to volatilize and transfer to the exit lines of furnace. This americium must be collected and removed from the glove box area to maintain exposure at acceptable levels. Since the amount of material processed as MOX fuel is about 20 times greater than the plutonia feed, additional cost savings would most likely be incurred by removing the americium from the plutonium instead of the MOX. A detailed evaluation of the costs to remove americium from the feed material should be performed. A comparison of these costs and the costs associated with providing additional shielding for the MOX facility, as well as the collection and storage of the americium from the sintering furnaces, must be made to determine the approach which provides the most benefit to the Pu dispositioning program.

\subsubsection{MOX Processing}

The powder blend process is utilized to convert the plutonium feed material for both island and full MOX fuel bundles, which can be used to provide reload fuel for existing BWRs. The processes utilized to fabricate fuel are similar for both island and full MOX assemble designs with the exceptions of throughput and storage quantities. The fuel for the island assemblies will be fabricated at a modified facility at LANL utilizing hands-on operation and maintenance with only limited automation of some processing steps. These processing details which are automated for the new MOX fabrication facility are summarized in the following sections. 
only limited automation of some processing steps. These processing details which are automated for the new MOX fabrication facility are summarized in the following sections.

\section{Plutonium Feed Material Receiving and Storage}

The plutonium feed material (in this case, plutonium dioxide) will be received in sealed containers from the facility which has converted the excess plutonium from retired weapons into the oxide form. Based on decay heat limits, 4.5 kilograms $(\mathrm{kg})$ of plutonium dioxide can be accommodated in a single package. If an exclusive-use shipment such as an SST is utilized, as many as 200 packages could be combined in a single shipment. Based on this shipment information, the MOX facility at LANL would receive four 50-kg shipments each year. Assuming that the plutonia arrives two months prior to the time required for blending in the fabrication process, the storage facility at LANL must be sized to store a maximum of $100 \mathrm{~kg}$ of material. The facility for fabrication of the full MOX assemblies will receive six $425-\mathrm{kg}$ shipments of plutonia each year. Utilizing the storage requirements developed at LANL, the storage facility for fabrication of full MOX assemblies is sized to store $650 \mathrm{~kg}$ of plutonia. Automated processing techniques will be utilized to receive and store the plutonia as well as transfer the material to the blending area.

\section{Blending and Pellet Fabrication}

In the pellet fabrication process, the feed oxides (plutonia, urania and gadolinia if required) will be transferred from the storage areas to geometrically safe holding bins in the blending and splitting area. Weight feeders will be utilized to load the appropriate amount of materials into a vibromill, where the powders are blended and milled together. The resulting blend will be split into as many as eight increments and, subsequently, the increments from the next eight blends will be cross blended to form a large uniform feed lot of powder. The blend splits will be stored in a geometrically safe arrangement and removed for continued processing one at a time. During reblending or cross blending, each blend split will be vibromilled to smooth blend differences and agglomerated as feed for the final pressing operation. Small hydraulic pellet presses developed for glove box applications will be used in the pellet pressing operations. Multi-cavity dies will be required in order to meet the throughput requirements. The MOX pellets will be loaded into molybdenum sintering boats with a capacity up to four kilograms each. Specially designed sintering furnaces, with removable refractory sections (to reduce downtime should a failure occur), will be utilized to sinter the MOX pellets. These furnaces will be designed with a capacity of $8 \mathrm{~kg} / \mathrm{hr}$ for MOX and $4 \mathrm{~kg} / \mathrm{hr}$ for MOX with gadolinia. The oxygen content of the 
After inspection for sintering characteristics, all acceptable pellets will be passed through a centerless grinding station to reduce the pellet diameter dimensions to final design tolerances. The grinder will have a control feedback loop which will automatically adjust the grinding operation to maintain the pellet diameter within specified limits. The column of pellets which exits the grinding operation passes directly to the automated pellet inspection station. At the station, the pellets are inspected and certified for diameter, length, surface finish and inspected for surface flaws such as cracks, chips and pits. After a statistical sampling for chemical characteristics, the pellets are placed in quarantine until final release by Quality Assurance. Acceptable pellets are transferred to the next station for loading into fuel rods while rejected pellets are sent to scrap recovery.

\section{Rod Processing}

Rod loading will be automatic but attended by an operator. The equipment will be properly shielded to minimize operator exposure levels. Vendor-supplied tubes with a serialized end plug welded on one end will be introduced into either the MOX or the MOX with gadolinia area from tube storage. Certified pellets will be arranged into fuel columns and the weight checked against the design specification. Following tube loading, the open end of the tube will be swabbed to clean the inside of any loose powder. Final welding will be done on an automated, operator attended, tungsten inert gas (TIG) welder. The rod is flushed, evacuated and backfilled with helium gas to a specific pressure, an end plug inserted, welded in place and ultrasonically inspected on line. After welding, the rod will be leak checked and examined for contamination. The rods will then be released and held in storage until required for bundle assembly. All in-process transport will be handled in shielded carts that will hold a critically safe quantity of rods.

In-process fuel rods will be stored in the rod storage racks. The storage racks will be designed to hold a critically safe batch of rods in the rack bins. A design for approximately 10,000 fuel rods will accommodate normal in-process fabrication activity. The storage area will be shielded and adequately ventilated to remove any generated heat. Trays will be moved into and removed from the storage rack bins by stacker-retriever robots on track rails. One robot will be located on the rod fabrication side of the facility and another on the bundle assembly side of the facility.

The in-process fuel rods will be removed from rod storage on the assembly side of the building. A certain percentage of the rods may be transferred to the X-ray station where final weld integrity is verified. Following $X$-ray, in-specification rods are returned to the rod storage area. Out-of-specification rods will be returned to the quarantined section of the rod storage area to 
The in-process fuel rods will be removed from rod storage on the assembly side of the building. A certain percentage of the rods may be transferred to the $\mathrm{X}$-ray station where final weld integrity is verified. Following $X$-ray, in-specification rods are returned to the rod storage area. Out-of-specification rods will be returned to the quarantined section of the rod storage area to await rework or further Quality Assurance disposition. All rods will be actively and passively scanned for fissile content, pellet integrity, fuel column consistency and gadolinia content. Scanners and X-ray equipment will be operator attended. Equipment will be automated to the maximum extent and shielded to minimize exposure levels.

\section{Bundle Assembly}

The bundle assembly operation will be performed at an automated assembly station. Selected rods required for the assembly of one bundle are collected at a rod accumulation station. The station is limited to a critically safe batch of 60 fuel rods. The accumulation and assembly stations will be operator attended and shielded to minimize operator exposure. The quantity of rods required by type will be mechanically removed from the holding trays, visually inspected and transferred to the automated bundle assembly station. The bundle assembly station is fixtured manually to accept bundle spacers and the water rod. Following fixturing and serial number reading, the rods are automatically indexed and positioned into the proper bundle grid location. A rod map of the loading locations is automatically transferred to a computerized Quality Assurance system for verification. This information is stored in a computerized data base for further Quality Assurance activities and bundle certification.

A "strong back" at the assembly station tilts the completed bundle to a vertical position from which an automated crane will move the bundle to a shielded in-process storage area awaiting final inspection. This in-process storage area is designed to hold a limited amount of critically safe fuel bundles. Bundle inspection will be done on an automated, operator attended, inspection station. Data accumulated from this inspection operation will be permanently stored in the Quality Assurance computerized database and, along with other data, will be used for customer certification. Bundles are automatically wrapped in a plastic sleeve for protection and transferred by remote control crane to the bundle storage area.

The final bundle storage area will be designed to store 750 bundles based on reload requirements. The bundles will be stored in critically safe storage containers nested in shielded and ventilated storage pits. All operations associated with bundle storage will be automated and remotely controlled. It is expected that the storage containers will serve as a shipping container to the reactor site. 


\section{Scrap and Waste Treatment}

One of the main characteristics of the MOX fuel manufacturing plant is the amount of plutonium handled, which requires safeguards and the establishment of an extremely precise material balance. When ensuring that this level of precision is obtained, one of the major challenges lies in controlling the residual quantities of plutonium which leave with the waste. Metal waste rich in plutonium (such as equipment and glove boxes) is carefully decontaminated and disposed. Scrap from rejected items created in the manufacturing operations are either recycled by a chemical conditioning operation or a dry recycle process. Waste created in the manufacturing operations that is combustible is collected and incinerated prior to plutonium recovered. Figure 2.4-2 is a typical flow diagram for scrap and waste from the MOX plant.

The main manufacturing operations can be represented by the following four stages:

- Powder preparation

- Pellet fabrication

- Rod fabrication

- Bundle assembly.

Scrap or reject materiul is mainly produced in the first two stages. Some waste is generated by all the plant manufacturing operations.

Manufacturing scrap or rejects containing plutonium are generated by:

- Glove box sweepings

- Laboratory and decontamination residues

- Rejected pellet batches

- Pellets from mechanical process rework (welding, loading, inspection)

- Filters

- Pellet grinding residue

- Equipment clean up residues. 


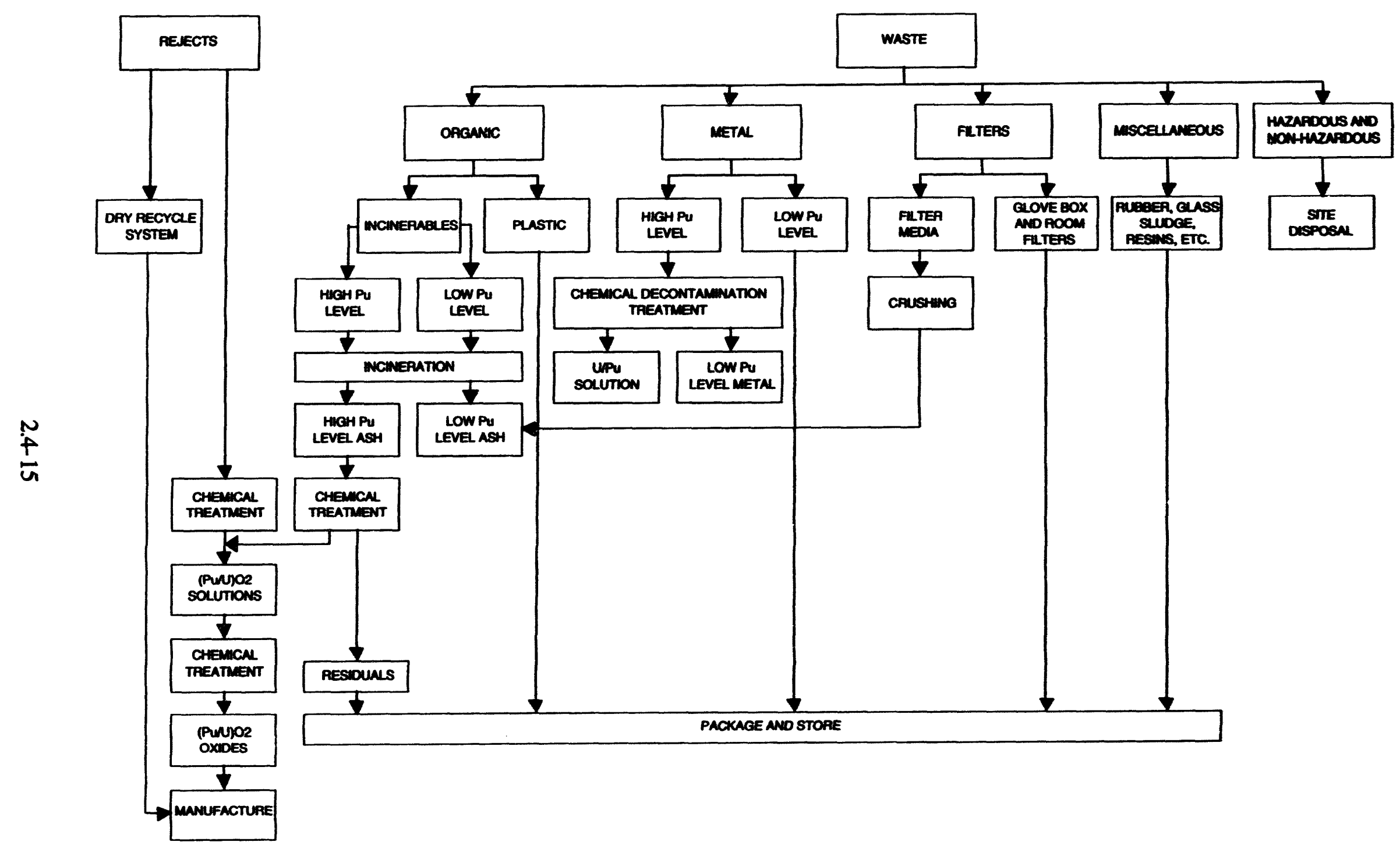

Figure 2.4-2. Treatment of Manufacturing Waste 
Pellet rejects are either chemically conditioned (for contaminated material) or recycled through a dry process and returned to the process through the blending operation. The other rejected material is chemically conditioned to produce powder which again can be directly recycled into the blending operation. In the early stages of the project, a typical quantity of rejected material to be recycled will vary from 10 - 15 MT per $100 \mathrm{MT}$ of MOX fuel processed. As the projects matures, a drop to 5 - $10 \mathrm{MT}$ per $100 \mathrm{MT}$ of MOX fuel processed can be expected.

The manufacturing waste consists of the following items:

- High and low-level plutonium incinerable organic waste

- Inactive plastic waste

- High and low-level plutonium containing metal waste

- Filter waste

- Miscellaneous waste.

The organic waste constitutes the major part of the manufacturing waste. Quantities estimated annually per $100 \mathrm{MT}$ of fuel manufactured are $45 \mathrm{MT}$ of high and low-level plutonium incineratable waste and $28 \mathrm{MT}$ of plastic waste based on preliminary studies performed at the Melox facility [Ref. 2-11]. Metal waste is estimated at 4 MT annually. Filter and miscellaneous waste is estimated to be about $6 \mathrm{MT}$ per year. The plutonium contained by these wastes is extracted chemically and can be directly recycled into the manufacturing process. It is estimated that the conditioning and chemical extraction of the plutonium from the scrap and waste will result in a processing and scrap loss of about 0.5 percent of the throughput. The Melox study indicated that the encapsulated wastes associated with $100 \mathrm{MT}$ of MOX fabrication annually will be in the $50 \mathrm{MT}$ range. This amount of waste corresponds to about 1150 fifty-five-gallon drums which must be discarded annually. Since the density of the wastes is relatively low, particularly for the plastic wastes, a compaction step could significantly reduce the volume. The MOX fuel fabrication facility is designed so that no liquids will be discharged to the environment. A liquid waste treatment area is equipped to collect the liquids and provide for chemical recycling. The main source of these liquids are several units operating in the solvent extraction area. The acidic and basic waste solutions are concentrated to $40 \%$ dissolved solids in concentrated nitric acid. The vapors from the concentrator are fed to a fractionating tower, where nitric acid is reclaimed for recycle within the plant. The solution from the concentrator is sent to a waste solidification area, where the remaining acid is driven off and the metal nitrates are converted to oxides. These 
oxides are collected in waste containers and stored for subsequent shipment to a government waste repository.

The fuel fabrication area produces three basic mixtures of gases which must be treated before discharge. The first group of these gas streams is $6 \%$ hydrogen in nitrogen mixed with some water vapor which results from various furnaces in the facility. All of these gases will be filtered hot to remove any potential uranium or plutonium particulate and routed to the building exhaust system for dilution and subsequent treatment. The second group of gases is primarily nitrogen with water vapor diluted with air supplied to fluidize the conversion beds in the wet scrap recycle area. These gases are filtered hot to remove any particulate and routed to a gas scrubber. The scrubber is designed to chemically react with any of the oxides of nitrogen present in the gas. The final group of gas streams is generated by the scrap dissolution and waste treatment areas and contain appreciable amounts of nitrogen oxides. These gases are sent to an absorber designed to remove 95 plus percent of the nitrogen oxides. The resulting liquid recovered from the absorber tower contains significant amounts of nitric acid and will be recycled within the facility. The gases from the absorber tower are scrubbed to remove any remaining oxides of nitrogen prior to discharge to the building exhaust system.

\subsubsection{MOX Facilities}

The TA-55 area at LANL will be modified by adding selected new equipment items for fabrication of island design fuel assemblies. The space allocations, equipment costs and schedule have been reviewed with LANL personnel.

A new facility has been designed to receive weapons grade plutonium in the oxide form and fabricate the material into full MOX fuel assemblies. This facility contains the necessary equipment and processing technology to receive and store all feed materials as well as fabricate fuel pellets, rods and bundles. Adequate space has been provided to store the assemblies required for the initial reactor cores and subsequent reloads. Provisions have been made to treat and minimize the process scrap and waste streams. A building layout is shown for the new PDC in Figure 2.4-3. The throughput requirements for the reference case, as well as all alternatives, is such that the facility will be the same size in each case. Any changes necessary to satisfy throughput adjustments can be accomplished by adding individual equipment items in available space or modifying the fabrication shift schedules.

The portions of the building housing plutonium material will be designed to DOE Order 6430.1A, Section 1304, "Plutonium Processing and Handling Facilities," and Section 1305, 


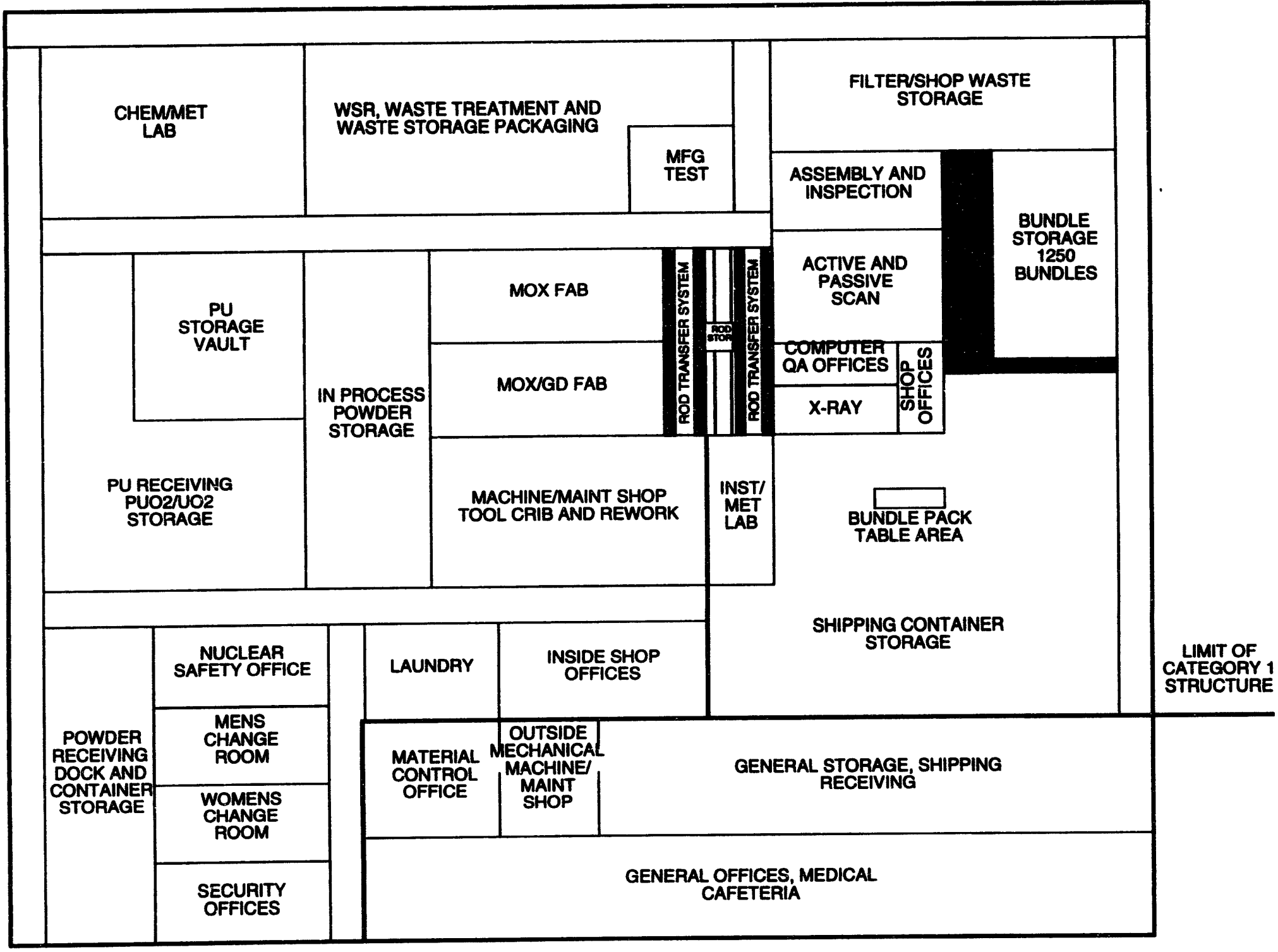

Flgure 2.4-3 MOX Facility Arrangement 
"Plutonium Storage Facilities." The parts of the building which normally or potentially contain plutonium or radionuclides will be reinforced concrete structures designed to Safety Class 1 (Category 1) standards to maintain their confinement and safety functions in the event of a design basis accident. Processing of the material which contains plutonium will be achieved in glove boxes with appropriate ventilation controls. Any transfers, movement or storage of material will be accomplished in sealed containers.

The staff required for the MOX facility was based on operating the process areas 24 hours a day for 300 days per year. The remaining time in each year will be utilized to perform off-line maintenance, inventory verifications and accountability checks. Weekly staffing in the processing areas was based on a five-shift per week rotation, which provides about 6 hours per week for training and certification on each shift. An organization chart for the staffing of the MOX facility is shown in Figure 2.4-4. A breakdown of the employees by both number and distribution is presented in Table 2.4-3 for the reference MOX fabrication facility during steadystate operations. It is anticipated that the overall site will share emergency, safety, security, regulatory compliance, facilities and disaster team personnel which have been excluded from the staffing estimate in Table 2.4-3. The staffing for the MOX facility is estimated to increase by $50 \%$ per year during the equipment installations and checkout phases of the program as defined in the schedule shown in Section 6.6. A full complement of personnel will be on-board at the end of the fourth year to initiate startup testing and subsequent operation. The uniqueness of the MOX facility operations and lack of qualified personnel will require an extensive training program to establish safety, radiation protection and health physics procedures to properly operate the facility and reduce the associated risks.

\subsubsection{Variation for Use of DOE Facilities}

The reference costing approach requires that a new MOX fuel fabrication facility be constructed at a DOE operated greenfield site. The utilization of existing DOE sites and facilities can greatly reduce the initial capital expenditures required for the MOX fabricaton facility, as well as reduce the time required to establish an operating facility. Location of the MOX facility at an established DOE site allows the utilization of existing infrastructure which includes design engineering, maintenance, analytical laboratories, waste treatment facilities and storage facilities. The existing functions which provide permitting, transportation, security, safeguards and accountability will also be of unquestioned value. A number of evaluations were made of 


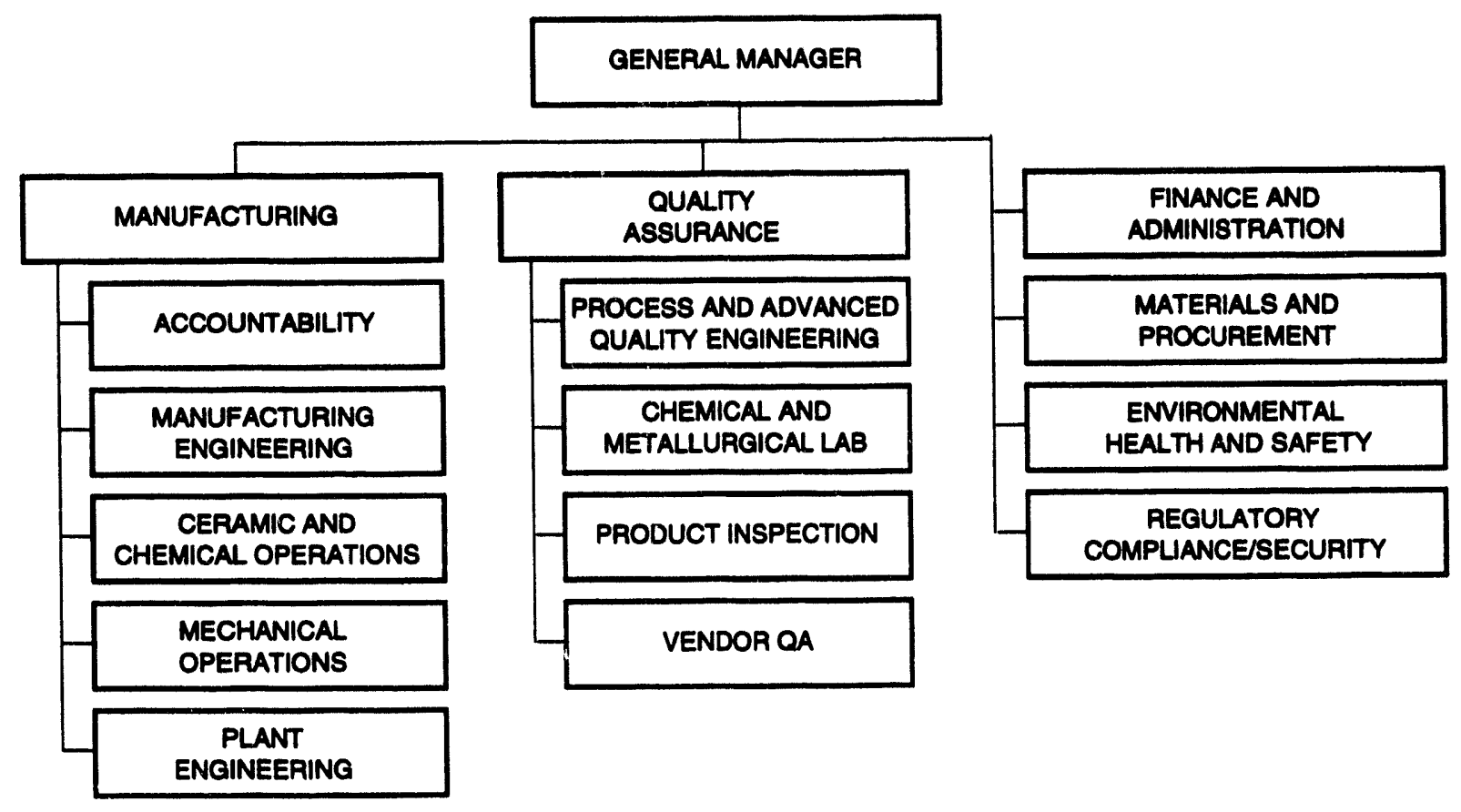

Figure 2.4-4. MOX Fuel Plant Organization Chart 
Table 2.4-3 MOX Plant Staff - Reference Disposition Option

\begin{tabular}{|c|c|c|c|c|c|c|}
\hline Function & $\begin{array}{c}\text { General } \\
\text { Manager } \\
\text { Staf } \\
\end{array}$ & $\begin{array}{c}\text { Manager } \\
\text { and } \\
\text { Supervisor }\end{array}$ & Professional & $\begin{array}{l}\text { Clerk and } \\
\text { Technician } \\
\end{array}$ & Hourly & $\begin{array}{l}\text { Total by } \\
\text { Function }\end{array}$ \\
\hline General Manager & 1 & & & 1 & & \\
\hline $\begin{array}{l}\text { Finance \& } \\
\text { Administration }\end{array}$ & $i$ & 1 & & 2 & & \\
\hline $\begin{array}{l}\text { Materials \& } \\
\text { Procurement }\end{array}$ & 1 & 3 & & 3 & 6 & \\
\hline $\begin{array}{l}\text { Environmental \& Health } \\
\text { Safety }\end{array}$ & 1 & & & 9 & & \\
\hline $\begin{array}{l}\text { Compliance \& } \\
\text { Licensing }\end{array}$ & 1 & & & 8 & & \\
\hline Total Administration & 5 & 4 & 0 & 23 & 6 & 38 \\
\hline $\begin{array}{l}\text { Manufacturing } \\
\text { Accountability }\end{array}$ & $\overline{1}$ & 3 & 3 & $\begin{array}{l}1 \\
9\end{array}$ & & \\
\hline Manufacturing Engineer & & 1 & 14 & 3 & & \\
\hline $\begin{array}{l}\text { Chemical \& Ceramic } \\
\text { Operations }\end{array}$ & & 9 & & 5 & 55 & \\
\hline $\begin{array}{l}\text { Mechanical Operations } \\
\text { Plant Engineering }\end{array}$ & & $\begin{array}{l}2 \\
3\end{array}$ & & 3 & $\begin{array}{l}10 \\
16\end{array}$ & \\
\hline Total Manufacturing & $\overline{1}$ & 18 & 17 & 21 & 81 & 138 \\
\hline $\begin{array}{l}\text { Quality Assurance } \\
\text { Vendor \& Process } \\
\text { Chemical Laboratory } \\
\text { Production Inspection } \\
\end{array}$ & $\overline{1}$ & $\begin{array}{l}1 \\
4 \\
1 \\
\end{array}$ & $\begin{array}{l}9 \\
2\end{array}$ & $\begin{array}{c}1 \\
2 \\
15 \\
8 \\
\end{array}$ & & \\
\hline Total QA & $\overline{1}$ & 6 & 11 & 26 & $\overline{0}$ & 44 \\
\hline Total by Title & $\overline{7}$ & 28 & 28 & $\overline{70}$ & 87 & 220 \\
\hline
\end{tabular}

existing DOE sites during this study and are summarized in Appendix F. A more detailed assessment of the buildings which might be utilized to convert weapons plutonium to feed material, and to process the feed material into MOX fuel assemblies for irradiation in a BWR is summarized in this section. The MOX fabrication processes arranged to fit into existing buildings are shown for several sites and the potential cost saving are presented in Section 6.2.3.

\section{Hanford Site}

The Fuels Materials Examination Facility (FMEF) was originally designed for examination of irradiated Fast Flux Test Reactor (FFTF) MOX fuel and then modified to fabricate MOX fuel for FFTF as well as other functions. Several years were spent procuring and installing equipment items for the Secure Automated Fabrication (SAF) facility to produce fuel utilizing automated processes which would reduce worker exposure levels. Since much of this support and processing equipment is still located in the FMEF, selected items which can be adapted to 
shaded areas in Figure 2.4-5. Much of the other unused space in this building could be utilized for conversion of plutonium to feed material, storage of feed material and fuel assemblies as well as scrap and waste processing. It should be noted that portions of this facility were specifically designed for processing MOX fuel and could be easily adapted for use during fabricaion of MOX fuel for existing BWRs.

\section{Idaho National Engineering Laboratory}

The Fuel Processing Restoration (FPR) facility at the Idaho Engineering National Laboratory (INEL) is a new building constructed to the Category 1 requirements necessary for plutonium handling. The facility is over $90 \%$ complete and nuclear material has not been introduced into the building. The MOX fabrication facilities for the reference dispositioning option could be installed on the mezzanine and upper levels leaving adequate space for the other operations required in the MOX facility as shown as shaded areas in Figures 2.4-6 and 2.4-7. The cells and rooms located on the four lower levels of this facility can be utilized for pit storage, metal-tooxide conversion, assembly storage as well as scrap and waste treatment. In addition, the fuel assembly area in the Zero Power Physics Reactor (ZPPR) facility could provide adequate space for the conversion of plutonium metal to oxide. This facility is also equipped with a plutonium storage vault. This vault could be utilized to store either plutonium metal or the oxide. The New Material Isotopic Separation (NMIS) area associated with the Advanced Test Reactor is a Category 1 facility which could provide space for plutonium conversion or rod assembly and inspection operations. Various other facilities at the INEL site have been utilized for both wet and dry rod storage operations which may be directly applicable to some of the operations in the MOX facility.

\section{Los Alamos National Laboratory}

The TA-55 area at LANL houses several glove box lines which have been used to fabricate mixed oxide and uranium nitride fuel [Ref. 2-8]. Both development and demonstration of automated technology for the disassembly of pits from retired weapons is underway at this location. Conceptual design of an Automated Retirement and Integrated Extraction System (ARIES) has been completed and long lead time equipment items ordered. An automated hydride-dehydride process has been selected for this facility. Additional facilities are also available for development and testing of plutonium-to-oxide conversion technology. The fuel fabrication facilities at LANL will be utilized for process verification, demonstration and 


\section{$\bullet$}
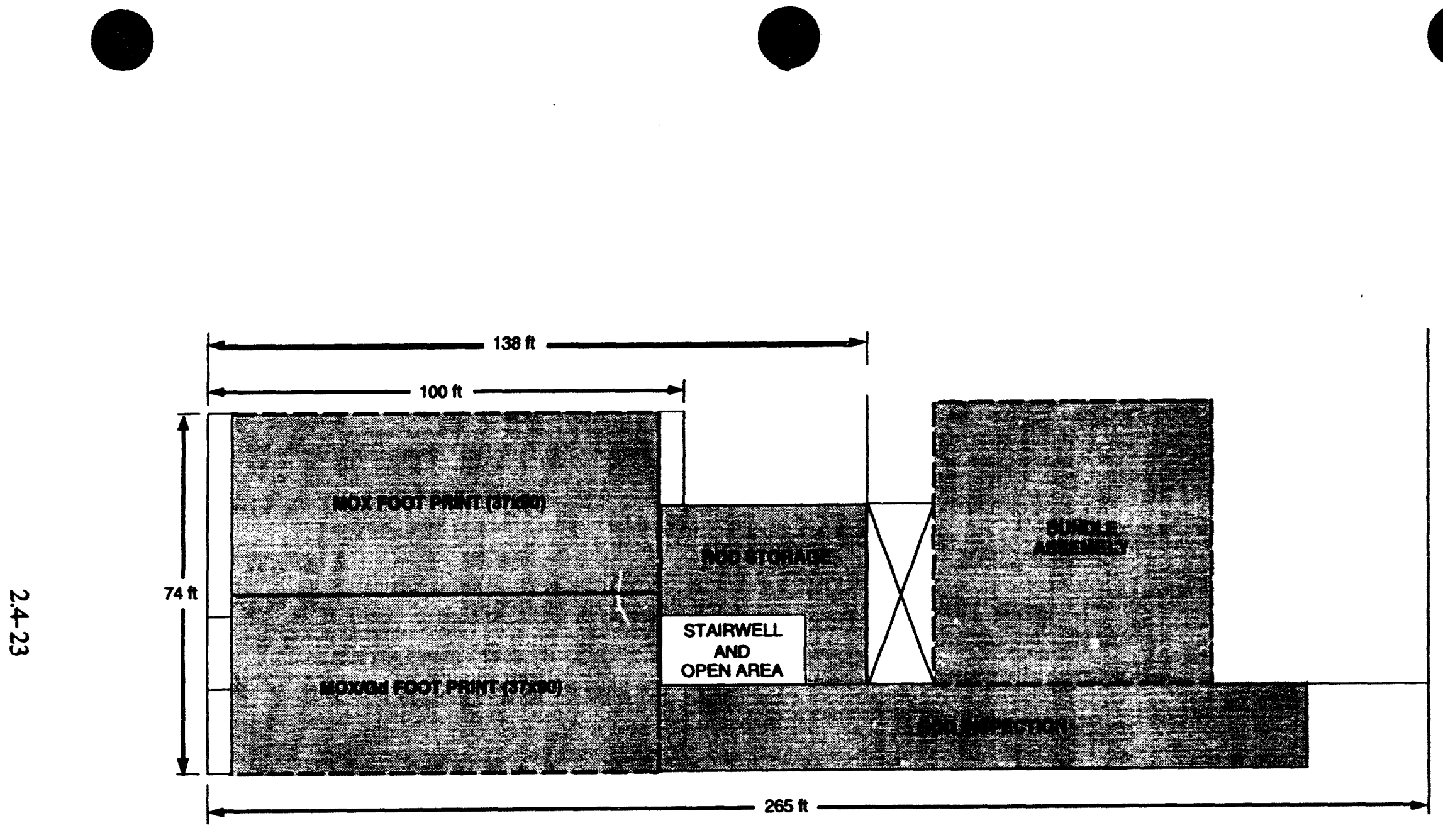

勇

Figure 2.4-5 Adaptation of FMEF 70 Foot Level Internal Area for MOX Fabrication 


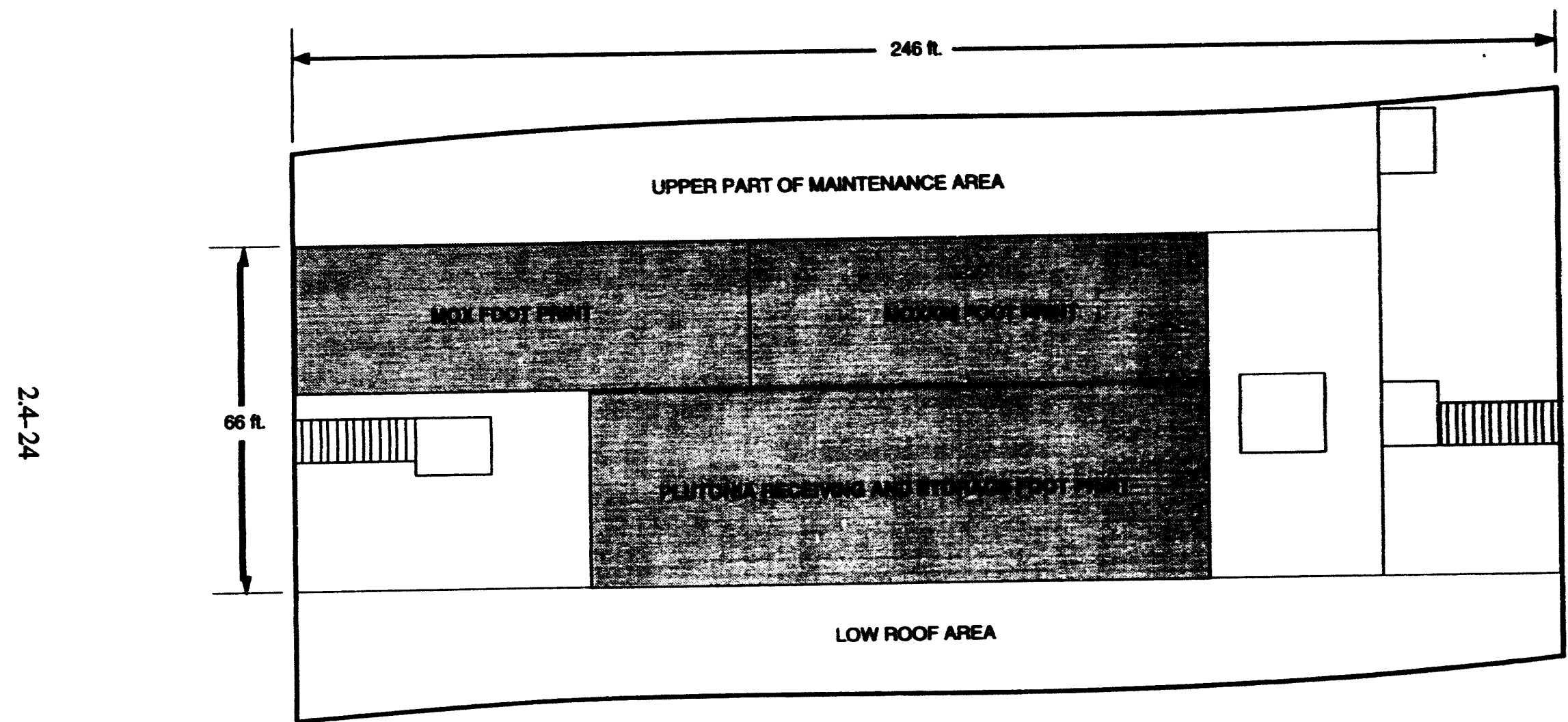

Figure 24-6 Upper Level of Fuel Processing Restoration Building 


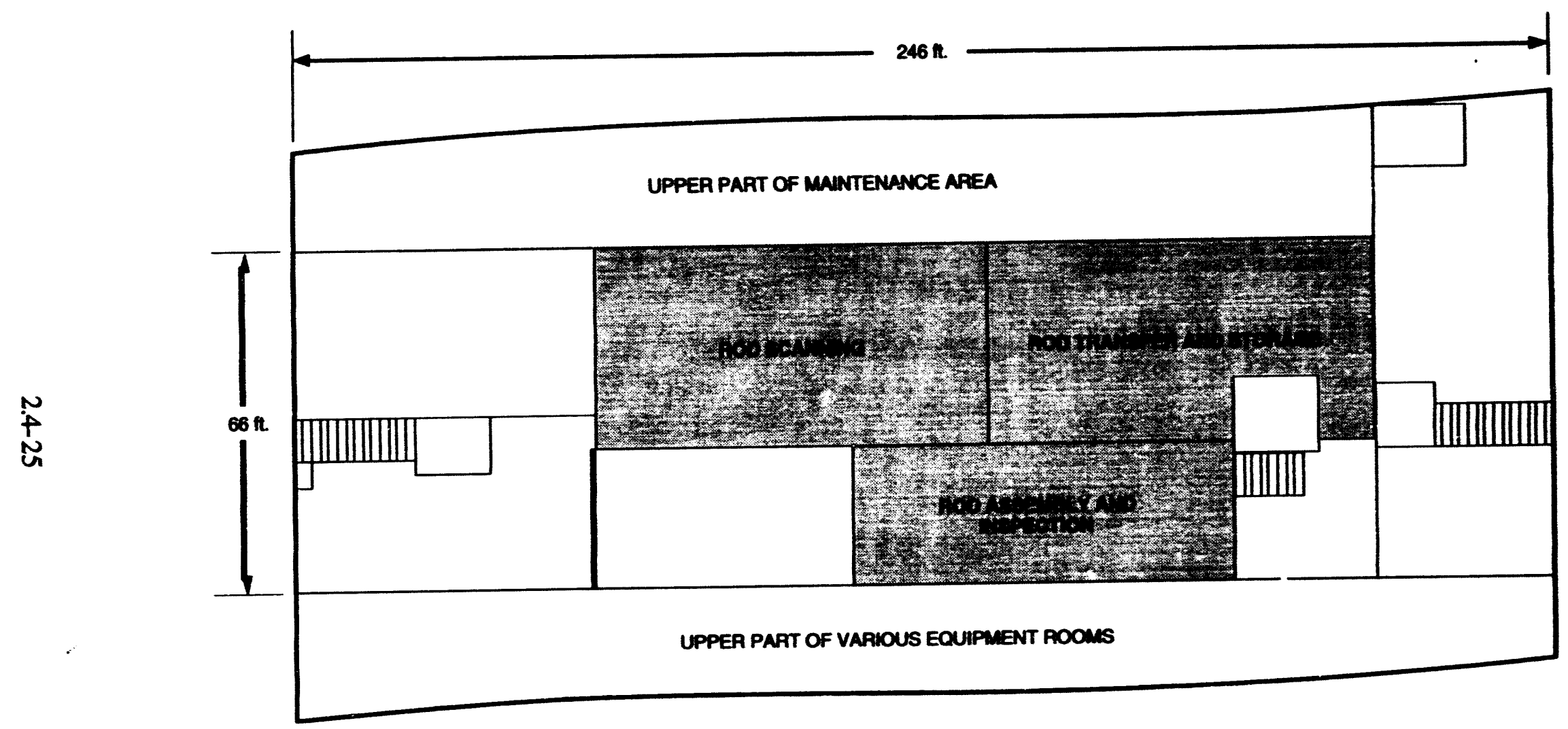

Figure 2.4-7 Mezzanine Level of Fuel Processing Restoration Bulling 
fabrication of test fuel assemblies. The modification of these facilities for fabrication of island design assemblies will require the addition of new milling, sintering, welding and rod inspection equipment. The current single shift operation will require modification to accommodate threeshift operation most likely on a five-day per week basis. The six-month delay between the final fabrication of island assemblies and start of fabrication of full MOX assemblies should allow movement of uncontaminated equipment items (welder, rod inspection equipment and analysis instrumentation) to the new MOX facility. Development of remote analysis techniques utilizing fiber optics technology will allow location of instrumentation outside of the glove box environment. This is a key activity at LANL which will reduce the product certification times associated with MOX fabrication. Processes which reduce the waste volumes associated with glove box related scrap and waste are also being developed and evaluated. Although the construction of production facilities at LANL may be inconsistent with their existing goals, the expertise and technology developed at this site, as well as the demonstration of island design fuel fabrication, will provide key elements necessary for the success of the plutonium dispositioning activities.

\section{Lawrence Livermore National Laboratory}

The facilities at LLNL have been instrumental in the development and demonstration of technology to recover plutonium from retired weapons. Several processes have been reviewed for plutonium recovery from weapons. Demonstration of the hydride-dehydride processing steps and the required robotics systems for remote operation are under evaluation. Molten salt type processes are under development for the handling and treatment of scrap and waste streams that result from the recovery of plutonium, conversion of the metal to oxide and MOX fuel fabrication. Although the location of LLNL in a populated area and the current goals may preclude location of any production facilites at this site, the expertise and technology developed at this location will provide key elements for design and operation of the necessary MOX facilities.

\section{Nevada Text Site}

The Engine Maintenance, Assembly and Disassembly (E-MAD) facility was used to assemble and prepare nuclear rocket engines for testing. After completion of the rocket program, the facility was used to evaluate the option of spent fuel storage in granite formations. Although this area could be utilized for plutonium conversion or MOX fabrication, extensive upgrading would be required before the facility could be used as part of thie plutonium disposition project. 


\section{Oak Ridge Site}

The Y-12 Building at the Oak Ridge Site has been used for the enrichment of uranium ore for the nuclear industry. Since the operation of this area has been terminated, the potential exists for upgrading this facility to meet plutonium handling criteria for this project.

\section{Savannah River Site}

The Barnwell Nuclear Fuel Plant (BNFP) is located adjacent to the Savannah River Site. The Waste Tank Exchange Gallery (WTEG) facility located within the Barnwell complex is capable of containing the MOX fuel fabrication facility for the reference dispositioning options, but additional space several times the current area must be added. This space will accommodate the powder receiving, rod inspection, bundle inspection and bundle assembly areas as shown in Figure 2.4-8. The New Special Recovery facility, which has not been contaminated, is capable of handling all of the scrap and waste from the MOX facility which is processed by aqueous techniques. The Plutonium Storage Facility can also be utilized for feed material receiving and storage. A great deal of automation technology has been developed and tested at this site for mechanized processing in canyon type areas. This technology should be directly applicable to automated MOX fabrication. 
NEDO-32361

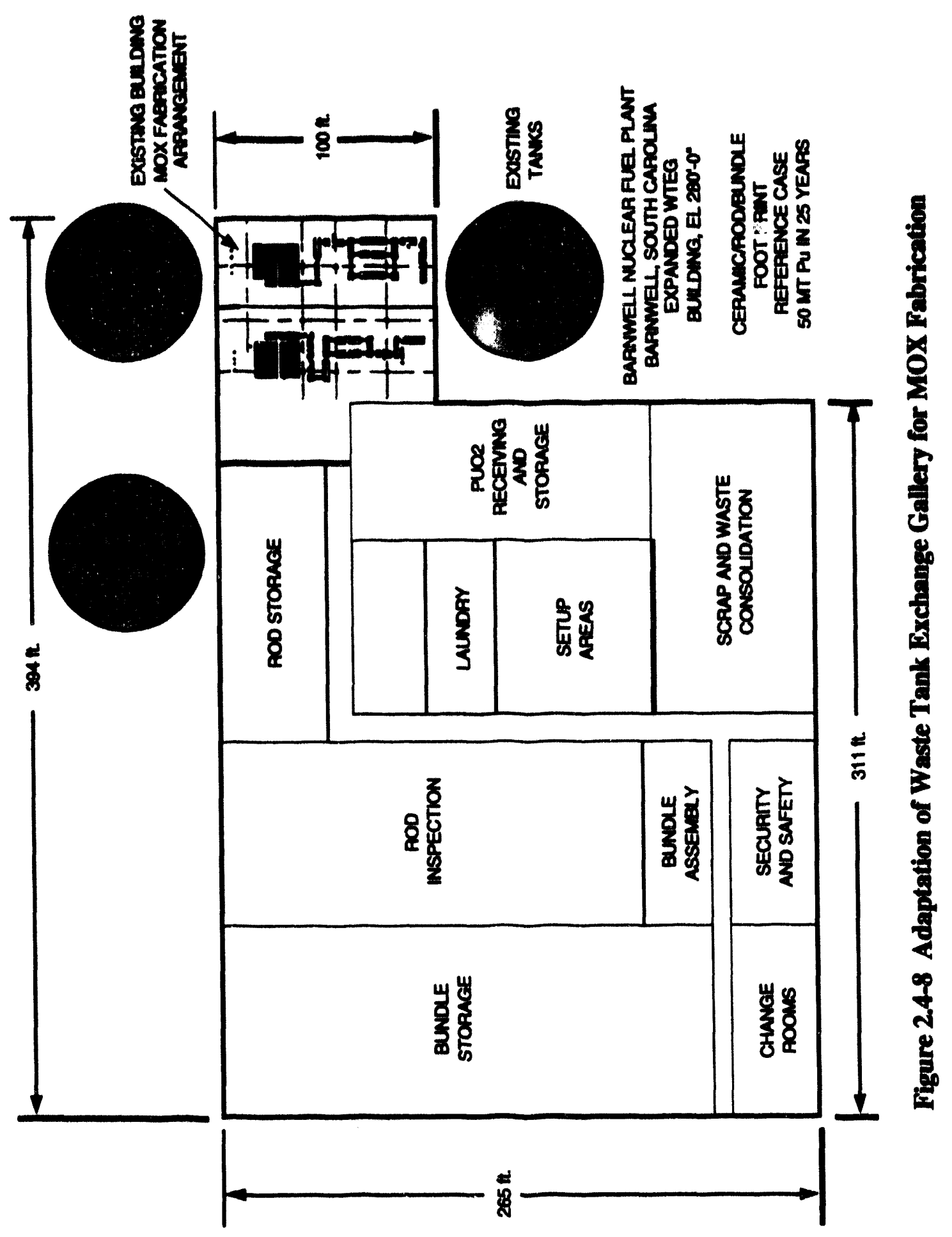




\subsection{Ex-Reactor Storage for Fresh and Spent Fuel}

Section 2.6 provides the amount of fresh MOX fuel needed and spent fuel generated for plutonium disposition in commercial BWRs. Because of the additional safeguards and security associated with plutonium (Section 7), there are some differences in the handling and storage of the fresh MOX fuel. Furthermore, since the spent fuel pools in some of the reactors under consideration will be nearing their csipacity in mid-1997 when the plutonium disposition mission is expected to start and the Federal Repository for spent fuel at Yucca Mountain may not be available, it is reasonable to consider that some amount of ex-reactor building spent fuel storage will be necessary.

\subsubsection{Fresh Fuel Storage Outside the Reactor Building}

The planning basis for the study is to provide a single, stand-alone complex for ex-reactor building storage for both fresh and spent fuel close to the reactor site. Security for the storage complex is provided by a double fence that incorporates both intrusion detection and assessment plus a hardened guard post with personnel surveillance, access control and communications. While this approach is applicable to any site, other configurations which include part or all of the storage within the plant security fence may be more cost effective, depending on the characteristics of the sites and plants selected.

Normal practice for operating BWRs is to stage the fresh $\mathrm{UO}_{2}$ fuel in its shipping containers at the power plant prior to the refueling outage. The fuel is then transferred to the refueling floor (Fuel Building in Mark III plants) as needed, removed from its shipping container, inspected and placed in the fuel storage pool.

As discussed in Section 2.7, the need to use SST's for transport of fresh MOX fuel from the fabrication plant combined with their limited payload $(10,000 \mathrm{lb})$ leads to approximately 18 shipments for each reload. In order to minimize the impacts of the increased MOX fuel security requirements during new fuel staging, an ex-reactor building fresh fuel storage vault is provided. In this way, the increased security associated with fresh MOX fuel is limited to the ex-reactor building storage area until the MOX fuel is transferred to the plant just prior to the actual refueling operation.

The alternative to an ex-reactor MOX fuel vault would be to enhance the security provisions for fresh fuel storage in the Reactor Building. This approach is technically feasible and eliminates the additional short distance shipment from the ex-reactor vault to the Reactor Builuing. 
However, since the fresh MOX fuel is expected to be delivered over a period of several months, the impacts on personnel access within the Reactor Building over such an extended period could be a significant problem, depending on plant configuration. For Mark III plants, which have a separate Fuel Building, the use of existing facilities may be more cost effective than an exreactor MOX fuel vault.

Figure 2.5-1 shows the layout of the proposed fresh MOX fuel storage vault. The storage area consists of a $40 \times 60$ foot reinforced concrete vault with an attached $45 \times 45$ foot conventional steel building. The storage area would be designed to meet the safety and security requirements of DOE Order 6430.1A for plutonium storage facilities. The function of the steel building (anteroom) is for weather protection and screening for unauthorized observation of the SST unloading operation.

The entire facility would be located within a double security fence (Figure 2.5-2) and would be provided with the required fire, radiation, criticality and security monitoring instrumentation. The wooden shipping overpacks will be stored away from the building. This, plus other administrative controls on combustible materials, should preclude the need for automatic fire suppression within the building.

The SST payload contains five fresh fuel shipping containers, each holding two BWR fuel bundles. The shipping containers would be unloaded from the SST into the anteroom, where the internal shipping container would be removed from its wooden overpack. The internal shipping containers would immediately be moved into the vault for storage. The vault is sized to hold 140 shipping containers (160\% of a normal reload) stacked in 2-wide by 5-high arrays with a 3-foot clear space on all sides for ease of inspection. The vault would be provided with a 2-ton overhead crane plus adequate laydown area for handling the shipping containers.

The criticality analysis for transportation accidents discussed in Section 8.4 confirmed that an infinite array of internal shipping containers flooded with water was subcritical. Further, as discussed in Section 7, the proposed SNM accountability concept is based on maintaining the MOX fuel in the internal shipping containers while in the storage vault. Thus, the internal shipping containers can be relied upon to provide adequate spacing and no additional criticality control features need to be incorporated into the vault design.

\subsubsection{Normal Fresh Fuel Handling at the Reactor}

Figure 2.5-3 illustrates the typical flow path for fresh fuel from the time the shipping container arrives in the Reactor Building until the fuel is loaded in the core for the Mark I and Mark II 


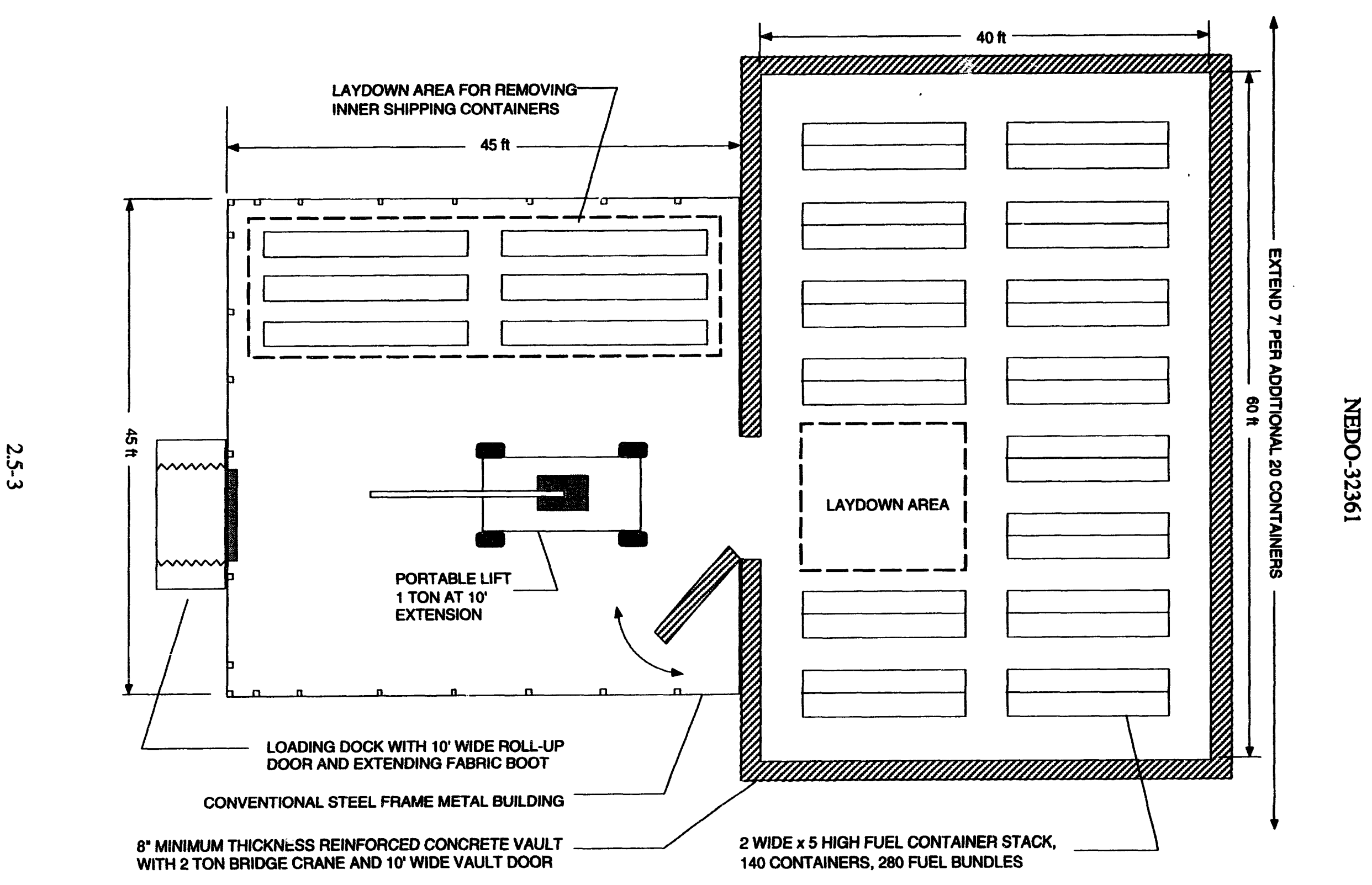

Figure 2.5-1 Fresh MOX Fuel Storage Vault 


\section{NEDO-32361}

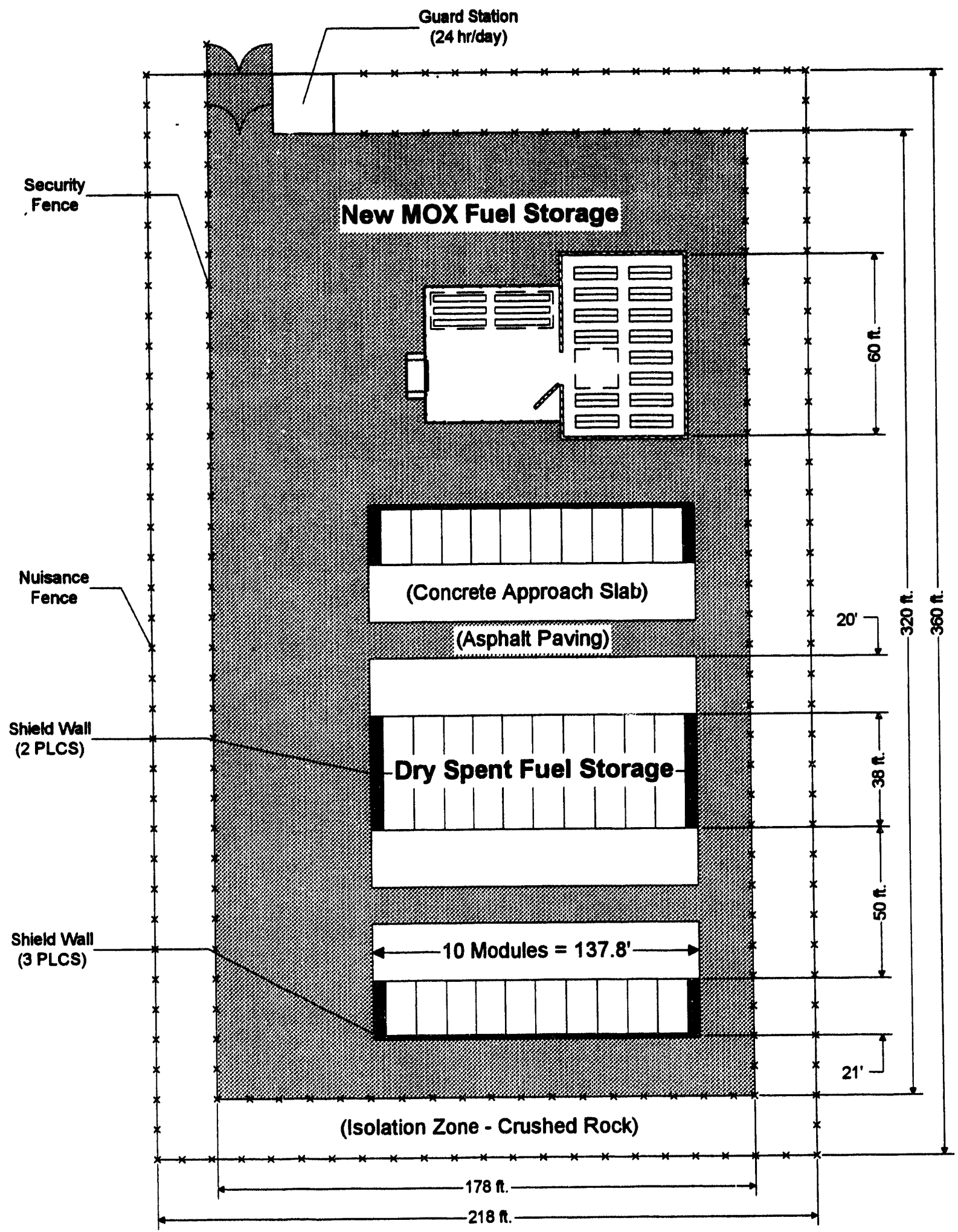

Figure 2.5-2 Ex-Reactor Fuel Storage 
NEDO-32361

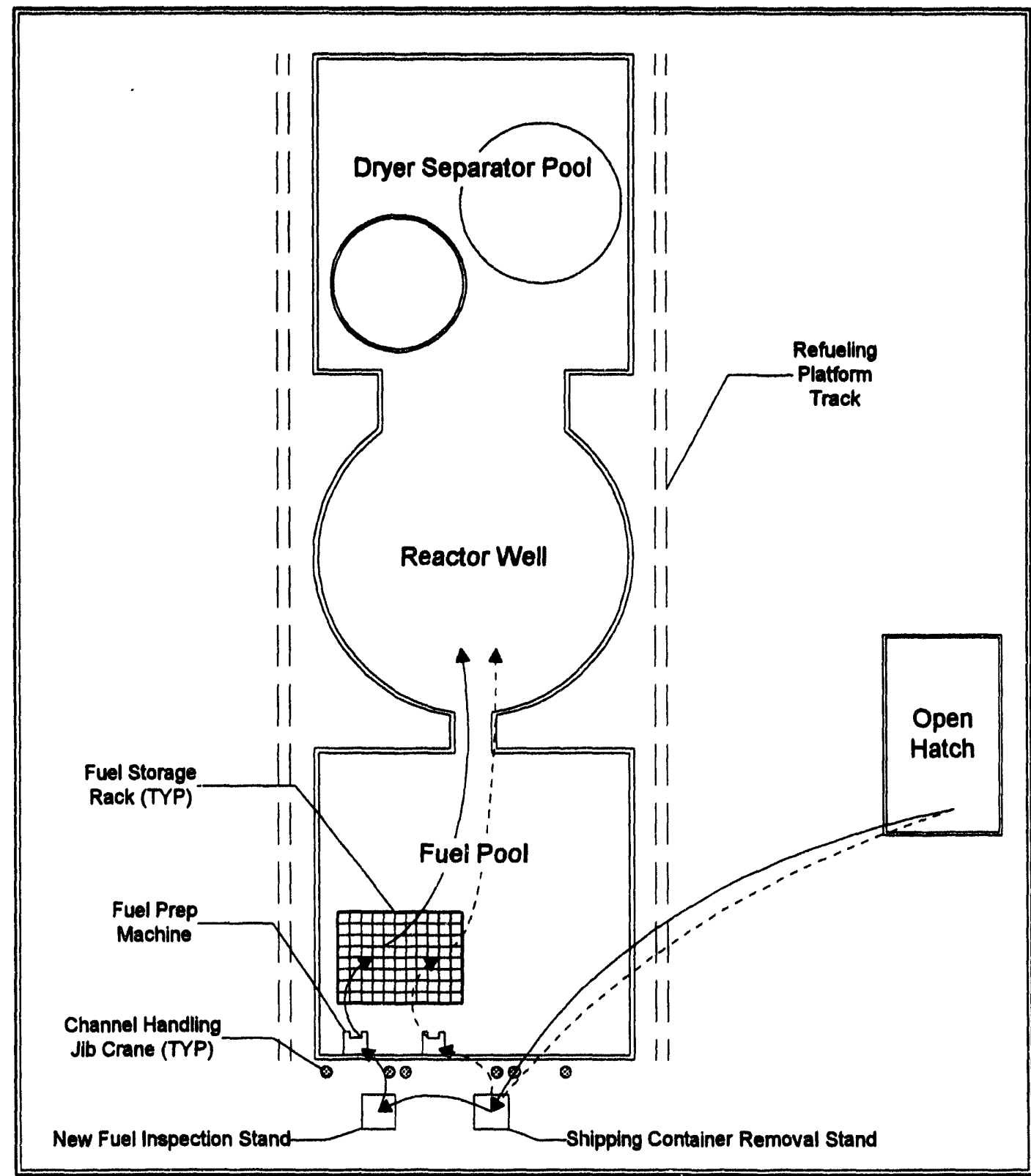

Alternate Fuel Handling

Normal Fuel Handling

Figure 2.5-3 Typical Refueling Floor Layout 
containment designs. The Mark III design contains an upper pool inside the primary containment boundary and a lower fuel storage pool located in a separate fuel building which is part of the secondary containment. Fresh and spent fuel is transferred from the lower pool to the upper pool and vice-versa through the Inclined Fuel Transfer System.

The following discussions are based on the Mark I/II containment designs. However, the basic fuel handling activities are the same for a Mark III design except that the fresh fuel receipt and preparation activities would be performed in the lower pool and the completed fuel bundles stored in the upper pool until loaded into the core.

Fuel shipping containers received from the ex-reactor vault are lifted from the transport vehicle to the refueling floor by the reactor building crane. Once on the refueling floor, the shipping container tamper indicating devices will be verified and the container identification information recorded. The shipping containers will then be uprighted, opened and the fuel elements transferred to the new fuel inspection stand.

The new fuel inspection stand serves as a support for the fuel bundles while undergoing inspection and provides a working platform for technicians engaged in performing the inspection. The new fuel inspection stand consists of a vertical guide column, a lift unit to position the work platform at any level, bearing seats and upper clamps to hold the fuel bundles in position.

Following inspection, the bundle is transferred to one of two fuel preparation machines attached to the spent fuel pool wall. The fuel preparation machine is used for stripping reusable channels from spent fuel and for rechanneling of the new fuel. Each fuel preparation machine consists of a work platform, a frame, and a movable carriage. The frame and moveable carriage are located below the normal water level in the pool, thus providing a water shield for the fuel assemblies being handled. The fuel preparation machine carriage has permanently installed up-travel-stops to prevent raising the fuel above the safe water shield level. The fuel preparation machines may also used with the fuel inspection fixture to provide underwater inspection capability.

Other standard BWR fuel servicing equipment includes:

- A channel bolt wrench, which is a manually operated device used for removing and installing the channel fastener assembly while the fuel assembly is held in the fuel preparation machine. The channel bolt wrench has a socket which mates and captures the channel fastener capscrew. 
- A channel-handling tool used in conjunction with the fuel preparation machine to remove, install and transport fuel channels in the spent fuel pool. The tool is composed of a handling shaft, angle guides, and clamp arms which engage the fuel channel.

- A general-purpose grapple tool that can be attached to the jib crane to handle fuel during channeling.

- A channel handling boom with a spring-loaded balance reel used to assist the operator in supporting a portion of the weight of the channel as it is removed from the fuel assembly. The boom is located between the fuel preparation machines. With the channel handling tool attached to the reel, the channel may be conveniently moved between the fuel preparation machines.

A schematic of the typical BWR refueling process is shown in Figure 2.5-4. Spent fuel removed from the reactor vessel is stored underwater while awaiting disposition. In addition, the pool provides interim storage for new fuel and for irradiated fuel channels. Spent fuel storage racks which are used for these purposes are located at the bottont of the fuel storage pool under sufficient water to provide radiological shielding. The spent fuel pool is a reinforced concrete structure with a stainless steel liner. The bottoms of all pool gates are sufficiently high to maintain the water level over the spent fuel storage racks and provide adequate shielding and cooling. All pool fill and drain lines enter the pool above the safe shielding water level. Antisiphon vacuum breakers preclude a pipe break from siphoning the water from the pool and jeopardizing the safe water level. The spent fuel storage pools of operating BWRs vary but typically provide for $\approx 10$ years of fuel discharge. In addition, reserve capacity must be maintained for a full core discharge.

Fuel storage racks within the pool are top entry, providing storage for fuel assemblies (with channels) and fuel bundles (without channels). The upper tie plate of the fuel elements rests against the rack to provide lateral support. The lower tie plate sits in the bottom of the rack, which supports the weight of the fuel. The rack modules are arranged side-by-side with minimal clearance. The racks are designed to protect the fuel assemblies from damage caused by impacting other fuel assemblies or other equipment, and to withstand all credible static and seismic loadings.

Following channeling of the bundles on the fuel preparation machines, the channel fastener assembly is installed to lock the channel and fuel bundle together as one unit. The assembly is then transferred to a specified storage rack position for interim storage until core loading begins. 


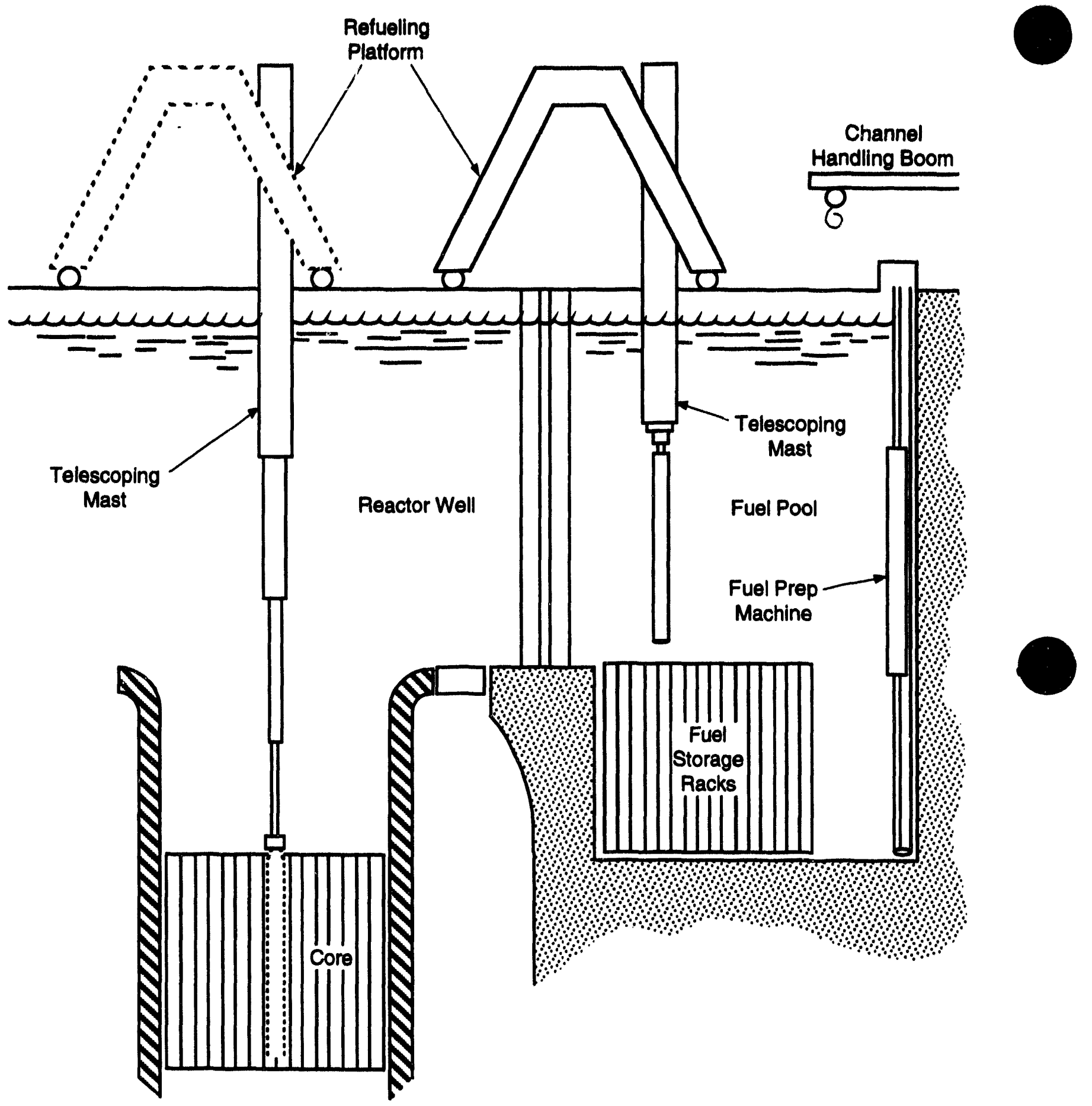

Figure 2.5-4 Typical BWR Refueling Process 
Fuel transfer from the pool to the reactor core is accomplished by use of the refueling platform. The refuel platform is a gantry crane that spans the spent fuel pool on bedded tracks in the refueling floor. A telescoping mast and grapple suspended from a trolley system is used to lift and orient fuel bundles for placement in the core or storage rack. Control of the platform is from an operator station on the platform.

\subsubsection{Fresh Fuel Handing With Increased Americium Levels}

As discussed in Section 8, the effect of 3,000 ppm of americium (Am) in the fresh MOX fuel is to increase the dose rate at the bundle surface from about $5 \mathrm{mr} / \mathrm{hr}$ to about $5.5 \mathrm{mr} / \mathrm{hr}$. Based on such low radiation levels, fresh fuel with high Am levels could be handled in the same manner as discussed in Section 2.5.2. However, an alternative to reduce personnel exposure for either case is to transfer the fresh fuel directly from the shipping container unloading area to the fuel preparation machine in the pool (Figure 2.5-3). The fuel preparation machine and associated equipment would be used to remove the shipping spacers, inspect the fuel and install the channel. After channeling, the fuel assembly would be handled in the same manner as any other channeled fuel.

\subsubsection{Fuel Tracking}

BWRs normally use a manual system of tracking fuel assemblies based on visual observation of bundle serial numbers. However, a computerized fuel tracking system (Lasertrac) is available if needed to support enhanced material control and accountability for the MOX fuel bundles from the point of receipt through residence in the fuel storage pool and insertion in the reactor.

Lasertrac, an "automated boundary zone protection and fuel positioning system", is an automatic refueling platform positioning system developed and tested by GE to reduce refueling time. With the addition of commercially available refueling tracking software, which manages fuel shuffling and provides criticality calculations for each step of the fuel handling operation, not only are the safeguards and tracking needs for the plutonium disposal mission be satisfied, but the process has the capacity to be automated to reduce the potential for error and to assure a more reliable refueling process. Further, addition of communications software could make it possible to communicate bundle locations to the DOE for safeguards provisions.

Once installed and calibrated, the automated fuel handling system would allow the operator to input a move command to any defined fuel or storage pool location and the system will move the bridge at the maximum allowed speed to that location. In refueling outages, sequences of moves 
may be pre-programmed on disk and copied into the computer so that the operator may command the system to perform sequential moves from a "File move" file. While in the "auto" mode, a file of move commands allows a pre-planned refuel shuffles of up to several thousand steps to be executed sequentially. After each step is executed, the file is marked "executed" and the tracking system is maintained in "realtime".

The basic system consists of two optical distance positioning systems, an industrial computer, a graphics monitor and logic cards for proper interface. Most of the equipment is installed on the trolley. Two reflectors are installed as reference points for the lasers, one small reflector on the end truck for the trolley laser and a larger reflector on a wall (or any sturdy object) for the bridge. By controlling $X, Y$ and $Z$ movements and mast load through interfaces with the computer, automatic fuel moves can be performed. The system, which has been tested at GE's Maintenance Training Center (MTC) in San Jose has demonstrated high accuracy and precision. With $\mathbf{Z}$ mast position and controlled automatic lowering of bundles to a position directly above the final position, nearly automatic grappling and transfer of bundles is possible. The degree of operator interaction required in the move can be set up based on utility desire.

\subsubsection{Spent Fuel Storage Outside the Reactor Building}

One of the study requirements is to "deliver to the government at the plant boundary ten-yearcooled fuel assemblies". While U.S. commercial reactors are typically designed to store at least 10-years of spent fuel, the storage pools in most power plants, including the BWRs of interest for this study, are expected to reach their capacity limit during the next decade. Thus, unless shipment of the existing spent fuel to the Federal Repository is initiated before the start of a plutonium disposition mission, some ex-reactor building spent fuel storage will be needed.

Further, the proposed plutonium disposition mission is expected to use the remaining life of the plants involved. As a result, at the end of the disposition mission, some ex-reactor building spent fuel storage would be needed to meet the "ten-year-cooled" requirement unless the in-reactor building pools, their essential support systems and the NRC license are maintained for ten years beyond the end of operations. While this later option is feasible, it has not been found to be cost effective by the Rancho Seco and Trojan power plants currently undergoing decommissioning.

While the need for ex-reactor spent fuel storage is not caused by the plutonium disposition mission, the fact remains that it will most likely be needed to carry out the mission. Thus, as indicated in Section 2.5.1, the planning basis for this study is to provide passive dry spent fuel storage for ten years of operation. This meets the "ten-year-cooled fuel" requirement, provides 
flexibility to accommodate delays in shipping the existing spent fuel to the Federal Repository and minimizes any potential impact on decommissioning due to continued in-reactor storage of the spent fuel.

There have been over 40 years of actual operating experience with dry storage and handling of spent fuel and over 20 years of research in passive dry storage technology in the United States and abroad. There are several options available for dry storage, including cask storage, surface field drywells, or surface vaults. Several methods of dry cask storage include vertical metal cask, horizontal metal cask, vertical concrete cask (silo), or modular horizontal concrete vaults using a shielded transfer cask.

Metal storage casks are essentially versions of proven spent fuel transportation cask designs. These casks are constructed of lead and steel or ductile iron; water or other materials provide additional radiation shielding. Cooling is provided by conduction of heat through the metal walls and natural convection to the atmosphere. Dual purpose transport/storage casks have been proposed to reduce fuel handling activities.

Concrete casks (silos) are similar to metal casks, although the designs differ because of the different materials of construction. Concrete is used for radiation shielding.

Drywells are excavated cavities or caissons into which sealed metal canisters containing spent fuel are placed. The field drywells are typically an array of caissons covering a large area. Radiation shielding and heat transfer medium are provided by the surrounding overpack material and the earth.

Vaults are typically large structures where spent fuel packages are stored and cooled by various means. In the open-cycle surface vault design, canisters of spent fuel are stored in large, shielded warehouse-type structures through which cooling air circulates by natural convection. Groups of smaller modular vaults provide for incremental increases to capacity.

Surface cask storage methods appear to be the most reasonable approach for the interim supplemental storage needed for the plutonium disposition mission because they require minimal site preparation and construction.

Among the surface cask options, metal casks are considerably more expensive than concrete casks. Concrete casks and horizontal modular vaults are very similar in concept. Several utilities are now use horizontal concrete storage modules at their plants (i.e., Calvert Cliffs, H. B. Robinson, and Oconee). Other utilities have selected vertical concrete cask storage. 
For the purposes of this study, a commercially available dry spent fuel management system was used as the basis for facility layout and cost estimating. This system by is currently licensed and in service at several commercial reactor sites and under construction or development at several more plants (i.e., Rancho Seco, Davis-Besse, FitzPatrick, and Oyster Creek). As a result, cost, schedule, operating and personnel exposure experience is available to provide a sound basis for planning.

The reference dry spent fuel management system is a complete system that provides for storage, transport and eventual disposal of canisterized spent nuclear fuel assemblies. It is used at the reactor site for the modular $c^{\prime}-v$ storage of spent fuel assemblies in an Independent Spent Fuel Storage Installation (ISFSI) in compliance with 10CFR72. The system is capable of meeting the life-of-plant dry spent fuel storage needs in a safe, simple and economical manner and can be implemented under the 10CFR72 Subpart K generic certification rule or under 10CFR72 Subpart C site-specific license.

The system uses a ventilated reinforced concrete Horizontal Storage Module (HSM) to store spent fuel assemblies that are sealed in a stainless steel Dry Shielded Canister (DSC). The prefabricated HSM provides radiological shielding and physical protection for the DSC against a wide range of postulated natural hazards. The HSM has internal air flow passages to provide natural convection cooling for removal of decay heat from the DSCs spent fuel. The DSC serves as the containment pressure boundary for the confinement of radioactive materials and provides a leak tight, inert atmosphere to ensure that the integrity of the fuel cladding is maintained.

In addition to the HSM and the DSC, the standard system includes a canister transfer cask and lifting yoke, cask support skid and transport trailer, and a hydraulic ram system. An onsite transfer cask is used to transfer the loaded DSC from the plant's spent fuel pool to an HSM located on the ISFSI site. The auxiliary equipment used in the plant's fuel building includes a canister vacuum drying system and a remote automatic welding system to facilitate canister closure operations. These standard components and equipment are all that are needed to place spent fuel assemblies in passive dry storage.

The standard 52B canister design uses fixed neutron absorbing materials. The design can be readily adapted to accommodate a particular BWR plant's fuel incorporating whatever criticality safety measures are required to demonstrate compliance.

The design of the system includes consideration of normal and off-normal operating conditions, and worst case hypothetical accidents. The system is designed in accordance with the 
requirements of 10CFR72, as well as Regulatory Guide 3.54, ANSIANS 57.9, Section III of the ASME Boiler and Pressure Vessel Code, ACI 349, and other applicable specifying documents, as described in the CSAR.

The ventilated reinforced concrete Horizontal Storage Module is the principal structural component to be located on the ISFSI site. Each HSM is a free-standing prefabricated unit. The HSMs are grouped to form arrays that are placed in service on a concrete basemat that is typically 18-36 inches thick, depending on soil conditions. The HSM provides physical protection for the Dry Storage Canister against all worst case postulated natural phenomena such as tornadoes, earthquakes, and floods; and provides highly effective biological shielding sufficient to reduce occupational doses consistent with ALARA objectives (less than $20 \mathrm{mr} / \mathrm{hr}$ contact) and to attenuate the site boundary doses to within regulatory limits (less than $2 \mathrm{mr} / \mathrm{hr}$ at site fence). The HSM also provides capability for future canister transfer to an existing multipurpose transportation cask for offsite shipment of intact canisters to a DOE facility for interim or final disposal.

The Dry Spent Fuel Storage portion of the Fuel Storage Complex shown in Figure 2.5-2 will consist of 40 Horizontal Storage Modules located in 4 arrays of 10 modules. This will provide for storage of ten years worth of spent fuel (176 assemblies per year) and includes optional capacity to accommodate a full core discharge if elected as part of the MOX fueling activities. Each Dry Storage Canister has a capacity of 52 BWR spent fuel assemblies.

Each HSM is $19 \mathrm{ft}-10$ in long by $9 \mathrm{ft}-8 \mathrm{in}$ wide and the adjacent units are spaced 6 inches apart to facilitate ventilation flow. An auxiliary shielding wall is installed along the exterior walls of the array. The system requires a concrete basemat (18-36 in. thick) for the HSM placement and a 20-ft wide approach pad (18 in. thick) for jacking and alignment of the transport trailer. A $50-\mathrm{ft}$ spacing between the arrays is required for turning and backing the transport cask into position. The transport trailer (max. gross weight less than 125 tons) has 32 pneumatic tires and is suitable for use on engineered asphalt paving. Access areas in and around the storage arrays will be paved. 


\subsection{Fuel Management}

This section discusses the management of fuel materials during the irradiation and power production cycle. It contains information on isotopics, fuel characteristics and recharging/ discharging strategies for the full MOX generic BWR-5 reference core. The general strategy is to utilize the island assembly design (partial MOX) for an early program demonstration and testing purposes. The full MOX design will complete the disposition mission.

Figure 2.6-1 illustrates the charged and discharged full MOX fuel assembly isotopic weight percentages being dispositioned and consumed. In the charged fuel assembly, approximately $2.6 \%$ of the isotopes is $\mathrm{Pu}$ - all the $\mathrm{Pu}$ is dispositioned, and approximately $40 \%$ is consumed. The consumption of Pu between a charged and discharged fuel assembly is calculated to be $1.95 \mathrm{~kg}$. Figures 2.6-2 and 2.6-3 show charged and discharged Pu isotopic compositions.

\subsubsection{Reactor Startup Plutonium Inventory and Recharge Rate}

The BWR inventory and rate of disposition for fresh fuel utilizing plutonium from the weapons program are shown in Table 2.6-1.

\subsubsection{Fuel Cycle Characteristics}

The characteristics defining the BWR full MOX fuel cycle are summarized in Table 2.6-2. It should be noted that these parameters are representative of an equilibrium cycle full MOX design.

The charging strategy defines the quantity and time schedule for both loading fresh assemblies into the core and unloading spent fuel assemblies from the core. This strategy is based on certain assumptions:

- The island design is initially loaded into each reactor. DOE facilities will manufacture the MOX rods necessary for this design.

- All fuel fabrication at the MOX facility is completed and the fuel is shipped to the reactor six months before refueling.

The specific charging and discharging schedules are presented in detail in the following two sections. 


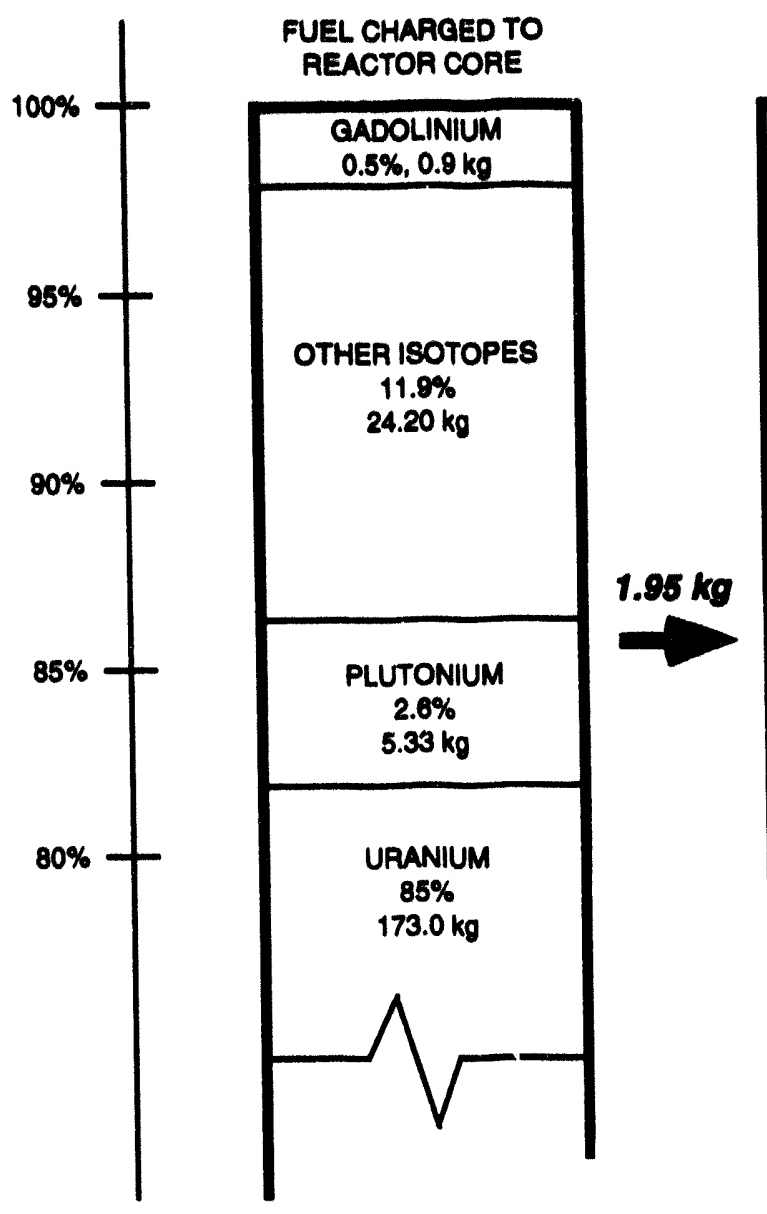

FUEL DISCHARGED FROM REACTOR CORE

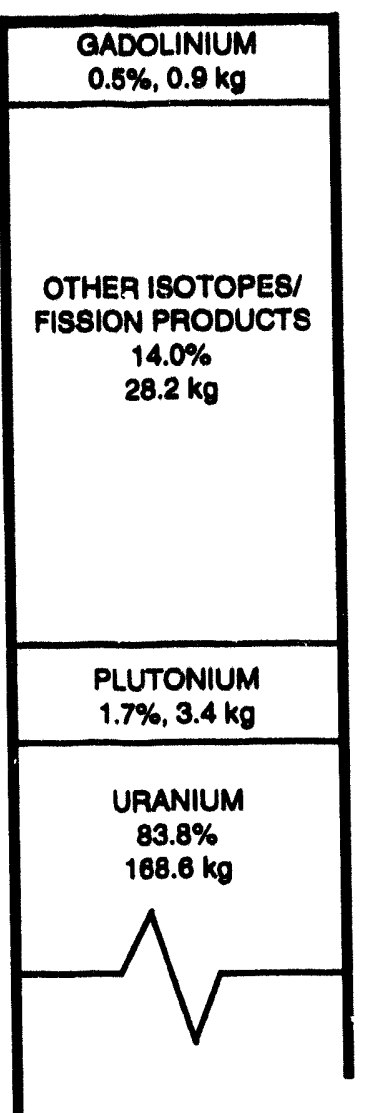

Figure 2.6-1 Composition of MOX Assemblies Charged to and Discharged from the Generic BWR 
NEDO-32361

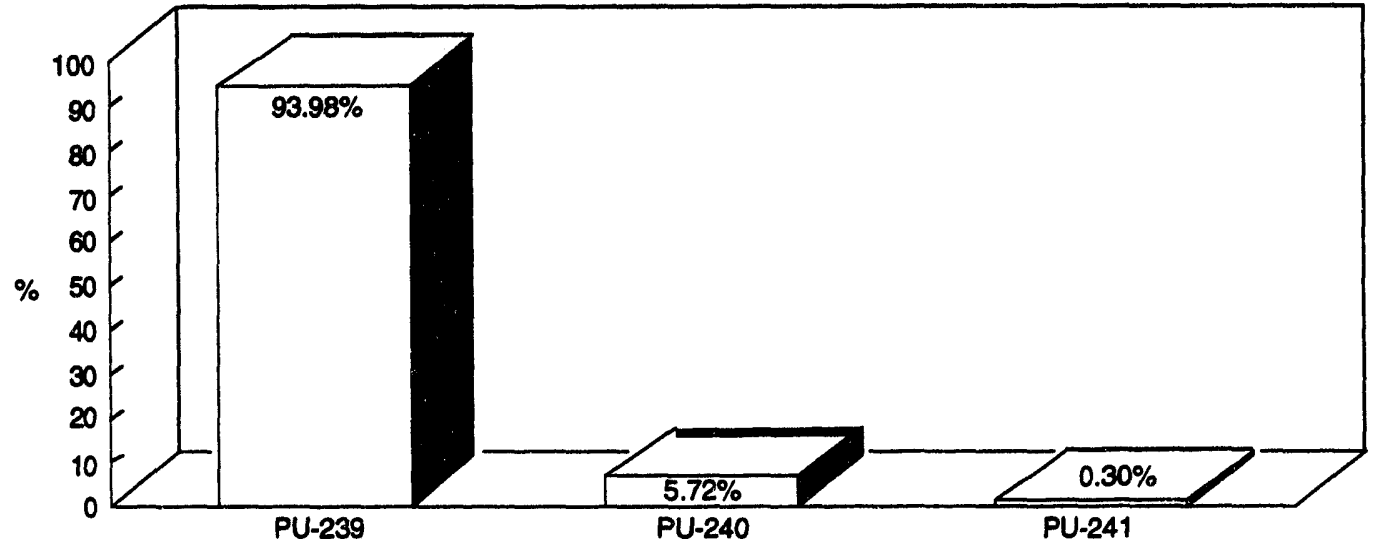

Figure 2.6-2 Isotopic Composition of MOX Fuel Charged - Generic BWR-5

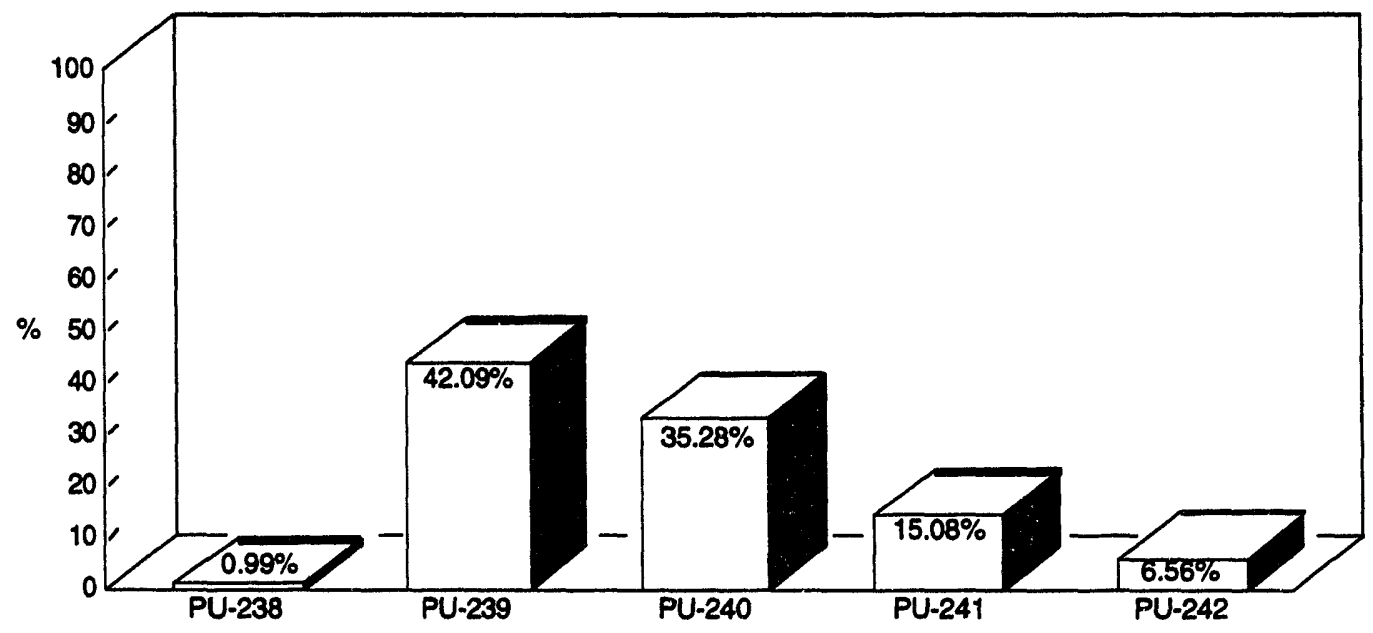

Figure 2.6-3 Isotopic Composition of MOX Fuel Discharged - Generic BWR-5 
NEDO-32361

Table 2.6-1 Plutonium Disposition Capacity and Rate for a Generic BWR-5

\begin{tabular}{|l|c|}
\hline Pu Per Assembly (kg) & 5.31 \\
\hline Pu Dispositioned Per Year (MT) & 0.75 \\
\hline Pu Dispositioned Per Cycle/Reload (MT) & 0.93 \\
\hline
\end{tabular}

Table 2.6-2 Fuel Cycle Characteristics

\begin{tabular}{|l|c|}
\hline Total Cycle Length (days) & 453 \\
\hline Effective Full Power Days (EFPD) (days) & 340 \\
\hline Outage Length/Refueling Length (days) & 113 \\
\hline Reload Batch Size (bundles) & 176 \\
\hline Full Core Size (bundles) & 764 \\
\hline Cycle Energy (GWd) & 1,184 \\
\hline Cycle Exposure (MWd/MT) & 7857 \\
\hline Average Discharge Exposure (MWd/MT) & 33.7 \\
\hline
\end{tabular}

\subsubsection{As-Charged Fuel Actinide Isotopic Composition}

This section focuses on the full MOX fuel composition as it is charged to the BWR. Table 2.6-3 shows the isotopic composition of fresh MOX fuel in the BWR. Also presented is the fraction of each isotope in the total element for uranium and plutonium.

The charging and discharging schedule for the BWR is shown in Table 2.6-4. It describes, as a function of time from Reactor 1 island design loading, the number of assemblies to be loaded into each reactor. There is no impact on the refueling outage because all of the assemblie: loaded into the core arrive at the site six months prior to the outage. The cumulative number of charged assemblies have been added to Table 2.6-4.

It should be noted that, since there are no reactor system or plant modifications to use MOX fuel, post-plutonium disposition power production can be achieved through the direct substitution of MOX with uranium-oxide fuel.

\subsubsection{Discharged Fuel Actinide Isotopic Composition}

As the reactor operates, a large number of isotopic changes occur. Plutonium and uranium are fissioned and transmuted, gadolinia is transmuted, and fission products decay. Table 2.6-5 shows the isotopic composition of a BWR discharged MOX fuel assembly. Also presented is the fraction of isotope in the total element for uranium and plutonium. 
The discharged MOX fuel assembly contains uranium, plutonium and gadolinium elemental mass contribution totals listed in Table 2.6-5. Tables 2.6-5 and 2.6-3 show the net consumption of plutonium between a charged and discharged fuel assembly to be $1.95 \mathrm{~kg}$, and $0.3 \mathrm{Mt}$ consumption for each reload.

Table 2.6-3 As-Charged Fuel Actinide Isotopic Composition

\begin{tabular}{|l|c|c|}
\hline Isotope (Weight \%) & kg/Assembly & MT/Reload \\
\hline U-234 & 0.009 & \\
\hline U-235 $(0.71 \%)$ & 1.23 & \\
\hline U-238 (99.29\%) & 172.03 & 30.50 \\
\hline Total Element Mass & 173.27 & \\
\hline & & \\
\hline PU-239 $(94.0 \%)$ & 5.01 & \\
\hline PU-240 (5.7\%) & 0.30 & \\
\hline PU-241 (0.3\%) & 0.01 & \\
\hline Total Element Mass & 5.33 & 0.938 \\
\hline & & \\
\hline AM-241 & 0.01 & \\
\hline & & \\
\hline GD-154 & 0.02 & \\
\hline GD-155 & 0.14 & \\
\hline GD-156 & 0.19 & \\
\hline GD-157 & 0.15 & \\
\hline GD-158 & 0.24 & \\
\hline GD-160 & 0.21 & \\
\hline Total Element Mass & 0.94 & \\
\hline & & \\
\hline O-16 & 24.20 & \\
\hline Total MOX (Kg) & 203.77 & \\
\hline
\end{tabular}


NEDO-32361

Table 2.6-4 MOX Charging/Discharging Schedule for Existing BWRs

\begin{tabular}{|c|c|c|c|c|c|c|}
\hline $\begin{array}{l}\text { Time from } \\
\text { MOX Load in } \\
\text { First Reactor } \\
\text { (Years) }\end{array}$ & $\begin{array}{c}\text { Reactor } 1 \\
\text { Assemblies } \\
\text { Loaded } \\
\end{array}$ & $\begin{array}{c}\text { Reactor } 2 \\
\text { Assemblies } \\
\text { Loaded }\end{array}$ & $\begin{array}{c}\text { Reactor } 3 \\
\text { Assemblies } \\
\text { Loaded }\end{array}$ & $\begin{array}{c}\text { Cumulative } \\
\text { Assemblies } \\
\text { Charged }\end{array}$ & $\begin{array}{c}\text { Cumulative } \\
\text { Disposed Pu } \\
\text { (MT) }\end{array}$ & $\begin{array}{l}\text { Cumulative } \\
\text { Assemblies } \\
\text { Discharged }\end{array}$ \\
\hline 0 & 58 & & & 58 & 0.08 & \\
\hline 1.24 & 58 & & & 116 & 0.17 & \\
\hline 2.24 & & 58 & & 174 & 0.25 & \\
\hline 2.48 & 58 & & & 232 & 0.33 & \\
\hline 3.48 & & 58 & & 290 & 0.42 & \\
\hline 3.72 & 176 & & & 466 & 1.35 & \\
\hline 4.48 & & & 58 & 524 & 1.42 & \\
\hline 4.72 & & 176 & & 700 & 2.37 & \\
\hline 5.21 & 176 & & & 876 & 3.31 & 58 \\
\hline 5.72 & & & 176 & 1052 & 4.24 & \\
\hline 6.21 & & 176 & & 1228 & 5.17 & \\
\hline 6.46 & 176 & & & 1404 & 6.11 & 116 \\
\hline 7.21 & & & 176 & 1580 & 7.04 & \\
\hline 7.46 & & 176 & & 1756 & 7.98 & 174 \\
\hline 7.70 & 176 & & & 1932 & 8.91 & 232 \\
\hline 8.46 & & & 176 & 2108 & 9.85 & \\
\hline 8.70 & & 176 & & 2284 & 10.78 & 290 \\
\hline 8.94 & 176 & & & 2460 & 11.72 & 466 \\
\hline 9.70 & & & 176 & 2636 & 12.65 & 524 \\
\hline 9.94 & & 176 & & 2812 & 13.58 & 700 \\
\hline 10.18 & 176 & & & 2988 & 14.52 & 876 \\
\hline 10.94 & & & 176 & 3164 & 15.45 & 1052 \\
\hline 11.18 & & 176 & & 3340 & 16.39 & 1228 \\
\hline 11.42 & 176 & & & 3516 & 17.32 & 1404 \\
\hline 12.18 & & & 176 & 3692 & 18.26 & 1580 \\
\hline 12.42 & & 176 & & 3868 & 19.19 & 1756 \\
\hline 12.66 & 176 & & & 4044 & 20.13 & 1932 \\
\hline 13.42 & & & 176 & 4220 & 21.06 & 2108 \\
\hline 13.66 & & 176 & & 4396 & 22.00 & 2284 \\
\hline 13.90 & 176 & & & 4572 & 22.93 & 2460 \\
\hline 14.66 & & & 176 & 4748 & 23.87 & 2636 \\
\hline 14.90 & & 176 & & 4924 & 24.80 & 2812 \\
\hline 15.14 & 176 & & & 5100 & 25.73 & 2988 \\
\hline 15.90 & & & 176 & 5276 & 26.67 & 3164 \\
\hline 16.14 & & 176 & & 5452 & 27.60 & 3340 \\
\hline 16.39 & 176 & & & 5628 & 28.54 & 3516 \\
\hline 17.14 & & & 176 & 5804 & 29.47 & 3692 \\
\hline 17.39 & & 176 & & 5980 & 30.40 & 3868 \\
\hline 17.63 & 176 & & & 6156 & 31.34 & 4044 \\
\hline 18.39 & & & 176 & 6332 & 32.27 & 4220 \\
\hline 18.63 & & 176 & & 6508 & 33.21 & 4396 \\
\hline 18.87 & 176 & & & 6684 & 34.15 & 4572 \\
\hline 19.63 & & & 176 & 6860 & 35.08 & 4748 \\
\hline 19.87 & & 176 & & 7036 & 36.01 & 4924 \\
\hline 20.11 & 176 & & & 7212 & 36.95 & 5100 \\
\hline
\end{tabular}


NEDO-32361

Table 2.6-4 MOX Charging/Discharging Schedule for Existing BWRs (Continued)

\begin{tabular}{|c|c|c|c|c|c|c|}
\hline $\begin{array}{l}\text { Time from } \\
\text { MOX Load in } \\
\text { First Reactor } \\
\text { (Years) }\end{array}$ & $\begin{array}{l}\text { Reactor } 1 \\
\text { Assemblies } \\
\text { Loaded }\end{array}$ & $\begin{array}{c}\text { Reactor } 2 \\
\text { Ascemblies } \\
\text { Loaded }\end{array}$ & $\begin{array}{l}\text { Reactor } 3 \\
\text { Assemblies } \\
\text { Londed }\end{array}$ & $\begin{array}{c}\text { Cumulative } \\
\text { Assemblies } \\
\text { Charged }\end{array}$ & $\begin{array}{l}\text { Cumulative } \\
\text { Disposed Pu } \\
\text { (MT) }\end{array}$ & $\begin{array}{l}\text { Cumulative } \\
\text { Assemblies } \\
\text { Discharged }\end{array}$ \\
\hline 21.87 & & & 176 & 7388 & 37.88 & 5276 \\
\hline 21.11 & & 176 & & 7564 & 38.82 & 5452 \\
\hline 21.35 & 176 & & & 7740 & 39.75 & 5628 \\
\hline 22.11 & & & 176 & 7916 & 40.69 & 5804 \\
\hline 22.35 & & 176 & & 8092 & 41.62 & 5980 \\
\hline 22.59 & 176 & & & 8268 & 42.56 & 6156 \\
\hline 23.35 & & & 176 & 8444 & 43.49 & 6332 \\
\hline 23.59 & & 176 & & 8620 & 44.43 & 6508 \\
\hline 23.83 & 176 & & & 8796 & 45.36 & 6684 \\
\hline 24.59 & & & 176 & 8972 & 46.30 & 6860 \\
\hline 24.83 & & 176 & & 9148 & 47.23 & 7036 \\
\hline 25.07 & 176 & & & 9324 & 48.16 & 7212 \\
\hline 25.83 & & & 176 & 9500 & 49.10 & 7388 \\
\hline 26.07 & & 176 & & 9676 & 50.03 & 7564 \\
\hline 26.31 & (2) & & & & & 7740 \\
\hline 27.07 & & & 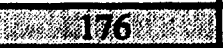 & & & 7916 \\
\hline 27.31 & & 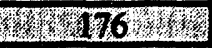 & & & & 8092 \\
\hline 27.56 & 37 & & & & & 8268 \\
\hline 28.31 & & & 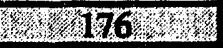 & & & 8444 \\
\hline 28.56 & & 196 & & & & 8620 \\
\hline 28.80 & 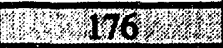 & & & & & 8796 \\
\hline 29.56 & & & 176 GYY & & & 8972 \\
\hline 29.80 & & 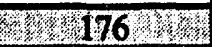 & & & & 9148 \\
\hline 30.04 & 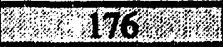 & & & & & 9324 \\
\hline 30.80 & & & 1786, 176 & & & 9500 \\
\hline 31.04 & & (5) 176 . & & & & 9676 \\
\hline
\end{tabular}


Table 2.6-5 Discharged Fuel Actinide Isotopic Composition (at shutdown)

\begin{tabular}{|c|c|c|}
\hline Isotope (Wt \%) & kg/Assembly & MT/Reload \\
\hline $\mathrm{U}-234$ & 0.006 & \\
\hline $\mathrm{U}-235$ & 0.38 & \\
\hline $\mathrm{U}-236$ & 0.15 & \\
\hline $\mathrm{U}-238(100 \%)$ & 168.11 & \\
\hline Total Element Mass & 168.65 & 29.68 \\
\hline PU-238(1\%) & 0.03 & \\
\hline PU-239(42\%) & $1 . \overline{43}$ & \\
\hline PU-240(35\%) & 1.20 & \\
\hline PU-241 (15\%) & 0.51 & \\
\hline PU-242(7\%) & 0.22 & \\
\hline Total Element Mass & 3.39 & 0.60 \\
\hline NP-237 & 0.029 & \\
\hline NP-239 & 0.010 & \\
\hline Total Element Mass & 0.04 & Negl \\
\hline AM-241 & 0.031 & \\
\hline AM-243 & 0.044 & \\
\hline Total Element Mass & 0.07 & Negl \\
\hline $\mathrm{TH}-230$ & negl & Negl \\
\hline CM-242 & 0.11 & \\
\hline $\mathrm{CM}-244$ & 0.12 & \\
\hline Total Element Mass & 0.23 & 0.04 \\
\hline GD-154 & 0.18 & \\
\hline GD-155 & 0.0009 & \\
\hline GD-156 & 0.33 & \\
\hline GD-157 & 0.001 & \\
\hline GD-158 & 0.39 & \\
\hline GD-160 & 0.21 & \\
\hline Total Element Mass & 0.95 & 0.17 \\
\hline PM-148 & 0.002 & \\
\hline PM-148M & 0.027 & \\
\hline PM-149 & 0.0002 & \\
\hline PM-151 & 0.0004 & \\
\hline Total Element Mass & 0.03 & Negl \\
\hline RU-101 & 0.17 & \\
\hline RU-103 & 0.007 & \\
\hline Total Element Mass & 0.18 & 0.03 \\
\hline $\mathrm{RH}-103$ & 0.12 & \\
\hline RH-105 & 0.00002 & \\
\hline Total Element Mass & 0.12 & 0.02 \\
\hline ND-145 & 0.12 & \\
\hline ND-143 & 0.14 & \\
\hline Total Element Mass & 0.26 & 0.05 \\
\hline
\end{tabular}


Table 2.6-5 Discharged Fuel Actinide Isotopic Composition (at shutdown) (Continued)

\begin{tabular}{|l|c|c|}
\hline Isotope (Wt \%) & kJ/Assembly & MT/Reload \\
\hline SM-147 & 0.017 & \\
\hline SM-148 & 0.00007 & \\
\hline SM-149 & 0.004 & \\
\hline SM-150 & 0.055 & \\
\hline SM-151 & 0.003 & \\
\hline SM-152 & 0.035 & \\
\hline Total Element Mass & 0.114 & 0.02 \\
\hline EU-153 & 0.025 & \\
\hline EU-154 & 0.009 & \\
\hline EU-155 & 0.004 & \\
\hline Total Element Mass & 0.038 & 0.01 \\
\hline O-16 & 24.20 & 4.26 \\
\hline KR-83 & 0.007 & Negl \\
\hline TC-99 & 0.14 & 0.02 \\
\hline CD-113 & 0.002 & Negl \\
\hline XE-131 & 0.091 & 0.02 \\
\hline CS-133 & 0.22 & 0.04 \\
\hline XE 133 & 0.001 & Negl \\
\hline XE-135 & 0.0002 & Negl \\
\hline I-135 & 0.000060 & Negl \\
\hline PR-143 & 0.0003 & Negl \\
\hline TB-159 & 0.015 & 0.04 \\
\hline Fission Products & 2.82 & 0.50 \\
\hline Total MOX & 201.32 & 35.43 \\
\hline
\end{tabular}




\subsection{Nuclear Material Transportation}

This section provides technical descriptions for the transportation of nuclear material, and discusses applicable governing environmental safety and health and criticality regulations. It characterizes the transportation of fresh fuel from the fuel fabrication plant, waste from the fuel fabrication plant, waste from the reactor, as well as spent fuel from the reactor. This study assumes that the uranium and plutonium oxide feed material is provided to the MOX fuel fabrication plant. Criticality safety-related considerations for fresh and spent fuel transport are addressed in Section 8.4, where it is demonstrated that all the required criteria for subcriticality are met.

Additional evaluations of nuclear material transport were previously performed that included a study on transportation of Pu materials to the MOX fabrication facility, as well as a study on the comparison of U.S. and Internationa transport regulations. These additional studies are included in NEDO-32351, Study of Pu Disposition Using the GE ABWR (see Appendix I of this report).

\subsubsection{Fresh Fuel from the Fuel Fabrication Plant}

The initial loading and the first few reloads of island fuel for an early demonstration pilot program can be supplied from existing facilities at the Los Alamos National Laboratory (LANL). Full MOX core fuel will be fabricated in a new Fuel Fabrication Facility with the location to be determined and will replace the island design fuel in later core reloads. For the purpose of this study, the distance between Los Alamos and the reactors is assumed to be 1500 miles. The LANL-produced island design fuel first used would consist of 12 MOX fuel rods per bundle. The island loading will consist of 58 of these MOX bundles plus 118 standard urania bundles contained in each reload of 176 bundles. Up to three selected reactors will receive their initial island reloads in a staggered schedule determined by the LANL fabrication capacity. This will result in two reload batches for the first reactor, one reload for the second reactor and only the initial loading of the third reactor before the new fuel fabrication facility is on line and all reload batches become full MOX bundle reloads continuing at 176 bundles/cycle per reactor. The typical cycle length is about 15 months.

Standard fuel assembly shipping containers typically used for transporting fresh $\mathrm{UO}_{2}$ assemblies are certified right rectangular boxes consisting of an outer container of wooden construction and a metal inner container separated by cushioning material. Two fuel assemblies are contained in each shipping container. From tile perspective of personnel exposure, this packaging is sufficient since the radiation from the $\mathrm{UO}_{2}$ has minimal exposure impact when being handled. 
While the physical size of the MOX fuel bundles is the same as $\mathrm{UO}_{2}$ fuel elements used in BWR reactors, the difference in plutonium content will affect the shipping requirements. The MOX elements designated GE9-8 $\times 8$ fuel bundles (Section 2.1.1) have the following characteristics: (1) total plutonium loading for the initial island loading is $1.44 \mathrm{~kg}$ and for the full core loading is $5.31 \mathrm{~kg}$; (2) the Pu-239 content is $93.7 \%$ or $1.35 \mathrm{~kg}$ for the island loading and $4.98 \mathrm{~kg}$ for the full core loading; and (3) mass of heavy metal for both loadings is $179 \mathrm{~kg}$. A BWR-5 has 764 bundles in the core.

Assemblies containing MOX fuel may have a slightly higher radiation level than $\mathrm{UO}_{2}$ assemblies, depending on the process used to prepare the weapon plutonium as feed material. It has been assumed, for the reference case, that the enrichment and impurities will be low and that the radiation level will be readily accommodated. Since the MOX fuel ownership will be retained by DOE throughout the life of the project, transportation must be in accordance with DOE Orders. Safeguards considerations require that fresh fuel, classified as Level IID per DOE Order 5633.3, be transported by Safe Secure Transport (SST). Safeguards requirements discussed in more detail in Section 7.6.

The annual number of shipments based on the fuel fabrication throughputs for the reference and alternate cases are summarized in Table 2.7-1. Route distances are assumed to be 1500 miles for the reference case. The SST has a payload limitation of $10,000 \mathrm{lb}$ and this, in turn, restricts the number of containers per shipment to five.

Island loading will require $6 \mathrm{SST}$ shipments to the new fuel vault per reactor. Full MOX reloads for each reactor will require 18 SST shipments to the new fuel vault. No limitations are

Table 2.7-1 Fresh Fuel Shipping Schedule

\begin{tabular}{|c|c|c|c|}
\hline Year & Reference & Alternate 1 & Alternate 2 \\
\hline 1 & 6 & 6 & 6 \\
\hline 2 & 6 & 6 & 6 \\
\hline 3 & 12 & 12 & 12 \\
\hline 4 & 24 & 24 & 24 \\
\hline 5 & 24 & 24 & 24 \\
\hline 6 & 36 & 54 & 36 \\
\hline 7 & 36 & 36 & 36 \\
\hline 8 & 54 & 88 & 54 \\
\hline 9 & 36 & 88 & 36 \\
\hline $10-26$ & $36-54(42 \mathrm{avg})$ & $54-106(86 \mathrm{avg})$ & $36-54(44 \mathrm{avg})$ \\
\hline $26-48$ & $\mathrm{n} / \mathrm{a}$ & $\mathrm{n} / \mathrm{a}$ & $44 \mathrm{avg}$ \\
\hline
\end{tabular}


anticipated with regard to the onsite shipment of MOX bundles from the new ex-reactor fuel vault (Section 2.5 ) to the reactor facility. It is expected that new fuel for each reload can be transferred to the reactor in 5-6 onsite shipments.

The use of SSTs is under the auspices of the Transportation Safeguards Division of the DOE Albuquerque Operations Office. As can be seen in the table, SST shipments will be required about weekly. With the current demand for SSTs, the current fleet of SSTs may not be sufficient to satisfy this demand. One or two additional dedicated SSTs may be required. The current cost of an SST is estimated at \$3 million. Movement of an SST includes escorts of field element couriers or contractor security forces and telecommunications tracking which results in a cost of approximately $\$ 65 \mathrm{~K}$ per round trip. The total cost for all shipments would be about $\$ 80$ million for the reference case.

\subsubsection{Waste from Fuel Fabrication Plant}

The fuel fabrication facility is described in Section 2.4 and waste generation from the MOX plant is discussed in Section 2.4.3. This section addresses the transport of waste from the fuel fabrication plant to offsite storage facilities.

Low level waste from the MOX plant is expected to consist of coveralls, shoe covers, gloves, and paper which can be placed in 55-gallon drums for storage and disposal. DOE Order 5480.3 addresses offsite shipments of radioactive and hazardous waste from DOE facilities. Shipments of low level waste are expected to be made to the Nevada Test Site by flat bed trucks or covered trucks. If 720 barrels $\left(144 \mathrm{~m}^{3}\right)$ are shipped per year in trucks with a capacity to carry 90 barrels, about 8 such shipments would be expected on an annual basis. The distance and transport time to the Nevada Test site, of course, depends on the site chosen for the fuel fabrication facility, but is assumed to be 1500 miles for the reference case.

Based on the estimated transuranic (TRU) waste generation from the MOX fabrication facility, a maximum of about 1330 barrels $\left(266 \mathrm{~m}^{3}\right)$ of TRU waste would be generated. The TRU 55gallon barrels must be packaged into TRU-PAC II shipping containers that hold 14 drums each. Three of these overpack shipping containers can be transported on a truck. This would permit 42 drums per shipment and a total of 32 shipments annually. The distance and transport time to the WIPP Site at Carlsbad, New Mexico, depends on the site chosen for the fuel fabrication facility, but is assumed to be 1500 miles for the reference case. 


\subsubsection{Waste from Reactor}

Section 8.3.1.1 provides annual waste quantities from a typical BWR. BWR waste shipments will be regulated by NRC specifications. Fifty-five-gallon drums and metal boxes can be used for the low-level waste as shipping containers. Shipments of low level waste are expected to be made to the respective Low Level Waste Repository of the Compact where the reactor is located by flat bed trucks or covered trucks. Resins are expected to be dried and packaged in metal canisters and radwaste-sludge will be shipped in disposal liners to the repository of the Compact where the reactor is located utilizing existing commercial reactor practices. If the reactors are DOE owned, then the disposal site would be the Nevada Test Site with a travel distance assumed to be $\mathbf{1 5 0 0}$ miles. A distance of $\mathbf{4 0 0}$ miles is assumed for the repository of the Compact where the reactor is located.

Based on the BWR waste generation, a total of about $245 \mathrm{~m}^{3}$ of waste will be generated from each reactor annually, although this number might be reduced with additional compaction. A typical mix of containers might be 300 55-gallon drums and 3095 -cu $\mathrm{ft}$ boxes of dry activated waste; 1 120-cu ft High Integrity Container (HIC) of cemented resins; and 17 207-cu ft liners of radwaste dried and cemented sludges. The waste shipments are made in trucks with a capacity to carry 90 barrels, 6 boxes, or 1 cask w/liner. About 27 shipments would be expected from each reactor on an annual basis for a total of 81 shipments annually.

\subsubsection{Spent Fuel from Reactor}

No significant concerns have been identified related to acceptance, handling, transportation, and disposal of spent MOX fuel from a BWR for plutonium disposition. The waste acceptance criteria of spent nuclear fuel for disposal in the Federal Repository are under revision. Although applicable regulations from DOE may change, design requirements assumed to apply to spent fuel shipments are summarized in Table 2.7-2

A revision to the current requirements is expected to be developed during 1994. This revision is expected to clarify, modify and add several new provisions, including changes to some of the standard nuclear fuel specifications. This is expected to include a possible modification of the spent nuclear fuel cooling time, establish criteria for and disposition of failed fuel, address priority for disposing of spent fuel from permanently shutdown reactors, address disposition of consolidated fuel rods, and other potential changes.

DOE specified shipping containers, meeting the new shipping requirements, are expected to be provided to the BWR operator for shipment of spent fuel to the offsite repository in accordance 
with 10CFR961. Although still under development, containers with 25, 75 and 125-ton loaded weight capacities are assumed to be available. It is expected that most BWR sites selected would have the capacity to use the 125-ton containers. Such containers would be able to transport up to 40 bundles of MOX fuel. The 25-ton and 75-ton containers are expected to transport 9 and 24 bundles, respectively.

The number of shipments for both MOX and the existing utility LEU fuel are summarized in Table 2.7-3 based on the discharge rate provided in Section 2.6 (176 bundles/yr per reactor). As indicated, storage capacities are provided such that no shipments are anticipated for 10 years (in accordance with the statement of work) after the initial reload of MOX fuel, and manageable numbers of shipments are forecast. A similar number of MOX shipments would be expected for an additional ten years after completion of the plutonium disposition mission. The commercial LEU shipments out of the dry storage facility is simply an annual average that would empty the dry storage area during the duration of the $\mathrm{Pu}$ disposition effort and is variable as long as MOX fuel is being shipped from the reactor pool after 10 years. Table 2.7-3 provides the number of shipments for the reference case and would be the same for Alternative 2, but doubled for Alternative 1.

Table 2.7-2 Spent Fuel Shipping Requirements

\begin{tabular}{|l|l|}
\hline $\begin{array}{l}\text { Maximum } \\
\text { physical } \\
\text { dimensions }\end{array}$ & $\begin{array}{l}\text { Overall length: } \\
\text { Active fuel length: } 14 \mathrm{ft}, 11 \text { in. } \\
\text { Cross section: } \quad 6 \text { in. } 6 \text { in. } 6 \text { in. (not including the channel) }\end{array}$ \\
\hline $\begin{array}{l}\text { Non-fuel } \\
\text { components }\end{array}$ & $\begin{array}{l}\text { Non-fuel components such as BWR channels that are an integral part of the } \\
\text { fuel assembly, which do not require special handling, may be included as part } \\
\text { of the spent nuclear fuel delivery for disposal. }\end{array}$ \\
\hline $\begin{array}{l}\text { Minimum } \\
\text { cooling time }\end{array}$ & $\begin{array}{l}\text { The minimum cooling time for fuel is five years (ten years has been specified } \\
\text { for evaluation of plutonium disposal). }\end{array}$ \\
\hline Failed fuel & $\begin{array}{l}\text { Assemblies shall be visually inspected and those which are structurally } \\
\text { deformed or have damaged cladding to the extent that special handling may } \\
\text { be required, or for any reason cannot be handled with normal fuel handling } \\
\text { equipment, shall be classified as failed fuel. }\end{array}$ \\
\hline
\end{tabular}

Table 2.7-3 Annual Spent Fuel Shipments after 10 Years

\begin{tabular}{|l|c|c|c|c|}
\hline \multirow{2}{*}{ Type of Fuel } & \multicolumn{4}{|c|}{ Shipping Container Type } \\
\cline { 2 - 5 } & $\begin{array}{c}\text { 25 Ton } \\
\text { Cask }\end{array}$ & $\begin{array}{c}\text { 75 Ton } \\
\text { Cask }\end{array}$ & $\begin{array}{c}\text { 125 Ton } \\
\text { Cask }\end{array}$ & $\begin{array}{c}\text { Dry } \\
\text { Ctorage } \\
\text { Canisters }\end{array}$ \\
\hline MOX Shipments & 60 & 21 & 15 & \\
\hline Commercial LEU Shipments & & & & 5 \\
\hline
\end{tabular}




\subsection{References}

2-1 W. N. Mobley, "Fuel Fabrication," Summary of Nuclear Fuels Seminar, Wrightsville Beach, North Carolina (May 14-17, 1972), pp. 41 to 4-10.

2-2 L. T. Hagie, et. al., Facilities Startup and Fabrication of Mixed Oxide Fuel for the Garigliano Reactor, NEDE-12457, General Electric Company (October 1973).

2-3 J. Krellmann, "Plutonium Processing at the Siemens Hanau Fuel Fabrication Plant," Nuclear Technology, Vol. 102 (April 1993), pp. 18-28.

2-4 G. Vanhellemont, "Fabrication of Plutonium Fuel," Nuclear Europe, Vol. 12 (1985), pp. 23-34.

2-5 W. J. Ross, "Fabrication or FFTF Fuel Pins at Babcock \& Wilcox," Journal American Nuclear Society, Vol. 21 (June, 1995), pp. 248-250.

2-6 S. Fukushima, et. al., "Thermal Conductivity of Near-Stoichiometry (U, Pu, Nd)O2 and (U, Pu, Eu)O2 Solid Solutions," Journal of Nuclear Material, Vol. 116 (1983), pp. 287-296.

2-7 S. Fukushima, et. al., "Thermal Conductivity of $\left(\mathrm{Pu}_{1-\mathrm{x}} \mathrm{Nd}_{\mathrm{y}}\right) \mathrm{O}_{2-\mathrm{x}}$ and $\left(\mathrm{PU}_{1-\mathrm{x}} \mathrm{Y}_{\mathrm{y}}\right) \mathrm{O}_{2-\mathrm{x}}$ Solid Solution," Journal of Nuclear, Voi. : 15 (1983), pp. 118-127.

2-8 R. B. Matthews, Ceramic Fuel Development for Space Reactors, Ceramic Bulletin, Vol. 71, No. 1 (1992) pp. 96-101.

2-9 W. Dworzak, et. al., ARIES Conceptual Design Report, NMT-DO:(U)93-041, Los Alamos National Laboratory (June 1, 1993).

2-10 Anonymous, Plutonium Storage Facility Lead Laboratory Interface Document, Los Alamos National Laboratory (September, 1993).

2-11 J. Malherbe, et. al., Characteristics and Management of Waste Produced by the MOX Fuel Cycle in Light Water Reactors, Catalogue No CDNA-13082-EN-C, Office for Official Publications of the European Communities (1990). 


\subsection{Technology Needs}

\subsection{Overview}

The proposed means of plutonium disposition, by conversion to mixed-oxide (MOX) fuel and utilization in existing BW/2s, entails no new technology and requires a minimum of development while embodying already developed methods for implementing verifiable safeguards provisions. Most of the elements that are required for implementation of this concept have been developed and tested in a large-scale/industrial setting and no areas have been identified that require research and development. For example, MOX fuel fabrication facilities exist in a number of countries [Ref. 3-1] and in the U.S., MOX fuel has been fabricated in large quantities as part of the Liquid Metal Reactor Programs [Ref. 3-2]. The use of MOX fuel in BWRs was tested more than 20 years ago at Big Rock Point [Ref. 3-3] and this study included a U.S. AEC sponsored integrated safeguards experiment to verify that sufficient redundancy could be incorporated into the fuel fabrication program to verify accountability provisions. MOX fuel fabrication plants employing recycled plutonium, which present a significantly greater technological challenge, are currently in operation in other countries [Ref. 3-4]. Therefore, the areas listed below, identified as technology needs, are primarily related to confirmatory testing and analyses rather than conventional research and development. These tasks, except those related to fuel testing, are for the most part, the same as those identified in the earlier report on plutonium disposition using the GE-ABWR [Ref. 3-5]. For the sake of completeness, all the task descriptions are reported here. The tasks related to fuel testing differ slightly because a demonstration program using an "island" design concept has been proposed in dispositioning with existing reactors, prior to the introduction of full MOX cores. Lead Use Assemblies (LUA) could be introduced as part of this demonstration program.

Since partial core loads of MOX fuel have been irradiated in BWRs in the past, the level of maturity for the island design is judged to be Level 7 as defined by Reference 3-6 and reproduced in Table 3.1-1. Further, as the full MOX core will be implemented in stages, progressing through partial MOX core loads, the level of maturity for the full MOX core is judged to be higher than Level 6, although, to-date, no BWR has been loaded with a full MOX core. 

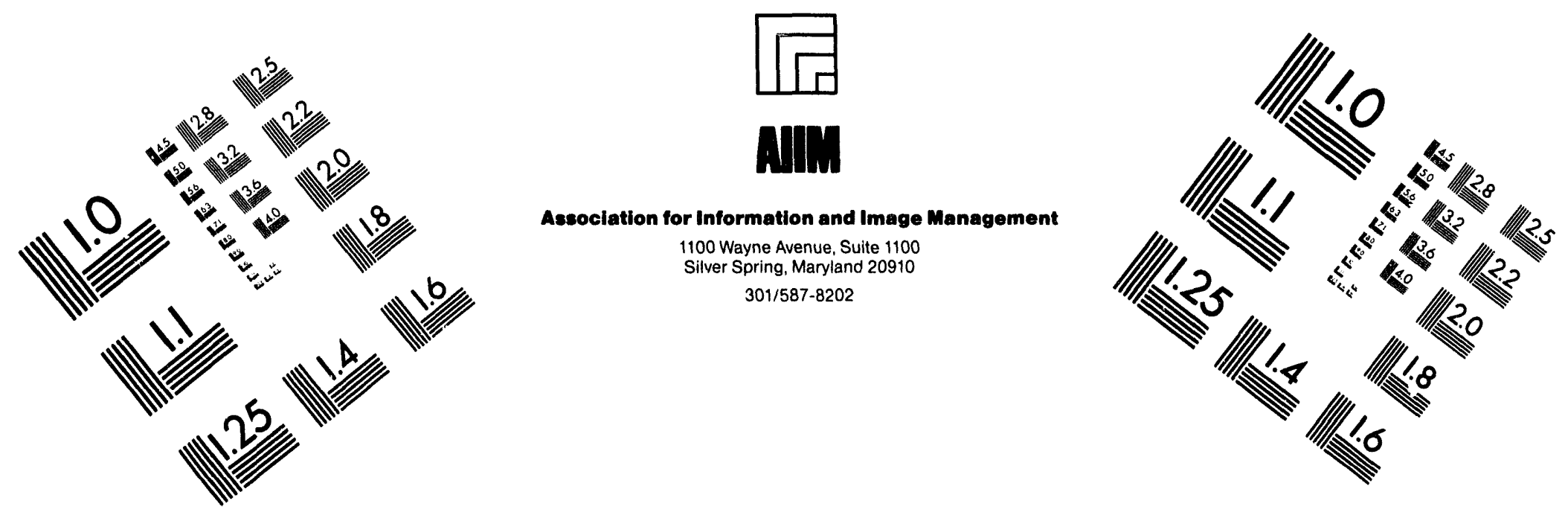

\section{Centimeter}

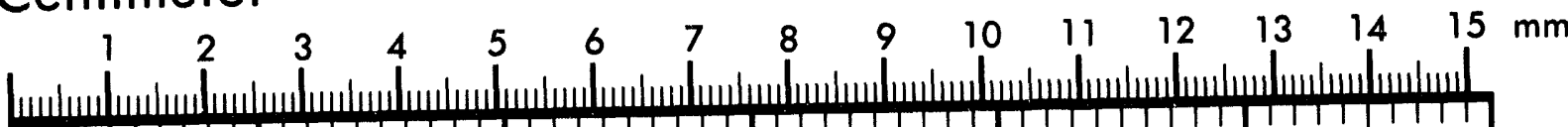

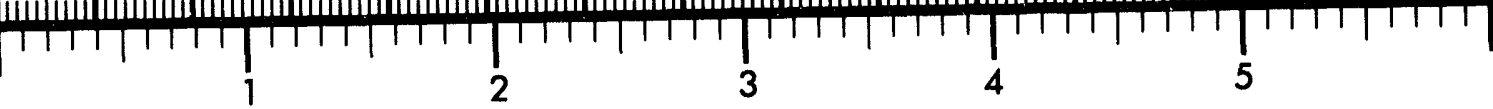
Inches

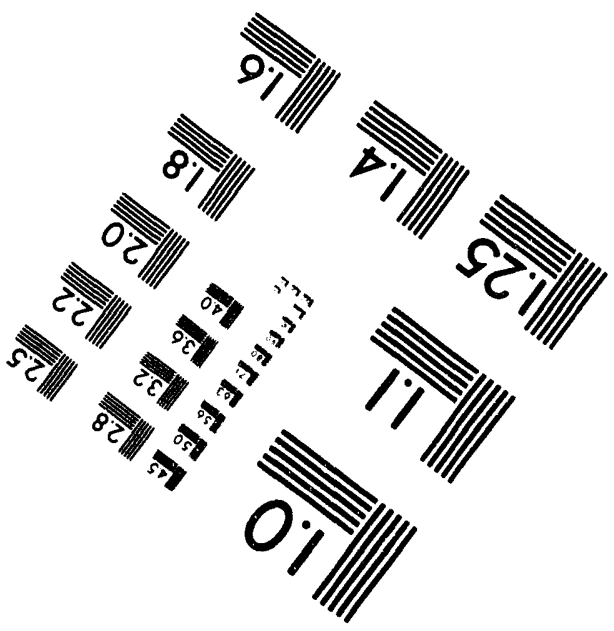

MANUFACTURED TO AIIM STANDARDS

$$
\text { BY APPLIED IMAGE, INC. }
$$

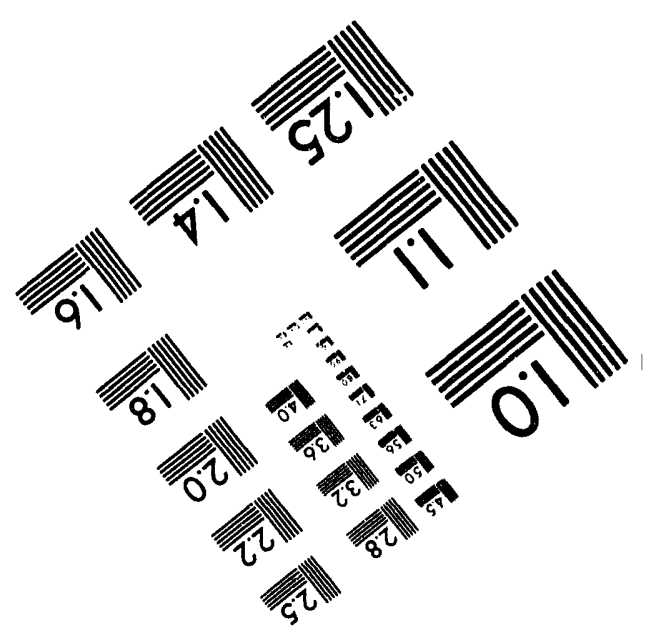



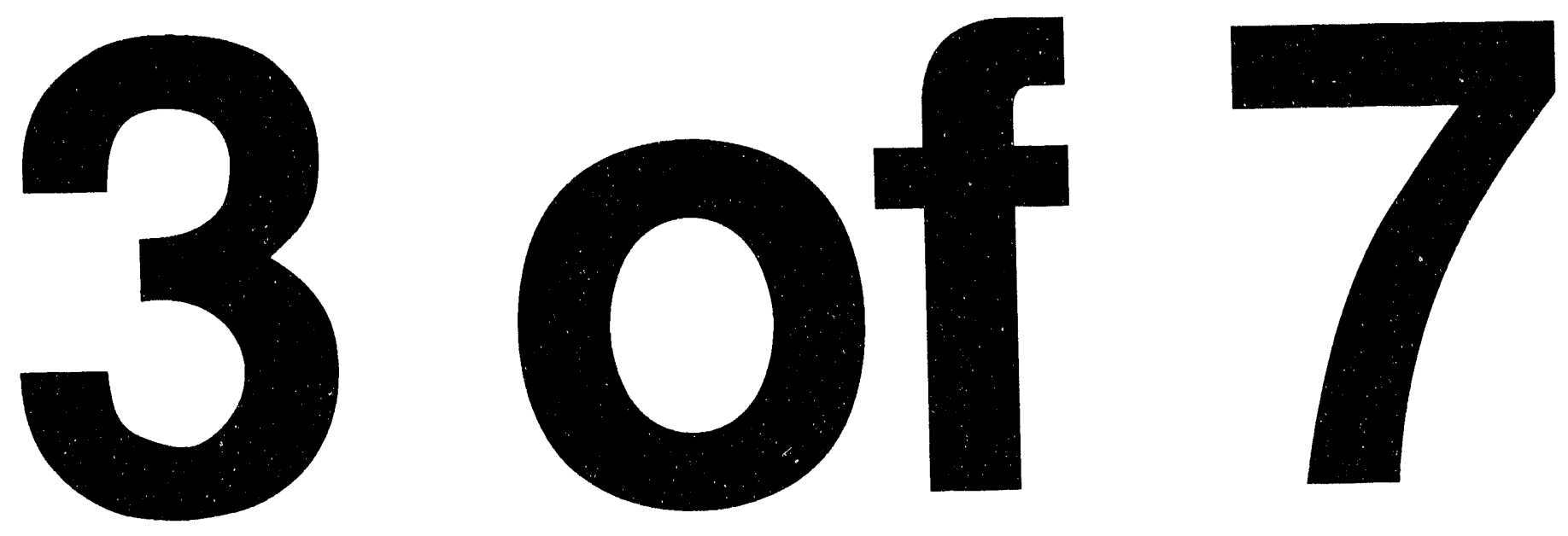
Table 3.1-1 Definition of Technological Readiness Levels

\begin{tabular}{|c|c|}
\hline $\begin{array}{l}\text { Level } 1 \\
\text { Basic principles observed and } \\
\text { reported }\end{array}$ & $\begin{array}{l}\text { This is the earliest level of technology readiness, at which the } \\
\text { basic principles of the concept, function, and potential } \\
\text { application have been identified. }\end{array}$ \\
\hline $\begin{array}{l}\text { Level } 2 \\
\text { Technology concept and } \\
\text { application formulated }\end{array}$ & $\begin{array}{l}\text { At this level of technology readiness, the basic engineering } \\
\text { system, which allows the concept to perform its function, has } \\
\text { been defined and preliminary analysis is underway to } \\
\text { determine if the system will in fact perform the function in } \\
\text { the specific application of interest. }\end{array}$ \\
\hline $\begin{array}{l}\text { Level } 3 \\
\text { Identification and verification } \\
\text { of critical functions }\end{array}$ & $\begin{array}{l}\text { At this level of technology readiness, requirements for the } \\
\text { engineering system have been defined and functions critical } \\
\text { to the performance of the system have been identified. These } \\
\text { functions are being verified with applicable computer codes } \\
\text { and general experimental data. }\end{array}$ \\
\hline $\begin{array}{l}\text { Level } 4 \\
\text { Conceptual design evaluation }\end{array}$ & $\begin{array}{l}\text { At this level of technology readiness, design tradeoffs for the } \\
\text { engineering system have been identified, and tradeoff } \\
\text { analyses are underway to establish a reference design } \\
\text { configuration against pre-established top-level mission } \\
\text { performance requirements. Initial safety analyses are being } \\
\text { performed, and general compliance discussions with licensing } \\
\text { organizations are being initiated. Information from earlier } \\
\text { tests or demonstrations is being incorporated into the design. }\end{array}$ \\
\hline $\begin{array}{l}\text { Level } 5 \\
\text { Design and component } \\
\text { demonstration in a relevant } \\
\text { environment }\end{array}$ & $\begin{array}{l}\text { At this level of technology readiness, the technology } \\
\text { development process begins transition into a technology } \\
\text { demonstration. System design descriptions are complete, and } \\
\text { integrated systems are being designed against pre-established } \\
\text { mission performance requirements. Safety analyses are being } \\
\text { conducted in accordance with a Standard Review Plan, and } \\
\text { compliance discussions are routinely held with the licensing } \\
\text { organization. Requirements for the design of major } \\
\text { components are being developed. }\end{array}$ \\
\hline $\begin{array}{l}\text { Level } 6 \\
\text { System performance } \\
\text { demonstrated in a relevant } \\
\text { environment }\end{array}$ & $\begin{array}{l}\text { At this level of technology readiness, the technology } \\
\text { development process has progressed to integrated system } \\
\text { demonstration, against pre-established performance } \\
\text { objectives, although possibly at less than full scale. Full- } \\
\text { scale preliminary designs are being developed against pre- } \\
\text { established mission performance requirements, using data } \\
\text { from the integrated system demonstration. }\end{array}$ \\
\hline $\begin{array}{l}\text { Level 7 } \\
\text { Integrated system performance } \\
\text { demonstrated }\end{array}$ & $\begin{array}{l}\text { This is the final level of technology readiness, at which a } \\
\text { final design is approved or approval is pending with no } \\
\text { outstanding issues of significance. An integrated system has } \\
\text { been demonstrated at a scale relevant to the final application } \\
\text { in the proper operating environment. }\end{array}$ \\
\hline
\end{tabular}


Fuel fabrication, transport and safeguards provisions have been demonstrated in a relevant environment, albeit in UK and France. Therefore, the maturity of the fuel technology, as defined by Reference 3-6, is judged to be higher than Level 6 and close to Level 7. Although individual components of the disposition process have been demonstrated to a high level of maturity, since the integrated system has not been demonstrated in this country at the scale required for this disposition, the overall technology readiness is rated at a level above 6 but below 7 . 


\subsection{Reactor}

No reactor system modifications are necessary to any of the existing BWRs listed as possible candidates in this-report to utilize full core loads of MOX fuel. Full MOX usage is accomplished through fuel design. However, because of the safeguards considerations involved in using MOX fuel and because a fresh fuel bundle will be slightly more radioactive, certain applied engineering level changes have to be made.

\subsubsection{Safeguards-Related Changes to MOX Fuel Handling}

Material accountability requirements indicate that the MOX fuel bundles should be handled with complete "near real-time" accountability. While handling and movement of fuel assemblies within the Reactor Building requires permissions and recording of such movement (even in the case of urania bundles), additional software and control will likely be required for MOX bundles, in particular, to tie these records to the overall material accounting system. This is not considered new technology, however, and is readily implemented. The physical security provisions associated with handling the fresh MOX bundle do not appear to pose any new problems, as a higl level of security and personnel security clearances are already required with urania bundle handling. A detailed discussion relative to the safeguards implementation associated with fresh fuel handling is given in Section 7.

\subsubsection{Fresh MOX Fuel Storage, Handling, and Inspection in the Reactor Building}

Evaluations of on-contact radiation dose rates associated with new MOX fuel would depend upon the level of americium present in the fuel. For americium content in the range of 3000 ppm in plutonium, the dose rate at the bundle midplane surface is expected to be approximately $5 \mathrm{mRem} / \mathrm{hr}$. This dose rate should pose no problem relative to worker exposure, in storage, handling or inspection of the bundles, when carried out using the procedures normally used with urania fuel.

The standard procedure for inspection of new uranium fuel upon receipt at the reactor site could require lengthy manual inspection activities. The inspection insures that no damage has occurred during shipment from the factory to the site. Aspects of the inspection include the following:

- Removal of shipping spacers; the spacers are required to provide vertical support for the fuel rods during horizontal shipment to prevent wear and damage to the fuel rod spacers.

- Visual inspection for cleanliness (fingermarks, oil smears, rust, dirt, etc.). 
NEDO-32361

- Visual inspection for foreign material (obstructions, rags, paper, etc.).

- Visual inspection of visible surface for burrs, hent surfaces, or other visual defects.

- Inspection of visible surfaces of fuel rods for scratches, nicks, or dents. A scratch depth gage is used when required.

- Visual inspection for fuel rod spacer damage.

- Numerous clearance and dimensional measurements using various gages and calipers.

The inspections outlined here are routine for transport of fresh urania fuel assemblies over long distances by commercial carriers. Even though the MOX factory might in some instances be colocated with an existing reactor complex, it is assumed in the reference case that the fresh bundles are transported using SSTs. If americium (Am) were removed from the feed material, the dose rates of fresh assemblies would be such that hands-on inspections are possible. Even for fuel containing americium in the range of $3000 \mathrm{ppm}$ (in plutonium), hands-on inspection is still possible. However, to lower the exposure as much as possible, the inspections of fresh MOX fuel will be performed underwater. GE has considerable experience in routine underwater inspection and adapting this experience to the present case should pose no problem. Recently, an entire core load of very slightly irradiated fuel bundles was transferred from the Shoreham plant to the Limerick plant. All the receiving inspections, de-channeling and removal of protective plastic separators were done under water in an automated fashion with tools and camera equipment specially designed for this purpose. These tools and procedures exist and are easily adapted to the requirements of this project.

\subsubsection{Additional Evaluations for Vessel and Core Internals Material Mechanical Behavior}

The number of prompt neutrons per fission is higher for plutonium fission compared to that from U-235 fission. At first sight, it would therefore appear that the surface of core internal structures would receive a higher flux of neutrons. However, a more detailed assessment indicates that any increase in the fluence on the surfaces of fixed internal structures such as the reactor vessel and shroud are likely to be small compared to the uncertainties in the fluence estimates at these locations. The primary reason for this result, as already pointed out in Section 2, is that the last two rows of assemblies in the reactor core which would be the main source of the neutrons that are incident upon the core internals, tend to be very similar to commercial urania assemblies that would be in these rows. It should be recalled that commercial urania assemblies are designed to maximize 
the energy output from self-generated plutonium. This plutonium fraction in the commercial urania core is at the highest near the end-of-life of the fuel assemblies and they would be located in the outer rows. If the fluence on the upper grid which is exposed to not only end-of-life fuel but also the start-of-life fuel is found to be too high, some blanket pellet material could be included near the top of the fuel rods to protect the upper grid. This will affect the plutonium throughput very slightly. In summary, no new technology or change to the core internals will be needed in using MOX fuel. However, it will be necessary to conduct more detailed analyses to document the actual flux levels at these surfaces.

\subsubsection{Fresh Fuel Shipping Container}

The fresh fuel shipping container has been licensed to ship BWR urania fuel bundles and to survive both transportation induced loads and a number of accident scenarios. Standard fuel assembly shipping containers typically used for transporting fresh urania fuel assemblies are certified right rectangular boxes consisting of an outer container of wooden construction and a metal inner container separated by cushioning material. Two fuel assemblies are contained in each shipping container. This container would be equally suited for MOX fuel shipments. Subcriticality analyses had been reported earlier for GE9 assemblies with a plutonium loading of $5.33 \mathrm{~kg} /$ bundle [Ref. 3-7] and $10.15 \mathrm{~kg} /$ bundle [Ref. 3-5], showing that no additional poison (control) materials are needed for MOX fuel shipments. If the shipping container has to be modified to provide additional shielding, detailed design will be carried out during the next phase of the project, and the modified fresh fuel shipping container will be licensed for transport of MOX fuel.

It should be noted that both French and Belgium fabricators have qualified shipping containers for routine transport of BWR and PWR MOX fuel assemblies around Europe. This MOX fuel utilizes plutonium from reprocessed commercial spent fuel and its radiation level is higher than any anticipated with the weapons plutonium. This European technology can be readily adapted to the design and licensing of a new shipping container. 


\subsection{Fuel}

\subsubsection{Lead Fuel Testing}

BWR MOX fuel is designed to be as interchangeable as possible with the standard uranium fuel it replaces. Based upon the design and operating experience accumulated to date on mixed-oxide fuel and the results of this study, it is anticipated that the candidate BWRs can safely and reliably operate with a full core of mixed-oxide bundles as well as urania fuel bundles. The thermalhydraulic characteristics of the various bundle designs currently in use have been extensively evaluated and have undergone NRC review. These characteristics will not change with the use of MOX fuel in the fuel rods. The peak plutonium enrichment has been limited to be below $10 \%$ based on an in-depth comparative review of MOX and urania fuel properties (Appendix G), so that the fuel rod thermal-mechanical performance will be comparable to urania fuel. The nuclear characteristics will be different, as most of the fissions will come from plutonium, rather than from uranium. However, the BWR fuel design for full MOX core takes into account these differences. As presently envisioned, disposition will start with a demonstration program using the available fabrication infrastructure at LANL and by utilizing an "island" design. Such a demonstration program will serve to identify any problem areas which can then be taken into account in full MOX fuel fabrication and disposition. Moreover, the transition from the urania core to full MOX will occur in stages with replacement of the discharge fraction of urania fuel with MOX fuel. For these reasons, the fuel testing program proposed here for disposition using existing reactors differs slightly from that proposed earlier in Reference 8-6 for disposition with an ABWR reactor.

A limited MOX fuel rod and Lead Use Assembly (LUA) testing program will be used for the following reasons:

- To ensure that the desired fuel microstructure is obtained with the proposed fabrication process and disposition-specific feed material interface; to confirm the performance of the MOX fuel.

- To obtain benchmark data on MOX fuel containing Gd burnable poison, on the radial burnout of the poison as a function of exposure, so that the nuclear analysis codes can be further calibrated. Benchmark data are available for MOX fuel and for urania fuel containing gadolinium (Gd), but data for MOX fuel containing Gd has not yet been generated. 
The test plan would consist of the following: (1) verification of the fuel fabrication process (discussed both here and in Section 3.3.2); (2) confirmation of individual fuel rod mechanical, thermal and nuclear performance principally by conducting tests in research reactors; and (3) confirmation of assembly performance through full-scale MOX assembly testing. The test plan in the first two areas noted here are the same as described for disposition with the ABWR [Ref. 8-6].

\section{Verification of Fuel Fabrication Process}

There are four reasons to include qualification of the fuel fabrication process in the fuel testing program. First, although MOX fuel has been irradiated in the past to 50,000 MWD/MT and beyond, and found to perform exceptionally well, it is necessary that the fuel be produced with the appropriate chemistry and microstructure to realize this performance. Verification that the proposed fuel fabrication process can produce fuel of the correct microstructure and prespecified chemistry, in conjunction with the disposition-specific feed stock, is essential to ensure satisfactory thermo-mechanical performance of the fuel. Second, there is little experience in fabricating MOX with gadolinia, although urania fuel with gadolinia has been fabricated by GE for a number of years and no problems are anticipated in fabricating MOX with gadolinia. Third, evaporation of americium has been noted during sintering of high americium MOX fuel. If the feed stock were to contain high levels of americium, data are needed on the rates of evaporation and location of americium condensate, so that appropriate levels of shielding and americium collection equipment could be designed into the MOX fuel fabrication facility. Finally, the gamma signature will be used to complement more positive interrogation techniques for plutonium assays in generating material accountability data. The considerations relevant to this topic are discussed in more detail in Section 7. It is useful to understand the variability of the gamma signature during the fabrication process to better implement the material accounting system.

\section{Confirmation of Rod Performance}

Data on gadolinium burnup as a function of exposure in the MOX fuel is appropriate to provide additional benchmark data for nuclear methods validation. Since gadolinium burns up, typically during the first cycle in commercial reactor operation, such data cannot be generated easily by lead assembly tests in a commercial BWR, as special "midcycle" outages would be needed and this would be highly expensive. Therefore, these "rod" tests will be carried out in a research reactor. In addition to providing the data on Gd burnup, these tests could provide lead confirmatory data on MOX fuel rod performance for fuel thermal and mechanical behavior. 


\section{Confirmation of Assembly Performance}

Full-scale MOX assembly tests will be conducted principally to provide goal exposure data on the thermo-mechanical performance of full length fuel pins. As lead assemblies, they will surface any problems arising from the thermo-mechanical response of the fuel long before a full MOX fuel system is deployed. However, the usefulness of the isolated full scale MOX assembly tests in a urania fueled BWR from the perspective of reactor safety or licensing is limited. This is because tests of isolated MOX assemblies in existing BWRs are unlikely to provide useful additional data on the overall dynamic (system) response of the MOX fueled core, as this - the overall system response in the test reactor - will clearly be dominated by the preponderance of urania assemblies in the core. Therefore, only a limited number of lead test assemblies are proposed. These are conveniently included as part of the island core demonstration program. In Section 2.4, 58 island assemblies are proposed for loading into each disposition reactor. About 6 to 12 of these assemblies can be specially designed to contain the range of fuel variables proposed for the full MOX disposition core. These special assemblies will be taken to an exposure slightly higher than the typical full MOX core discharge exposure. Fuel rods at goal exposure from such assemblies will be available for post-irradiation examination by the time the second reload of full MOX assemblies are introduced into the reactor. These tests therefore will provide the lead data on fuel behavior at goal exposure.

The full MOX core is implemented in stages, as MOX fuel bundles replace the discharge fraction of urania bundles. A nearly full MOX core is achieved by the fourth cycle after the start of full MOX bundle design loading. The nuclear response of the core can be evaluated during each cycle as the fraction of MOX in the core is increased in a gradual fashion to provide full validation of the predictive methods employed in the nuclear calculations.

Details of the proposed plan for lead fuel testing are given in Appendix H. The test plan elements include fabrication of MOX fuel from representative plutonium oxide derived from weapons plutonium by the desired process, an assessment of the pre-irradiation fuel chemistry and microstructure, the conformance of the fuel to specifications, generation of data on the sintering response of fuel containing high and low americium, in-reactor tests and postirradiation examinations to generate nuclear benchmark data for additional calibrations of existing methods/codes and for confirmation of fuel thermo-mechanical performance to goal exposures. The results of these tests will further correlate the models used for predicting fuel performance and the nuclear analysis methods. 


\subsubsection{Development Requirements for MOX Fabrication Facility}

The facility for fabrication of MOX fuel is on the critical path associated with the disposition process. The technology for processing the MOX fuel currently exists and is being implemented in several foreign countries. Nevertheless, the following areas require applied engineering development and are considered long-lead items that could fall in the category of development:

\section{Automation}

The proposed fabrication facility is highly automated to minimize worker exposure, yet provides for non-remote maintainability. Both the GE-Wilmington and the JNF facility in Japan, which fabricate urania fuel, are an examples of facilities which employing a high level of automation. A number of automation steps used in these facilities could be scaled down and used in the MOX facility proposed as part of the plutonium dispositioning activities. The areas where this automation technology could be implemented include pellet fabrication, rod fabrication and bundle assembly. A critical long-lead item is the preliminary engineering required to (1) identify the areas where automation is readily available, (2) evaluate its applicability for MOX usage as well as develop and test the required modifications, and (3) integrate these aspects with other MOX fabrication systems.

\section{Modular Concept Evaluation}

Engineering scale fabrication processes have traditionally involved a "series" production line arrangement. Fabrication efforts involving radioactive material, particularly in cases involving plutonium, is generally carried out using modular or glove box type arrangements. During MOX fuel fabrication, accountability and criticality considerations require that a material balance areas be completely verified by a physical inventory. These inventories are required on predetermined time intervals, as well as before starting operations with a new plutonium enrichment. A concept being proposed by GE involves the use of a modular or hybrid accountability concept whereby an individual glove box or module can be taken off-line and verified by a physical inventory. Completion of these physical inventories in a sequential fashion allows a minimization of the effects on processing capacity, particularly for redundant operations, while satisfying accountability requirements. Further development of this concept requires (1) an assessment of the areas where this concept could be readily and usefully implemented, (2) the associated cost/benefit tradeoffs resulting from equipment redundancy and improved capacities, (3) the extent to which this approach satisfies material accountability requirements, and (4) the ease with which this concept can be accomplished in practice. 


\section{Confirmatory Tests for MOX Pellet Fabrication}

The majority of the MOX fabrication activities completed to date have utilized oxalate derived plutonium feed powders. Since a direct metal-to-oxide conversion process is being considered for the plutonium disposition activities, the viability of the feed in the developed MOX fabrication process needs to be verified. The plutonium homogeneity resulting from the blending and milling operations after the completion of the sintering process is the key item which requires verification.

In fabricating MOX fuel from plutonium containing high americium levels, it has been found that there is a certain amount of evaporation of the americium during the sintering process. Generally, this material will plate-out (condense) in the exhaust portions of the sintering system. Additional features are required in the design to trap the americium in a predictable manner to minimize worker exposure. An acceptable procedure and process for disposal of this material is also required. Americium has not been a problem when processing small quantities of MOX fuel or when the level is extremely low. Processing up to $50 \mathrm{MT}$ of $\mathrm{Pu}$, particularly if the americium is not removed during feed material conversion, could result in the accumulation of considerable quantities of material in the sintering area. Simulated sintering tests with americium doped urania is recommended to precisely monitor the extent of evaporation, determine the location of the condensate, and verify the frequency with which the condensate has to be removed and disposed. Several sintering furnaces which have been used with plutonium are available in many national laboratories and these could be used for this testing.

\section{Confirm Fabrication Procedures for MOX Fabrication with Gadolinia}

Urania fuel with gadolinia is now routinely fabricated by GE as well as other foreign fuel fabricators. The addition of plutonium to fabricate MOX fuel with gadolinia is not expected to pose any problems. The oxygen partial pressure must be carefully controlled to ensure that the pellets are produced with the required stoichiometry and micro cracking is not a problem. Given that MOX can exist in a wider band of stoichiometries (compared to urania) and that plutonium tends to act as a sintering aid, it should be less difficult to sinter MOX with gadolinia additions. However, it is appropriate to confirm these facts.

The technology needs defined in the two previous paragraphs will be completed as part of the lead fuel testing for performance validation outlined in Section 3.3.1. 


\section{Disposition Process Simulation}

A computer simulation of the plutonium flow during the disposition process that includes all the processing operations, from the removal of the pit to final disposition as irradiated bundle, appears to be needed. The program is aimed at simulating the flow of plutonium through the various process steps and should include downtime for maintenance and accountability inspections, as well as the admissible range of statistical errors in assays.

The output of this computer simulation is expected to provide the following information for the detailed design:

- Statistical sampling requirements in regard to accountability

- Redundancy needs for material accountability

- Storage requirements at various points in the process

- Points of maximum vulnerability (i.e., points where the loss of material might be hardest to detect because of margins of error in assay and transit times)

- Possible impact of levels of transparency and frequency of complete physical inventory. Complete physical inventory with the process lines in the off condition would tend to increase the downtime but is expected to provide the best verification for accountability.

- Detailed areas relative to lead/lag requirements and reciprocity in bilateral agreements if disposition were to occur in the U.S. and CIS simultaneously, and if the disposition rates were to differ due to unavailability of process equipment.

\section{Development and Validation of Surveillance Equipment}

The technology for fabricating MOX fuel is already being implemented in foreign countries. Evaluations have been conducted on adapting and improving this technology for weapons $\mathrm{Pu}$ disposition. One area which has been identified for further evaluation involves the surveillance equipment used for safeguards and security control. Unlike plutonium from reprocessed fuel, which is highly $\gamma$ active, weapons plutonium has a low level of $\gamma$ activity. For this reason, material accountability or surveillance cannot be dependent upon instrumentation based on $\gamma$ activity per se. Alternative, positive interrogation measures have to be implemented, including the use of Californium (Cf) scanners. While such scanners have been used in the past, their use has principally been for fully assembled fuel rods. The simplest means of utilizing the already available technology would be to sample small quantities of a batch of material by placing it in a 
cladding tube and extrapolating the plutonium content in the batch by ratio of the weights. The application to on-line measurements is expected to require some limited additional development and engineering verification. Similarly, magnetic sensors have been used for identifying gadolinia bearing-rods and, once again, some additional development and engineering validation for application in the proposed MOX factory will be needed.

\subsubsection{Compatibility in Geologic Repository}

A complete discussion of the acceptability of the discharged MOX fuel for permanent repository storage can be found in Reference 3-5. Acceptability to the extent that criteria are available for the repository was evaluated for fuel assemblies containing an initial Pu loading of 5.33 $\mathrm{kg} /$ bundle in Reference 3-7 and for fuel assemblies containing an initial Pu loading of 10.15 $\mathrm{kg} /$ bundle in Reference 3-5. These evaluations are repeated here for the reader's convenience.

The Nuclear Waste Policy Act of 1982 made the DOE responsible for developing an underground repository for the highly radioactive waste from civilian and DOE sites. Amendments to this act in 1987 directed the DOE to investigate only the Yucca Mountain site for this repository. Currently, the utilities have signed the standard contract given in 10CFR Chapter III Part 961, Standard Contract for Disposal of Spent Nuclear Fuel [Ref. 3-8]. Article VI.A.l of this contract provides the criteria for spent fuel acceptance. The applicability of this contract as stated in Part 961.2 extends to "spent nuclear fuel or high level radioactive waste, of domestic origin, generated in a civilian nuclear power reactor". It is reasonably concluded that the spent fuel generated by the disposition program will qualify under the category of "spent fuel generated in a civilian nuclear power plant" and the rest of this section briefly examines whether the general criteria for acceptance of spent fuel are satisfied when MOX fuel is used instead of the standard urania fuel.

The general criteria for acceptance given in Article VI of 10CFR Part 961 (which, in turn, refers to Appendix E of this Part), as well as the information which the purchaser of the contract is to provide, are specified in this and succeeding Articles. These criteria have been evaluated for potential applicability to spent fuel deploying MOX fuel. It has been found that all the general criteria are met by the spent fuel to be discharged from the disposition program.

\subsubsection{General Criteria for Disposal}

The general criteria stated in Appendix E of 10CFR Part 961 is attached as Appendix I of this report. The spent fuel from the disposition program will meet all the requirements enunciated for this "standard fuel". These criteria do not place any limit on the fuel burnup. Appendix I also 
contains data on the discharge exposures of spent fuel, both those already discharged as well as potential fuel discharges. The average discharge exposure at this time is close to $30,000 \mathrm{MWd} / \mathrm{MT}$, while the expected average for future discharges in the year 2010 is 36,000 MWd/MT.

All waste forms to be disposed of must be extensively characterized so that their behavior during disposal is understood. Currently, there is an extensive characterization program ongoing that will continue for several years on LWR urania fuel. It will be necessary to show that the MOX fuel behaves the same way. Ongoing studies include oxidation, dissolution, cladding and gaseous release. A preliminary assessment for these areas is given below.

\subsubsection{Waste Acceptance System Requirements}

DOE document DOE/RW-0351P [Ref. 3-9] issued by the Office of Radioactive Waste Management in January 1993 and Yucca Mountain Site Characterization Project Change Directive CR No. DCP-060, dated 2/5/93 [Ref. 3-10] describe the functions to be performed and the technical requirements for a Waste Acceptance System for accepting Spent Nuclear Fuel (SNF) and high-level radioactive waste (HLW) into the Civilian Radioactive Waste Management System (CRWMS). As a starting point, it is worthwhile noting that the only significant difference between the standard spent fuel from urania fueled assemblies and those from the MOX fueled disposition reactor, consists of the higher fraction of transuranics, specifically $\mathrm{Pu}$ isotopes, in the discharged bundle. The decay heat is at a maximum just prior to the placement of the bundle in the repository after a cooling period of 10 years. At this time, the full MOX bundle decay heat is only slightly higher, by about $5 \%$, compared to a urania bundle with similar exposure. Therefore, decay heat is not considered limiting for repository disposal.

Table F1.1.1 of Reference 3-7, reproduced in Appendix I, defines the Waste Acceptance Criteria. These requirements have been reviewed in detail and only the following criteria are considered to be affected by the presence of higher amounts of transuranics:

- 10CFR60.135(a) High-Level Waste Package:

“(1) Packages for HLW shall be designed so that the in situ chemical, physical, and nuclear properties of the waste package and its interactions with the emplacement environment do not compromise the function of the waste package or the performance of the underground facility or the geologic setting. 
(2) The design shall include but not be limited to considerations of the following factors: solubility, oxidation/reduction reactions, corrosion, hydriding, gas generation, thermal effects, mechanical strength, mechanical stress, radiolysis, radiation damage, radionuclide retardation, leaching, fire and explosion hazards, thermal loads, and synergistic interactions."

- 10CFR60.43: "License conditions shall include items in the following: restrictions as to the physical and chemical form and radioisotopic content of the radioactive waste."

- 10CFR60.131 Criticality Control: “All systems for processing, transporting, handling, storage, retrieval, emplacement and isolation of radioactive waste shall be designed to ensure that a nuclear criticality accident is not possible unless at least two unlikely, independent, and concurrent or sequential changes have occurred in the conditions essential to nuclear criticality safety. Each system shall be designed for criticality safety under normal and accident conditions. The calculated effective multiplication factor (Keff) must be sufficiently below unity to show at least a 5\% margin, after allowance for the bias in the method of calculation and the uncertainty in the experiments used to validate the method of calculation."

- 10CFR72.124 Criteria for Nuclear Criticality Safety: "Design for criticality safety. Spent fuel handling, packaging, transfer, and storage systems must be designed to be maintained to be subcritical and to ensure that, before a nuclear criicality accident is possible, at least two unlikely, independent, and concurrent or sequential changes have occurred in the conditions essential to criticality safety. The design of handling, packaging, transfer, and storage systems must include margins of safety for the nuclear criticality parameters that are commensurate with the uncertainties in the data and methods used in calculations and demonstrate safety for the handling, packaging, transfer and storage conditions and in the nature of the immediate environment under accident conditions."

Of the requirements listed above, 10CFR60.131 and 10CFR72.124 are addressed in Section 8.4 of this report, where it is shown that subcriticality is maintained for proposed geometries under consideration for repository disposal. In modeling for subcriticality, a configuration of $10 \mathrm{BWR}$ assemblies within a single container was chosen to provide bounding analyses. 10CFR60.43 and 10CFR60.135 requirements primarily pertain to making sure that the engineered barrier system (EBS) performs as intended. Containment of the waste within the EBS is expected to be complete for 300 to 1000 years and limited to less than 1 part in $10^{5}$ per year of the 1000 year inventory beyond this period. The nuclides of interest in this regard are ${ }^{14} \mathrm{C},{ }^{85} \mathrm{Kr}$ and ${ }^{3} \mathrm{H}$ and 
short-lived isotopes such as ${ }^{90} \mathrm{Sr},{ }^{137} \mathrm{Cs}$ during the containment period and ${ }^{99} \mathrm{Tc},{ }^{129} \mathrm{I}$, and ${ }^{135} \mathrm{Cs}$ during the post-containment period. Of these, $\mathrm{Sr}, \mathrm{Cs}, \mathrm{Tc}$ and I are of interest because they are expected to move to the fuel grain boundaries or the pellet-cladding gap region. The inventory of these isotopes should be comparable for MOX fueled and conventional urania fueled discharges for the same level of exposure, although there are small differences in the fission product yields from plutonium. Typical urania fueled assemblies could contain up to $1 \% \mathrm{Pu}$ at discharge. While the $\mathrm{Pu}$ in the spent fuel from MOX fueled assemblies would be higher, there is considerable data from MOX fuel developed for Liquid Metal Fast Breeder Reactors which indicate that the movement of these fission products is not correlated with $\mathrm{Pu}$ enrichment. Therefore, the release rates of spent fuel from MOX fueled assemblies should be no worse than that from the conventional assemblies. The higher level of Pu content in the discharged MOX assemblies is not considered to be consequential except as it affects subcriticality requirements.

\subsubsection{Other Disposal Criteria}

Heat load from a single waste container (or canister) would appear to be a key criterion for disposal. With regard to spent fuel, the repository is likely to be required to package and dispose of a variety of fuel configurations. Container materials would have to be selected based on material properties as well as its corrosion characteristics. The higher Pu content of the SNF is judged unlikely to affect the corrosion characteristic of the container material. A key parameter in this regard is the decay heat load of the container and the manner in which the waste form will be distributed within the container. One approach, proposed by Lawrence Livermore National Laboratory (LLNL), would seek to keep the emplacement hole walls above the unconfined boiling point of water in the unsaturated zone (about $97^{\circ} \mathrm{C}$ at the repository elevation), while others have proposed keeping the package as cool as practical. In any case, it is likely that the spent fuel will be "packaged" within the outer container to achieve the required objectives. While "geometric tailoring" of the waste products (SNF) within the container to achieve these objectives has been proposed, "receipt tailoring" or managing the waste inventory to obtain the required levels of waste heat has also been suggested. In the latter case, a single container may contain not only disposition spent fuel but other SNF or non-fuel components as well. By such combination of measures, it will be possible to maintain the heat load within adequate limits.

Studies and field work are still ongoing and the set of requirements for the acceptability of a given spent fuel form or composition might undergo changes in the future. The design information for the repository given below was taken from DOE document DOEIRW-0198, 
Nuclear Waste Policy Act, Site Characterization Plan Overview, Yucca Mountain Site, USDOE-OCRWM, December 1988:

- Repository Total Area:

- Effective Repository Area:

- Repository Heat Limit:

- Container Separation along Tunnel:

- Separation between Tunnels:

- Container Area allocation:

- Number of Containers per acre:

- Heat Limit per container (average): $2.5 \mathrm{~kW}$
2100 Acres

1400 Acres

$57 \mathrm{~kW} /$ Acre

$15 \mathrm{ft}$

$126 \mathrm{ft}$

$1890 \mathrm{ft}^{2}$

23

However, a recent private communication (T. Doring, B\&W to $S$. Vaidyanathan, GE) indicates that the heat load requirement is undergoing further review and has not been fixed at this time.

The SNF assemblies from the disposition reactor will be able meet the design constraints above equally as well as conventional urania fueled SNF assemblies. No specific requirement on the allowable quantities of actinides or fission products per container was found.

\subsubsection{Spent Fuel Characteristics from Pu Disposition}

The isotopics of the spent fuel are discussed in Section 2 of this report. After interim storage for 10 years prior to its placement in the repository, the heat load is mainly due to long-lived fission products. The heat load per assembly as a function of time following discharge is also discussed in Section 2. The heat load per assembly is less than 250 watts if transport to repository commences immediately after 10 years of reactor site storage. If the heat load is limited to $2.5 \mathrm{~kW}$, a maximum of ten assemblies could be stored in each container. This is not very different from the disposal scheme proposed for typical commercial spent fuel, as the heat load contribution at this time is principally from the fission products and the fission product content is related to the exposure. 


\subsubsection{Conclusions}

Based on the information available to-date, it is judged that the spent nuclear fuel assemblies from the disposition reactor could be stored in the permanent repository and that all the applicable requirements will be met. The slightly higher transuranics in the spent fuel does not preclude its disposal in the permanent repository. While ongoing development and evaluation for permanent repository storage have to be completed, no additional technology needs have been identified as arising from the use of MOX fuel assemblies.

\subsubsection{Development Issues}

The proposed disposition process relies upon an already established technology. The technology needs identified so far are principally to verify aspects of this already established technology. No development issues which need to be resolved by research and development in the conventional sense have been identified for the proposed disposition process. 


\subsection{New Development and Test Facilities}

No new development and test facilities have been identified as being necessary to complete the technology needs: Existing facilities, particularly at the national laboratories, are more than sufficient to implement the technology needs identified here. These existing facilities include:

\section{- Fuel Fabrication Facility}

Plutonium handling has been carried out at a number of national laboratories. The TA-55 facility at Los Alamos National Laboratory (LANL) is particularly well suited to making MOX fuel from weapons Pu for two major reasons. First, LANL will be demonstrating plutonium removal from the pits in their planned ARIES facility, so the material will be available within the same location and no new transportation issues have to be faced. Second, all the equipment required for small scale (test scale) fabrication of MOX fuel such as milling, blending, and sintering equipment are already available. In addition, the asfabricated fuel can be evaluated for quality using metallographic and chemical analysis equipment, which are readily available at LANL.

\section{- Fuel Testing}

Fuel rod tests should be implemented at an early date in a research reactor. A number of research reactors are available to conduct these tests, including those at ORNL and INEL. No specific recommendations are made here as to a preferred facility.

\section{- PIE Facilities}

Post-irradiation facilities are readily available at Hanford, Oak Ridge and INEL. In addition, examination facilities for BWR rods are also available at GE-Vallecitos. In particular, for assembly tests, the GE-Vallecitos facility has the necessary equipment to handle full length rods in its hot cells.

\section{- Full-Scale Assembly Testing}

Full-scale lead assembly tests could be included as part of the island fuel demonstration program or conducted separately as LUA tests in the candidate BWR. The fuel required for this purpose can also be fabricated in the LANL facility. No new test facilities are needed. 
NEDO-32361

\subsection{References}

3-1 Nuclear Technology, Vol. 102, April 1993, H. MacLeod and G. Yates, Development of Mixed-Oxide Fuel Manufacture in the United Kingdom; J. Kellerman, Plutonium Processing at the Siemens Hanau Fuel Fabrication Plant; P. Deramaix, D. Haas and J. Van De Velde, In-Pile Performance of Mixed-Oxide Fuel with Particular Emphasis on MIMAS Fuel.

3-2 T.T. Claudson and R.E. Bardsley, The FFTF Fuel Story, JSD-SA-1959, October 1979.

3-3 GE-NEDC-10747, Plutonium Utilization in Boiling Water Reactors, Phase II, November 1972.

3-4 M. Zendel, IAEA, Table I in Experiences and Trends in Safeguarding Plutonium MixedOxide Fuel Fabrication Plants, JNMM, February 1993.

3-5 Disposition of Weapons Plutonium Using the GE Advanced Boiling Water Reactor, A report prepared for the U.S. DOE, GE NEDO-32351, April 1994.

3-6 R.P. Omberg and C.E. Walter, Disposition of Plutonium from Dismantled Nuclear Weapons: Fission Options and Comparison, LLNL, UCRL-ID-113055, February 1955.

3-7 GE NEDO-31314, Study of Pu Consumption in Advanced Light Water Reactors, Evaluation of GE Advanced Boiling Water Reactor Plants, Compilation of Phase 1C Task Reports, January 15, 1994.

3-8 10CFR Ch. III Part 961, Standard Contract for Disposal of Spent Nuclear Fuel.

3-9 DOE document DOE/RW-0351P, issued by the Office of Radioactive Waste Management, January 1993.

3-10 Yucca Mountain Site Characterization Project Change Directive CR No. DCP-060, February 5, 1993. 


\subsection{Operations}

This section describes the government, utility, and private industry interfaces that will impact the plutonium disposition reference case for existing reactors which has unique differences from the evolutionary reactor report. The preoperational checkout and startup testing of the MOX fuel fabrication facility for island and full MOX fuels is included in this section and is similar in scope to the evolutionary reactor report. The preoperational checkout and startup testing of the existing BWR plant is covered anc is less extensive than the evolutionary plant, since the plant is built, licensed, has commercial operating experience; and except for fuel, no systems changes are required to support burning MOX. Information on BWR fleet-wide average availability and capacity factor performance is provided to show that the program goal of $75 \%$ is achievable.

\subsection{Government, Utility, and Private Industry Interfaces}

The completion of the Reference Case disposition mission requires the combined use of three of the later generation power plants (BWR-4,5,\&6) to meet the $50 \mathrm{MT}$ disposition goal. The reference case assumes that MOX fuel will be fabricated at a Government-owned facility. The reactor(s), and their operating licenses are assumed to remain under the control of the current utility owners. The general agreements necessary for any use of commercial power plants will then focus on (1) the design, fabrication, and supply of the MOX fuel, and (2) the payment of fees to the utility for utilization of the reactor(s) in the disposition mission. This section reviews and discusses possible resolution of the key issues in each of these areas based on utility interviews conducted as part of this study. Where applicable, the utility specific input will be discussed.

\subsubsection{MOX Fuel Supply Agreements}

For the reference case, the Government is responsible for delivery of the MOX fuel to the reactor sites, similar to that of the commercial fuel suppliers currently contracted by operating utilities. Implicit in this overall responsibility for fuel supply is the requirement for the fuel to meet the reliability and operability requirements of the utility, normally covered via a commercial warranty provision. These provisions will be particularly important to those utilities who purchase the MOX fuel from the Government, and apply the commercial incentive to other financial accounts within their corporations. These responsibilities are best handled in fuel 
supply contracts negotiated between the selected utilities and the Government. Examples of the provisions that may be necessary are as follows:

- Warranty for defects in manufacture, design, materials, and workmanship

- Warranty for minimum energy content

- Guarantee for supply, including quantities and schedule and provisions for alternate supply

- Remedies for failure to meet contract requirements, including early termination

- Indemnification against third party intervention in the contract supply

None of these provisions is unexpected in any current commercial fuel contract, and will be negotiated as part of the overall contract covering the involvement of any commercial utilities. However, to implement this agreement, standard Government contracting practices will require review and modification to provide the utilities with adequate legal and financial protection against the potential risks associated with involvement in the disposition program.

\subsubsection{Commercial Incentives and Utility Involvement}

To determine the potential for utility involvement in the disposition program, and attempt to quantify the incentives that would be required to gain their commitment to the program, several BWR utilities were contacted to discuss the program. The feedback provided covered the entire spectrum, from strong interest in being selected for the mission to no interest in discussing any part of the program. This section provides an overview of this utility feedback on possible incentive structures, and discussion of the detailed feedback received from selected utilities on the program.

\subsubsection{General Structure for Commercial Incentives}

The overall structure of commercial incentive packages is primarily dependent on the utility ownership structure, specifically if the utility is a private investor-owned or a government (state or federal) affiliated agency. This will impact any incentive structure for involvement in the mission based on the utility's need to provide a return on investment to any shareholders, as well as the utility rate structure utilized by the utility for the sale of power from the facility.

For the private investor-owned utility, the general incentive structure will need to repay any incremental costs incurred by the utility in implementing the MOX fuel cycle, as well as 
incremental operational costs incurred by the program. All additional fee would be necessary to pay the utility for 'use' of the facility and to compensate the investors for any added business risk associated with involvement in the disposition program. However, based on the individual utility's regulated rate structure, the Government may be paid a fee for the MOX fuel supply, as most fuel costs are allowed to be passed directly through on the power rates, with no return to the utility. This fee would be equivalent to the current market price for nuclear fuel (approx. $\$ 0.005$ per $\mathrm{kWh}$ ) and would offset the Government costs in the program. In general, any incentive structure negotiated with a private utility would be used to offset their current investment in the plant, not as an offset in the costs of power production that are currently allowed to be recovered through the rate structure.

This would not be the case for the Government affiliated power agency. As many of these agencies sell their power at its production cost, without the need to provide investors a return, the 'incentives' for program involvement would generally be simpler to establish. The general program would include the incremental costs for program implementation, the supply of fuel at no cost to the agency, and a fee that would provide some portion of the debt service that the agency would be incurring on the investment in the nuclear plant. These fees would directly reduce the cost of power production, and result in a reduction in the rates charged by the agency. These public agencies also have the ability to establish an optional structure, where the government would pay all costs of the facility operation (fuel, operation and maintenance, and debt service) and in return receive the proceeds from the power sale to offset these costs. The agency would retain plant ownership and the operating license to minimize complications involved with the transfer of the operation license, but in all other aspects act as the contractor in a traditional Government Owned, Contractor Operated agreement.

\subsubsection{Utility Specific Feedback}

The basis structure for commercial incentive packages described above was developed from a summary of all discussions with operating utilities. In each discussion, utility specific issues were discussed that would impact the detailed structure of any incentives, and be the focus of detailed negotiations between the utility and the Government if their plant was to be utilized for the mission. The following paragraphs present details received from several of the utilities contacted, as well as many of the issues raised during the specific discussions. The summaries are representative of the varying interest levels found with the operating utilities.

Washington Public Power Supply System: The Supply System is very interested in supporting the disposition program. They have independently developed a program, titled the Dual Use 
Concept, that would support the mission by converting their operating BWR, WNP-2, to the MOX fuel cycle and subsequently completing the construction of WNP-1 (a Babcock \& Wilcox PWR) for added capacity in the disposition mission. This concept has the Supply System retaining the license and ownership of the plants, with the DOE providing funding for the following:

- Costs to convert WNP-2 plant

- Completion of the WNP-1 plant

- Operation and maintenance costs for both plants

- Fuel supply costs for both plants

- Repayment of the investment by the Bonneville Power Authority in the facilities, estimated at $\$ 2$ Billion, at a rate of $\$ 500$ Million per year for 5 years.

The power sales would be handled by the Bonneville Power Authority, and all revenues from the power sales (proceeds minus any fees required) would be returned to the DOE to defray the costs incurred for the program.

This concept has the benefit of utilizing plants that are located within a current DOE reservation, in an area where the public is openly receptive to nuclear power and involvement in the program. (NOTE: the capability of the dual Use Concept to meet the mission goal cannot be conclusively determined in this study due to the undetermined capacity for Pu disposition of the WNP-1 plant.)

Commonwealth Edison Company: Commonwealth Edison was interested in supporting the program provided specifics issues of the program could be negotiated at some point in time. Their overall position was that if the potential impacts of plant operation could be minimized, and the financial incentives were sufficient to offset the business risk of committing to the program, then they would strongly consider utilizing any, or all, of their nuclear plants (6 BWRs and 6 PWRs) to support the mission goal. The specific economic incentives would be negotiated, and would follow the structure outlined above for investor-owned utilities. There were not enough programmatic details available at the time of the discussion to quantify the necessary incentives.

A key issue associated with minimizing the program impact and risk is the implementation of safeguards requirements for the MOX fuel, once received on site for use in the reactor. The 
utility's position is that the MOX fuel bundles should be treated as normal reactor fuel once inside the facility protected area, and that existing plant security plans and handling processes are sufficient for protection of the fuel. This condition can be accommodated by modifying the existing DOE regulations to exempt the MOX fuel for this program from the current safeguards requirements. If these exemptions cannot be made for the program, Commonwealth Edison would not elect to participate in the program. If the safeguards issues can be resolved, the Commonwealth Edison BWRs would provide enough capacity to meet the mission goal to disposition $50 \mathrm{MT}$ of plutonium, thus limiting the contractual arrangements and negotiations to a single utility organization.

Tennessee Valley Authority - Browns Ferry Plants: Discussions were held with the TVA due to their unique position of being a federal government agency with ownership of a plant site with three licensed BWRs. Additionally, they are currently evaluating the potential to recover a minimum of 21 years on the operating licenses for the three units, due to the extended shutdowns of the plants by the NRC for regulatory issues. This recovered operating time would increase the throughput capacity of the units such that the entire $50 \mathrm{MT}$ goal with the three plants. The Browns Ferry staff would pursue approval to support the disposition mission from their Board of Directors, provided the following costs would be paid by the program:

- Cost to convert the units to the MOX cycle

- Cost to complete the planned restart of Browns Ferry Unit 1 (estimated at \$1.2 Billion)

- Fuel costs for all MOX fuel used by the three units

The ability of the Browns Ferry site to meet the mission goals is contingent on the license recovery effort and successful restart of Unit 1. Based on a technical and legal review of the license recovery issues, approval by the NRC of the application for the recovery is considered a high probability by both TVA and GE Nuclear Energy (GENE). There are several unique benefits to the possible utilization of the Browns Ferry units, including (1) the use of a single site for the mission, (2) location in an area with positive public attitudes toward nuclear power, (3) ownership of the plants by the federal government, and (4) availability of other units (PWRs) for the mission if the disposition requirements are increased. The site also appears to be suitable for a Greenfield MOX fabrication facility.

Other Responses: Other utilities were contacted to discuss the program, but detailed discussions could not be scheduled due to other priorities at the utilities involving the operation of their units. Most demonstrated some interest in discussing the program at a later time, and one specifically 
indicated there was no interest in being involved in the program at this time. None of the utilities contacted indicated an interest in the sale of their BWRs to the Government at this point in time. 


\subsection{MOX Fuel Facility Preoperational Checkout and Startup Test Program}

The preoperational checkout and startup test programs utilized for the island fuel fabrication at LANL and the new MOX facility for the full MOX fabrication will be somewhat different. Since the LANL facility has been operated for fabrication of both uranium nitride and MOX fuel in the past, only new equipment items will require testing. This checkout will be accomplished prior to installation of the equipment items in the glove boxes. A qualification test program will be used to verify the process operation after equipment installation is completed. The more detailed checkout and startup programs for the new MOX facility are summarized in the following sections.

\subsubsection{Preoperational Testing}

In the time period 12 to 18 months prior to the completion of the fuel fabrication facility, equipment installation and operational testing will be performed. During this phase of activities, the equipment items, many of which have been cold tested, and the glove boxes will be installed in the completed building structure. The operations associated with each of the equipment items will be verified and any nonoperational items repaired or replaced. The glove boxes will be connected to the ventilation system and the flow patterns verified. Subsystem level equipment for movement of material and process control will be checked out. The construction activities will be scheduled so that individual processing areas (i.e., plutonia feed receiving, MOX fabrication) will be completed in a sequential fashion allowing minimization of the preoperational staff. A schedule of major activities is provided in Figure 4.2-1.

\subsubsection{Startup Testing}

The startup testing and process qualification activicies will be completed during the final six months of preoperational testing and the following six months which will lead up to full-scale operation. Depleted or natural urania will be utilized to evaluate the operation of each equipment item and verify the design capacities. Key processes such as blending and sintering will require special verifications with mixed oxide prior to release for full scale fuel fabrication. The first batch(s) of MOX fabricated will be qualification lots to verify the operation of each equipment item. If the lot(s) are acceptable, they will be utilized as fuel for the first reactor core initial loading. A schedule for the activities associated with the startup is also shown in Figure 4.2-1. 
NEDO-32361

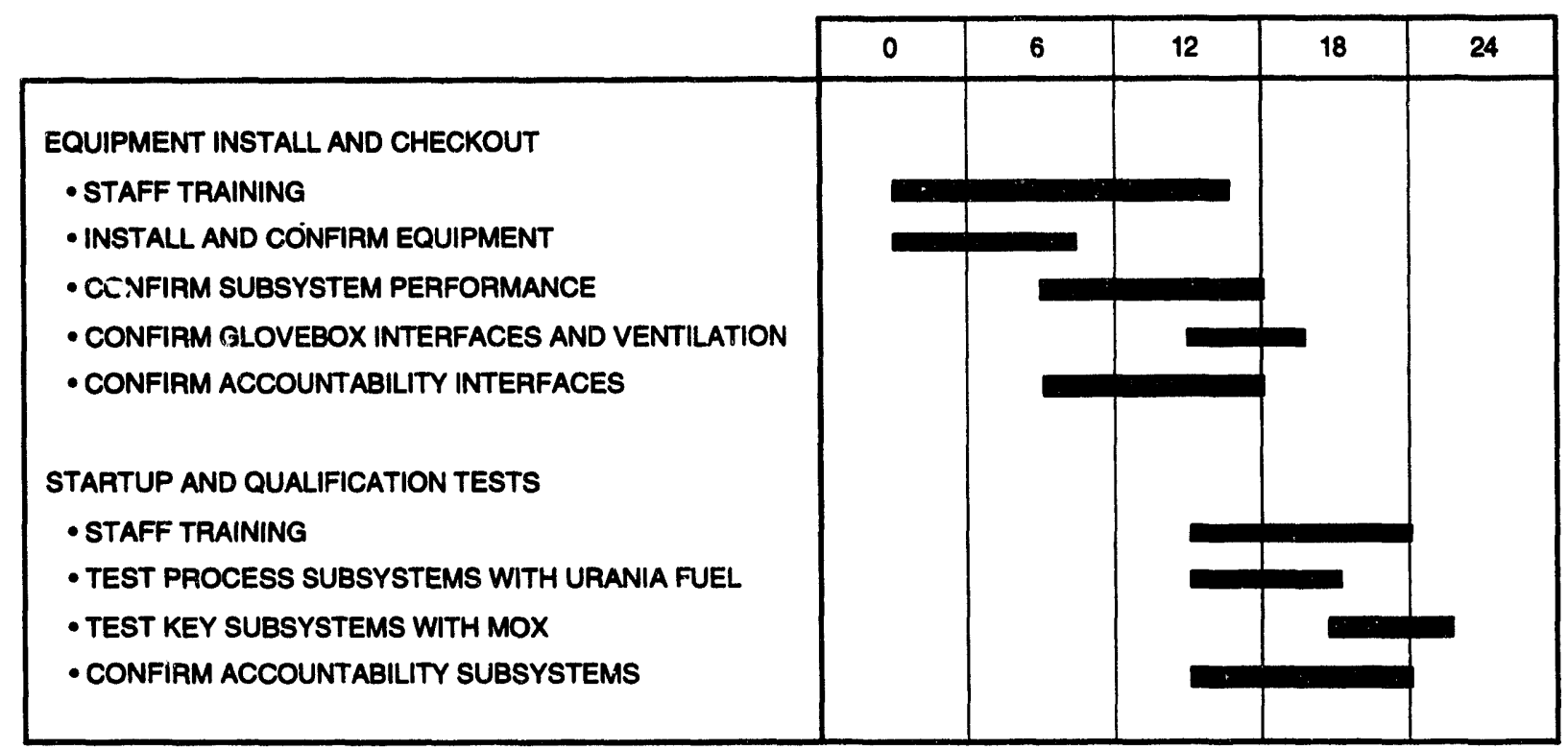

Figure 4.2-1 Fuel Fabrication Facility Startup Schedule Prior to Production (mo) 


\subsection{Initial Startup and Checkout}

\subsubsection{Operating Plant Cycle and Outage}

BWR plants operate at power for 12 to 18 months, then shutdown to conduct a refueling outage. The outage lasts from 2 to 4 months and allows time for refueling the reactor, plant maintenance, equipment inspections, and modifications. Testing is performed after any system or equipment maintenance or modifications to verify proper design characteristics and operation. Most testing is done prior to reloading fuel in the reactor and hydrostatic testing of the reactor vessel. Testing which requires reactor operation is done at predetermined power levels during ascension to full power.

Outage work on systems that support core reload are completed first. These systems will support all technical specifications, nuclear safety requirements and operational needs that will allow the fuel both exposed and new to be safely transferred from the fuel storage pool back to the correct location in the reactor core for power operation. These systems provide a variety of functions such as decay removal, vessel water clarity, reactivity control, radioactivity containment, monitoring core neutron activity, personnel radiation exposure, and coolant temperature and pressure. Reloading the core follows some general steps to achieve all nuclear safety requirements and stay within technical specification limitations. First, the once exposed fuel is removed (most reactive). Next, the remaining exposed fuel is rearranged. Then the new fuel (MOX reload) is inserted while the required shutdown margin is maintained according to technical specifications. The partial reloading is verified and the once exposed fuel is reloaded. A full core verification is done prior to reinstalling the reactor vessel head. This involves video taping the core load to document the reload using an underwater camera to read the identification numbers of the reloaded bundles. The verification of each bundle's orientation and serial number is performed during the video taping.

Control rod drive testing is done after moving fuel above the core to verify that the reloaded bundles do not impede control blade motion. It is also important to test the operability of any control rod drive that has been replaced.

\subsubsection{Power Ascension}

Power ascension involves a controlled startup of the reactor with deliberate checks and tests to verify that the reactor is responding to operator control as it was designed in a consistent and safe manner. The use of MOX fuel that is designed to perform within the existing license will not 
require any additional testing. The following tests are typically performed during power ascension and are described below:

- Reactivity Anomaly Demonstration: This is performed to determine if the core loading, control rod system, and other reactivity control and monitoring systems are correctly configured to provide the expected nuclear response to operation of the core. This is accomplished by recording the control positions when criticality is achieved along with plant conditions and making comparisons with historical data with the same operating conditions and overall trends over the operating cycle. A significant deviation from historical data or trends will indicate that a problem exists and needs to be investigated.

- Shutdown Margin Calculation: This is performed to determine that the core loading configuration will have adequate shutdown reactivity insertion using only the Control Rod Drive System and assuming a system failure of the strongest worth rod.

The following Neutron Monitoring Systems are checked for proper indication and response and to ensure that the correct signals are provided to the safety system for protective trip functions to prevent operating limits for neutron level or power from being exceeded given the operating conditions of the core:

- Source Range Monitor Operability

- Intermediate Range Monitor Operability

- Local Power Range Monitor Calibration

- Average Power Range Monitor Calibration

- Process Computer Verification: With a new reload of fuel, a new data bank is loaded in the process computer. The process computer runs programs that check important aspects of the core's performance throughout the operating cycle. The new data bank must be checked for proper program execution and correct results compared to previous results obtained with the program.

- Core Flow Calibration and Data Acquisition of Recirculation System Drive Flow versus Total Core Flow Correlation: These tests establish a correct indication and input from instrumentation monitoring the performance of the recirculation system and core flow. The determination of core performance depends upon accurate and correct core flow information. Drive flow which is generated by recirculation pumps is a fraction of total core flow and can 
be correlated to total flow. This correlation allows drive flow be used as measurement of total flow.

- Thermal Limits Determination: These are specific programs run by the process computer to evaluate the core during the power operating cycle. The programs obtain core performance data on void fraction, fuel bundle and segment power, fuel exposure, gross power distribution, operating status of Local Power Range Monitors (LPRM), and fuel thermal limits.

- Rod Pattern Control Operability: This test checks that the rod pattern controller is providing rod blocks upon violating of the programmed sequence for rod withdrawal during a startup. It is required to mitigate the consequences of a rod drop reactivity addition event at low power levels.

After power ascension testing is completed, the plant will normally operate at full power for much of its cycle. Surveillance testing and core performance monitoring programs will be done regularly to check that required safety systems and instrumentation are working properly and that the core is being operated for optimum performance and safety. Power operation with MOX fuel is expected to fit easily into the current operating routine and schedule of the utility plant staff. 


\subsection{Related Operating Experience on Availability and Capacity Factor}

Availability and capacity factor are performance measurements which can be used as a basis for setting goals and determining incentives which is done by some state utility rate regulators. Averages for U.S. BWRs from 1989 through 1993 are shown on Figure 4.4-1. More than a decade's worth of operating experience has been accumulated on most plants being considered for the mission. Various factors influence or affect plant operations and can be categorized into four basic areas: (1) systems and equipment, (2) design, (3) procedures and controls, and (4) human factors. An example of a system problem: a fuel defect occurs raising offgas activity and requiring power to be reduced. The derating in plant output reduces capacity factor. An example of a human factor problem: during maintenance on a control system which can initiate a reactor scram, an operator error is made which triggers the scram and shuts the plant down, reducing plant availability and capacity factor. The enforcement actions of regulatory agencies such as the NRC can also affect operations by imposing a shutdown order on plants that violate regulations to the extent that they affect the plant license. Historically, plant of identical or similar configuration can exhibit a wide disparity in their capacity factors. This can generally be attributed to management of and investments made in variables which contribute to higher capacity factor such as training, maintenance, planning, quality assurance, etc.

The normal plant operating cycle includes refueling outages, which are periods for conducting plant maintenance, modifications, and refueling the reactor. Outage lengths vary due to changes in work scope from outage to outage which directly affect availability and capacity factors. Operations are normally done at full power for long periods of time to provide base load capacity to the utilities customers. Some plants may choose to load follow (change power with demand on the grid ) which is reflected in lower capacity factor. In this instance, any favorable cost advantages provided under the plutonium disposition mission could simulate more demand, which can improve future capacity factor performance. The changes and impacts due to the use of MOX fuel do not affect critical path activities in plant operations and therefore do not impact availability or capacity factor. 
NEDO-32361

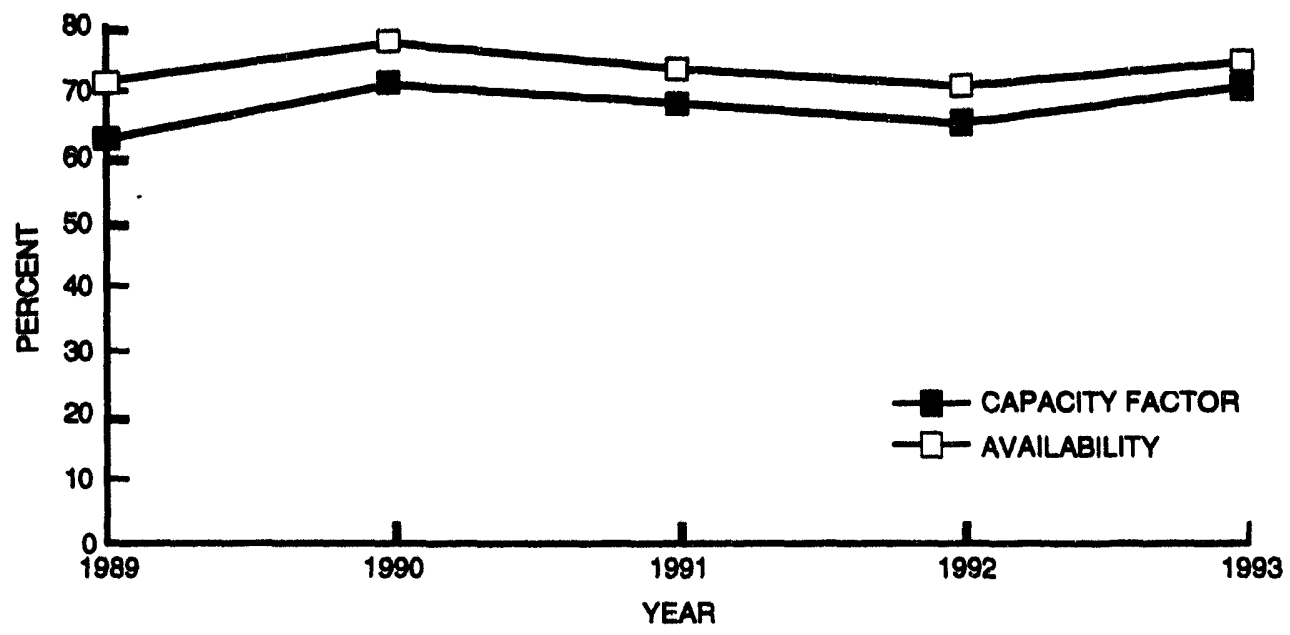

Figure 4.4-1 U.S. BWR Fleet Performance Averages 


\subsection{BWR Reactor Complex Safety and Licensing}

This study includes a review of the requirements and procedures for obtaining safety approvals for the facilities supporting the plutonium disposition program. The licensing activities in the report include the existing BWR reactor plant, new and spent fuel storage facilities, MOX fuel, and the MOX fuel fabrication facility. The licensing and license amendment processes are discussed along with the fundamental technical issues relative to the nuclear safety and licensing necessary for successful implementation of this project. The study concludes that there are no unresolvable issues that would impede the safety approval of the facilities for plutonium disposition. The evaluation that form the bases for these conclusion are discussed in the following subsections.

Integrated licensing planning with the NRC, DOE and other participating organizations in the plutonium disposition program will be initiated early in the proposed schedule. The purpose of these meetings is to establish the licensing process and schedule to be followed for all components of the project which require a license or license modification. Since all issues are unlikely to be resolved in one meeting, it is expected that this planning process will be achieved in a series of gatherings. By obtaining early concurrence on the licensing plan among the involved members, expectations will be set, and the licensing activities can proceed accordingly.

\subsection{BWR Transient and Accident Analysis}

The Final Safety Analysis Report (FSAR) of an operating nuclear power plant contains a chapter on transient and accident analysis evaluated for the plant. This chapter investigates two groups of events pertinent to safe operation of the plant: (1) anticipated operational transients, and (2) accidents.

Anticipated operational transients (also referred to as abnormal operational occurrences) are the result of postulated malfunctions of a single piece of equipment or control device or a single operator error assumed to occur during normal and planned modes of plant operation. The spectrum of anticipated operational transients evaluated represents those which tend to challenge the fuel design limits and those which challenge the reactor coolant pressure boundary (RCPB) design conditions. NRC acceptance criteria require that any radiological release from an operational transient does not exceed the concentration limits of 10CFR20.

An accident is a very low probability event for which the plant design and siting are evaluated to confirm that the radiological consequences of the accident are within the guidelines set forth in 
10CFR100. For reasons discussed in Section 5.1.2, the postulated loss-of-coolant accident (LOCA) is always the most severe accident for a light water reactor.

Although the mixed-oxide (MOX) fuel may change the results of the existing transient and accident analyses, it can be shown that the radiological consequences will be comparable to those resulting from uranium oxide fuel. Thus, the level of protection provided to the public health and safety is not significantly changed. A more detailed description of the transient and accident analysis is provided in Section 2.3 of this study, including a comparison of the core inventory with $\mathrm{UO}_{2}$ and a full MOX core.

\subsubsection{Transients}

The MOX fuel used in a BWR will be designed such that the challenge to the fuel cladding integrity during transients is always acceptable. This is accomplished by establishing a maximum duty level for the fuel cladding during normal steady-state operation prior to the onset of a transient. The maximum duty level for the fuel cladding is established by the Safety Limit Minimum Critical Power Ratio (SLMCPR), which prescribes that $99.9 \%$ of the fuel rods would be expected to avoid boiling transition, a condition which most severely challenges the fuel cladding integrity. The plant-unique MCPR operating limit is established by the addition of the largest reduction in the CPR (determined by consideration of all the operational transients) to the SLMCPR. Thus, if the plant operates within the plant-unique MCPR operating limit requirement, the plant will meet the $10 C F R 20$ limits during and after a transient.

It is expected that the difference between SLMCPR of uranium oxide fuel and SLMCPR of MOX fuel would be very small. This is because the design characteristics of the MOX fuel will resemble that of the current $\mathrm{UO}_{2}$ fuel. Once the SLMCPR of MOX fuel is determined, the CPR calculation is straightforward because it relates to thermal hydraulics and is dominated by plant characteristics. Thus, the plant-unique MCPR operating limit can be established for the MOX fuel. Section 2.3 evaluates limiting transients for a full MOX core and establishes that nuclear performance criteria are satisfied.

\subsubsection{Accidents}

The dose calculations for accidents are based on conservative deterministic assumptions taken from NRC Regulatory Guides and Standard Review Plans. The most severe dose from any design basis accident at the site boundary is the Design Basis LOCA. Radiological consequences are based on the source terms of Regulatory Guide 1.3, which includes an assumption that $100 \%$ of the noble gases and $25 \%$ of the iodines for a full power equilibrium core be available for 
release from the primary reactor containment. These source terms imply severe core damage, and, thus, the Regulatory Guide does not appear to give full credit for the mitigating effects of the BWR's Emergency Core Cooling Systems (ECCS), which -- with even a single failure -would significantly limit and possibly prevent fuel damage.

A change to a mixed-oxide core will result in little change to the full power equilibrium core inventory of iodine and noble gases, and the chemical composition of the iodine would not change. Little variation is expected in the required source term. This fact, combined with the knowledge that the resultant doses from accidents in the SARs of operating plants are sufficiently below 10 CFR 100 requirements, minimizes any challenges to the established limits. 


\subsection{Licensing the BWR Plant}

\subsubsection{Discussion on Reactor Plant Licensing}

\subsubsection{Applicabillty of MOX Fuel Use Under Current Operating Licenses}

Two fuel assembly designs have been developed for this study. The first is an "island" design, which is a minor variation on existing $\mathrm{UO}_{2}$ fuel designs and contains limited plutonium. This design resembles previous lead use assemblies (LUAs) and was developed to utilize the early fabrication capability available at Los Alamos National Laboratory for the production of MOX fuel rods. The second design is the full MOX bundle design, which contains a greater amount of plutonium and would be introduced into the reactor after one or more island reloads. The full MOX design maximizes the consumption of plutonium in order to accomplish the full disposition mission. A more detailed description of the fuel designs are given in Section 2.1 of this study, and the process by which these designs will be licensed is discussed in Section 5.2.3.

It is important to note that MOX fuel usage could be permitted under a BWR's current operating license, which generally contains these words: "The Commission hereby licenses [utility name], pursuant to the Act and 10CFR Part 70, to receive, possess and use at any time special nuclear material as reactor fuel, in accordance with the limitations for storage and amounts required for reactor operation, as described in the FSAR... ." Special nuclear material, according to the definitions given in \$70.4 of 10CFR70 Domestic Licensing of Special Nuciear Material, includes plutonium. Therefore, it could be concluded that any nuclear power plant with a valid operating license is permitted to utilize MOX fuel.

Although this clause generically allows the use of MOX fuel, consideration must be given to the specific impact of the fuel design on other licensing bases and issues of the reactor plant. If there are significant variations to the plant's current safety analysis, operating procedures, administrative or security plans as described in the FSAR, then additional requirements must be met to allow for the use of such a fuel. Therefore, the impact of each MOX bundle design on the BWR system must be evaluated. The resulting changes, if any, to such items as the power-flow map and technical specifications of a plant determines the process through which the current operating license of an existing BWR will permit the use of MOX fuel. These impact and processes are described in the following sections. 


\subsubsection{Evaluating Changes to the Reactor Plant}

\subsection{Impact of MOX Fuel Bundle Designs}

As indicated previously, the island fuel bundle design is intended to be an interim step between the start of a plutonium disposition program and a full MOX core loading of the selected BWRs. The island design allows more immediate demonstration of plutonium disposition in these reactors, as well as the opportunity for personnel training, while the capabilities to fabricate a full MOX core is developed and the full MOX fuel design and analysis are reviewed.

The island design was developed to have no significant impact on BWR operation. Its low plutonium content means that the reactor's performance is dominated by the urania fuel. Accordingly, the core characteristics and, thus the transient and accident results, are similar to those resulting from $\mathrm{UO}_{2}$ fuel. No changes to the plant-unique technical specifications appear to be required.

The full MOX fuel bundle design, however, has greater impact on the reactor plant than the island design due to its increased plutonium content. The principal difference between the full mixed-oxide core described in this study and current uranium cores is the degree to which the composition of the fuel contains various plutonium isotopes. The introduction of the full MOX bundle design to a BWR will modify several aspect of the reactor's current operation, including the power-flow map.

In the case of both MOX bundle designs, the plutonium content of the fuel and the consequent concern for diversion of the material requires greater attention to other non-technical aspects of the plant. In particular, additional precautions and protection against sabotage in the handling and storage of MOX fuel will be necessary.

\subsection{Review of BWR Plant FSAR}

The safety analysis topics contained in the plant's FSAR provide the licensing basis for the BWR plant. Any change to the plant, such as a new fuel bundle design, necessitates an examination of this licensing basis with respect to the proposed change. The specific areas that are most likely to be affected by BWR operation with MOX fuel are listed below:

- Irradiated fuel source terms

- New fuel storage 
- Spent fuel storage design basis

- Protection against sabotage

- New fuel handling

Such a review would determine if the proposed change results in an unreviewed safety question or changes to the plant's technical specifications. Discussion of the new fuel vault and dry spent fuel storage is included in Sections 5.2.4 and 5.2.5, respectively, and issues of safeguards and security are discussed in Section 7 of this study.

\subsection{CFR50.59 Process}

If no changes to the plant's technical specifications are necessary and no unreviewed safety question is involved, then the proposed change in fuel design can be incorporated in the operating license through the process defined in 10CFR50.59. This allows changes to be made in the facility as it is described in the FSAR without prior NRC approval, provided the conditions specified in $\mathbf{5 0 . 5 9}$ are satisfied. The licensee is required simply to submit a written report describing the change, as well as a safety evaluation report which provides the basis for determining that the change does not involve an unreviewed safety question.

\subsection{License Amendment Process}

If it is determined that the change will result in an unreviewed safety question or a change in the technical specifications, then the licensee is required to amend the operating license according to the procedures of 10CFR50.90-50.92. This process is well-known by utilities because of the frequency with which amendments are made to a reactor's operating license. Hundreds of amendment applications are submitted to the NRC each year.

To amend a reactor's operating license, the licensee files an application with the NRC which describes and justifies the proposed changes to the technical specifications or safety analysis. This description includes design changes and the re-analysis resulting from the proposed change. The amendment application is also accompanied by a safety evaluation concerning the issue of no significant hazards considerations under the standards of 10CFR50.92. Section 5.2.1.3 discusses the regulations and regulatory guidance that provides the criteria with which the changes and re-analysis must comply.

After the proposed amendment is filed, the NRC reviews the application and prepares a draft safety evaluation report (SER). This process generally involves a question and answer exchange 
between the NRC and the licensee. The NRC then issues a notice of proposed action that contains the staff's proposed determination and a brief description of the amendment and the facility involved. The staff also solicits public comments, providing at least 30 days for this comment period. Only if the NRC determines that a significant hazards consideration is involved and a request is made by an affected party will the Commission provide an opportunity for a hearing prior to the issuance of the license amendment. Once the NRC concludes that there is no significant hazards consideration, it issues its final determination and final SER. The amendment is effective immediately upon issuance.

If there has been sufficient public involvement at DOE's programmatic and site-specific EIS stage of the plutonium disposition project, then only minor public intervention would be expected for a MOX fuel design amendment.

\subsection{Application of Existing Processes to MOX Fuel Designs}

While a complete evaluation of the items of the plant's FSAR applicable to a change in fuel design is not possible in this phase of the study, this study determined that the impact of the full MOX bundle design will probably result in some minor changes to a plant's safety analysis and technical specifications. Consequently, a licensee would amend the BWR's current operating license in accordance with 10CFR50.90-50.92, as described above. Although it appears that the island design does not require any changes to the plant's technical specifications, an unequivocal determination of no unreviewed safety question cannot be made at this point. When there is doubt as to whether or not a change in a reactor plant involves an unreviewed safety question, a utility will often seek counsel with the NRC. Similarly, the Integrated Licensing Planning Meetings described in the introduction to this section provides the means for settling such an issue upfront. This joint NRC, licensee, and contractor licensing planning effort would determine specifically the type, extent, and schedule of licensing for both the island and full MOX fuel designs. The BWR licensee could then proceed with the appropriate process for incorporating the change in fuel design into his/her operating license.

\subsubsection{Regulatory Rules and Guidance to be Reviewed due to MOX Usage}

The main regulatory rules and guidance used by an operating license applicant for a nuclear power plant are:

- 10 CFR20

- 10CFR50 and its appendices 
- 10CFR100 and Appendix A

- Regulatory Guides

- NUREG-0800 Standard Review Plan (SRP)

- NUREG-0933 Prioritization of Generic Safety Issues

These regulations should be reviewed to assure that a MOX-fueled BWR will remain within all established criteria for safe reactor operation. Some regulations may be found to inadequately provide for appropriate methods and acceptance criteria for BWR operating with MOX fuel. If so, new criteria can be provided by the NRC, or alternative methods for meeting existing criteria can be suggested by the licensee and provided as part of the licensing process. This would include justification that the modification meets the intent of the original NRC requirement. A general discussion of the regulations and regulatory guidance listed above follows.

\section{- Code of Federal Regulations and Regulatory Guides}

10 CFR Parts 20,50 and 100 and their appendices constitute the bulk of the NRC requirements for an applicant to gain an NRC nuclear power plant construction permit and operating license. These regulatory rules must be strictly adhered to by a licensee. The Regulatory Guides provide guidance and specific interpretation for implementing parts of the NRC's rules, including the General Design Criteria (GDC) for Nuclear Power Plants in Appendix A of 10CFR50.

\section{- Standard Review Plan (SRP)}

The Standard Review Plan (SRP) in NUREG-0800 provides technical review guidance to the NRC staff who perform safety reviews of applications to construct and operate nuclear power plants. In general, SRPs were issued by the NRC to improve the quality and uniformity of the NRC staff reviews, and to present a well-defined base from which to evaluate proposed changes to the scope and requirements of reviews. The SRP is written to cover a variety of site conditions and plant designs and is revised as the need arises. Sections of the SRP follow closely with the standard format of the SAR, which is detailed in Regulatory Guide 1.70, and provide the NRC review procedure and acceptance criteria for all areas of review pertinent to that section. It is possible that the NRC staff may elect to revise some sections of the SRP to accommodate MOX fuel usage. If this is done, the licensee would need to address these staff concerns. 


\section{- Prioritization of Generic Safety Issues (GSI)}

NUREG-0933, A Prioritization of Generic Safety Issues (GSI), is a publication of the NRC that prioritizes some of the known nuclear power plant related issues. This includes the Three Mile Island (TMI) issues and the new generic issues which consist of those on the old USI (unresolved safety issues) and GSI lists. There are several issues in NUREG-0933 that may be impacted by MOX fuel usage. These have to do with new fuel handling, personnel training, and risk assessment. Also, the subject of turbine or tornado missile onto the new fuel handling and storage areas should be addressed. The stability issue for the current operating plants has not been completely closed, but is being resolved. This issue would be similar for MOX-fueled BWRs.

\subsubsection{Plant License Renewal Considerations}

\subsubsection{Overview}

One of the factors that may impact the plant selection process for the plutonium disposition mission is the amount of time remaining on the initial 40-year licensed life of the plant. Part of the selection process will eliminate those plants that have insufficient remaining license life to perform the plutonium disposition in support of the DOE mission objectives. The ability to renew a reactor's operating license could justify the selection of certain BWR plants that would otherwise be eliminated.

Although the Atomic Energy Act of 1954 limits a nuclear power plant's license to a maximum of 40 years, it also permits the renewal of an operating license. However, it does not outline any standards or procedures for determining when or under what conditions a plant's operating license should be renewed. In response to this lack of criteria and a clear process, the NRC proposed a rule in July 1990 that was intended to allow the extension a reactor's license beyond 40 years of operation. The final rule - 10CFR54 - was issued in December 1991.

However, industry experience with 10CFR54 so far is that the requirements for life extension are still not clearly defined by this rule. Although a few nuclear plants have pursued studies on life extension, no lead plants have yet submitted applications for license renewal. While some lead plants (e.g., Northern States Power Co's Monticello, the BWR lead plant) have expended considerable time and resources on license renewal feasibility studies, nuclear utilities have been reluctant to submit license renewal applications because of the ongoing uncertainty surrounding the 10CFR54 process. The NRC commissioners have directed the Staff to revise and/or clarify the requirements of 10CFR54 by the end of 1994. The revised license renewal rule is expected 
to make the process of applying for plant life extension simple, cost effective, and predictable. The expectation is that the license renewal granted by the NRC would be for a period of 20 years. Additionally, it is anticipated that the NRC's revised license renewal rule will rely heavily on the maintenance rule (which is scheduled to take effect in July 1996) as a way for utilities to handle most aging issues.

The above expected changes and clarification of the requirements specified by 10CFR54 would potentially open the door to utilities seeking license renewal. If the guidelines are clarified, it may be a reasonable assumption that a plutonium disposition selectee could initiate and complete the license renewal process and have a license renewal in place within a five-year time frame. Prioritization of the plutonium disposition program could potentially improve this time.

\subsubsection{Plant License Recovery Variation}

With the uncertainty of license extension availability, there is an optional approach available to Tennessee Valley Authority's (TVA) Browns Ferry units that would extend their current license life and provide sufficient added mission time to allow the three units to meet the plutonium disposition goal of 50 MT. This program, known as Out of Service Time Recovery Program, is currently under evaluation by TVA for implementation on Browns Ferry 1, 2, and 3. The basis of the program is to recover the time associated with the extended shutdown of the Browns Ferry units on the operating licenses, adding approximately 13,8 , and 10 years to the Unit 1, 2 and 3 operating licenses, respectively. The program is considered both technically and legally feasible, based on the existing precedence of several operating plants recovering the elapsed time between receipt of their operating license and first power operations. In recent weeks, such a program was approved by the NRC for implementation at Turkey Point, resulting in a license time recovery associated with this plant's construction period. When considered in conjunction with the significant upgrade programs, such as the Environmental Qualification Program, required to be completed prior to the restart of any Browns Ferry unit, approval by the NRC is considered a high probability by TVA and GE. Costs to implement the program are under final development but are considered to be less than a license renewal application. 


\subsubsection{MOX Fuel Reload Licensing}

\subsubsection{MOX Fuel Reload Licensing Process}

\subsection{Background Information}

In existing commercial reactors, the licensing of new fuel designs is performed using an NRC approved process which utilizes generic acceptance criteria. A new fuel design is considered to be equivalent to a formally NRC approved design if all of the acceptance criteria are met. NRC review is limited to specific areas of noncompliance. The licensing acceptance criteria applicable to GE new fuel designs are documented in Amendment 22 to the GE Licensing Topical Report NEDE-24011-P-A, General Electric Standard Application for Reactor Fuel (GESTAR II). These criteria, which include such areas as thermal mechanical, nuclear, thermal hydraulic, stability and critical power performance, were developed for uranium fuel, so their applicability to and adequacy for approval of MOX fuel must be addressed. In a comparative study of uranium and MOX fuel (see Appendix G), it was found that the differences in material properties between the two fuel types are not significant up to $10 \%$ plutonium enrichment. Based on these similar behavioral findings and the knowledge that no individual fuel rod of the MOX fuel designs considered by this study exceeds $10 \%$ plutonium enrichment, Amendment 22 provides a satisfactory structure for validating MOX fuel.

Thus, for a plutonium disposition program utilizing GE BWRs, the Amendment 22 process for licensing the new GE9 MOX fuel will be used. It should be noted here that the currently used GE9 urania fuel design was licensed by the NRC prior to the advent of Amendment 22. The introduction of MOX to GE9 fuel can be considered a new fuel design within the context of Amendment 22 and, therefore, requires verification that the licensing criteria are still met. The discussion of the licensing acceptance criteria in Table 5.2-1 summarizes an assessment that the MOX design can be licensed under Amendment 22. The island design, which contains MOX fuel with no gadolinium, will be evaluated separately from the full MOX design in which MOX fuel rods contain gadolinium.

\subsection{Process Description}

The Amendment 22 process requires a step-by-step assessment of the new fuel design against the acceptance criteria. This sometimes involves the design, fabrication and insertion of LUAs. Once the requirements have been met and a report has been generated documenting the compliance with the Amendment 22 criteria, the NRC is notified and the fuel is presumed to be licensed. Although no formal NRC review is required, the NRC would be expected to audit the 
technical basis for the analysis. Should any of the criteria of Amendment 22 not be met for a new fuel design, that aspect must be submitted for a separate review by the NRC. Prior to fuel loading, a cycle specific reload analysis will be performed, as is routinely done for all reloads.

An evaluation of the Amendment 22 steps for introducing MOX fuel was performed and a summary of this assessment is included in Table 5.2-1. Unless otherwise indicated, the assessment applies to both island design and full MOX design.

\subsubsection{Mixed-Oxide Fuel Experience Base for BWRs}

Mixed-oxide fuel bundles have been designed utilizing the same principles outlined in this report and have undergone irradiation in a broad range of reactor environments. Some of the programs which have contributed to the BWR experience base with mixed-oxide fuel are summarized in Table 5.2-2. These programs range from those involving a few fuel rods to a full reload batch of mixed-oxide bundles at the Garigliano reactor. The Garigliano bundles were operated to exposure levels similar to those planned for the bundles designed in this study, making this MOX irradiation experience particularly relevant. The results of these irradiation programs have confirmed the high degree of interchangeability between $\mathrm{MOX}$ and $\mathrm{UO}_{2}$ bundles for operation in BWRs. Thermal performance of mixed-oxide fuels has been found to be comparable to that of $\mathrm{UO}_{2}$ fuel.

The EPRI sponsored program at Quad Cities 1 has included a substantial number of nuclear and mechanical performance measurements which provide benchmark data for verifying analytical models. Featured in the measurements are fuel rod isotopic distributions at low exposure (one cycle), nominal discharge burnup bundle rod-by-rod and axial gamma scans at cycle refueling outage to assess power/exposure distributions, and a full range of fuel rod nondestructive tests and profilometry to verify mechanical integrity. The five test assemblies of this program were operated satisfactorily for a core residence time of six years.

Based upon the design and operating experience accumulated to date on mixed-oxide fuel, it is concluded that BWRs can be safely and reliably operated with mixed-oxide bundles as well as $\mathrm{UO}_{2}$ bundles. The data and experience obtained from these programs will fulfill the LUA requirements for the island design. 
Table 5.2-1 Evaluation of Amendment 22 Criteria in Regard to MOX Fuel

\begin{tabular}{|c|c|}
\hline Amendment 22 Criteria & Evaluation In Regard to MOX Fuel \\
\hline General Criteria & 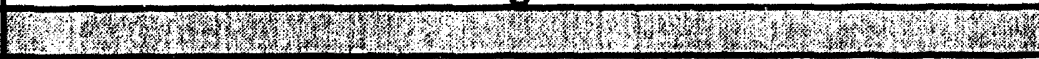 \\
\hline NRC approved models & $\begin{array}{l}\text { Fine-tuning to nuclear methods for MOX-Gd fuel rods of } \\
\text { full MOX design }\end{array}$ \\
\hline Lead use assemblies & $\begin{array}{l}\text { Planned for full MOX; previous experience applies to island } \\
\text { design. }\end{array}$ \\
\hline $\begin{array}{l}\text { Post-irradiation fuel } \\
\text { examinations }\end{array}$ & Will be included in LUA program for full MOX \\
\hline $\begin{array}{l}\text { New fuel-related licensing } \\
\text { issues }\end{array}$ & $\begin{array}{l}\text { Pu content restricted to }<10 \% \text {; existing criteria/limits remain } \\
\text { applicable }\end{array}$ \\
\hline NRC separate review & NRC independently reviews findings of above criteria \\
\hline $\begin{array}{l}\text { Fuel rod thermal-mechanical } \\
\text { design }\end{array}$ & Limits will be verified by analytical models and LUA \\
\hline Fretting & Unaffected by use of MOX; met by GE9 design \\
\hline Metal thinning & Unaffected by use of MOX; met by GE9 design \\
\hline $\begin{array}{l}\text { Fuel rod internal hydrogen } \\
\text { content }\end{array}$ & Compliance will be demonstrated by LUA for full MOX \\
\hline Fuel rod channel bow & Unaffected by use of MOX; met by GE9 design \\
\hline Control rod insertion & Unaffected by use of MOX; met by GE9 design \\
\hline Cladding collapse & Unaffected by use of MOX; met by GE9 design \\
\hline Nuclear & 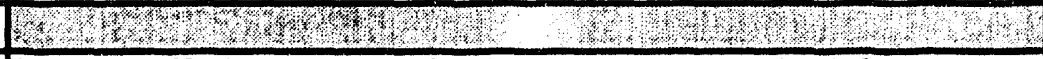 \\
\hline Doppler reactivity coeff. & Neg. coeff. demonstrated using approved methodology \\
\hline Moderator void coefficient & Neg. coeff. demonstrated using approved methodology \\
\hline $\begin{array}{l}\text { Moderator temperature } \\
\text { coefficient }\end{array}$ & Neg. coeff. demonstrated using approved methodology \\
\hline Prompt reactivity feedback & Neg. coeff. demonstrated using approved methodology \\
\hline Power coefficient & Neg. coeff. demonstrated using approved methodology \\
\hline Cold shutdown margin & Has been demonstrated using approved methodology \\
\hline Fuel storage & $\begin{array}{l}\text { All requirements will be met; detailed analysis performed } \\
\text { later }\end{array}$ \\
\hline New fuel licensing evaluation & $\begin{array}{l}\text { Compliance analysis will be performed when cycle-specific } \\
\text { data is available; no problems anticipated }\end{array}$ \\
\hline Flow pressure drop characteristics & $\begin{array}{l}\text { Unaffected by use of MOX; will be included in cycle- } \\
\text { specific analysis }\end{array}$ \\
\hline Safety Limit MCPR & No change expected due to use of MOX \\
\hline $\begin{array}{l}\text { Operating limit MCPR licensing } \\
\text { evaluation }\end{array}$ & Cycle-specific analysis will be conducted later \\
\hline Critical Power Correlations & 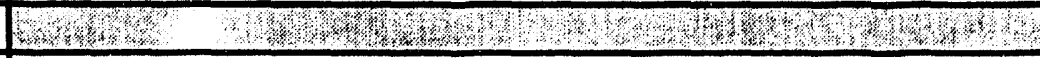 \\
\hline New fuel design features & Unaffected by use of MOX \\
\hline New correlation data & Unaffected by use of MOX \\
\hline $\begin{array}{l}\text { Critical power correlation } \\
\text { calculation }\end{array}$ & Not applicable \\
\hline
\end{tabular}


NEDO-32361

Table 5.2-1 Evaluation of Amendment 22 Criteria in Regard to MOX Fuel (Continued)

\begin{tabular}{|c|c|}
\hline Amendment 22 Criteria & Evaluation In Regard to $M 0 X$ Fuel \\
\hline Stability Licensing Acceptance Criteria & 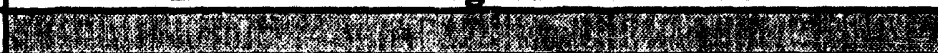 \\
\hline $\begin{array}{l}\text { Comparison with previous } \\
\text { designs }\end{array}$ & Stability analysis has been conducted \\
\hline Overpressure protection analysis & $\begin{array}{l}\text { Bounding analysis performed; cycle-specific analyses } \\
\text { carried out when data is available }\end{array}$ \\
\hline LOCA Analysis & 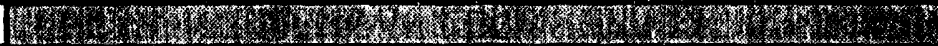 \\
\hline ECCS Criteria & Compliance has been demonstrated \\
\hline Plant MAPLHGR & Adjustment factors will be re-evaluated \\
\hline $\begin{array}{l}\text { Rod drop accident analysis licensing } \\
\text { evaluation }\end{array}$ & $\begin{array}{l}\text { Plants not following generically approved CRDA } \\
\text { withdrawal sequence will have cycle-specific analysis } \\
\text { performed }\end{array}$ \\
\hline Refueling Accident & $\begin{array}{l}\text { Consequences similar to urania fuel; detailed analysis } \\
\text { will be done }\end{array}$ \\
\hline Anticipated Tra & 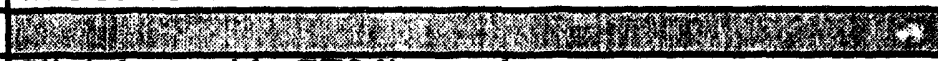 \\
\hline Void reactivity coefficient range & Slightly outside GE9 licensed range \\
\hline Plant evaluation & S criteria \\
\hline
\end{tabular}

\subsubsection{New Fuel Vault}

As previously discussed in Section 5.2.1, operating reactors have incorporated into the operating license a provision pursuant to part 10CFR70 which permits them to possess (store) and use special nuclear materiai as reactor fuel in accordance with the FSAR. It was this license provision that enabled testing of MOX fuel in operating BWRs during the 1970's. While the existing license may have to be amended to use MOX fuel as described in Section 5.2.1, it does not appear that a separate or "special" license is required for the new fuel vault. However, the licensee would be required to address 10CFR50.59(a)(2) as to the potential for an unreviewed safety question being introduced before allowing the new MOX fuel vault to be constructed on its property. This is true whether the vault is within the existing security perimeter or nearby or even if it were constructed and owned by DOE.

As the licensee had not previously identified to the NRC the potential hazards of nearby storage of MOX fuel, it is likely that an unreviewed safety question would be identified requiring a request to amend the operating license in accordance with 10CFR50.59(c) and pursuant to 10CFR50.90.

It is important to understand that the issue is not whether the storage of new MOX fuel would represent a meaningful risk to public health and safety. 10CFR50.59(a)(2) indicates that an unreviewed safety question is introduced "if the probability for an accident or malfunction of a 
NEDO-32361

Table 5.2-2 GE BWR Mixed-Oxide Fuel Experience Base

\begin{tabular}{|c|c|c|}
\hline Program & Scope & Key Results \\
\hline $\begin{array}{l}\text { Euratom Sponsored } \\
\text { Irradiation of Pu Fuel } \\
\text { in Vallecitos BWR } \\
(1960-1966) \text {. }\end{array}$ & $\begin{array}{l}\text { Pu fuel in } 1 / 4 \text { of a fuel assembly } \\
\text { was irradiated. }\end{array}$ & $\begin{array}{l}\text { Determination of isotopic content } \\
\text { as a function of fuel burnup. }\end{array}$ \\
\hline $\begin{array}{l}\text { Plutonium Recycle } \\
\text { Test Reactor } \\
\text { (Hanford) }\end{array}$ & $\begin{array}{l}80 \text { rods of hot pressed and } 3 \text { rods } \\
\text { of cold-pressed pellets were } \\
\text { irradiated to } 11,000 \mathrm{MWd} / \mathrm{MT} \text {. }\end{array}$ & $\begin{array}{l}\text { Fuel subjected to specific powers } \\
\text { in excess of } 21 \mathrm{~kW} / \mathrm{ft} \text { to provide } \\
\text { operating experience under severe } \\
\text { environmental conditions }\end{array}$ \\
\hline $\begin{array}{l}\text { Garigliano } \mathrm{MO}_{2} \\
\text { Reload Fuel (1975- } \\
\text { 1981) }\end{array}$ & $\begin{array}{l}\text { Reload fuel batch of } 46 \mathrm{MO}_{2} \\
\text { bundles ( } 32 \mathrm{MO}_{2} \text { rods per } \\
\text { bundle). }\end{array}$ & $\begin{array}{l}\text { Bundles operated satisfactorily and } \\
\text { reached a discharge exposure of } \\
>25.000 \mathrm{MWd} / \mathrm{MT} \text {. }\end{array}$ \\
\hline $\begin{array}{l}\text { Big Rock Point-EEI } \\
\text { Programs (1969- } \\
\text { 1974) }\end{array}$ & $\begin{array}{l}\text { Irradiation of } 32 \mathrm{MO}_{2} \text { rods in } 16 \\
\mathrm{UO}_{2} \text { carrier bundles and } \\
\text { irradiation of } 3 \mathrm{MO}_{2} \text { reload } \\
\text { bundles. }\end{array}$ & $\begin{array}{l}\text { Evaluation of annular and solid } \\
\text { pellet } \mathrm{MO}_{2} \text { fuel performance } \\
\text { including destructive examina- } \\
\text { tions. Peak pellet burnups to } \\
36,000 \text { MWdMT. }\end{array}$ \\
\hline $\begin{array}{l}\text { Halden Plutonium } \\
\text { Program }\end{array}$ & $\begin{array}{l}\text { MO2 fuel rods with annular } \\
\text { pellets were irradiated at } 17 \text { to } \\
22 \mathrm{~kW} / \mathrm{ft} \text {. }\end{array}$ & $\begin{array}{l}\text { Measurements of the effects of } \\
\text { pellet-clad interaction showed } \\
\text { acceptable behavior of the } \mathrm{MO}_{2} \\
\text { rods. }\end{array}$ \\
\hline Kritz Program & $\begin{array}{l}\text { A series of hot critical } \\
\text { experiments was completed } \\
\text { including power distribution and } \\
\text { other measurements for } \\
\text { Garigliano } \mathrm{MO}_{2} \text { reload bundles. }\end{array}$ & $\begin{array}{l}\text { Benchmarking of data to analytical } \\
\text { predictions produced good } \\
\text { agreement. }\end{array}$ \\
\hline $\begin{array}{l}\text { Quad Cities 1 EPRI } \\
\text { Program (1974-1982) }\end{array}$ & $\begin{array}{l}4 \mathrm{MO}_{2} \text { bundles around the center } \\
\text { control blade and } 1 \mathrm{MO}_{2} \text { bundle } \\
\text { on the core periphery underwent } \\
\text { irradiation in a highly controlled } \\
\text { situation with extensive non- } \\
\text { destructive measurements. }\end{array}$ & $\begin{array}{l}\text { Center bundles operated } \\
\text { successfully for } 8 \text { years of core } \\
\text { residence to burnups of } 57,000 \\
\text { MWd/MT. Detailed benchmark } \\
\text { data has been obtained on local } \\
\text { power distributions, fuel isotopics } \\
\text { and annular } \mathrm{MO}_{2} \text { pellet } \\
\text { mechanical integrity. }\end{array}$ \\
\hline
\end{tabular}

different type than any evaluated previously in the safety analysis report may be created." This question contains no mention of acceptable risk. As such, the NRC has the opportunity to agree or disagree that the storage of new MOX fuel in an ex-reactor vault represents a minimal risk.

\subsubsection{Dry Spent Fuel Storage}

As discussed in Section 2.5, the NUHOMS independent spent fuel storage installation (ISFSI) was selected as the planning basis for this study, since it has already been licensed by the NRC and is in operation at several reactor sites in the U.S. The design of this system includes consideration of normal and off-normal operating conditions, and worst case hypothetical accidents. It is designed in accordance with the requirements of 10CFR72, as well as Regulatory 
Guide 3.54, ANSIANS 57.9, Section III of the ASME Boiler and Pressure Vessel Code, ACI 349 , and other applicable specifying documents, as described in the CSAR.

A system with a Certification of Compliance - such as the NUHOMS - can be implemented under the 10CFR72 Subpart $K$ generic certification rule or under 10CFR72 Subpart $C$ site specific license. It appears that implementation under the general certification rule would be a simpler process than the site specific license. However, although the current security conditions are conservative with respect to the requirements of 10CFR72, Subpart $K$, including the new MOX fuel vault within the same secured area as the ISFSI is a departure from what was intended under the generic certification and would need prior NRC concurrence with this approach. This is another issue which could be addressed in the early integrated licensing planning described in the introduction to this section. 


\subsection{Licensing the MOX Fuel Fabrication Facility}

\subsubsection{Fuel Fabrication Facillty Safety Approval Process}

The safety approval of the design, construction and operation of the MOX fuel fabrication facility could be performed by either the DOE or the NRC. The following sections provide a brief summary of the DOE and NRC review approval processes, the applicable requirements and regulations, the experience base and other considerations involved in the selection of the approach to be used.

\subsubsection{DOE Procedures for Fuel Fabrication Facility Safety Approval}

The fuel fabrication facility could be a government-owned/contractor-operated nuclear facility. This approach would place the responsibility of operational readiness verification and startup approval upon the existing system of DOE Orders which has been used for similar nuclear facilities.

This MOX fuel fabrication facility would be designed in accordance with DOE 6430.1A, General Design Criteria, which has special sections applicable to Plutonium and Uranium Processing and Handling Facilities (Sections 1304, 1305, 1306, 1319, 1322). The Safety Analysis Report would be preparer in accordance with DOE 5480.23, Nuclear Safety Analysis Reports. General facility safety would be in accordance with DOE 5480.5, Safety of Nuclear Facilities. Training for the facility personnel would be in accordance with DOE 5480.20, Personnel Selection, Qualification, Training and Staffing Requirements at DOE Reactor and Non-Reactor Nuclear Facilities. An Operational Readiness Review (ORR) would be performed per DOE-STD-3006-93, Planning and Conduct of Operational Readiness Reviews. Startup of the facility would be authorized in accordance with DOE 5480.31, Startup and Restart of Nuclear Facilities.

DOE Order 4700.1, Project Management System, establishes the DOE project management system and provides implementing instructions, formats, and procedures. It also sets forth the principles and requirements which govern the development, approval, and execution of DOE's outlay program acquisitions as embodied in the project management system. After the decision has been made to initiate a Major System Acquisition and an Acquisition Officer has been named by the Secretary of Energy, there are four Key Decision points during the project. These Key Decisions are described below: 


\section{Key Decision \#1 - Approval of New Start}

- Input to decision process includes conceptual design report, project data sheet, justification for new start document and budget validation.

- Approval occurs in internal review budget process and is documented by the forwarding of project to OMB.

\section{Key Decision *2 - Approval to Commence Title I, or FinalDetalled Deaign}

- Scheduled prior to start of Title II, or final/detailed design.

- Approval of project plan signifies accomplishment of Key Decision 2.

- Input to decision process includes completed project plan (cost, schedule, and technical baselines).

- Approval to begin long-lead procurement, if applicajole.

\section{Key Decision \#3 - Approval to Commence Construction}

- Scheduled prior to date in approved project plan schedule for starting construction.

- Input to decision process is evidence of readiness to proceed, appropriateness of timing, and firm baseline.

- Approval of updated project plan signifies authorization to starting construction or committing major resources.

\section{Key Decision \#4 - Approval to Commence Operation/Production}

- Scheduled prior to date in approved project plan schedule for transition from project to operation/production; transition is not formally made until demonstrated capability to meet technical performance goals approved in baseline.

- Input to decision process is evidence of operational readiness.

- Approval is necessary precondition to transitioning out of the project management system.

The Key Decisions are indicated in Figure 5.3-1 along with significant project activities and other requirements that must occur during a major system acquisition process. Since the fuel is 


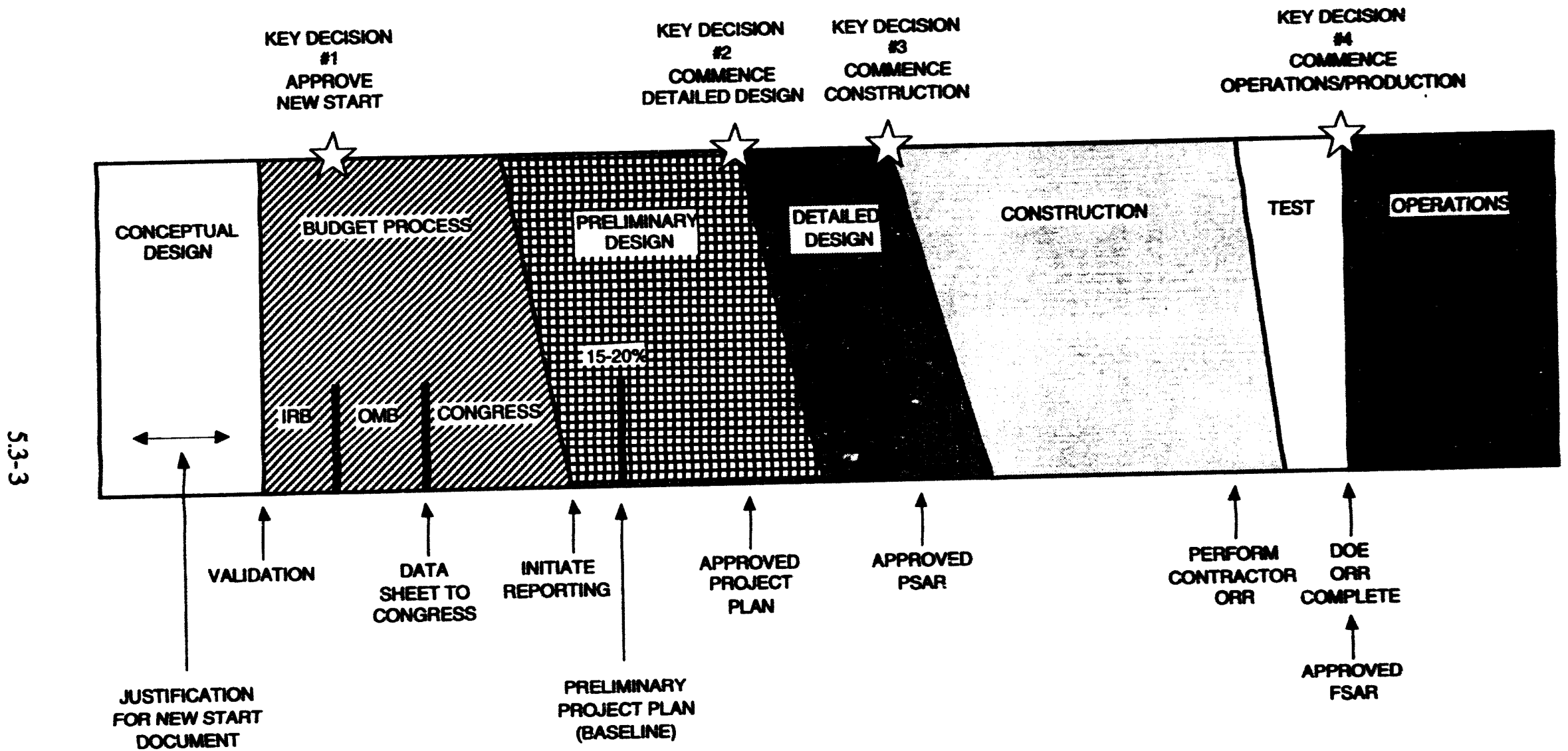

Figure 5.3-1. Key Events in the Acquisition Process 
intended for use in an NRC licensed reactor, it will be necessary to establish interfaces between DOE and NRC to provide for NRC review of the fabrication quality assurance.

\subsection{Deaign Criteria and Requirements}

DOE Order 6430.1A, General Design Criteria, provides mandatory, minimally acceptable requirements for non-reactor nuclear facility design. Criteria are included to cover a large range of facility types and uses. The MOX fuel fabrication facility falls into several of the categories, specifically as a Plutonium Processing and Handling Facility.

The basis for the selection of applicable criteria from DOE Order $6430.1 \mathrm{~A}$ is predicated by the facility type and classification, and engineering interpretation of the criteria in the GDC. Criteria selection is intended to include all potential considerations for special facilities and non-reactor nuclear facilities. The information that normally is considered and influences the criteria selection process includes DOE facility hazard classification $(1,2$, or 3; High, Moderate, or Low), DOE facility classification (whether the facility is considered critical), and DOE program classification (whether the program is considered vital).

In addition, other facility based safety-related issues influence the criteria selection process. Information and issues to be considered during the criteria selection process for the fuel fabrication facility include:

- DOE facility hazard classification (assumed High)

- DOE facility classification (assumed critical)

- DOE program classification (assumed vital)

- Safety class structures, systems, and components

- Environmental (protection) systems (process and monitoring systems) associated with routine discharges

- Industrial (worker) safety provisions

- Operational human factors considerations for safety-class related systems.

The GDC contains a wealth of facility specific criteria, both for safety class systems as well as for general building design, construction and operation. The criteria for DOE nuclear, non-reactor type facilities are listed specifically and organized by facility type in the 1300 and 
-99 series sections. The criteria in these sections, for the most part, are generally focused on the systems and components that are applicable for evaluation (based on consideration and selection of the above facility characteristics).

DOE 6430.1A and the approximately 350 referenced national codes, standards and other Orders provide sufficient design criteria and requirements to adequately design the facilities required to fabricate MOX reactor fuel. Also available for design guidance is DOE/TIC 11603, Nonreactor Nuclear Facilities: Standards and Criteria Guide, which provides a comprehensive source for identifying applicable guides, codes, and standards. Many elements of this guide were incorporated in the original DOE 6430.1 .

\subsection{Safety Analysis Report (SAR)}

Safety Analysis for nuclear facilities is currently governed by DOE Order 5480.23, Nuclear Safety Analysis Reports, which establishes and evaluates the adequacy of the safety bases of the facilities. The Nuclear SAR required by this order documents the results of the safety analysis. The safety analysis is a documented process which (1) provides systematic identification of hazards within a given DOE operation; (2) describes and analyzes the adequacy of measures taken to eliminate, control, or mitigate identified hazards; and (3) analyzes and evaluates potential accidents and their associated risks. The safety basis is the combination of information relating to the control of hazards at a nuclear facility (including design, engineering analyses, and administrative controls) upon which DOE depends for its conclusion that activities at the facility can be conducted safely.

It is the policy of the DOE that nuclear facilities and operations be analyzed to identify all hazards and potential accidents associated with the facility and the process systems, components, equipment or structures, and to establish design and operational means to mitigate these hazards and potential accidents. The results of these analyses are to be documented in the SAR. The identified hazards and the SAR are to be approved by DOE.

The contractor responsible for the design, construction, or operation of DOE nuclear facilities is required to perform a safety analysis that develops and evaluates the adequacy of the safety basis for each facility. Contractors are required to prepare and submit to DOE for approval the SARs documenting safety analyses for each DOE facility under its cognizance.

The scope and content of the SAR is described in DOE 5480.23. SARs define the safety basis, document the logic of its derivation, demonstrate adherence to the safety basis and justify its adequacy. The SAR will include thorough documentation of the assumptions employed in the 
safety analysis, as well as identify the dominant contributors to the risk of the facility so that these vulnerabilities can be better managed.

Contractors are also required to perform a hazard analysis of the nuclear activities and classify the processes, operations or activities into Categories 1, 2, or 3 based on potential for significant offsite, onsite or localized consequences respectively. The Hazard Analysis shall be based on an inventory of hazardous materials enveloping all radioactive and non-radioactive hazardous materials that are stored, utilized, or may be formed within the nuclear facility and shall identify energy sources. The Hazard Analysis shall be submitted to DOE for approval in accordance with an approved Safety Analysis Plan.

DOE Standard 3009-93, Preparation Guide for U.S. Department of Energy Nonreactor Nuclear Facility Safety Analysis Reports, provides more detailed guidance for SAR preparation, but is still in Draft form. DOE Order 5480.24, Nuclear Criticality Safety, describes the requirements of the contractor criticality safety program for nuclear facilities. It is the implementation of this program that will form the basis for the assessment in the SAR along with design and geometric controls that are implemented for the prevention of inadvertent criticality.

The SAR for new DOE nuclear facilities requires Program Secretarial Officer (PSO) approval of the PSAR prior to undertaking procurement of materials and components, construction, and preoperational testing. A PSAR documents the adequacy of the safety basis for a new nuclear facility and provide assurance that the facility can be constructed, operated, maintained, and shut down safely and in compliance with applicable laws and regulations. The contractor is required to submit the FSAR to the PSO for approval and authorization to operate the DOE nuclear facility. For new facilities, authorization will generally constitute approval of the FSAR, and DOE may document its review with an SER. DOE may decide to impose conditions of approval, which might include constraints on technical safety requirements (TSRs), or alterations to other commitments. The approved FSAR must incorporate the necessary modifications to reflect DOE-imposed conditions of authorization.

\subsection{Nuclear Facility Safety Requirements}

DOE Order 5480.5, Nuclear Facility Safety, addresses the following areas: ES\&H Program Requirements, Contractor Independent Review and Appraisal System, Personnel selection and Training, Nuclear Criticality Safety Elements, Nuclear Criticality Safety Control Parameters, and Safe Storage Criteria for Unirradiated Fissionable Material. 
NEDO-32361

\subsection{Personnel Training and Staffing Requirements}

DOE Order 5480.20, Personnel Selection, Qualification, Training, and Staffing Requirements at DOE Reactor and Non-Reactor Nuclear Facilities, establishes requirements for personnel involved in operation, maintenance, and technical support of DOE-owned Category A and B reactors and non-reactor nuclear Facilities.

DOE Order 5480.18A, Accreditation of Performance-Based Training for Category A Reactors and Nuclear Facilities, establishes a performance-based training process for reactor and non-reactor nuclear facilities.

\subsection{Operational Readiness Review (ORR)}

DOE Order 5480.31, Startup and Restart of Nuclear Facilities, establishes the requirement to conduct ORRs or Readiness Assessments (RAs) prior to startup of a new nuclear facility. DOE 5480.31 is discussed in Section 5.3.1.1.6.

DOE-STD-3006-93, Planning and Conduct of Operational Readiness Reviews, describes a consistent approach to the conduct of ORRs and RAs for new starts and restarts of DOE nuclear facilities.

\subsection{Nuclear Facility Startup Approval}

DOE Order 5480.31, Startup and Restart of Nuclear Facilities, establishes the actions to be taken and assigns the responsibilities and authorities necessary for authorizing the startup or restart of DOE nuclear facilities. It is the policy of DOE that new nuclear facilities will be started up only after documented reviews of readiness have been conducted and the approvals specified in this order have been received. The readiness review will in all cases demonstrate that it is safe to startup (or restart) the applicable facility. DOE 5480.31 provides explicit requirements for approval of the various documents and stages of the ORR that are discussed in Section 5.3.1.1.5.

For the initial startup of this facility (Hazard Category 1 or 2), the Secretary of Energy has startup authority. Final approval for facility startup cannot be granted until the FSAR has been approved by DOE Headquarters and the contractor ORR and DOE ORR have been completed and all pre-start findings have been resolved. Following completion of the DOE ORR and resolution of restart findings, DOE management will recommend to the approval authority that startup approval be granted. 


\subsubsection{NRC Procedures for Fuel Fabrication Facility Licensing}

The Regulations for NRC approval of the fuel fabrication facility have not been applied to a plutonium facility in recent years. A significant level of effort would likely be required to update them and the Regulatory Guides applicable to plutonium fabrication facilities.

The body of NRC regulations, guidance, and review criteria (sometimes termed the "regulatory base") applicable to the licensing of mixed-oxide fuel fabrication activities was going through significant change and upgrading in the late 1970's when most of the operations listed in Table 5.3-1 began to be closed or scaled back. The regulations of 10CFR70 have continued to be revised and updated regularly because Part 70 covers all nuclear fuel fabrication operations, not just those involving plutonium. Thus, it is generally agreed that the current regulations

Table 5.3-1 Mixed (Pu-U) Fuel Fabrication Facilities Licensed In the U.S.

\begin{tabular}{|l|c|l|}
\hline Facility/Location & Docket/License No. & \multicolumn{1}{|c|}{ Comments } \\
$\begin{array}{l}\text { Babcock \& Wilcox, } \\
\text { Leechburg, PA }\end{array}$ & $70-364$ & $\begin{array}{l}\text { Production scale, MOX, produced } \\
\text { FFTF driver fuel of 25\% Pu. } \\
\text { Decommissioned. }\end{array}$ \\
\hline $\begin{array}{l}\text { Babcock \& Wilcox } \\
\text { Apollo, PA }\end{array}$ & $70-135$ & $\begin{array}{l}\text { Low and High enriched U, some Pu } \\
\text { work. }\end{array}$ \\
\hline $\begin{array}{l}\text { Babcock \& Wilcox } \\
\text { Lynchburg, VA }\end{array}$ & $70-824$ & $\begin{array}{l}\text { Exxon Nuclear, } \\
\text { Richland, WA }\end{array}$ \\
\hline $\begin{array}{l}\text { Westinghouse PFDL, } \\
\text { Cheswick, PA }\end{array}$ & $70-1257$ & $\begin{array}{l}\text { Production scale, MOX, LWR Fuel } \\
\text { of about 3 - 5.5\% Pu. } \\
\text { Decommissioned. }\end{array}$ \\
\hline $\begin{array}{l}\text { Kerr-McGee, } \\
\text { Cimarron, OK }\end{array}$ & $70-1143$ & $\begin{array}{l}\text { Operated 1970-75, MOX, produced } \\
\text { FFT driver fuel of } ~ 25 \% \text { Pu. } \\
\text { Decommissioned. }\end{array}$ \\
\hline $\begin{array}{l}\text { GE Advanced Fuel } \\
\text { Laboratory, Vallecitos, CA }\end{array}$ & $\begin{array}{l}\text { Pilot scale, MOX, produced LWR } \\
\text { fuel of 10 - 25\% Pu. } \\
\text { Decommissioned. }\end{array}$ \\
\hline $\begin{array}{l}\text { Atomics International } \\
\text { NMDF, Santa Susana, CA }\end{array}$ & License No. SNM-21 & $\begin{array}{l}\text { Developinent Facility, mixed } \\
\text { carbide fuels. Decommissioned. }\end{array}$ \\
\hline $\begin{array}{l}\text { General Atomic } \\
\text { San Diego, CA }\end{array}$ & $70-734$ & \\
\hline $\begin{array}{l}\text { Nuclear Fuel Services } \\
\text { Erwin, TN }\end{array}$ & $70-143$ & $\begin{array}{l}\text { Manufactured some LWR MOX } \\
\text { fuel. Decommissioned. }\end{array}$ \\
\hline
\end{tabular}

themselves would need little or no revision to allow NRC's Office of Nuclear Materials Safety and Safeguards (NMSS) to accept an application and to issue a license for a MOX fuel fabrication operation. 
Unlike the regulations, however, the Regulatory Guides that pertain specifically to operations with plutonium have not been maintained. The Regulatory Guides provide the applicant with details of the format, content, data requirements, and technical approach that the NRC staff will view favorably. Of thirteen Division 3 (Fuels and Materials Facilities) Regulatory Guides, listed in Table 5.3-2, that deal specifically with plutonium operations, only one has been updated since 1979. Regulatory Guide 3.39, Standard Format and Content of License Applications for Plutonium Processing and Fuel Fabrication Plants, has not been updated since its initial issuance in 1976. An SRP, such as exists for power reactor license applications, has never been issued by the NRC for fuel fabrication license applications. An SRP sets forth the methods and acceptance criteria that the NRC staff will use in reviewing the application. Therefore, it can serve as a valuable guide to preparing a license submittal that will meet most of the tests and conditions likely to be placed on it by the NRC reviewers. Submitting a license application without the benefit of a current Standard Content and Format Guide and an SRP will necessitate a more involved iterative process with the NRC staff than would otherwise be expected.

\subsubsection{Status of MOX Plant Safety Approval}

While either DOE or the NRC could perform the safety review and approval of the MOX fuel fabrication facility by itself, a hybrid approach which utilizes the DOE process and requirements with support from the NRC in specific areas appears best suited for the plutonium disposition program. This conclusion is based on the following considerations.

There is no economic value for the Government to obtain an NRC license for the MOX fuel fabrication plant, and there is a potential incentive to avoid the perception that the U.S. Government is revitalizing the infrastructure required for plutonium recycle. The MOX fuel fabrication facility would have no value outside the plutonium disposition program.

While neither DOE nor the NRC has issued safety approval for a MOX fuel fabrication in many years, DOE experience in plutonium handling and safety review of its nuclear activities has continued to evolve with changes in the technology and ES\&H requirements. 
Table 5.3-2 NRC Division 3 Regulatory Guides That Specincally Address Plutonium Operations

\begin{tabular}{|l|l|c|}
\hline $\begin{array}{l}\text { Guide } \\
\text { Number }\end{array}$ & \multicolumn{1}{|c|}{ Title } & $\begin{array}{c}\text { Latest } \\
\text { Rev. Date }\end{array}$ \\
\hline 3.3 & $\begin{array}{l}\text { Quality Assurance Program Requirements for Fuel Reprocessing } \\
\text { Plants and for Plutonium Processing and Fuel Fabrication Plants }\end{array}$ & $3 / 74$ \\
\hline 3.7 & $\begin{array}{l}\text { Monitoring of Combustible Gases and Vapors in Plutonium } \\
\text { Processing and Fuel Fabrication Plants }\end{array}$ & $3 / 73$ \\
\hline 3.10 & $\begin{array}{l}\text { Liquid Waste Treatment System Design Guide for Plutonium } \\
\text { Processing and Fuel Fabrication Plants }\end{array}$ & $6 / 73$ \\
\hline 3.12 & $\begin{array}{l}\text { General Design Guide for Ventilation Systems of Plutonium } \\
\text { Processing and Fuel Fabrication Plants }\end{array}$ & $8 / 73$ \\
\hline 3.14 & $\begin{array}{l}\text { Seismic Design Classification for Plutonium Processing and Fuel } \\
\text { Fabrication Plants }\end{array}$ & $10 / 73$ \\
\hline 3.16 & $\begin{array}{l}\text { General Fire Protection guide for Plutonium Processing and Fuel } \\
\text { Fabrication Plants }\end{array}$ & $1 / 74$ \\
\hline 3.21 & $\begin{array}{l}\text { Quality Assurance Requirements for Protective Coatings Applied } \\
\text { to Fuel Reprocessing and to Plutonium Processing and Fuel } \\
\text { Fabrication Plants }\end{array}$ & $3 / 74$ \\
\hline 3.28 & $\begin{array}{l}\text { Welder Qualification for Welding in Areas of Limited } \\
\text { Accessibility Fuel Reprocessing and in Plutonium Processing and } \\
\text { Fuel Fabrication Plants }\end{array}$ & $5 / 75$ \\
\hline 3.29 & $\begin{array}{l}\text { Preheat and Interpass Temperature Control for the Welding of } \\
\text { Low-Alloy Steel for Use in Fuel Reprocessing Plants and in } \\
\text { Plutonium Processing and Fuel Fabrication Plants }\end{array}$ & $5 / 75$ \\
\hline 3.35 & $\begin{array}{l}\text { Assumptions Used for Evaluating the Potential Radiological } \\
\text { Consequences of Accidental Nuclear Criticality in a Plutonium } \\
\text { Processing and Fuel Fabrication Plant }\end{array}$ & $7 / 79$ \\
\hline 3.39 & $\begin{array}{l}\text { Standard Format and Content of License Applications for } \\
\text { Plutonium Processing and Fuel Fabrication Plants }\end{array}$ & $1 / 76$ \\
\hline $\begin{array}{l}\text { Design Basis Floods for Fuel Reprocessing Plants and for } \\
\text { Plutonium Processing and Fuel Fabrication Plants }\end{array}$ & $\begin{array}{l}\text { Nuclear Criticality Control and Safety of Homogeneous } \\
\text { Plutonium-Uranium Fuel Mixtures Outside Reactors }\end{array}$ & $7 / 81$ \\
\hline 3.40 & & \\
\hline
\end{tabular}

Since the MOX fuel would be used in NRC licensed reactors, NRC will need to assure itself that the quality of the fuel produced meets the established requirements. However, this is a product quality function which can be separated from the safety approval of the facility design, construction and operation.

It is widely perceived that public acceptance of DOE nuclear activities would be enhanced by independent external oversight, and this was the basis for the creation of the Defense Nuclear Facilities Safety Board (DNFSB) by Congress. Further, DOE has established a goal that any new DOE nuclear facilities will be "licensable" by the NRC. Implementation of this goal has usually involved incorporation of NRC safety requirements and guidance into DOE implementing documents. In the case of the Fast Flux Test Facility (FFTF), it also included 
included NRC staff reviews, formal resolution of NRC questions and public hearings involving the Advisory Council on Reactor Safeguards (ACRS). A similar approach could be implemented for the MOX fuel fabrication facility if it were considered desirable to use the NRC rather than the DNFSB for this function.

\subsubsection{Nuclear Facility Safety Approval Experience}

\subsection{Recent MOX Licensing Experience}

As discussed in Section 5.3.1.1, the MOX fuel fabrication facility would most likely be operated as a government-owned/contractor-operated facility. The fuel fabrication facility would be considered a Plutonium Processing and Handling Facility according to DOE Criteria. DOE has continuous and recent experience in approval of plutonium processing facilities. It is expected that this DOE experience and the recent licensing experience with a commercial MOX fuel plant overseas (Sellafield) would provide a technical base for the approval of the MOX fabrication facility in accordance with the requirements described in Section 5.3.1.

A summary of DOE facility design and startup experience is provided in Table 5.3-3.

\subsection{Plutonium Processing Experience in the U.S.}

At least 10 different mixed (plutonium-uranium) nuclear reactor fuel fabrication operations were licensed in the 1960's and 1970's by the NRC or its predecessor agencies under 10CFR70. Nine of the licensed operations produced oxide (MOX) fuels on a pilot or small production scale while the tenth was an experimental facility for the development of mixed carbide fuels. The nine MOX facilities were identified in Table 5.3-1. Nearly all mixed fuel development and fabrication activity was phased out in the late 1970's when plans for commercial reprocessing and recycling of plutonium produced in the U.S. nuclear power program were abandoned. While there have been many changes that have since taken place in nuclear licensing and environmental regulation in the past 20 to 30 years, there is precedent for the licensing and operation of MOX fabrication facilities in the US.

\subsection{Adapting Commercial MOX Fuel Fabrication Experience}

In Phase 1C of this study, a Hazard and Operability Study (HAZOP) was held to discuss the implications of processing plutonia derived from weapons, or "A", grade plutonium using a 
Table 5.3-3 DOE Facility Design and Startup Experience

\begin{tabular}{|l|c|c|c|c|c|c|}
\hline Name & Location & Facility Type & Design Criteria & SAR & ORRR & Date \\
\hline Grout Facility & Hanford & Solid Waste & 6430.1 & $5481.1 \mathrm{~B}$ & 5480.5 & 1990 \\
\hline $\begin{array}{l}\text { Super } \\
\text { Compactor }\end{array}$ & Rocky Flats & $\begin{array}{c}\text { Compactor } \\
\text { Module }\end{array}$ & $6430.1 \mathrm{~A}$ & (USO) & 5480.5 & 1991 \\
\hline S59Laboratory & Rocky Flats & Laboratory & (existing fac.) & $5481.1 \mathrm{~B}$ & 5480.5 & 1992 \\
\hline HBB Line & SRS & Pu Processing & DuPont Specs. & $5481.1 \mathrm{~B}$ & 5480.5 & 1992 \\
\hline RTF & SRS & Tritium & DuPont6430.1A & $5481.1 \mathrm{~B}$ & 5480.5 & 1993 \\
\hline RWMC & Idaho Falls & Solid Waste & (existing fac.) & 5480.23 & NA & 1994 \\
\hline $\begin{array}{l}\text { 242-A } \\
\text { Evaporator }\end{array}$ & Hanford & Evaporator & (existing fac.) & $5481.1 \mathrm{~B}$ & 5480.5 & 1994 \\
\hline DWPF & SRS & Vitrification & 6430.1A & $5481.1 \mathrm{~B}$ & 5480.5 & 1995 \\
\hline Pit 9 & Idaho Falls & Solid Waste & $6430.1 \mathrm{~A}$ & 5480.23 & 3006 & 1996 \\
\hline
\end{tabular}

commercial MOX fabrication process such as that currently being developed for the Sellafield MOX Plant (SMP). The SMP process is based on the conversion of civil plutonia powder to mixed-oxide fuel elements. The study concluded that the use of weapons grade plutonia either does not affect or decreases the level of requirements for most aspects of fuel fabrication. Only the instrument-based protection systems may require modification. A complete description of the study and its main findings are included in Appendix M.

\subsubsection{MOX Plant Design Safety Features}

A conceptual MOX facility has been designed for this study which incorporates numerous safety features to provide public and environmental protection from the hazards associated with nuclear material processing. The nuclear material - in this case, plutonium and uranium - is hazardous to the workers as well as the public if it finds its way into the body. The two primary paths through which plutonium or uranium can enter the body are by ingestion from airborne contamination and penetration through a broken area in the skin. However, the facility includes multiple barriers and special procedures to minimize the chance of such an occurrence. Because plutonium has a higher alpha activity, it is more toxic than uranium. Therefore, the safety features of the MOX fuel fabrication facility are designed to address this material, and consequently provide sufficient protection for uranium as well.

\section{- Facility Design}

All facilities which handle plutonium or plutonium containing materials must be designed to meet DOE Order 6430.1A, Section 1304, Plutonium Processing and Handling Facilities, and Section 1305, Plutonium Storage Facilities. This building acts as the first layer of containment for the plutonium and has been designed to prevent breach during such natural disasters as floods, earthquakes and tornadoes. 
Within the building, a series of isolation areas, or rooms for processing, provides a second barrier for contamination control. The air flow within these areas is regulated so that movement is from "clean" (uncontaminated) areas to "dirty" (contaminated) areas. As an example, air flow would move sequentially from the areas which contain bundle assembly to rod loading and then sintering. This type of flow pattern limits the spread of contamination, should it occur, and protects the workers who are located in clean areas.

A third barrier for contamination control is the glove boxes in which all nuclear material processing is accomplished. The glove boxes are maintained at a negative pressure relative to the processing rooms so that air flow is into the glove box, helping to prevent plutonium containment spread in the event of a breach. Similar to the isolation areas, the air movement within the glove boxes is also from cleanest to dirtiest areas. Absolute filters are located at the ventilation exit of each glove box as well as at a central location to capture any particulate contamination. All filter areas and glove boxes are equipped with fire detectors in order to detect a potential fire that could breach a filter system. A sand filter is utilized as a final method to capture particulate contamination prior to discharge to a stack. The stack is monitored to detect any radioactivity and maintain releases within environmental limits.

- Administrative Controls

In addition to the facility design based on the toxicity of plutonium, the MOX facility was designed to prevent a criticality accident by the utilization of administrative controls for each glove box and material control area. The allowable criticality limit is based on $45 \%$ of a critical mass to allow for a double batching error. A computerized accountability system is utilized to monitor and approve all material transfers. In many cases, in-line safeguards instrumentation verifies the concentration and weight of any nuclear material that is transferred within and between material control areas. The criticality limits for each material control area are adjusted based on the relative concentration of plutonium, as well as the potential presence of water in the material control area. The application of these criteria provide different limits for plutonium feed, mixed-oxide powder and process scrap in the recovery area.

Each of the glove boxes in the MOX facility is shielded with either lead sheet or lead glass to reduce the exposure to individual operators. Special provisions are made to provide lead impregnated gloves and glove port covers to further reduce exposure in key areas (e.g., plutonia receiving and powder blending). In processing areas where material is concentrated (e.g., blended powder storage and sintered pellet storage), localized shielding is added to 
further reduce worker exposure. Work locations are controlled to maintain individual exposure limits as low as possible below the allowable limit, currently $500 \mathrm{mRem}$ per year. 


\subsection{Environmental Impact Review Process}

\subsubsection{National Environmental Policy Act (NEPA) Requirements}

The environmental impact review process for plutonium disposition follows the requirements and guidelines of the National Environmental Policy Act (NEPA). The procedures for obtaining a project Record of Decision (ROD) and approval to proceed through the NEPA process are described in this section. The environmental impact review requires two Environmental Impact Statements (EIS) - a Programmatic EIS for the concept prepared by DOE and a site specific EIS submittal for each of the new individual facilities used for the plutonium disposition mission.

Operation of current BWRs with MOX fuel will probably not require any change to their NEPA approval. All reactors considered for this study were built after NEPA was implemented and are expected to have successfully completed the NEPA process. Therefore, most of this section will apply to a new MOX fuel fabrication facility.

The programmatic environmental impact review (PEIR) is a determination of the preferred concept for plutonium disposition. For the utilization option, it is prepared using generic information at a generic site. No actual site data is used for the assessments.

For the site-specific EIS, NEPA requires identification and assessment of impacts to the environment from all major projects proposed. In order to fulfill this requirement, the EIS collects and develops information for assessment of the impact. A variety of issues are to be addressed and characterized for determining the project impact. Among these are:

Land/geologic Resources

Air Resources and Noise

Water Resources

Land use, recreation and visual environment

Biotic Resources and Endangered Species

Cultural Resources

\section{Radiological Impacts}

Hazardous Materials

Socioeconomics

Transportation

Waste Management

Decontamination and Decommissioning

For operating BWRs and existing DOE facilities which could be adapted for the production of MOX fuel, these issues are characterized in existing EIS documentation. An outline of the areas that are typically addressed in the EIS reports are indicated in Table 5.4-1, along with an 
evaluation of areas needing further assessment. As shown, significant modification to existing EIS documentation is not anticipated. A portion of the EIS for existing DOE facilities may need modification if the "facility mission" is deemed to have changed. For operating BWRs only the impact of MOX fuel transportation and separate new fuel storage facilities to the site should require public hearings. Individual environmental assessments would be needed to document any changes in radiological impacts, non-radioactive hazardous materials, transportation and waste management. A discussion of these issues is provided in Section 8 with the conclusion that no significant environmental impacts will result from the disposition of plutonium in BWRs.

\subsubsection{Other Regulatory Requirements}

The following are the individual State and Local permits that may be needed or to be modified for the plutonium disposition program Table 5.4-2 provides a listing of permits which are typically applicable to sites considered for the mission. A brief description of each permit follows, along with a discussion of applicability to facilities.

\subsubsection{Clean Water Act Section 404/Section 10 Permit}

Generally, the U.S. Army Corps of Engineers (COE) is involved in projects when construction occurs in a waterway or wetland. Any project which has the potential to discharge dredged or fill material into the waters of the U.S. is required to obtain a permit from the COE as authorized by Section 404 of the Federal Clean Water Act (CWA). Section 404(h) of the CWA allows transfer of administration of this permit program to qualified states. There is provision for public notice and opportunities for public input in the permitting process, as the regulatory purpose of Section 404 is to balance public and private benefits and interests against resulting impact on aquatic environment.

Section 10 of the Rivers and Harbors Act authorizes COE to regulate activities and issue permits for projects involving construction in navigable waters of the U.S. after notice and opportunity of public inputs, similar to Section 404.

The time requirement for review and approval is approximately one year. This permit should not require modification for BWRs. The MOX facility may need this permit, depending on the site. 
Table 5.4-1 Environmental Report Impact Evaluation

\begin{tabular}{|c|c|c|c|}
\hline \multicolumn{2}{|c|}{ Report Section } & \multirow{2}{*}{$\begin{array}{l}\text { MOX Facllity } \\
\text { The extent of } \\
\text { onsite plutonium } \\
\text { included in the } \\
\text { existing EIS } \\
\text { should be defined. } \\
\text { No change from }\end{array}$} & \multirow[b]{2}{*}{$\begin{array}{l}\text { BWRSlte } \\
\text { No change in the } \\
\text { power production } \\
\text { purpose of the } \\
\text { facility }\end{array}$} \\
\hline $1.0^{\circ}$ & Purpose of the raclity & & \\
\hline $\begin{array}{l}2.0 \\
2.1 \\
2.2 \\
2.3 \\
2.4 \\
2.5 \\
2.6\end{array}$ & $\begin{array}{l}\text { Site and Envrronmental Interiaces } \\
\text { Geography and Demography } \\
\text { Ecology } \\
\text { Meteorology } \\
\text { Hydrology } \\
\text { Geology } \\
\text { Regional, Historic, Scenic, Cultural and Natural } \\
\text { Features }\end{array}$ & & $\begin{array}{l}\text { No change from } \\
\text { existing EIS. }\end{array}$ \\
\hline $\begin{array}{l}3.0 \\
3.1 \\
3.2 \\
3.3 \\
3.4 \\
3.5 \\
3.6 \\
3.7\end{array}$ & $\begin{array}{l}\text { Facility Description } \\
\text { External Appearance } \\
\text { Facility Systems } \\
\text { Station Water Use } \\
\text { Heat Dissipation System } \\
\text { Water Management } \\
\text { Transmission Facilities } \\
\text { Conduct of Operations }\end{array}$ & $\begin{array}{l}\text { Some } \\
\text { modification } \\
\text { needed for } \\
\text { changes in } \\
\text { existing } \\
\text { buildings. }\end{array}$ & $\begin{array}{l}\text { Some change } \\
\text { from existing EIS } \\
\text { to include } \\
\text { description of } \\
\text { new fuul storage } \\
\text { facility. }\end{array}$ \\
\hline $\begin{array}{l}4.0 \\
4.1 \\
4.2 \\
4.3 \\
4.4 \\
4.5\end{array}$ & $\begin{array}{l}\text { Dnvtronmental Ehects of Construction } \\
\text { Site Preparation and Plant construction } \\
\text { Transmission Facilities Construction } \\
\text { Resources Committed } \\
\text { Radioactivity } \\
\text { Construction Impact Control Program }\end{array}$ & $\begin{array}{l}\text { Not applicable } \\
\text { unless a new } \\
\text { facility is } \\
\text { selected. }\end{array}$ & $\begin{array}{l}\text { Not applicable } \\
\text { unless completion } \\
\text { of plant } \\
\text { construction } \\
\text { considered. } \\
\text { Construction of } \\
\text { fuel storage area } \\
\text { may need to be } \\
\text { considered. }\end{array}$ \\
\hline $\begin{array}{l}5.0 \\
5.1 \\
5.2 \\
5.3 \\
5.4 \\
5.5 \\
5.6 \\
5.7 \\
5.8\end{array}$ & $\begin{array}{l}\text { Envronmental arects or Plant Operation } \\
\text { Effects of the Heat Dissipation System } \\
\text { Radiological Impacts from Routine Operation } \\
\text { Effects of Liquid chemical and Biocide } \\
\text { discharges } \\
\text { Effects of Sanitary Waste Discharges } \\
\text { Effects of Operation of the Transmission System } \\
\text { Other Effects } \\
\text { Irretrievable Commitments of Resources } \\
\text { Decommissioning }\end{array}$ & $\begin{array}{l}\text { Some } \\
\text { modification } \\
\text { needed for } \\
\text { modified facility }\end{array}$ & $\begin{array}{l}\text { Some change } \\
\text { from existing EIS } \\
\text { to include MOX } \\
\text { fuel transport. }\end{array}$ \\
\hline
\end{tabular}


Table 5.4-1 Environmental Report Impact Evaluation (Continued)

\begin{tabular}{|c|c|c|}
\hline $\begin{array}{l}\text { Leport Section } \\
\text { 6.0 } \\
\text { Monluent and Environmental Measurement and } \\
\text { 6.1 Pre-operational Environmental Program } \\
6.2 \text { Operational Environmental Program } \\
6.3 \text { Environmental Measurement and Monitoring } \\
\text { 6rogram } \\
\text { Monitoring Data } \\
\end{array}$ & $\begin{array}{l}\text { MOX racllity } \\
\text { No change from } \\
\text { existing EIS. }\end{array}$ & $\begin{array}{l}\text { BWLSIte } \\
\text { No change trom } \\
\text { existing EIS. }\end{array}$ \\
\hline $\begin{array}{l}\text { 7.0 Environmental Ehiects of Accidents } \\
\text { 7.1 Plant Accidents Involving Radiation } \\
7.2 \text { Transportation Accidents Involving Radiation } \\
7.3 \text { Other Accidents (Chemical, fire, fuel oil etc.) }\end{array}$ & $\begin{array}{l}\text { Some } \\
\text { modification } \\
\text { needed for } \\
\text { modified facility }\end{array}$ & $\begin{array}{l}\text { Some } \\
\text { modification } \\
\text { needed for } \\
\text { transportation } \\
\text { impact }\end{array}$ \\
\hline $\begin{array}{l}\text { 8.0 Economic and social briects of the Project } \\
8.1 \text { Benefits of Operation } \\
8.2 \text { Costs of Operation }\end{array}$ & $\begin{array}{l}\text { No change from } \\
\text { existing EIS. }\end{array}$ & $\begin{array}{l}\text { No change from } \\
\text { existing EIS. }\end{array}$ \\
\hline 9.0 Alternates and sites & $\begin{array}{l}\text { Addressed in } \\
\text { PEIR }\end{array}$ & $\begin{array}{l}\text { Addressed in } \\
\text { PEIR }\end{array}$ \\
\hline 10.0 Plant Design Alternatives & $\begin{array}{l}\text { Addressed in } \\
\text { PEIR }\end{array}$ & $\begin{array}{l}\text { Addressed in } \\
\text { PEIR }\end{array}$ \\
\hline 11.0 Coot-Beneift Summary & $\begin{array}{l}\text { Addressed in } \\
\text { PEIR }\end{array}$ & $\begin{array}{l}\text { Addressed in } \\
\text { PEIR }\end{array}$ \\
\hline $\begin{array}{l}\text { 12.0 Environmenta Approvals } \\
12.1 \text { Facility environmental Operating Specifications } \\
12.2 \text { Facility Environmental Permits }\end{array}$ & See Section 3.4 .2 & See Section 5.4 .2 \\
\hline
\end{tabular}

\subsubsection{National Pollutant Discharge Elimination System (NPDES) Permit}

The NPDES Permit is administered under Section 402 of the Federal Clean Water Act (CWA) provisions promulgated by the U.S. Environmental Protection Agency (EPA). Under Section 402(b) of the Act, states can administer their own permit programs under the delegated authority from the EPA, providing a regulatory framework to enforce standards for protecting water quality.

The NPDES Permit regulates the point source discharge of pollutants into the waters of the United States. Typically, industrial discharges regulated under the NPDES program include process wastewaters, contaminated area drainage, and stormwater during construction. Criteria and standards for the NPDES permit system are described in 40CFR125. The permit application requires information on water use, wastewater flow, characteristics and disposal methods, planned treatment and improvements, stormwater treatment, plant operation, material and chemical used, and other pertinent information. Depending on project complexity, the 
Table 5.4-2 Potential Environmental Permit Requirements

\begin{tabular}{|l|}
\hline Section 404 (Clean Water Act)/ Section 10 (Rivers and Harbors ACt) Permit \\
\hline NationalPollutant Discharge Elimination System Permit (NPDES) \\
\hline Air Quality Construction Permit \\
\hline Section 401 Water Quality Certíication \\
\hline Storm water Permit \\
\hline Hazardous Air Pollutants Emission Permit (NESHAP) \\
\hline Permit for Construction/Operation of a Public Water Supply System \\
\hline Underground Storage Tank Construction Permit \\
\hline Permit to Construct Sewage Treatment Plant \\
\hline Sanitary Landfill Permit \\
\hline Radioactive Waste Transpurt Permit \\
\hline Hazardous Waste Permit (RCRA Parts A\&B) \\
\hline Notice of Construction / Alteration to FAA \\
\hline Radioactive Material Transport Permit (DOT) \\
\hline Permit to Construct Solid Waste Management System. \\
\hline Permit for Onsite Incinerator \\
\hline
\end{tabular}

processing time for an NPDES permit varies from 6-12 months. A public hearing may be required.

The agency specifies conditions in the NPDES permit on issuance, which include technologybased effluent limitations for the waste-streams as well as water-quality based limitations for the receiving water. New industrial facilities are generally required to meet best conventional technology (BCT) for conventional pollutant parameters (e.g., COD, BOD, TSS, pH, oil and grease), and best available technology (BAT) for toxins and nonconventional pollutants. The permit conditions will include monitoring requirements and provisions for additional technical requirements to check conformance.

Review of this permit would be needed for the plutonium disposition program at an operating BWR. An NPDES permit would be required for the MOX facility.

\subsubsection{Air Quality Construction Permit}

For the protection of air quality, the Environmental Protection Agency (EPA) sets air pollution standards that apply nationally through Clean Air Act (CAA) and its subsequent amendments. Additionally, state and local governments, through air pollution control districts (agency), have broad responsibilities for implementing air pollution control standards and regulations within their jurisdictional boundaries.

Each proposed new or modified air contaminant source must undergo a new source review. As part of this review, Prevention of Significant Deterioration (PSD) applicability is determined. If 
PSD review is required (generally applicable for facilities emitting more than 100 tons per year of a regulated pollutant, or as designated by the local agency), a PSD application must be submitted and a permit obtained before beginning project construction.

The air permit requires identification of all stationary sources in the facility, type and amounts of pollutants produced, and air pollution control equipment used. The permit processing time (for review and approval) ranges from 6 weeks (no PSD) to 6 months (with PSD) assuming no additional data collection. The construction permit as issued is generally in effect until the completion of construction, after which the agency maintains compliance through the issuance of operating permits.

An operating permit will not need to be obtained for operating BWRs. The MOX facility will probably have to obtain a permit for construction.

\subsubsection{Section 401 - Water Quality Certification}

A Water Quality Certification is required for a Federal License or Permit to conduct any activity that may result in a discharge into surface waters, pursuant to Section 401 of the CWA. The federal agency is provided a certification from the state that the said discharge complies with the discharge requirements of federal law and the aquatic protection requirements of state law. Generally, it takes 4-6 months to obtain the Water Quality Certification.

Activities requiring this certification include construction in navigable waters and discharge of dredged or fill materials into state waters, including wetlands. COE will be the federal agency to request this certification, the timing of which will be tied to the Corps permit application review. Public notice for the water quality certification is included with the Corps public notice.

This permit should not require modification for operating BWRs. However, a certification may be required for the MOX facility.

\subsubsection{Stormwater Permit}

The stormwater permit will be required to address the water quality concerns related to any stormwater discharges associated with industrial activities. This permit requirement implements the regulations set forth by the Environmental Protection Agency (EPA) in Section 301 and Section 402(p) of the CWA, primarily contained in 40CFR122.26, and is administered by most states under the delegated authority of the EPA. Time required to obtain a stormwater permit may vary from state to state; however, a maximum of $6-8$ months is presently estimated. 
Development of a stormwater management plan is required for the facility under this permit, to cover both construction and operation.

This permit should not require modification for the BWRs. The fuel storage facility will require review of the existing permits. The MOX facility will need to obtain this permit.

\subsubsection{Hazardous Air Pollutants Emission (NESHAP) Permit}

This permit, administered by the EPA, covers construction of any new source of radionuclides or modification to any existing source, under the National Emission Standards for Hazardous Air Pollutants (NESHAP) implemented in 40CFR61, subpart H. States retain the right to adopt or enforce standards that are more stringent than the applicable federal standards. The Subpart $H$ standard for DOE facilities specifies that activities causing radionuclide emissions should not result in an effective dose to the public greater than 10 millirems per year from airborne sources. Section 8 of this report provides an estimate of the emissions from the MOX fabrication facility and shows that this standard is easily met.

This permit would be applicable to the fuel fabrication facility to support the plutonium disposition mission.

\subsubsection{Permit for Construction/Operation of Domestic Wells}

Construction, modification, and/or expansion of any domestic water well are activities that, if undertaken for this facility, will require this permit. The permit is administered by the State Department of Health, and regulated under the State and Federal Drinking Water Act, and primary drinking water regulations as applicable.

Any monitoring wells or dewatering wells do not require a permit, although a water well record may be necessary for submittal to the agency, if dewatering wells pump more than $\mathbf{7 0}$ gallons per minute (gpm). Also, a statement to the effect that the well will be drilled by a state-certified well driller is required to be furnished. Total time required to obtain this permit is approximately 3 months.

Modification of existing permits should not be required for the BWRs. The MOX facility may need this permit if a well is drilled. 


\subsubsection{Permit for Construction/Operation of a Public Water Supply System}

As above, the legal authority for the administering of this permit are the State and Federal Drinking Water Act, and primary drinking water regulations. Construction, modification, or expansion of a public water system as well as its operation are covered by this permit.

For the facilities used for plutonium disposition, water supply systems include fire water, domestic water, demineralized water, cooling water (makeup and circulating), chilled water and heavy water. The permit application will be required to furnish complete information regarding general location plans, surface and/or ground water sources, quantity and quality of water use, water treatment plant, water distribution systems including improvements and appurtenances, design criteria and calculations.

During operation, chemical and bacteriological self-monitoring requirements are generally imposed on the facility. A surface water and ground water supply operation report form (including water quality and water production information) is required to be submitted to the agency, at a frequency determined by the nature of the system and as designated in the permit.

Modification of existing permits are not required for BWRs. A permit would only be required for the MOX facility if it required development of its own water system.

\subsubsection{Underground Storage Tank (UST) Construction Permit}

Installation of new underground storage tanks which will store regulated substances (including petroleum) are covered under this permit, the administering of which is the responsibility of the State Department of Health. The objective is primarily protection of drinking water and environmental health and safety. The legal authority is the Safe Drinking Water Act, and the activity is also regulated by the applicable State Underground Storage Tank Regulations. The entire permitting process takes 4-6 weeks.

Modification of existing permits are not required for BWRs. The MOX facility will need these permits for any new underground storage tanks.

\subsubsection{Permit to Construct Sewage Treatment Plant}

Construction of an onsite sewage treatment system for this facility, for the handling, treatment and disposal of sanitary wastewater generated at the facility rest rooms, showers and dining areas will necessitate this permit to be acquired. The applicable state departments of Health and Environmental Control, responsible for the control of water pollution, will administer this permit. 
State Pollution Control Act and applicable EPA guidelines will be the regulatory bases for this permitting process.

The application will be required to include physical site description, nature, quantity and characteristics of the waste, treatability of the waste, details of the treatment system, point of discharge and its impact on the receiving water, and other information relevant to the proposed treatment method as it relates to NPDES or other permits.

Construction permit for a sewage treatment plant typically takes 4-6 months for issuance. However, a Permit to Construct will not be issued until the NPDES permit (Section 5.4.2.2) becomes effective. A Permit to Operate must be issued by the agency prior to startup of the sewage treatment plant at the facility, and the level of operator required will be based upon the classification received in the Permit to Construct.

Modification of existing permits should not be required for BWRs. The MOX facility would need a permit if a sewage treatment plant were added as part of the facility.

\subsubsection{Sanitary Landfill Permit}

As the facility generates solid waste, both during construction and operation, onsite disposal of such waste at a new or modified sanitary landfill area will necessitate acquiring this permit. The administering authority for this permit will be the State, in accordance with the existing State Landfill Regulations. Time requirements for review and approval is approximately 6 months to a year, depending on the complexity of the issue and related factors, such as the public hearing process.

Modification of existing permits should not be required for existing BWRs. The MOX facility may need this permit. This permit applies only to non-radioactive waste.

\subsubsection{Radioactive Waste Transport Permit}

Transportation of radioactive waste from the facility offsite within and/or out of state will require this permit, as well as a 72-hour advance, written notification of such waste shipments. DOE, as the owner of the facility, may have special agreements with the State, as the administering agency of this permit, to delineate the conditions for conformance to the permit requirements. The conditions to fulfill such special agreement are considered open at this time. The time required for review and approval of this permit is postulated to be about 3 months. 
Modification of existing permits should not be required for BWRs. The MOX facility may need this permit if wastes are transported over a public road.

\subsubsection{Hazardous Waste (Resource Conservation and Recovery Act (RCRA) - Parts A and $B$ )}

Storage of any waste, designated as hazardous, for longer than 90 days onsite, as well as any treatment and/or disposal of such waste inside the boundary of the facility are activities that will require the acquisition of this permit. Wastes generated at the facility will need to be characterized at the source, and categorized as either RCRA or non-RCRA wastes. RCRA and/or potential RCRA wastes will be collected in RCRA tanks and the project will have to decide if any of the above activities (storage over 90 days, treatment, disposal) will take place onsite to trigger this permit.

The State organization responsible for solid and hazardous waste management within the state will be the administering agency for this permit, regulated under the legal authority of the State Hazardous Waste Management Act and applicable regulations.

Generally, Parts A and B applications will be reviewed within 60 days. A draft permit (or denial) is subject to a public review and comment period of $\mathbf{4 5}$ days, when a public hearing may be requested, prior to the Department's final decision.

Modification of existing permits should not be required for existing BWRs. A Part A permit will be required for the MOX facility.

\subsubsection{Notice of Construction / Alteration to FAA}

A notification will be required to the Federal Aviation Administration (FAA) for any construction or alteration in the facility at more than 200 feet in height above ground level. Existence of cooling towers (especially natural-draft type) and/or stacks(s) for exhaust emissions, if any, will have the potential to fall in this category.

The notification will be required to include the location of the said structure with respect to the nearest city/town and airport(s), its height and elevation above MSL, and other prominent terrain features in the vicinity. Unless informed otherwise, the notification must be submitted at least 30 days before the earlier of a) the start date of proposed construction/alteration, or b) the date a state construction permit is to be filed.

This permit should not be required for any facility in the plutonium disposition mission. 
NEDO-32361

\subsubsection{Radioactive Material Transport (DOT) Permit}

This permit will apply to the activity of transporting the plutonium from its storage in different parts of the country to the fuel fabrication complex. However, a special agreement between DOE and the Department of Transportation (DOT) may be made to cover this permit requirement by imposing special conditions unique to this facility for compliance during the operating phase. In that case, the review and approval time required will be expected to be minimal. This assumption will be confirmed as more information becomes available regarding this issue.

\subsubsection{Permit to Construct Solid Waste Management System}

This permit will be required in conjunction with a Sanitary Landfill Permit to cover construction or modification of any solid waste disposal unit onsite. The State Solid Waste Management Regulations will govern the requirements of this permitting process, and the administering authority for this permit will be the State Department of Health and Environmental Control.

Modification of existing permits should not be required for the BWRs. The MOX facility may require this permit if such waste management activities are involved.

\subsubsection{Permit to Operate Incinerator}

This permit is required by the applicable state air control authority and/or the EPA. The permit will be required to operate the onsite incinerators as part of the waste management programs for the MOX fuel fabrication facility.

Modification of existing permits should not be required for BWRs. This permit will be required if an incinerator is part of the MOX facility. 


\subsection{Estimated Cost and Schedule}

As discussed in other sections of this report, the overall economics of using existing plants to perform the plutonium disposition project may not be directly related to identifiable or quantifiable costs. The commercial incentives that may be required to encourage a utility to convert plants for the project may be based on considerations that are not directly related to the cost of that conversion or subsequent operation. Each plant and utility will have unique economic considerations that will have to be evaluated in arriving at compensation agreements. Therefore, the cost and cash flow estimates supplied in this report provide only a part of the information needed to evaluate the relative economics of the use of existing plants for the plutonium disposition project. Discussed in Sections 1 and 4 are some of the commercial incentive considerations and their implications on the relative economics of different options available for the plutonium disposition project.

\subsection{Summary of Life Cycle Costs}

As in the Advanced Boiling Water Reactor (ABWR) study [Ref. 6-1], it is expected that life cycle costs will be evaluated by ORNL based on cost inputs provided in this report. GE has compared the relative economics of the life cycle costs and revenues for the plutonium disposition reference cases for existing BWRs and for ABWRs. Results of that comparison show that if DOE were to make an outright purchase of three existing BWRs for the project, the maximum acquisition price that would make the economics favorable relative to building two new ABWRs would be about $900 \$ / \mathrm{kW}$ (based on revenues of $4 \mathrm{c} / \mathrm{kWh}$, real discount rate of $5 \%$, capacity factors of $75 \%$, and $1 / 94$ base date for present worth values). That price is significantly below the book values of the existing plants under consideration. GE's analysis is preliminary and the results are highly dependent on projected revenue rates, present-worth discount rates, and assumed capacity factors. Figure 6.1-1 shows the ranges of break-even capital values for the existing plants based on varying assumptions. Since the ABWR is expected to achieve higher capacity factors than existing BWRs, Figure 6.1-1 also shows the comparison if the ABWR achieves $85 \%$ capacity factor while existing BWRs are assumed to achieve $75 \%$. 

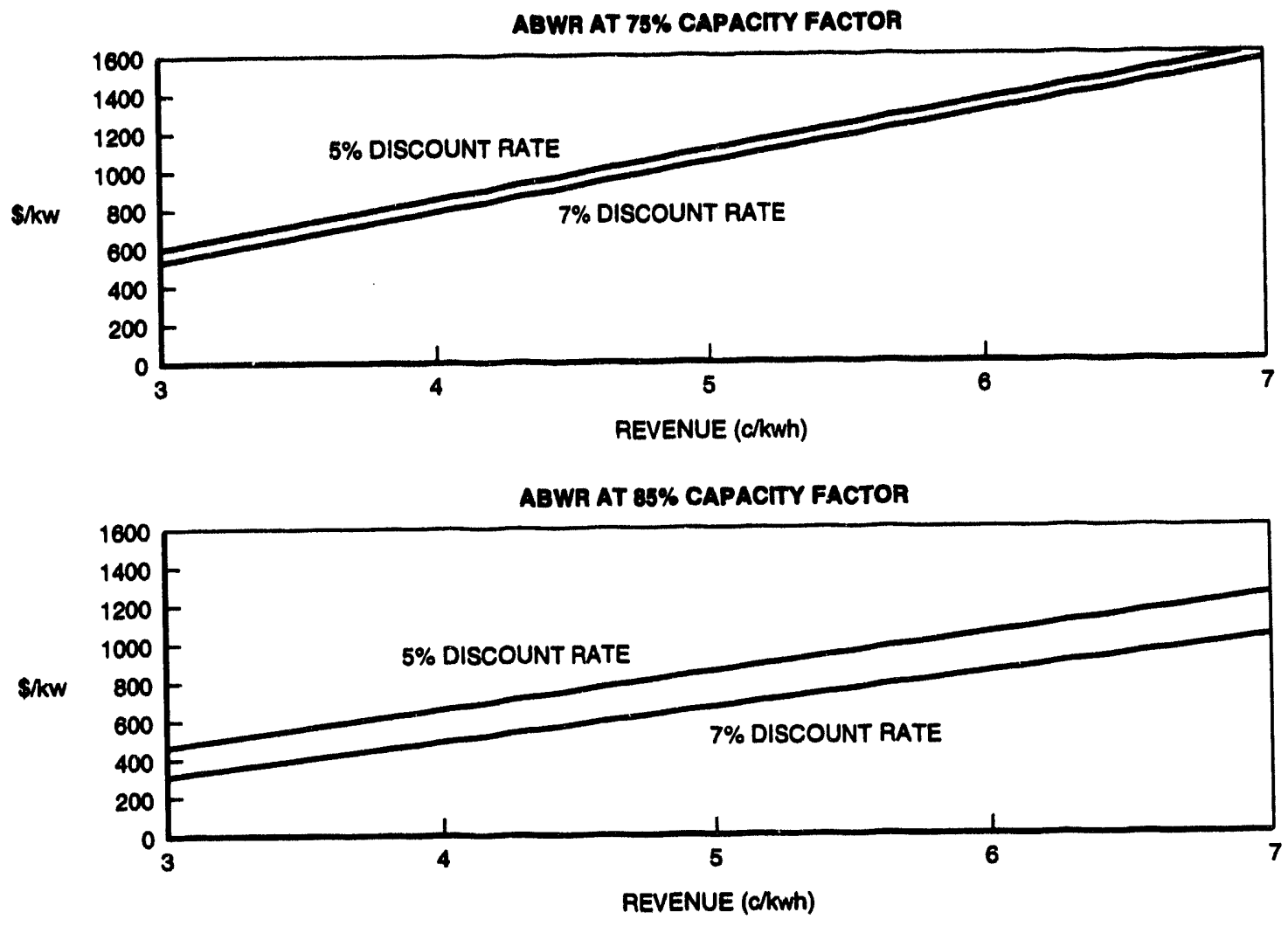

Figure 6.1-1 Break-Even Capital Value for Existing BWRs

Compared to Building New ABWRs

(\$/kw, 1/94 Present Worth) 


\subsection{Capital Cost Detall}

\subsubsection{Capital Cost Bases and Assumptions}

Each existing plant has unique combinations of design, licensing, and operating status that have unique cost considerations. This report identifies generic costs that are likely to be applicable for most of the BWR units under consideration. As agreed with DOE in the April 19, 1994 kickoff meeting, analysis of costs related to plant-unique conditions is not within the scope of this study.

The reference case plant parameters assumed for cost estimates are as described in Section 1.4. For estimating purposes, three BWR units on two sites were assumed: one site with a single BWR unit and one site with a dual BWR unit. The MOX fuel fabrication plant was assumed to be located 1500 miles away from each of the BWR plant sites, and all new MOX fuel transportation costs were based on the 1500 mile transportation distance. Each site is assumed to have the same labor cost basis as the Kenosha, Wisconsin site specified in the ORNL guidelines [Ref. 6-2, 6-3, and 6-4]. As in the ABWR disposition study [Ref. 6-1], it is expected that ORNL will develop cost adjustment factors for other site locations. All costs were estimated as overnight cost with a base date of January 1993.

In general, only estimates of incremental capital costs related to implemewiation of the MOX fuel cycle have been generated for this study. For information purposes, Table 6.2-1 provides indications of capital investments currently reported for most BWR plants that are likely candidates for this project. The numbers in Table 6.2-1 are from Federal Energy Commission Reports (FERC Form 1) and Energy Information Agency reports (EIA 412) and represent the cumulative capital investments reported by utilities to date. Assuming continued utility ownership of the plants, new capital investment for the plutonium disposition project would presumably be additive to these reported capital bases. Capital costs allowed by state Public Utility Commissions (PUCs) for inclusion in utility rate bases will likely be different from the total FERC Form 1 values. Information relative to PUC allowances is published by Regulatory Research Associates, Inc. (RRA). Although GE does have available some of the RRA reports, analysis of PUC rate-making decisions for individual plants is a complex process and is considered to be beyond the scope of this report.

Assuming continuation of utility ownership of the plants and depending on the structure of the commercial arrangements between the Government and utility, new capital investments for the plutonium disposition project may or may not affect the capital investments now allowed by state 
Table 6.2-1 Total Capital Cost for Selected BWR Plants (From Year of Commercial Operation to 1992)

\begin{tabular}{|c|c|c|c|c|c|}
\hline Plant Name & $\begin{array}{c}\text { Year of } \\
\text { Date }\end{array}$ & $\begin{array}{l}\text { Land } \\
\text { Cost** }\end{array}$ & $\begin{array}{l}\text { Structures } \\
\text { and Improv. } \\
\text { Cont** }\end{array}$ & $\begin{array}{l}\text { Equilpment } \\
\text { Cost** }\end{array}$ & $\begin{array}{l}\text { Total } \\
\text { Capltal } \\
\text { Coot** }\end{array}$ \\
\hline $\begin{array}{l}\text { BROWNS FERRY } 1 \\
\text { BROWNS FERRY } 1 \& 2 * \\
\text { BROWNS FERRY } 1,2 \& 3 * \\
\text { BROWNS FERRY } 1,2 \& 3 \\
\text { BROWNS FERRY } 1,2 \& 3 \\
\text { BROWNS FERRY } 1,2 \& 33 \\
\text { BROWNS FERRY } 1,2 \& 3 \\
\text { BROWNS FERRY } 1,2 \& 3 \\
\text { BROWNS FERRY } 1,2 \& 3 \\
\text { BROWNS FERRY } 1,2 \& 3 \\
\text { BROWNS FERRY } 1,2 \& 3 \\
\text { BROWNS FERRY } 1,2 \& 3 \\
\text { BROWNS FERRY } 1,2 \& 3 \\
\text { BROWNS FERRY } 1,2 \& 3 \\
\text { BROWNS FERRY } 1,2 \& 3 \\
\end{array}$ & $\begin{array}{ll}1974^{*} & \\
1975^{*} & \\
1977^{*} & \\
1981 \\
1982 \\
1983 \\
1984 \\
1985 \\
1986 \\
1987 \\
1988 \\
1989 \\
1990 \\
1991 \\
1992 \\
\end{array}$ & $\begin{array}{r}\mathbf{\$ 8 9 0 , 0 0 0} \\
\mathbf{\$ 8 9 0 , 2 7 0} \\
\mathbf{\$ 8 9 0 , 2 6 9} \\
\mathbf{\$ 1 , 2 2 2 , \mathbf { 5 0 5 }} \\
\mathbf{\$ 1 , 2 2 2 , 8 6 6} \\
\mathbf{\$ 9 4 0 , 6 3 1} \\
\mathbf{\$ 9 4 0 , 6 3 1} \\
\mathbf{\$ 9 4 0 , 6 3 1} \\
\mathbf{\$ 9 4 0 , 6 3 1} \\
\mathbf{\$ 8 9 0 , 6 3 1} \\
\mathbf{\$ 8 9 0 , 6 3 1} \\
\mathbf{\$ 8 9 0 , 6 3 1} \\
\end{array}$ & $\begin{array}{l}\mathbf{\$ 1 8 9 , 0 8 1 , 0 0 0} \\
\mathbf{\$ 1 9 2 , 2 1 7 , 0 4 4} \\
\mathbf{\$ 2 0 0 , 9 8 8 , 1 4 3} \\
\mathbf{\$ 2 3 6 , 9 8 3 , 8 8 7} \\
\mathbf{\$ 2 4 2 , 1 6 5 , 8 2 7} \\
\mathbf{\$ 2 5 0 , 5 7 6 , 3 5 2} \\
\mathbf{\$ 2 6 9 , 4 2 4 , 1 8 5} \\
\mathbf{\$ 2 7 1}, 111,083 \\
\mathbf{\$ 4 9 4 , 3 7 4 , 4 1 9} \\
\mathbf{\$ 5 0 4 , 1 2 6 , 0 8 2} \\
\mathbf{\$ 6 2 6 , 4 7 3 , 3 2 3} \\
\mathbf{\$ 6 3 3 , 0 3 4 , 0 1 7} \\
\end{array}$ & $\begin{array}{r}\$ 702,744,000 \\
\$ 722,407,000 \\
\$ 727.611,853 \\
\$ 799,584,094 \\
\$ 900,369,725 \\
\$ 895,198,395 \\
\$ 905,163,668 \\
\$ 957,984,794 \\
\$ 924,659,340 \\
\$ 947,126,452 \\
\$ 1, \$ 11,562,871 \\
\$ 1,499,931,869 \\
\end{array}$ & 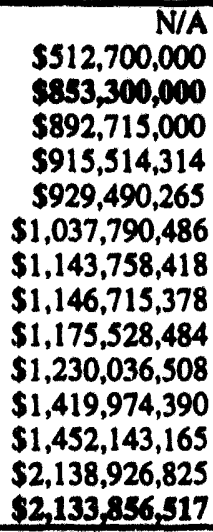 \\
\hline $\begin{array}{l}\text { CLINTON } \\
\text { CLINTON } \\
\text { CLINTON } \\
\text { CLINTON } \\
\text { CLINTON } \\
\text { CLINTON } \\
\end{array}$ & $\begin{array}{r}1987 * \\
1988 \\
1989 \\
1990 \\
1991 \\
1992 \\
\end{array}$ & $\begin{array}{r}\$ 13,533,974 \\
\$ 13,533,683 \\
\$ 0 \\
\$ 0 \\
\$ 0 \\
\$ 0 \\
\end{array}$ & $\begin{array}{r}\$ 1,308,866,934 \\
\$ 1,313,658,233 \\
\text { SO } \\
\text { SO } \\
\text { SO } \\
\text { SO } \\
\end{array}$ & $\begin{array}{r}\$ 3,054,022,846 \\
\$ 3,068,656,955 \\
\$ 0 \\
\$ 0 \\
\$ 0 \\
\$ 0 \\
\end{array}$ & $\begin{array}{l}\$ 4,376,423,754 \\
\$ 4,395,848,871 \\
\$ 4,391,105,592 \\
\$ 4,298,740,608 \\
\$ 4,304,915,446 \\
\$ 4,301,100, \$ 88 \\
\end{array}$ \\
\hline $\begin{array}{l}\text { FERMI } 2 \\
\text { FERMI } 2 \\
\text { FERMI } 2 \\
\text { FERMI } 2 \\
\text { FERMI } 2 \\
\end{array}$ & $\begin{array}{r}1988^{*} \\
1989 \\
1990 \\
1991 \\
1992 \\
\end{array}$ & $\begin{array}{l}\$ 1,728,250 \\
\$ 1,902,141 \\
\$ 1,900,999 \\
\$ 1,900,999 \\
\$ 1,900,999 \\
\end{array}$ & $\begin{array}{l}\$ 730,294,310 \\
\$ 737,566,075 \\
\$ 673,880,967 \\
\$ 674,641,551 \\
\$ 673,967,451 \\
\end{array}$ & $\begin{array}{l}\$ 2,969,058,809 \\
\$ 2,975,940,392 \\
\$ 3,132,897,068 \\
\$ 3,148,177,441 \\
\$ 3,168,667,333 \\
\end{array}$ & 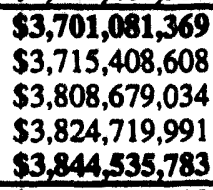 \\
\hline $\begin{array}{l}\text { GRAND GULF } \\
\text { GRAND GULF } \\
\text { GRAND GULF } \\
\text { GRAND GULF } \\
\text { GRAND GULF } \\
\text { GRAND GULF } \\
\text { GRAND GULF } \\
\text { GRAND GULF }\end{array}$ & $\begin{aligned} & 1985 * \\
& 1986 \\
& 1987 \\
& 1988 \\
& 1989 \\
& 1990 \\
& 1991 \\
& 1992 \\
&\end{aligned}$ & $\begin{array}{r}\$ 965,556 \\
\$ 965,556 \\
\$ 1,002,003 \\
\$ 0 \\
\$ 0 \\
\$ 1,002,003 \\
\$ 1,002,003 \\
\$ 1,002,003 \\
\end{array}$ & $\begin{array}{r}\$ 1,265,783,386 \\
\$ 1,227,697,242 \\
\$ 1,446,752,097 \\
\$ 0 \\
\$ 0 \\
\$ 1,452,785,698 \\
\$ 1,453,496,550 \\
\$ 1,459,221,760 \\
\end{array}$ & $\begin{array}{r}\mathbf{\$ 2 , 3 7 5 , 9 4 2 , 3 5 7} \\
\$ 2,452,805,965 \\
\$ 2,266,235,872 \\
\$ 0 \\
\$ 0 \\
\$ 2,361,458,404 \\
\$ 2,359,886,687 \\
\$ 2,343,504,426 \\
\end{array}$ & $\begin{array}{l}\mathbf{\$ 3 , 6 4 2 , 6 9 1 , 2 9 9} \\
\mathbf{\$ 3 , 6 8 1 , 4 6 8 , 7 6 3} \\
\mathbf{\$ 3 , 7 1 3 , 9 8 9 , 9 7 2} \\
\mathbf{\$ 3 , 7 3 5 , 4 1 1 , 0 7 5} \\
\mathbf{\$ 3 , 8 1 3 , 9 3 4 , 5 2 4} \\
\mathbf{\$ 3 , 8 1 5 , 2 4 6 , 1 0 5} \\
\mathbf{\$ 3 , 8 1 4 , 3 8 5 , 2 4 0} \\
\mathbf{\$ 3 , 8 0 3 , 7 2 8 , 1 8 9} \\
\end{array}$ \\
\hline $\begin{array}{l}\text { HOPE CREEK } \\
\text { HOPE CREEK } \\
\text { HOPE CREEK } \\
\text { HOPE CREEK } \\
\text { HOPE CREEK } \\
\text { HOPE CREEK } \\
\text { HOPE CREEK }\end{array}$ & $\begin{array}{r}1986^{*} \\
1987 \\
1988 \\
1989 \\
1990 \\
1991 \\
1992 \\
\end{array}$ & $\begin{array}{l}\$ 1,441,823 \\
\$ 1,424,166 \\
\$ 1,425,629 \\
\$ 1,425,629 \\
\$ 1,425,629 \\
\$ 1,425,629\end{array}$ & $\begin{array}{l}\$ 1,257,614,956 \\
\$ 1,271,379,485 \\
\$ 1,280,528,632 \\
\$ 1,294,766,295 \\
\$ 1,304,404,032 \\
\$ 1,305,841,267\end{array}$ & $\begin{array}{l}\$ 2,759,884,673 \\
\$ 2,844,994,155 \\
\$ 2,874,029,611 \\
\$ 2,884,036,849 \\
\$ 2,926,584,178 \\
\$ 2,955,093,713\end{array}$ & $\begin{array}{r}\text { N/A } \\
\$ 4,018,941,452 \\
\$ 4,117,797,806 \\
\$ 4,155,983,872 \\
\$ 4,180,228,773 \\
\$ 4,232,413,839 \\
\$ 4,262,360,609\end{array}$ \\
\hline 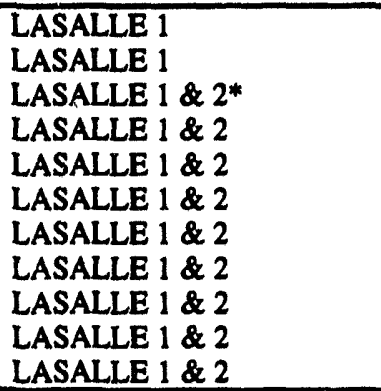 & $\begin{aligned} & 1982 * \\
& 1983 \\
& 1984 \\
& 1986 \\
& 1987 \\
& 1988 \\
& 1989 \\
& 1990 \\
& 1991 \\
& 1992 \\
&\end{aligned}$ & $\begin{array}{l}\mathbf{\$ 5 , 3 7 8 , 1 3 5} \\
\mathbf{\$ 5 , 3 7 8 , 1 3 5} \\
\mathbf{\$ 5 , 3 7 8 . 1 3 5} \\
\mathbf{\$ 5 , 3 7 8 , 1 3 5} \\
\mathbf{\$ 5 , 3 7 8 , 1 3 5} \\
\mathbf{\$ 5 , 3 7 8 , 1 3 5} \\
\mathbf{\$ 5 , 3 7 8 , 1 3 5} \\
\mathbf{\$ 5 , 3 7 8 , 1 3 5} \\
\mathbf{\$ 5 , 3 7 8 , 1 3 5} \\
\mathbf{\$ 5 , 3 7 8 , 1 3 5} \\
\mathbf{\$ 5 , 3 7 8 , 1 3 5}\end{array}$ & $\begin{array}{l}\$ 398,725,720 \\
\$ 400,579,076 \\
\$ 516,551,409 \\
\$ 520,710,449 \\
\$ 476,226,167 \\
\$ 485,193,532 \\
\$ 486,433,598 \\
\$ 488,503,871 \\
\$ 489,515,521 \\
\$ 492,360,025 \\
\$ 498,117,466\end{array}$ & $\begin{array}{r}\$ 932,062,478 \\
\$ 938,095,926 \\
\$ 1,895,984,006 \\
\$ 1,909,084,130 \\
\$ 1,975,369,984 \\
\$ 2,005,223,740 \\
\$ 2,011,503,277 \\
\$ 2,018,246,622 \\
\$ 2,061,254,262 \\
\$ 2,094,960,276 \\
\$ 2,111,336,433 \\
\end{array}$ & $\begin{array}{l}\mathbf{\$ 1 , 3 3 6 , 1 6 6 , 3 3 3} \\
\mathbf{\$ 1 , 3 4 4 , 0 5 3 , 1 3 7} \\
\mathbf{\$ 2 , 4 1 7 , 9 1 3 , 5 5 0} \\
\mathbf{\$ 2 , 4 3 5 , 1 7 2 , 7 1 4} \\
\mathbf{\$ 2 , 4 5 6 , 9 7 4 , 2 8 6} \\
\mathbf{\$ 2 , 4 9 5 , 7 9 5 , 4 0 7} \\
\mathbf{\$ 2 , 5 0 3 , 3 1 5 , 0 1 0} \\
\mathbf{\$ 2 , 5 1 2 , 1 2 8 , 6 2 8} \\
\mathbf{\$ 2 , 5 5 6 , 1 4 7 , 9 1 8} \\
\mathbf{\$ 2 , 5 9 2 , 6 9 8 , 4 3 6} \\
\mathbf{\$ 2 , 6 1 4 , 8 3 2 , 0 3 4}\end{array}$ \\
\hline
\end{tabular}


NEDO-32361

Table 6.2-1 Total Capital Cost for Selected BWR Plants (From Year of Commercial Operation to 1992) (Continued)

\begin{tabular}{|c|c|c|c|c|c|}
\hline Plans Name & $\begin{array}{c}\text { Year of } \\
\text { Data }\end{array}$ & $\begin{array}{l}\text { Land } \\
\text { Coat** }\end{array}$ & $\begin{array}{l}\text { Structures } \\
\text { and Improv. } \\
\text { Coot }\end{array}$ & $\begin{array}{l}\text { Equipment } \\
\text { Cont** }\end{array}$ & $\begin{array}{l}\text { Total } \\
\text { Capltal } \\
\text { Cost }\end{array}$ \\
\hline $\begin{array}{l}\text { LIMERICK } 1 \\
\text { LIMERICK } 1 \\
\text { LIMERICK } 1 \\
\text { LIMERICK } 1 \\
\text { LIMERICK } 1 \& 2 * \\
\text { LIMERICK } 1 \& 2 \\
\text { LIMERICK } 1 \& 2\end{array}$ & 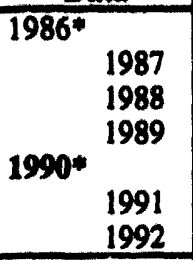 & $\begin{array}{r}\mathbf{\$ 7 , 8 9 7 , 6 5 3} \\
\mathbf{\$ 7 , 9 0 3 , 5 0 4} \\
\mathbf{\$ 7 , 9 0 3 , 5 0 4} \\
\mathbf{S 0} \\
\mathbf{S 0} \\
\mathbf{S 0} \\
\mathbf{S 0} \\
\end{array}$ & $\begin{array}{r}\$ 809,591,781 \\
\$ 813,637,843 \\
\mathbf{\$ 8 3 2 , 2 3 0 , 4 2 4} \\
\$ 0 \\
\$ 0 \\
\$ 0 \\
\$ 0 \\
\end{array}$ & $\begin{array}{r}\mathbf{\$ 2 , 9 7 8 , 7 7 0 , 5 5 2} \\
\mathbf{\$ 3 , 0 3 0 , 2 8 0 , 5 2 8} \\
\mathbf{\$ 3 , 0 4 9 , 6 7 2 , 5 0 8} \\
\mathbf{S 0} \\
\mathbf{\$ 0} \\
\mathbf{S 0} \\
\mathbf{S 0} \\
\end{array}$ & $\begin{array}{l}\mathbf{\$ 3 , 7 9 6 , 2 3 9 , 9 8 6} \\
\mathbf{\$ 3 , 8 5 1 , 8 2 1 , 8 7 5} \\
\mathbf{\$ 3 , 8 8 9 , 8 0 6 , 4 3 6} \\
\mathbf{\$ 4 , 2 3 2 , 0 3 4 , 0 5 0} \\
\mathbf{\$ 7 , 3 0 9 , 5 3 8 , 8 7 9} \\
\mathbf{\$ 7 , 2 9 7 , 0 2 1 , 4 6 1} \\
\mathbf{\$ 7 , 3 2 9 , 6 8 1 , 8 3 1} \\
\end{array}$ \\
\hline $\begin{array}{l}\text { NINE MILE POINT } 2 \\
\text { NINE MILE POINT } 2 \\
\text { NINE MILE POINT } 2 \\
\text { NINE MILE POINT } 2 \\
\text { NINE MILE POINT } 2\end{array}$ & $\begin{array}{r}1989 \\
1989 \\
1990 \\
1991 \\
1992 \\
\end{array}$ & $\begin{array}{r}\text { So } \\
\text { so } \\
\text { so } \\
\text { so } \\
\text { so } \\
\end{array}$ & $\begin{array}{l}\text { so } \\
\text { so } \\
\text { so } \\
\text { so } \\
\text { so } \\
\end{array}$ & $\begin{array}{l}\text { So } \\
\text { SO } \\
\$ 0 \\
\$ 0 \\
\text { SO } \\
\end{array}$ & $\begin{array}{r}\text { N/A } \\
\text { N/A } \\
\text { N/A } \\
\text { N/A } \\
\text { N/A } \\
\end{array}$ \\
\hline $\begin{array}{l}\text { PERRY } 1 \\
\text { PERRY } 1 \\
\text { PERRY } 1 \\
\text { PERRY } 1 \\
\text { PERRY } 1 \\
\text { PERRY } 1 \\
\end{array}$ & $\begin{array}{r}1987 * \\
1988 \\
1989 \\
1990 \\
1991 \\
1992 \\
\end{array}$ & $\begin{array}{l}\$ 0 \\
\$ 0 \\
\$ 0 \\
\$ 0 \\
\$ 0 \\
\$ 0\end{array}$ & $\begin{array}{l}\text { s0 } \\
\$ 0 \\
\$ 0 \\
\$ 0 \\
\$ 0 \\
\$ 0 \\
\$ 0\end{array}$ & $\begin{array}{r}\text { So } \\
\text { so } \\
\text { so } \\
\text { so } \\
\text { so } \\
\text { So } \\
\end{array}$ & $\begin{array}{r}\$ 5,971,355,207 \\
\$ 5,895,201,704 \\
\text { N/A } \\
\text { N/A } \\
\text { N/A } \\
\text { N/A }\end{array}$ \\
\hline $\begin{array}{l}\text { RIVER BEND } \\
\text { RIVER BEND } \\
\text { RIVER BEND } \\
\text { RIVER BEND } \\
\text { RIVER BEND } \\
\text { RIVER BEND } \\
\text { RIVER BEND } \\
\end{array}$ & $\begin{array}{r}1986 * \\
1987 \\
1988 \\
1989 \\
1990 \\
1991 \\
1992 \\
\end{array}$ & $\begin{array}{r}\mathbf{\$ 1 2 , 1 5 1 , 9 4 0} \\
\mathbf{\$ 1 2 , 2 8 3 , 6 9 7} \\
\mathbf{\$ 8 , 7 0 5 , 2 9 6} \\
\mathbf{\$ 8 , 7 0 5 , 2 9 6} \\
\mathbf{\$ 8 , 7 0 5 , 2 9 6} \\
\mathbf{\$ 8 , 7 0 5 , 2 9 6} \\
\mathbf{\$ 8 , 7 0 5 , 2 9 6} \\
\end{array}$ & $\begin{array}{l}\$ 1,883,565,155 \\
\$ 1,905,756,828 \\
\$ 1,908,707,416 \\
\$ 1,918,549,687 \\
\$ 1,921,095,106 \\
\$ 1,924,775,781 \\
\$ 1,968,805,284 \\
\end{array}$ & $\begin{array}{l}\mathbf{\$ 2 , 4 6 3 , 9 3 3 , 4 9 0} \\
\mathbf{\$ 2 , 4 9 5 , 8 9 4 , 2 4 7} \\
\mathbf{\$ 2 , 4 9 8 , 3 3 9 , 6 2 9} \\
\mathbf{\$ 2 , 4 9 4 , 7 3 1 , 3 3 8} \\
\mathbf{\$ 2 , 5 0 8 , 7 8 2 , 6 5 4} \\
\mathbf{\$ 2 , 5 1 2 , 3 3 8 , 2 7 0} \\
\mathbf{\$ 2 , 4 7 2 , 4 2 3 , 1 5 0} \\
\end{array}$ & $\begin{array}{l}4,359,650,585 \\
\$ 4,413,934,772 \\
\$ 4,415,752,341 \\
\$ 4,421,986,321 \\
\$ 4,438,583,056 \\
\$ 4,445,819,347 \\
\$ 4,449,933,730 \\
\end{array}$ \\
\hline 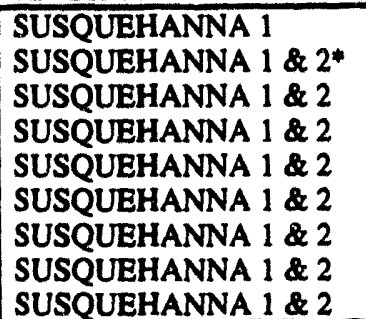 & $\begin{aligned} & 1983^{*} \\
& 1984^{*} \\
& 1986 \\
& 1987 \\
& 1988 \\
& 1989 \\
& 1990 \\
& 1991 \\
& 1992\end{aligned}$ & $\begin{array}{r}\$ 6,028,686 \\
\$ 6,448,362 \\
\$ 6,138,782 \\
\$ 6,138,794 \\
\$ 16,216,094 \\
\$ 17,601,470 \\
\$ 17,659,055 \\
\$ 17,751,128\end{array}$ & $\begin{array}{r}\$ 993,239,421 \\
\$ 952,461,097 \\
\$ \$ 981,617,979 \\
\$ 983,655,678 \\
\$ 992,759,660 \\
\$ 1,000,936, \$ 28 \\
\$ 1,003,024,359 \\
\$ 1,005,605,455\end{array}$ & $\begin{array}{l}\mathbf{\$ 3}, 094,092,399 \\
\mathbf{\$ 3}, 142,914,792 \\
\mathbf{\$ 3 , 2 5 2 , 4 1 9 , 8 4 6} \\
\mathbf{\$ 3 , 2 8 5 , 3 6 3 , 6 0 9} \\
\mathbf{\$ 3}, 321, \mathbf{5 4 7 , 9 6 9} \\
\mathbf{\$ 3}, 333,978,574 \\
\mathbf{\$ 3}, 334,097,467 \\
\mathbf{\$ 3}, 355,113,273\end{array}$ & $\begin{array}{l}\$ 1,947,000,000 \\
\$ 4,093,360, \$ 06 \\
\$ 4,101,824,251 \\
\$ 4,240,176,607 \\
\$ 4,275,158,081 \\
\$ 4,330,523,723 \\
\$ 4,352,516,572 \\
\$ 4,354,780,881 \\
\$ 4,378,469,856\end{array}$ \\
\hline $\begin{array}{l}\text { WNP 2 } \\
\text { WNP 2 } \\
\text { WNP 2 } \\
\text { WNP 2 } \\
\text { WNP 2 } \\
\text { WNP 2 } \\
\text { WNP 2 } \\
\text { WNP 2 }\end{array}$ & $\begin{aligned} & 1985 * \\
& 1986 \\
& 1987 \\
& 1988 \\
& 1989 \\
& 1990 \\
& 1991 \\
& 1992 \\
&\end{aligned}$ & $\begin{array}{r}\$ 200,642 \\
\$ 200,643 \\
\$ 200,643 \\
\$ 200,642 \\
\$ 0 \\
\$ 0 \\
\$ 0 \\
\$ 0 \\
\end{array}$ & $\begin{array}{l}\$ 1,109,557,851 \\
\$ 1,110,119,450 \\
\$ 1,116,302,898 \\
\$ 1,125,993,911 \\
\$ 1,095,230,044 \\
\$ 1,097,156,372 \\
\$ 1,097,156,372 \\
\$ 1,092,854,748 \\
\end{array}$ & $\begin{array}{l}\$ \$ 2,231,343,189 \\
\$ 2,284,247,470 \\
\$ 2,312,585,595 \\
\$ 2,319,818,799 \\
\$ 2,130,425,564 \\
\$ 2,136,090,367 \\
\$ 2,136,187,654 \\
\$ 2,033,384,724 \\
\end{array}$ & $\begin{array}{l}\$ 3,341,101,682 \\
\$ 3,394,567,563 \\
\$ 3,429,089,136 \\
\$ 3,446,013,352 \\
\$ 3,225,655,608 \\
\$ 3,233,246,739 \\
\$ 3,233,344,026 \\
\$ 3,126,239,472 \\
\end{array}$ \\
\hline \multicolumn{6}{|c|}{ 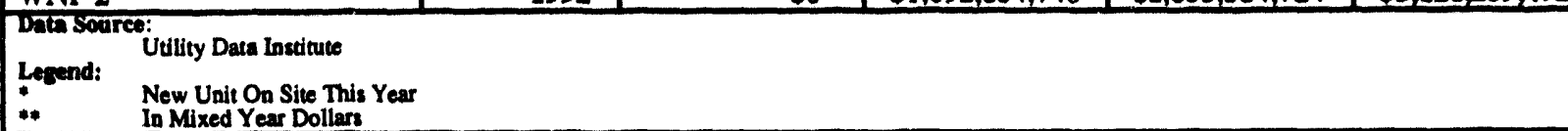 } \\
\hline
\end{tabular}


PUCs for inclusion in utility rate bases. If entire or partial Government ownership of the plants is an assumed option, then the capital investments shown in Table 6.2-1 should be of some value as an indication of possible bases for purchase negotiations when combined with data on depreciation and rate base capital allowances.

Cost estimates for the fuel fabrication facilities at Los Alamos National Laboratories (LANL) and the full MOX fabrication facility are based on the assumption that americium is removed from the plutonium material supplied for fuel fabrication. If americium levels and consequent radiation levels are higher than assumed, the capital and $O \& M$ cost will be significantly higher.

\subsubsection{BWR Complex Capital Cost}

The incremental capital costs and associated cost related to the extension of outage time for conversion to MOX operation are summarized in Table 6.2-2. No outage extension is required for plant modification since the expected modifications can be accomplished during operation or during outages prior to initial MOX fuel loading. Three-month outage extensions are expected to be required for the core physics testing during the initial startup following loading of full MOX bundles. Table 6.2-2 includes cost for replacement power which may be required as a result of the extended outage durations. A typical replacement power cost of three cents $/ \mathrm{kWh}$ has been assumed, but such cost will vary significantly, depending upon individual utility and plant circumstances.

\section{Capital Cost for New MOX Fuel and Spent Fuel Storage Facilities}

A detailed description of these facilities is presented in Sections 2.5 of this report. Table 6.2-3 shows the capital cost required for a single installation on a single reactor site. It has been assumed that some of the indirect costs such as engineering and owners costs will be shared between the two sites and among the three units. Therefore, the cost for three units at two sites is substantially less than the costs for a single unit site plus the costs for a dual unit site.

Capital costs for the new fuel storage facility were developed from conceptual design sketches and descriptions of the facility design, material handling and support equipment. 
Table 6.2-2 Reference Case BWR Plant Incremental Costs for Conversion to Full MOX Fuel Cycle (1/93 base date)

New and Spent Fuel Storage Facilities

Plant Modifications

BWR Process Systems and Building

Safeguards and Security Systems

Fuel Handling Equipment

Engineering \& Licensing

Total Capital Costs*

Plant Outage Cost for MOX Fuel Testing

Replacement Power (3 mo at 3c/kWh)

Total Conversion Cost

*Contingency and Owner's costs included 
Table 6.2-3 Capital Cost Estimate for MOX and Spent Fuel Storage Facilities (1/93 base date)

\begin{tabular}{|c|c|c|c|c|c|c|c|}
\hline \multirow[b]{2}{*}{ ITEM and DESCRIPTION } & \multirow[b]{2}{*}{ QTY } & \multirow[b]{2}{*}{ UNIT } & \multirow{2}{*}{$\begin{array}{l}\text { DQPT \& } \\
\text { MaTpis } \\
\text { (ax) }\end{array}$} & \multicolumn{2}{|c|}{ SITE LABOR } & \multirow{2}{*}{$\begin{array}{l}\text { SUB. } \\
\text { CONT. } \\
\text { (ks) }\end{array}$} & \multirow{2}{*}{$\begin{array}{c}\text { Total } \\
\text { cost }\end{array}$} \\
\hline & & & & $\mathbf{M} \mathbf{A}$ & $(\mathbf{K S})$ & & \\
\hline 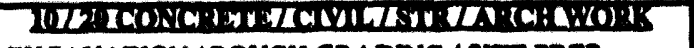 & & & & & & & \\
\hline $\begin{array}{l}\text { EXCAVATION / ROUOH ORADNO / SITE PREP } \\
360^{\prime} \times 220^{\prime} \text { FOR VAULT BLDO / STORAGE AREA }\end{array}$ & 1 & LT & 0 & 0 & 0 & 30 & 50 \\
\hline 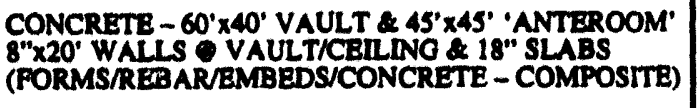 & 400 & CY & 80 & 4,800 & 120 & 0 & 200 \\
\hline 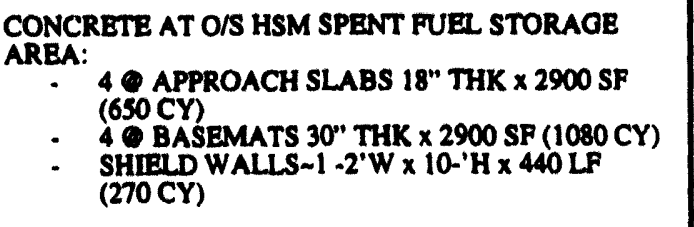 & 2000 & CY & 300 & 12,000 & 300 & 0 & 60 \\
\hline $\begin{array}{l}\text { STR STEELROOF \& BLDG FINISH } \\
\text { VAULT/ANTEROOM (INCL 'BUTLER' BLDO } \\
\text { ANTEROOMMDOCK \& BOOT }\end{array}$ & 1 & LT & 20 & 2,000 & 56 & 100 & 176 \\
\hline SECURITY FENCE-(INTERIOR FENCE) & 1000 & $\mathbf{L F}$ & 0 & 0 & 0 & 15 & 15 \\
\hline 'NUISANCE' FRNCE FACILITY PERIMETER & 1160 & LF & 0 & $\mathbf{0}$ & 0 & 17 & 17 \\
\hline 20' DOUBLE-SWING GATES EACH FENCE/ENTRY & 2 & EA & 0 & 0 & 0 & 10 & 10 \\
\hline FINISH ORADING/ORAVEL & 1 & LT & 0 & 0 & 0 & 25 & 25 \\
\hline ASPHALT PAVING & 1 & $\mathbf{L T}$ & 0 & 0 & 0 & 73 & 75 \\
\hline OTHER - SITE FINISHDRAINAOE & 1 & LT & 10 & 400 & 10 & 75 & 95 \\
\hline$-20^{\prime} \times 20^{\prime}$ OUARDHOUSE - ALLOW $\$$ S200/SF & 1 & EA & 0 & 0 & 0 & 80 & 80 \\
\hline 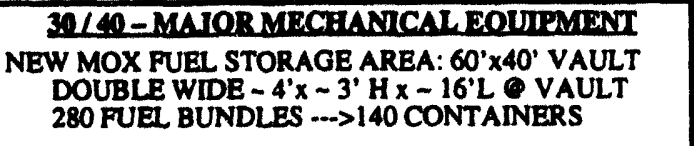 & 140 & EA & 840 & & & & 840 \\
\hline $\begin{array}{l}\text { HSMs -PREFAB DRY SPENT FUEL STORAGE } \\
\text { MODULES: SET IN PLACE (4 ROWS OF } 10 \text { EACH) }\end{array}$ & 40 & EA & 6,000 & 800 & 20 & 0 & 6,020 \\
\hline 1 - TON PORTABLE LIFT RIG (NO SPECS) & 1 & EA & 100 & 0 & 0 & 0 & 100 \\
\hline 2 - TON BRIDGE CRANE/ - 40 FT SPAN 9 VAULT & 1 & EA & 100 & 200 & 5 & 0 & 105 \\
\hline HVAC SYSTEMHEPA FILTERS - ALLOW & 1 & $\mathbf{L T}$ & 80 & 500 & 13 & 20 & 113 \\
\hline FIRE PROTECTION @ VAULT/ANTEROOM AREA & 1 & LT & 20 & 200 & 5 & 20 & 45 \\
\hline DRY SHIELDED CANISTERS (DSCs) FOR HSMs & 40 & EA & 12,000 & 0 & 0 & 0 & 12,000 \\
\hline OTHER XFERAUX EQUIPMENT & 1 & LT & 2,350 & 0 & $0]$ & 0 & 2,350 \\
\hline $\begin{array}{l}\text { SO PIPINGAVALVSSAGIRS } \\
\text { FIRE PROTECTION VAULT AREA - INCL ABOVE } \\
\text { FIRE PROTECTION OUTSIDE AREA \& } \\
\text { HYDRANTS/FIRE PROT SPECIALTIES (FIREWATER } \\
\text { MAIN AVAILABLE } @ \text { SITE FENCE) }\end{array}$ & 1 & LT & 100 & 400 & 10 & 0 & 110 \\
\hline $\begin{array}{l}\text { EONSTURUMINTATION } \\
\text { MISCELLANEOUS - DEVICES/TIE WITH } \\
\text { DETECTION SYSTEMS INCL W / ELEC: }\end{array}$ & 1 & LT & 50 & 200 & 5 & 0 & 55 \\
\hline
\end{tabular}


The new MOX fuel storage facility is sized for receipt and storage of 280 new MOX fuel bundles. The MOX assemblies are assumed to be shipped to the facility in standard BWR LEU shipping containers in Safe Secure Transporters (SSTs). The bundles will be stored in the facility in the internal shipping containers after removal from a standard wooden overpack and inspection of tamper indicating seals. It is assumed that the containers are never opened until the fuel bundles are transferred to the reactor refueling floor. The costs for shipping containers is included in the estimate for the new and spent fuel storage facility and are based on current costs for standard BWR LEU fuel bundle containers.

The new fuel storage vault is a nuclear Category I structure designed to survive the site design basis events. Fire, criticality, and radiation alarms and a standard warehouse ventilation system equipped with exhaust HEPA filters have been included. A conventional steel frame anteroom provides an area to receive the new MOX fuel shipping containers, remove the internal container from the wooden overpacks and transfer them to the storage vault. A truck loading dock is equipped with a fabric boot to prevent unauthorized observation of the unloading operation from the SSTs. A portable lift vehicle with an extended boom will lift and remove the shipping containers from the SSTs. This lift will also be used to assist in removal of the internal shipping containers from the wooden overpacks. A two-ton bridge crane is provided to move the containers inside the vault.

A commercially available NRC licensed ex-reactor dry cask spent fuel storage system has been used as the basis of the capital cost estimate for the ex-reactor spent fuel storage facility to supplement existing fuel storage capacity at existing reactor sites. This system is described in more detail in Section 2.5 of this report. Capital costs for the spent fuel storage facility prefabricated horizontal storage modules and associated canister handling and welding equipment were developed from budgetary cost guidelines provided by a commercial dry cask storage system vendor. Costs for associated concrete basemats, approach pads and engineered asphalt paving were developed from conceptual design sketches.

The system consists primarily of prefabricated ventilated reinforced concrete horizontal storage modules containing the spent fuel bundles in dry shielded stainless steel canisters. The concrete horizontal storage modules are designed to remain functional after all design basis events including tornadoes, earthquakes, floods, etc. and provide biological shielding consistent with current regulatory ALARA objectives. Concrete basemats up to 30 inches thick are provided to support the modules and approach loading areas. Transport equipment including a transfer cask, 
cask skid, transfer trailer, hydraulic positioning system, and hydraulic ram system is provided as well as a canister welding and vacuum drying system.

Both the new MOXX fuel storage and spent fuel storage facilities are located within a security protected area as defined in 10CFR73. An isolation zone of 20 feet interior and 20 feet exterior to the protected area security fence has been included. A nuisance fence is installed 20 feet outside the security fence. The isolation zone is provided with perimeter intrusion detection systems which meet the requirements of USNRC Regulatory Guide 5.44. The system assumed is a combination microwave, infrared system. A closed circuit television (CCTV) system equipped with appropriate lighting, communications equipment, alarm systems, access control systems, and a continuously manned guardhouse equipped with appropriate monitoring and guard support and protection systems are provided.

Safeguards and security (S\&S) system costs were based on conceptual design descriptions and sketches and historical cost data from recent detailed designs of S\&S systems for similar nuclear projects. Other support systems included in the estimate for these facilities include fire detection/protection, emergency power generation, radiation and criticality monitors and alarms, and tie-ins to existing on-site monitoring, control, utilities and service systems.

Per the DOE cost guidelines, a minimum contingency of $15 \%$ has been applied to the vendorbased cost information provided for the spent fuel dry cask storage modules and support equipment, while a contingency factor of $25 \%$ has been used for the remainder of the facility since the current designs are considered conceptual.

\subsubsection{Capital Costs for MOX Fuel Fabrication Facility}

\subsubsection{Capital Costs for Full MOX Fuel Fabrication Facility}

Table 6.2-4 shows the capital cost estimate for the full MOX fuel fabrication facility assuming a 'greenfield' basis. Table 6.2-5 shows the distribution of manual labor estimated for construction of this facility. Although fuel throughput rates are different for this reference case than for the 
Table 6.2-4 MOX Fuel Fabrication Facility Pre-Conceptual Cost Estimate Reference Case

(1/93 base date)

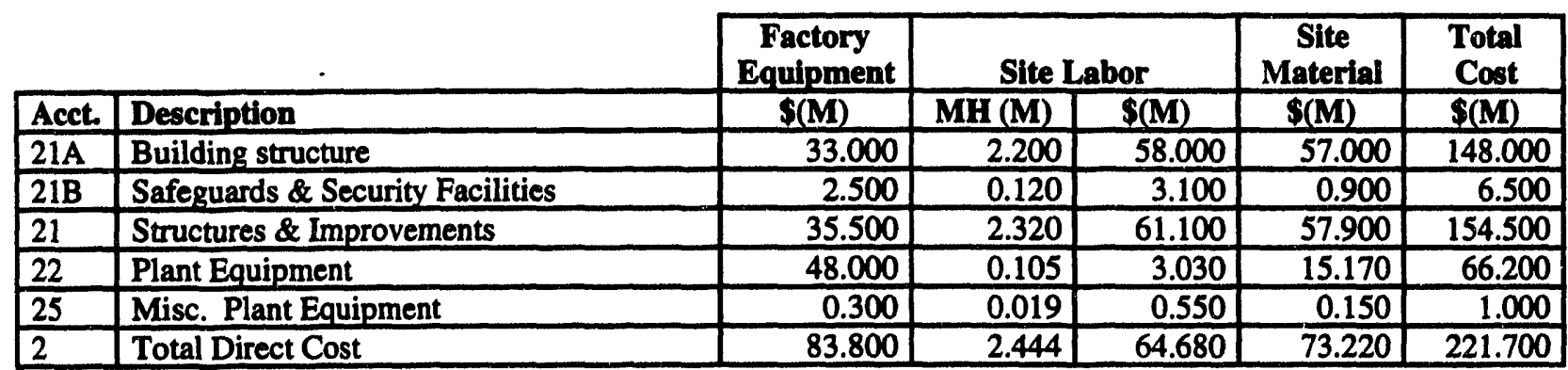

\begin{tabular}{|c|c|c|c|c|c|c|}
\hline 91 & Construction Services & & 0.770 & 29.000 & 17.700 & 46.700 \\
\hline 92 & Engineering \& Home Office & 76.000 & incl. & incl. & incl. & 76.000 \\
\hline 93 & Field Supervision \& Field Office Services & & .660 & 30.000 & 6.000 & 36.000 \\
\hline 94 & Owner's Cost & 57.000 & íncl. & incl. & incl. & 57.000 \\
\hline 9 & Total Indirect Cost & 133.000 & 1.430 & 59.000 & 23.700 & 215.70 \\
\hline
\end{tabular}

\begin{tabular}{|l|r|r|r|r|r|}
\hline Sub-total Direct \& Indirect Cost & 216.800 & 3.874 & 123.680 & 96.920 & 437.400 \\
\hline
\end{tabular}

Contingency

Total Base Construction Cost and Contingency

525.000

Table 6.2-5 MOX Fuel Fabrication Facility Estimated Manual Labor Distribution

\begin{tabular}{|l|c|c|}
\hline Craft & $\begin{array}{c}\text { Manual Labor Distribution } \\
(\mathbf{\%})\end{array}$ & $\begin{array}{c}\text { Manhours } \\
(\mathbf{1 0 0 0} \text { manhours })\end{array}$ \\
\hline Boilermakers & 2.0 & 64 \\
\hline Carpenters & 15.0 & 482 \\
\hline Electricians & 15.0 & 482 \\
\hline Ironworkers & 15.0 & 482 \\
\hline Laborers & 18.0 & 579 \\
\hline Mkillwrights & 3.0 & 96 \\
\hline Operating Engineers & 10.0 & 322 \\
\hline Pipefitters & 12.0 & 386 \\
\hline Teamsters & 5.0 & 161 \\
\hline All Other & 5.0 & 161 \\
\hline Total & 100.0 & 3,215 \\
\hline
\end{tabular}

ABWR reference case [Ref. 6-1], capital costs for these facilities are relatively insensitive to capacity. Therefore, these estimates are the same as the estimates for the fabrication facility included in the $A B W R$ report. As noted in the ABWR report, if existing facilities can be used instead of developing a 'greenfield' site, then significant savings (in the order of $\$ 100-\$ 150$ million) could be achieved. 
NEDO-32361

\subsubsection{Capital Costs for MOX Fuel Fabrication at Los Alamos}

Table 6.2-6 shows the capital cost estimate for conversion of the Los Alamos National Laboratories (LANL) facility for pilot production of fuel rods for assembly into 'island' design fuel bundles.

Table 6.2-6 Capital Cost Estimate for LANL Island Fuel Design Fabrication (1/93 base date)

\begin{tabular}{|l|r|r|r|r|}
\hline \multirow{2}{*}{ Item } & \multicolumn{2}{|c|}{ Equipment and Materia Costs (K\$) } & Total \\
\cline { 2 - 5 } Cost (K\$)
\end{tabular}

\begin{tabular}{|l|r|r|r|r|}
\hline Total Cost (K\$) & 1,575 & & 1,942 & 3,517 \\
\hline
\end{tabular}




\subsection{Operations and Maintenance (O\&M) Cost Detail}

\subsubsection{O\&M Bases and Assumptions}

As indicated in Section 6.0, only incremental costs related to MOX operation are included in this report. Table 6.3-1 is included for reference to give an indication of current levels of reported O\&M costs now being incurred by the BWR plants under consideration for the plutonium disposition project. Table 6.3-1 shows reported annual O\&M costs for the years 1983-1992 and, where applicable, average costs are also shown. It should be recognized that the O\&M costs normally reported by utilities by station do not include some overhead costs that are normally accounted for at the corporate level and that are not allocated to the costs reported for individual stations. The ORNL guidelines for calculation of O\&M costs prescribe factors for corporate overheads. Therefore, the reported costs in Table 6.3-1 are different in scope from those costs calculated using the ORNL guidelines (and those included in the ABWR report [Ref. 6-1]).

It needs to be emphasized that the industry has made $O \& M$ cost reduction and capacity factor improvement high priorities and significant improvements have been occurring. For example, the capacity factors for large nuclear plants (1020 MW or greater) have improved from a median value of $65.8 \%$ in $1988-1990$ to $72 \%$ in $1991-1993$. Since capacity factors have been improving over the last few years, O\&M cost per kilowatt are being reduced from the historical levels indicated in Table 6.3-1.

\subsubsection{O\&M Costs for BWR Plants}

Incremental O\&M cost estimates for the BWR plant due to MOX operations are shown in Table 6.3-2. These incremental O\&M costs would be additive to the current levels of O\&M expenditures indicated in Table 6.3-1. The new and spent fuel storage facilities will be new additions at each site, all additional O\&M costs related to these facilities are included in the estimates shown in Table 6.3-3.

\subsubsection{O\&M Costs for Operation of the MOX Fuel Fabrication Facility for Supply of Full MOX Core Fuel}

O\&M costs for the full MOX fuel fabrication facility are shown in Table 6.3-4.

\subsubsection{O\&M Costs for Operation of the Los Alamos Fuel Fabrication Facilities for Producing Initial Island Design Fuel}

O\&M costs for the 'island' MOX fuel fabrication facility are shown in Table 6.3-5. 
Table 6.3-1 Average O\&M Costs for Selected BWR Plants

\begin{tabular}{|c|c|c|c|c|c|}
\hline $\begin{array}{l}\text { PLANT NAME } \\
\text { (LOCATION) }\end{array}$ & Year & $\begin{array}{c}\text { MW } \\
\text { (Gross) }\end{array}$ & $\begin{array}{c}\text { MW Hours } \\
\text { (net) }\end{array}$ & $\begin{array}{c}\text { O\&M COSTS } \\
\text { (expensed) }\end{array}$ & $\begin{array}{c}\text { \$/MWHR } \\
\text { (mixed Year \$) }\end{array}$ \\
\hline BROWNS FERRY 1,2 \& 3 (AL) & 1983 & 3456 & $16,115,228$ & $\$ 108,946,000$ & $\$ 6.8$ \\
\hline BROWNS FERRY 1,2 \& 3 (AL) & 1984 & 3456 & $11,768,207$ & $\$ 129,996,000$ & $\$ 11.0$ \\
\hline BROWNS FERRY $1,2 \& 3$ (AL) & 1985 & 3456 & $5,543,822$ & $\$ 99,913,000$ & $\$ 18.0$ \\
\hline BROWNS FERRY $1,2 \& 3$ (AL) & 1986 & 3456 & 0 & $\$ 143,268,000$ & N/A \\
\hline BROWNS FERRY $1,2 \& 3$ (AL) & 1987 & 3456 & 0 & $\$ 178,661,000$ & N/A \\
\hline BROWNS FERRY $1,2 \& 3$ (AL) & 1988 & 3456 & 0 & $\$ 149,512,000$ & N/A \\
\hline BROWNS FERRY $1,2 \& 3$ (AL) & 1989 & 3456 & 0 & $\$ 90,378,000$ & N/A \\
\hline BROWNS FERRY 1,2 \& 3 (AL) & 1990 & 3456 & 0 & $\$ 42,713,000$ & N/A \\
\hline BROWNS FERRY $1,2 \& 3$ (AL) & 1991 & 3456 & $1,559,739$ & $\$ 101,407,000$ & $\$ 65.0$ \\
\hline BROWNS FERRY $1,2 \& 3$ (AL) & 1992 & 3456 & $8,517,243$ & $\$ 102,850,000$ & $\$ 12.1$ \\
\hline Ave. O8xM Cost: (1983-92) & & & $\mathbf{N} / \mathbf{A}$ & $\mathbf{N} / \mathbf{A}$ & $\mathbf{N} / \mathbf{A}$ \\
\hline CLINTON (IL) & 1987 & 985 & $1,803,044$ & $\$ 65,156,000$ & $\$ 36.1$ \\
\hline CLINTON (IL) & 1988 & 981 & $5,852,705$ & $\$ 72,017,000$ & $\$ 12.3$ \\
\hline CLINTON (IL) & 1989 & 985 & $2,830,046$ & $\$ 91,228,000$ & $\$ 32.2$ \\
\hline CLINTON (IL) & 1990 & 985 & $3,571,431$ & $\$ 118,068,000$ & $\$ 33.1$ \\
\hline CLINTON (IL) & 1991 & 985 & $6,038,223$ & $\$ 86,664,000$ & $\$ 14.4$ \\
\hline CLINTON (IL) & 1992 & 985 & $4,909,386$ & $\$ 99,900,000$ & $\$ 20.3$ \\
\hline Ave, O8M Cost: (1987-92) & & & $\mathbf{4 . 1 6 7 . 4 7 3}$ & $\$ 88.839 .000$ & $\$ 21.3$ \\
\hline FERMI 2 (MI) & 1988 & 1094 & $4,230,186$ & $\$ 138,804,000$ & $\$ 32.8$ \\
\hline FERM' 2 (MI) & 1989 & 1094 & $5,193,096$ & $\$ 139,400,000$ & $\$ 26.8$ \\
\hline FERMI 2 (MI) & 1990 & 1094 & $7,089,841$ & $\$ 108,725,000$ & $\$ 15.3$ \\
\hline FERMI 2 (MI) & 1991 & 1094 & $6,157,125$ & $\$ 114,009,000$ & $\$ 18.5$ \\
\hline FERMI 2 (MI) & 1992 & 1094 & $7,337,965$ & $\$ 109,001,000$ & $\$ 14.9$ \\
\hline Ave, OsM Cost: (1988-92) & & & 6.001 .643 & $\$ 121.988 .000$ & $\$ 20.3$ \\
\hline GRAND GULF (MS) & 1985 & 1373 & $2,669,899$ & $\$ 68,350,000$ & $\$ 25.6$ \\
\hline GRAND GULF (MS) & 1986 & 1373 & $4,098,054$ & $\$ 110,127,000$ & $\$ 26.9$ \\
\hline GRAND GULF (MS) & 1987 & 1373 & $7,727,978$ & $\$ 99,529,000$ & $\$ 12.9$ \\
\hline GRAND GULF (MS) & 1988 & 1373 & $9,593,732$ & $\$ 83,427,000$ & $\$ 8.7$ \\
\hline GRAND GULF (MS) & 1989 & 1373 & $7,848, j 54$ & $\$ 105,984,000$ & $\$ 13.5$ \\
\hline GRAND GULF (MS) & 1990 & 1373 & $7,407,120$ & $\$ 107,639,000$ & $\$ 14.5$ \\
\hline GRAND GULF (MS) & 1991 & 1373 & $9,132,933$ & $\$ 85,826,999$ & $\$ 9.4$ \\
\hline GRAND GULF (MS) & 1992 & 1373 & $8,174,867$ & $\$ 117,893,000$ & $\$ 14.4$ \\
\hline Are, Os M Cost: (1985-92) & & & $\mathbf{7 , 0 8 1 . 6 1 7}$ & $\$ 97.347 .000$ & $\$ 13.7$ \\
\hline HOPE CREEK (NJ) & 1987 & 1168 & $7,218,342$ & $\$ 69,422,000$ & $\$ 9.6$ \\
\hline HOPE CREEK (NJ) & 1988 & 1168 & $6,951,407$ & $\$ 85,027,000$ & $\$ 12.2$ \\
\hline HOPE CREEK (NJ) & 1989 & 1168 & $6,588,563$ & $\$ 88,491,000$ & $\$ 13.4$ \\
\hline HOPE CREEK (NJ) & 1990 & 1168 & $8,081,647$ & $\$ 85,666,000$ & $\$ 10.6$ \\
\hline HOPE CREEK (NJ) & 1991 & 1168 & $7,375,578$ & $\$ 97,394,000$ & $\$ 13.2$ \\
\hline HOPE CREEK (NJ) & 1992 & 1168 & $7,033,399$ & $\$ 107,486,000$ & $\$ 15.3$ \\
\hline Ave, O8.M Cost: (1987-92) & & & 2.208.156 & $\$ 88.914 .000$ & $\$ 12,3$ \\
\hline
\end{tabular}


Table 6.3-1 Average O\&M Costs for Selected BWR Plants (Continued)

\begin{tabular}{|c|c|c|c|c|c|}
\hline $\begin{array}{l}\text { PLANT NAME } \\
\text { (LOCATION) }\end{array}$ & Year & $\begin{array}{c}\mathbf{M W} \\
\text { (Gross) }\end{array}$ & $\begin{array}{c}\text { MW Hours } \\
\text { (net) }\end{array}$ & $\begin{array}{c}\text { O\&M COSTS } \\
\text { (expensed) }\end{array}$ & $\begin{array}{c}\text { \$MWHR } \\
\text { (mixed Year \$) }\end{array}$ \\
\hline $\begin{array}{l}\text { LASALLE } 1 \text { (IL) } \\
\text { LASALLE } 1 \text { \& } 2 \text { (IL) } \\
\text { LASALLE } 1 \text { \& } 2 \text { (IL) } \\
\text { LASALLE } 1 \text { \& } 2 \text { (IL) } \\
\text { LASALLE } 1 \text { \& } 2 \text { (IL) } \\
\text { LASALLE } 1 \text { \& } 2 \text { (IL) } \\
\text { LASALLE } 1 \text { \& } 2 \text { (IL) } \\
\text { LASALLE } 1 \text { \& } 2 \text { (IL) } \\
\text { LASALLE } 1 \text { \& } 2 \text { (IL) } \\
\text { LASALLE } 1 \text { \& } 2 \text { (IL) } \\
\text { AYe. O\&M Cog: (1983-92) }\end{array}$ & $\begin{array}{l}1983 \\
1984 \\
1985 \\
1986 \\
1987 \\
1988 \\
1989 \\
1990 \\
1991 \\
1992\end{array}$ & $\begin{array}{l}1170 \\
2341 \\
2341 \\
2341 \\
2341 \\
2341 \\
2341 \\
2341 \\
2341 \\
2341\end{array}$ & $\begin{array}{r}1,604,210 \\
8,408,610 \\
8,240,293 \\
7,735,131 \\
8,618,451 \\
11,100,841 \\
12,653,228 \\
14,821,165 \\
15,537,477 \\
12,234,374 \\
10,095,378 \\
\end{array}$ & $\begin{array}{r}\$ 35,381,000 \\
\$ 59,982,000 \\
\$ 74,801,000 \\
\$ 80,417,000 \\
\$ 101,455,000 \\
\$ 137,789,000 \\
\$ 123,010,000 \\
\$ 134,039,000 \\
\$ 150,438,000 \\
\$ 177,176,000 \\
\$ 107,499,000 \\
\end{array}$ & $\begin{array}{r}\$ 22.1 \\
\$ 7.1 \\
\$ 9.1 \\
\$ 10.4 \\
\$ 11.8 \\
\$ 12.4 \\
\$ 9.7 \\
\$ 9.0 \\
\$ 9.7 \\
\$ 14.5 \\
\$ 10.6 \\
\end{array}$ \\
\hline $\begin{array}{l}\text { LIMERICK } 1 \text { (PA) } \\
\text { LIMERICK } 1 \text { (PA) } \\
\text { LIMERICK } 1 \text { (PA) } \\
\text { LIMERICK } 1 \text { (PA) } \\
\text { LIMERICK } 1 \text { \& } 2 \text { (PA) } \\
\text { LIMERICK } 1 \text { \& } 2 \text { (PA) } \\
\text { LIMERICK } 1 \text { \& } 2 \text { (PA) } \\
\text { Ave. O\&M Cost: (1986-92) }\end{array}$ & $\begin{array}{l}1986 \\
1987 \\
1988 \\
1989 \\
1990 \\
1991 \\
1992\end{array}$ & $\begin{array}{l}1092 \\
1092 \\
1092 \\
1092 \\
2184 \\
2184 \\
2184\end{array}$ & $\begin{array}{r}6,848,745 \\
5,319,387 \\
6,674,070 \\
5,214,118 \\
12,853,807 \\
15,274,488 \\
14,718,242 \\
2.557,551 \\
\end{array}$ & $\begin{array}{r}\$ 48,687,000 \\
\$ 135,986,000 \\
\$ 124,896,000 \\
\$ 192,083,000 \\
\$ 184,283,000 \\
\$ 185,299,000 \\
\$ 196,798,000 \\
\$ 152,576,000 \\
\end{array}$ & $\begin{array}{r}\$ 7.1 \\
\$ 25.6 \\
\$ 18.7 \\
\$ 36.8 \\
\$ 14.3 \\
\$ 12.1 \\
\$ 13.4 \\
\$ 16.0 \\
\end{array}$ \\
\hline $\begin{array}{l}\text { NINE MILE POINT } 2 \text { (NY) } \\
\text { NINE MILE POINT } 2 \text { (NY) } \\
\text { NINE MILE POINT } 2 \text { (NY) } \\
\text { NINE MILE POINT } 2 \text { (NY) } \\
\text { NINE MILE POINT } 2 \text { (NY) } \\
\text { AYe. O\&M C0.4:(1988-92) }\end{array}$ & $\begin{array}{l}1988 \\
1989 \\
1990 \\
1991 \\
1992\end{array}$ & $\begin{array}{l}1214 \\
1214 \\
1214 \\
1214 \\
1214\end{array}$ & $\begin{array}{l}2,755,012 \\
4,290,994 \\
4,125,001 \\
6,547,710 \\
5,118,087 \\
4.567,361 \\
\end{array}$ & $\begin{array}{r}\$ 69,999,000 \\
\$ 86,546,000 \\
\$ 134,640,000 \\
\$ 111,765,000 \\
\$ 133,754,000 \\
\$ 107,341,000 \\
\end{array}$ & $\begin{array}{l}\$ 25.4 \\
\$ 20.2 \\
\$ 32.6 \\
\$ 17.1 \\
\$ 26.1 \\
\mathbf{\$ 2 3 . 5} \\
\end{array}$ \\
\hline $\begin{array}{l}\text { PERRY } 1(\mathrm{OH}) \\
\text { PERRY } 1(\mathrm{OH}) \\
\text { PERRY } 1(\mathrm{OH}) \\
\text { PERRY } 1(\mathrm{OH}) \\
\text { PERRY } 1(\mathrm{OH}) \\
\text { PERRY } 1(\mathrm{OH}) \\
\text { Are. O\&NM Cost: (1987-92) } \\
\end{array}$ & $\begin{array}{l}1987 \\
1988 \\
1989 \\
1990 \\
1991 \\
1992\end{array}$ & $\begin{array}{l}1205 \\
1205 \\
1253 \\
1253 \\
1253 \\
1253\end{array}$ & $\begin{array}{l}1,330,353 \\
7,255,514 \\
5,342,811 \\
6,608,626 \\
8,980,005 \\
7,169,866 \\
6.114 .529 \\
\end{array}$ & $\begin{array}{r}\$ 40,486,000 \\
\$ 118,350,000 \\
\$ 160,318,000 \\
\$ 156,211,000 \\
\$ 118,642,000 \\
\$ 117,351,000 \\
\$ 118,560,000 \\
\end{array}$ & $\begin{array}{l}\$ 30.4 \\
\$ 16.3 \\
\$ 30.0 \\
\$ 23.6 \\
\$ 13.2 \\
\$ 16.4 \\
\$ 19.4 \\
\end{array}$ \\
\hline $\begin{array}{l}\text { RIVER BEND (LA) } \\
\text { RIVER BEND (LA) } \\
\text { RIVER BEND (LA) } \\
\text { RIVER BEND (LA) } \\
\text { RIVER BEND (LA) } \\
\text { RIVER BEND (LA) } \\
\text { RIVER BEND (LA) } \\
\text { Ave. O\&M Cost: (1986-92) }\end{array}$ & $\begin{array}{l}1986 \\
1987 \\
1988 \\
1989 \\
1990 \\
1991 \\
1992\end{array}$ & $\begin{array}{l}1035 \\
1036 \\
1036 \\
1036 \\
1036 \\
1036 \\
1036\end{array}$ & $\begin{array}{l}3,038,203 \\
4,898,191 \\
7,248,981 \\
4,785,009 \\
5,592,554 \\
6,687,201 \\
2,762,680 \\
\mathbf{5 . 0 0 1 . 8 3 1} \\
\end{array}$ & $\begin{array}{r}\$ 64,651,000 \\
\$ 120,891,000 \\
\$ 109,374,000 \\
\$ 127,640,000 \\
\$ 108,597,000 \\
\$ 138,093,000 \\
\$ 166,445,000 \\
\$ 112,384,000 \\
\end{array}$ & $\begin{array}{l}\$ 21.3 \\
\$ 24.7 \\
\$ 15.1 \\
\$ 26.7 \\
\$ 19.4 \\
\$ 20.7 \\
\$ 60.2 \\
\$ 23.9 \\
\end{array}$ \\
\hline
\end{tabular}


NEDO-32361

Table 6.3-1. Average O\&M Costs for Selected BWR Plants (Continued)

\begin{tabular}{|c|c|c|c|c|c|}
\hline $\begin{array}{l}\text { PLANT NAME } \\
\text { (LOCATION) }\end{array}$ & Year & MW (Gross) & MW Hours(net) & $\begin{array}{c}\text { O\&M COSTS } \\
\text { (expensed) }\end{array}$ & $\begin{array}{c}\text { \$MWHR } \\
\text { (mixed Year \$) }\end{array}$ \\
\hline SUSQUEHANNA 1 (PA) & 1983 & 1152 & $3,536,373$ & $\$ 34,305,000$ & $\$ 9.7$ \\
\hline SUSQUEHANNA 1 (PA) & 1984 & 1152 & $6,088,141$ & $\$ 70,502,000$ & $\$ 11.6$ \\
\hline SUSQUEHANNA $1 \& 2$ (PA) & 1985 & 2291 & $12,218,478$ & $\$ 123,031,000$ & $\$ 10.1$ \\
\hline SUSQUEHANNA $1 \& 2$ (PA) & 1986 & 2291 & $11,278,509$ & $\$ 131,373,000$ & $\$ 11.6$ \\
\hline SUSQUEHANNA $1 \& 2$ (PA) & 1987 & 2291 & $14,761,343$ & $\$ 133,580,000$ & $\$ 9.0$ \\
\hline SUSQUEHANNA $1 \& 2$ (PA) & 1988 & 2291 & $14,296,639$ & $\$ 159,847,000$ & $\$ 11.2$ \\
\hline SUSQUEHANNA $1 \& 2$ (PA) & 1989 & 2304 & $13,239,807$ & $\$ 163,520,000$ & $\$ 12.4$ \\
\hline SUSQUEHANNA $1 \& 2$ (PA) & 1990 & 2304 & $14,726,700$ & $\$ 160,279,000$ & $\$ 10.90$ \\
\hline SUSQUEHANNA $1 \& 2$ (PA) & 1991 & 2304 & $15,857,002$ & $\$ 180,180,000$ & $\$ 11.4$ \\
\hline SUSQUEHANNA 1 \& 2 (PA) & 1992 & 2304 & $13,573,637$ & $\$ 170,304,000$ & $\$ 12.5$ \\
\hline Ave O8M Cost: (1983-92) & & & 11.957 .663 & $\$ 132.692 .000$ & $\$ 11.1$ \\
\hline WNP 2 (WA) & 1985 & 1200 & $2,556,559$ & $\$ 40,299,000$ & $\$ 15.8$ \\
\hline WNP 2 (WA) & 1986 & 1200 & $4,363,364$ & $\$ 67,358,000$ & $\$ 15.4$ \\
\hline WNP 2 (WA) & 1987 & 1200 & $5,519,482$ & $\$ 67,106,000$ & $\$ 12.2$ \\
\hline WNP 2 (WA) & 1988 & 1200 & $5,944,781$ & $\$ 72,213,000$ & $\$ 12.1$ \\
\hline WNP 2 (WA) & 1989 & 1200 & $6,034,275$ & $\$ 84,114,000$ & $\$ 13.9$ \\
\hline WNP 2 (WA) & 1990 & 1200 & $6,497,925$ & $\$ 92,291,000$ & $\$ 14.2$ \\
\hline WNP 2 (WA) & 1991 & 1200 & $5,670,059$ & $\$ 95,436,000$ & $\$ 16.8$ \\
\hline WNP 2 (WA) & 1992 & 1200 & $3,799,206$ & $\$ 101,398,000$ & $\$ 26.7$ \\
\hline Axe. O\&M Cost: (1985-92) & & & 5.048 .206 & $\$ 77.527 .000$ & $\$ 15.4$ \\
\hline
\end{tabular}

Source Of Data:

Utility Data Institute 
Table 6.3-2 BWR Plant Incremental O\&M Costs for Full MOX Core Operations

(Over and above normal O\&M cost levels for LEU operation)

(1/93 base date)

Onsite Staff

\begin{tabular}{|r|}
\hline $\mathbf{K} \$ \mathbf{y r}$ \\
\hline 3,020 \\
868 \\
\\
\\
813 \\
0 \\
TBD \\
TBD \\
703 \\
\hline 5,404 \\
\hline
\end{tabular}

Total O\&M

(1) Estimates for costs related to IAEA inspections not available.

(2) Insurance estimates not available, costs may be higher than for LEU operation.

(3) Includes average cost of acquisition \& maintenance of ' $Q$ ' clearances. 
Table 6.3-3 Estimate of O\&M Costs for MOX and Spent Fuel Storage Facillties Three Unit Reference Case

(1/93 base date)

\begin{tabular}{|c|c|c|c|}
\hline \multirow[b]{2}{*}{ Item and Description } & \multicolumn{2}{|c|}{ Site Labor } & \multirow{2}{*}{$\begin{array}{l}\text { Total } \\
\text { Cost } \\
\text { (K\$) }\end{array}$} \\
\hline & $\begin{array}{l}\text { Man- } \\
\text { hours }\end{array}$ & $\$ \times 1000$ & \\
\hline $\begin{array}{l}\text { Annual Operation Hours: Spent Fuel Storage } \\
\text { - } 10 \text { people for 5-day operation @ } 4 \text { times/yr/Rx } \\
\text { - includes SS/INS/Overhend @ } 70 \% \\
\text { - includes Pension / Benefits @ 25\% }\end{array}$ & 1600 & 93.6 & 93.6 \\
\hline $\begin{array}{l}\text { Annual Operation Hours: MOX Fuel Storage } \\
\text { - } 6 \text { people for 1-day operation @ } 18 \text { times/yr/Rx } \\
\text { - includes SS/INS/Overhead @ } 70 \% \\
\text { - includes Pension/Benefits @ 25\% }\end{array}$ & 1100 & 64.4 & 64.4 \\
\hline $\begin{array}{l}24 \text { Hour Guard Service @ Gatehouse ( } 365 \text { days) } \\
\text { - includes } 10 \% \text { for SS/INS } \\
\text { - includes Pension/Benefits @ 25\% }\end{array}$ & 8760 & 177.4 & 177.4 \\
\hline $\begin{array}{l}\text { Other Administration/General Expenses } \\
\text { - includes Materials, Supplies, etc. }\end{array}$ & & 67.1 & 67.1 \\
\hline
\end{tabular}

\begin{tabular}{|l|l|l|l|}
\hline TOTAL O\&M COSTS (Single Unit) & 11,460 & 402.4 & 402.4 \\
\hline TOTAL O\&M COSTS (Three Unit Reference Case) & 26,600 & 996.8 & 996.8 \\
\hline
\end{tabular}


Table 6.3-4 Reference Case MOX Fuel Fabrication Facility Operating and Maintenance Cost Summary

( $1 / 93$ base date)

\begin{tabular}{|l|c|}
\hline Item & Estimated Cost (WM $/ \mathbf{y r}$ \\
\hline Manpower & 23.4 \\
\hline Direct Manpower & 3.4 \\
\hline Benefits & 2.3 \\
\hline Site Support & 0.5 \\
\hline Supplies & \\
\hline Maintenance Materials & 0.8 \\
\hline Equipment Replacement & 0.6 \\
\hline Maintenance Materials & 13.3 \\
\hline Assembly Hardware & 1.1 \\
\hline LWW Processing and Disposal & 5.8 \\
\hline TRU Waste Processing and Disposal & $\mathrm{N} / \mathrm{A}^{*}$ \\
\hline Decommissioning Cost & 51.2 \\
\hline TOTALO\&M COST & \\
\hline
\end{tabular}

Note: TRU waste disposal costs are assumed to be approximately equivalent to the $1 \mathrm{mill} / \mathrm{kW}$-hr charge for high level waste since TRU waste will be disposed of in a geological repository (WIPP) similar to the planned Yucca Mountain Repository for high level waste.

* Decommissioning costs are estimated separately (Section 6.4.4.2)

Table 6.3-5 Estimated O\&M Expenditures for LANL Island Fuel Fabrication Facility (1/93 base date)

\begin{tabular}{|l|c|c|c|c|c|c|}
\hline \multirow{2}{*}{\multicolumn{1}{|c|}{ Item }} & \multicolumn{5}{|c|}{ O\&M Expenditures } & Total \\
\cline { 2 - 6 } & 1995 & 1996 & 1997 & 1998 & 1999 & Cost (K\$) \\
\hline Labor: Staff & 2,500 & 3,250 & 4,000 & 4,000 & 4,000 & 17,750 \\
Labor: Technicians & 3,300 & 4,500 & 5,700 & 5,700 & 5,700 & 24,900 \\
\hline
\end{tabular}

\begin{tabular}{|l|l|l|l|l|l|l|}
\hline Total Cost $(\mathbf{K} \$)$ & $\mathbf{5 , 8 0 0}$ & $\mathbf{7 , 7 5 0}$ & $\mathbf{9 , 7 0 0}$ & $\mathbf{9 , 7 0 0}$ & $\mathbf{9 , 7 0 0}$ & 42,650 \\
\hline
\end{tabular}

Note: An additional $\$ 5$ million O\&M allowance is included in the reference and alternate case cash flows (Table 6.4-3 and Table 10.3-2) to account for island fuel fabrication demobilization. 


\subsection{Other Life Cycle Cost Detall}

This section covers data requested by DOE for life cycle cost analyses of the proposed reference case. The general format and content follows the ABWR study [Ref. 6-1] with appropriate adjustments and modifications to the data to reflect the current study bases of the use of existing reactors and early use of government facilities at LANL.

\subsubsection{Preoperational Costs}

Preoperational costs as defined in the ABWR study Requirements Document are presented in Table 6.4-1. Separate estimates are shown for the BWR plant and the MOX fuel fabrication facility. The bases for the various elements of preoperational costs provided in the tables include:

- Pre-Title I Engineering Costs - These costs apply only to the MOX fuel fabrication facility for a brief period of conceptual design as shown on the project schedule. The estimate is based on the combined actual experience of BNFL with MOX fuel fabrication facilities in the UK and GE/Bechtel experience with private and government fuel fabrication facilities and similar fuel cycle projects in the United States.

- Safety and Environmental Approval Costs - These costs are estimated based on the combined experience of GE, Bechtel, and BNFL with both the NRC and the UK licensing processes. Experience with the safety/environmental approval process in both DOE government projects (including the uavannah River Plant) and commercial projects.

- Startup/Operational Readiness Review - These costs are based on GE, Bechtel, and BNFL experience for similar government and commercial nuclear projects.

Table 6.4-1 Preoperational Costs

\begin{tabular}{|l|c|c|}
\hline \multirow{2}{*}{ Item } & \multicolumn{2}{|c|}{ Estimated Cost (\$n millions, 193) } \\
\cline { 2 - 3 } & BWR Plant & FullMOX Fuel Facility \\
\hline Pre-Title I Engineering (Conceptua) & 0 & 7 \\
\hline Safety/Environmental Approvals* & 10 & 15 \\
\hline Startup/Op. Readiness Rev. & $5^{* *}$ & 26 \\
\hline
\end{tabular}

* For the BWR plant, safety/environmental approval costs are included in the engineering and licensing estimate shown in Table 6.2-2.

** Per unit. 


\subsubsection{Development Costs}

As stated in Section 3 of this report, the BWR plutonium disposition project does not require any basic research and only limited confirmation testing due to the inherent flexible design of the reactor, the extensive MOX fuel testing previously done in the U.S. and abroad, and the actual use of MOX fuel in light water reactors.

Table 6.4-2 summarize s the costs for the few development programs required. Separate costs are shown for the BWR Plant and the MOX fuel fabrication facility.

\subsubsection{Capital Replacement Cost}

Only very small incremental capital replacement costs are expected as a result of conversion of any of the existing BWRs to MOX operation. For the BWR plant, an allowance of $\$ 0.5$ million per year per unit has been provided to account for such items as replacement of security devices and computers and for replacement of fuel handling tools in excess of those replacements that would be necessary under LEU operation. For the MOX facility, allowances for capital replacement are the same as those implemented in the ABWR study [Ref. 6-1].

Table 6.4-2 BWR Development Program Costs (1/93 base date)

\begin{tabular}{|l|r|}
\hline Item Description & $\begin{array}{c}\text { Estimated Cost } \\
\text { (K\$) }\end{array}$ \\
\hline BWR Plant & 240 \\
- Lasertrac System & 1,200 \\
- Automated Assembly Inspection & 120 \\
- Reactor Internals Radiation Effects & 2,600 \\
- Full MOC New Fuel Shipping Containers & $\mathbf{4 , 1 6 0}$ \\
Total - BWR Plant & 14,700 \\
\hline MOX Fuel Fabrication Facility & 13,300 \\
- Fuel Testing: Rod Testing & 1,200 \\
- Fuel Testing: Lead Use Assembly & 240 \\
- MOX Plant Automation Evaluations & 350 \\
- Line-Glove Box Hybrid Evaluations & 710 \\
- Disposition Process Simulation & $\mathbf{3 0 , 5 0 0}$ \\
- Accountability Equipment & \\
Total - MOX Facility &
\end{tabular}

\subsubsection{Decommissioning Cost}

\subsubsection{BWR Plant Decommissioning}

For the purposes of this study, the reference BWR plant is assumed to have approximately the same decommissioning costs whether it has operated totally on LEU fuel or whether it has 

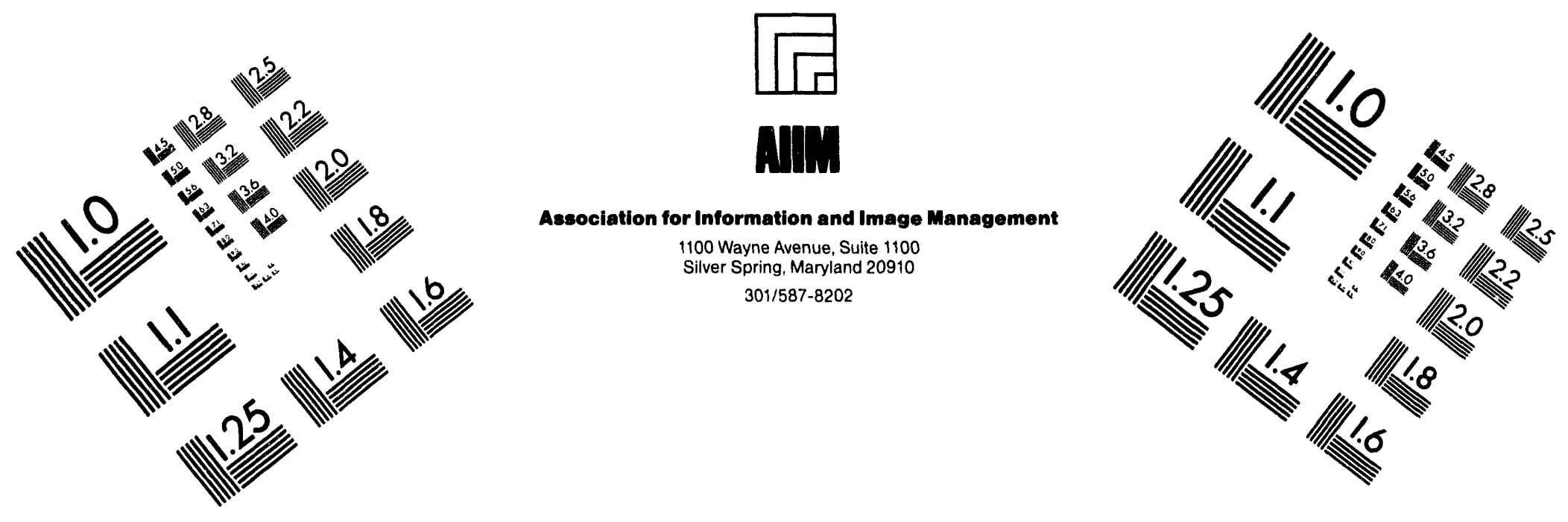

\section{Centimeter}

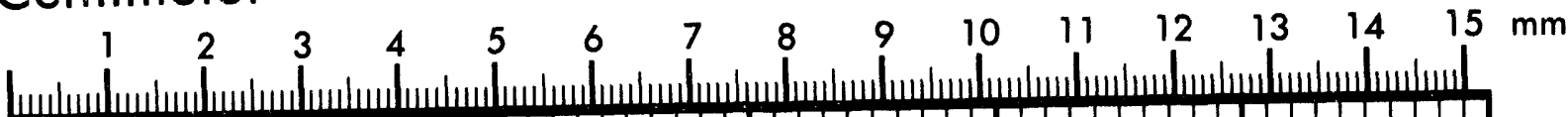

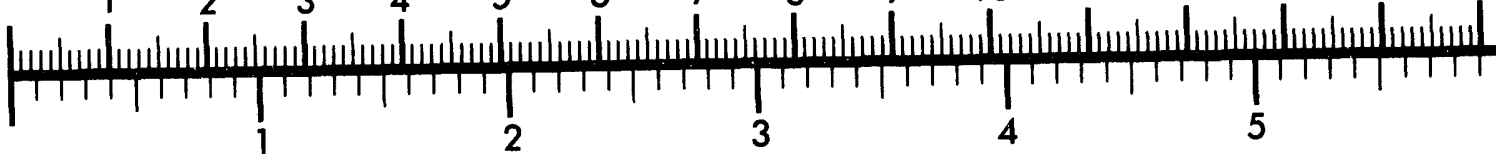
Inches

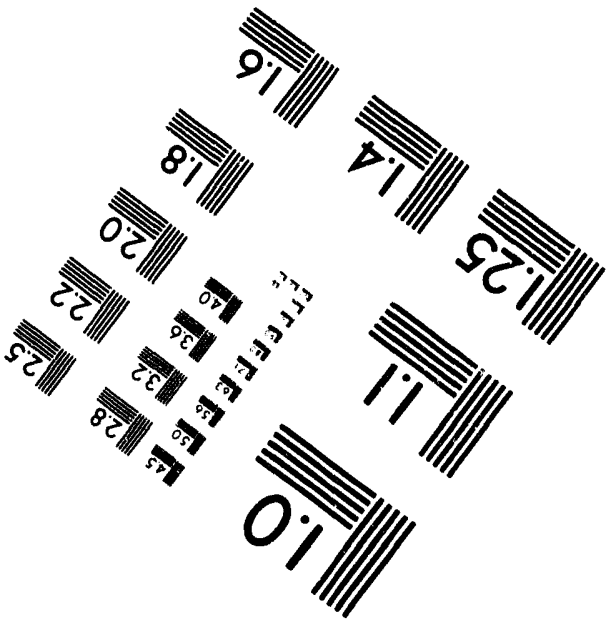

MANUFACTURED TO AIIM STANDARDS

$$
\text { BY APPLIED IMAGE, INC. }
$$

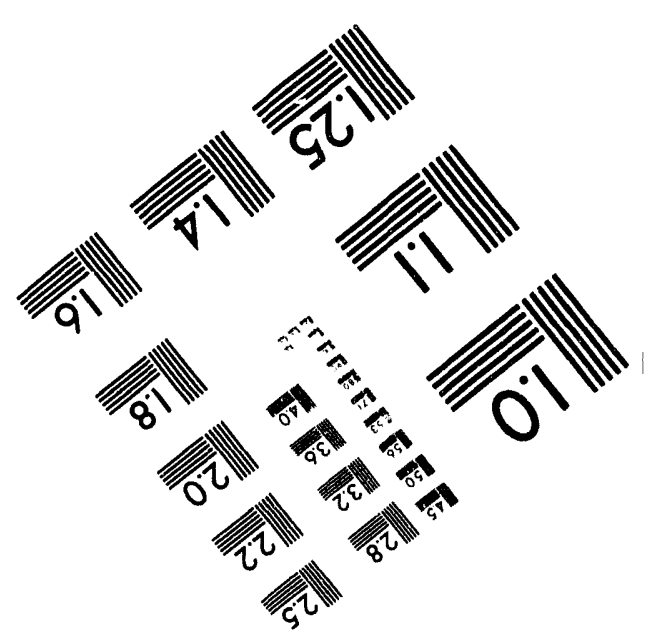



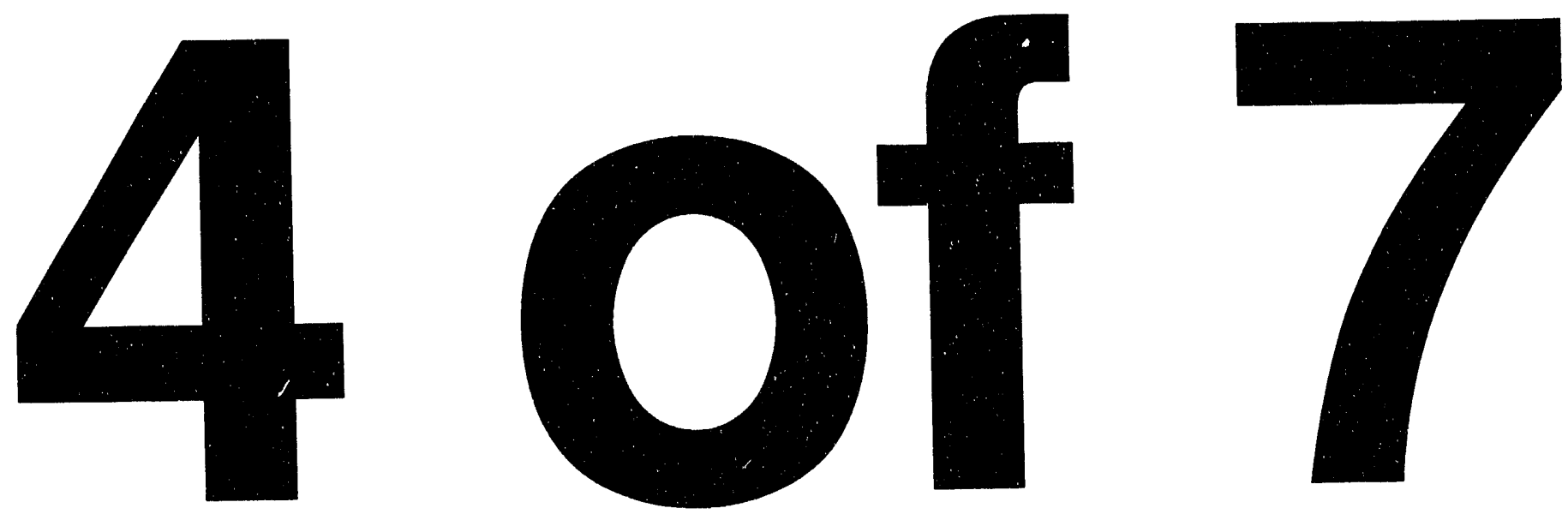
operated for part of its lifetime on MOX fuel. Therefore, incremental decommissioning costs for MOX fueled plants are assumed to be zero. If during the plant lifetimes there are fuel leaks, the decontamination costs are likely to be similar for either LEU or MOX fueled plants. Likewise, if there are no fuel leaks, decontamination will be essentially the same. For the ABWR disposition study, the formulas from the ORNL guidelines were used to estimate ABWR decommissioning costs since more definitive decommissioning estimates are not now available. At this point in time, the relative uncertainties involved in decommissioning cost estimates are high enough that discrimination between LEU and MOX burning plant costs is not within the accuracy of the available data. Also D\&D costs for existing plants will be significantly dependent upon operating histories before conversion to MOX operation and upon plant unique configurations. Therefore, each existing plant will have uniquely different decommissioning conditions and costs.

Recent decommissioning cost studies have indicated that the D\&D operation cost has remained essentially unchanged over the last ten years. However, the cost of low level waste disposal has shown an increase. This is a local (regional) issue that will likely depend on the timing of the plutonium disposition project.

\subsubsection{MOX Fuel Fabrication Facility Decommissioning}

An allowance of $10 \%, \$ 53$ million of initial capital cost, has been made for decommissioning of the full MOX fuel fabrication facility. This cost estimate is approximately the same as the estimate included in the ABWR disposition report. It is assumed that there will be no additional costs for decommissioning of the LANL fabrication facility, since that facility will have to undergo decontamination and decommissioning whether or not it is used in this particular program.

\subsubsection{New and Spent Fuel Storage Facility Decommissioning}

An allowance of $10 \%, \$ 4$ million of initial capital cost (per unit), has been made for decommissioning of the fuel storage facility. It is assumed that the facility will be decommissioned ten years after the BWR plant since there is a requirement for ten years fuel storage after removal from the reactor and before shipment to a long-term storage facility. This cost allowance may be conservatively high since no residual radioactive contamination is expected in the facility after removal of the stored fuel. 


\subsubsection{Cash Flows}

Table 6.4-3 shows the overall cash flow for the three unit reference case for 50 MT of plutonium disposition. The fuel cycle lengths and durations of reactor operations utilized to estimate the costs of uranium fuel and transportation shown in the cash flow table are approximate.

\subsubsection{Plant License Renewal Considerations}

\subsubsection{Overview}

The Atomic Energy Act of 1954 limits a nuclear power plant's license to a maximum of 40 years. Although the act permits the renewal of an operating license, it does not outline any standards or procedures for determining when or under what conditions a plant's operating license should be renewed. The Nuclear Regulatory Commission (NRC) proposed a rule in July 1990 , finalized in December 1991, that provided provisions to license a plant beyond 40 years of operation.

One of the factors that may impact the plant selection process for the plutonium disposition project is the amount of time remaining on the initial 40-year licensed life of the plant. Part of the selection process will eliminate those plant(s) that have insufficient remaining license life to perform the plutonium disposition project in support of the DOE objectives.

It is possible that a license renewal program, if implemented quickly, could justify the selection of certain BWR plants that would otherwise be eliminated because of insufficient remaining license life to carry out the project to completion with potentially fewer reactors and/or sites. For the purposes of the plutonium disposition project, such a program would have to be available near term in order to derive any benefits. The expectation is that the license renewal granted by the NRC would be for a period of 20 years.

While a few nuclear plants have pursued studies on life extension, no lead plants have yet submitted applications for license renewal, and industry experience so far with the rule 10 Code of Federal Regulations Part 54 (10CFR54), is that the requirements for life extension are not clearly defined by 10CFR54. Consequently, while some lead plants (e.g., Northern States Power Co's Monticello, the BWR lead plant) have expended considerable time and resources on license 
Table 6.4-3 Cash Flow - Incremental Cost

Reference Case - 3 BWR Units, 50MT Disposition

( $\$$ in millions, $1 / 93$ base date)

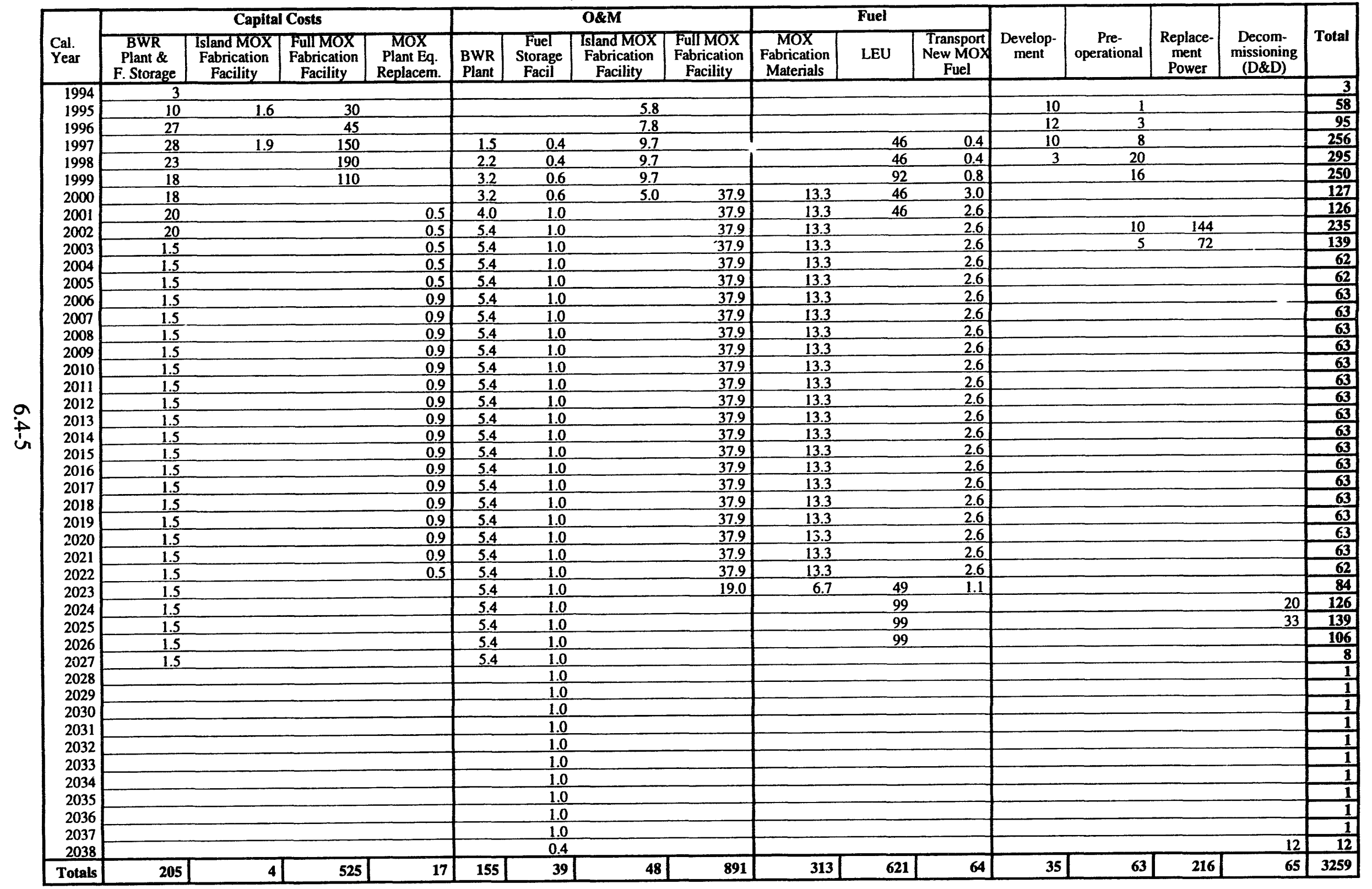


renewal feasibility studies, nuclear utilities have been reluctant to submit license renewal applications because of the ongoing uncertainty surrounding the 10CFR54 process. The NRC Commissioners have directed the Staff to revise and/or clarify the requirements of 10CFR54 by the end of 1994. The revised license renewal rule is expected to make the process of applying for plant life extension simple, cost effective, and predictable. NRC's revised license renewal rule is supposed to lean heavily on the maintenance rule (scheduled to take effect in July 1996) as a way for utilities to handle most plant aging issues. Although the above expected changes and clarification of the requirements specified by 10CFR54 would potentially open the door to utilities seeking license renewal, it would be premature to conclude that the new regulation guidelines could be implemented in the near term. If the guidelines are clarified, it may be a reasonable assumption that a plutonium disposition selectee could initiate and complete the license renewal process and have a license renewal in place within a five year time frame.

\subsubsection{Costs of License Renewal}

The nuclear industry, the U.S. Department of Energy, and many other entities have evaluated the benefits and costs of nuclear plant life extension (NUPLEX) for many years. These studies have attempted to estimate the likely costs associated with NUPLEX and the break-even costs. Relevant life-cycle costs of NUPLEX include the following:

- All typical operating and maintenance costs.

- Additional costs of refurbishment required to meet regulatory standards as well as desired operating performance.

- Incremental license renewal requirements imposed by NRC. This includes ISTM (inspection, surveillance, testing, and monitoring) of structures and systems that are affected by age.

- Replacement power costs associated with any down time due to refurbishment.

- $\quad$ Allowance for funds used during construction (AFUDC).

The pilot plant life extension studies of capital costs indicated that total costs could range from $\$ 237$ to $\$ 635 / \mathrm{kW}$ (1989 dollars). The costs for refurbishment ranged from $\$ 82$ to $\$ 301 / \mathrm{kW}$. The balance of the costs include replacement power ( $\$ 111$ to $\$ 233 / \mathrm{kW}$ ) and AFUDC which ranged from $\$ 44$ to $\$ 101 / \mathrm{kW}$ [Ref. 6-5]. 


\subsubsection{Current Status and Cost Estimate for a Typical BWR}

Many utilities are working with their owners groups to assess the license renewal program. For example, the GE BWR Owners Group (BWROG) formed a generic license renewal committee in June 1993. Due to the rule uncertainty, there is no commitment from a plant for an application at this time. The program plan appears to be structured on working with NRC on a reference plant basis.

The Nuclear Energy Institute, acting on behalf of the industry, is working to simplify the license renewal rule [Ref. 6-6] and to take credit for effective maintenance programs in place at operating plants. This effort to simplify the rule is being supported by the NRC Commissioners and staff.

The expenditures necessary for licensing depend on the complexity of the NRC regulation and the amount of time for a public hearing. Northern States Power expended approximately $\$ 11$ million to prepare its application and anticipated spending an equivalent amount to obtain a license for an additional 20 years of operation at Monticello. Baltimore Gas \& Electric (BG\&E) is reported to have expended about $\$ 15$ million on a combined life cycle management and license renewal program, with $\$ 8$ million on life extension work. BG\&E projected that it will cost another $\$ 4$ million to get an application to the NRC.

It is estimated that, if the new license renewal rule is successful, the costs for an application would amount to between $\$ 5$ and $\$ 10$ million.

Additional operating and maintenance (O\&M) and capital costs can be anticipated depending on the physical condition of the plant and the effectiveness of existing aging management programs. The Monticello program estimated that there would be no increase in operating and maintenance costs and that the additional capital costs needed to support extended operation would amount to $\$ 30$ million over the last 20 years of operation. No extended outages would be necessary because the work could be done during normal refueling and maintenance outages.

The estimated order of magnitude capital costs for extending the operating term of a typical BWR facility could be between $\$ 80$ million to $\$ 120$ million in 1993 dollars. Additional capital costs may be necessary for potential replacement power costs and AFUDC on the overnight cost. To minimize replacement power costs, refurbishment could be timed to take place during normal plant downtime to the maximum extent feasible. However, some normal maintenance periods will likely be extended. Also, some additional O\&M costs will be required for using full core MOX fuel. 
The owner of a BWR utility might derive an economic benefit from a decision to renew a license by potentially reducing annual contributions to the decommissioning trust, since the fund will not be needed for 20 more years.

\subsubsection{Plant License Recovery Variation}

With the uncertainty of license extension being available for the project, there is an optional approach available to Tennessee Valley Authority's (TVA) Browns Ferry units that would extend their current license life and provide sufficient added project time to allow the three units to meet the $50 \mathrm{MT}$ goal for the project. This program, known as license recovery, is currently under evaluation by TVA for implementation on Browns Ferry 1, 2, and 3. The basis of the program is to recover the time associated with the extended shutdown of the Browns Ferry units on the operating licenses, adding approximately 13,8 , and 10 years to the Unit 1,2 and 3 operating licenses, respectively. The program is considered both technically and legally feasible, based on the existing precedence of several operating plants recovering the elapsed time between receipt of their operating license and first power operations. When considered in conjunction with the significant upgrade programs (Appendix R, Environmental qualification, etc.) required to be complete prior to the restart of any Browns Ferry unit, approval by the NRC is considered a high probability by TVA and GE. Costs to implement the program are under final development, but are considered to be less than a life extension application. 
NEDO-32361

\subsection{Anticipated Cost Risks}

The use of existing reactors to dispose weapons plutonium may involve lower cost risks than other disposition options. Since the various cost categories associated with the reactor plant can be evaluated with a relatively high level of confidence, the risk associated with the disposition cost should be minimal. Similarly, the costs associated with the construction and operation of the MOX fuel plant can be evaluated with confidence based on European experience. This should in turn minimize the cost risks associated with the MOX plant. In this context, the demonstration program with the 'island' core design proposed in this study should surface any serious discrepancies from the initial project estimates when full scale disposition with the full MOX core is implemented and corrective actions could be implemented. While this observation is valid in general, there are specific areas where cost risks could arise and they are described below.

\subsubsection{Funding Availability for the Project}

Although the National Academy of Sciences (NAS) study [Ref. 6-7] came to the conclusion that the method for disposition of weapons plutonium should not be chosen based on cost per se, funding availability to implement the project should be considered high on the list of potential cost risks. A shortfall in funding anytime during the project implementation phase will impact the schedule and this in turn, could leai to increased overall cost to complete the project. Any expectation of funding shortfalls could also impact the willingness of a prospective utility to participate in the program.

\subsubsection{MOX Fuel Fabrication Plant Construction and Operation}

MOX fuel is currently being fabricated in Europe. A detailed evaluation of the construction and operating costs in the European plants and their applicability to this project should be undertaken in the follow-on phase of this study to arrive at the final cost estimate. A study by BNFL on the differences between MOX fuel fabrication using weapons plutonium and reprocessed plutonium indicated that higher MOX plant availability and lower worker exposure could result from the use of weapons plutonium as it is less radioactive than reprocessed plutonium. It is therefore judged that the cost risk, once a more definitive estimate is made based on the European experience, is minimal.

The O\&M costs for the MOX facility are dependent on the worker radiation exposure levels and the degree of equipment mechanization for normal operation. The use of equipment items of modular construction which can be easily and quickly replaced with minimal exposure to 
workers should tend to reduce maintenance activities. Key equipment items (i.e., sintering furnace) have been designed to minimize maintenance activities providing for further reduction in maintenance costs. The performance of the MOX factory is expected to be better than European experience for worker exposure and plant availability and therefore the cost risks computed based on the European experience should bound the anticipated risks in this category for the disposition project.

\subsubsection{Reactor O\&M Costs}

The O\&M costs for operating the reactor in the disposition mode reported here reflect only the additional costs arising from MOX fueled reactor operation. The underlying O\&M costs for commercial urania fueled operations are highly plant specific and will have been taken into account in the economic incentive agreements between the reactor owner and the government. Cost risks that could be ascribed to the additional costs incurred for operating with the MOX fueled core are considered minimal. In general, plant O\&M costs per kilowatt are being reduced by improvement in capacity factor and by stabilization and reduction of plant staffing levels. Therefore, continuing this trend in O\&M cost reduction should be viewed as a positive potential.

\subsubsection{Completion of Incomplete Plant, Renewal of Plant License, Replacement or Retrofit of Capital Equipment in the Reactor Plant}

In considering the existing plants that can be used for disposition, two broad categories can be distinguished. The first category consists of plants which are currently operating (with urania fuel) and the second category consists of partially completed plants as well as plants that are not operational at this time. In the case of the partially completed plants, while the construction license might permit completion of these plants, an operating license might still be required, as the one-step licensing procedure for both construction and operation was not implemented for the older plants.

The costs associated with bringing the second category of plants on-line, should one or more of these be chosen for the disposition project, cannot be estimated with precision at this time. The cost will be highly plant specific. More quantitative data in this regard can only be generated in a detailed phase of the study after specific plants (of the second category) are identified for this project.

\subsubsection{Licensing Costs}

Licensing the reactor plant for MOX fuel, if the plant is currently operating with urania fuel, should be relatively straightforward. The MOX fuel has been designed so that it will stay within 
the overall licensing envelope for urania fuel. The evaluations in this report indicate that the urania licensing criteria are valid for MOX fuel. However, if the plant is not currently operating, a licensing cost should be associated for use with the disposition project. The licensing cost will be very nearly what it would take to license urania fuel, as the additional expenses to license MOX fuel are considered minimal. The risk associated with the licensing cost of a plant not operational at the present time is plant specific and can only be evaluated after specific plants are identified for the disposition project. A significant licensing cost increase and schedule delay could occur if the licensing process and requirements are changed from the basis currently assumed, e.g. if expanded NRC review or additional public hearings are required.

\subsubsection{Non-Availability of MOX Fuel}

It is conceivable that MOX fuel may not be available on the projected schedule for a variety of reasons including delays in MOX plant construction or non-availability of plutonium feed material. The cost risk associated with such a scenario is easily estimated. This would be the cost of replacement urania fuel to continue running the reactor until such time MOX fuel becomes available. If MOX fuel is unavailable at the planned start date for the disposition operations, it is possible to delay the start of disposition operations and incur only a minor cost risk. If, however, MOX fuel becomes unavailable during the disposition project, it is likely that the cost risks may have to be shared for the replacement urania fuel. These costs are offset by the revenues from the sale of electricity generated by the plant, regardless of the type of fuel burned.

\subsubsection{Capacity Factor}

The study assumes a capacity factor of $75 \%$. Most of the recent plants equal or better this value. However, it is conceivable that some of the older plants may not have this high a capacity factor. There is certainly a schedule risk associated with a lower capacity factor as the plutonium will be disposed of at a slower rate.

\subsubsection{Schedule Delays}

Schedule delays and extensions can result in significant increases in cost. The risks of schedules delays are discussed in Section 6.7. 
r.

\subsection{Plutonium Disposition Project Schedule}

The mission for the reference case of the plutonium disposition project is to disposition $50 \mathrm{MT}$ of weapons-grade plutonium utilizing safe, effective technology and methods that are acceptable to both the United States and Russia. The recommended approach includes a preliminary mixed oxide island design fuel loading in July 1997, which allows for an early demonstration of U.S. commitment to the plutonium disposition mission and a full MOX fuel design loading in April 2001 to achieve the mission's desired throughput. Effective project management and thorough activity coordination are required to complete the mission in a timely manner. Thorough analysis of project's activities resulted in the integrated project schedule detailed in Table 6.6-1. Based on this detail of the project's major element, activities, and milestones, Figure 6.6-1 provides an overview of the project schedule which is both realistic and achievable.

\subsubsection{Schedule Development and General Bases and Assumptions}

\subsubsection{Schedule Development}

The plutonium disposition project schedule (Figure 6.6-1 and Table 6.6-1) was developed for dispositioning $50 \mathrm{MT}$ of weapons-grade plutonium using existing BWR capabilities. This project requires the adaptation of existing national laboratory capacity to fabricate the initial fuel rods and assemblies required for the preliminary rod testing program, the lead use assembly (LUA) program, and a demonstration program utilizing a MOX island design. Full MOX fuel assembly fabrication requires design, licensing, construction, and pre-operational testing of a MOX fuel fabrication facility. Figure 6.6-2 shows the schedule of these fuel related activites.

The project schedule was developed using the following methodology:

- Major project elements and associated assumptions were defined.

- Tasks associated with each major project element were specified.

- Previous schedules and experience with similar tasks were reviewed and, literature surveys were conducted.

- The duration of the each specific task was estimated based on the integrated information.

- The schedule was reviewed with plutonium disposition project and outside experienced personnel. 


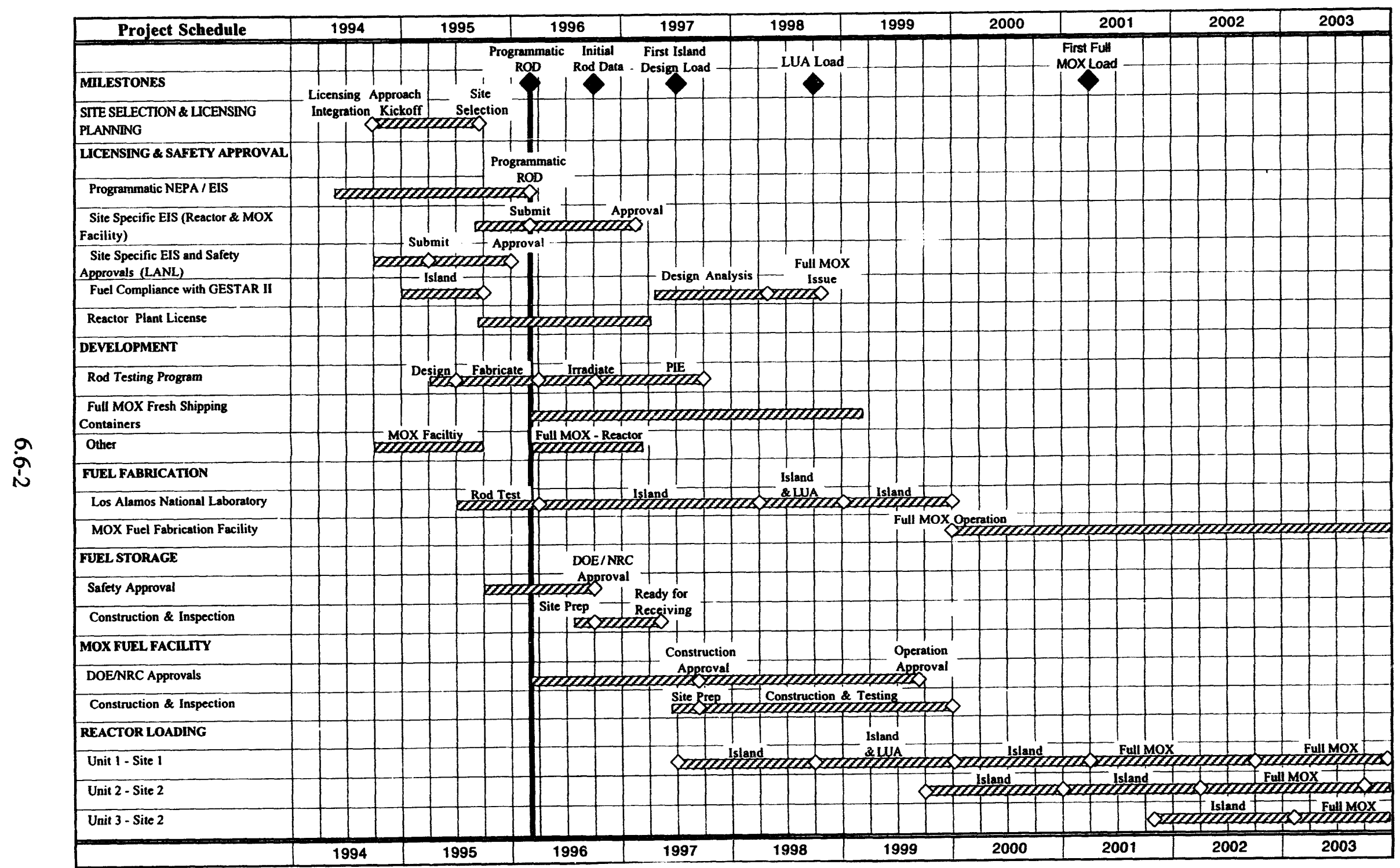

Figure 6.6-1 Overall Project Schedule for Dispositioning 50 MT Plutonium using Three BWRs 


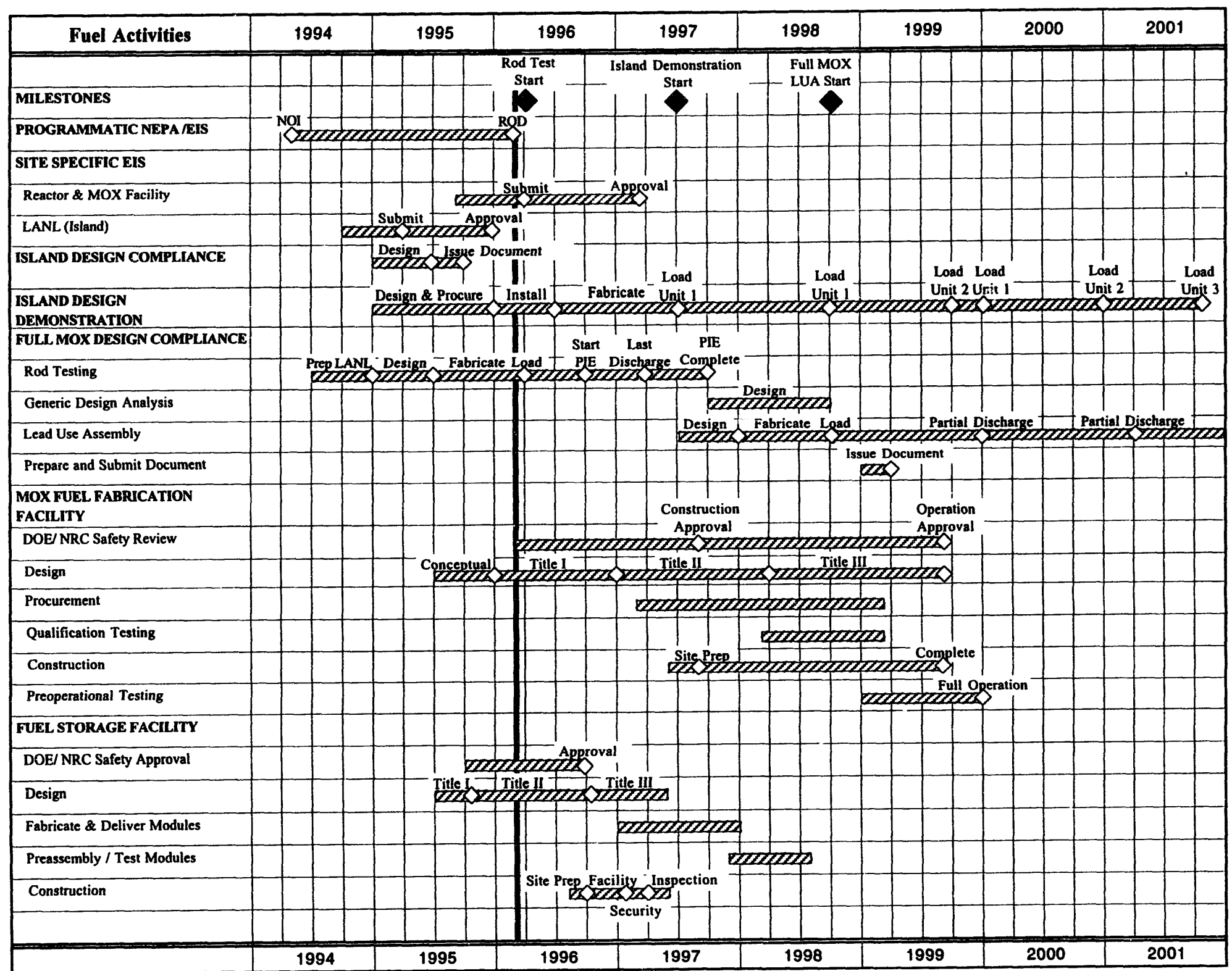

Figure 6.6-2 Fuel Activities Schedule 
NEDO-32361

Table 6.6-1 Existing BWR Project Schedule Activities Breakdown

\begin{tabular}{|c|c|c|c|}
\hline Schedule Activity & Duration & Start & End \\
\hline \multicolumn{4}{|l|}{ NEPA/EIS PROCESS } \\
\hline \multicolumn{4}{|l|}{ PROGRAMMATIC } \\
\hline Notice of Intent & & Present & $6 / 94$ \\
\hline Implementation Plan & 4 months & 6/94 & $10 / 94$ \\
\hline Draft EIS & 8 months & $10 / 94$ & $6 / 95$ \\
\hline Public Comment & 3 months & $6 / 95$ & 9/95 \\
\hline Final EIS & 3 months & $9 / 95$ & $12 / 95$ \\
\hline Record of Decision & & & $3 / 96$ \\
\hline \multicolumn{4}{|l|}{ SITE SPECIFIC } \\
\hline Selection and Utility Negotiation & & present & 9/95 \\
\hline Reactor and MOX Fuel Facility & 18 months & 9/95 & 3/97 \\
\hline - Prepare EIS & 6 months & $9 / 95$ & $3 / 96$ \\
\hline - DOE/NRC and Public Review & 12 months & $\begin{array}{l}3 / 96 \\
10 / 94\end{array}$ & $\begin{array}{l}3 / 97 \\
1 / 96\end{array}$ \\
\hline $\begin{array}{l}\text { Los Alamos National Laboratory } \\
\text { Prepare EIS Amendment }\end{array}$ & $\begin{array}{l}15 \text { months } \\
6 \text { months }\end{array}$ & $10 / 94$ & $4 / 95$ \\
\hline DOE Review and Approval & 9 months & $4 / 95$ & $1 / 96$ \\
\hline Licensing Integration Planning & 11 months & $10 / 94$ & 9/95 \\
\hline \multicolumn{4}{|l|}{ DEVELOPMENT } \\
\hline Lasertrac & 6 months & $3 / 96$ & 9/96 \\
\hline Automated Assembly Inspection & 12 months & $3 / 96$ & 3/97 \\
\hline Core Internals Radiation Surveillance & 6 months & $3 / 96$ & 9/96 \\
\hline Full MOX Fresh Shipping Container & 36 months & $3 / 96$ & 3/99 \\
\hline \multicolumn{4}{|l|}{ Fuel Rod Testing Program } \\
\hline - Design Test & 3 months & 4/95 & $7 / 95$ \\
\hline - Fabricate Rods & 9 months & $7 / 95$ & $4 / 96$ \\
\hline - Load and Irradiate & 12 months & 4/96 & $4 / 97$ \\
\hline - Post Irradiation Tests & 12 months & $10 / 96$ & $10 / 97$ \\
\hline Automation Evaluation & 12 months & $10 / 94$ & $10 / 95$ \\
\hline Line-Glove Box Hybrid Evaluation & 12 months & $10 / 94$ & $10 / 95$ \\
\hline Process Simulation & 12 months & $3 / 96$ & $3 / 97$ \\
\hline Accountability Equipment & 12 months & $3 / 96$ & $3 / 97$ \\
\hline \multicolumn{4}{|l|}{ FUEL RELATED ACTIVITIES } \\
\hline ISLAND DESIGN COMPLIANCE & & & \\
\hline Fuel Design Analysis & 6 months & $1 / 95$ & $7 / 95$ \\
\hline Prepare and Submit Document & 3 months & $7 / 95$ & $10 / 95$ \\
\hline \multicolumn{4}{|l|}{ FULL MOX DESIGN COMPLIANCE } \\
\hline Rod Testing Program & 36 months & $10 / 94$ & $10 / 97$ \\
\hline Full MOX Design Analysis & 12 months & $10 / 97$ & $10 / 98$ \\
\hline \multicolumn{4}{|l|}{ Lead Use Assembly (Full MOX) } \\
\hline - Design & 6 months & 7/97 & $1 / 98$ \\
\hline - Fabricate Bundles & 9 months & $1 / 98$ & $10 / 98$ \\
\hline - Load and Irradiate (Cycle I) & 15 months & $10 / 98$ & $1 / 00$ \\
\hline - Partial Discharge (Cycle II) & 15 months & $1 / 00$ & $4 / 01$ \\
\hline - Partial Discharge (Cycle III) & 18 months & 4/01 & $10 / 02$ \\
\hline - Final Discharge (Cycle IV) & 15 months & $10 / 02$ & $1 / 04$ \\
\hline Prepare and Submit Document & 3 months & $1 / 99$ & 4/99 \\
\hline
\end{tabular}


Table 6.6-1 Existing BWR Project Schedule Activities Breakdown (Continued)

\begin{tabular}{|c|c|c|c|}
\hline Schedule Activity & Duration & Start & End \\
\hline \multicolumn{4}{|l|}{ PLANT LICENSE ACTIVITIES } \\
\hline Prepare Proposed License Submittal & 6 months & 9/95 & 3/96 \\
\hline NRC Review & 12 months & 3/96 & 3/97 \\
\hline Site Specific EIS (Reactor and MOX Facility) & 18 months & 9/95 & $3 / 97$ \\
\hline NRC Final Approval & 1 month & $3 / 97$ & $4 / 97$ \\
\hline Revise Safety Evaluation Report & 1 month & 4/97 & $5 / 97$ \\
\hline \multicolumn{4}{|l|}{ STORAGE FACILITY } \\
\hline DOE/NRC Safety Approvals & 12 months & $10 / 95$ & $10 / 96$ \\
\hline Design (I, II, and III) & 22 months & $7 / 95$ & $5 / 97$ \\
\hline Fabricate and Deliver Modules & 12 months & $1 / 97$ & $1 / 98$ \\
\hline Preassembly/Test Modules & 8 months & $12 / 97$ & $8 / 98$ \\
\hline Construction & & & \\
\hline - Site Prep & 2 months & $8 / 96$ & $10 / 96$ \\
\hline - ISFSI Concrete Pad and Walls & 3 months & $10 / 96$ & $1 / 97$ \\
\hline Facility Structure & 4 months & $10 / 90$ & $3 / 97$ \\
\hline $\begin{array}{l}\text { Fencing and Security } \\
\text { Utilities }\end{array}$ & 1 month & $2 / 97$ & $3 / 97$ \\
\hline Place Modules/Canisters & 5 months & $12 / 97$ & $5 / 97$ \\
\hline Testing/Inspection & 2 months & $3 / 97$ & 5/97 \\
\hline \multicolumn{4}{|l|}{ LOS ALAMOS \& CTIVITIES } \\
\hline Funding & & & \\
\hline - Funding Proposal & 3 months & $\begin{array}{l}7 / 94 \\
10 / 94\end{array}$ & $10 / 94$ \\
\hline \multicolumn{4}{|l|}{ Programmatic EIS/NEPA } \\
\hline - Prepare EA and NEPA Document & 6 months & $10 / 94$ & $3 / 95$ \\
\hline $\begin{array}{l}\text { - DOE Review and Approval } \\
\text { Safety Review and Approval }\end{array}$ & \multicolumn{3}{|c|}{$\begin{array}{l}\text { Safety Review and Approval } \\
\text { - Prepare Unreviewed Safety Oualification (Rc } J\end{array}$} \\
\hline $\begin{array}{l}\text { - Prepare Unreviewed Safety Qualification (Rc } J \\
\text { Testing and LUA) }\end{array}$ & 3 months & $10 / 94$ & $1 / 95$ \\
\hline - Full Site Specific EIS/NEPA (Island) & 6 months & $10 / 94$ & 4/95 \\
\hline \multicolumn{4}{|l|}{ Design/Procurement for Fabrication } \\
\hline - Rod Testing Program & 6 months & $10 / 94$ & $4 / 95$ \\
\hline - Island Design Demonstration & 12 months & $1 / 95$ & $1 / 96$ \\
\hline \multicolumn{4}{|l|}{ Equipment Installation and Checkout } \\
\hline - Island Design Demonstration & 6 months & $1 / 96$ & $7 / 96$ \\
\hline \multicolumn{4}{|l|}{ Fabrication } \\
\hline - Rod Testing Program & 9 months & $7 / 95$ & $4 / 96$ \\
\hline - Lead Use Assembly Program & 9 months & $\begin{array}{l}1 / 98 \\
7 / 96\end{array}$ & 10/98 \\
\hline - Island Design (First Reload) & 12 months & $\begin{array}{l}7 / 96 \\
7 / 97\end{array}$ & $\begin{array}{l}7 / 97 \\
1 / 98\end{array}$ \\
\hline \multicolumn{4}{|l|}{ MOX FUEL FACILITY } \\
\hline DOE/NRC Safety Review & 42 months & 3/96 & 9/99 \\
\hline Design & 50 months & $7 / 95$ & 9/99 \\
\hline Construction Review and $\mathrm{A}$ & 18 months & $3 / 96$ & 9/97 \\
\hline Operation Review and Approval & 24 months & 9/97 & 9/99 \\
\hline
\end{tabular}


Table 6.6-1 Existing BWR Project Schedule Activities Breakdown (Continued)

\begin{tabular}{|l|c|c|c|}
\hline Schedule Activity & Duration & Start & End \\
\hline Equipment Procurement & 24 months & $3 / 97$ & $3 / 99$ \\
Equipment Qualification Testing & 12 months & $3 / 98$ & $3 / 99$ \\
Site Prep & 3 months & $6 / 97$ & $9 / 97$ \\
Construction & 24 months & $9 / 97$ & $9 / 99$ \\
Pre-Operational and Startup Testing & 12 months & $1 / 99$ & $1 / 00$ \\
Operation & 23.5 years & $1 / 00$ & $7 / 23$ \\
REACTOR LOADISG & & & \\
UNIT 1 & 15 months & $7 / 97$ & $10 / 98$ \\
- Island Load & 15 months & $10 / 98$ & $1 / 00$ \\
- Island Load & 15 months & $1 / 00$ & $4 / 01$ \\
- Island Load & 18 months & $4 / 01$ & $10 / 02$ \\
- Full MOX Load & & $7 / 22$ & $10 / 23$ \\
- Final Full MOX Load & & & $7 / 27$ \\
- Final Full MOX Discharge & & & \\
UNIT 2 & 15 months & $10 / 99$ & $1 / 01$ \\
- Island Load & 15 months & $1 / 01$ & $4 / 02$ \\
- Island Load & 18 months & $4 / 02$ & $10 / 03$ \\
- Full MOX Load & & $7 / 23$ & $10 / 24$ \\
- Final Full MOX Load & & & $7 / 28$ \\
- Final Full MOX Discharge & & & \\
UNIT 3 & 15 months & $11 / 01$ & $2 / 03$ \\
- Island Load & 18 months & $2 / 03$ & $8 / 04$ \\
- Full MOX Load & & $4 / 23$ & $7 / 24$ \\
- Final Full MOX Load & & & $4 / 28$ \\
- Final Full MOX Discharge & & & \\
\hline
\end{tabular}

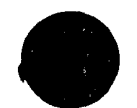

Completion of schedule development resulted in the project schedule shown in Figure 6.6-1 and was based on the following schedule basis and assumptions. Critical schedule items and risks are discussed and assessed in Section 6.7, Anticipated Schedule Risks.

\subsubsection{General Bases and Assumptions}

The following overall bases and assumptions were used for developing the project schedule. Specific bases and assumptions for major project elements are provided in following subsections that discuss schedule elements.

- The project objective is to disposition $50 \mathrm{MT}$ weapons-grade plutonium.

- The project start date is the date of the DOE's anticipated programmatic ROD issuance, March 1996.

- Preliminary utility selection and contract work begins immediately to insure that site selection occurs early in the program by September 1995. 
- Funding is available at the beginning of fiscal year 1995, October 1994, before the project start date, to support the development activities and licensing and permitting preparation. Submittal of licensing and permitting documents will not occur prior to programmatic ROD issuance.

- Post-ROD issuance funding is available as required to support uninterrupted licensing, design, testing, construction, installation, checkout, and operation.

- The MOX fuel fabrication facility is located on an existing precharacterized site. The effect of this assumption is that the time associated with environmental permitting is significantly decreased.

- Three BWRs are used to disposition the plutonium.

- The BWR reactor complex is subject to the US NRC Code of Federal Regulations for licensing approvals. The MOX fuel fabrication facility is subject to the DOE Safety Review process and the NRC licensing criteria.

- The DOE assumes the role of Lead Agency for NEPA and is responsible for interagency coordination.

- The schedule assumes a beneficial and effective public involvement program as part of the EIS process. The effect of this assumption is to minimize the risk of significant delays resulting from intervention in the licensing process.

\subsubsection{Schedule Elements, Activities, and Durations}

\subsubsection{Development Activities}

Development activities associated with this project do not require conventional research and development. These tasks are for the purposes of applying proven technology, updating quality assurance procedures, and monitoring design performance and identify only the additional design requirements due to the introduction of MOX fuel. Funding for development activities is assumed available in October 1994, prior to the project start date. Figure 6.6-3 details the schedule for the development of existing technologies for MOX application. The development tasks critical to the schedule begin on the October 1994 funding availability date. 


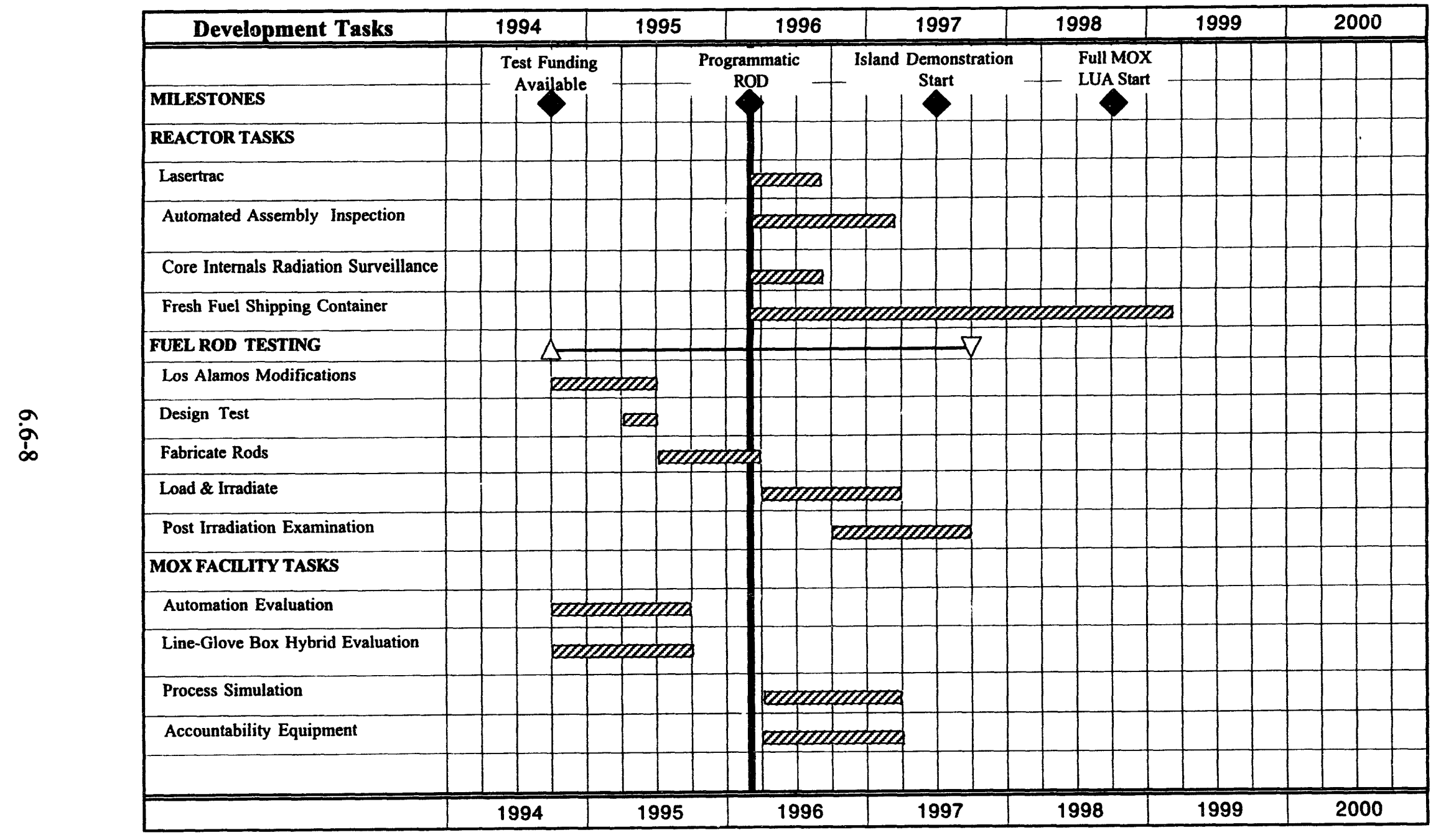

Figure 6.6-3 Project Development Schedule 
The duration of the overall development schedule is dependent primarily on the fuel rod testing program. The fuel rod testing program will provide mixed oxide - gadolinium fuel rod physical and performance characteristics at various exposures. This data will verify that the fabrication process and fuel rod design yield the calculated performance characteristics. Los Alamos National Laboratory (LANL) will be used to fabricate the pins needed for this rod testing program, as well as the assemblies for the island design demonstration and lead use assemblies programs. Figure 6.6-4 details the LANL activities, and additional discussion of these activities is included in a following subsection and in Section 2.4 of this report.

Durations for the other development activities were determined to be sufficient to complete the application of proven technologies for the plutonium disposition project using existing BWRs. Lasertrac, the automated assembly inspection equipment, core internal radiation effects, and the fresh fuel shipping containers are development items associated with reactor operations for using the full MOX fuel design. Because of the small quantity of plutonium in the island design and the small number of assemblies used, these development items are not required for the demonstration program. The process automation and glove box hybrids evaluation programs, scheduled to start with the funding availability date, were estimated to require twelve months to complete. These programs are required as input into the MOX fuel fabrication facility Title I design, scheduled to begin July 1995. Process simulation and accountability equipment development are required for the MOX fuel fabrication facility and will be based on overseas and Los Alamos fabrication experience.

\subsubsection{Licensing, Environmental Permitting, and Safety Approvals}

The licensing and safety approvals documents required for project implementation are the programmatic Environmental Impact Statement (EIS), the reactor and MOX fuel fabrication facility site specific EIS, the reactor plant operating licenses, fuel compliance documents for both the island and full MOX design, safety approvals for the construction and operation of the MOX fuel fabrication facility, and the Los Alamos National Laboratory's Unreviewed Safety Qualification (USQ) and site specific EIS. The process for obtaining each of these documents is discussed briefly below and is detailed in Section 5.0 of this report.

The schedule includes a licensing integration planning and site selection period, discussed in Section 5.0 of this report, beginning with the funding start date October 1994. Eleven months should be adequate time to establish a licensing plan acceptable to the project participants and to select the existing BWR candidate sites for project implementation. However, these two 


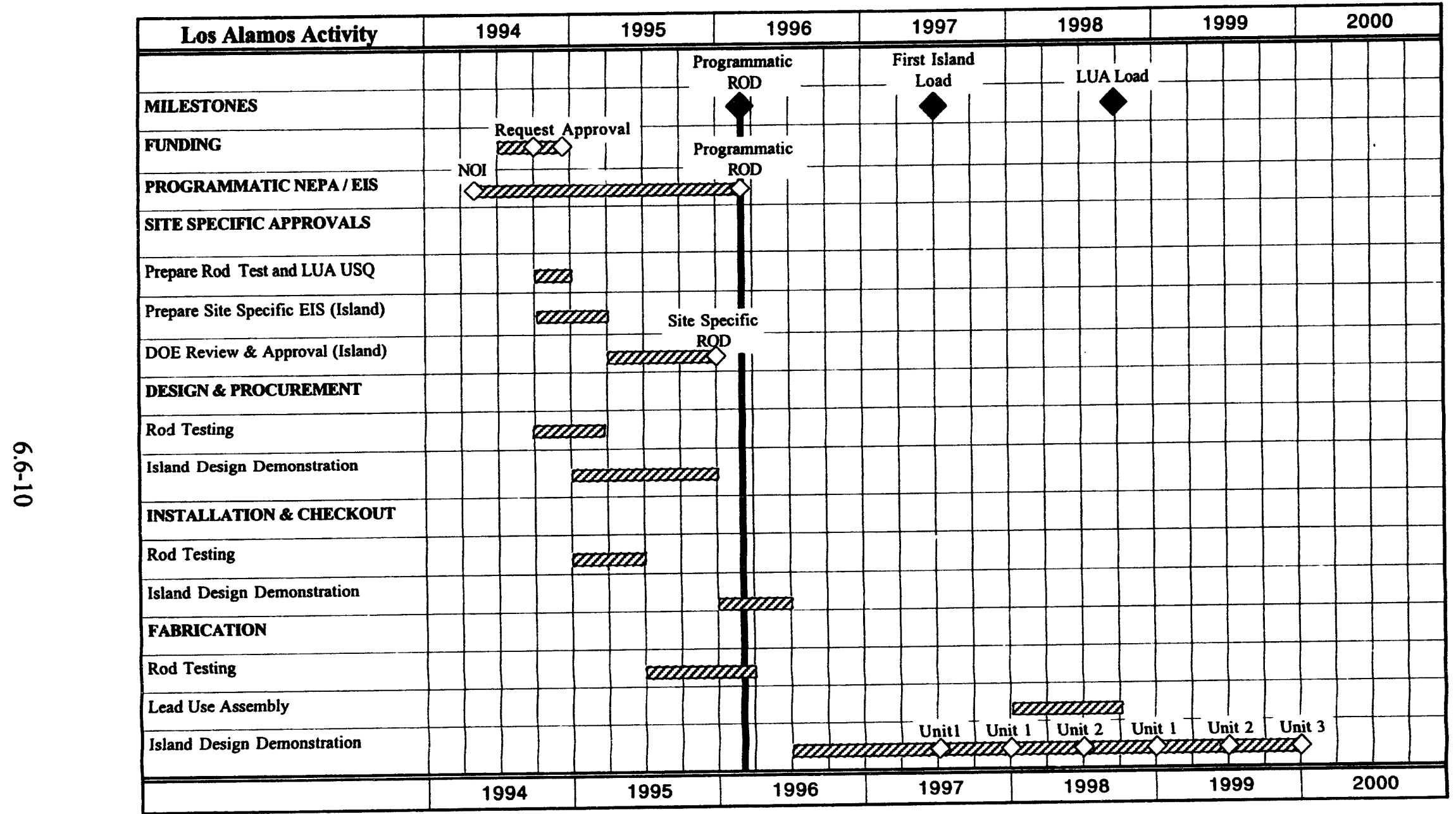

Figure 6.6-4 Los Alamos National Laboratory Activities Schedule 
activities should begin immediately if more time is determined to be necessary.

\subsection{Programmatic Environmental Impact Statement}

The National Environmental Policy Act (NEPA) requires the identification and assessment of the impact to the environment for all major projects proposed. In order to meet this requirement, the DOE, as lead agency coordinating the environmental activities, will prepare the programmatic Environmental Impact Statement (EIS). The purpose of this NEPA programmatic EIS process is to specify the technology option to be used for completing the disposition mission and identify sites for use with the specified option. The process begins with a Notice of Intent issued in mid1994. Following an implementation plan submitted in October 1994 and a draft EIS issued in June 1995, the public comment period begins. NEPA requires a 90-day public comment period after publication of the Draft EIS in the Federal Register. During this period, no decision on the proposed action can be made or recorded. However, because potential sites have been identified and discussed in the public comment period, early site specific EIS preparation may begin. Preparation of the final programmatic EIS follows. NEPA has a similar requirement of 30 days after publication of the final EIS, prior to the Record of Decision (ROD). Final EIS preparation will last three months with final publication set for December 1995. In addition to the required 30-day public comment time, two additional months were added to the schedule before the final Record of Decision will be issued in March 1996.

\subsection{Site-Specific Environmental Impact Statement}

Following the public comment period of the programmatic EIS ending in September 1995, preparation of the site specific EIS on existing, pre-characterized sites will begin. This sitespecific EIS will take a total of 18 months to complete and will include amendments to the sitespecific EISs for the reactors to be used, as well as for the precharacterized site of the MOX fuel fabrication facility. A description of each permit, the permitting process, and the expected permitting duration are discussed in more detail in Section 5.4 of this report. This licensing duration is consistent with similar projects where extensive site data is available. The sitespecific ROD for the reactor sites and the MOX fuel fabrication facility site, satisfying both the DOE and the NRC environmental requirements, will be issued March 1997, 18 months after the September 1995 starting date. This is 12 months after programmatic ROD issuance.

\subsection{Reactor Plant License Amendment}

The process for modifying a current reactor operating license for the island and full MOX design applications will be determined during the licensing integration planning period. The duration 
specified in the project schedule details the conservative amendment approach. Section 5.2 of this report discusses an additional, simpler approach that might be available for incorporating the island design into a reactor operating license.

Preparation activities supporting the proposal of amendments to the reactor operating license begin at the same time site specific EIS activities start in September 1995 and are conservatively estimated to take six months. In March 1996, following the programmatic EIS ROD issuance, reactor plant license modifications will be submitted to the NRC for review. This question and answer period between the operators and the NRC is scheduled to last twelve months, which includes time for public comment. The NRC will issue its final determination and amendment approval following the NRC review period and the six month public comment period. Should the licensing integration planning team determine that separate licensing processes are appropriate for the island and full MOX fuel designs, ample schedule time is available.

Based on the requirements associated with introducing mixed oxide fuel into an operating plant, the amendments to the reactor license pertain to safeguards, security, and public health. Safety approval by the DOE and NRC will be required for the construction and operation of a secure storage area to receive and store fresh mixed oxide fuel and spent fuel from the spent fuel pool. This secure area is described in detail in Section 2.5 of this report and in the schedule shown in Table 6.6-1. This area, located on the reactor site, consists of a double fence, surveillance equipment, concrete pads for placing the spent fuel storage containers, and a fresh fuel receipt building used for storage and inspection purposes. DOE/NRC approval is expected in October 1996. This is an aggressive approval schedule considering that the site specific EIS is not complete until March 1997. However, this risk is low and acceptable because of the importance of this facility to safeguards and security, the low cost of this facility relative to the overall project cost, the proven and simple technology utilized, and the fact that most utilities will require this type of facility regardless of this project due to the unavailability of a long term spent fuel storage facility.

\subsection{GESTAR II Amendment 22 Fuel Compliance}

The licensing process established for introducing new fuel designs to the reactor fleet is well established and is specified in Amendment 22 of the General Electric Standard Application for Reactor Fuel (GESTAR II) document. Section 5.2 .3 provides a detailed description of the process. To summarize, Amendment 22 establishes a set of criteria that must be met before a generic new fuel design may be used in a reactor on a full scale reload basis. According to Amendment 22, the new fuel design is acceptable for use after all criteria are met. Usually, a 
document detailing compliance with the established criteria is issued to the NRC for notification purposes. No official NRC review period is required although the NRC may decide to audit the analysis supporting the compliance document. This project schedule allows time for this type of NRC audit which is estimated to last six to nine months. As with all reloads, a cycle specific reload document is issued prior to actual fuel load.

Amendment 22 states that all new fuel designs incorporating new design features must load lead use assemblies prior to using the fuel for an actual reload. Lead use assemblies are typically irradiated for four cycles or five-plus years depending on the cycle length. Even if, as is typically done, a full scale reload of the new fuel design is loaded after the first two LUA cycles, two-plus years have passed. The plutonium disposition project with GE BWRs uses two mixed oxide fuel designs, the island and full MOX design. The island design, fully described in Section 2.1.1, does not serve to disposition vast quantities of plutonium - the island design enables a quick program startup. In addition to overseas MOX experience, the Quad Cities plant had a lead use assembly program that used a similar mixed oxide design from 1974-1982. Therefore, no lead use assemblies are required for the island design. The issuance of the island design's compliance document is conservatively estimated to take nine months, with six months for generic design analysis and three months for document preparation. This period may begin at any time but, should be completed at least six months prior to the fuel loading in July 1997 to allow for a potential NRC audit.

The full MOX design will probably require a lead use assembly program in addition to the extensive rod testing program. The assemblies for this program will be fabricated at Los Alamos after the rod testing program is complete. Lead use assemblies will be ready for loading with the first unit is second island design reload in October 1998. Two of the eight lead use assemblies will be discharged from the core at the end of each of the next four cycles for inspection purposes. The compliance document is issued after the lead use assemblies are loaded. As with the island design compliance, sufficient time for an NRC audit is provided in the project schedule.

\subsection{MOX Fuel Fabrication Facility Licensing and Environmental Approval Schedule}

The MOX fuel fabrication facility will be licensed and approved using the DOE Safety Review process subject to NRC regulations. The DOE experience for approving both weapons and test facilities that handle plutonium is directly applicable to the MOX fuel fabrication facility. Consistent with current administration policy, the NRC will play a significant role. Because the proposed MOX fabrication facility license is expected to be similar to the BNFL Sellafield MOX 
plant license, the safety reviews will start immediately following the programmatic ROD issuance. This project expects to issue the required DOE submittals, conduct safety reviews, and obtain construction approval by September 1997. Fuel production operations begins 27 months later in January 2000.

\subsection{Los Alamos Licensing and Safety Approval Schedule}

Los Alamos National Laboratory will fabricate the fuel rods required for the rod testing program, the assemblies needed for the lead use assembly program, and the island design assemblies for the early demonstration program. The existing LANL authorizations allow for the fabrication of the fuel rods and the lead use assemblies provided an Unreviewed Safety Qualification (USQ) is issued prior to starting program activities. This USQ takes three months to prepare and issue, and is expected by January 1995 according to current LANL plans. The fabrication of island design assemblies may not be an approved activity in the current LANL authorizations and may require an amendment to the site specific EIS. Should an EIS be required, six months of preparation activities, beginning in October 1994, will be followed by a nine month DOE review period. Approval is expected January 1996. An update of the LANL SAR is scheduled in parallel.

\subsubsection{Fuel Fabrication Activities}

\subsection{Los Alamos National Laboratory Fabrication and Related Programs}

\section{Preparation and Operation}

The ability to fabricate the required rods and assemblies for the initial fuel related programs is necessary to the project's quick and effective implementation. Los Alamos National Laboratory is capable of meeting this requirement with few adjustments. Figure 6.6-4 shows the activities required for the fabrication capability. This schedule was developed with LANL personnel and is realistic and achievable.

\section{Rod Test Program}

The purpose of the fuel rod testing program is to provide data on mixed oxide - gadolinium fuel rod physical and performance characteristics at various exposures. This data will verify that the fabrication process and fuel rod design yield the calculated performance characteristics. Los Alamos National Laboratory's TA-55 will be used to fabricate the fuel rods needed for this testing program. The previous discussion of LANL's activities describes the activities prior to faabrication. After the installation and checkout period of six months, fabrication for the fuel rod 
testing program will begin in July 1995. This fabrication process will take nine months to complete and fuel will be loaded in April 1996 in a test reactor. The fuel will be irradiated and examined at a variety of exposures. The total irradiation period is one year, and initial test data from the first post irradiation examinations will be available six months after the test start date in October 1996. Full rod characterization test data is expected a year later in October 1997.

\section{Island Design Demonstration}

The purpose of the demonstration program using the island design is to introduce MOX fuel at the earliest possible time to demonstrate the U.S. Government's commitment to dispositioning plutonium to the public and foreign governments while allowing time for the MOX fuel fabrication facility licensing and construction. In addition, the demonstration program provides an important opportunity to train personnel at each site for the new accountability, handling, inspection, storage, and safeguards procedures associated with mixed oxide fuel. Following fabrication for the rod testing program and the final installation and checkout of the island design equipment, fabrication of the island design fuel begins in July 1996. Los Alamos estimated that six months is required to make each island design reload of 58 assemblies. However, one year is provided for the first island batch. This first reload will be loaded in July 1997. Each of the three disposition reactors will be loaded with at least one partial reload of 58 island design bundles primarily for training purposes as mentioned earlier. Figures 6.6-1 and 6.6-2 show the loading scheme for the island design and Figure 6.6-4 shows the fabrication schedule.

\subsection{MOX Fuel Fabrication Facility Schedule}

\section{MOX Fuel Fabrication Facility Design Schedule}

This plutonium disposition project's proposed MOX fuel fabrication facility is based on the existing technology used to fabricate nuclear fuel elements world wide during the last 25 years. The use of this existing technology significantly contributes to quick completion of Title I and Title II design. Design begins prior to the project start date towards the end of the evaluation programs described in the development schedule discussion. After the site specific ROD issuance date, Title I design is complete after 12 months. Title II design lasts 15 months. Title III activities which verify conformity with the approved design coincide with the facility construction. 


\section{MOX Fuel Fabrication Facility Construction Schedule}

The fuel fabrication facility construction schedule is 24 months with limited site preparation three months prior to construction approval. Pre-operational activities and startup testing will last 12 months extending three months after construction is completion. Operation will begin January 2000. This schedule appears realistic due to the fact that all foreign and domestic schedules for MOX fuel fabrication facilities reviewed allowed a two year time period for construction. These other facilities were designed to fabricate mixed oxide from reactor grade plutonium and were more complex. Six to nine months after the start of building construction, equipment installation will be initiated on a system by system basis. As part of installation activities, operation checkouts of each equipment item will be performed.

As shown in the fuel activities schedule (Figure 6.6-2), the authorization for start of construction of the MOX fuel fabrication facility is scheduled for September 1997, 18 months after the project start date. This schedule is predicated on expeditious design and procurement based on the overseas experience. The schedule for this facility is achievable with agency commitment and existing technology utilization from the U.S. and abroad. As noted earlier, the technology and infrastructure for MOX fuel fabrication exist at both DOE and foreign sites and may be drawn upon to expedite the schedules. Although licensing approvals for some modern facilities in other countries were extensive, that experience should facilitate the safety reviews and clearly demonstrate that there are no unresolved technical issues that would delay approval for the operation of this facility on an environmentally reviewed and approved DOE site.

\section{MOX Fuel Fabrication Facility Pre-operational Activities Schedule}

The MOX fuel fabrication facility begins operation January 2000. Pre-operational activities are scheduled during the last nine months of the construction period and continue for an additional three months after construction is complete. Qualification testing will be initiated with urania in applicable situations as systems are completed. Key systems, such as blending and milling, require verification testing with MOX prior to full scale operation. This schedule allows ample time for fabricating the first full MOX reload, scheduled for loading in April 2001. The facility is capable of fabricating approximately 200 assemblies every six months which satisfies the standard reload requirement of 176 bundles. Fuel fabrication operation will continue at the design rate of $97 \mathrm{MT} / \mathrm{yr}$ of mixed oxide for 23.5 years until July 2023 to support the $50 \mathrm{MT}$ plutonium disposition project. Parallel activities are necessary to assure tha the converted plutonium supplied by the government is available for use in the fabrication facility. 
BNFL reviewed this schedule based on their previous design and construction experience in the UK. They verified that the plant construction schedule is achievable and that operation could begin within the fifth year after start of design assuming that the project will be a DOE program and that environmental/safety approvals can be obtained early. Design information is available from BNFL for conceptual design activities. Again, it is emphasized that effective program management of the plutonium disposition program must be maintained in order to meet the MOX fuel fabrication facility schedule.

\subsubsection{Reactor Loading Schedule}

The overall project schedule (Figure 6.6-1) shows the reactor loading schemes for the three BWR units used in the plutonium disposition project. The first unit using MOX fuel in the island design is loaded with 58 island design and 118 urania assemblies in July 1997 with a cycle length of 15 months. Two additional partial island design reloads are irradiated until the 176 full MOX assemblies are ready for loading in April 2001. The second reactor loads two island design batches of 58 bundles before its first full MOX reload in April 2002. The third reactor, on the same site as the second reactor, is loaded with island design fuel in November 2001 and full MOX one cycle later in February 2003. 


\subsection{Anticipated Schedule Risks}

The following section addresses the key schedule uncertainties associated with the major project elements. In addition, overall schedule risks are identified and discussed. Government policy, public involvement, and funding present the most significant challenges and uncertainties to meeting the project schedule. As discussed earlier, a proven NRC licensing process exists for introducing new (urania) fuel in the BWR and this process will be applied to license the proposed MOX design. The MOX fuel has been designed to meet the existing fuel licensing criteria and this licensing envelope has been evaluated to apply equally well to MOX and urania fuels. No changes or modifications to the reactor system are needed. MOX fuel will be introduced in stages to replace discharged urania fuel. For these reasons, schedule risks should be minimal, when disposition is conducted using BWRs which are currently operational. If a reactor plant which is not currently operational (either because the reactor is only partially completed or because of some other reason) is chosen, schedule risks will be highly plant specific. Effective project management and planning will minimize these uncertainties. No schedule risks have been identified in the reactor system area that are unique to the plutonium disposition mission.

The plutonium disposition project schedule includes numerous contingencies to accommodate minor delays in major project element durations. In addition, an initial demonstration program using an "island" design concept has been proposed which could serve to surface any schedule risk associated with the various program elements prior to the implementation of the full MOX program. Although the overall schedule risk has been evaluated to be minimal, specific categories which have major impact on the schedule are discussed below in more detail.

\subsubsection{Non-Technical Factors}

The following information identifies potential schedule uncertainties that arise from political, financial, managerial, and institutional factors. When possible, these are quantified. However, it should be noted that the nature of the majority of potential schedule uncertainties is such that no quantitative assessment is possible.

- Government Reviews and Approvals - The schedule developed for the NEPA process is realistic, based on the DOE NEPA process plan. It is assumed that the MOX facility will be located at one of the DOE sites. For all the DOE sites under consideration, site specific information necessary for the NEPA process is already available. Revisions might be needed at the reactor site to previously approved EIS, however, these are judged to be minor as the 
quality of the air, water or other environmental discharges will not be affected by MOX operation. However, contingencies were added to account for potential minor delays. In addition, schedule delays could result if licensing activities are not effectively coordinated and integrated with the appropriate Government agencies. Although quantification of the risk associated with this critical process is not possible, it is a significant risk to the project schedule.

- Public Acceptance - In view of the urgency of the need for disposition of weapons plutonium from the perspective of national and international security, it is anticipated that a majority of the public will fully support this project. Nevertheless, schedule risks could arise in this process.

- Regulation Revision - Additional delays could result if new regulations are necessary. This risk is minimal because current regulations were determined to either be sufficient or readily updated to reflect technical improvements.

- Funding - If timely appropriation of funding by the Government is not available for the various stages of the project, uncertainty will be introduced into the schedule. It is not possible to adequately account for this element in the schedule risk assessment.

- Early Project Site Selection - Early identification of the project sites will serve to decrease the time associated proportional program activities. Issuance of the programmatic ROD will determine the project and project site. Activities scheduled prior to ROD issuance involve preparation activities for the identified potential sites; therefore, no direct schedule delay is associated with having to wait for the ROD issuance.

- Other - Schedule delays and uncertainties due to natural disasters or unanticipated legal action have not been analyzed.

\subsubsection{Specific Project Schedule Uncertainties}

The following is a discussion of the major project element schedule uncertainties with specific consideration given to technical considerations. Effects of the overall schedule risks, such as political, financial, managerial, or institutional, will be mentioned here only on a limited basis.

\subsubsection{Conversion}

The conversion of plutonium into a form usable as MOX fuel feed stock is an element defined by the DOE to be outside the project scope for the purposes of this study. However, the conversion 
process is a key element and requires immediate attention at project start. The process is based on mature technology and national laboratory experience. In addition, the equipment and facilities required for the conversion process are much less complex than that required by the MOX fuel fabrication facility. Early decision should be made as to whether americium will be removed from the feed stock or will be left with the feed stock for disposition. The start date for fuel fabrication for the testing and demonstration programs is October 1994. The MOX fuel fabrication facility starts full operation in January 2000. Considering the mature status of the technology, the capability of the national laboratories, and the complexity of the process equipment used in the conversion process, the potential for schedule impact due to the conversion process is considered minimal. However, required amounts of feed stock made by qualified process must be made available.

\subsubsection{Transportation}

The proposed method of transportation of MOX fuel bearing special nuclear material is only a minor deviation from current practices. Currently, plutonium materials and derivatives are commonly transported between DOE laboratories, and spent fuel and radwaste transportation infrastructures exist. The transport of fresh fuel assemblies will require only minor modification to the new fuel shipping containers already in use, as discussed in Section 3 on development issues. An early demonstration program using the island design would surface any problems in this area. There should be no risks to the schedule concerning this issue.

Unlike disposition using activities where the MOX facility and the reactor could be co-located, surface transportation over reasonably large distances could be anticipated when dispositioning with existing reactors. Transport of MOX assemblies is being carried out routinely in Europe without any adverse impact on safety or environment. Although transport of plutonium bearing materials will not pose a schedule risk from a technical standpoint, there is the possibility of schedule risk arising from having to obtain approvals from local and state governments, and secure public acceptance for surface transport on the intervening route.

\subsubsection{Identification of Reactor Plants for Disposition and Agreements for Economic Incentives}

The reactor plants that will be used for disposition will have to be identified by application of a number of screening criteria such as those discussed in Section 1.2 of this report. If plants that are not currently operational are chosen, there is a schedule risk associated with bringing plants into operation which will be plant specific. Such schedule risks should be assessed based on what might be required to bring the plant on-line for urania fueled operations as the additional 
schedule risk from MOX fueled operation is judged to be minimal. The process of identifying potential reactors for use in disposition and negotiations to arrive at economic incentives should be started early and in parallel with a number of reactor owners/utilities to minimize schedule risks in this area.

\subsubsection{Licensing for MOX Fuel, Reactor Operations}

The licensing of the BWR reactor for MOX fueled operation is considered relatively straightforward. The NRC has a process for licensing new fuel which should be used for the MOX application. Partial core loads of "island" design MOX fuel have already been irradiated successfully in BWRs and therefore, the early demonstration using this concept could go forward without delays. Full MOX core implementation is expected to occur in stages and, therefore, once again, no delays should occur as a result of licensing the fuel. The schedule contingencies added should provide adequate time to resolve any potential conflicts associated with using MOX fuel.

For some plants, an ex-reactor, secure fresh MOX fuel storage may be needed. This can be implemented in time for the full MOX operations. The reloads for the demonstration program involve only 58 assemblies and could proceed without the ex-reactor storage, by direct transfer of the assemblies to the in-reactor fuel storage pool. The safeguards provisions for the demonstration program can be implemented in time for the start of this program in 1997 and no risks to the schedule were identified.

\subsubsection{Capacity Factor}

There is significant variability among existing plants in plant availability and capacity factors. The reasons are plant specific and arise as a result of a number of factors including efficiency of operations, forced or planned outages, management, and age of the plant. Most recent plants have a capacity factor of $75 \%$ or higher. A capacity factor (during disposition) of less than $75 \%$ could lead to a stretch in the overall disposition program. Plant-specific assessments should be made as to the potential for lower than anticipated capacity factors and the economic incentive agreements should be structured to reward early disposition with improved capacity factors.

\subsubsection{MOX Fuel Fabrication}

The primary element with a potential risk to the schedule concerns the political and institutional effects on licensing and constructing this facility. A licensing basis is available with the DOE 
Safety Approval process utilizing NRC Regulations and assistance. However, institutional and political risks exist and could potentially influence the licensing process and duration.

The estimated four and a half year schedule represents the best estimate for construction and startup of for the MOX fuel fabrication facility based on European experience. Possible delays could be accommodated by MOX fabrication capacity at LANL after completion of the island demonstration program. The current schedule requires that about 200 assemblies will have to be fabricated every six months for disposition of 50MT plutonium using three reactors. By applying additional manpower during the first two years, the production could be increased sufficiently such that loading of the first reactor will be delayed by a maximum of six months and no delays will be incurred in the loading of subsequent reactors. The following areas require attention:

- Labor Agreements - There is considerable experience in the U.S. on labor scheduling to ensure availability of the necessary skills, such as specialty welders, electricians, boiler makers, and iron workers, to insure consistency between schedule and productivity (shift capacity), and to avoid labor disputes. Proper attention to labor agreements and labor management relations will assure that labor disputes do not impact the schedule.

- Long Lead Time Item Procurement - The construction techniques used today permit shorter and more predictable schedules. Detailed assessments of the European experience in constructing and operating MOX plants and its applicability for the present application, will help obviate any potential schedule risk. Early attention toward vendor certification and planning at the project start will decrease the potential uncertainties related to procurement issues. 
NEDO-32361

\section{$6.8 \quad$ References}

6-1 Disposition of Weapons Plutonium Using the GE Advanced Boiling Water Reactor, Report for the US DOE, GE NEDO-32351, April 1994.

6-2 ORNL Letter, J.G. Delene to R.J. Neuhold, "Transmittal of Economic Factors for Disposal of Weapons Plutonium in Existing Reactors," April 26, 1994.

6-3 ORNL Letter, K.A. Williams to R.J. Neuhold, "Transmittal of Letter Reports on Business Model, Description and Detailed Guidelines for Cost Estimating: Plutonium Disposition," February 14, 1994.

6-4 J.G. Delene and C.R. Hudson II, "Cost Estimating Guidelines for Advanced Nuclear Power Technologies," ORNL/TM10071/R4, May 1993.

6-5 Generic Environmental Impact Statement for License Renewal of Nuclear Plants, NUREG-1437, August 1991.

6-6 Requirements of Renewal of Operating Licenses for Nuclear Power Plants, 10CFR54, November 1992.

6-7 Management and Disposition of Excess Weapons Plutonium, National Academy of Sciences, National Academy Press, Washington DC, 1994. 


\section{NEDO-32361}

\subsection{Safeguards and Security}

\subsection{Overview}

Much of the safeguards and security related issues for disposition of plutonium using existing reactors is identical to that discussed in an earlier report [Ref. 7-1] which examined plutonium disposition using the GE-ABWR. For the sake of completeness, the discussion on issues that are common to both is repeated here as well. The areas where differences arise primarily relate to transportation and handling of new fuel.

Permanently safeguarding the plutonium from weapons usage is the paramount reason for this study. In the context of the present study, it is necessary to distinguish between two related but different definitions that can be ascribed to the word "safeguards." In one case, we are referring to the permanent long-term disposition of plutonium derived from dismantling the weapons. Here, we are concerned with the relative efficacies of different disposition options for long-term safeguarding of plutonium. In the second case, we are referring to the safeguards provisions that should be implemented during the various stages in a given disposition process to prevent theft or diversion. Although much of this section is concerned with this second definition of safeguards, it is useful to briefly examine the long-term disposition advocated in this study.

Dispositioning the plutonium as irradiated fuel assemblies by using this material as fuel in a light water reactor represents one of the most permanent, long-term, readily implemented means of safeguarding this material. The literature on safeguards, both at the system level and in its functional details, is extremely large [Ref. 7-2, 7-3, 7-4]. The conclusions of the Office of Technology Assessment (OTA) [Ref. 7-2] published in 1977 and later in 1993, as well as the results of the more recent NAS study [Ref. 7-5], indicate that Pu disposition as spent fuel has the least technical risk and would result in a product with acceptable proliferation resistance. The earlier OTA study concluded that (1) massive spiking with a material such as radioactive cobalt was neither cost effective nor useful in the restraint of proliferation, (2) the concept of 'denaturing' plutonium where Pu-239 is converted into higher atomic mass plutonium isotopes was fallacious, and (3) storage and transport in dilute mixed-oxide form does present a certain level of resistance, as it requires time-consuming chemical separation activities to reclaim the plutonium. The OTA study advocated real-time material control and accountability, citing work done in this area at Los Alamos National Laboratory (LANL) and Lawrence Livermore National Laboratory (LLNL). Overall, a majority of the reports on safeguarding plutonium conclude that the use of fissile material in dilute form as fuel in LWRs poses the lowest risk, provided appropriate safeguards are implemented to counter diversion. Recovering plutonium from 
irradiated assemblies will be time and capital intensive, requiring heavily shielded facilities; and monitoring programs, whether it be IAEA standards or bilaterally agreed upon standards, will easily detect such diversion in a timely manner.

In addition to the high diversion resistance characteristic of the final irradiated fuel assemblies, a certain level of diversion resistance is imparted as the plutonium is taken through the various process steps of fuel fabrication. Diversion and efforts towards redeployment will be detected quickly under the proposed safeguards structure, since on-line material accountability assessment is implemented every step of the way. Resistance increases because the time and infrastructure needed to reclaim the plutonium increase as the plutonium becomes progressively more dilute. At certain stages, the plutonium is encased in a metallic tube or assembly that is almost 15 feet long and cannot be removed without attracting considerable attention. A fuel assembly weighs nearly $300 \mathrm{~kg}$ and is likely to require machinery for lift and transport. Given the security and controls implemented as part of the safeguard provisions, diversion of fresh fuel assemblies is highly improbable.

A number of reasons exist for a reexamination of the conventional provisions for safeguarding special nuclear materials:

- The quantity of plutonium involved that has to be processed is much larger than the typical quantities dealt with in the past.

- The radioactivity of weapons plutonium is lower than reactor grade plutonium. Conventional gamma signature based assaying methods may have to be supplemented or replaced by positive interrogation techniques.

- This would be the first time that dismantlement of nuclear weapons and disposition of plutonium would have been undertaken in a concerted way and therefore requires a consideration of broad bilateral and (in future) potentially multilateral issues and agreements. Further, inasmuch as this disposition could be the forerunner of future weapons reductions, any problems in the safeguards area - whether they be technical or institutional - could lead to major setbacks for future efforts.

- Unique to this project, safeguards should be effective as measured against the backdrop of possible social and political changes in the CIS. In this sense, it is important that the material be dispositioned with speed, and in parallel at comparable rates in both the U.S. and CIS. 
- For simultaneous disposition in the U.S. and CIS, issues of transparency and reciprocity in safeguards have to be taken into account. The safeguards structure associated with the use of plutonium as MOX fuel is well understood, is currently being applied in other countries, and, as a result, the transparency and reciprocity provisions can be readily taken into account.

\subsubsection{Institutions Avallable for Implementing Safeguards Provisions}

It is useful at this point to examine the institutions which might be called on to provide the safeguards provisions that would be applied to the disposition project. Safeguards implementation requires not only the technology but also the necessary institutional agreements. Three different institutions could be distinguished:

- Domestic Safeguards as applied to Special Nuclear Materials are principally enforced per NRC regulations on the commercial side. For Special Nuclear Materials controlled by government agencies or in DOE facilities which are exempt from licensing and regulation by NRC, DOE Orders apply for the handling, processing, transport and utilization of these materials.

- International Safeguards, principally enforced per international treaties such as the NonProliferation Treaty through the IAEA.

- Bilateral Safeguards agreements between the U.S. and CIS, which are yet to be negotiated. (At the time of this writing, some bilateral agreements appear to have been reached.)

Conventional safeguards are primarily administered through the first two institutions. It should be noted that the provisions of international safeguards, as applied through IAEA or a similar arrangement, will still be required after discharge of irradiated assemblies from the reactor to the extent that any spent fuel assembly could be processed to yield plutonium for use in weapons.

\subsubsection{Domestic Safeguards}

Domestic safeguards provisions for handling special nuclear materials have been very successful to-date in preventing theft or diversion. The processes envisioned in the proposed disposition scheme - plutonium metal to oxide to MOX powder to MOX pellets to fuel bundles to reactor loading - are all well covered by existing DOE Orders for Special Nuclear Materials (SNM) or by NRC regulations. DOE Order 5633.2A (September 1992), for instance, specifies the Responsibilities and Authorities for the Control and Accountability of Nuclear Materials. There 
are two specific areas where additional work appears to be needed for this project application. These arise as a result of the exceptions to conventional safeguards noted earlier:

- The admissible level of discrepancies in material accountability arising from the natural scatter in measurement techniques needs further scrutiny in view of the large amounts of plutonium flow-through involved. Although it is unlikely that diversion will take place by the removal of very small quantities of material over a long period of time, this cannot be discounted. No new assay techniques with a lower scatter are needed but the results of material accountability and the statistics of the control measurements should be correlated more precisely and any errors that result in material accountability which are within the statistically admissible region should be tracked by trend analysis. If the results indicate that the unaccounted material is dispersed in a normal fashion over a period of time, no action may be warranted; however, if the unaccounted material is always biased (towards a possible removal of material from the stream), additional actions may be warranted.

- A second area where additional work is needed relates to the use of conventional assay techniques. A number of different assay techniques are available to determine the quantity of plutonium in a given setting. Some of them depend not on a positive interrogation for Pu-239 but on the signature of other isotopes which are $\gamma$ emitters such as americium (Am). The quantity of Pu-239 is then computed knowing the ratio of the various isotopes in the batch. A comparison of the radioactivity of various Pu isotopes and Am is shown in Table 7.1-1, which indicates an order of magnitude lower radioactivity in weapons plutonium compared to reactor grade plutonium. Therefore, these "indirect" methods should be supplemented by more positive interrogation techniques. The Non-Destructive Test assay method using Cf-252 developed at LANL and successfully used to assay MOX fuel pins fabricated for the Fast Flux Test Facility would appear to be the most suitable. Although developed for nondestructive assaying of Pu in fuel pins, the method could be extended for other configurations used in the production process.

A preliminary review of the applicable DOE Orders for Special Nuclear Material (SNM) indicates that existing domestic safeguards provisions can be readily implemented and are sufficient to safeguard the plutonium through the proposed disposition process. 
Table 7.1-1 Radioactivity of Plutonium (Curies/gm)

\begin{tabular}{|l|c|c|c|c|c|c|c|c|}
\hline & \multicolumn{3}{|c|}{ Reactor Grade Plutonium } & \multicolumn{3}{c|}{ Weapons-Grade Plutonium } \\
\cline { 2 - 9 } Isotope & t\% & Gamma & Neutron & Total & wt\% & Gamma & Neutron & Total \\
\hline Pu-238 & 2 & 0.046 & & 0.337 & & & & - \\
Pu-239 & 54 & 0.002 & & 0.036 & 93.7 & 0.003 & & 0.062 \\
Pu-240 & 25 & 0.009 & $6.2 E-9$ & 0.067 & 5.7 & 0.002 & $1.4 \mathrm{E}-9$ & 0.015 \\
Pu-241 & 11 & 0.004 & & 11.34 & 0.3 & 0.000 & & 0.308 \\
Pu-242 & 7 & 0.000 & & & & & & \\
AM-241 & 1 & 0.023 & & 0.061 & 0.3 & 0.006 & & 0.017 \\
TOTAL & 100 & 0.084 & $6.2 E-9$ & 11.84 & 100.0 & 0.011 & $1.4 E-9$ & 0.402 \\
\hline *Total radioactivity also includes alpha and beta particle emissions. & & \\
\hline
\end{tabular}

\subsubsection{International Safeguards}

Of the various disposition options that have been suggested, only disposition as spent fuel has been subject to international scrutiny through IAEA safeguards standards. In this respect, only disposition as spent MOX fuel has the attractiveness that an international body could be brought in to independently verify the safeguards for weapons plutonium. It also provides the U.S. with the opportunity to provide a direct input to the IAEA for the standards by which the IAEA should measure and safeguard plutonium usage which is already taking place in some foreign countries. Safeguards administered through the IAEA or by bilateral provisions would also be required to safeguard the spent fuel.

The IAEA safeguards parallel domestic safeguards in a number of respects, particularly as it concerns provisions related to material characterization, accountability and physical security. There has been extensive cooperation between the U.S. agencies such as DOE and NRC and the IAEA to bring to bear the latest in available technology to international safeguard systems design and implementation. There are two key features where IAEA safeguards differ from those in the U.S. First, it is a voluntary system in that the nation-states declare the sites where special nuclear materials may be involved and open them to inspection, and, further, these states are voluntary parties to the NPT. The inspection efforts are principally aimed at detecting diversion if it occurs from these declared sites. The second key feature relates to the fact that it is a two-tier system, with separate provisions for those who currently possess nuclear weapons and those who do not. This feature would normally not be of concern, except that the nation-states of the CIS who will participate in this disposition project might claim the status of a nuclear weapons-state and therefore claim certain exemptions from IAEA safeguards accorded to this tier. 


\subsubsection{Bilateral Safeguards Agreements Between the U.S. and CIS}

At present, no bilateral agreements appear to exist between the U.S. and CIS for the safeguards provisions to be applied in the disposition operation. The first step in disposition is the dismantlement of the weapon, removal of the pit and the destruction of its shape. There are a number of issues in this area, related to the levels of transparency and reciprocity and the manner in which each country would ascertain that the plutonium that is made available to this project did in fact come from a dismantled weapon. The details relative to this area are being worked out by a designated U.S. agency and will not be discussed any further in this report. (More recent information would appear to indicate that bilateral agreements have been reached in this area.)

The key issues to be decided for bilateral safeguards agreements are:

- Extent to which U.S. provisions for safeguards (embodied in the U.S. institutions such as NRC regulations and DOE Orders) are made part of the bilateral safeguards: This will entail the transfer and/or the supply of procedures and machinery that are used in materials characterization and accountability such as assay techniques. Detailed studies on the potential for theft, diversion or redeployment would be needed if the currently accepted U.S. safeguards provisions are not incorporated in toto in the bilateral agreement. Evaluation and qualification of the CIS safeguards practices to have them equivalent to the U.S. DOE Orders is a possibility.

- Lead/lag provisions, if any, during disposition: It is unlikely that disposition will occur at the same rate in both countries. Agreements would be needed to ensure that disposition is occurring at comparable rates. Given that the U.S. policy precludes reprocessing, continued disposition as spent fuel should depend upon commensurate disposition in the CIS. This area would be more difficult to resolve if two entirely different processes for disposition were adopted by the U.S. and CIS.

- Reciprocity and Transparency Provisions: Issues pertain to mutual access to the "near real-time" material accounting system, provision for the presence of U.S./CIS personnel during transport or physical inventory, provision for the presence of U.S./CIS personnel in the MOX fuel fabrication factory, for shared monitoring of portal entries/exits for nuclear materials, and others. 


\subsection{Fuel Fabrication Facility}

The safeguards and security measures to be implemented for the fuel fabrication plant will be developed to meet applicable U.S. and international regulations. In this context, it is useful to note that a number of provisions related to material accountability, control, surveillance and security standards adopted by IAEA were originally formulated in the U.S. and are applied through NRC regulatory standards. Safeguards and security requirements will form an integral part of the design requirements for the fuel fabrication plant and would be maintained throughout the design, construction, operation and decommissioning phases. Multiple layers of safeguards and security to assure redundancy will be provided for the plutonia that is delivered to the fuel fabrication plant. The various stages in the processing of plutonium oxide into MOX fuel assemblies are discussed in more detail in Section 2.4. A schematic flow chart is given in Figure 7.2-1 for ease of following the security and safeguards provisions implemented in the fuel fabrication facility and described below.

\subsubsection{Physical Security in the Fuel Fabrication Facility}

Implementation of physical security measures will meet all applicable DOE Orders for safeguarding SNM. The security practices being adopted in foreign countries with similar plutonium operations, such as by BNFL at UK, were reviewed and will also be incorporated, as appropriate. Three physical areas of security were identified for the MOX plant:

- An inner area where plutonium in various forms is stored or handled and will require a high level of security, permission and control for entry and exit of personnel.

- An intermediate area where general security will be maintained but where there will be no SNM.

- The outer periphery which will require a high level of security and surveillance.

The layers of physical security provisions that will be implemented are described below:

- The fuel fabrication plant will be within a double-fenced area that is within the fenced site area, and access will be controlled and surveillance maintained for the outside area.

- The construction requirements for the portion of the fuel fabrication facility that houses the plutonia storage area, the pellet and rod process, the fuel pin and bundle assembly areas, and 


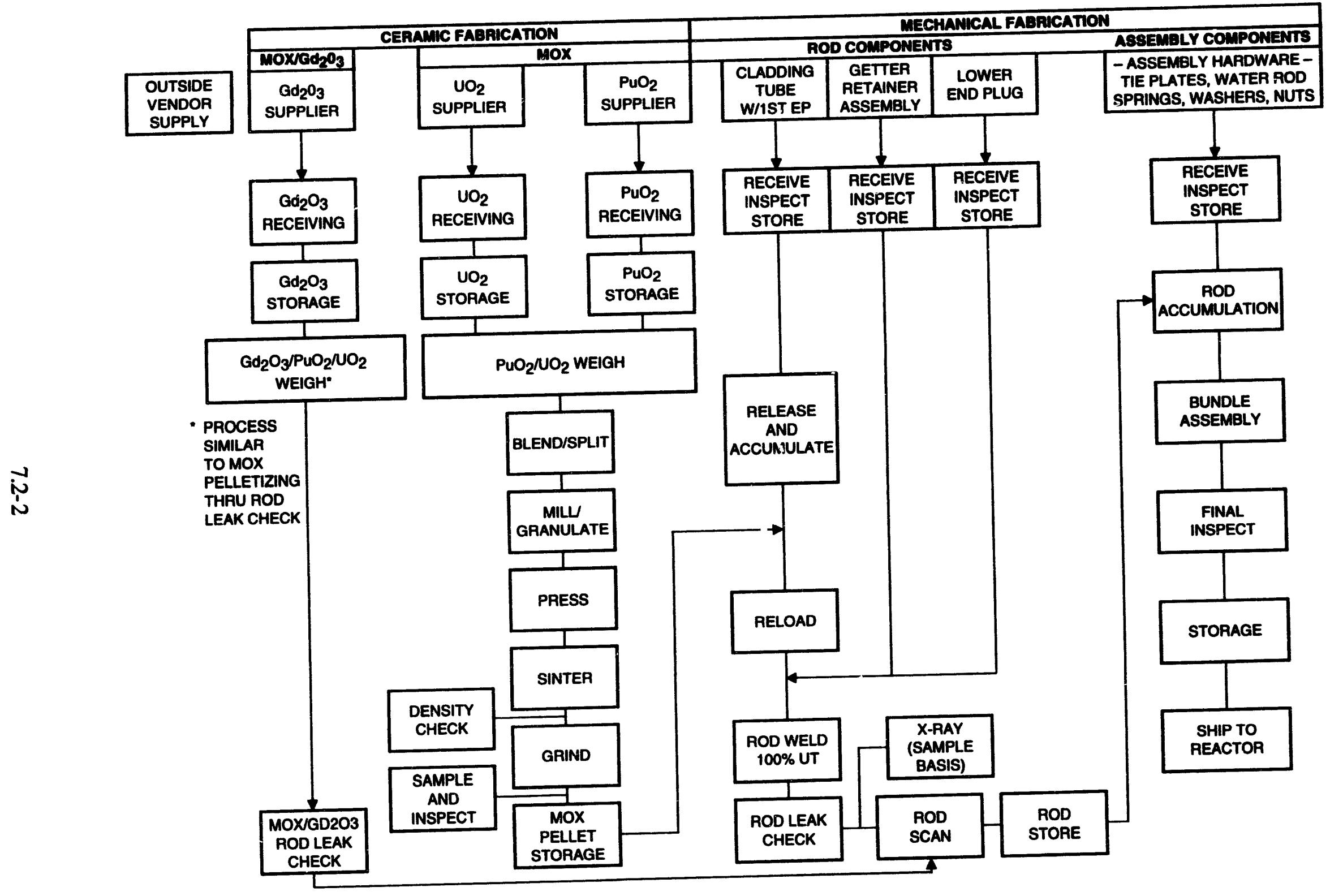

Figure 7.2-1 MOX Fuel Fabrication Process Operations 
the completed bundle storage area shall be similar to that for the reactor containment building, where a high level of security will be enforced.

- Within the fabrication facility, the initial plutonia storage area shall be within a vault which will have limited, computer-controlled access and continuous surveillance.

- In the batch processing area, the plutonia will be controlled in glove-boxes with no direct access to the material during normal operation. Any potential access during service or maintenance will be pre-planned and controlled with continuous surveillance and portal entry/exit monitors to the area involved.

- After the fuel pellet loading step, the plutonia will be hermetically sealed into zircaloy tubes, which are subsequently clustered into bundles with no direct access during normal operation.

- Two fuel bundles would be loaded into a steel shipping container and moved by a computercontrolied crane into a shielded, vault-like storage area, with no direct access during normal operations.

- The site security force will monitor the area outside the fuel fabrication plant fences and will control access of personnel and materials into and out of the double-fenced area. Personnel access into and out of the actual fabrication process areas will also be limited.

- Surveillance equipment will be used in all parts of the facility and tied to the central security command post so that any movement of personnel or material can be detected, and the permission for such movement confirmed and checked for control/permissions from the material accountability system.

\subsubsection{Safeguards Implementation in the Fuel Fabrication Facility}

The layers of safeguards would be provided by redundant capabilities to verify and monitor the amounts and locations of plutonium on a near real-time basis. The safeguards would include:

- An integrated computerized system would (1) log-in each container of plutonium received by scanning the unique identification codes on each shipping container, (2) collect and compare the verification data from each of the automated verification stations, (3) monitor and record the movement of the containers of special nuclear materials into and out of each material balance area, and (4) monitor and record the weighing of each container into each material balance area. 
- Initial verification of the contents of the shipping containers received will be done by passive gamma scan to establish its radioactive signature, which will be compared against previously measured database of acceptable signatures, and diverse verification of container contents will be by the.Californium-252 scanner technique [Ref. 7-6], which was developed for nondestructive assay of fast flux test facility fuel pins, and random samples of container contents.

- Assay and evaluation of decontaminated waste materials to determine the amounts of trace plutonium being lost with the waste materials.

- Material balance areas to include the initial plutczila receiving and storage area, the batch powder processing area, the pellet production area, the fuel pin assembly area, the fuel bundle assembly and storage area, and the scrap recycle area.

- Verification of fuel contents by chemical analysis of random samples taken from various stages in the fuel fabrication process.

- Verification of the contents of assembled fuel pins by the Cf-252 scanner technique.

- Quarterly material balance audits of the entire fuel fabrication facility.

- Space and resources for onsite independent inspectors to audit material balance and verification data for accuracy and timeliness, and to audit the methods of data collection and analysis.

A preliminary division of the Material Balance Areas (MBA) within the MOX plant is shown in Figure 7.2-2. Four major MBAs are envisioned: (1) in the plutonia storage area, (2) in the process area from oxide to storage as fuel rods, (3) in the fuel assembly fabrication area and (4) in the assembly storage area. In addition, minor quantities of plutonia in various forms will be tied up in filter waste, waste treatment and instrumentation/metallurgical laboratory space. The provisions of the DOE Orders for SNM accountability will be fully implemented, including all provisions related to the frequencies for physical inventory, checking of standards, material characterization and software validation of computer codes used in material accounting.

\subsubsection{Security and Safeguards in the Storage Areas}

These areas are the plutonium receiving and storage area and the assembled fuel bundle storage area. For safeguards reasons, the plutonium receiving and storage area will be a vault, which also provides extremely good shielding. Movement of plutonium into and out of the vault will 


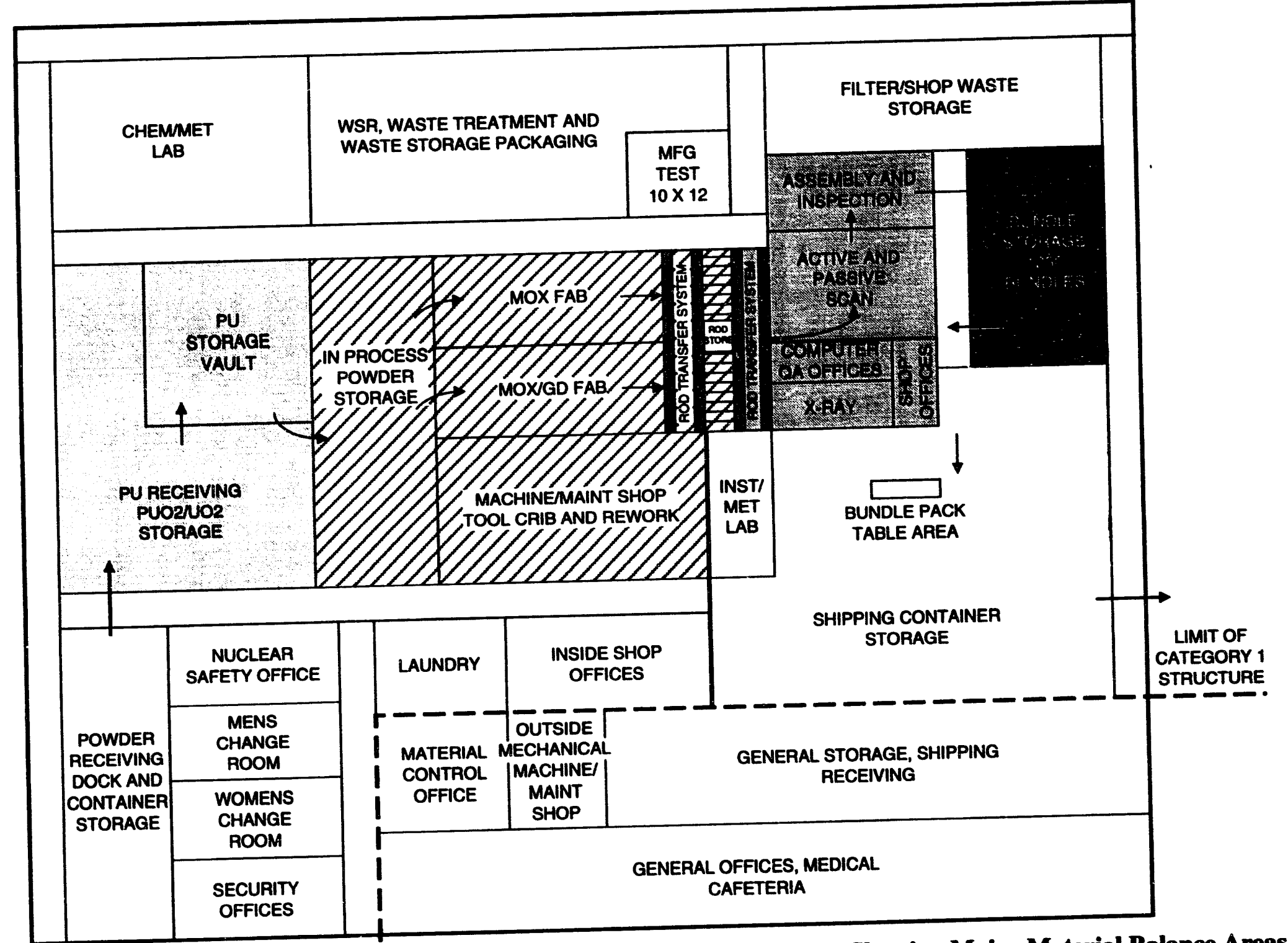

Figure 7.2-2 MOX Facility Block Layout for 35,000 Rods/Year Reference Case Showing Major Material Balance Areas 
be accomplished by using a computer-controlled robot. Within the fuel fabrication facility, when the plutonium oxide and uranium oxide are in powder form, they will be contained in hermetically sealed (welded) steel containers. These containers will only be opened after entrance into the sealed-off glove-box system for processing these powders into the pellet form. Required permissions and controls will be implemented into the software for initiating any movement. As with the material balance areas, material accountability will be maintained by automatic records of weights transferred and positive interrogation of samples of the material, if necessary. After completing bundle assembly, two bundles would be loaded into a steel storage and transfer container. The container would be moved by a computer-controlled overhead crane into a shielded storage area. As with the initial feed material vault, permissions and control will be needed for movement of these assemblies, and their movement will be automatically recorded.

In summary, the security and safeguards systems to be implemented in the fuel fabrication plant will meet all existing standards to prevent diversion or theft. Near real-time material accountability will be implemented. Specific areas where additional factors such as positive interrogation techniques have to be implemented have been identified. With these provisions and the use of surveillance equipment in all parts of the facility tied to the security system, the safeguards of this material in the fuel fabrication facility is assured. 


\subsection{New Fuel Receipt, Handling and Storage}

By the time the plutonium has been converted into MOX fuel assemblies, its diversion resistance would have increased significantly, as a typical fuel bundle weighs about $300 \mathrm{~kg}$ and some type of machinery would be required to move such a bundle. When the MOX facility and the reactor are not co-located, additional provisions will have to be made to ensure that bundles are not diverted during transport, and incoming inspections performed to verify that the reported quantities of plutonium are present in the bundle. This requirement could be met in two ways. First, tamper-proof container designs will be implemented to provide evidence for this on receipt. Second, a gross check of the plutonium inventory of the incoming material at the bundle level can be performed.

Existing safeguards provisions would appear to require verification of plutonium inventory upon receipt to confirm that the quantity specified on the shipping manifest has been received. However, a reexamination of the usefulness of this provision for the disposition operation is recommended. The level of physical security and real-time tracking of the SST used for the transport may provide sufficient assurance for safeguards, and verification of Pu inventory upon receipt may not be necessary. A gross nondestructive assay of an entire bundle is still possible. If a more detailed inventory assessment upon receipt is found to be needed, a selected number of pins could be removed and examined using positive interrogation techniques such as the one using Cf-252 developed by LANL to assay MOX fuel pins. From a statistical analysis, it is possible to confirm the plutonium inventory.

Prior to loading the new fuel bundles into the stainless steel inner shipping container at the fuel fabrication facility, all necessary assays will have been performed and the MC\&A records necessary to correlate fissile material content with fuel bundle serial number will have been verified. As soon as the fuel bundles are loaded into the inner shipping container, Tamper Indicating Devices (TIDs) will be applied to the container. These seals can be separate or combined with the cup seals utilized by the IAEA as part of their verification. The internal container will then be enclosed in the wooden overpack and loaded into an SST for shipment to the reactor site. The MOX fuel bundles will remain in the sealed shipping containers within protected facilities (i.e., SST or a vault) until they arrive on the reactor refueling floor just prior to refueling. With this fuel handling concept, which is described in more detail in Section 2.5, the use of TIDs on the stainless steel inner shipping containers should be adequate for verification of shipper-receiver transfer and eliminate the need for plutonium assay measurements at the reactor site. 
At the reactor site, the SST will be taken to the fresh fuel storage vault. This vault will be constructed as an SNM vault, meeting the criteria specified in the DOE General Design Criteria (6430.1A) and co-located with the ex-reactor spent fuel storage facility. This allows both facilities to utilize the same security and delineates a concise area for the protective force to defend. The vault and the spent fuel storage facility will be located in the equivalent of a protected area as defined by the DOE. Intrusion detection will be at the dual fence line surrounding the facilities. Response will be provided by an appropriate trained and armed protective force meeting the DOE requirements. Access into this area will be through an access control facility providing positive identification and search procedures as specified by DOE Orders (5600 series).

The SST will be unloaded in the anteroom of the vault by a portable lift. Security during the unloading process will consist of Q-cleared protective force personnel stationed appropriately around the storage building while the vault and SST are open. The container overpack will be removed in the anteroom and the inner container moved into the vault. The identity of the inner shipping container and the integrity of its TIDs is verified at this point. The containers provide the necessary criticality spacing and protection of the fuel bundle during storage. All persons with access to the vault, as well as anyone handling the fuel containers, must be Q-cleared.

The vault is a windowless enclosure with walls, floor and ceiling constructed of material that affords penetration resistance equivalent to 8 inches of reinforced concrete. Openings, other than the door, are protected by imbedded steel bars or are less than 96 square inches in area with no dimension over six inches. The door will meet Class 5 standards as set forth in FS AA-D-6008 of the Federal Specification and Standards cited in 41CFR101. Volumetric alarms and door alarms will be part of the security system. The vault is sized for 280 fuel bundles and the anteroom is sized to provide laydown, unloading and handling one SST shipment at a time. A bridge crane with a capacity of two tons will be used to move the containers within the vault. The containers will be stacked in clusters with noncombustible spacers or a pallet between them with a three-foot clear space on all sides to permit TID and container inspection.

In preparation for the fuel to be moved to the refueling floor, the containers will be loaded into an enclosed vehicle at the vault anteroom and moved by convoy the short distance to the reactor building. The vehicle used for movement will be provided with limited fuel capacity, possibly an engine kill switch, and speed limiting devices. The convoy consists of lead and follow vehicles occupied by armed protective force members. Actual numbers of persons will depend on the results of a specific vulnerability analysis. 
Once in the building, the containers will be taken to the reactor refueling floor, where the TIDs are verified again, the containers opened and fuel bundle serial numbers verified. The sequence of operations from this point to the completion of refueling depends to some degree on the americium level in the fuel and whether a new or irradiated fuel channel is to be used. However, as discussed in Section 2.5, most of these operations will be performed in the fuel pool. The total duration, from receipt of the first MOX fuel in the Reactor Building until completion of refueling, is estimated to be 9 to 14 days. For purposes of relaxing the enhanced MOX fuel security requirements, completion of refueling is defined as the point where the time required to access the fresh MOX fuel is longer than that required for the security force to successfully respond to the design basis threat. This is discussed further in Section 7.4.

Security on the refueling floor throughout this period will be provided by Q-cleared protective force members. Fuel handlers will also have to be Q-cleared and will conduct the fuel handling operation under two-person control. Two-person control for fuel handling operations is the normal practice even today for safety and to verify that the correct bundle is being moved to its proper destination and orientation. Other persons on the refueling floor (pipe fitters, electricians, etc.) should not require $Q$ clearances and will remain outside the exclusion zone around the fuel handling activities.

The effect of the requirement for $Q$ clearances is similar in nature to other constraints that currently exist on the use of personnel for critical tasks (e.g., background checks, training and certification and periodic drug and alcohol testing). Its impacts are primarily the time and cost associated with the investigation and the small risk that sufficient numbers of Q-cleared personnel will not be available for a specific critical path activity during refueling operations. The cost of a $Q$ clearance is $\approx \$ 8,500$ over 20 years, including the periodic reinvestigations. The time required for the initial investigation is typically six months. From a utility perspective, if the DOE assumes the cost of the investigations, the only remaining impact is the risk of increased personnel unavailability. This risk can be minimized by increasing the pool of Q-cleared personnel.

Access to the refueling floor will be controlled by positive identification using non-intrusive biometric devices [i.e., hand geometry and Personal Identification Number (PIN)]. Final resolution of who must have $Q$ clearances and the configuration of access controls will be established as a result of detailed vulnerability analyses of the plants selected. However, the procedures outlined above should be sufficient to meet DOE requirements for protection of the 


\subsection{Reactor Operations}

Considerable perimeter security and surveillance are already in place in existing reactors to meet applicable NRC regulations, as well as other applicable local, state and federal mandates. It is likely that these provisions will satisfy all applicable guidelines for handling Special Nuclear Material (SNM) with attractiveness levels of Class D and E. (The reader is referred to Section 7.8 for a discussion of the attractiveness level of SNM handled at the reactor site.) If the existing security provisions are found not to meet the applicable DOE Orders/NRC Regulations, security and surveillance will be upgraded as needed. It is envisioned that enhanced security would be maintained on the refueling floor during the sequence of activities shown in Figure 7.4-1. This period of time is estimated to be 9 to 14 days and is bounded on the front end by arrival of the first bundle and at the back end by the completion of steam dryer installation. Steam dryer installation is judged to be the point where the time to gain access to and remove fresh MOX fuel would be longer than needed for the response force to successfully engage potential adversaries. However, the actual point in the sequence will be based on the results of a detailed vulnerability analysis. During reactor operation, there are no special security requirements, provided that any fresh MOX fuel not loaded into the reactor is returned to the fresh fuel storage facility and not stored in the fuel pool. 
NEDO-32361

TIME (days)

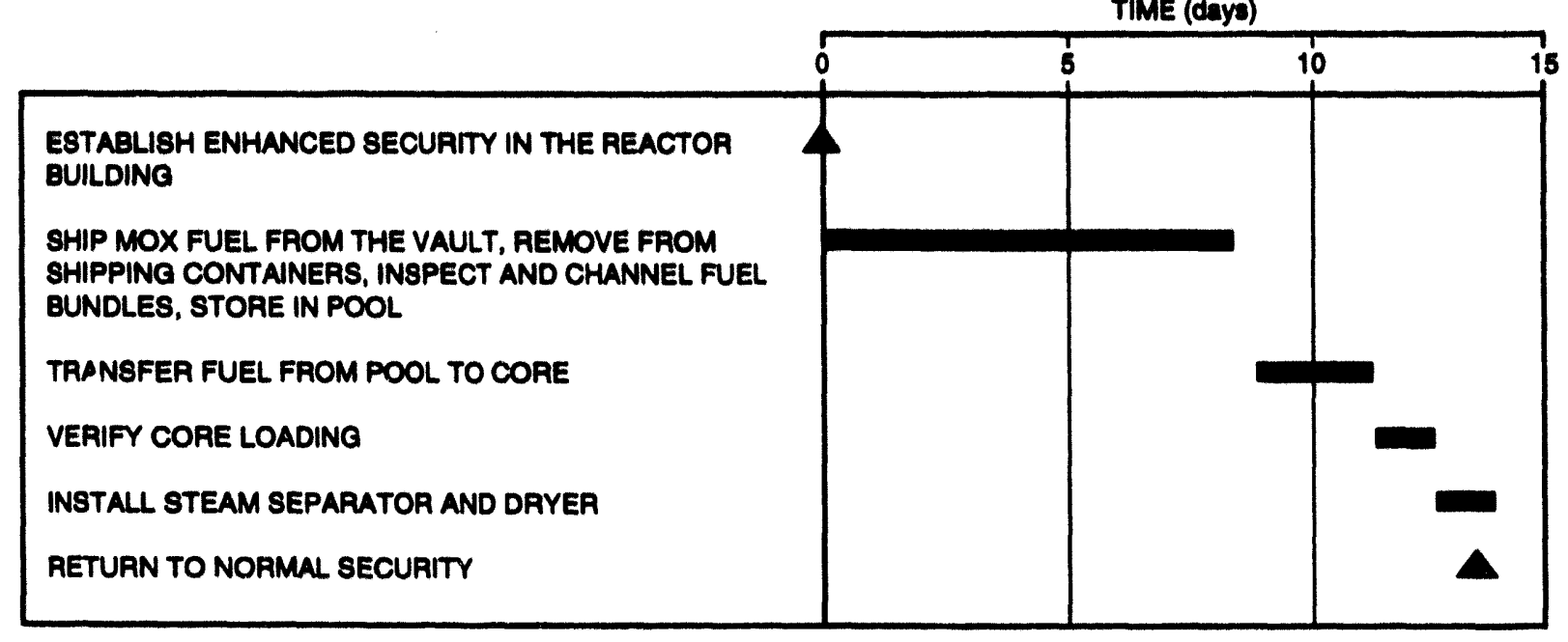

Figure 7.4-1 Period of Enhanced MOX Fuel Security 


\subsection{Spent Fuel Handiling and Storage}

Security requirements for spent fuel are no different than for normal spent BWR fuel. The radiation doses from the spent MOX fuel are high enough to classify it as self-protecting and, thus, no additional security is needed. Following refueling, the spent fuel will be stored in the fuel pool for $\approx 5$ years until it has decayed sufficiently for transfer to the ex-reactor spent fuel storage. It will then be loaded into dry shielded canisters (Section 2.5.3) which are welded closed, backfilled with helium and transported to the ex-reactor storage site. This site is situated in the same enclosure as the fresh fuel vault. 


\subsection{Safoguards Risks in Shipping and Handling}

Shipments of Pu feed material for MOX fabrication do not appear to present a logistics problem other than the requirement for Safe Secure Transport (SST). Each material shipment will be classified either Category IB or IC SNM, depending upon the shipment form (metal is $\mathrm{IB}_{1} \mathrm{PuO}_{2}$ is IC) as shown in Figure 7.6-1, reproduced from DOE Order 5633.3A [Ref. 7-7]. Intersite shipments will be made under the auspices of the Transportation Safeguards Division of the Albuquerque Operations Office (SST); intrasite shipments will escorted by field element couriers or contractor security forces as specified in Site Master Safeguards and Security Agreements required by Order $5632.2 \mathrm{~A}$ [Ref. 7-8].

If the fuel fabrication facilities and the reactor are co-located, shipments of MOX assemblies from the fabrication facility to the reactor may be made under the supervision of site protective forces; SST is not required. If the reactor and the fuel cycle facilities are not co-located, assembly shipments will be treated as Category ID SNM [Ref. 7-9] and SST is required by DOE Order 5632.2A [Ref. 7-8].

The security risks associated with the handling, shipment, and storage of fresh MOX fuel are related to the threats of theft and radiological sabotage as defined within DOE Orders and NRC regulations. The threat of state-sponsored adversaries is probably the higher threat to be addressed in the protection of this material. However, it is important to recognize that from the IAEA's perspective the host nation (i.e., the United States) is a potential diversion threat and that this is the focus of much of their safeguards efforts.

During the shipping phase, the fuel bundles are under the protection of the SST and its escort team. During storage in the fresh fuel vault, the risk is related only to the threat of theft. The fuel element design makes the threat of radiological sabotage minimal. The plutonium present is in the form of high density uranium-plutonium oxide (ceramic) pellets sealed within in the fuel element cladding. The dispersion of this form of material to the extent that it would exceed 10CFR100 limits is not considered credible within the DOE guidelines.

The quantity of material being shipped or stored could make this material attractive to a statesponsored terrorist organization, although the actual threat of theft of this material is probably small. However, according to federal law and DOE Orders, appropriate safeguards must be in place to assure low risk for this event, as defined in DOE Order 5630.13B. The above security 


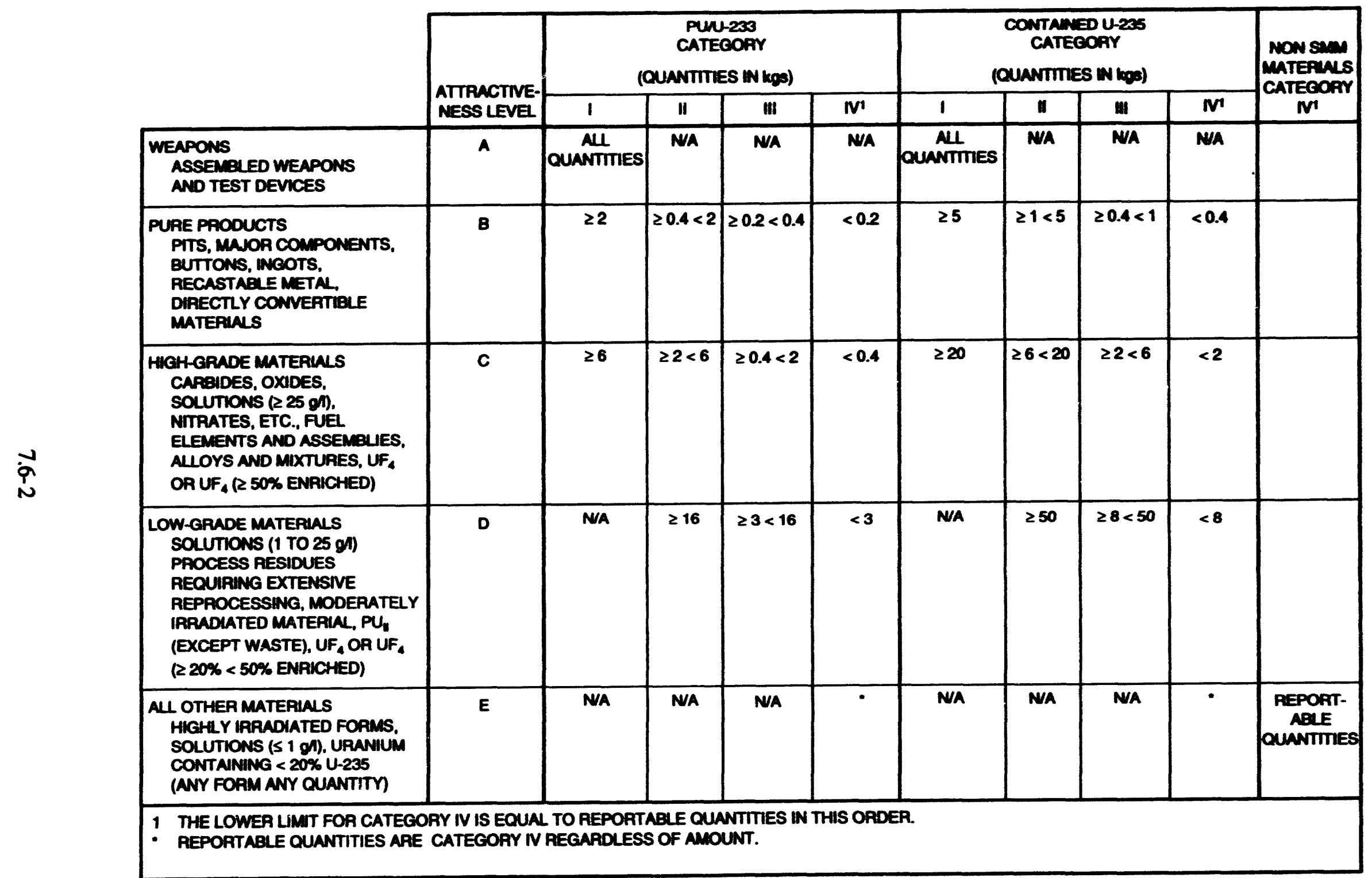

Figure 7.6-1 Nuclear Material Safeguards Categories 
considerations were derived from DOE Order prescriptive requirements as delineated in DOE Orders 5632.2B, 5633.3B, and DOE M 5632.2B-1 (Draft) and experience with vulnerability assessments.

The actual risks associated each stage must be determined by a detailed vulnerability assessment when the specific plants/sites have been selected.

Each assembly will weigh about $300 \mathrm{~kg}$ in the crated shipping condition, with each shipping crate holding two assemblies. Since SST trailer loadings are limited to $10,000 \mathrm{lb}(\sim 4500 \mathrm{~kg})$, approximately 10 assemblies could be shipped in a single SST. For the reference case, the three reactors require a combined total of approximately 528 assemblies per year or 4.5 SST shipments per month. This is fully within the present capability. In view of the highly improbable nature of diversion during shipment of assemblies while under SST transport, considerable flexibility is available in choosing the reactor sites for carrying out the disposition mission, nearly independent of the fuel fabrication facility site.

This analysis has been carried out in accordance with the specific requirements of current DOE Orders (5632.2A and 5633.3A) governing protection, control and accountability of SNM. But current orders do not specifically address the unique circumstances presented by the use of $\mathrm{Pu}$ in LWRs. Both Orders 5632.2A and 5633.3A provide for the granting of exceptions in accordance with Order 5633.2 [Ref. 7-10] such that more flexibility could be introduced into the SST requirement for the shipment of MOX assemblies. 


\subsection{Vulnerability Assessment}

DOE Order 5630.14A requires that all facilities using Category I and Category II quantities of SNM and facilities having a radiological or toxicological threat which could cause an unacceptable impact on the health and safety of the public, employees, or the environment must produce a Site Safeguards and Security Plan (SSSP). The SSSP provides the basis and justification for Safeguards and Security (S\&S) program development, budget, and staffing requirements. The SSSP process (1) examines, describes, and documents improvement priorities; (2) describes the site and facility protection philosophy and strategy; and (3) provides an estimate of resources required to accomplish security.

The SSSP is implemented to ensure that the graded protection of Category I and Category II material is in compliance with existing DOE policy and protection requirements. The core of the SSSP is the vulnerability analysis report (VAR) and the associated vulnerability assessment (VA). The VA is based on protection programs currently in place or anticipated to be in place in order to reduce risks from the design basis threat to an acceptable level. Separate VAs must be conducted for each facility, whether they are co-located or at individual sites. VAs conducted for co-located sites can be combined into a site wide SSSP; otherwise, individual SSSPs must be completed.

In addition to reactor vital area security definitions, VAs must be completed for the risks associated with transportation, fresh fuel storage, and fuel fabrication. Typically, these VAs require information about the infrastructure of the site security, protective force response and detailed procedures and are initiated at the Title I stage of facility design. If the prescriptive requirements of the DOE Orders (5600 series and the DOE General Design Criteria) are considered early in the planning process, the major features of the facility will in all probability be acceptable.

The security features for an existing reactor which meets all current NRC criteria will probably also meet DOE requirements, at least in terms of vital areas and their associated security. Overall site security may be somewhat different for MOX fuel, but these differences will not be discernible until the design and operating procedures for specific plants/sites are examined in detail. 


\subsection{Description of Plutonium During Various Stages of Disposition}

In order to properly design the safeguards system, it is valuable to know the form and quantity of materials at any time during the various stages of disposition. This would include a description of the plutonium during various stages of disposition by form, quantities, isotopics, location and inventory at various points as well as the final state of plutonium in respect to isotopics, quantity and form. The material at various stages can be categorized based on its form by an attractiveness level (for diversion). These quantities are described below.

\subsubsection{Plutonium Distribution During Disposition}

The process steps required to dispose of the weapons program plutonium were briefly reviewed in this section on a functional and architectural level. (More detailed descriptions of the facilities and processes are found in the report sections dealing with each segment of the process.) This section will describe the various forms and the diversion categories of the plutonium-bearing materials as they move through the disposition infrastructure.

The disposition infrastructure is composed of the following major segments, which could conceivably be located at several sites:

- Weapons storage and dismantlement facility

- Pit destruction and storage facility

- Conversion to $\mathrm{PuO}_{2}$ facility

- Fuel fabrication and storage facility

- Reactor and fuel storage facilities

- DOE SNF repository

- Transportation modes and facilities

As the plutonium moves through these segments and is processed for disposition, it exists in various forms and becomes an increasingly less attractive target for diversion. The decreasing attractiveness to diversion initially results from increasing dilution of the plutonium among nonfissile materials. Later, after the fuel is placed in the reactor, the plutonium becomes highly unattractive and inaccessible. When discharged from the reactor, the plutonium is enveloped in a 
heavy and highly radioactive fuel bundle. Table 7.8-1 indicates the various plutonium forms and lists their attractiveness levels as determined by DOE Order 5633.3.

L.zference 7-9 details the methods for determining target quantities and attractiveness levels for nuclear materials. It also provides a "decision tree" structure for determination of the attractiveness levels. Classification of the fuel rods and fuel assemblies as category IID material was based on this decision tree and the explicit designation on page 11 of this referenced guide, which identifies "mixed oxides (SNM content $<100 \mathrm{~g} / \mathrm{kg}$ )" as Attractiveness Level D material. Level D materials are defined as "those materials that are bulkier or of lower purity than Level C materials, and require greater processing time or greater processing complexity to convert to metal than Level $\mathbf{C}$ materials. Diversion or theft of a very large quantity of bulk material is required to obtain a goal quantity of SNM, or processing requires extensive precautions for protection against radioactive emissions." Any amount greater than $16 \mathrm{~kg}$ with this attractiveness level is Category II. Shipment of the MOX fuel assemblies will therefore conform to DOE Orders applicable to Category II, Level D material.

To aid in the visualization of the plutonium distribution, Figure 7.8-1 was developed to show the time-dependent mass distribution of plutonium forms based on the major disposition processes. Table 7.8-2 presents the same data in tabular format in case these quantities are needed for further computation. For the reference option, $50 \mathrm{MT}$ of weapons plutonium is transformed among the eight forms as indicated. The form labeled "Destroyed Pu" tracks the plutonium which is consumed during reactor operations (i.e., there is a net loss of plutonium during the reactor cycle). The Government schedule for dismantling of weapons and ultimate production of plutonia powder is not known. Plutonia powder production has been assumed herein to meet the MOX fuel fabrication needs. To provide the last integral core reload, an additional $0.033 \mathrm{MT}$ of plutonium was required. Hence, the total plutonium inventory in the chart is $50.033 \mathrm{MT}$.

Table 7.8-1 Characteristics of Various Plutonium Forms During Disposition

\begin{tabular}{|l|c|c|c|}
\hline Physical Form & $\begin{array}{c}\text { Attractiveness } \\
\text { Level }\end{array}$ & $\begin{array}{c}\text { Pu Density } \\
\text { (g/cc in form) }\end{array}$ & $\begin{array}{c}\text { Unit Form } \\
\text { Pu Mass (kg) }\end{array}$ \\
\hline Weapons Pu & B & 19.0 & $*$ \\
\hline PuO2 powder & C & 2.0 & $*$ \\
\hline MOX pellet & C & 0.2 to 1.0 & 0.001 (max.) \\
\hline Fuel rod & D & 0.2 to 1.0 & 0.1 to 0.4 \\
\hline Fuel bundle, new & D & 0.2 to 1.0 & 10.15 \\
\hline Fuel bundle, irradiated & D, E & 0.2 to 1.0 & 6.3 \\
\hline * Unit form mass is criticality limited & \multicolumn{3}{|l}{} \\
\hline
\end{tabular}


Table 7.8-2 Plutonium Distribution During Reference Disposition of 50MT Using 3 BWRs

\begin{tabular}{|c|c|c|c|c|c|c|c|c|c|c|c|c|c|c|c|c|}
\hline \multicolumn{17}{|l|}{ Pu Mass (kg) } \\
\hline & & & & & & & & & & & & & & & . & \\
\hline Time, Years & 1994 & 1996 & 1998 & 2000 & 2002 & 2004 & 2006 & 2008 & 2010 & 2012 & 2014 & 2016 & 2018 & 2020 & 2022 & 2024 \\
\hline & & & & & & & & & & & & & & & & \\
\hline Exces Pu, Metal & 50032 & 50032 & 49698 & 48469 & 44221 & 39973 & 35725 & 31477 & 27229 & 22981 & 18733 & 14485 & 10237 & 5988.9 & 1740.9 & 0 \\
\hline Oxide & 0 & 0 & 42 & 531 & 531 & 531 & 531 & 531 & 531 & 531 & 531 & 531 & 531 & 531 & 531 & $\mathbf{0}$ \\
\hline Pellets & 0 & 0 & 42 & 531 & 531 & 531 & 531 & 531 & 531 & 531 & 531 & 531 & 531 & 531 & 531 & 0 \\
\hline Fresh Bundles & 0 & 0 & 167 & 167 & 2379 & 3889 & 2464 & 2974 & 1614 & 2124 & 1699 & 1274 & 1784.2 & 424.8 & 934.56 & $\mathbf{0}$ \\
\hline In Reactor Core & 0 & 0 & 84 & 334 & 2370 & 5970 & 10421 & 11880 & 12171 & 12171 & 12171 & 12171 & 12171 & 12171 & 12171 & 11236 \\
\hline Irradiated Bundles in Reactor Pool & 0 & 0 & 0 & 0 & 0 & 89 & 231 & 1689 & 5092 & 7485 & 10440 & 13288 & 14169 & 15551 & 14953 & 14953 \\
\hline DOE Repository & 0 & 0 & 0 & 0 & 0 & 0 & 0 & $\mathbf{0}$ & 0 & 0 & 35 & 177 & 1689 & 3895.8 & 6886.4 & 9877 \\
\hline Pu Destroyed & $\mathbf{0}$ & 0 & 0 & 0 & 0 & 50 & 130 & 950 & 2864 & 4210 & 5892 & 7574 & 8920.2 & 10939 & 12285 & 13967 \\
\hline Total & 50032 & 50032 & 50032 & 50032 & 50032 & 50032 & 50032 & 50032 & 50032 & 50032 & 50032 & 50032 & 50032 & 50032 & 50032 & 50033 \\
\hline & & & & & & & & & & & & & & & & \\
\hline Cumulative Reactor Loading & 0 & 0 & 84 & 334 & 2370 & 6108 & 10781 & 14520 & 20127 & 23865 & 28538 & 33211 & 36949 & 42556 & 46295 & 50033 \\
\hline
\end{tabular}




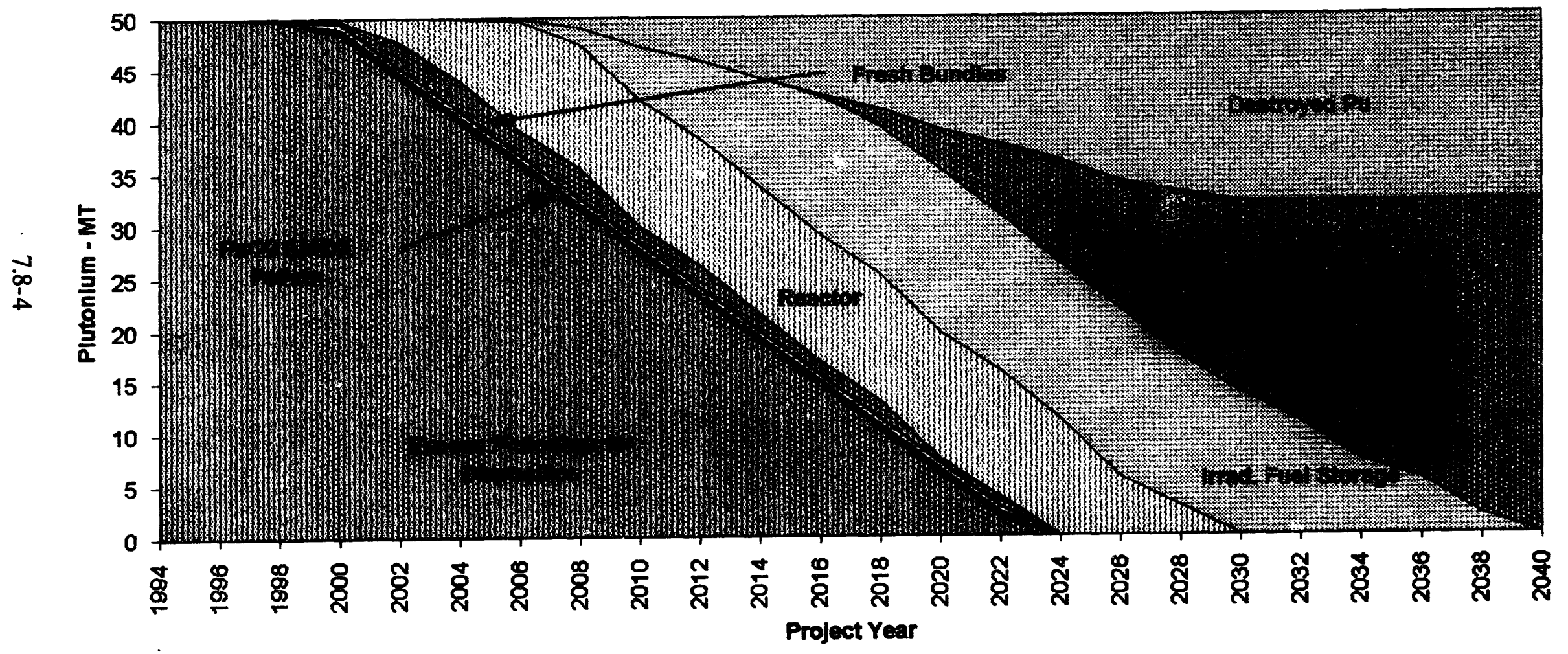

Figure 7.8-1 Plutonium Distribution During Reference Disposition Using Three BWRs 
The schedule calls for initial loading of full MOX bundles in the year 2001. All 50MT of plutonium would have been loaded into the reactor(s) by the year 2024, within 23 years after this initial full MOX loading. The last of the MOX fuel will be discharged five years hence, and after a residence time of 10 years in the storage pool, all the excess plutonium will be deposited in the DOE repository, in the form of spent fuel, -38 years after the start of full MOX bundle loading. Figures 7.8-2 and 7.8-3 show the distribution of Pu halfway through the disposition program, and at the end of the disposition process.

Similar information on plutonium distribution is shown for disposition of 100 MT (Alternate 1) in Figure 7.8-4. This option employs six BWRs. The initial full MOX bundle loading is scheduled for the year 2001, as before. Final MOX fuel load takes place in the year 2025, or 24 years after the start of full MOX bundle loading. The final MOX fuel discharge will occur five hears hence and, after a 10-year cooldown period, the spent MOX fuel assemblies will be placed in the DOE repository 39 years after the start of full MOX bundle loading, in the year 2040. 
NEDO-32361

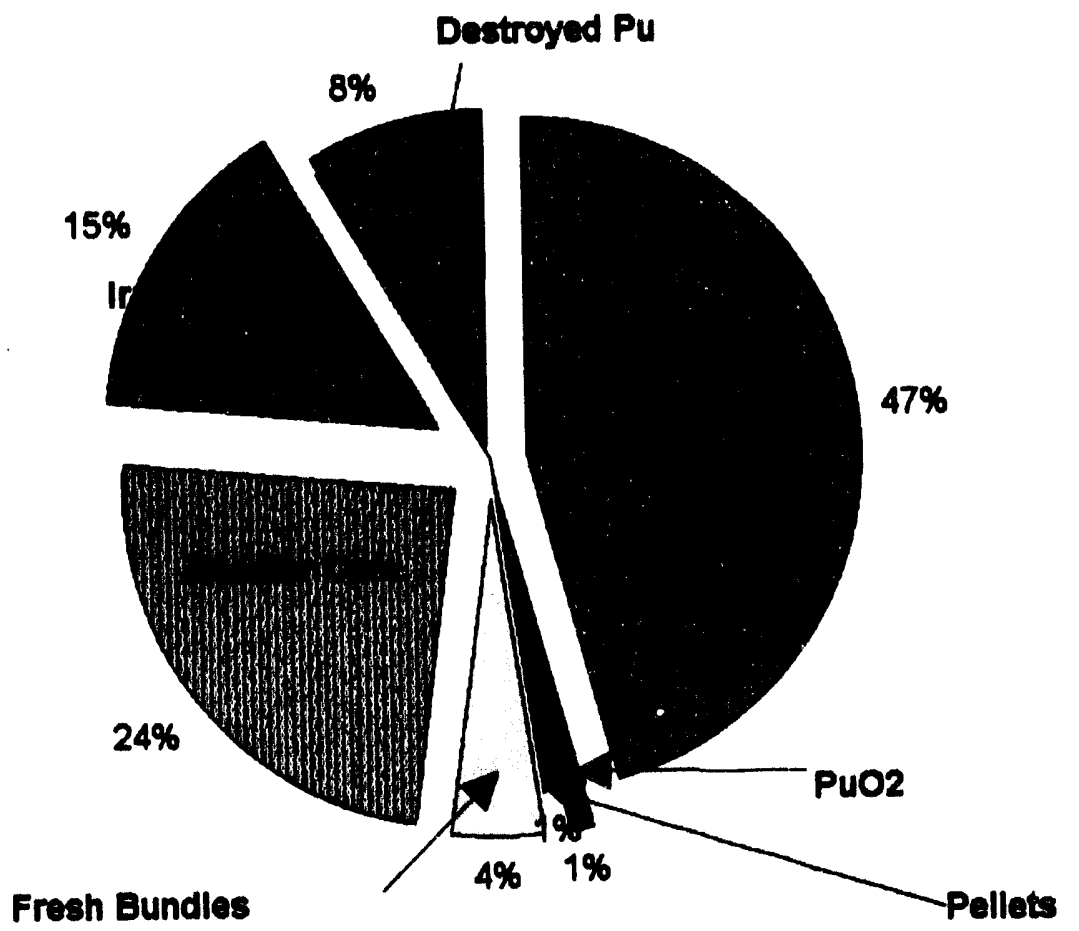

Figure 7.8-2 Plutonium Distribution in Year 2012, Reference Option Using Three BWRs

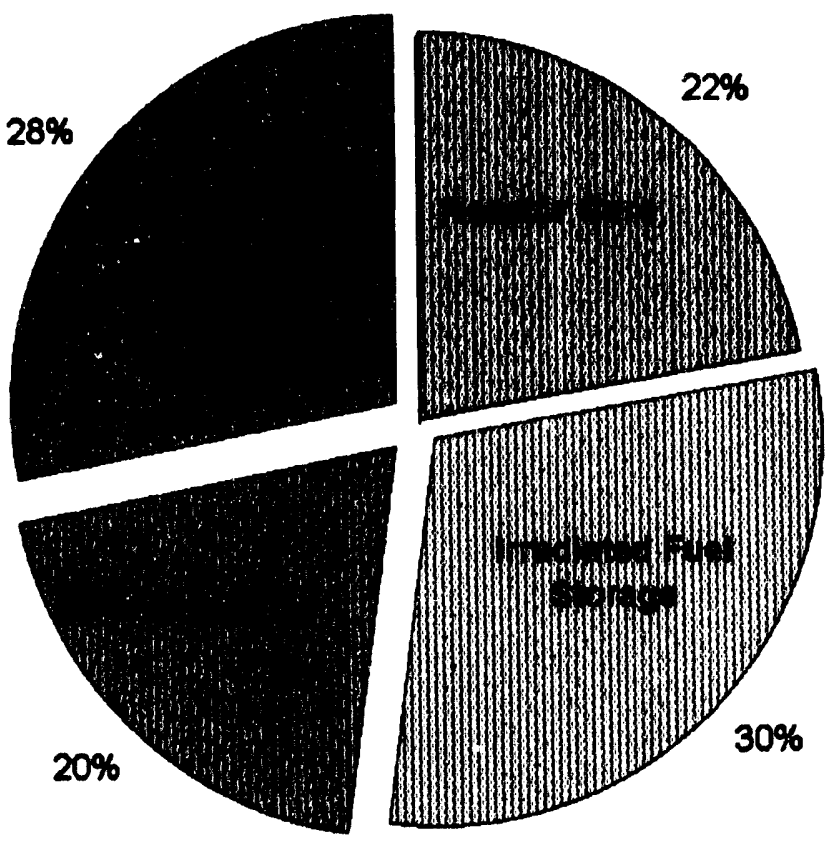

Figure 7.8-3 Plutonium Distribution in Year 2024, Reference Option Using Three BWRs 


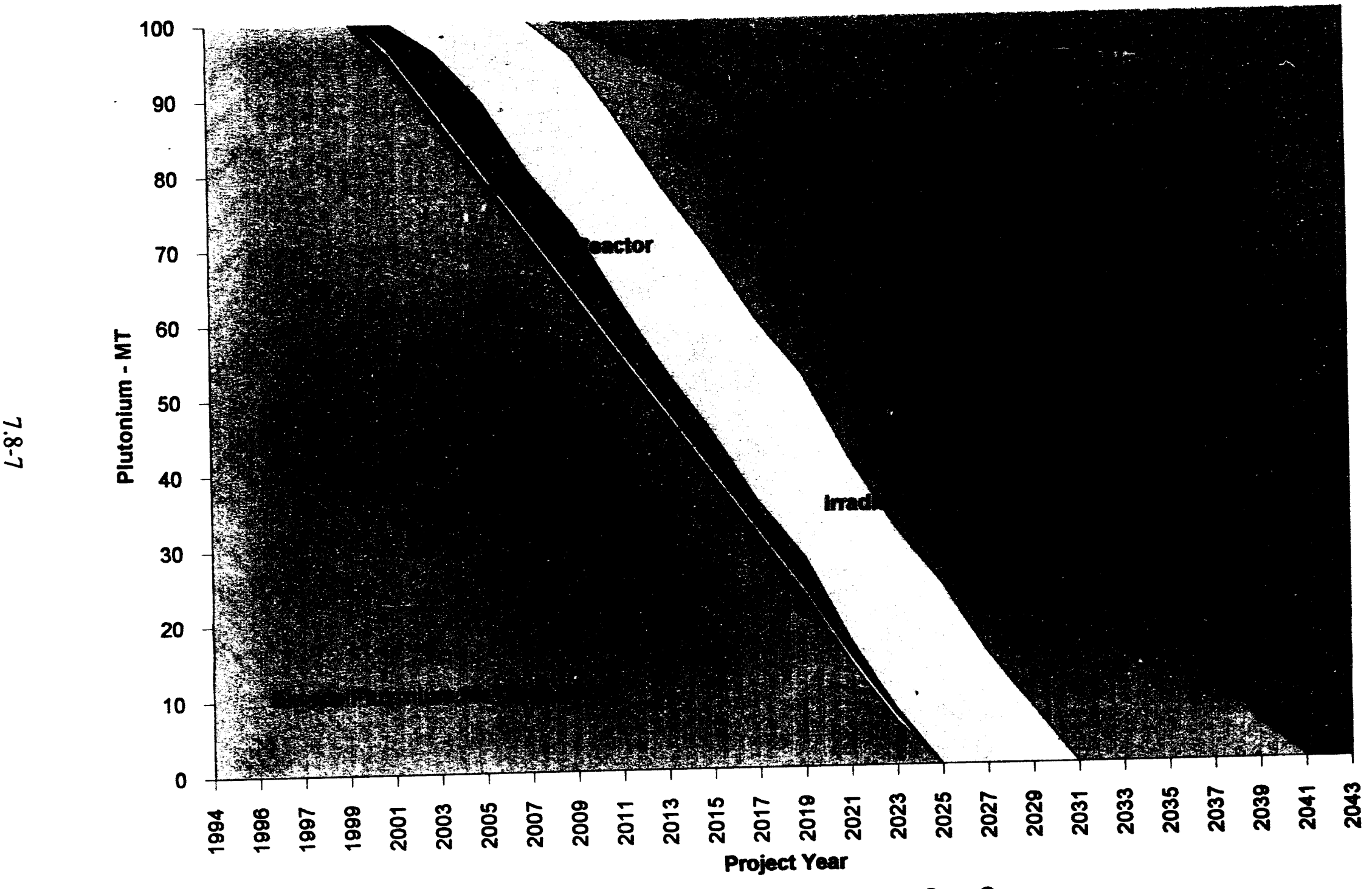




\subsection{International Safeguards, Role of IAEA and IAEA Monitoring}

\subsubsection{Feasibility and Effectiveness of IAEA Monitoring of Operations}

This section discusses the feasibility and effectiveness of the International Atomic Energy Agency (IAEA) to monitor all operations involved in the plutonium disposition program.

\subsubsection{Background}

The IAEA's role in safeguards is established through provisions in agreements between sovereign states that have signed the Treaty on the Non-proliferation of Nuclear Weapons (called the NPT). These countries have agreed to accept safeguards on sources of special fissionable materials used in all commercial nuclear activities within their territory. The exclusive purpose of safeguards is verifying that such material is not diverted for nuclear weapons or explosive purposes. These states agree to implement an effective national safeguards system, to report specified nuclear material transactions to the IAEA, and to permit IAEA inspectors access to facilities and records for purposes of verifying the reported data. The general provisions are described in one document for states that already have nuclear weapons - INFCIRC/66/Rev. 2. The IAEA has also issued another general document (INFCIRC-153). This later document is used to negotiate safeguard agreements with non-nuclear weapon states such as Japan. Specific provisions of the IAEA agreement with an individual country are in a second document that IAEA negotiates with each country. The negotiated agreement with the U.S. can be found in Nuclear Regulations 0980 A 33.1, Vol 2 \#2. The latest renewal of the agreement was in October 1993.

The IAEA has had considerable experience in monitoring nuclear reactor installations, fuel fabrication facilities and spent fuel handling and storage facilities. In addition, they have monitored plants reprocessing spent nuclear fuels and converting uranyl and plutonyl nitrates to oxides, including mixtures of plutonium and uranium oxides. One of the significant limitations in the NPT is that the provisions apply only to commercial nuclear activities. Therefore, only those plutonium disposition activities that were handled commercially would be subject to IAEA inspection. While all the above activities have been done commercially, the IAEA has had no experience with the conversion of plutonium pits to oxides, as that has been an activity under a country's defense program. IAEA has had inspectors in foreign plants that were owned and operated by government corporations such as British Nuclear Fuels Ltd. (BNFL) in England and Power Reactor \& Nuclear Fuel Development Corp. (PNC) in Japan. Therefore, the U.S. could negotiate an agreement with IAEA for monitoring any of the steps for an operation that is owned 
and operated by the U.S. Government or government contractors. However, this has not been done in the U.S. before.

\subsubsection{Basis of Safeguards}

IAEA safeguards are predicated on the existence of a state system of accounting for and control of nuclear materials. The traditional safeguards activities focus on data originated by the plant operators and periodically forwarded to the state safeguards system. The state safeguard system forwards that information to the IAEA. Both the IAEA and the state safeguards system conduct onsite inspections at the operator's plant to collect information that will later be used for verification of the operator's reported data. Within this framework, the IAEA safeguards activities are essentially auditing functions combined with onsite verification which enable tests of the hypotheses that the operator's declared materials transactions did occur as reported.

In order for the IAEA to develop a safeguards approach for an installation, relevant design information must be provided to the IAEA through the state system. When agreement has been reached on the appropriate safeguards approach, the IAEA can perform design verification inspections during the facility construction to assure that the actual design conforms to the reported design data.

As part of the agreement on the safeguards approach, a Material Balance Area (MBA) structure and the related Key Measurement Points (KMPs) are established in which the facility is divided into compatible and manageable sections. Specifications are written for which data must be supplied by the operator. Information on measurement systems to be used by the operator, measurement control programs, and estimates of measurement errors must also be provided. The types of analyses to be applied to the reported accounting data are described and various strategies for data verification by the inspectors are identified.

Agreement must also be reached on the containment and surveillance (C/S) methods to be used by the IAEA. In each instance, several basic questions must be arswered, such as:

- What safeguards issue or concern must be addressed?

- What information or data is needed?

- What $\mathrm{C} / \mathrm{S}$ equipment can obtain the information/data? 
- Is that $\mathrm{C} / \mathrm{S}$ equipment compatible with the particular application?

- What impacts on the facility and the operator will result from the use of the C/S equipment?

Finally, agreement must be reached on how the inspections at the facility will be conducted and what access considerations are involved. Also, the level of inspection effort must be established. After all the preliminary agreements are in place, the flow of the information between the operator, the state safeguards system, and the IAEA is initiated.

\subsubsection{Elements of IAEA Involvement}

There are several established technical elements of IAEA involvement. These fall into two categories: (1) conventional materials accounting and (2) conventional containment and surveillance.

\section{Conventional Materials Accounting}

Conventional Materials Accounting is a basic element of IAEA safeguards. The IAEA receives from the state system monthly inventory change reports for each MBA which are used to adjust the operators declared inventory for each MBA. In addition, the IAEA receives from the state system a declared material balance report for each MBA following the operator's physical inventory taking (PIT) at the facility. In most cases, the physical inventory is required only once per year. If done only once per year, there is a quite long interval between inventories and, therefore, it is difficult to detect, in a timely way, any prompt diversion of nuclear material. In order to overcome this deficiency, Near-Real-Time-Accountancy (NRTA) methods are being developed and employed at some sites. The IAEA evaluates the material balance reports relative to the consistency of the declared information collected and also makes comparative checks between the declared information and independent information collected by IAEA inspection at the facility.

Further assessments of the reported values of the Inventory Difference (ID) statistic for each MBA are made to determine the material accounting sensitivity for detection of material losses. At present, the criteria for Limit of Error for the Inventory Difference (LEID) for an MBA must be less than $0.8 \%$ of the plutonium throughput for that MBA during that accounting period. The LEID is defined as twice the value of the estimated standard deviation for the reported ID for the MBA. 
The IAEA sends inspectors to the facility whose general objective is verifying the accuracy and completeness of the materials reporting accounting data declared by the operator. To accomplish this objective, the inspector is authorized to perform certain functions, including:

- Witness measurements for the KMP transactions.

- Obtain from the operator independent samples prepared for analysis (usually done offsite).

- Make independent measurements of nuclear materials.

- Verify the functions and calibrations of operators measurements systems used to obtain safeguards data.

- Other mutually agreed functions.

The allowable level of IAEA inspections is determined by the type of the facility and amount of the materials involved. Also, the availability of IAEA resources is an important factor. The maximum inspection efforts for facilities is specified in the IAEA document INFCIRC 66 in a Table in Article 57. The number of inspections is dependent on the amount of "effective kilograms" of nuclear material in whichever is larger-facility inventory, annual throughput or maximum potential annual production. An example was selected for a fuel fabrication facility corresponding to the reference core design case with a bundle average of $0.69 \%$ U-235 enrichment containing 78,180 kg/year of uranium and $2373 \mathrm{~kg}$ of plutonium. This number is far in excess of the Article 57 limit of 60 effective $\mathbf{~ k g}$ of nuclear material, so according to the table in Article 57, this amount of plutonium would require right of access to the plant at all times. The reactor would have similar amounts, so the IAEA would also have right of access to the reactor at all times.

\section{Conventional Containment and Survellance (C/S)}

This element compliments the basic materials accounting element. The purpose of $\mathrm{C} / \mathrm{S}$ measures are to ensure the integrity of materials accounting data by providing continuity of knowledge about contained material and to verify the completeness and accuracy of declared material transactions. C/S measures-if effectively applied-can contribute to the safeguards goals for timeliness. For conventional $\mathrm{C} / \mathrm{S}$ applications, the safeguards inspectors may use seals on containers, CCTV surveillance, equipment status monitors, and access indicators. The hardware is usually provided by IAEA, installed by the operators, and used by inspectors. While effective $\mathrm{C} / \mathrm{S}$ measures are very important for achieving the IAEA objectives, implementation of $\mathrm{C} / \mathrm{S}$ in a 
facility must minimize interference with facility operations. To the maximum extent possible, the $\mathrm{C} / \mathrm{S}$ measures should be totally transparent to the operators.

The IAEA has also issued a Safeguard Criteria 1991-1995, Document \# 1990-11-21. These criteria are used for the planning of safeguards implementation activities in the field and at headquarters for all facilities and locations outside facilities. These criteria also apply to the evaluation of inspection goals attainment at facilities and safeguards evaluation at the level of Entire States. The criteria cover eleven types of facilities. These criteria also define the normal frequency of inspection. Applicable sections of the criteria are Section 1 (Light Water Reactors), Section 6 [Fabrication Plants Handling Direct-Use Material (MOX, HEU)] and Section 9 (Storage Facilities and certain Annexes). Each section covers the following:

\section{Examination of Records}

- Verification of the following:

- Design information, the operator's measurement system, physical inventory, transfers, other inventory changes, at other strategic points, at interim inspections for timely detection.

- Criteria covering confirmation of the following: absence of unrecorded production of direct use material subject to safeguards, the absence of nuclear material borrowing and transfers, discrepancy and anomaly followup.

- The following activities:

- When the inventory is less than one significant quantity (1 SQ) and used for the evaluation of partial attainment of the inspection goal when the inventory is greater than 1 SQ.

- Items related to non-nuclear material under safeguards.

- Equipment and facilities under safeguards facilities.

- The preparation and maintenance of inventories and lists of information.

Some of the significant portions of the criteria applicable for each of the facilities in the plutonium disposition program are presented below: 


\section{Reactor}

Design verification for safeguards provisions is done prior to any proposed transfer of material to the facility. It is also reexamined at least once per year in the light of any facility modifications or changes in operating conditions.

A physical inventory verification (PIV) must be taken by the operator once each calendar year. The period of time does not normally exceed 14 months. This PIV is scheduled during the time that the core is refueled or opened for another reason; if the reactor core is not reopened, the PIV can still be done. For multi-core facilities, the PIV is taken when one core is refueled, provided the other cores are maintained under acceptable $\mathrm{C} / \mathrm{S}$.

For low enriched uranium cores, fresh fuel is verified by counting the assemblies and any separated fuel pins with high detection probability for gross defects or by serial number identification. Note: The IAEA's system for detection probability is: High $=90 \%$, Medium $=$ $50 \%$ and Low $=20 \%$.

The use of MOX fuel at the reactor site will require additional verification. For example, verification of fresh MOX fuel is required to be carried out 12 times/calendar year at monthly intervals. With the proposed ex-reactor storage facility, this will not be a problem, as no fresh MOX bundles are stored in the Reactor Building except for 15 days during refueling.

Fuel in the reactor cores is verified as follows:

- If the core is opened, the items are item counted and thereafter until the core is sealed. $\mathbf{C} / \mathrm{S}$ is maintained either on the open core or on removal routes for the fuel assemblies.

- For closed cores under $\mathrm{C} / \mathrm{S}$, the $\mathrm{C} / \mathrm{S}$ system is evaluated by one of the following methods or a combination of them: verification of seals on the reactor closure compartment, evaluation of surveillance records or evaluation of results from another type of C/S system.

Spent fuel is maintained under $\mathrm{C} / \mathrm{S}$ and verified the following way, as applicable:

- For that part of the spent fuel under dual C/S, both C/S systems are evaluated. Seals are verified with medium detection probability. In addition, spent fuel may require item counting, dependent on certain conditions.

- For that part of spent fuel under a single $\mathrm{C} / \mathrm{S}$ based on surveillance, the $\mathrm{C} / \mathrm{S}$ is evaluated and the spent fuel is item counted. Verification is required with medium detection probability for 
gross defects under some of the following conditions: (1) the contents of all full spent fuel casks shipped during the previous PIV had not been at least item counted, (2) core refueling has taken place or (3) spent fuel assemblies can be dismantled at the facility. In the mid1980's a Cerenkov glow viewing device was developed. The early models required either darkness or subdued light but several authorities did not want that condition over the spent fuel ponds, so today instruments are available that operate in normal light. These devices are capable of determining that the material is spent fuel. For the long cooled fuel, which emits only weak glow, a new device, a spent fuel attribute tester, has been developed and is in use. The type of instrument is owned by IAEA. Formerly, there was no method to reverify any MOX which was stored in spent fuel water-filled pools. However, in recent years a gross defect instrument has been developed which will determine that the material is MOX fuel.

- If the $\mathrm{C} / \mathrm{S}$ system is based on seals only, the seals require verification with medium detection probability and the sealed items selected are item counted.

- Spent fuel not under $\mathrm{C} / \mathrm{S}$ is item counted and verified with high detection probability for gross defects.

- Any irradiated fuel pins in canisters that were not under $\mathrm{C} / \mathrm{S}$, or where $\mathrm{C} / \mathrm{S}$ evaluation was inconclusive, are counted and their content verified with high detection probability for gross defects.

- Any transfers into or out of the facility are verified by item counting and under the same verification techniques as described above.

\section{MOX Fuel Fabrication}

Design verification procedures for MOX fuel fabrication plants are the same as stated for reactors. Criteria for these plants also require a physical inventory to be taken by the operator each calendar year with the interval not to exceed 14 months between inventories.

The basic steps in the MOX fuel fabrication process are (1) storage of the $\mathrm{UO}_{2}$ and $\mathrm{PUO}_{2}$ powders, (2) blending the powders, (3) pressing and sintering the powders into pellets, (4) grinding and drying the pellets, (5) loading the pellets into fuel rods, (6) decontaminating and welding the end closures to the fuel rods, (7) inspection of the fuel rods, (8) assembly of the fuel rods into bundles or subassemblies, (9) storage of the new fuel assemblies, and (10) recovery of process scrap materials containing fissile material. 
IAEA inspectors would use several different instruments and techniques to monitor the operation. Some of these are described below.

For direct use nuclear material, such as mixed oxides under $\mathrm{C} / \mathrm{S}$, an evaluation is made of the $\mathrm{C} / \mathrm{S}$ and seal verification is made with medium detection probability. If material has been under $\mathrm{C} / \mathrm{S}$ for one month or more, the iiems are counted, identified and remeasurement is performed with $10 \%$ detection probability for gross and partial defects for bulk material and for gross defects for fuel items. For dual C/S systems, both systems are evaluated. The IAEA has relied on Minolta Camera systems for several years as the surveillance instrument of areas in fabrication plants and oiler facilities. These systems are owned and operated by them. Inspectors periodically review the film to ascertain if any unauthorized movement of material has taken place. In 1991 IAEA had 225 photo surveillance units in operation.

If the direct use nuclear material is not under a $\mathrm{C} / \mathrm{S}$ system, items are counted and verified as follows: (1) plutonium metal, plutonium alloys and plutonium compounds are verified with high detection probability for gross partial and bias defects; (2) fuel rods, fuel assemblies and other fuel items are verified with detection probability for gross and partial defects and serial number identification is made; and (3) other bulk material, such as waste, is verified with high detection probability for gross defects.

Any transfers into or out of the facility are verified by item counting and under the same verification techniques as described above.

In a fuel fabrication plant there are other operations that require verification such as blending of process steams or powders. This involves the examination of records and reports to confirm the amounts and constituents of the blending operations, including the weight fractions, to determine if it agrees with the information in the advance notification that was made to the IAEA.

IAEA has employed a variety of NDA methods and instruments in their inspections. These include low resolution gamma ray measurements, gamma ray measurements using portable multichannel analyzers, high resolution gamma spectrometry and neutron coincidence measurements samples of pellets are taken at the rod loading station for bias defect verification. This is done four times/year, normally during inspections for other purposes. If the plant has remote and/or automated fabrication lines, measurements with a rod scanner can replace the pellet sampling if the nuclear material in the rods is determined to be within a standard deviation of not greater than 0.06 (using IAEA standards for calibration). The use of automated systems is growing, as designers try to limit operator exposures. Most of the plants in operation today 
utilize reprocessed material that has higher radiation levels, because of fission products associated with it, than the material from the plutonium pits in the plutonium disposition program will need to handle. Therefore, it is believed that the program's MOX plant will not need the heavy shielding and automated operation of the currently operated plants, and verification can be made by sampling the pellets.

\section{Storage Facilities}

Since storage facilities embody some of the operations at a reactor or in a fuel fabrication plant, the criteria for the storage are identical to that stated above. In particular, design verification, verification of procedures for spent fuel storage, uranium and plutonium materials and mixed oxides storage, transfers in and out of storage and containment and surveillance systems are the same as described above.

\section{NRC Involvement}

In the U.S. our state system of safeguards is administered by the U.S. Nuclear Regulatory Commission (NRC). Therefore, the implementation of safeguards agreement with the IAEA has been handled by rules and regulations issued by the NRC. Title 10 Chapter 1 Code of Federal Regulations Part 75 is the applicable document.

This document adopts the same wording in the U.S. agreement with the IAEA and in the IAEA Safeguards Criteria. Since the NRC is the responsible agency for implementation, it provides that, in addition to the IAEA inspectors, NRC inspectors can accompany them on their inspections, or carry out their own inspections.

\section{Conclusions}

In view of the above experience and the establishment of agreed upon procedures between the IAEA and the U.S., the U.S. could allow the IAEA inspection of the reactor, MOX fuel fabrication plant, any storage facilities connected with the plutonium disposition program, as well as plutonium pit to oxide conversion facilities, since those operations, from a technical standpoint, are similar to other operations in which the IAEA has been involved. A reexamination of some of the IAEA provisions for the disposition operation, particularly in the areas of audit frequency and resident inspectors, is recommended.

Normally, a country will submit a list of facilities that they would allow IAEA inspectors to inspect. The U.S. list includes reactors and fuel fabrications plants. Currently, it is not believed 
that IAEA has inspectors in any U.S. facilities, as the IAEA has been concentrating their inspectors in Non-Weapon Countries such as Japan (Estimates have been made that $80 \%$ of the inspectors are concentrated in Japan). The British and French have Euratom inspectors in their large reprocessing plants and the IAEA accepts the Euratom inspections as their own. The principal advantage of IAEA involvement is that it paves the way for better control of both civilian and (dismantled) weapons plutonium for the future. The involvement of IAEA would require added expense such as office space and possibly a laboratory and computer connections. 
NEDO-32361

\subsection{Conclusions}

An evaluation of the safeguards requirements for the plutonium disposition process has been completed. Discharging the plutonium as spent fuel is seen to be a proven method of providing long-term diversion resistance for this material. This approach utilizes the already existing national and international infrastructure for safeguards. Although the existing technology and infrastructure are sufficient for safegarding the plutonium during the disposition process, specific areas have been identified where new initiatives might be beneficial.

Clarification at the system level is needed relative to bilateral issues and the role of the IAEA. For the most part, the IAEA provisions are predicated on working with a "state" agency and it should be adapted for plutonium disposition with commercial reactors. At the functional level, an examination of some of the existing safeguards provisions appears to be needed. Specific areas requiring a reassessment include the provisions that apply to the transportation of large numbers of mixed-oxide fuel assemblies and the requirements for handling of these assemblies at the reactor site. The present regulations would appear to require SSTs for shipment, as well as (both nondestructive and destructive) assays to verify the plutonium inventory upon receipt. It is not clear that inventory verification upon receipt in the bundle would be needed after SST transport. By contrast, the current limits for MUF (Material Unaccounted For) may need to be reduced. The provision of a secure ex-reactor storage building will greatly alleviate any adverse impact of safeguards implementation for fresh fuel assemblies on routine reactor operation.

A complete and thorough review of the IAEA safeguards standards and experience indicates that the proposed disposition process is extremely amenable to IAEA involvement. The IAEA requirements can be accommodated at all the disposition related sites, namely at the MOX fuel fabrication facility, at the storage sites and at the reactor. 
NEDO-32361

\subsection{References}

7-1 Study of Plutonium Disposition Using the GE Advanced Boiling Water Reactor (ABWR), Evaluation of Pu consumption in Advanced Light Water Reactors, Prepared for the U.S. DOE, GE, NEDO-32351, April 1994.

7-2 Nuclear Proliferation and Safeguards, Congress of the United States, Office of Technology Assessment, Praeger Publishers, New York, 1977; also, Dismantling the Bomb and Managing the Nuclear Materials, OTA, September 1993.

7-3 Safeguarding Nuclear Materials, Vols. I and II, Proceedings of a Symposium held in October 1975, IAEA, Vienna, 1976.

7-4 Measurement Technology for Safeguards and Material Control, NBS Special Publication 582, U.S. Department of Commerce, National Bureau of Standards, Proceedings from ANS Topical Meeting held in November 1979 in South Carolina, June 1980.

7-5 Management and Disposition of Excess Weapons Plutonium, National Academy of Sciences, National Academy Press, Washington D.C., 1994.

7-6 Goris, P., DeMarschman, Fast Flux Text Facility (FFTF) Fuel Pin Non-Destructive Assay Measurement, Safeguarding Nuclear Materials, Vol. II, IAEA, Vienna, 1976.

7-7 DOE Order 5633.3A, Control and Accountability of Nuclear Materials, February 12, 1993.

7-8 DOE Order 5632.2A, Physical Protection of Special Nuclear Material and Vital Equipment, January 17, 1989.

7-9 USDOE, Office of Safeguards and Security, Guide to DOE Order 5633.3, Control and Accountability of Nuclear Materials, Draft Guidance, April 1990.

7-10 DOE Order 5633.2, Control and Accountability of Nuclear Materials: Responsibilities and Authorities, January 29, 1988. 


\subsection{Environment, Safety and Health (ES\&H)}

This section provides a summary of environmental, safety and health (ES\&H) impacts from utilizing MOX fael in currently operating BWR reactors. The impacts of the plutonium disposition mission, including fuel manufacture and transportation, are also assessed. The assessments are not as comprehensive as would normally be conducted for an environmental impact statement. However, sufficient information was developed to determine the health, safety, and environmental impacts of using of BWRs for plutonium disposition. Before detailed discussions are conducted, however, the potential hazards associated with the process materials are briefly described.

\section{Radiological Hazards}

Plutonium Feed: The plutonium feed material can present a variety of radiological hazards to personnel throughout all plutonium disposition activities. Table 8.0-1 provides information on the variety and relative severity of occupational hazards that could be encountered during these activities.

The characteristics of the MOX feed can have a significant impact on the occupational radiation doses at the fuel facility and, to a lesser extent, at the reactor location. Pu-239 basically presents no external radiation hazard. However, over time, impurities within the plutonium material decay into radioisotopes that can present an external radiation hazard. For instance, the few percent of Pu-241 present in the feed material decays (with a 14.4 year half-life) into Am-241, which then decays (with a 432 year half-life) with a $60 \mathrm{keV}$ photon emission $35.7 \%$ of the time. Feed material that has aged for several years without an americium stripping process normally

Table 8.0-1 Relative Occupational Hazards

\begin{tabular}{|l|l|}
\hline Material & \multicolumn{1}{|c|}{ Potential Hazards } \\
\hline Plutonium-239 & $\begin{array}{l}\text { 1) Internal radiation hazard (H) } \\
\text { 2) External radiation hazard (L) } \\
\text { 3) Criticality (H) }\end{array}$ \\
\hline $\begin{array}{l}\text { Other Plutonium } \\
\text { Isotopes }\end{array}$ & $\begin{array}{l}\text { 1) Internal radiation hazard (H) } \\
\text { 2) External radiation hazard (M) }\end{array}$ \\
\hline Americium and Other & 1) Internal radiation hazard (H) \\
Radioactive Impurities & 2) External radiation hazard (H) \\
\hline Natural Uranium & 1) Internal radiation hazard (M) \\
& $\begin{array}{l}\text { 2) External radiation hazard (M) } \\
\text { 3) Toxological hazard (L) }\end{array}$ \\
\hline \multicolumn{1}{|c|}{ Relative Hazard } & (L)-Low \\
& (M)-Medium \\
& (H)-High
\end{tabular}


has a relatively high external radiation dose component. Am-241 can also present a significant neutron dose component through the alpha/neutron reaction when americium atoms are in intimate contact with light elements (e.g., beryllium). Spontaneous fissions and neutron-induced fissions also contribute a small neutron component of the overall radiation dose. However, this hazard can typically be controlled to a very small percentage of the radiation dose.

Throughout this discussion, two different feed material variations are considered. The first (referred to as low Am feed) has a very low Am-241 content (low ppm concentration of americium). The second variation considers MOX feed manufactured from plutonium feed that has not undergone an americium stripping process (containing $\sim 3000 \mathrm{ppm}$ americium) prior to being manufactured into MOX fuel (referred to as high Am fuel). High Am fuel presents a higher external radiation dose contribution from the americium that must be considered in the facility design, the fuel handling process, and in the personnel protection program. This is more significant in the MOX fuel facility, where concentrated plutonium in the oxide form is processed.

Fresh Fuel: Material in fuel bundles has the advantage of (1) clad shielding, (2) self-shielding in dense pellets, and (3) a lower average Pu concentration of $2.79 \mathrm{wt} \%$. To confirm this advantage, the gamma dose rate for a fresh fuel bundle containing 3000 ppm Am-241 was evaluated. A typical fuel assembly was modeled with discrete modeling of the central water rod and the bundle channel. A two-dimensional transport calculation was performed to determine the gamma flux distribution, which was subsequently used to compute the gamma dose rate, based on the standard energy-dependent flux-to-dose rate conversion factors (ANSI/ANS-6.1.11977). Two different types of fresh fuel bundles, one with $3000 \mathrm{ppm}$ of Am-241 and one without Am-241, were used in the calculation.

Without Am-241, the maximum gamma dose rate at the fuel assembly surface is $5.0 \mathrm{mrem} / \mathrm{hr}$. The addition of Am-241 increases the dose rate to $5.5 \mathrm{mrem} / \mathrm{hr}-\mathrm{a} 10 \%$ increase. This is in contrast to the doubling of the photon source with the addition of Am-241. The increase results because Am-241 in general contributes to higher energy photons ( $50 \mathrm{keV})$ than the low energy $(\sim 10 \mathrm{keV})$ photons generated by the plutonium isotopes and the flux-to-dose conversion factors are significantly lower at $50 \mathrm{keV}\left(2.92 \times 10^{-7}\right.$, compared to $5.15 \times 10^{-6}$ at $\left.10 \mathrm{keV}\right)$. 


\subsection{Transportation Safety}

This section addresses the safe transport of fresh MOX fuel from the MOX fabrication facility to the BWR site and the transport of radioactive and hazardous waste from the BWR site. The transport of plutonium material feed from the U.S. Government Storage to the MOX manufacturing facility is assumed to be conducted by the DOE and is not within the scope of this section.

As discussed in Section 10, there is only one deployment scenario in which co-location of the MOX fuel fabrication plant on an existing BWR site could potentially avoid shipment of fresh MOX fuel over public roads. Thus, the planning basis for this study is that the fresh fuel will be transported over public roads and require DOT approved shipping containers and the use of Safe Secure Transport (SST) vehicles.

The General Electric RA Series fresh fuel shipping container used for the past 20 years provides adequate protection $\mathrm{c} f$ the fuel for normal shipping conditions and limits the damage to the inner stainless steel container and fuel bundle during the 10CFR71 hypothetical accidents. Since the MOX fuel bundle is no different than a typical BWR urania fuel ndle from a mechanical standpoint, the RA Series shipping container should still provide adequate rhysical totection for normal and accident shipping conditions. While this container is not licensed for MOX fuel and would need to be reevaluated, any modifications required to accommodate the additional radiological hazards associated with MOX fuel are not expected to reduce the number of shipping containers that can be accommodated in an SST. In a MOX bundle, the difference between a high Am and low Am fuel is only about $0.5 \mathrm{mrem} / \mathrm{hr}$. Therefore, the difference between the two types will only be discussed where the impact is significantly different.

The fresh MOX fuel is expected to be stored in secured bunkers upon delivery to the reference BWR site. For low Am fuel, the onsite transfer of fresh fuel from the storage bunker to the reactor is assumed to be performed under supervision of the site protective forces using the normal shipping containers designed for low enriched uranium BWR fuel bundles. While an analysis of the radiological impacts of onsite transportation accidents needs to be performed, it is expected that these containers will be adequate because of the reduced severity of potential accidents for controlled access, low speed, onsite transfers compared to the over-the-road transportation conditions for which these containers were designed. The public impact of onsite transportation of MOX fuel is considered insignificant. 
Hazardous and low-level radioactive wastes from the BWR and the MOX fuel fabrication plant are not unique and will be packaged in the appropriate DOT approved waste containers. Use of such containers eliminates the need to perform additional assessments of worker or public safety because those assessments were prerequisite to obtaining DOT approval. Transuranic waste will be packaged in Type B containers such as the TRU-PAC ${ }^{\oplus}$, which the DOE currently uses for shipments of TRU waste between DOE sites.

Spent MOX fuel from the BWR sites is assumed to be transported in casks provided by the DOE in accordancs with 10CFR96. A brief review of the status of the Civilian Radioactive Waste Management System (CRWMS) to be established under 10CFR96 identified no significant concerns related to transportation of spent MOX fuel bundles from the BWR. While few firm design requirements exist for the CRWMS at inis time, because the physical, thermal and criticality characteristics of the spent MOX fuel from the BWR are very similar to current BWR spent fuel, it is expected that compatibility with the CRWMS can be achieved. The impacts on radiological hazards due to increased plutonium content would need to be evaluated, but should be minimal, since spent fuel shipping casks are designed to maintain their integrity during transportation accidents.

No ES\&H issues have been identified that would adversely affect the transport of BWR hazardous materials or wastes. Continued interface with the CRWMS should be established to assure compatibility with any spent MOX fuel. Although an insignificant risk from transportation of MOX fuel is projected, the reaction of state and local governments to transport of hazardous material though their jurisdictions is also unknown. Recently enacted laws and court rulings have given these entities greater control over transportation issues within their jurisdictions. 


\subsection{Environment, Safety, and Health Impacts of Fuel Activities}

This section addresses the environmental, health, and safety impacts of utilizing MOX fuel. Specifically, the impacts associated with the design and operating features of BWRs and the MOX fabrication facility are examined. Both occupational and environmental impacts are discussed along with projected impacts and comparisons to currently operating facilities.

Because of the design and operating features of the facilities studied, no health, safety, or environmental protection issues exist for dispositioning plutonium in current BWRs or from the MOX facility.

\subsubsection{Design and Operating Features}

\subsubsection{Current BWR Design and Operating Features}

GE BWR designs provide a safe, reliable system for producing electric power from nuclear fuels. Plant arrangements and administrative controls are established to maintain worker exposures as low as reasonably achievable (ALARA).

Liquid Radwaste Management System: The Liquid Radwaste Management System collects, monitors, and treats liquid radioactive wastes for return of coolant to the primary system whenever practicable. The BWR is designed to operate as a closed system with no routine discharges of liquid effluents from the reactor coolant and associated systems. The radwaste processing equipment is located in the Radwaste Building. Processed waste volumes discharged are expected to be small. Any discharge is such that concentrations and quantities of radioactive material and other contaminants are maintained "as low as reasonably achievable (ALARA)" and in accordance with applicable local, state, and federal regulations.

All potentially radioactive liquid wastes are collected in sumps or drain tanks at various locations in the plant. These wastes are transferred to collection tanks in the radwaste facility for future waste processing.

Waste processing is done on a batch basis. Each batch is sampled as necessary in the collection tanks to determine concentrations of radioactivity and other constituents. Equipment drains and other low-conductivity wastes are treated by filtration and demineralization and are transferred to the condensate storage tank for reuse. The BWR plant may be permitted to batch release small quantities of waste water; however, this is not a routine release pathway. Protection against inadvertent release of liquid radioactive waste is provided by design redundancy, instrumentation 
for the detection and alarm of abnormal conditions, automatic isolation, and administrative controls. Waste processing equipment is selected, arranged, and shielded to permit operation, inspection, and maintenance with minimum radiation exposure to personnel.

Solid Radwaste Management System: The Solid Radwaste Management System provides for the safe handling, packaging, and short-term storage of radioactive solid and concentrated liquid wastes that are produced. Wet waste produced by this system is transferred to the solidification system, where it is solidified and loaded into waste containers. Dry active waste is surveyed and disposed of whenever possible via the provisions of 10CFR20.302(a). Combustible waste is normally compacted and shipped for offsite disposal. Noncombustible dry waste also is compacted and loaded into containers for offsite disposal.

Ofigas System: The main condenser Offgas System removes the noncondensible gases from the main condenser and discharges them to the gaseous radwaste (offgas) system. The Offgas System consists of two $100 \%$ capacity, multiple-element, multi-stage steam jet air ejectors (SJAEs) with intercondensers, for normal station operation, and mechanical vacuum pumps for use during startup.

The Offgas System receives air and noncondensible gases from the SJAEs and processes the effluent by decay and/or removal of gaseous and particulate radioactive isotopes. The system processes the offgas and releases it to the environs through the plant stack, keeping the dose to the environment as low as practicable.

The Offgas System also reduces the possibility of an explosion from the buildup of radiolytic hydrogen. This is accomplished by the recombination of the radiolytic hydrogen and oxygen under controlled conditions within a recombiner. The system strips the condensible gases and reduces the total volume. The remaining noncondensible (principally air with traces of krypton and xenon) are passed through activated charcoal beds which provide a holdup volume to allow time for the krypton and xenon to decay. After this processing, the remaining gas is discharged through the monitored plant stack.

The plant gaseous effluents are released through an elevated stack. The stack also vents the Reactor Building, Turbine Building, Radwaste Building, Control and Service Buildings, as well as discharge from the Standby Gas Treatment System. 


\subsubsection{MOX Fuel Facility Deaign and Operating Features}

Fabrication of plutonium-bearing fuels in the MOX facility is highly controlled because of the potential hazards associated with plutonium and americium radioisotope work. Confinement systems are designed to maintain a nearly contamination free work environment in order to protect facility workers. In addition, facility confinement systems collect nearly all airborne contaminants generated within the process areas to minimize the potential for environmental releases. The MOX facility uses design and engineered features as the primary means to control hazards. These are backed by proven or improved operating procedures and administrative controls to ensure safe working conditions and minimal impacts on the environment. The potential for significant environmental releases from this plant during normal operations is very small.

Several of the key MOX facility features that help minimize occupational worker and environmental impacts are listed below:

- Nearly pure feed plutonium will eliminate most of the external radiation doses associated with Am-241. However, the facility design can handle relatively high Am-241 impurity levels in the feed stream with shielding and layout modifications.

- All process operations are performed remotely or are automated-minimal hands-on Pu work is anticipated that could cause radiation exposures or skin contaminations.

- All glove-box access points to the process are shielded such that the nonextremity portions of the body receive less than $0.5 \mathrm{mrem} / \mathrm{year}$.

- All Pu processes are isolated both physically and by ventilation from the ambient environment, thus minimizing the potential for internal exposures.

- All required hands-on maintenance will occur only after all radioactive process material has been removed and the area decontaminated to the extent possible.

- Ventilation systems and duct work have no accumulation points for particulate contaminants (except for filters).

- Worker occupied areas are segregated from process materials in piping, tanks, cells, or storage locations.

- All material process cells have remote decontamination capability. 
- The MOX facility has no liquid discharge to the environment.

- Gaseous effluents go through several scrubbing and filtration stages prior to being released to the environment.

- High utilization and recovery efficiency of the process drastically decreases the amount of waste generated, treated, and handled; thus decreasing occupational exposures and the volume of waste shipped offsite.

\subsubsection{Island MOX Fuel Concept}

An early demonstration of the fission option is the manufacture of the BWR island fuel concept. This proposed concept calls for small-scale MOX fuel production by modifying the LANL TA-55 facility. Because of the relatively small amount of MOX fuel to be produced, quantitative projection of health, safety, and environmental impacts were not performed. However, some relative impacts can be projected.

The amount of fuel processed in this manner is very small, involving only about $334 \mathrm{~kg}$ of plutonium over an eight-year period. This indicates that the majority of fuel manufacture work may be conducted in a small-scale process with hands-on techniques involving multiple glovebox operations. These types of operations are acceptable from an occupational dose standpoint for low-Am plutonium feed; however, the high-Am feed would cause external radiological conditions that would be difficult to control and work with.

With respect to environmental impact, the island fuel manufacturing process is expected to operate within the existing facility safety envelope. That would include the existing environmental impact statement and the Safety Analysis Report, with limited modifications, if needed. Therefore, the environmental impacts would have already been conducted and found to be acceptable.

\subsubsection{Radiological Exposures}

The following sections provide projections of the occupational and environmental radiological doses that may be experienced during operation of current BWRs with MOX fuel and of the MOX facility. 


\subsubsection{Radiological Impacts Associated with the Current BWRs}

This section details the projected environmental and occupational radiological impacts to the public and to facility workers. One design criterion of current BWRs has been the control of radiological impacts during operation, and decontamination and decommissioning activities. This section provides detailed information on the radiological impacts of using MOX fuel in these reactors and concludes that the additional environmental impact is within safety standards.

Occupational and Environmental Dose Limitations: Current BWRs are licensed and operated in accordance with the requirements of 10CFR20, Standards for Protection Against Radiation. These requirements include (1) establishment of an operational radiation protection program which achieves occupational radiation doses as low as reasonably achievable (ALARA) and

(2) administrative controls to limit individual doses to less than:

- $5 \mathrm{rem} / \mathrm{yr}$ total effective dose equivalent (tede)

- $50 \mathrm{rem} / \mathrm{yr}$ to individual organs

- $15 \mathrm{rem} / \mathrm{yr}$ dose equivalent (de) to the eye

- $50 \mathrm{rem} / \mathrm{yr}$ de to the skin and extremities.

The offsite radiation dose for individual members of the public from each reactor is limited by a design objective in 10CFR50, Appendix I, to:

- An annual dose of 0.003 rem to the total body or 0.01 rem to any organ from liquid effluents

- An annual air dose of $0.01 \mathrm{rad}$ from gamma or $0.02 \mathrm{rad}$ from beta radiation

Occupational Dose Assessment: Typical occupational doses to BWR workers have been estimated from external exposure rates and projected exposure times experienced in operating BWRs. The occupational exposures for the BWRs, which are the predominant contributors to the $\mathrm{Pu}$ disposition occupational dose, are presented in Table 8.2-1. These doses are a typical annual average based on expected refueling cycles in the BWR. Operation of a BWR with MOX fuel will slightly increase the occupational exposures from higher exposures encountered during new fuel storage, inspection, and movement activities. The additional exposures projected from the use of MOX fuel (MOX impact) are also provided in Table 8.2-1. As can be seen, the increases are not significant. 
NEDO-32361

Table 8.2-1 BWR Occupational Dose Assessment

\begin{tabular}{|c|c|c|c|c|}
\hline BWR Plant Area & $\begin{array}{l}\text { Person } \\
\text { Hours } \\
\text { per year }\end{array}$ & $\begin{array}{c}\text { Person } \\
\text { Rem }\end{array}$ & $\begin{array}{l}\text { MOX Impact } \\
\text { Person Hours } \\
\text { per Year }\end{array}$ & $\begin{array}{l}\text { MOX Impact } \\
\text { Person Rem } \\
\text { per Year }\end{array}$ \\
\hline Fresh Fuel Storage Activities & $\sim 0$ & $\sim 0$ & 500 & 1.0 \\
\hline $\begin{array}{rr}\text { Drywell } & \text { MSIV } \\
\text { SRV, Pumps, } \\
\text { Valves } \\
\text { CRD } \\
\text { LPRM/TIP } \\
\text { ISI }\end{array}$ & $\begin{array}{l}9,000 \\
1,450 \\
1,500 \\
200 \\
2,400 \\
5,500\end{array}$ & $\begin{array}{l}72.0 \\
24.7 \\
25.5 \\
12.0 \\
38.8 \\
93.5\end{array}$ & & \\
\hline $\begin{array}{r}\text { Refueling } \\
\text { MOX Fuel Movement } \\
\text { Vessel Access } \\
\text { Vessel Reassembly } \\
\text { Refueling } \\
\text { Fuel Sipping } \\
\text { Jet Pump Work }\end{array}$ & $\begin{array}{c}\sim 0 \\
4,500 \\
4,400 \\
950 \\
600 \\
2,400\end{array}$ & $\begin{array}{l}\sim 0 \\
13.5 \\
11.0 \\
3.2 \\
3.0 \\
4.5\end{array}$ & 300 & 0.6 \\
\hline $\begin{array}{rr}\text { Reactor Building } & \\
\text { RHR/RWCU } & \text { CRD } \\
\text { Instruments } \\
\text { Other }\end{array}$ & $\begin{array}{c}1,550 \\
600 \\
1,000 \\
5,000\end{array}$ & $\begin{array}{c}25.6 \\
3.0 \\
5.0 \\
16.3\end{array}$ & & \\
\hline $\begin{array}{cr}\text { Turbine Building } & \text { Valves } \\
& \text { Turbine } \\
\text { Condensate } \\
\text { Other }\end{array}$ & $\begin{array}{l}1,150 \\
18,250 \\
2,000 \\
13,150\end{array}$ & $\begin{array}{c}10.9 \\
5.5 \\
15.0 \\
1.3\end{array}$ & & \\
\hline $\begin{array}{l}\text { Radioactive Waste } \\
\text { Management }\end{array}$ & 6,700 & 36.9 & & \\
\hline Work At Power & 5,000 & 33.0 & & \\
\hline Total (1 Plant) & 87,300 & 454.2 & 800 & 1.6 \\
\hline
\end{tabular}

Environmental Dose Assessment: The environmental impact from radioactive materials in gaseous effluents from current BWRs is historically very low. A summary for typical BWRs is provided in Table 8.2-2. BWRs typically have no planned liquid effluents containing radioactive contaminants.

No increase in environmental exposure is anticipated from the use of MOX fuel in BWRs. In fact, a slight decrease in the airborne pathway dose is expected due to the change in noble gas generation between U-235 and Pu-239 fission yields. 
NEDO-32361

Table 8.2-2 BWR Offsite Radiological Impact (rem/yr ede - 1 BWR Unit)

\begin{tabular}{|l|c|c|c|}
\hline Source of Dose & Typical BWR & MOX Delta & $\begin{array}{c}\text { 10 CFR 50 } \\
\text { Appendix I }\end{array}$ \\
\hline $\begin{array}{l}\text { All Airborne Dose } \\
\text { Contributors }\end{array}$ & $0.1 \mathrm{mrem}$ & Negligible & $\begin{array}{c}5 \text { mrem } \\
\text { to total body or } \\
\text { any organ }\end{array}$ \\
\hline $\begin{array}{l}\text { All Liquid Pathway } \\
\text { Dose Contributors }\end{array}$ & $0.15 \mathrm{mrem}$ & $\sim 0$ & $\begin{array}{c}3 \mathrm{mrem} \\
\text { to total body or } \\
\text { any organ }\end{array}$ \\
\hline $\begin{array}{l}\text { Radioiodines and } \\
\text { radioactive } \\
\text { particulates }\end{array}$ & $0.0057 \mathrm{mrem}$ & Negligible & $\begin{array}{c}15 \mathrm{mrem} \\
\text { to any organ }\end{array}$ \\
\hline
\end{tabular}

\subsubsection{Radiological Hazards from the MOX Fuel Facility}

Occupational and Environmental Dose Limitations: The MOX Fuel Facility is assumed to be constructed and operated in accordance with the occupational protection standards specified in DOE Orders, 29 CFR, and/or applicable State regulations. These Order requirements must be met in order to obtain approval to operate through the DOE readiness review process. The DOE Orders require that annual occupational doses be limited to 5 rem effective dose equivalent (ede), $15 \mathrm{rem} / \mathrm{yr}$ dose equivalent (de) to the lens of the eye, $50 \mathrm{rem} / \mathrm{yr}$ de to extremities and $50 \mathrm{rem} / \mathrm{yr}$ de to individual organ or tissue. However, DOE has adopted a design and operating goal of less than $0.5 \mathrm{rem} / \mathrm{yr}$ ede for individual workers and requires DOE Program Secretarial Official approval prior to exceeding $2 \mathrm{rem} / \mathrm{yr}$ for any individual worker.

With respect to environmental regulatory limits, DOE facilities are subject to a site dose limit of $0.01 \mathrm{rem} / \mathrm{yr}$ ede to members of the public from all airborne radionuclides (40CFR61, Subpart H). For liquid effluents, the Clean Water Act specifies a dose limit of $4 \mathrm{mrem} / \mathrm{year}$ to individuals. In addition, DOE Orders also specify a maximum dose of $100 \mathrm{mrem} / \mathrm{year}$ from all DOE sources. However, the DOE has a policy that radiation doses to the public shall be maintained as low as reasonably achievable.

Occupational and Environmental Dose Assessment: Occupational exposures from the MOX facility were estimated from dose rates, worker exposure time, annual throughput, and other exposure conditions during operation of the facility. The estimated occupational exposures are provided in Table 8.2-3 for the low Am fuel. As can be seen, a total facility dose of 21.65 person-rem per year is projected. The average dose among the 106 facility workers is about $204 \mathrm{mrem} /$ year, well below the DOE annual goal of 500 mrem. 
Table 8.2-3 MOX Fuel Facility Occupational Radiation Doses - Low Am Fuel

\begin{tabular}{|l|c|c|c|c|}
\hline Function & $\begin{array}{c}\text { Number } \\
\text { of } \\
\text { Radiation } \\
\text { Workers }\end{array}$ & $\begin{array}{c}\text { Average } \\
\text { Dose Rate } \\
\text { (mrem/hr) }\end{array}$ & $\begin{array}{c}\text { Annual } \\
\text { Exposure } \\
\text { Hours }\end{array}$ & $\begin{array}{c}\text { Total Annual } \\
\text { Dose } \\
\text { (Person-rem) }\end{array}$ \\
\hline ES\&H Technicians & 9 & 0.25 & 9,000 & 2.25 \\
\hline Process Operators & 62 & 0.2 & 62,000 & 12.4 \\
\hline Mechanical Operations & 10 & 0.3 & 10,000 & 3.0 \\
\hline Chemistry/Lab & 15 & 0.1 & 15,000 & 1.5 \\
\hline QA/Inspection & 5 & 0.1 & 5,000 & 0.5 \\
\hline Waste Processing & 5 & 0.4 & 5,000 & 2.0 \\
\hline Total & 106 & & 106,000 & 21.6 \\
\hline
\end{tabular}

Dose projections for the high Am case are provided in Table 8.2-4. As can be seen, despite the additional shielding, the total facility dose is projected to be $55.8 \mathrm{rem} / \mathrm{year}$ for a facility average of 0.53 rem per individual. This exceeds the DOE annual dose goal by a small margin. However, certain worker groups (e.g., mechanical operations which includes maintenance, and ES\&H personnel) may experience annual doses approaching $1 \mathrm{rem}$. The greatest portion of this dose is expected to be incurred during infrequent operational and maintenance activities involving hands-on or close proximity work.

Environmental doses for the MOX Fuel Facility were calculated using an EPA-approved compliance computer code. Because of the effective air cleaning systems in the facility and the fact that most of the Pu scrap will be recovered and recycled back into the fabrication stream, very little radioactive material (Section 8.3.2) is expected to be released into the environment. Environmental doses from the MOX facility were projected based on the assumption that 2.0 E-04 curies per year of Pu-239 is released in the plant gaseous effluent for the

Table 8.2-4 MOX Fuel Facility Occupational Radiation Doses - High Am Fuel

\begin{tabular}{|l|c|c|c|c|}
\hline Function & $\begin{array}{c}\text { Number } \\
\text { of } \\
\text { Radiation } \\
\text { Workers }\end{array}$ & $\begin{array}{c}\text { Average } \\
\text { Dose Rate } \\
\text { (mrem/hr) }\end{array}$ & $\begin{array}{c}\text { Annual } \\
\text { Exposure } \\
\text { Hours }\end{array}$ & $\begin{array}{c}\text { Total Annual } \\
\text { Dose } \\
\text { (Person-rem) }\end{array}$ \\
\hline ES\&H Technicians & 9 & 0.7 & 9,000 & 6.3 \\
\hline Process Operators & 62 & 0.5 & 62,000 & 31.0 \\
\hline Mechanical Operations & 10 & 0.9 & 10,000 & 9.0 \\
\hline Chemistry/Lab & 15 & 0.3 & 15,000 & 4.5 \\
\hline QA/Inspection & 5 & 0.2 & 5,000 & 1.0 \\
\hline Waste Processing & 5 & 0.8 & 5,000 & 4.0 \\
\hline Total & 106 & & 106,000 & 55.8 \\
\hline
\end{tabular}


Low Am fuel. The High Am fuel includes an additional 4.0 E-4 curies of Am-241 in the source term. The results of the dose assessment are listed in Table 8.2-5. The MOX fabrication facility has no liquid effluents containing radioactive contaminants.

\subsubsection{Relative Impacts of using High- and Low-Americium Plutonium Feed}

A relative comparison of the occupational dose impacts between the two $\mathrm{Pu}$ feed alternatives is provided in Table 8.2-6. This information indicates that removal of the americium component early in the process would eliminate the majority of the external radiation exposures in subsequent processing and fuel handling activities. A cost/benefit analysis for americium removal probably would find that the radiation dose avoided throughout the $\mathrm{Pu}$ disposition

Table 8.2-5 MOX Fuel Facility Offsite Radiological Impact

\begin{tabular}{|l|c|c|}
\hline & $\begin{array}{c}\text { Projected Ofisite Dose } \\
\text { (mrem/yr ede) }\end{array}$ & $\begin{array}{c}\text { EPA Ofisite Dose Limit } \\
\text { (mrem/yr ede) }\end{array}$ \\
\hline $\begin{array}{l}\text { Fuel Fabrication Facility- } \\
\text { Low Am Fuel }\end{array}$ & 0.016 & 10 \\
\hline $\begin{array}{l}\text { Fuel Fabrication Facility- } \\
\text { High Am Fuel }\end{array}$ & 0.048 & 10 \\
\hline
\end{tabular}

Table 8.2-6 Impact Comparison of High- and Low-Americium Content Feed Plutonium

\begin{tabular}{|c|c|}
\hline Low-Americium Plutonium & High Americium Content \\
\hline 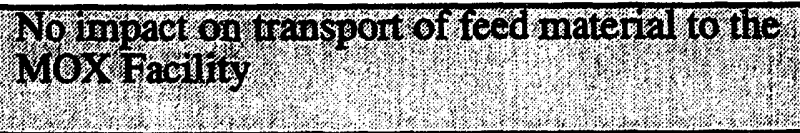 & $\begin{array}{l}\text { Will have increased impact on external } \\
\text { radiation doses during transport to the MOX } \\
\text { Facility }\end{array}$ \\
\hline $\begin{array}{l}\text { No deritional limpact on facility operations or } \\
\text { he cost of facility shielding }\end{array}$ & $\begin{array}{l}\text { Will have significant impact on facility } \\
\text { operations and on the cost of facility shielding }\end{array}$ \\
\hline Oecupational tadiation doses will be low and & $\begin{array}{l}\text { External occupational radiation doses will be } \\
\text { at least twice that of the low-Am case and } \\
\text { meeting DOE dose goals will be difficult. }\end{array}$ \\
\hline $\begin{array}{l}\text { No impact on transport of fresh } M O X \text { fuel to } \\
\text { he reactor sites }\end{array}$ & $\begin{array}{l}\text { Occupational and public radiation doses will } \\
\text { be slightly higher }\end{array}$ \\
\hline $\begin{array}{l}\text { Tief storage and handling activities at the } \\
\text { reactor will not increase dose }\end{array}$ & $\begin{array}{l}\text { Fuel storage and handling activities at the } \\
\text { reactor will involve slightly increased dose }\end{array}$ \\
\hline $\begin{array}{l}\text { Reactor operation and waste management } \\
\text { activities will have no impact from americium } \\
\text { content }\end{array}$ & $\begin{array}{l}\text { Reactor operation and waste management } \\
\text { activities will have no impact from americium } \\
\text { content }\end{array}$ \\
\hline $\begin{array}{l}\text { Safeguards activities preventing diversion of } \\
\text { plutonium are less effective }\end{array}$ & $\begin{array}{l}\text { Sofreguards activities preventing diversion of } \\
\text { plitonium are nore effective }\end{array}$ \\
\hline $\begin{array}{l}\text { The early removal of americium creates an } \\
\text { additional waste stream }\end{array}$ & $\begin{array}{l}\text { Processing Pu with all impurities does not } \\
\text { create an aditional waste stream }\end{array}$ \\
\hline
\end{tabular}

Shade indicates the lesser impact of the two. 
process would justify the americium removal costs. The DOE Health Physics Manual of Good Practices for Plutonium Facilities (DOE 1988) recommends that plutonium that contains "more than about 5\% Pu-241 and is several years old should be reprocessed to remove the Am-241 before processing the plutonium".

\subsubsection{Other Complex Hazards}

As with any large industrial facility, $\mathrm{Pu}$ disposition facilities will present certain worker and environmental hazards based solely on the presence of hazardous materials and industrial hazards. These hazards will initially be controlled or eliminated in the definitive design of the MOX plant. Where they cannot be eliminated, the safety program will further control the hazards to ensure they are minimized. For operating BWRs, these hazards are currently controlled to within industry standards. The utilization of MOX fuel should have no impact on the current level of safety.

Occupational Exposures to Hazardous Materials: The operation of commercial BWRs and the MOX facility will require use of hazardous materials such as acids, bases, hydrocarbon fuels and lubricants, and solvents. The occupational safety program will control worker exposures to hazardous materials to within the levels listed in Title 29 Code of Federal Regulations, Labor, Occupational Safety and Health Administration. In addition, the safety program will extend the ALARA concept to ensure worker exposures to hazardous chemicals are also as low as reasonably achievable. The $\mathrm{Pu}$ disposition facilities will present no additional material hazards beyond those present at similar commercial facilities.

Occupational Exposures to Industrial Hazards: The presence of workers in any industrial setting creates the potential for occupational injuries. Hazards such as tripping, falling, electrical shock, etc. can be safely engineered to a certain point. However, both the safety program and worker commitment to safety must be fully utilized to minimize the frequency and severity of occupational injuries. BWRs and the MOX facility will present no additional industrial hazards beyond those present at similar commercial facilities.

Environmental Releases of Hazardous Materials: DOE Orders specify that hazardous pollutants in environmental discharges will be driven by the DOE ALARA policy which is implemented with Best Available Technology (BAT) criteria. In addition, EPA and applicable state regulations specify limits on discharges of regulated materials to the environment. Discharges are usually regulated by a permit such as the National Pollution Discharge Elimination System, which is obtained prior to plant construction or startup. Utilization of MOX 
NEDO-32361

fuel in BWRs and operation of the MOX facility should result in no increase in regulated pollutants being discharged to the environment. 


\subsection{Waste Streams}

Operation of the plutonium disposition facilities consisting of one MOX facility and currently operating BWRs will generate a volume of various waste types at different locations. This section will define those types expected to be generated and will project the volumes and characteristics of the wastes to be stored, treated, and or disposed. This report uses the following DOE definitions for the various waste types:

Spent Nuclear Fuel - Spent nuclear fuel is fuel that has been withdrawn from a nuclear reactor following irradiation, but that has not been reprocessed to remove its constituent elements. The disposition of this waste is the responsibility of the DOE as specified in the Nuclear Waste Policy Act of 1982. The volume of this waste generated annually is discussed in Section 2.6.4. Current plans are to transport it to a national repository.

Transuranic Waste - Transuranic waste (TRU) is waste contaminated with alpha-emitting radionuclides with half-lives greater than 20 years and concentrations greater than $100 \mu \mathrm{Ci} / \mathrm{gram}$. TRU wastes are transported to the Waste Isolation Pilot Plant (WIPP) in the State of New Mexico for disposal.

Low-Level Radioactive Waste - Low-Level Radioactive Waste (LLW) is waste containing radioactive materials that is not high-level waste, spent nuclear fuel, or has a TRU concentration greater than $100 \mu \mathrm{Ci} / g r a m$. Low-level radioactive waste generated at the complex will be compacted or incinerated, packaged, and transported to a DOE facility for disposal.

Hazardous Waste - Hazardous waste is defined in 40CFR261. Some examples are used oils, lubricants, hydraulic fluids, and various chemical compounds. All hazardous wastes will either be rendered non-hazardous at the site or will be shipped to an offsite contractor for treatment and disposal.

Mixed Radioactive and Hazardous Wastes - Mixed wastes are those that contain characteristics of both radioactive wastes and hazardous wastes. Examples are contaminated oils and lubricants and solvents used to decontaminate surfases. The very small volume of mixed waste will be transported to and stored at an approved DOE mixed waste storage facility.

Solid and Sanitary Waste - Solid and sanitary wastes are those that are nonregulated. Solid waste consists of garbage, trash, and items normally disposed at a community landfill. It will be disposed at permitted faci ity either at or near the site. Sanitary waste will be disposed either in site septic systems or will enter existing municipal systems for treatment and disposal. 


\subsubsection{Waste Minimization Measures}

Currently operating BWRs typically have waste minimization programs in place to minimize both the volume and cost impacts of waste generation. The MOX facility will be designed and operated to minimize all waste streams.

\subsubsection{BWR Plant Radioactive Waste Management}

Low-level radioactive waste from the reactor primarily consists of concentrated waste from the reactor water cleanup and condensate demineralizer systems and waste generated during maintenance activities (e.g., protective clothing, replaced equipment, etc.). A breakdown of the major contributors is provided in Table 8.3-1. The annual volume of radioactive waste shipped from a typical BWR unit is projected to be $245 \mathrm{~m}^{3}$. The BWR does not produce TRU or mixed waste.

Waste minimization is an operational goal of all commercial BWRs in order to reduce operating and maintenance costs and to avoid disposal site restrictions. The amount of waste generation is reported by each utility to INPO on a quarterly basis. Operation with MOX fuel is not expected to increase the amount or change the content of waste generated in BWRs.

\subsubsection{MOX Plant Radwaste Management}

This section provides a description of the radioactive waste management activities for the MOX fuel fabrication facility. The operation of the MOX fuel facility generates a volume of radioactive waste each year. The waste types generated are LLW, TRU, and perhaps a very small volume of mixed waste.

MOX Fabrication Radioactive Waste Experience: During operation of the MOX fabrication facility, some minimum amount of waste will be generated that cannot be recycled within the plant. Some of the material must be recycled because it does not meet final pellet specifications. This would include materials required for setting up machine parameters, material for test

Table 8.3-1 BWR Radioactive Waste Generation (1 Unit)

\begin{tabular}{|l|c|}
\hline Waste Source & $\begin{array}{c}\text { Estimated Waste Volume } \\
\left(\mathbf{m}^{\mathbf{3}} / \mathbf{y r}\right)\end{array}$ \\
\hline Filter Sludge, spent resins, etc. & 100.5 \\
\hline $\begin{array}{l}\text { Dry Active Wastes, } \\
\text { contaminated equipment }\end{array}$ & 139.7 \\
\hline Other Wastes & 4.9 \\
\hline Total & 245.1 \\
\hline
\end{tabular}


specimens, material removed during pellet grinding, and pellets with surfaces defects such as cracks. Either a dry recycle or wet recycle can be used depending on the purity of the material. In the dry method, if the material is out of specification because of damage, such as chips or cracks, it can be ball milled and the powder returned to the fabrication process. A wet method is used if the waste is powder from cleanup of the glove boxes which could contain impurities. This wet method consists of dissolving the material and processing the solution by solvent extraction or ion exchange.

Three categories of nuclear waste result from fuel fabrication: (1) solid glove-box waste, (2) solid waste from retired equipment, and (3) liquid waste from scrap recovery and from work in the plant analytical laboratory. This waste can be classified as low level wastes, mixed wastes (radioactive and hazardous waste), and transuranic (TRU) waste.

Before disposal of the waste, it may be treated for (1) volume reduction of the waste, (2) reduction of the plutonium contained in the waste, and (3) solidification of the liquid waste. A typical plant will usually use compaction or incineration to reduce the volume. Plutonium recovery can be accomplished by ash leaching, acid digestion and a washing process. Solidification of the waste can be by cementation or bitmunization, such as is practiced in French and German plants.

Based on the European experience, the majority of a fabrication plant waste is expected to be a low-level waste, due to a possible difference in waste definitions. In the U.S., the majority of that waste would probably meet the definition of TRU waste. TRU waste would be disposed at the Waste Isolation Pilot Project (WIPP).

Low Level Waste: Fuel fabrication waste will include low-level radioactive waste (LLW) from facility filters and purification columns. Techniques that minimize the generation and assist in the treatment of radioactive wastes from the fuel fabrication plant will be implemented. Improved housekeeping and operating practices and techniques will aid in reducing waste generation. Treatment of water and gases which may contain radioactive elements can be processed with currently available proven technologies. Incineration of appropriate combustible wastes, compaction of noncombustible wastes and solidification of wastes in cement-glass will all be utilized to stabilize and reduce the volume of wastes.

In the chemical laboratory, utilization of accurate nondestructive assay techniques will reduce the number of the chemical analyses required and thereby reduce the amount of contaminated chemicals that must be recovered. 
Transuranic Waste: Operation of the fuel fabrication facility will result in a small volume of transuranic (TRU) waste each year. Transuranic wastes will be packaged, possibly compacted, certified, and transported to the Waste Isolation Pilot Plant (WIPP) in New Mexico for disposal.

In the scrap fuel area, the amount of TRU waste generated is expected to be minimal because the amount of scrap fuel will be very low. Because most scrap fuel will be recaptured for recycling into the fabrication process, the major source of transuranic waste would be generated during infrequent equipment or process upsets with the majority of that material being recycled. Most equipment or process upsets should not generate scrap fuel because the planned on-line computer monitoring and control of the equipment or process should initiate actions to prevent damage to the fuel being fabricated. Additionally, if needed, special treatments, such as re-sintering if pellet density were found to be low, can be used to recover the affected fuel.

Mixed Radioactive/Hazardous Waste: Despite the fact that nearly all of the MOX facility waste will be recovered, a very small volume may be considered both radioactive and hazardous waste. This may be generated when lubricants become contaminated or where decontamination fluids also become radioactively contaminated and chemical or physical recovery methods are not effective. The quantities of waste and a description of waste characteristics are summarized in Table 8.3-2. These are the waste volumes projected to be sent offsite for storage/disposal each year.

Hazardous and Non-Regulated Wastes: Operation of the plutonium disposition complex will create a volume of hazardous and non-regulated wastes each year. Some examples of hazardous materials are used oils, lubricants, hydraulic fluids, and various chemical compounds. All hazardous wastes will either be rendered non-hazardous at the site or will be shipped to an offsite contractor for treatment and disposal.

Solid and sanitary wastes are those that are non-regulated. Solid waste consists of garbage, trash, and items normally disposed at a community landf.ll. It is currently disposed at a permitted facility either at or near the site. Sanitary wastes are disposed either in site septic systems or enter existing municipal systems for treatment and disposal. There are no additional impacts to the types, quantities, or volumes of waste as a result of using MOX fuel in BWRs.

Table 8.3-3 presents a summary of each type of hazardous waste generated at the complex each year. 
NEDO-32361

Table 8.3-2 MOX Fuel Facility Radioactive Waste Generation Low and High Am Cases

\begin{tabular}{|l|c|c|c|}
\hline $\begin{array}{l}\text { Waste } \\
\text { Characteristic }\end{array}$ & $\begin{array}{c}\text { Low-Level Waste } \\
(\mathbf{m} 3 / \mathbf{Y r})\end{array}$ & $\begin{array}{c}\text { Transuranlc } \\
\text { Waste } \\
(\mathbf{m} 3 / \mathbf{y r})\end{array}$ & $\begin{array}{c}\text { Mixed } \\
\text { Waste } \\
(\mathbf{m} 3 / \mathbf{y r})\end{array}$ \\
\hline Miscellaneous Waste & 142 & & 2 \\
\hline Ash Waste & & 0.62 & \\
\hline Plastic Waste & & 264 & \\
\hline Pulped Waste & 1.72 & 0.94 & \\
\hline Metallic Waste & 144 & & 266 \\
\hline Total & & 266 & 2 \\
\hline
\end{tabular}

Table 8.3-3 Hazardous and Non-Regulated Wastes Reference Case

\begin{tabular}{|l|c|c|}
\hline Individual Facility & $\begin{array}{c}\text { Hazardous Waste } \\
(\mathbf{m} 3 / \mathbf{y r})\end{array}$ & $\begin{array}{c}\text { Sold and Sanitary } \\
\text { Wastes (Per Year) }\end{array}$ \\
\hline Fuel Fabrication Facility & 100 & $\begin{array}{c}30 \mathrm{~m}^{3} \\
(2.1 \mathrm{E} 6 \mathrm{gal})\end{array}$ \\
\hline BWR (1 Unit) & 200 & $\begin{array}{c}170 \mathrm{~m}^{3} \\
(3.2 \mathrm{E} 6 \mathrm{gal})\end{array}$ \\
\hline
\end{tabular}

\subsubsection{Plutonium Carryover}

This section summarizes the estimated plutonium contained in the waste streams from the BWR and MOX fuel facility.

\subsubsection{BWR Plutonium Carryover}

Fuel materials including plutonium are expected to be fully contained within the fuel rods. Significant operational data has been collected to demonstrate that fuel materials do not migrate into the reactor systems even when rods experience a leak. Therefore, no plutonium is considered as a carryover from the BWR.

\subsubsection{MOX Plant Plutonium Carryover}

It is expected that less than $1 \%$ of the throughput of the plutonium will be present in the MOX plant waste, leaving more than 99\% of the feed plutonium which is processed in the manufacturing process into fuel rods. Based on the European estimate of plutonium carryover, the MOX plant waste for the various options is summarized in Table 8.3-4. 
NEDO-32361

Table 8.3-4 Summary of Plutonium Carryover Quantities

\begin{tabular}{|c|c|c|}
\hline & $\begin{array}{c}\text { Reierence and } \\
\text { Alternate } 1\end{array}$ & Alternate 2 \\
\hline MOX plant throughput & $2.13 \mathrm{Mr} / \mathrm{yr} \mathrm{Pu}$ & $4.06 \mathrm{MIT/yr} \mathrm{Pu}$ \\
\hline 1\% Plutonium Carryover & $21.3 \mathrm{~kg} / \mathrm{yr}$ & $40.6 \mathrm{~kg} / \mathrm{yr}$ \\
\hline MOX Facility Life & $497 \mathrm{~kg}$ & $994 \mathrm{~kg}$ \\
\hline
\end{tabular}




\subsection{Criticality Safety}

This section summarizes the criticality safety analyses conducted for MOX fuel bundles during transportation as both fresh fuel and spent fuel bundles. In addition, preliminary investigation of spent fuel storage in the repository was conducted. Criticality safety issues for the MOX facility are also discussed.

\subsubsection{Criticality S fety of Fresh Fuel Transportation}

RA series shipping containers have been used by GE Nuclear Energy (GENE) for more than 20 years to ship BWR fuel elements to domestic and international customers. Extensive analyses have been performed to demonstrate criticality safety of these containers for the transport of a wide range of $7 \times 7,8 \times 8,9 \times 9$, and $10 \times 10 \mathrm{BWR}$ fuel assemblies. This section provides analysis of the GE9 bundle design with MOX fuel, as used in the reference spent fuel alternative for $\mathrm{Pu}$ disposition. This bundle design analysis is based on a high environment core and bounds the bundles designed for commercial BWRs.

\subsubsection{Criticality Safety Requirements}

Two criticality safety requirements for shipping containers are applicable to the shipment of fresh MOX fuel: (1) 10CFR71 Requirements for Fissile Class I Shipping Container and (2) IAEA Requirements for Arrays of Shipping Packages.

The criticality safety criteria for shipping containers meeting the requirements for Fissile Class I containers and the IAEA Regulations can be summarized as follows:

Individual Undamaged Container - An individual undamaged container must be subcritical when optimally moderated and fully reflected by water.

Infinite Array of Undamaged Containers - An infinite array of undamaged containers must be subcritical with optimum interspersed moderation between containers.

Array of Damaged Containers - An array of 250 Fissile Class I containers each subject to the Hypothetical Accident Conditions must be subcritical when closely reflected by water and when arranged in the most reactive configuration. For the IAEA regulations, an array of " $2 \mathrm{~N}$ " containers, each subject to the Hypothetical Accident Conditions, must be subcritical when closely reflected by water and when arranged in the most reactive configuration. The number $\mathbf{N}$ sets the Transport Index and is defined as the allowable number of containers which may be transported in any shipment. 

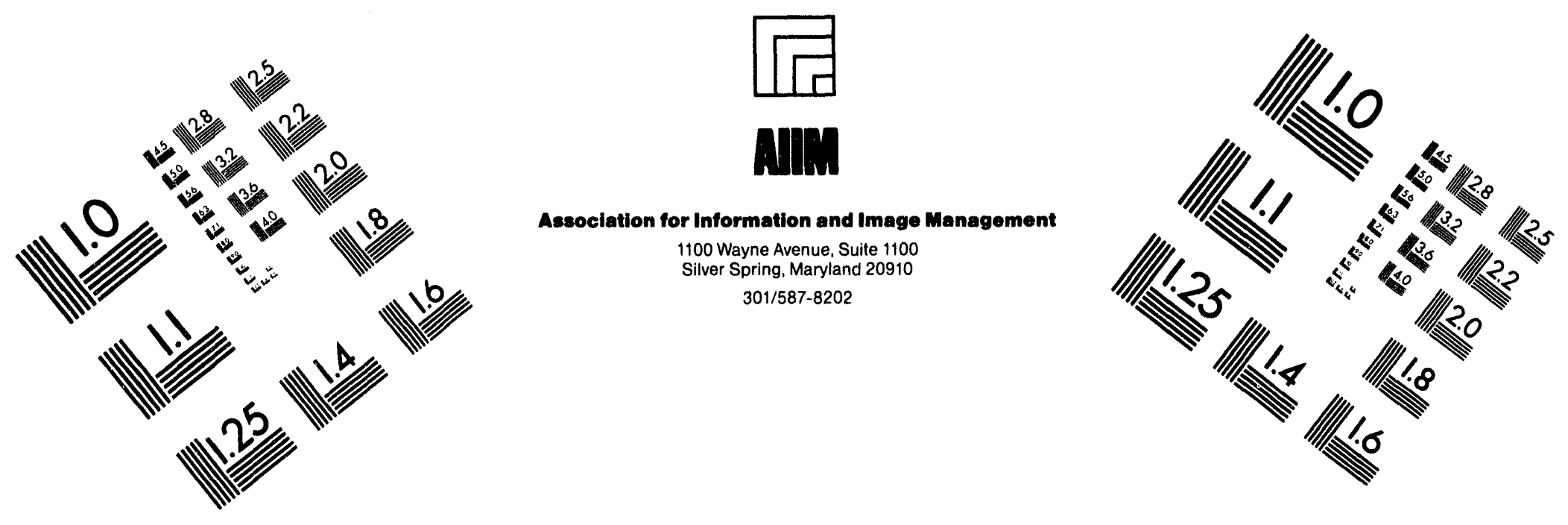

\section{Centimeter}

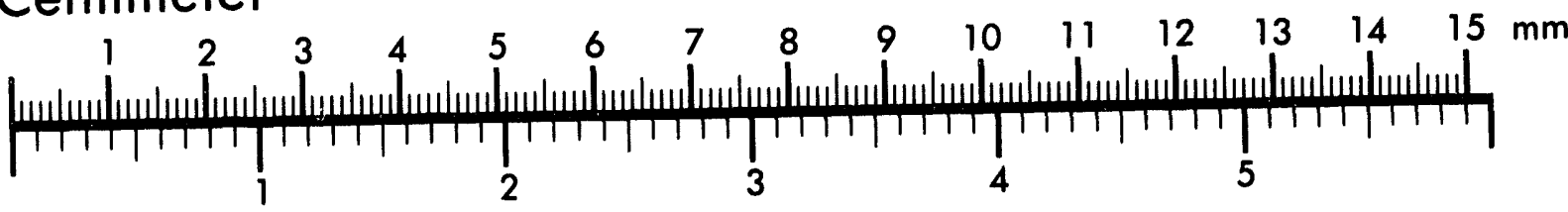
Inches
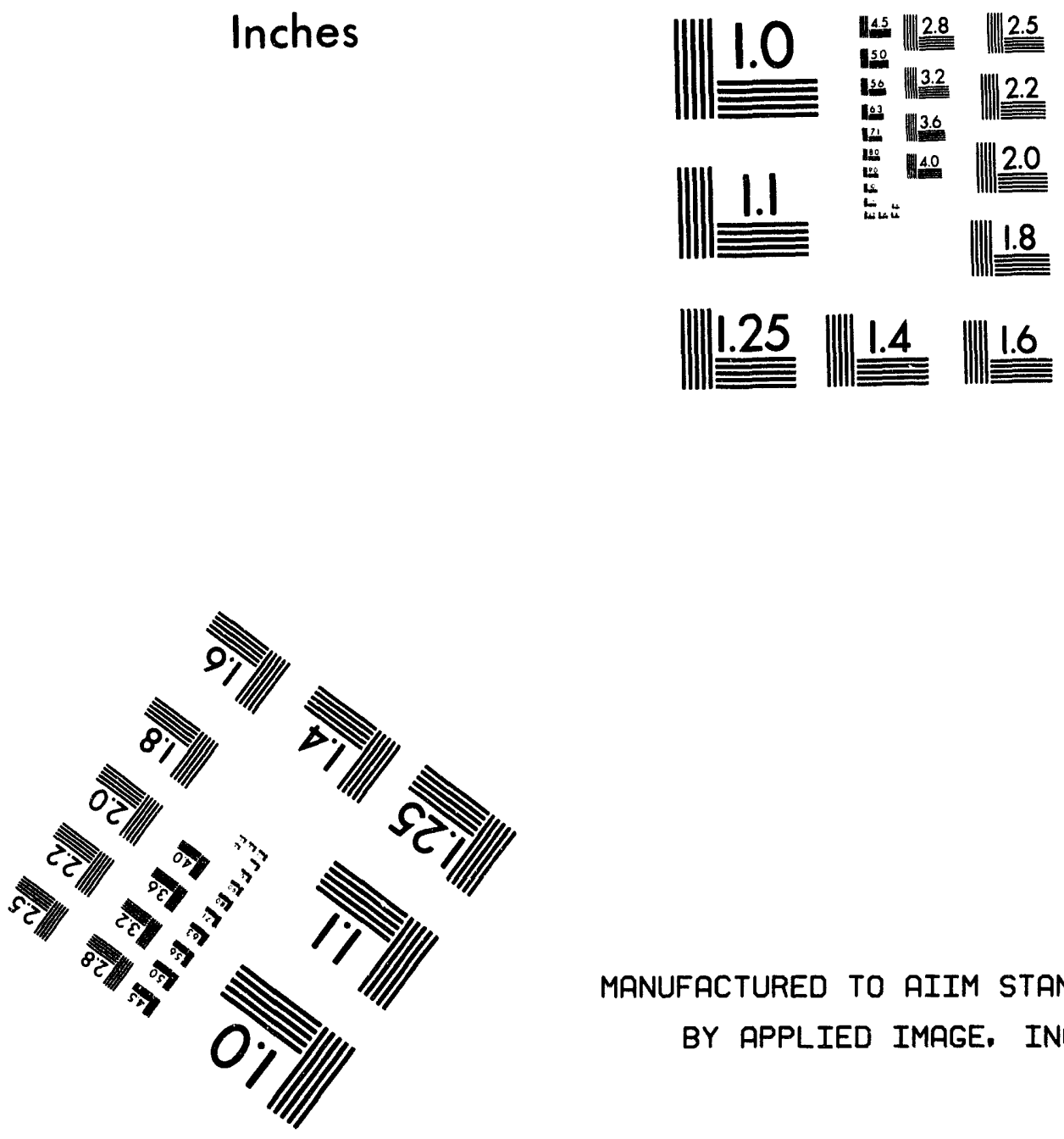

MANUFACTURED TO AIIM STANDARDS BY APPLIED IMAGE, INC.

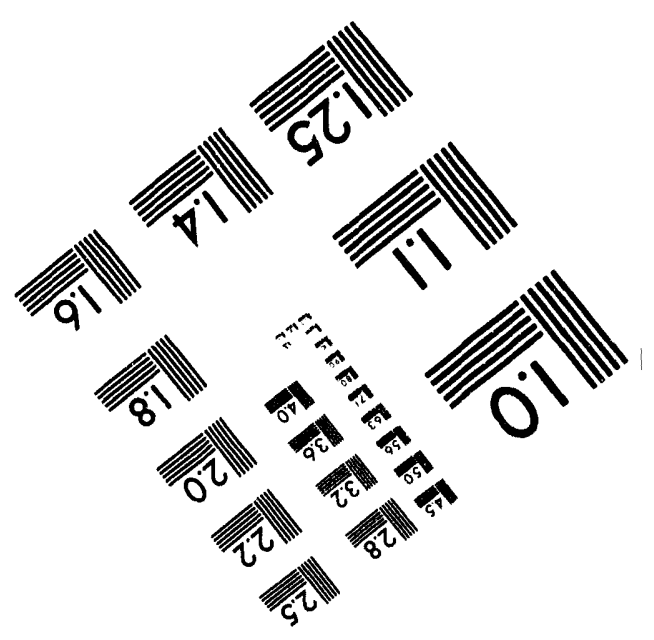



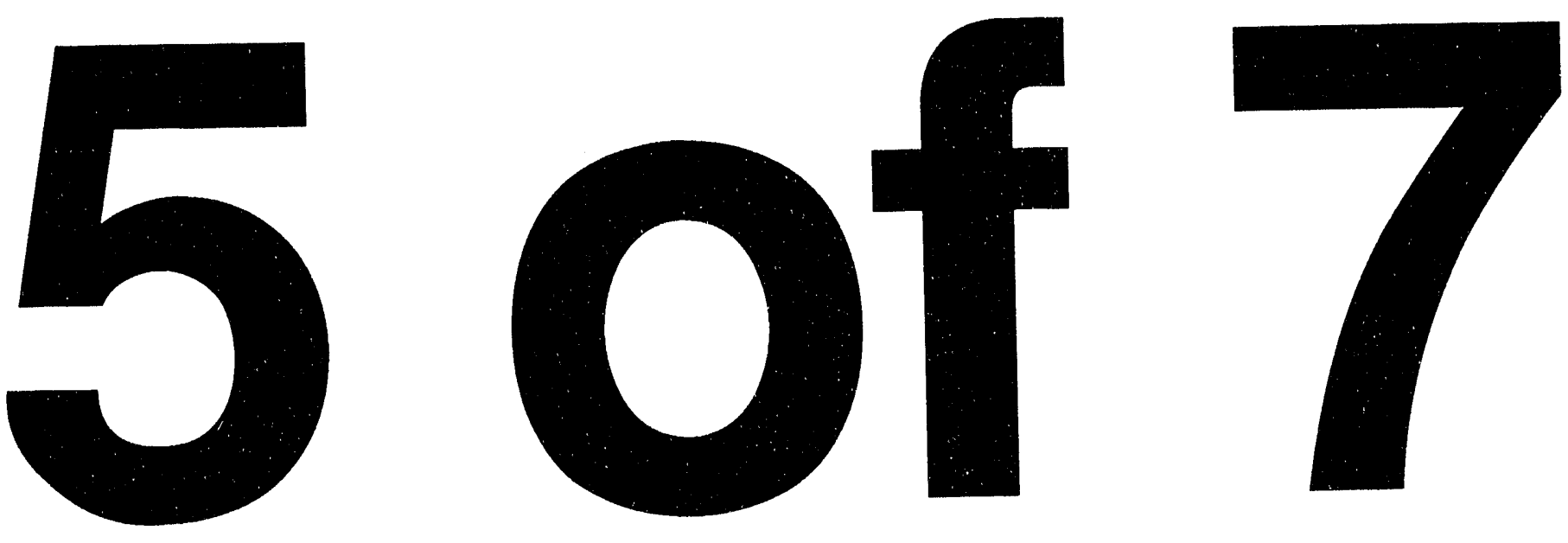
Since for the array of damaged containers, if the allowable number of containers to be shipped is taken to be infinite, the requirements for Fissile Class I packages are less conservative than the IAEA Regulations, the IAEA requirement is evaluated for the analysis of infinite array of damaged containers.

The Hypothetical Accident Conditions are summarized below:

Table 8.4-1 Hypothetical Accident Conditions

\begin{tabular}{|l|l|}
\hline Accident Test & Condition \\
\hline Drop Test & 30 foot free drop \\
\hline Free Drop and Puncture Test & 1 meter free drop and puncture \\
\hline Fire Test & $\begin{array}{l}\text { Exposure to } 800^{\circ} \mathrm{C} \text { for at least } \\
30 \text { minutes, }\end{array}$ \\
\hline Immersion Test & $\begin{array}{l}\text { At least } 50 \text { feet of water for at least } \\
8 \text { hours. }\end{array}$ \\
\hline
\end{tabular}

Extensive criticality safety analyses performed previously for BWR fuel bundles in the RA series containers have shown that the accident arrays have significantly higher multiplication factors than any of the undamaged container arrays or the single container and hence are the limiting cases in the criticality safety analysis. Therefore, only the accident arrays were investigated.

\subsubsection{Description of RA Series Shipping Containers}

For more than 20 years, the RA series shipping containers have been used by GENE to ship BWR fuel elements to domestic and international customers. The RA series containers consist of rectangular steel inner containers transported in wooden outer overpacks. The wooden overpack containers are designed with ethafoam and honeycomb cushioning between the metal inner container and the inside walls of the wooden overpack. The inner metal container has two internal ethafoam-cushioned channel sections each of which can hold a single fuel assembly.

The metal containers are constructed of Stainless Steel 321 with a minimum 16-gauge outer shell and structural and reinforcing components. An inner basket inside the metal container is formed from two perforated metal channels and is held in place by six thick angled supports welded to the inner wall of the outer shell. Within the inner basket, fuel assemblies rest on additional ethafoam cushioning. At the upper end of the inner container, a removable end cap is bolted onto the main body. The inner container is sealed by a lid and rubber gasket which are held in place by 14 stainless steel clamps. A pressure relief valve installed on the inner container is designed to pass up to $2 \mathrm{cfm}$ of air if the pressure differential between the inside and outside of the container exceeds $0.5 \mathrm{psi}$. 
The outer container is a rectangular wooden box fabricated of $2 \times 4$ inch wooden studs, wood planks, and plywood. The box is lined with 8.5 to 9.0 inch thick phenolic resin impregnated honeycomb and 3 to 4 inch thick ethafoam pads. Cutouts are made in the ethafoam and honeycomb to accommodate the handles and lifting lugs on the inner container. The ethafoam and honeycomb cushioning are arranged in the outer container to minimize vibrational effects on the fuel assemblies being transported in the inner container.

During the packaging and handling of the container, BWR fuel assemblies are placed in the chambers in the inner container after installing plastic separators between rows and columns of the fuel rods and by enclosing the entire fuel assembly in a thin plastic sheath.

The RA series shipping containers are currently licensed as Fissile Class I shipping packages for the transport of $7 \times 7,8 \times 8,9 \times 9$, and specific $10 \times 10 \mathrm{BWR}$ fuel assemblies. The container also is currently licensed as a Type-A package in accordance with the Regulations for the Safe Transport of Radioactive Materials, 1985 edition (Supplement 1990) of the International Atomic Energy Agency (IAEA) for generic 9x9 BWR fuel assemblies.

\subsubsection{Analytical Technique}

In this analysis, neutron multiplication factors ( $k_{\infty}$ 's or $k_{e f f}$ 's) have been calculated with the MCNP [Ref. 8-3] Monte Carlo code. MCNP is a general-purpose, continuous-energy, generalized-geometry, time-dependent, coupled neutron/photon Monte Carlo transport code. It solves neutral particle transport problems and may be used in any of three modes: neutron transport only, photon transport only, or combined neutron/photon transport, where the photons are produced by neutron interactions. The neutron energy regime is from $10-11 \mathrm{MeV}$ to $20 \mathrm{MeV}$, and the photon energy regime is from $1 \mathrm{keV}$ to $100 \mathrm{MeV}$. The capability to calculate k-effective eigenvalues for fissile systems is also a standard feature.

MCNP uses continuous-energy nuclear data libraries. The primary sources of nuclear data are evaluations from the Evaluated Nuclear Data File (ENDF) system, the Evaluated Nuclear Data Library (ENDL) and the Activation Library (ACTL) compilations from Livermore National Laboratory, and evaluations from the Applied Nuclear Science (T-2) Group at Los Alamos National Laboratory. Evaluated data are processed into a format appropriate for MCNP by codes such as NJOY. The processed nuclear data libraries retain as much detail from the original evaluations as is feasible.

Nuclear data tables exist for neutron interaction, photon interaction, neutron dosimetry or activation, and thermal particle scattering $S(\alpha, \beta)$ kernels. Over 500 neutron interaction tables are 
available for approximately 100 different isotopes or elements. Photon interaction tables exist for all elements from $Z=1$ through $Z=94$. The data in the photon interaction tables allow MCNP to account for coherent and incoherent scattering, photoelectric absorption with the possibility of fluorescent emission, and pair production. Cross sections for nearly 2000 dosimetry or activation reactions involving over 400 target nuclei in ground and excited states are part of the MCNP data package. These cross sections may be used as energy-dependent response functions in MCNP to determine reaction rates. Thermal data tables are appropriate for use with the $S(\alpha, \beta)$ scattering treatment in MCNP. The data include chemical binding and crystalline effects that become important as the neutron's energy becomes sufficiently low. Data are available for light and heavy water, beryllium metal, beryllium oxide, benzene, graphite, polyethylene, and zirconium and hydrogen in zirconium hydride.

\subsubsection{Modeling}

Inner Container - A geometry model of the inner container is shown in Figure 8.4-1. The metal in the shell, the inner basket, and the angled basket supports are modeled as stainless steel. The perforated inner basket is modeled as metal with a reduced density (85\% of the normal stainless steel density). The ethafoam cushioning between the fuel assembly and the inner basket as well as the plastic sheathing around the fuel assemblies are not included. Eliminating this internal moderating material is conservative for the following reasons:

- Arrays of undamaged containers are over-moderated by the cushioning material and wood in the outer container. Therefore, the omission of moderating materials will result in increasing the calculated $\mathrm{k}_{\infty}$ 's.

- For accident condition arrays, the fire test (which is part of the Hypothetical Accident Conditions) completely burns away all internal flammable materials (except plastic separators). Even were this not the case, the accident arrays are analyzed with interspersed moderation within the inner container which is varied to determine the optimum amount. The presence of additional ethafoam or the plastic sheaths around the assemblies will therefore not result in greater $k_{\infty}$ 's but will only cause a slight change in the optimum interspersed moderator density 
NEDO-32361

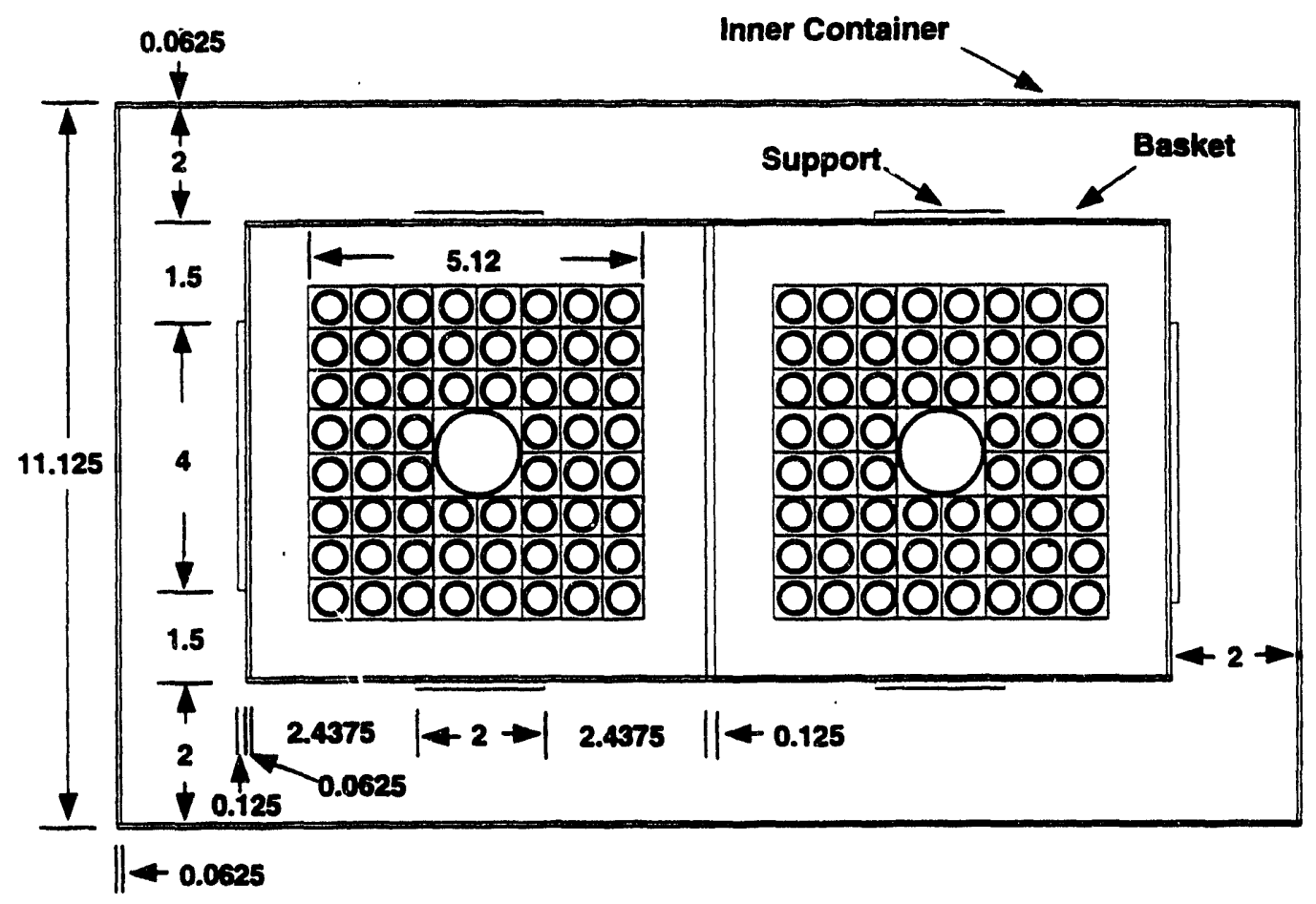

Dimensions in inches

Fuel Rod Cladding Zirconium

Fuel Within Fuel Rod Cladding

Polyethylene Separators Between Fuel Rods
Basket is $\mathbf{8 5} \%$ Density Stainless Steel

Inner Container and Supports are Full Density Stainless Steel

Other Open Areas are Interspersed Water

Figure 8.4-1 RA-3D Inner Container Geometry Model 
Outer Container - The outer container is modeled as shown in Figure 8.4-2. This model is also conservative since it does not include all of the moderating cushioning materials that are actually present in the package. Note in particular that portions of the regions between the inner and outer containers are empty (i.e., void) in the model. The model corresponds in this regard to is known as the "Minimum Packaging Model". The Minimum Packaging Model also includes a $50 \%$ reduced material density of the ethafoam to permit some flexibility in the arrangement of the cushioning.

Fuel Assembly - As discussed in Section 2.1.1, distinct MOX fuel rods are defined for the island and full MOX designs which contain different $\mathrm{Pu}$ enrichments and $\mathrm{Gd}$ loadings. The highest enrichment rods contained $4.2 \% \mathrm{Pu}$ with $1 \% \mathrm{Gd}$. The highest enrichment without gadolinium is $2.8 \% \mathrm{Pu}$.

The analysis conservatively modeled an average peripheral rod containing $2.79 \% \mathrm{Pu}$ without $\mathrm{Gd}$ and an average interior rod containing $8.25 \% \mathrm{Pu}$ and $3 \% \mathrm{Gd}$. A second bounding case assumed the most reactive combination of $\mathrm{Pu}$ and $\mathrm{Gd}$ in the peripheral rods and the interior rods. For this case, it was assumed that the 28 peripheral rods contain $4.00 \% \mathrm{Pu}$ without $\mathrm{Gd}$ and the 32 interior rods contain $9.00 \% \mathrm{Pu}$ (maximum) and $2 \% \mathrm{Gd}$ (minimum). All rods were assumed to contain $0.2 \%$ U-235 for the average case and $0.71 \%$ of U-235 in the bounding case.

\subsubsection{Discussion of Results}

This section documents the results of criticality safety analysis for an infinite array of damaged containers containing fresh fuel bundles.

From a criticality safety perspective, the key issues are water in-leakage (which is assumed to be optimum) and damage to the fuel assemblies or inner container leading to a more reactive configuration. For the RA series containers, the Hypothetical Accident Conditions are assumed to result in the burning of the wooden outer container and all of the internal ethafoam, honeycomb, rubber, and plastic. The failure of the rubber sealing gasket in the inner container results in the in-leakage of water during the immersion test. Because of the loss of the burnable materials, arrays of damaged containers are no longer over-moderated and the addition of interspersed water may cause the array $k_{\infty}$ to increase. The Hypothetical Accident Condition tests does not result in any changes in the fuel assemblies or the inner container that could have significant impact to the criticality safety results. Minor changes in geometry due to the drop test and the fire test result in a configuration that is less hazardous than the close-packed accident 


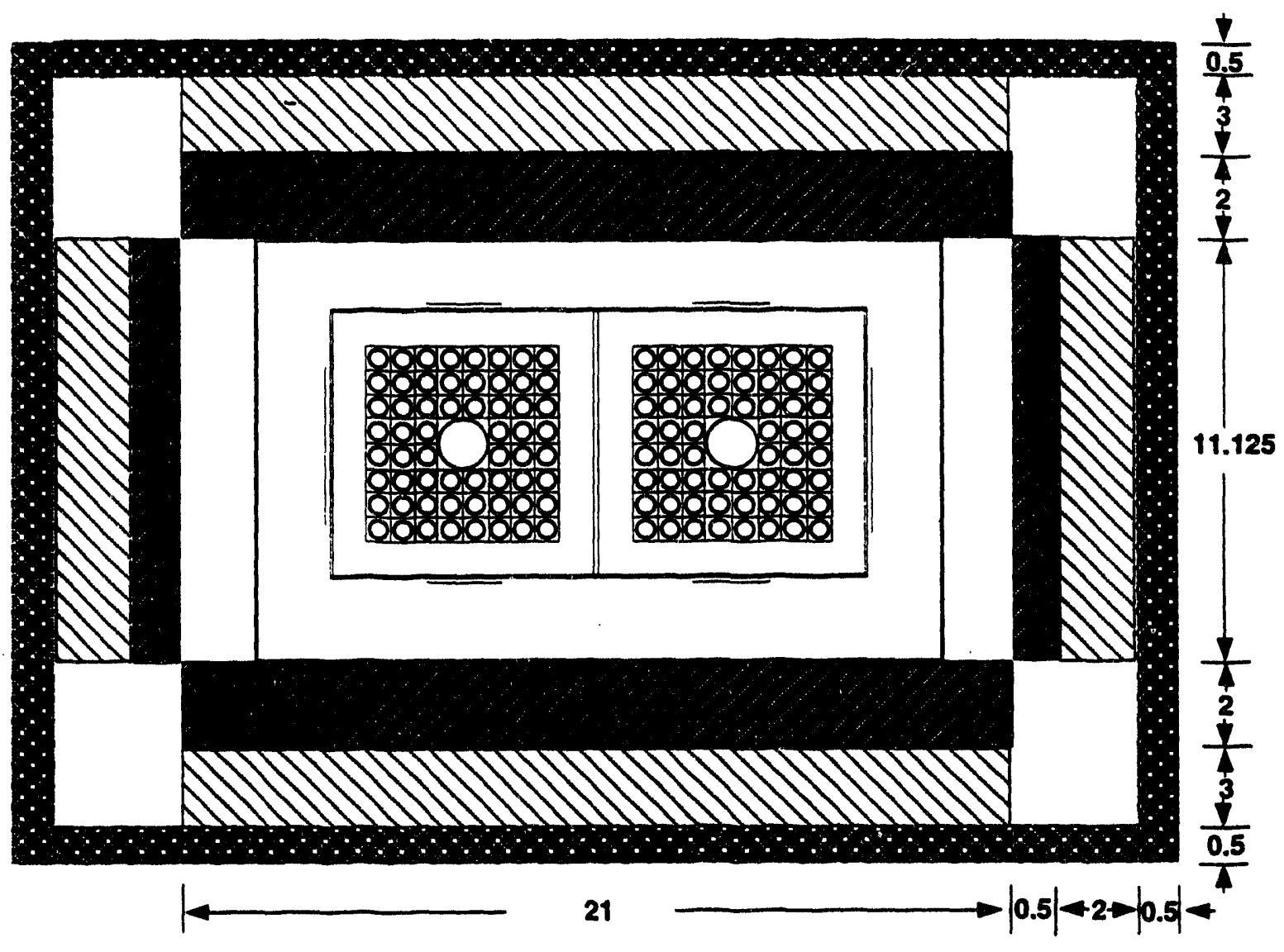

Dimensions in inche $s$

\section{LEGEND}

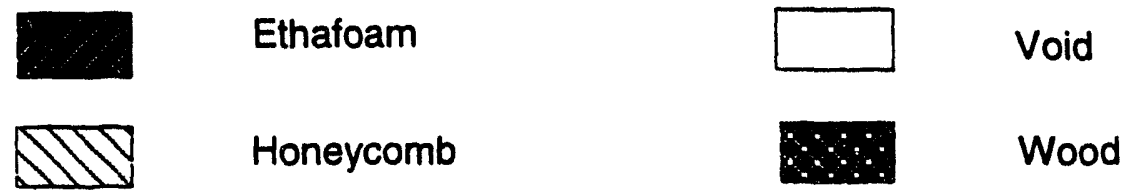

Figure 8.4-2 RA-3D Outer Container Geometry Model 
arrays assumed in criticality safety analyses. No loss of neutron absorbing materials occurs in the inner metal container or in the gadolinium rods.

The results of the analysis are presented in Table 8.4-2. The results show neutron multiplication factors as a function of varying interspersed moderator density for the accident condition container arrays. Both the average case and the bounding case results are presented. For the average case, the results indicate that the damaged array is significantly subcritical for all moderator densities. The maximum $\mathrm{k}_{\infty}$ is found to be $0.7829 \pm 0.0020$, or 0.7869 , including a $2 \sigma$ uncertainty, at a moderator density of 0.075 . For the bounding case, the results show, as expected, the increase of $\mathrm{Pu}$ enrichment and the reduction of $\mathrm{Gd}$ concentration raise the neutron multiplication by about $0.06-0.09$, depending on the moderator density. However, the results indicate that the damaged array is also significantly subcritical for all moderator densities. The maximum $k_{\infty}$ is found to be $0.8705 \pm 0.0020$, or 0.8745 , including a $2 \sigma$ uncertainty, at a moderator density of 0.05 .

\subsubsection{Criticality Safety of Spent Fuel Transportation}

This section summarizes criticality safety analysis performed for an infinite array of damaged containers for spent fuel bundles based on a geometry model similar to that described in Section 8.4.1 for fresh fuel bundles. The principal differences are (1) the removal of Gd (burnout in spent fuel), (2) change of $\mathrm{Pu}$ compositions that reflect the designed fuel burnup of $40,000 \mathrm{MWd} / \mathrm{t},(3)$ inclusion of bundle channel ( 0.1 in thick) and, (4) removal of plastic separator between fuel rods.

Table 8.4-2 Fresh Fuel Bundle Neutron Multiplication Factors for Infinite Arrays of RA-3D Containers in Accident Conditions

Average Bundle

\begin{tabular}{|l|c|c|c|c|}
\hline $\begin{array}{l}\text { Fractional Water } \\
\text { Density }\end{array}$ & $\begin{array}{c}\text { Nominal } \\
\text { K-infinity }\end{array}$ & $\begin{array}{c}1 \sigma \\
\text { Uncertainty }\end{array}$ & $\begin{array}{c}\text { Nominal } \\
\text { K-infinity }\end{array}$ & $\begin{array}{c}1 \sigma \\
\text { Uncertainty }\end{array}$ \\
\hline 0.00 & 0.6978 & 0.0025 & 0.7745 & 0.0018 \\
\hline 0.050 & 0.7812 & 0.0027 & 0.8705 & 0.0020 \\
\hline 0.075 & 0.7829 & 0.0020 & 0.8690 & 0.0024 \\
\hline 0.100 & 0.7732 & 0.0025 & 0.8651 & 0.0025 \\
\hline 0.125 & 0.7619 & 0.0022 & 0.8483 & 0.0025 \\
\hline 0.150 & 0.7437 & 0.0024 & 0.8320 & 0.0021 \\
\hline 1.000 & 0.5306 & 0.0041 & 0.5950 & 0.0032 \\
\hline
\end{tabular}


Spent fuel is assumed to contain waste in the quantities shown below based on the evaluation of isotopes provided in Section 2.6. Other TRU isotopes and fission products are conservatively ignored in the model.

It is noted that spent fuel shipping casks are of different design features than the fresh fuel shipping containers. Nevertheless, the results done with the shipping containers provide a clear indication of whether criticality safety is a concern for the spent fuel shipping.

The results of the analysis are presented in Table 8.4-4 based on MCNP calculations. These results are for the accident condition container arrays and show array neutron multiplication factors as a function of varying interspersed moderator density for both the average case and the bounding case with the spent fuel bundle that has as-built $\mathrm{Pu}$ enrichments and $\mathrm{Gd}$ concentrations corresponding to the cases discussed in the fresh fuel shipping.

The results indicate that, for the average case, the damaged array is significantly subcritical for all moderator densities. The maximum $k_{\infty}$ is found to be $0.7875 \pm 0.0027$, or 0.7929 , including a $2 \sigma$ uncertainty, at a moderator density of 0.10 . For the bounding case, the results show, as expected, the increase of $\mathrm{Pu}$ enrichment raises the neutron multiplication by about $0.02-0.04$, depending on the moderator density. However, the results indicate that the damaged array is also significantly subcritical for all moderator densities. The maximum $\mathrm{k}_{\infty}$ is found to be $0.8175 \pm 0.0030$, or 0.8235 , including a $2 \sigma$ uncertainty, at a moderator density of 0.10 .

\subsubsection{Criticality Safety of Spent Fuel Storage ir. Repository}

Monte Carlo calculations were performed on discretely modeled spent fuel storage arrays to determine the neutron multiplication factors of the Yucca Mountain repository filled with spent MOX fuel containers in normal and accident (water flooded) conditions.

Table 8.4-3 Spent Fuel Waste Quantities

\begin{tabular}{|l|c|}
\hline Isotope & Weight (\%) \\
\hline $\mathrm{Pu}-239$ & 45.96 \\
\hline $\mathrm{Pu}-240$ & 27.60 \\
\hline $\mathrm{Pu}-241$ & 11.23 \\
\hline $\mathrm{Pu}-242$ & 3.93 \\
\hline $\mathrm{Am}-241$ & 0.91 \\
\hline $\mathrm{Cm}-244$ & 7.65 \\
\hline
\end{tabular}


Table 8.4-4 Neutron Multiplication Factors for Spent Fuel Bundles Infinite Arrays of RA-3D Containers in Accident Conditions

\begin{tabular}{|l|c|c|c|c|}
\multicolumn{2}{c}{ Average Bundle } & \multicolumn{2}{c|}{ Bounding Case } \\
\hline $\begin{array}{l}\text { Fractional Water } \\
\text { Density }\end{array}$ & $\begin{array}{c}\text { Nominal } \\
\text { K-infinity }\end{array}$ & $\begin{array}{c}1 \sigma \\
\text { Uncertainty }\end{array}$ & $\begin{array}{c}\text { Nominal } \\
\text { K-infinity }\end{array}$ & $\begin{array}{c}1 \sigma \\
\text { Uncertainty }\end{array}$ \\
\hline 0.000 & 0.3885 & 0.0026 & 0.4226 & 0.0019 \\
\hline 0.050 & 0.7281 & 0.0022 & 0.7553 & 0.0024 \\
\hline 0.075 & 0.7755 & 0.0023 & 0.8002 & 0.0021 \\
\hline 0.100 & 0.7875 & 0.0027 & 0.8175 & 0.0030 \\
\hline 0.125 & 0.7856 & 0.0027 & 0.8164 & 0.0025 \\
\hline 0.150 & 0.7744 & 0.0032 & 0.8056 & 0.0023 \\
\hline 1.000 & 0.6499 & 0.0032 & 0.6724 & 0.0037 \\
\hline
\end{tabular}

For purpose of evaluation, a spent fuel storage container was assumed to hold $10 \mathrm{BWR}$ fuel assemblies for long-term repository storage. The container is assumed to be a $15-\mathrm{ft}$ steel drum with a 200 -mil wall thickness and an outside diameter of 28 inches. It is buried vertically in the ground, with adjacent containers spaced 15 feet away in a respective tunnel. Individual tunnels are spaced 126 feet apart. Figure 8.4-3 shows a cross-sectional view of the proposed single container geometry with $10 \mathrm{BWR}$ fuel assemblies that was used in this analysis.

The spent fuel assemblies are discretely modeled for the criticality analysis, incorporating endof-life heavy metal isotopics, as discussed in the previous section. The average case has been used in this analysis to demonstrate the criticality safety of the repository. The numeric models used reflective boundary conditions on the sides and bottom of the container arrays to simulate infinite tunnels, both in length and number. The water flooded scenario incorporates a 10-ft tall water region above the containers.

Two models were used in this analysis. The first one is normal spent fuel bundles and the second one is with the central water rods replaced by natural boron carbide. The purpose of the second model is to investigate the effectiveness of boron carbide for criticality safety. Table 8.4-5 shows neutron multiplication factors for both cases with varying water density.

The normal environment (non-flooded) Monte Carlo analysis yields a repository eigenvalue of $0.3415 \pm 0.0040$ (1-sigma). The accident (water flooded) environment analysis yields a repository eigenvalue of $0.9055 \pm 0.0027$ (1-sigma). Replacing the water in the central water rod position of each of the ten assemblies in each container with $\mathrm{B}_{4} \mathrm{C}$ yields a water flooded repository eigenvalue of $0.8050 \pm 0.0029$ (1-sigma). The results also show that the reactivity worth of natural $\mathrm{B}_{4} \mathrm{C}$ is about $10 \% \mathrm{~K}$ with water and $4 \% \mathrm{~K}$ without water. 


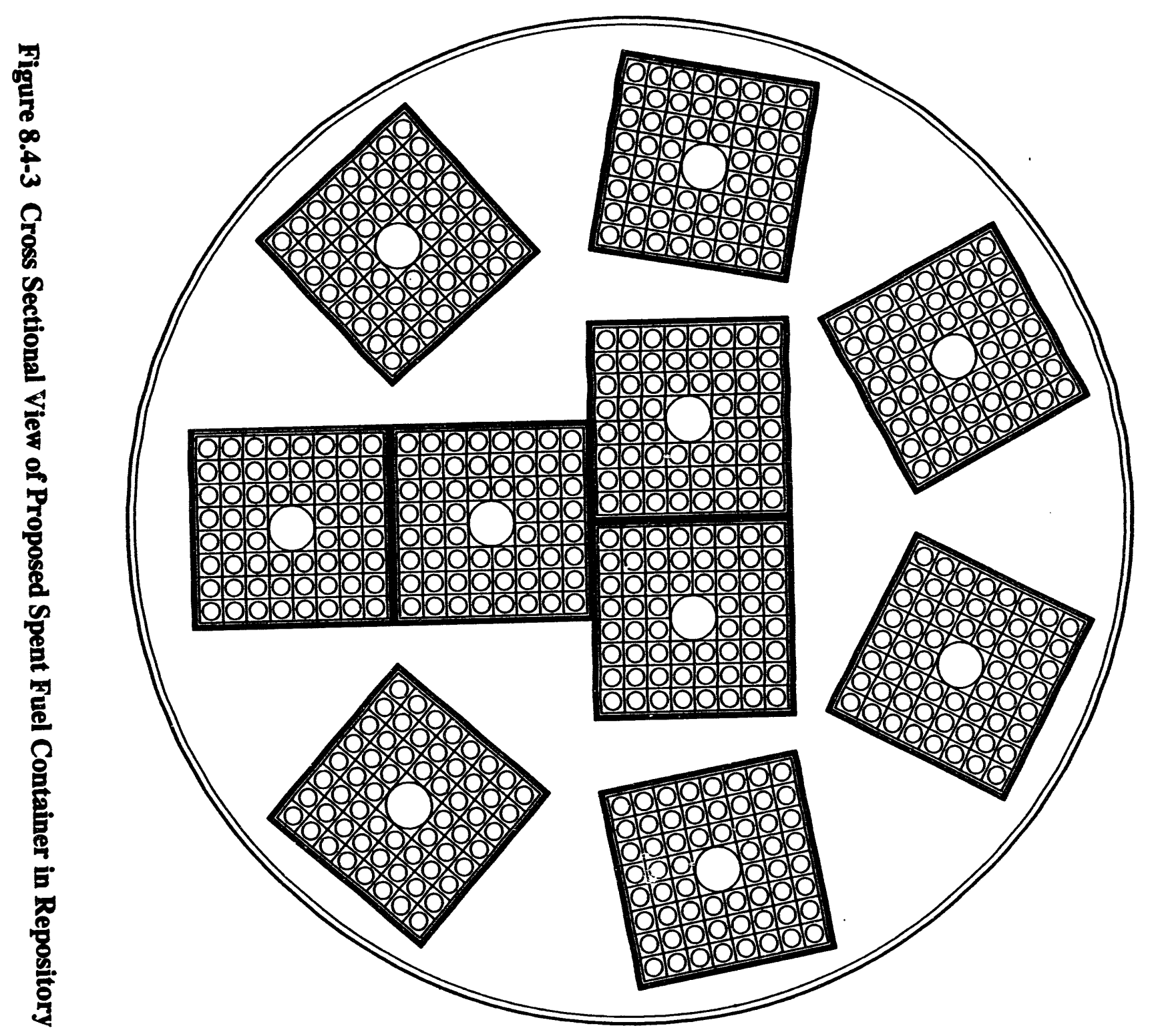

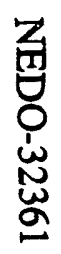


NEDO-32361

Table 8.4-5 Neutron Multiplication Factors for Repository

(10 BWR Spent Fuel Bundles)

\begin{tabular}{|l|c|c|c|c|}
\multicolumn{4}{c}{} & \multicolumn{3}{c}{ Without $\mathbf{B}_{\mathbf{4}} \mathbf{C}$} & \multicolumn{2}{c|}{$\begin{array}{c}\text { Water Rods Replaced } \\
\text { by } \mathbf{B} \text { C }\end{array}$} \\
\hline $\begin{array}{l}\text { Fractional Water } \\
\text { Density }\end{array}$ & $\begin{array}{c}\text { Nominal } \\
\text { K-infinity }\end{array}$ & $\begin{array}{c}1 \sigma \\
\text { Uncertainty }\end{array}$ & $\begin{array}{c}\text { Nominal } \\
\text { K-infinity }\end{array}$ & $\begin{array}{c}1 \sigma \\
\text { Uncertainty }\end{array}$ \\
\hline 0.000 & 0.3415 & 0.0040 & 0.2992 & 0.0036 \\
\hline 0.100 & 0.5690 & 0.0028 & 0.4698 & 0.0032 \\
\hline 0.200 & 0.7163 & 0.0032 & 0.6059 & 0.0034 \\
\hline 0.400 & 0.8440 & 0.0023 & 0.7469 & 0.0029 \\
\hline 0.600 & 0.8861 & 0.0025 & 0.7872 & 0.0025 \\
\hline 0.800 & 0.9021 & 0.0025 & 0.8000 & 0.0025 \\
\hline 1.000 & 0.9055 & 0.0027 & 0.8050 & 0.0029 \\
\hline
\end{tabular}

The comprehensive Monte Carlo criticality analysis indicates that long-term repository storage of spent fuel MOX assemblies, utilizing the indicated repository geomc '.y scheme, poses no criticality concerns.

\subsubsection{Criticality Safety in the MOX Facility}

The MOX facility is described in Section 2.4. The MOX facility has been designed to prevent a criticality accident by the utilization of facility design and administrative controls for each glovebox or material control area. The allowable criticality limit is based on $45 \%$ of a critical mass to allow for a double batching error. A computerized accountability system will be utilized to monitor and approve all material transfers. In many cases, in-line safeguards instrumentation will verify the concentration and weight of any nuclear material that is transferred between material control areas. The criticality limits for each material control area are adjusted based on the relative concentration of plutonium in each specific area, as well as the potential concentrations of water in the material control area. The application of these criteria provides different limits for plutonium feed, MOX powder and process scrap in the recovery area.

\subsubsection{Criticality Safety in BWR Fuel Pools}

The design of the BWR fuel pools and provisions to address criticality is described in Section 2.5. Fuel assembly location monitoring and individual subcriticality checks are made associated with each movement of fuel within the pool and the reactor. High density storige racks are designed to assure subcritical configurations are achieved for fully loaded fuel storage racks. 


\subsubsection{Conclusions}

The criticality safety requirements for classification as a Fissile Class I shipping container and for qualification under the 1985 IAEA Regulations for the Safe Transport of Radioactive Materials have been applied to the RA-3D container with Pu-based GE9 8x8 fuel assemblies. These have included requirements for the subcriticality of single undamaged containers, infinite arrays of undamaged containers, and infinite arrays of damaged containers.

For an infinite array of damaged containers, it has been shown that when optimally moderated by water, the system of such packages is subcritical with the specified Pu-based GE9 fuel assembly for $\mathrm{Pu}$ disposition application. The maximum $\mathrm{k}_{\infty}$, including $2 \sigma$ uncertainty, is 0.7869 for fresh fuel bundles and 0.7929 for spent fuel bundles.

Previous criticality safety analyses have shown that accident arrays have significantly higher multiplication factors than any of the undamaged container arrays or the single container and hence for the RA-3D are the limiting cases in the criticality safety analysis. Therefore, although analyses for a single RA-3D container and an infinite array of undamaged containers were not performed in this study, meeting the criticality safety requirements for these conditions should not be a problem based on prior experience.

The comprehensive Monte Carlo criticality analysis indicates that long-term repository storage of spent fuel MOX assemblies, utilizing the indicated repository geometry scheme, poses no criticality concerns. 


\subsection{References}

8-1. Title 10, Code of Federal Regulations, Part 71, United States of America.

8-2. Safety Series No. 6, Regulations for the Safe Transport of Radioactive Materials, 1985 Revised Edition, published by the International Atomic Energy Agency (IAEA) Vienna, Austria.

8-3. MCNP - A General Monte Carlo Code for Neutron and Photon Transport, J.F. Briesmeister, Editor, LA-7396-M, Los Alamos National Laboratory, September 1986. 


\subsection{Non-Plutonium Dispositioning Operations}

\subsection{Commercial Fuel Operation}

Disposition of weapons plutonium, in terms of the last core reload of MOX fuel into the reactor, is completed within 23 years after initial full MOX loading in the reference case. An additional four cycles or five years is required before the last $\mathrm{Pu}$ bundles are removed from the core. The BWR is licensed for 40 years of reactor operation so unless a license renewal program has been implemented, the plant will be at or near the end of its licensed life. If the deployment option has any remaining licensed life, the reactor will be operated in the power production mode using urania fuel as a commercial reactor.

The transition from MOX fueled operations to urania fueled operations for the BWR will be straightforward. As noted on several occasions in this study, there are no reactor system or plant level modifications to use MOX fuel and there are no changes to the number of control rods. Therefore, transitioning to urania fuel operations can be accomplished readily.

The above scenario whereby the flexibility in the fuel design is used to accommodate urania fuel also applies under other instances, not specifically called out in the study. For example, if only a partial MOX core is available for loading for unspecified reasons (e.g., a deliberate slowdown in the disposition rate for reciprocity reasons), urania fuel of enrichment typical of commercial fuel can be used to complement the MOX fuel in the BWR core. 


\subsection{Spent Fuel Storage (final ten years)}

As discussed in Section 9.1, after the plutonium disposition mission is complete, the inherent flexibility of the BWR core design facilitates transition from the full MOX core to a $\mathrm{UO}_{2}$ core if there is any remaining license life for the plant. Spent MOX bundles are discharged to the spent fuel pool or ex-reactor spent fuel storage and allowed to cool for ten years before shipment to the waste repository. Table 9.2-1 shows the approximate schedule for shipment of the remaining MOX bundles after completion of the mission. It should be noted that the behavior of MOX bundles in the fuel pool is not different from $\mathrm{UO}_{2}$ bundles and no special considerations are required.

While U.S. commercial reactors are typically designed to store at least 10 years of spent fuel, the storage pools in most power plants, including the BWRs of interest for this study, are expected to reach their capacity limit during the next decade. Thus unless shipment of the existing spent fuel to the Federal Repository is initiated prior to the start of a plutonium disposition mission some ex-reactor spent fuel storage will be needed. Further, the proposed plutonium disposition mission is expected to utilize the remaining life of the plants involved. At the end of the mission, some ex-reactor spent fuel storage would be needed to meet the "ten-year-cooled" requirement unless the in-reactor pools, their essential support systems and the NRC license are maintained for ten years beyond the end of operations. While this later option is feasible, it has not been found to be cost effective by the Rancho Seco and Trojan power plants currently undergoing decommissioning.

Thus, the planning basis for this study is to provide passive dry spent fuel storage for ten years of operation. This meets the "ten-year-cooled fuel" requirement, provides ten years of flexibility to accommodate delays in the availability of the Federal Repository and minimizes the potential impacts on decommissioning due to continued in-reactor storage of the spent fuel. For a detailed discussion of dry fuel storage options, refer to Section 2.

During the final ten years, as $\mathrm{UO}_{2}$ bundles are accumulated either in the fuel pool or in passive dry spent fuel storage, additional shipments of $\mathrm{UO}_{2}$ bundles may be made to the waste repository (depending on availability and bundle decay heat) to provide for flexibility in future shipments. The MOX discharging schedule is shown in Table 9.2-1. MOX assembly loading would be complete in the year 2023 and would be followed by the loading of $\mathrm{UO}_{2}$ assemblies as long as the plant remained licensed to operate. For the reference case, all MOX bundles would be discharged in the year 2028. 
Table 9.2-1 MOX Discharging Schedule and Spent Fuel Storage (Final Ten Years)

\begin{tabular}{|c|c|c|c|c|c|}
\hline $\begin{array}{l}\text { Time from MOX } \\
\text { Load in First } \\
\text { Reactor (Years) }\end{array}$ & $\begin{array}{c}\text { Reactor 1 } \\
\text { Assemblies } \\
\text { Loaded }\end{array}$ & $\begin{array}{l}\text { Reactor } 2 \\
\text { Assemblies } \\
\text { Loaded }\end{array}$ & $\begin{array}{c}\text { Reactor } 3 \\
\text { Assemblies } \\
\text { Loaded }\end{array}$ & $\begin{array}{l}\text { Cumulative } \\
\text { Assemblies } \\
\text { Charged }\end{array}$ & $\begin{array}{l}\text { Cumulative } \\
\text { Assemblies } \\
\text { Discharged }\end{array}$ \\
\hline 17.39 & & 176 & & 7564 & 5452 \\
\hline 17.63 & 176 & & & 7740 & 5628 \\
\hline 18.39 & & & 176 & 7916 & 5804 \\
\hline 18.63 & & 176 & & 8092 & 5980 \\
\hline 18.87 & 176 & & & 8268 & 6156 \\
\hline 19.63 & & & 176 & 8444 & 6332 \\
\hline 19.87 & & 176 & & 8620 & 6508 \\
\hline 20.11 & 176 & & & 8796 & 6684 \\
\hline 20.87 & & & 176 & 8972 & 6860 \\
\hline 21.11 & & 176 & & 9148 & 7036 \\
\hline 21.35 & 176 & & & 9324 & 7212 \\
\hline 22.11 & & & 176 & 9500 & 7388 \\
\hline 22.35 & & 176 & & 9676 & 7564 \\
\hline 22.59 & 176 & & & & 7740 \\
\hline 23.35 & & & 1762 & & 7916 \\
\hline 23.59 & & 18176 & & & 8092 \\
\hline 23.84 & 40176 & & & & 8268 \\
\hline 24.59 & & & 780176 & & 8444 \\
\hline 24.84 & & 90176 & & & 8620 \\
\hline 25.08 & 176.2 & & & & 8796 \\
\hline 25.84 & & & 176 & & 8972 \\
\hline 26.08 & & 30176 & & & 9148 \\
\hline 26.32 & 176 & & & & 9324 \\
\hline 27.08 & & & $6=176$ & & 9500 \\
\hline 27.32 & & $1 / 0$ & & & 9676 \\
\hline
\end{tabular}

Note: Shading indicates urania assemblies required to discharge all MOX from reactors.

As can be seen from Table 9.2-1, the discharge of the last MOX bundle to the spent fuel pool occurs about 5 years after the last loading. For Alternates 1 and 2, in which disposition occurs during the plant lifetimes, the discharge of the last MOX bundle would occur at 29 and 45 years, respectively, after initial full MOX loading of the first reactor. If continued plant operation were available as a result of a license renewal program, any additional storage requirements could be accommodated through the use of dry storage. If continued plant operation were not pursued because of license expiration, the requirement for ten years cooling of the MOX fuel could require a period of approximately eight years beyond the discharge of the last $\mathrm{UO}_{2}$ bundle which would require operation of fuel pool cooling and cleanup systems. This requirement to continue operation of plant systems could be relaxed much earlier if the dry storage option is fully implemented to accommodate spent bundle discharge. 


\subsection{Other Applications of this Technology}

The proposed disposition process is a once-through fuel cycle utilizing existing plutonium. Although the U.S. will not be reprocessing commercial spent nuclear fuel, facilities and plans to do this are ongoing in foreign countries. Both the OTA and NAS studies have acknowledged the importance of managing "civilian" plutonium in spent fuels so that non-proliferation objectives are assured while these operations are conducted.

If an alternate disposition process such as deep bore holes or vitrification is chosen for a U.S. Pu disposition program, the U.S. technology for managing the plutonium will be significantly different from the "civilian" or weapons plutonium management ongoing in other countries (including the former Soviet Union). As a result, the U.S. could become isolated from the plutonium management process in other countries and leadership in proliferation resistance would be extremely difficult. The technology used in the disposition process addressed by this study, particularly in the area of safeguards, could form the basis for U.S. Leadership in the technology, procedures, and practices applied to safeguards and in establishing international standards and practices for non-proliferation.

Past approaches to influence the international management of plutonium have included convincing foreign governments to adopt the no-reprocessing policy of the U.S., more stringent enforcement of IAEA safeguards/accountability provisions, and studies and recommendations of new fuel cycles which are inherently more proliferation resistant. These approaches have not been entirely successful. While a number of variables need to be taken into account in deciding the specific disposition process that should be employed in the U.S., disposition of plutonium in existing reactors has the advantage of maintaining U.S. technological parity with other nations while demonstrating adherence to responsible non-proliferation practices. 


\subsection{Alternative Cases}

\subsection{Introduction}

The purpose of this section is to evaluate two alternative cases which dispose of twice as much plutonium $(\mathrm{Pu})$ as the reference case. Other variations and disposition options for the reference case are also discussed. Each of the alternate cases was evaluated and compared with the reference case discussed in Sections 2 through 9 of this report.

The two alternative cases that were evaluated are:

Alternative 1 - $100 \mathrm{MT}$ weapon plutonium disposition using six reactors

Alternative 2-100 MT weapon plutonium disposition using three reactors

The remaining plant license life for all plant(s) under consideraition is much less than the original 40-year licensed lifetime. A license renewal program will play a key role in achieving the necessary plant operating lifetime required for alternative 2. Each of the alternative cases for $\mathrm{Pu}$ disposition was evaluated using the reference core design and found to be technically acceptable.

If larger amounts of disposition are desired, the selection of a particular alternative for disposition will primarily depend upon cost and the acceptability of the selected timeframe for plutonium disposition. As discussed in Section 4, irrespective of the disposition timeframe, it is desirable to formulate the government/ utility/owner interface agreements and arrangements for the remaining licensed life of the plant.

With each alternative, the impact of the disposition time and plutonium tonnage on other disposition areas such as MOX factory size, safeguards, licensability, transport, repository storage of spent fuel, cost, schedule and infrastructure was evaluated. The areas of safeguards, reactor technology base, reactor and fuel facility permitting/licensability, repository storage of spent fuel and overall infrastructure were unaffected by the specific alternative chosen. The major areas affected were the MOX plant throughput requirements and the cost and schedule.

Based on the comparison of each alternative and the reference case, the areas where differences were noted are described below. Because of the similiarities between the reference case and alternative 2 , the evaluation focused primarily on the six reactor case. 


\subsection{Technical Descriptions for Alternative Cases}

A comparison of the major parameters for each of the alternative cases as compared with the reference case is provided in Tables 10.2-1. The number of reactors selected for each alternative is based on ensuring that the peak plutonium enrichment percentage does not exceed $10 \%$. As noted in Table 10.2-1, the alternative and the reference cases are designed using an average plutonium concentration of $5.3 \%$.

Table 10.2-1 Characteristics of Reactor Deployment and Fuel Fabrication Capacity

\begin{tabular}{|c|c|c|c|}
\hline Parameter & Ref & Alt 1 & Alt 2 \\
\hline $\begin{array}{l}\text { Weapons Plutonium, MT } \\
\text { Number of Reactors } \\
\text { Defined Project Time, yrs } \\
\text { Reactor Years to Disposition }\end{array}$ & $\begin{array}{c}50 \\
3 \\
29.3 \\
26.3\end{array}$ & $\begin{array}{c}100 \\
6 \\
30.8 \\
27.8\end{array}$ & $\begin{array}{c}100 \\
3 \\
51.4 \\
48.4\end{array}$ \\
\hline $\begin{array}{l}\text { Tsland Core: } \\
\text { Fuel Pins, no. } \\
\text { Plutonium, kg }\end{array}$ & $\begin{array}{c}12 \\
1.44\end{array}$ & $\begin{array}{c}12 \\
1.44\end{array}$ & $\begin{array}{c}12 \\
1.44\end{array}$ \\
\hline $\begin{array}{l}\text { Full MOX Core } \\
\text { Fuel Pins, no. } \\
\text { Plutonium, kg }\end{array}$ & $\begin{array}{c}60 \\
5.33\end{array}$ & $\begin{array}{c}60 \\
5.33 \\
\end{array}$ & $\begin{array}{c}60 \\
5.33 \\
\end{array}$ \\
\hline $\begin{array}{l}\text { Fuel Fabrication Requirements } \\
\text { Island Core: } \\
\text { Fuel Pins, no./yr } \\
\\
\text { Bundles, no./yr } \\
\text { Full MOX Core: } \\
\text { Fuel Pins, no./yr } \\
\text { Bundles, no./yr } \\
\end{array}$ & $\begin{array}{c}1392 \\
(1400)^{1} \\
116 \\
(24,000)^{1} \\
24000 \\
400 \\
\end{array}$ & $\begin{array}{c}1392 \\
(1400)^{1} \\
116 \\
(24,000)^{1} \\
47214 \\
774 \\
\end{array}$ & $\begin{array}{c}1392 \\
(1400)^{1} \\
116 \\
(25,000)^{1} \\
24960 \\
416 \\
\end{array}$ \\
\hline $\begin{array}{l}\text { MOX Fabrication Design Rates } \\
\text { Island Core: } \\
\text { MOX Fab at } 120 \%, \mathrm{MT} / \mathrm{yr} \\
\text { Fab Duration, years } \\
\text { Full MOX Core: } \\
\text { MOX Fab at } 120 \%, \mathrm{MT} / \mathrm{yr}\end{array}$ & $\begin{array}{l}6 \\
3.5 \\
97 \\
\end{array}$ & $\begin{array}{c}6 \\
3.5 \\
189 \\
\end{array}$ & $\begin{array}{c}6 \\
3.5 \\
101 \\
\end{array}$ \\
\hline Fab Duration, years & 23.5 & 24.5 & 45.5 \\
\hline
\end{tabular}

1 Rod totals utilized for capacity estimates 


\subsection{Estimated Incremental Cost and Schedule for Alternative Cases}

Cost and schedule differences from the reference case are presented in this section.

\subsubsection{Alternative Case Costs}

Table $10.3-1$ is a summary of the estimated major cost components for Alternative 1. As mentioned before, because of the similarities between the reference case and Alternative 1, the cost evaluation was focused on the six-reactor case.

For Alternative 1, six BWR units at four sites (two single unit sites and two dual unit sites) and three sites (dual units at each site) were considered. However, for cost estimating purposes, six BWR urits at three different sites are assumed to provide the lowest incremental cost. Estimates for the dual unit plant developed for the reference case were utilized with appropriate adjustments to derive cost estimates for the six BWR units in Alternative 1. All costs are estimated as overnight cost with a base date of January 1993.

Development costs are assumed to be the same as the reference case for Alternative 1 (see Section 6.4.2 for discussion of development cost).

\subsubsection{Alternative Case Schedules}

Schedules for Alternatives 1 and 2 are shown in Figures 10.3-1, and 10.3-2, respectively.

\subsubsection{Alternative Case Cash Flows}

Table 10.3-2 shows the cash flow for the BWR complex, MOX fuel fabrication facility and Los Alamos facility for the six-unit Alternative 1. The fuel cycle lengths and durations of reactor operations utilized to estimate the costs of uranium fuel and transportation shown in the cash flow table are approximate. 
NEDO-32361

Table 10.3-1 Alternative Case Costs

\begin{tabular}{|c|c|c|}
\hline Item & $\begin{array}{c}\text { Ref } \\
3 \text { Units }\end{array}$ & $\begin{array}{l}\text { Alt 1 } \\
6 \text { Units }\end{array}$ \\
\hline $\begin{array}{l}\text { BWR CompleX } \\
\text { Capital Cost }(\$ M) * \\
\text { O\&M Cost }(\$ M / Y r) * \\
\text { Capital Replacement Cost }(\$ M / Y r) \\
\text { Decommissioning Cost }(\$ M)\end{array}$ & $\begin{array}{l}167 \\
6.4 \\
1.5 \\
12\end{array}$ & $\begin{array}{c}306 \\
11.3 \\
3 \\
24\end{array}$ \\
\hline $\begin{array}{l}\text { MOX Fuel Fabrication Facility } \\
\text { Capital Cost }(\$ M) \\
\text { O\&M Cost }(\$ M / Y r) \\
\text { Capital Replacement Cost }(\$ M / y r) \\
\text { Decommissioning Cost }(\$ M)\end{array}$ & $\begin{array}{c}525 \\
51.2 \\
0.5 \text { for yrs } 1 \text { to } 5 \\
0.9 \text { for yrs } 5+ \\
53 \\
\end{array}$ & $\begin{array}{c}525 \\
99 \\
0.5 \text { for yrs } 1 \text { to } 5 \\
0.9 \text { for yrs } 5+ \\
53 \\
\end{array}$ \\
\hline $\begin{array}{l}\text { LANL Island Fuel Fabrication } \\
\text { Capital Cost }(\$ M) \\
\text { O\&M Cost }(\$ M / Y r)\end{array}$ & $\begin{array}{c}4 \\
\text { Variable ** }\end{array}$ & $\begin{array}{c}4 \\
\text { Variable ** } \\
\end{array}$ \\
\hline
\end{tabular}

* Includes BWR Plant and Fuel Storage Facility

** See reference and alternate 1 cash flows: Table 6.4-3 and Table 10.3-2, respectively. 


\section{0}

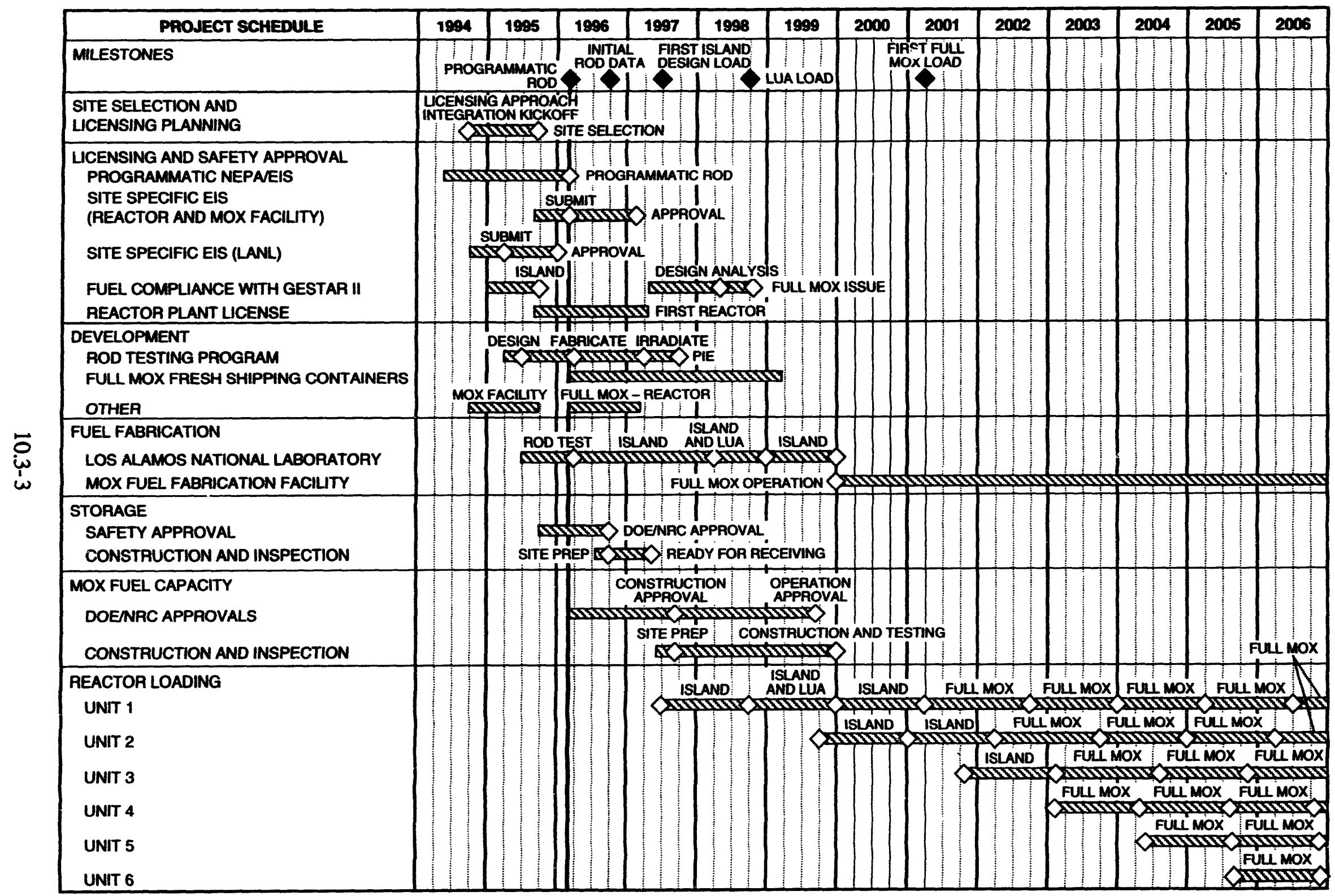

Figure 10.3-1 Schedule for Alternative 1 


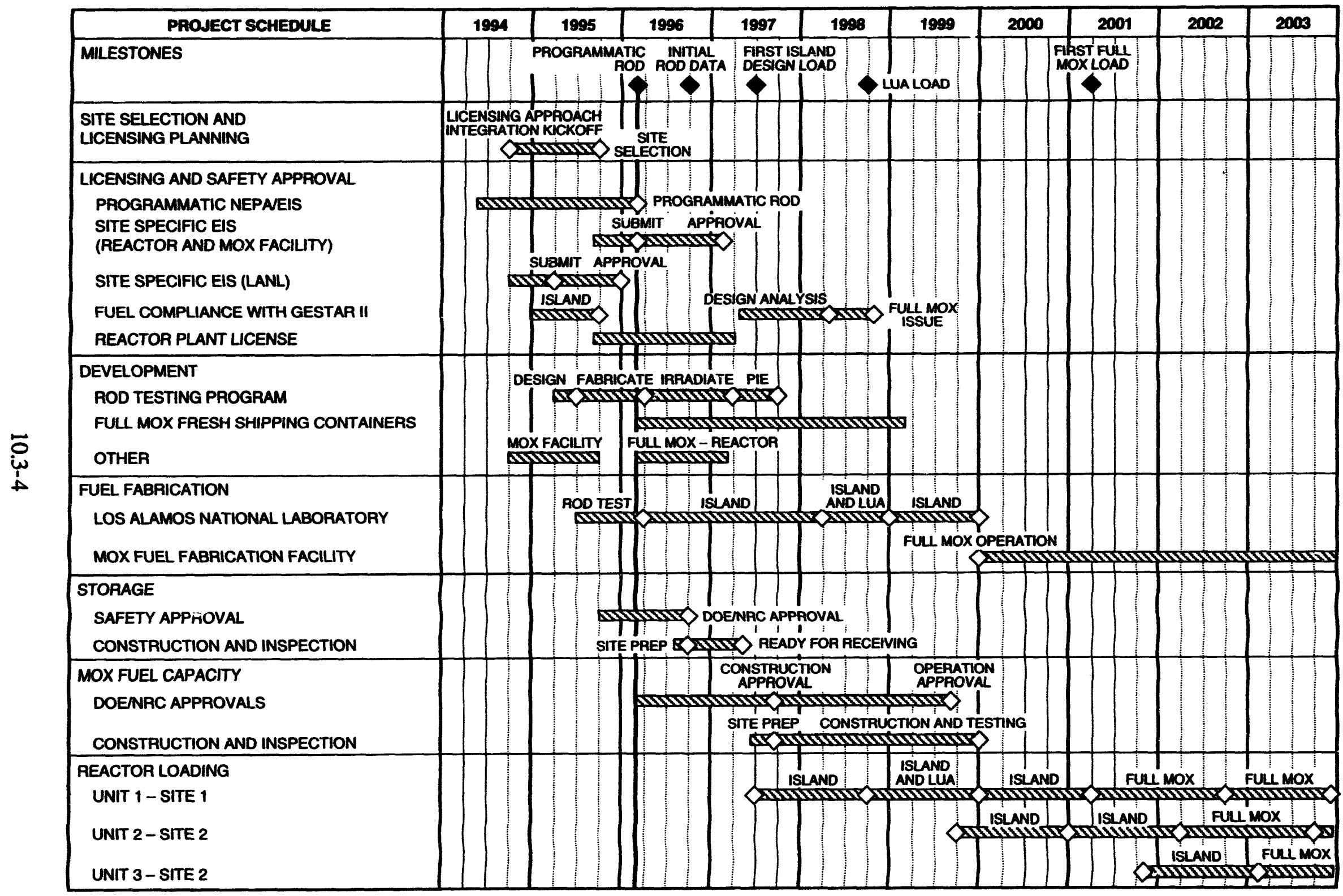

Figure 10.3-2 Schedule for Alternative 2

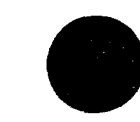


Table 10.3-2 Cash Fiu, - Incremental Cost

Alternate 1 - 6 BWR Units, 100 MT Disposition

( $\$$ in millions, 1/93 base date)

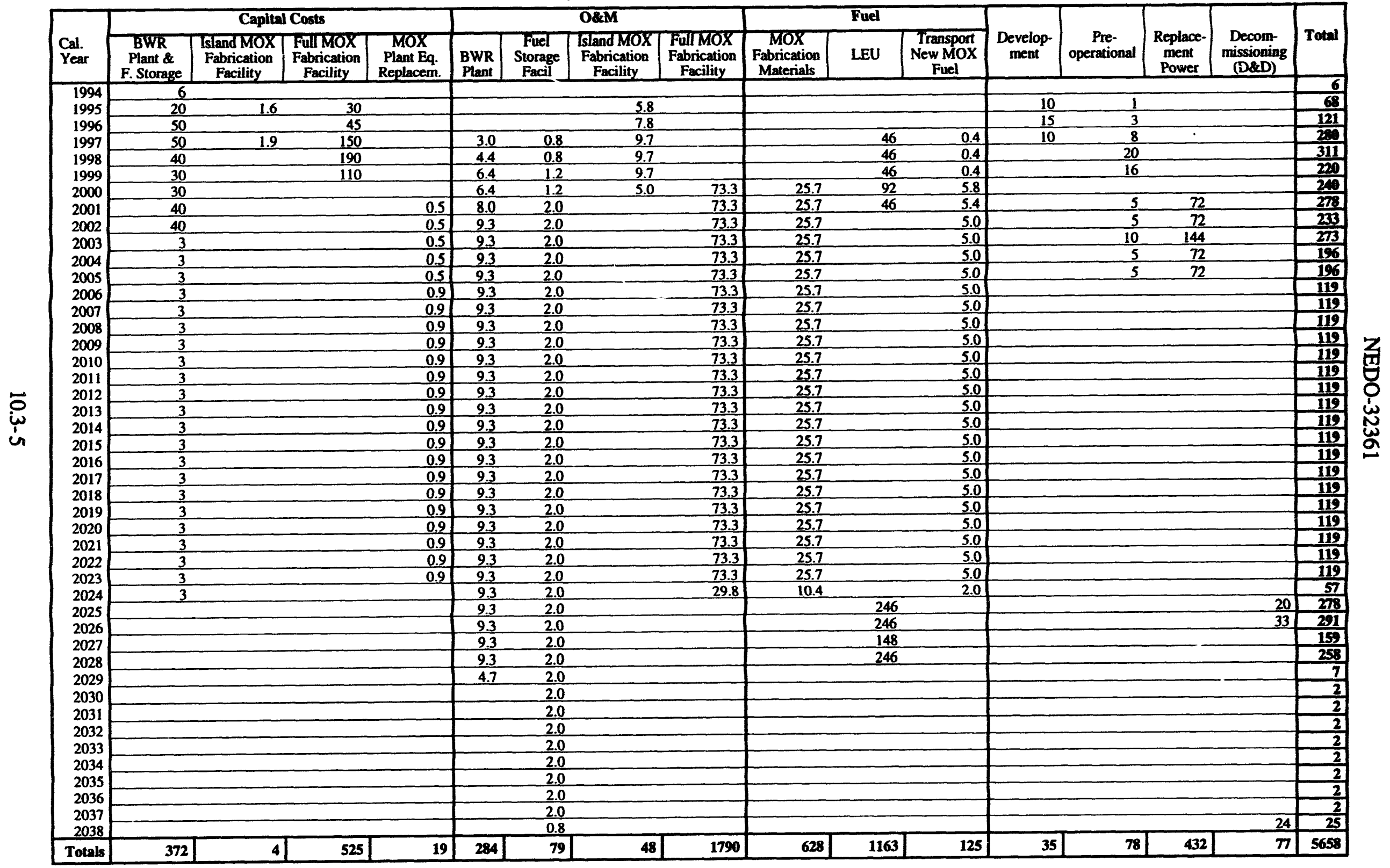




\subsection{Plutonium Disposition for the Alternative Cases}

The MOX facility functional layout for the alternative cases is detailed in Section 2.4. The utilization of two separate lines for the MOX plant was evaluated for meeting the throughput requirements for the different alternatives considered. Based on the evaluation, the MOX plant design is capable of providing the fuel requirements for all of the alternative cases. Because a minimum of two lines are required, the initial capital cost for the MOX plant is not expected to differ significantly for the various alternatives. The O\&M costs, however, will differ based on the throughput.

In Section 7.8, the form and quantity of Pu during the various stages of disposition was discussed for the reference case. As the plutonium is processed for disposition, it exists in various forms and becomes an increasingly less attractive target for diversion. The decreasing attractiveness to diversion initially results from increasing dilution of the plutonium among nonfissile materials. Later, after the fuel is placed in the reactor, the plutonium becomes highly unattractive and when discharged from the reactor, the plutonium is in a heavy and highly radioactive fuel bundle. Figures 10.4-1 and 10.4-2 show the plutonium distribution for the reference and Alternative case 1 , respectively. 

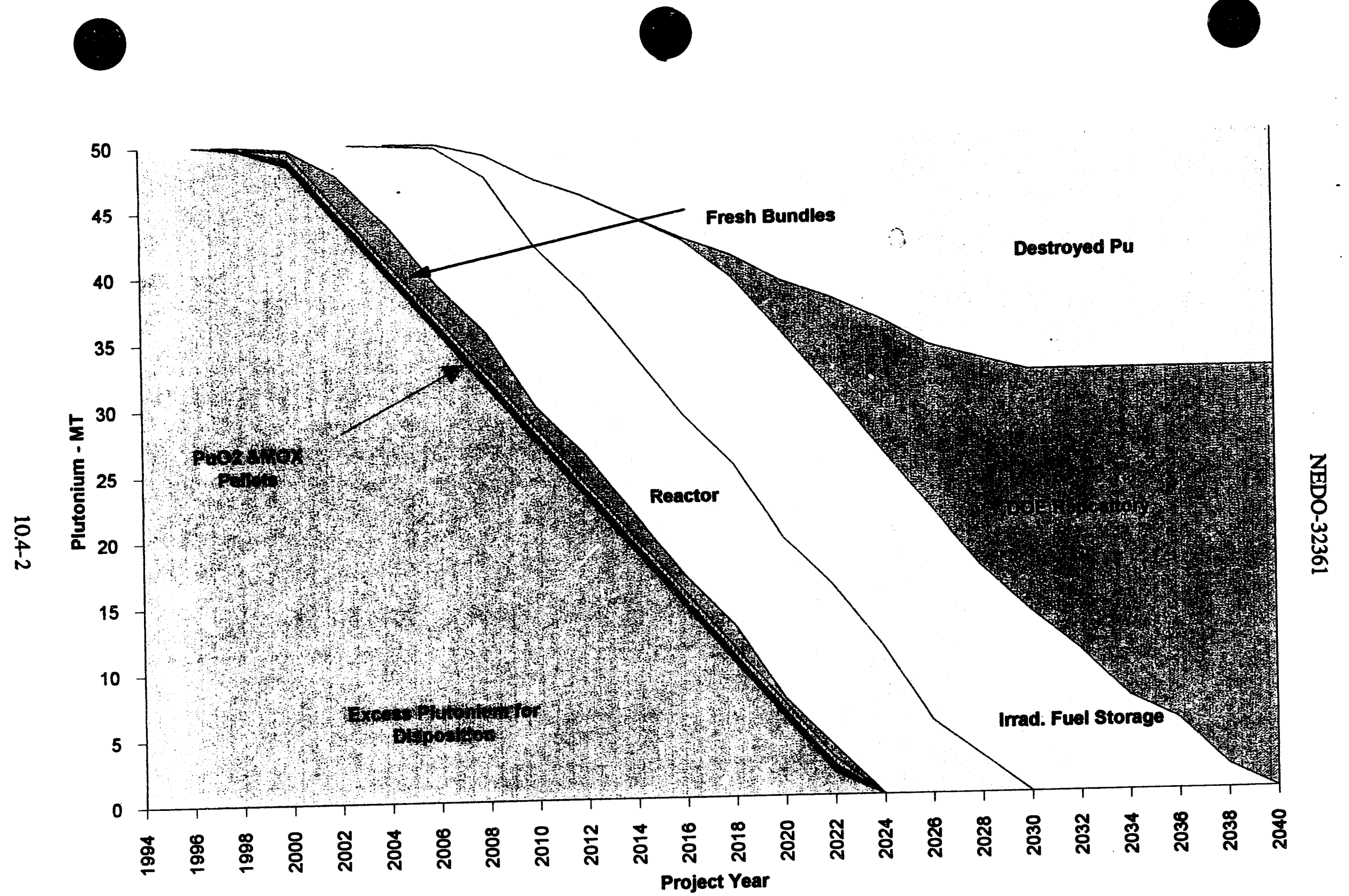

Figure 10.4-1 Plutonium Distribution During Reference Disposition Using 3 BWRs 


\section{NEDO-32361}

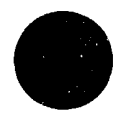




\subsection{Effect of Availability and Capacity Factor on the Alternative Cases}

Recent BWR experience in the U.S. has proven that availabilities at or greater than $75 \%$ are achievable. A number of factors can impact plant availability. For example, the plant management team is a key factor to ensuring a successful and well operated utility with a high availability factor. The availability of the BWR will be similar if MOX fuel is used instead of urania, since the use of MOX fuel does not adversely affect any parameters (such as cycle length) that might reduce plant availability. Since plant outage lengths are normally not determined by fuel handling activities, handling of MOX fuel is not expected to impact plant availability.

Capacity factor, as opposed to availability, represents the extent to which the reactor is producing the full rated power to the grid when it is available. If the plant is operated at a capacity factor which exceeds the $75 \%$ used as a basis for this study, a savings in terms of the number of years required to disposition the $\mathrm{Pu}$ would be realized. Achievement of a high capacity factor is not only a function of efficient plant operation but is contingent upon the commercial incentive provisions discussed in Section 4.1.2. Higher capacity factors also can affect the MOX fabrication schedule as well as SST transport schedules. Operational coordination of these activities is therefore needed to avoid undesirable delays. 


\subsection{Plant License Renewal Considerations}

\section{Overview}

The Atomic Energy Act of 1954 limits a nuclear power plant's license to a maximum of 40 years. Although the act permits the renewal of an operating license, it does not outline any standards or procedures for determining when or under what conditions a plant's operating license should be renewed. The Nuclear Regulatory Commission (NRC) proposed a rule (10CFR54) in July 1990 that was supposed to provide a license beyond 40 years of operation. The final rule was issued in December 1991.

One of the factors that may impact the plant selection process for the Pu disposition mission is the amount of time remaining on the initial 40-year licensed life of the plant. Part of the selection process will eliminate those plant(s) that have insufficient remaining license life to perform the Pu mission in support of the DOE objectives.

It is possible that a license renewal program, if implemented quickly, could be used to justify the selection of certain BWR plants that would otherwise be eliminated because of insufficient remaining license life to carry out the mission with potentially fewer reactors and/or sites. For the purposes of the $\mathrm{Pu}$ mission, such a program would have to be available near term in order to derive any benefits. The expectation is that the license renewal granted by the NRC would be for a period of 20 years. Clearly, for disposition of $100 \mathrm{MT}$ of plutonium with a limited number of plants (Alternative 2), license renewal would be necessary.

While a few nuclear plants have pursued studies on life extension, no lead plants have yet submitted applications for license renewal and industry experience is that the requirements for license renewal are not clearly defined by 10CFR54. Consequently, while some lead plants (e.g., Northern States Power- Monticello, the BWR lead plant) have expended considerable time and resources on license renewal feasibility studies, nuclear utilities have been reluctant to submit license renewal applications because of the ongoing uncertainty surrounding the 10CFR54 process.

The NRC commissioners have directed the Staff to revise and/or clarify the requirements of 10CFR54 by the end of 1994 . The revised license renewal rule is expected to make the process of applying for plant life extension simple, cost effective, and predictable. The NRC's revised license renewal rule is supposed to rely heavily on the maintenance rule (which is scheduled to take effect in July 1996 ) as a way for utilities to handle most aging issues. Although the above expected changes and clarification of the requirements specified by 10CFR54 would potentially 
open the door to utilities seeking license renewal, it would be premature to conclude that the new regulation guidelines could be implemented in the near term. If the guidelines are clarified, it may be a reasonable assumption that a plant selected for $\mathrm{Pu}$ disposition could initiate and complete the license renewal process and have a license renewal in place within a five-year time frame.

\section{Costs of License Renewal}

The nuclear industry, the U.S. Department of Energy, and many other entities have evaluated the benefits and costs of nuclear plant life extension (NUPLEX) for many years. These studies have attempted to estimate the likely costs associated with NUPLEX and the break-even costs. Relevant life-cycle costs of NUPLEX, include the following:

- All typical operating and maintenance costs

- Additional costs of refurbishment required to meet regulatory standards as well as desired operating performance

- Incremental license renewal requirements imposed by the NRC. This includes ISTM (inspection, surveillance, testing, and monitoring) of structures and systems that are affected by age.

- Replacement power costs associated with any downtime due to refurbishment.

- Allowance for funds used during construction (AFUDC)

The pilot plant life extension studies of capital costs indicated that total costs could range from $\$ 237$ to $\$ 635 / \mathrm{kW}$ (1989 dollars). The costs for refurbishment ranged from $\$ 82$ to $\$ 301 / \mathrm{kW}$. The balance of the costs include replacement power ( $\$ 111$ to $\$ 233 / \mathrm{kW}$ ) and AFUDC, which ranged from $\$ 44$ to $\$ 101 / \mathrm{kW}$ [Ref.10-1]

\section{Current Status and Cost Estimate for a Typical BWR}

Many utilities are working with their owners groups to assess the license renewal program. For example, The GE BWR Owners Group formed a generic license renewal committee in June 1993. Due to the rule uncertainty, there is no commitment from a plant for an application at this time. The program plan appears to be structured on working with NRC on a reference plant basis. 
The Nuclear Energy Institute, acting on behalf of the industry, is working to simplify the license renewal rule [Ref. 10-2] and to take credit for effective maintenance programs in place at operating plants. This effort to simplify the rule is being supported by the NRC Commissioners and staff.

The expenditures necessary for licensing depend on the complexity of the NRC regulation and the amount of time for a public hearing. Northern States Power expended approximately $\$ 11$ million to prepare its application and anticipated spending an equivalent amount to obtain a license for an additional 20 years of operation at Monticello. Baltimore Gas \& Electric (BG\&E) is reported to have expended about $\$ 15$-million on a combined life cycle management and license renewal program $-\$ 8$ million on life extension work. BG\&E projected that it will cost another $\$ 4$ million to get an application to the NRC.

It is estimated that, if the new license renewal rule is successful, the costs for an application would amount to between $\$ 5$ and $\$ 10$ million.

Additional operating \& maintenance (O\&M) and capital costs can be anticipated, depending on the physical condition of the plant and the effectiveness of existing aging management programs. The Monticello program estimated that there would be no increase in operating and maintenance costs and that the additional capital costs needed to support extended operation would amount to $\$ 30$ million over the last 20 years of operation. No extended outages would be necessary because the work could be done during normal refueling and maintenance outages.

The estimated order of magnitude capital costs for extending the operating term of a typical BWR facility could be between $\$ 80$ million to $\$ 120$ million in 1993 dollars. Additional capital costs may be necessary for potential replacement power costs and AFUDC on the overnight cost. To minimize replacement power costs, refurbishment could be timed to take place during normal plant downtime to the maximum extent feasible. However, some normal maintenance periods will likely be extended. Also, some additional O\&M costs will be required for using full core MOX fuel.

The owner of a BWR utility might derive an economic benefit from a decision to renew a license by potentially reducing annual contributions to the decommissioning trust, since the fund will not be needed for 20 more years. 


\subsection{Utilization of Existing DOE Facilities}

This section describes DOE facilities which may be used to different extents for fuel fabrication and/or Pu processing. The description material is contained in Section 2.4.5 and is reproduced here for convenience.

Following the descriptive material is an order of magnitude estimate of the potential cost savings from the use of these facilities.

\section{Variation for Use of DOE Facilities}

The reference costing approach requires a new MOX fuel fabrication facility be constructed at a greenfield site. The utilization of existing DOE sites and facilities can greatly reduce the initial capital expenditures required for the PDC, as well as reduce the time required to establish an operational MOX fabrication facility. Location of the Plutonium Disposition Complex (PDC) activities at an established DOE site allows the utilization of existing infrastructure which includes design engineering, maintenance, analytical laboratories, waste treatment facilities and storage facilities. The existing functions which provide permitting, transportation, security, safeguards and accountability can also be of unquestioned value to the PDC. A number of evaluations were made of existing DOE sites during this study. Those are summarized in Appendix F. A more detailed assessment of the buildings which might be utilized to convert weapons plutonium to feed material, and to process the feed material into MOX fuel assemblies for irradiation in a BWR is summarized in this section. The MOX fabrication processes arranged to fit into existing buildings is shown for several sites.

\section{Hanford Site}

The Fuels Materials Examination Facility (FMEF) was originally designed for examination of irradiated Fast Flux Test Reactor (FFTF) MOX fuel and then modified to fabricate MOX fuel for FFTF as well as other functions. Several years were spent procuring and installing equipment items for the Secure Automated Fabrication (SAF) facility to produce fuel utilizing automated processes which would reduce worker exposure levels. Since much of this support and processing equipment is still located in FMEF, selected items which can be adapted to fabrication ur fuel for the PDC could be utilized. The required MOX processing areas for the reference dispositioning option (97 MT/yr) were arranged in the FMEF building as shown as shaded areas in Figure 10.7-1. Much of the other unused space in this building could be utilized for conversion of plutonium to feed material, storage of feed material and fuel assemblies as well as scrap and waste processing. It should be noted that portions of this facility were specifically 
designed for processing MOX fuel and could be easily adapted for use during fabrication of MOX fuel for existing BWRs.

\section{Idaho National Engineering Laboratory}

The Fuel Processing Restoration (FPR) facility at the Idaho Engineering National Laboratory (INEL) is a new building constructed to the Category 1 requirements necessary for plutonium handling. The facility is over $90 \%$ complete and nuclear material has not been introduced into the building. The MOX fabrication facilities for the reference dispositioning option could be installed on the mezzanine and upper levels leaving adequate space for the other operations required in the MOX facility as shown as shaded areas in Figures 10.7-2 and 10.7-3. The cells and rooms located on the four lower levels of this facility can be utilized for pit storage, metal-tooxide conversion, assembly storage as well as scrap and waste treatment. In addition, the fuel assembly area in the Zero Power Physics Reactor (ZPPR) facility could provide adequate space for the conversion of plutonium metal to oxide. This facility is also equipped with a plutonium storage vault. This vault could be utilized to store either plutonium metal or the oxide. The New Material Isotopic Separation (NMIS) area associated with the Advanced Test Reactor is a Category 1 facility which could provide space for plutonium conversion or rod assembly and inspection operations. Various other facilities at the INEL site have been utilized for both wet and dry rod storage operations which may be directly applicable to some of the operations in the MOX facility.

\section{Los Alamos National Laboratory}

The TA-55 area at LANL houses several glove-box lines which have been used to fabricate mixed oxide and uranium nitride fuel. Both development and demonstration of automated technology for the disassembly of pits from retired weapons is underway at this location. Conceptual design of an Automated Retirement and Integrated Extraction System (ARIES) has been completed and long lead time equipment items ordered. An automated hydride-dehydride process has been selected for thi $i_{i}$ facility. Additional facilities are also available for development and testing of plutorium to oxide conversion technology. The fuel fabrication facilities at LANL will be utilized for process verification, demonstration and fabrication of test fuel assemblies. The modification of these facilities for fabrication of island design assemblies will require the addition of new milling, sintering, welding and rod inspection equipment. The current single shift operation will require modification to accommodate three shift operation 


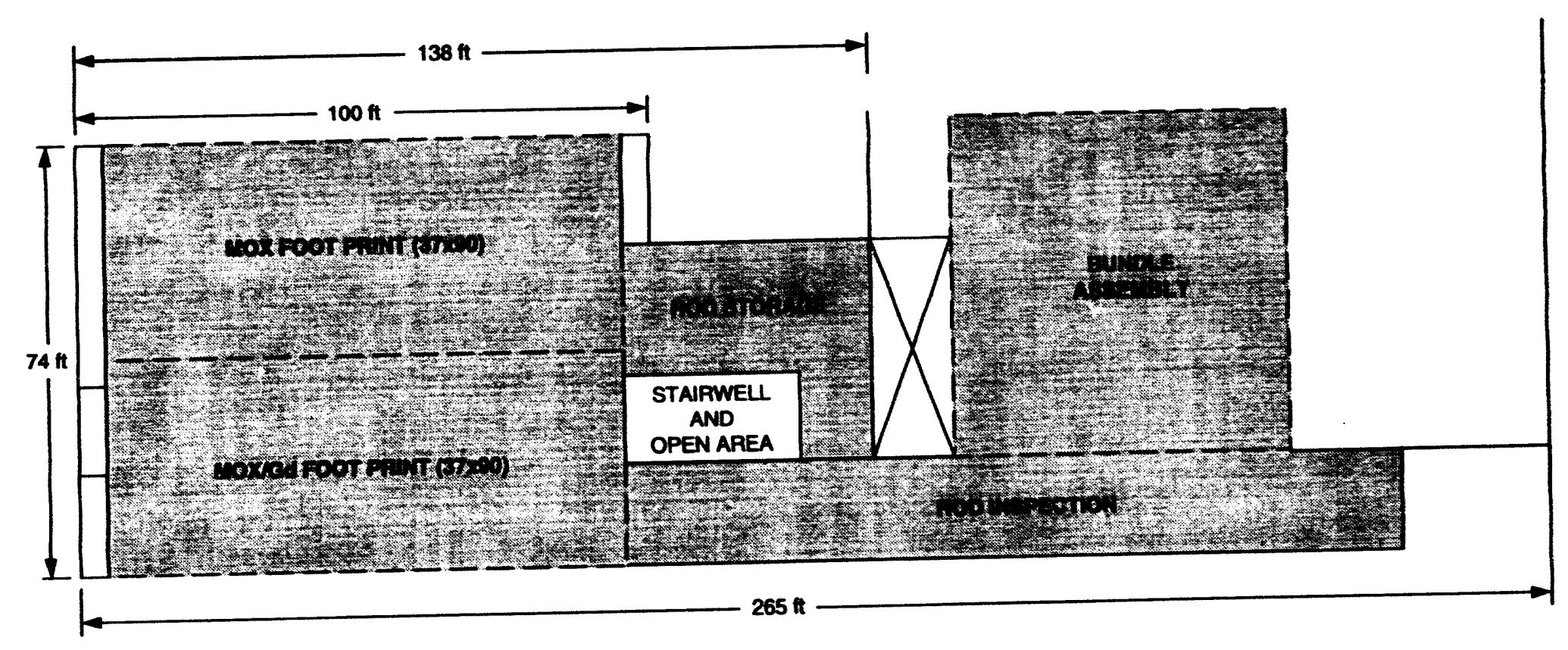

Figure 10.7-1 Adaptation of FMEF 70 Foot Level Internal Area for MOX Fabrication 


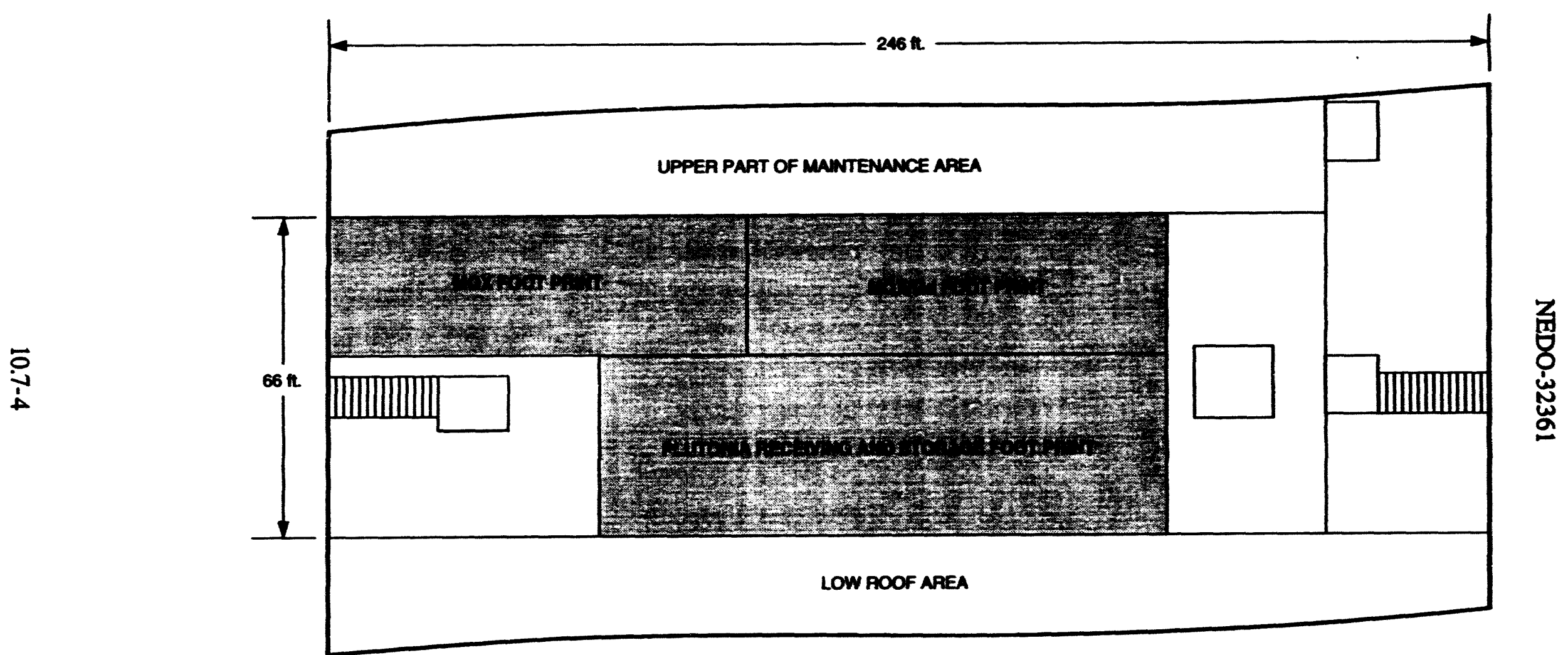

Figure 10.7-2 Upper Level of Fuel Processing Restoration Building 

most likely on a five-day per week basis. The six-month delay between the final fabrication of island assemblies and start of fabrication of full MOX assemblies should allow movement of selected equipment items (welder, rod inspection equipment and analysis instrumentation) to the new MOX facility. Development of remote analysis techniques utilizing fiber optics technology will allow location of instrumentation outside of the glove box environment. This is a key activity at Los Alamos which will reduce the product certification times associated with MOX fabrication. Processes which reduce the waste volumes associated with glove box related scrap and waste are also being developed and evaluated. Although the construction of production facilities at LANL may be inconsistent with their existing goals, the expertise and technology developed at this site, as well as the demonstration of island design fuel fabrication, will provide key elements necessary for the success of the plutonium dispositioning activities.

\section{Lawrence Livermore National Laboratory}

The facilities at LLNL have been instrumental in the development and demonstration of technology to recover plutonium from retired weapons. Several processes have been reviewed for plutonium recovery from weapons. Demonstration of the hydride-dehydride processing steps and the required robotics systems for remote operation are under evaluation. Molten salt type processes are under development for the handling and treatment of scrap and waste streams that result from the recovery of plutonium, conversion of the metal to oxide and MOX fuel fabrication. Although the location of LLNL in a populated area and the current goals which may preclude location of any production facilities at this site, the expertise and technology developed at this location will provide key elements for design and operation of the necessary MOX facilities.

\section{Nevada Text Site}

The Engine Maintenance, Assembly and Disassembly (E-MAD) facility was used to assemble ard prepare nuclear rocket engines for testing. After completion of the rocket program, the facility was used to evaluate the option of spent fuel storage in granite formations. Although this area could be utilized for plutonium conversion or MOX fabrication, extensive upgrading would be required before the facility could be used as part of this plutonium disposition project.

\section{Oak Ridge Site}

The Y-12 Building at the Oak Ridge Site has been used for the enrichment of uranium ore for the nuclear industry. Since the operation of this area has been terminated, the potential exists for upgrading this facility to meet plutonium handling criteria for this project. 


\section{Savannah River Site}

The Barnwell Nuclear Fuel Plant (BNFP) is located adjacent to the Savannah River Site. The Waste Tank Exchange Gallery (WTEG) facility located within the Barnwell complex is capable of containing the MOX fuel fabrication facility for the reference dispositioning options, but additional space several times the current area must be added. This space will accommodate the powder receiving, rod inspection, bundle inspection and bundle assembly areas as shown in Figure 10.7-4. The New Special Recovery facility, which has not been contaminated, is capable of handling all of the scrap and waste from the MOX facility which is processed by aqueous techniques. The Plutonium Storage Facility can also be utilized for feed material receiving and storage. A great deal of automation technology has been developed and tested at this site for mechanized processing in canyon type areas. This technology should be directly applicable to automated MOX fabrication.

A potential cost savings may be realized by utilization of existing DOE facilities. The infrastructure available at various DOE facilities for implementing this project has been discussed in Appendix F. Table 10.7-1 summarizes the DOE facilities that were evaluated for cost savings and possible use. An order of magnitude estimate of $\$ 100$ to 150 million could be expected as a potential cost savings for use of the following:

- Building structures and utilities

- Applicable equipment

- Other site-related services such as radwaste capability and safeguards and security infrastructure

- Engineering or facility support

These savings will vary between sites and include savings in both direct and indirect costs when compared with a new facility construction. 


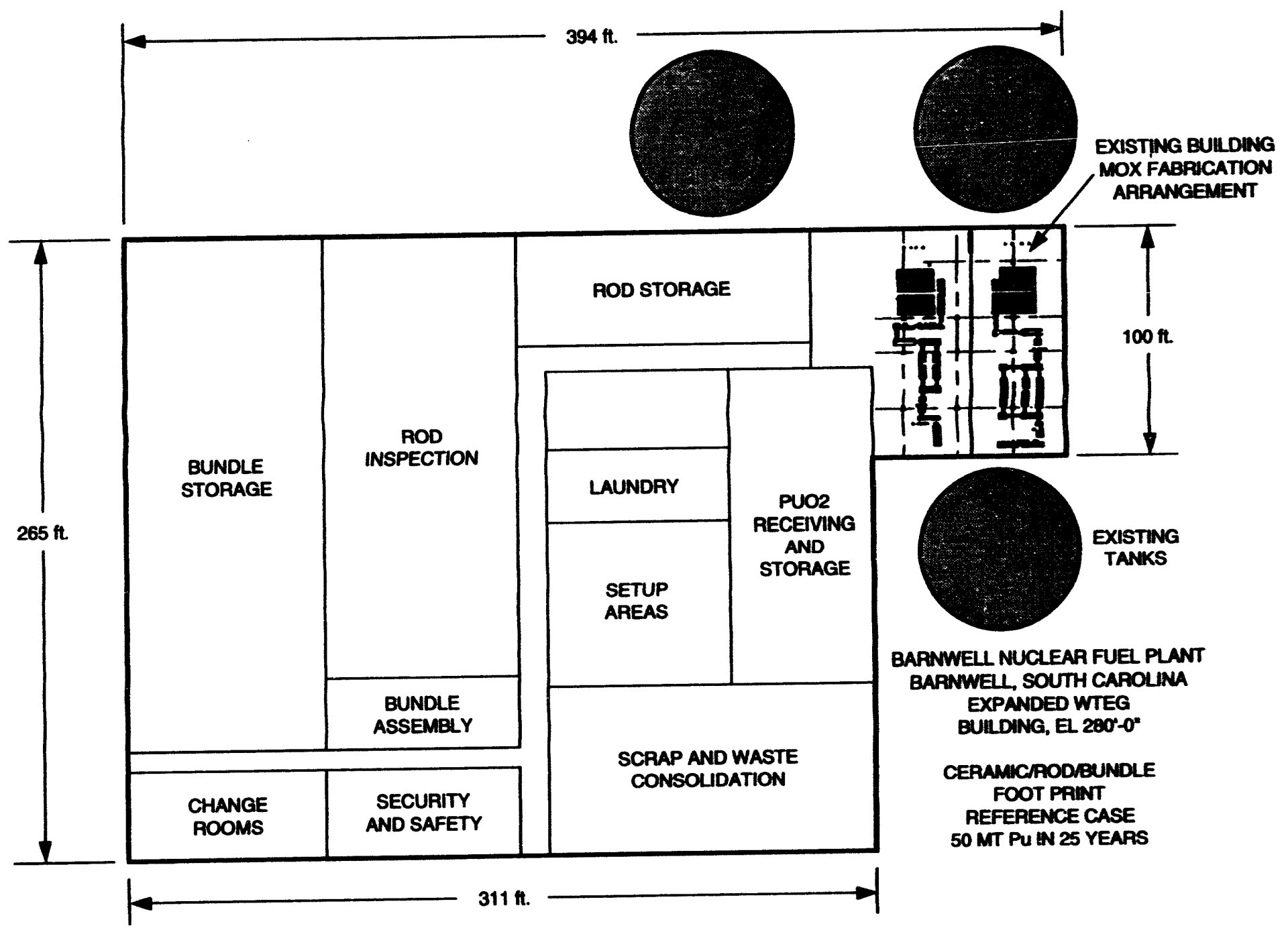

Figure 10.7-4 Adaptation of Waste Tank Exchange Gallery for MOX Fabrication 
Table 10.7-1 Potential Cost Savings from the Use of Existing DOE Facilities

\begin{tabular}{|c|c|c|c|c|}
\hline Site & Facility & Capability & $\begin{array}{l}\text { Cost } \\
\text { Savings } \\
(\$ M)^{*}\end{array}$ & Basis/Remarks \\
\hline Hanford & $\begin{array}{l}\text { Fuels Materials } \\
\text { and Examination } \\
\text { Facility (FMEF) }\end{array}$ & $\begin{array}{l}\text { Category I building } \\
\text { for Pu Storage, Pu } \\
\text { conversion MOX } \\
\text { Fuel Fab; also has } \\
\text { partially installed } \\
\text { MOX fuel fab line } \\
\text { for breeder fuel } \\
\text { (SAF) }\end{array}$ & $*$ & $\begin{array}{l}\text { Category I building needs } \\
\text { modifications of internal } \\
\text { walls and floors to } \\
\text { accommodate fuel } \\
\text { fabrication. Partial use of } \\
\text { SAF of line equipment } \\
\text { may be possible. }\end{array}$ \\
\hline INEL & $\begin{array}{l}\text { Fuel Processing } \\
\text { Restoration (FPR) } \\
\text { Facility }\end{array}$ & $\begin{array}{l}\text { Category I nuclear } \\
\text { building for } \mathrm{Pu} \\
\text { Storage, } \mathrm{Pu} \\
\text { Conversion, and } \\
\text { MOX Fuel Fab }\end{array}$ & * & $\begin{array}{l}\text { Need } \\
\text { upgrade/modifications of } \\
\text { existing FPR for Pu } \\
\text { conversion and fuel fab. }\end{array}$ \\
\hline Oak Ridge & $\begin{array}{l}\text { Y12 HEU } \\
\text { Processing } \\
\text { Facility or } \\
\text { Complex } 21 \\
\text { Facilities }\end{array}$ & $\begin{array}{l}\text { Facilities avail. for } \\
\text { upgrading for Pu } \\
\text { Storage, Conversion } \\
\text { and MOX } \\
\text { Fabrication }\end{array}$ & * & $\begin{array}{l}\text { Can use existing Y12 } \\
\text { facilities with upgrades to } \\
\text { current standards and } \\
\text { modifications for } \mathrm{Pu} \\
\text { storage, conversion, and } \\
\text { fuel fabrication. }\end{array}$ \\
\hline $\begin{array}{l}\text { Savannah } \\
\text { River Site }\end{array}$ & $\begin{array}{l}\text { Pu Storage } \\
\text { Facility (PSF) } \\
\text { New Special } \\
\text { Recovery Facility } \\
\text { (NSR) } \\
\text { AGNS Barnwell } \\
\text { Facilities }\end{array}$ & $\begin{array}{l}\text { Category I nuclear } \\
\text { buildings for } \mathrm{Pu} \\
\text { Storage, } \mathrm{Pu} \\
\text { Conversion, and } \\
\text { MOX Fuel Fab }\end{array}$ & \# & $\begin{array}{l}\text { Data available in SRS } \\
\text { report WSRC-RP-92-1004. } \\
\text { "Strategies for Developing } \\
\text { Denaturing of Weapons } \\
\text { Grade Pu." October } 1992 \\
\text { for use of existing SRS } \\
\text { facilities. }\end{array}$ \\
\hline
\end{tabular}

*An order of magnitude estimate of $\$ 100-150 \mathrm{M}$ could reasonably be expected as potential cost savings from the use of these existing facilities. Reference Appendix F for more details on existing DOE facilities. 


\subsection{Disposition Options}

The reference cases and alternates described in this report are considered to be representative of those operating BWRs which have sufficient time left within their operating license to fulfill the mission objectives. In order to be more specific in the evaluation and discussion of the options available, a number of disposition options are discussed below. These options have been selected because they have some attractiveness by virtue of their location, the number of units on a single site or U.S. Government ownership. The owners of these plants may not have been directly contacted, and may or may not be interested in participating in the program. This evaluation is intended to provide some range of options; however, it is not comprehensive, and there may be a number of other more attractive options identified in the future as the project progresses.

\subsubsection{Browns Ferry}

The Browns Ferry Nuclear Power Facility is a three unit nuclear power station owned and operated by the Tennessee Valley Authority (TVA). Each unit is a single cycle, forced circulation, boiling water reactor (BWR-4) rated at $3293 \mathrm{MW}$ thermal. The design gross electrical output is $1098 \mathrm{MW}$ electric. The Browns Ferry units are located in an agricultural area on a 840 acre site on the north shore of Wheeler Lake in Limestone County, Alabama.

The TVA power system is one of the largest in the United States. TVA is primarily a wholesaler of power, operating generating plants, and transmission facilities, and does not have any retail distribution systems. TVA has various large generating units such as the Paradise Steam Plant, the Cumberland Steam Plant, Sequoyah Nuclear Plant (PWR), Watts Bar Nuclear Plant (PWR) and numerous individual steam generating units.

Since Browns Ferry is the only single BWR site capable of carrying out the mission within 30 years, it is identified here as a deployment option. The site has the following additional advantages relative to other locations:

- The site is owned by an Agency of the U.S. Government.

- The site is within about 300 miles of potential fuel fabrication sites at Oak Ridge and Savannah River.

- The site is located as favorably as any with regard to public acceptance of nuclear programs. 
At the present time, Browns Ferry Unit 2 is operating and Browns Ferry Unit 3 is in a "restart" program with a planned return to service about January 1996. Browns Ferry Unit 1 is not operating and would require considerable reengineering and physical upgrading before it could return to service. - This work would be similar to the work performed on Unit 2, and currently underway on Unit 3, and would complete the modifications required by the NRC to allow the plant to restart. This work is not currently a high priority with TVA. An estimate of the cost and schedule for this scope of work is $\$ 1.2$ Billion and 3 to 5 years. Notwithstanding this cost and schedule, it would appear that Browns Ferry Unit 1 could be available in the circa 2002 timeframe if the decision were made to use the Browns Ferry plants for the mission. If the restart costts of BF-1 were prohibitive, an alternate plant such as WNP-2 could be used. Table 10.8-1 shows the schedule for the disposition of $50 \mathrm{MT}$ of plutonium at the Browns Ferry site.

The Browns Ferry 1,2, and 3 plant operating licenses are scheduled to expire in 2013, 2014, and 2016, respectively. With the uncertainty of license extensions, there is another approach available to TVA's Browns Ferry units that would extend their current license life and provide sufficient added time to allow the three units to meet the $50 \mathrm{MT}$ mission goal. This program, known as Out of Service Time Recovery Program, is currently under evaluation by TVA for implementation on Browns Ferry 1,2, and 3. The basis of the program is to recover the time associated with the extended shutdown of the Browns Ferry units on the operating licenses, adding approximately 13,8 , and 10 years to their respective operating licenses. The program is considered both technically and legally feasible, based on the existing precedence of several operating plants recovering the elapsed time between receipt of their operating license and first power operations. For example, Florida Power \& Light was recently able to recapture plant construction time in the operating licenses of Turkey Point Units 3 and 4. When considered in conjunction with the significant upgrade programs (Appendix R, Environmental Qualification, etc.) required to be complete prior to the restart of any Browns Ferry unit, approval by the NRC is considered a high probability by TVA and GE. Costs to implement the program are under final development, but are considered to be less than that for a license renewal application.

\section{MOX Facility}

Since the Browns Ferry site has sufficient reactor capacity by itself to carry out the plutonium disposition mission, one option would be to construct the new MOX fuel fabrication plant described in Section 2.4 on the Browns Ferry site. Co-location of the reactors and the MOX fuel fabrication facility avoids transportation of the fresh MOX fuel over public roads and minimizes 
Table 10.8-1 Browns Ferry Disposition Capability Based on 75\% C.F.

\begin{tabular}{|c|c|c|c|c|}
\hline \multicolumn{5}{|c|}{ Number of MOX Fuel Assemblies Loaded } \\
\hline Year & BF-2 & BF-3 & BF-1 & $\begin{array}{c}\text { CUMAMT } \\
\text { (Pu) }\end{array}$ \\
\hline 1997 & 58 & & & 0.06 \\
\hline 1998 & 58 & & & 0.12 \\
\hline 1999 & 58 & 58 & & 0.25 \\
\hline 2000 & & 58 & & 0.32 \\
\hline 2001 & 176 & & 58 & 1.8 \\
\hline 2002 & 176 & 176 & & 3.9 \\
\hline 2003 & 176 & 176 & 176 & 6.1 \\
\hline 2004 & 176 & 176 & 176 & 8.2 \\
\hline 2005 & & 176 & 176 & 10.3 \\
\hline 2006 & 176 & & 176 & 12.5 \\
\hline 2007 & 176 & 176 & & 14.6 \\
\hline 2008 & 176 & 176 & 176 & 16.7 \\
\hline 2009 & 176 & 176 & 176 & 18.9 \\
\hline 2010 & & 176 & 176 & 21.0 \\
\hline 2011 & 176 & & 176 & 23.1 \\
\hline 2012 & 176 & 176 & & 25.3 \\
\hline 2013 & 176 & 176 & 176 & 27.4 \\
\hline 2014 & 176 & 176 & 176 & 29.5 \\
\hline 2015 & & 176 & 176 & 31.7 \\
\hline 2016 & 176 & & 176 & 33.8 \\
\hline 2017 & 176 & 176 & & 35.9 \\
\hline 2018 & 176 & 176 & 176 & 38.1 \\
\hline 2019 & 176 & 176 & 176 & 40.2 \\
\hline 2020 & & 176 & 176 & 42.3 \\
\hline 2021 & 176 & & 176 & 44.5 \\
\hline 2022 & 176 & 176 & & 46.6 \\
\hline 2023 & 176 & 176 & 176 & 48.7 \\
\hline 2024 & 176 & 176 & 176 & 50.8 \\
\hline
\end{tabular}

the number of sites and shipments requiring enhanced security. This approach also eliminates the need for separate ex-reactor storage of the fresh MOX fuel and provides the opportunity to optimize the safeguards and security for the entire mission.

If it were desired to fabricate the MOX fuel at a DOE site, the closest would be Oak Ridge at about $\mathbf{2 0 0}$ miles and Savannah River at about $\mathbf{3 0 0}$ miles. Both sites have staff experienced in handling plutonium plus the necessary safeguards and security infrastructure and facilities that potentially could be modified for MOX fuel fabrication. Use of an existing DOE site and facilities for MOX fuel fabrication may be more acceptable to the public than establishing a new site. Further, modifying an existing DOE building that provides the required space and physical protection (seismic, wind, etc.) would be less expensive than a greenfield facility as discussed in Section 6. 
Since Browns Ferry is the only BWR site with sufficient existing reactor capacity to conduct the entire mission, and it is located relatively close to two DOE sites which are under consideration for the reconfigured weapons complex, it was used as the baseline for comparison of other BWR deployment options.

\subsubsection{WNP-2 with LaSalle 1 and 2}

Of the four deployment options discussed in Section 10.8, the following case most closely resembles the reference case discussed in this report. This similarity includes the number of reactors (3), the number of sites (2), the location of one reactor on a DOE site, and the average new fuel shipping distance 1000 miles vs. 1500 miles in the reference case.

The Washington Public Power Supply System (WPPS) Nuclear Project No. 2 (WNP-2) is a single cycle, forced circulation boiling water reactor (BWR-5) rated at $3484 \mathrm{MW}$ thermal. The design gross electrical output is $1205 \mathrm{MW}$ electric. The containment is of the Mark II design. WNP-2 is located on the southeast part of the Hanford Reservation of the U.S. Department of Energy, in Benton County, Washington, approximately 12 miles northwest of the city of Richland. Since WNP-2 is located on the DOE Hanford site and WPPS has indicated an interest in participating in the mission, that reactor is a logical deployment option. Since WNP-2 alone cannot fulfill the mission objectives within a reasonable period of time, this option combines WNP-2 with the Commonwealth Edison LaSalle Units 1 and 2. The LaSalle units are the most proximate BWRs to WNP-2, and Commonwealth Edison has also indicated some interest in participating in the mission (Section 4).

LaSalle County Station is the fourth dual reactor station in the Commonwealth Edison network that services the city of Chicago and the northern part of Illinois. The station has two single cycle forced circulation boiling water reactors (BWR-5) each rated at $3293 \mathrm{MW}$ thermal. The gross electrical power output of each unit is $1122 \mathrm{MWe}$. The containment is the GE BWR/Mark II design. The plant exclusion area consists of approximately 640 acres within the 3060 acre site. This power station is located in an agricultural area of LaSalle County, Illinois, approximately 55 miles southwest of Chicago and 20 miles west of Commonwealth Edison's Dresden Nuclear Power Station. 
Table 10.8-2 shows the schedule for the disposition of 50 MT of plutonium at the WNP-2 and LaSalle sites. It should be noted that the LaSalle Unit 1 license is scheduled to expire in the year 2022 , one year before the last reload batch is scheduled to be loaded in 2023 . For the purpose of this evaluation it is assumed that, if necessary, the plutonium which would be contained in that last batch, could be loaded earlier by further optimization of the bundle design.

Table 10.8-2 WNP-2, LaSalle 1/2 Disposition Capability Based on $75 \%$ C.F.

\begin{tabular}{|l|c|c|c|c|}
\hline Number of MOX Fuel Assemblies Loaded & \\
\hline Year & WNP-2 & LaSalle 1 & LaSalle 2 & CUM-MT (Pu) \\
\hline 1997 & 58 & & & 0.06 \\
\hline 1998 & 58 & & & 0.12 \\
\hline 1999 & 58 & 58 & & 0.25 \\
\hline 2000 & & 58 & & 0.32 \\
\hline 2001 & 176 & & 58 & 1.8 \\
\hline 2002 & 176 & 176 & & 4.0 \\
\hline 2003 & 176 & 176 & 176 & 6.2 \\
\hline 2004 & 176 & 176 & 176 & 8.4 \\
\hline 2005 & & 176 & 176 & 10.6 \\
\hline 2006 & 176 & & 176 & 12.7 \\
\hline 2007 & 176 & 176 & & 14.9 \\
\hline 2008 & 176 & 176 & 176 & 17.1 \\
\hline 2009 & 176 & 176 & 176 & 19.3 \\
\hline 2010 & & 176 & 176 & 21.4 \\
\hline 2011 & 176 & & 176 & 23.6 \\
\hline 2012 & 176 & 176 & & 25.8 \\
\hline 2013 & 176 & 176 & 176 & 28.0 \\
\hline 2014 & 176 & 176 & 176 & 30.2 \\
\hline 2015 & & 176 & 176 & 32.3 \\
\hline 2016 & 176 & & 176 & 34.5 \\
\hline 2017 & 176 & 176 & & 36.7 \\
\hline 2018 & 176 & 176 & 176 & 38.9 \\
\hline 2019 & 176 & 176 & 176 & 41.0 \\
\hline 2020 & & 176 & 176 & 43.2 \\
\hline 2021 & 176 & & 176 & 45.4 \\
\hline 2022 & 176 & 176 & & 47.6 \\
\hline 2023 & 176 & 176 & 176 & 49.8 \\
\hline 2024 & 176 & 176 & 176 & 51.9 \\
\hline & & & & \\
\hline & 176 & & & \\
\hline
\end{tabular}




\section{MOX Facility}

The WNP-2 plant is located on DOE's Hanford site within a few miles of the Fuels and Materials Examination Facility (FMEF). The FMEF is a modern plutonium handling facility that was constructed to examine MOX fuel from the Fast Flux Test Facility (FFTF) and the Clinch River Breeder Reactor project (CRBR). It also contains the Secure Automated Fabrication (SAF) line that was designed to manufacture fast reactor MOX fuel. While the FFTF and CRBR fuel had higher plutonium contents and was of a slightly different design than the MOX fuel for a BWR, significant portions of the equipment in the SAF line could probably be modified for use in the plutonium disposition mission since it was never contaminated and is generic in function.

Combining WNP-2 with the dual unit LaSalle station located 1500 miles east of Hanford minimizes the total shipment of fresh MOX fuel when utilizing FMEF as the BWR fuel fabrication facility. However, the total shipment-miles for this deployment option would be approximately three times as much as the Browns Ferry option with MOX fuel fabrication at the Savannah River or Oak Ridge sites.

\subsubsection{Grand Gulf, River Bend and Clinton}

The Grand Gulf Nuclear Station is a single unit nuclear power station owned by System Energy Resources Inc. and South Mississippi Electric Power Association, and operated by Entergy Operations Inc. Grand Gulf is a single cycle, forced circulation boiling water reactor (BWR-6) rated at $3833 \mathrm{MW}$ thermal with design gross electrical output of $1306 \mathrm{MW}$ electric. The containment is a GE BWRMark III design. The station is located in Clairborne County, Mississippi, on the east side of the Mississippi River, approximately 25 miles south of Vicksburg. The site consists of about 2100 acres in a flat to rolling recreational area.

The River Bend Station is a single unit nuclear power station owned by Gulf States Utilities and Cajun Electric Power Cooperative. Entergy Operations Inc. is also responsible for the design, construction and operation of the River Bend Station. River Bend is a single cycle, forced circulation, boiling water reactor of BWR-6 design rated at $2887 \mathrm{MW}$ thermal. The containment is the GE BWR/Mark III design. The River Bend Station is located east of the Mississippi River on a site in West Feliciana Parish, Louisiana approximately 24 miles northwest of Baton Rouge.

The Clinton nuclear power station is a single unit reactor, $80 \%$ of which is owned by Illinois Power (IP). IP is responsible for the design, construction, and operation of the station. Clinton is a single cycle, forced circulation boiling water reactor BWR-6 rated at $2894 \mathrm{MW}$ thermal with a gross electrical output of $950 \mathrm{MW}$. The containment is a GE BWR/Mark III design. Clinton is 
located in DeWitt County, about 6 miles east of Clinton, Illinois. The Clinton exclusion area includes about 150 acres on a site of about 14,000 acres.

These three sites are identified as deployment options because they are all relatively new units and therefore have maximum remaining license lifetime, and they are all within approximately 600 miles of Oak Ridge, Tennessee, which would minimize new fuel shipping costs.

Table 10.8-3 shows the schedule for the disposition of $50 \mathrm{MT}$ of plutonium at the Grand Gulf, River Bend, and Clinton plants.

\section{MOX Facility}

The Grand Gulf and River Bend sites are located within 100 miles of each other and the Clinton station is approximately 800 miles to the north. DOE's Oak Ridge site would be centrally located to support this deployment option (approximately 400 to 600 miles from any of the three reactor sites). Visits conducted and evaluated during an earlier phase of the project [Ref. 10-3] indicated that modification of existing facilities $Y-12$ or use of the proposed Complex 21 site for a new MOX facility were both viable alternatives for the Oak Ridge site. All three reactor sites are relatively remote from populated areas and could be candidates for a new MOX fuel fabrication plant. The total fresh fuel shipment miles with a new MOX fuel plant at any of the three reactor sites would be comparable to the Browns Ferry deployment option with the fuel fabrication located at the Oak Ridge or Savannah River sites. Locating the MOX fuel fabrication plant at the Oak Ridge site to support this option would increase the shipment-miles by about $50 \%$ relative to the Browns Ferry baseline.

\subsubsection{Perry 1 with Limerick 1 and 2}

The Perry Nuclear Power Plant is a single unit station owned and operated by Cleveland Electric Illuminating Company (CEI). Perry is a single cycle forced circulation boiling water reactor (BWR) rated at $3579 \mathrm{MW}$ thermal with a gross electrical output of $1205 \mathrm{MW}$. The containment is the GE BWR/Mark III design. CEI is also the major owner of Unit 1 of the Davis-Besse Nuclear Power Station and part owner of the Beaver Valley Nuclear Power Station (both are PWRs).

The Perry site is located on Lake Erie, in Lake County Ohio, approximately 35 miles northeast of Cleveland. 
Table 10.8-3 Grand Gulf, River Bend and Clinton Disposition Based on 75\% C.F.

\begin{tabular}{|c|c|c|c|c|}
\hline \multicolumn{5}{|c|}{ Number of MOX Fuel Assemblles Loaded } \\
\hline Year & Grand Gulf & River Bend & Clinton & $\underset{(\mathrm{Pu})}{\operatorname{Con}}$ \\
\hline 1997 & 60 & & & 0.07 \\
\hline 1998 & 60 & & & 0.14 \\
\hline 1999 & 60 & 36 & & 0.26 \\
\hline 2000 & & 36 & & 0.31 \\
\hline 2001 & 184 & & 36 & 1.8 \\
\hline 2002 & 184 & 164 & & 3.9 \\
\hline 2003 & 184 & 164 & 164 & 6.0 \\
\hline 2004 & 184 & 164 & 164 & 8.0 \\
\hline 2005 & & 164 & 164 & 10.1 \\
\hline 2006 & 184 & & 164 & 12.2 \\
\hline 2007 & 184 & 164 & & 14.3 \\
\hline 2008 & 184 & 164 & 164 & 16.3 \\
\hline 2009 & 184 & 164 & 164 & 18.4 \\
\hline 2010 & & 164 & 164 & 20.5 \\
\hline 2011 & 184 & & 164 & 22.6 \\
\hline 2012 & 184 & 164 & & 24.6 \\
\hline 2013 & 184 & 164 & 164 & 26.7 \\
\hline 2014 & 184 & 164 & 164 & 28.8 \\
\hline 2015 & & 164 & 164 & 30.9 \\
\hline 2016 & 184 & & 164 & 32.9 \\
\hline 2017 & 184 & 164 & & 35.0 \\
\hline 2018 & 184 & 164 & 164 & 37.1 \\
\hline 2019 & 184 & 164 & 164 & 39.2 \\
\hline 2020 & & 164 & 164 & 41.2 \\
\hline 2021 & 184 & & 164 & 43.3 \\
\hline 2022 & 184 & 164 & & 45.4 \\
\hline 2023 & 184 & 164 & 164 & 47.4 \\
\hline 2024 & 184 & 164 & 164 & 49.3 \\
\hline 2025 & & 164 & 164 & 31.6 \\
\hline
\end{tabular}

The Limerick Generating Station is a dual unit nuclear power station owned and operated by PECO Energy. Each reactor is a single cycle, forced circulation boiling water reactor (BWR-4) rated at $3293 \mathrm{MW}$ thermal with a gross electrical output of $1092 \mathrm{MW}$. The containment is a GE BWR/Mark II design. PECO also operates the two Peach Bottom BWRs (1098 MWe). Limerick is located on the east bank of the Schuylkill River in Limerick Township, Pennsylvania. The site consists of about 595 acres in rural Chester and Montgomery counties.

The Perry and Limerick sites were selected as potential deployment options because the are located within about 500 miles of Oak Ridge, they have sufficient capacity and license lifetime to fulfill the mission and the opportunity to complete Perry-2, if that should prove desirable. 
Table 10.8-4 shows the schedule for the disposition of $50 \mathrm{MT}$ of plutonium at the Perry and Limerick sites.

\section{MOX Facility}

These two stations are located approximately 400 miles from each other and about 400 to 600 miles from Oak Ridge, the nearest active DOE site. The DOE enrichment facilities near Portsmouth, Ohio and the former West Valley, New York fuel reprocessing site are closer to these reactors than Oak Ridge. However, since the West Valley site is in the process of environmental restoration and closure and the DOE's enrichment facilities are being turned over to a new entity, these sites were not considered as viable locations for a new MOX fuel fabrication facility.

Table 10.8-4 Perry and Limerick Disposition Based on 75\% C.F.

\begin{tabular}{|c|c|c|c|c|}
\hline Year & Perry-1 & Limerick-1 & Limerick-2 & CUMAMT(Pu) \\
\hline 1997 & 58 & & & 0.06 \\
\hline 1998 & 58 & & & 0.13 \\
\hline 1999 & 38 & 38 & & 0.26 \\
\hline 2000 & & 58 & & 0.32 \\
\hline 2001 & 176 & & 58 & 1.9 \\
\hline 2002 & 176 & 176 & & 4.1 \\
\hline 2003 & 176 & 176 & 176 & 6.3 \\
\hline 2004 & 176 & 176 & 176 & 8.4 \\
\hline 2005 & & 176 & 176 & 10.6 \\
\hline 2006 & 176 & & 176 & 12.8 \\
\hline 2007 & 176 & 176 & & 15.0 \\
\hline 2008 & 176 & 176 & 176 & 17.2 \\
\hline 2009 & 176 & 176 & 176 & 19.4 \\
\hline 2010 & & 176 & 176 & 21.6 \\
\hline 2011 & 176 & & 176 & 23.8 \\
\hline 2012 & 176 & 176 & & 26.0 \\
\hline 2013 & 176 & 176 & 176 & 28.2 \\
\hline 2014 & 176 & 176 & 176 & 30.4 \\
\hline 2015 & & 176 & 176 & 32.6 \\
\hline 2016 & 176 & & 176 & 34.8 \\
\hline 2017 & 176 & 176 & & 37.0 \\
\hline 2018 & 176 & 176 & 176 & 39.2 \\
\hline 2019 & 176 & 176 & 176 & 41.4 \\
\hline 2020 & & 176 & 176 & 43.6 \\
\hline 2021 & 176 & & 176 & 45.8 \\
\hline 2022 & 176 & 176 & & 48.0 \\
\hline 2023 & 176 & 176 & 176 & 50.1 \\
\hline
\end{tabular}


If a new MOX fuel fabrication plant were located at the Limerick site, the fresh fuel-miles would be approximately $50 \%$ less than the Browns Ferry option with fuel fabrication at the Oak Ridge or Savannah River sites. Using Oak Ridge as the fuel fabrication site to support the Limerick and Perry plants involves about 50\% more shipment-miles than the Browns Ferry baseline.

\subsubsection{Other Poselbillities}

Beyond the deployment options described above, there are a large number of other plants both BWRs and probably BWR/PWR combinations that could meet the plutonium disposition mission objectives. It may turn out that as further discussions with plant owners take place, there may be advantages to negotiate with a single owner for all the plants needed for the program. For example:

- TVA could deploy some combination of Browns Ferry, Sequoya, Watts Bar, and Bellafont units.

- CECO could deploy some combination of LaSalle, Quad Cities, Dresden, Zion, and Braidwood units.

Other possibilities include U.S. Government completion of Perry Unit 2 for use in combination with Perry Unit 1 alone or with Perry Unit 1 and other units. This option would have the advantage of providing the full 40-year licensed lifetime of Perry Unit 2 for the mission. Similarly, WNP Units 1 and/or 3 could be completed for use with WNP-2. This combination would minimize new MOX fuel shipping distances if the MOX fabrication facility were located on the Hanford reservation.

Another alternative that minimizes the number of reactors required for disposition would be to lower the discharge exposure. For example, by discharging the fuel around 24,000 MWd/MT instead off the reference design exposure of $37,600 \mathrm{MWd} / \mathrm{MT}$, only two reactors will be needed in the reference case for disposing $50 \mathrm{MT}$ of plutonium. As seen in Appendix I, which lists the discharge exposures of BWR fuel assemblies, the lower value is typical of earlier BWR fuel designs. Although it is judged that at this lower exposure level the reactor plant could still operate at $75 \%$ capacity factor and the discharged fuel would meet the definition of spent fuel, no additional evaluations were carried out for this altemative as one of the premises of this study was to design the reference fuel for a discharge exposure similar to current generation BWR fuel. 


\subsubsection{Summary}

The GE BWR has the capability to fulfill the plutonium disposition mission requirements with no system changes and a minimum of licensing requirements. It would appear that mission implementation would be based primarily on the logistics of MOX fabrication and transportation and the willingness of the plant owners to participate, and not on the capability of the BWR to safely, efficiently and economically convert weapons plutonium to spent nuclear fuel. 
NEDO-32361

\subsection{References}

10-1 NUREG-1437, Generic Environmental Impact Statement for License Renewal of Nuclear Plants, August 1991.

10-2 Requirements of Renewal of Operating Licenses for Nuclear Power Plants, 10 CFR Part 54, November 1992.

10-3 Study of $\mathrm{Pu}$ Consumption in Advanced LIght water Reactors, NEDO-32351, Compilation of Phase 1C Task Reports, Prepared for U.S. Department of Energy, January 1994. 


\subsection{Public and Political Acceptance}

Effective campaigns for public acceptance of nuclear power projects has gained recognition as a key factor in the success of a project in the post-TMI era. Experience has shown that failure to consider the reaction of the public to a project deployment will directly impact the completion of a contested project on schedule and within the allocated budget, due to the ability of intervenors to halt a project through litigation. Thus, to increase the probability of the project's success, a public information campaign to inform, educate, and gain the support of the public and special interest groups should be implemented for the Plutonium Disposition project.

\subsection{Approach for Achieving Public Acceptance}

The keys to establishing public acceptance for any program of this type is to establish positive support from the public on two levels, the national level for support of the necessary funding, and the local level to ensure support of the project in the location selected for deployment. These programs must be initiated early in the process to allow for the time necessary to develop a supportive public opinion at both levels. The focus of each of the "tiers" of this program will be different, but there should be an overriding theme to integrate the entire program and generate support at both the national and local levels.

The need for support at the national level is to ensure public support, and the resultant Congressional budgetary backing, is developed at project inception and continues throughout the programs life. One method of developing this support would be to establish a clear policy from the Administration that details the need for elimination of weapons plutonium, and solidly backs a practical technical solution for the problem. This position should be developed in conjunction with bipartisan congressional input to ensure there is a single government solution for the problem. It is only after this unified policy is developed that it is announced publicly, in a high level forum like the State of the Union Address, or national press conference dedicated to the announcement. The clear message of this policy is that the program will benefit the national goals of proliferation deterrence, assist the conversion of the National Laboratory resources from weapons production to peaceful industrial research, while demonstrating fiscal responsibility through utilization of existing facilities. The fission option defined in this report has that capability, and the cost benefit of utilizing the existing facilities for the near term initiation of the program should be used to demonstrate the new vision of government that includes fiscal responsibility. 
At the local level, these issues will be specific to the facility selected for implementation of the mission. The site selection should account for the current public perception of nuclear power, as well as overall administration of government programs. A likely scenario would be to select a utility site in close proximity to one of the existing National Laboratory sites as the reactor facility. This laboratory site would serve as the site for the fuel fabrication facility. This would minimize the potential for anti-nuclear sentiment and provide the local economy with a positive boost as a result of the facility construction and operation. The local "image" could also be boosted by committing to a project that is key to implementation of the National policy, while continuing the conversion support of the laboratory resources to limit the impact on the local economy. 


\subsection{Likelihood of National Political Acceptance}

Several key features of the fission program must be publicized for the program to gain the political support and acceptance necessary for implementation. The goal will be to describe the total program in such a way that it elevates the importance of the program to a level of national importance to avoid "pork barrel" politics. The key issues to be addressed will be:

- Cost effective nature of the fission option

- Environmentally neutral (compared to other disposition options like vitrification)

- Proliferation safe vs other technology available today

- Utilization of National Laboratory resources for peaceful technologies

- Available for rapid implementation

- Capable of integration with a reciprocal Russian program, resulting in an improvement in global security from the threat of nuclear weapons

This political acceptance will be accomplished as part of the development of a bipartisan policy position developed to gain the acceptance of the public. 


\subsection{Likelihood of Local or Regional Acceptance}

The probability of local acceptance will be a result of the criteria applied in the site selection process. The key will be to select a location/region that meets the following guidelines:

- Utility site near, or on, an existing national laboratory facility

- Close proximity with plutonium processing and/or nuclear power production to ensure familiarity with the technology

- Local economic conditions that would benefit from conversion of the laboratory to peaceful research purposes and construction of the fuel fabrication facility

- Location relatively distant from a major metropolitan area to ensure limited impact of the emergency planning zone.

These features, coupled with the boost in the local public image for supporting a key national program, will result in conditions that will maximize the potential for local public acceptance. 


\subsection{Conclusions and Recommendations}

The proposed method to disposition excess plutonium as spent nuclear fuel by utilizing it in existing BWRs involves no new technology and presents a low risk approach compared to alternative disposition methods. Satisfactory performance of MOX fuel in U.S. BWRs was demonstrated more than twenty years ago. Full core loading with MOX fuel is readily accomplished in BWRs evaluated in this study through fuel design and does not require reactor system modification. Large-scale MOX fuel fabrication has been demonstrated in Europe.

This study recommends an early demonstration of the proposed disposition process using an "island" MOX fuel assembly design that requires only a limited plutonium fabrication capability. A demonstration program is the best means to define and establish both the technical and nontechnical infrastructure while proceeding with the full MOX disposition program. Although most of the fission option elements have been tested and validated in the past in this country, and MOX fuel is currently being produced and used in light water reactors in Europe, a demonstration program is the most cost-effective and expeditious way to enunciate the interfaces and responsibilities for the various program elements in such areas as fuel fabrication, transportation, storage, safeguards, and fuel licensing. Although the $\mathrm{Pu}$ throughput is small (about 0.2 - $0.8 \mathrm{MT} / \mathrm{yr}$.), the mixed-oxide material requirements for the demonstration program are approximately 6 to $8 \mathrm{MT}$ of MOX fuel per year; well within the capabilities of the LANL TA-55 facility. This report has concluded that an initial island design reload of MOX fuel could be ready as early as July 1997 . The same facility could be used to produce fuel qualification test rods and assemble the fuel bundles for the full MOX design lead use (fuel) assemblies (LUAs).

Full MOX fuel utilization can be implemented in the candidate reactors in stages as the urania fuel is discharged. The interchangeability of urania and MOX fuel designs in the BWR allows replacement of the reload batches of urania fuel without requiring a full core replacement. Licensing of the reactor to operate with partial or full core loading of MOX fuel is straightforward, as each reload would be designed to satisfy existing fuel design safety criteria.

The challenges to implementing this project are expected to be mainly non-technical. In this regard, the five key technical challenges outlined below should be recognized as key areas which are judged to be on the critical success path, and not as "open" issues requiring an advance in technology. A major "non-technical" challenge in using existing reactors is in identifying potential reactors for use in the disposition mission which satisfy a number of desirable criteria 
such as (1) a single site with multiple reactors; (2) a single utility or owner to expeditiously conclude economic incentive issues; and (3) possibility of co-locating the MOX plant or at least finding a suitable DOE site in the near vicinity of the reactor(s) to minimize transportation.

Five key advantages of utilizing existing BWRs are presented below. This disposition process, using the fission option also provides the U.S. with the opportunity to develop key technologies for safeguards and security, which upon implementation would establish a U.S. leadership role in this vital area.

\subsection{Five Key Challenges}

(1) An adequate public acceptance and political support base must be established:

Because of the public sensitivity to nuclear weapons in general, and in some areas to nuclear power, it is absolutely essential that disposing of plutonium from nuclear weapons using existing power plants be recognized as safe and accepted as an important national priority. The extent to which this can be achieved will have a significant impact on all areas of the program including facility siting and licensing, transportation of nuclear materials, financial support in the congress and the interest of utilities and their regulators to support the program. Without adequate support there will be many opportunities to delay implementation of any plutonium disposition program leading to increased costs.

(2) Definitive agreement(s) including economic incentives must be defined and negotiated:

Issues that need to be resolved early in this process are the definitive agreement(s) established with the utility(s), the nature of the financial risks involved, and the extent to which these will be mitigated by the incentives offered. As discussed in Section 1.2.3, there was no interest at this time among the few utilities contacted in selling their existing BWRs to the government.

Assuming adequate public acceptance and political support at the national level, establishing the incentives necessary to acquire the use of the power plants desired for this mission will be aided by the following considerations:

The Government has considerable flexibility in choosing the reactor site(s), since any of the identified BWRs can utilize full core MOX loading by appropriate fuel design without modification to the plant systems. 
There are a number of multi-unit BWR sites and utilities with multiple plants (BWRs and PWRs) that provide the opportunity to minimize the number of sites and/or organizations required to accomplish the mission.

The utilities operating BWRs include both investor-owned, non-public and public utilities which encompasses the full range of financial and rate-regulating structures. As discussed previously, the investor-owned and public utilities will likely have different perspectives with regard to incentives providing the Government with a wider range of options.

\section{(3) An effective NRC licensing plan must be established:}

While the safety of MOX fuel utilization in LWRs was demonstrated more than 20 years ago using partial core loads, there has been no intervening work in the U.S. for licensing MOX fuel. An overall licensing plan must therefore be agreed upon early by the DOE, NRC and participating utilities. While this study has shown that full MOX core utilization in BWRs is safe and that the current NRC-approved methodology is appropriate, additional fuel testing appears prudent. A related challenge would be to implement a limited early program for fuel testing after initial discussions with NRC to confirm the licensing process for a full MOX core.

\section{(4) A feed material specification needs to be established:}

Two issues - the concentration of americium and the acceptable levels for other constituents in the $\mathrm{PuO}_{2}$ feed material - need to be resolved as soon as possible. High levels of americium will be a significant contributor to worker exposure in the MOX fuel fabrication process. Even with a shielded, highly automated fabrication line and a welldesigned maintenance program, some contact work will be necessary for decontamination and replacement of equipment. For this type of work, hand and arm exposure is unavoidable as well as some whole body dose. High americium levels are not a significant issue in handling the finished fuel rod or bundle because of dilution and self-shielding by the urania plus shielding from the cladding and the fuel bundle channel. The challenge here is to perform the tradeoff studies necessary to arrive at an early decision on the americium level so that the necessary features can be incorporated in the facility and equipment design to achieve ALARA goals. The other nonradioactive constituents are not 
expected to cause a problem but represent an uncertainty in the fabrication and in-reactor behavior of the MOX fuel. These issues are planned to be resolved through the limited testing program discussed above.

\section{(5) Existing safeguards and security practices need to be reexamined:}

The existing practices for implementing safeguards and security for Special Nuclear Materials (SNM) need to be reexamined in the context of the proposed mission. Based on preliminary evaluations, the key issues that need to be addressed include: (a) existing accountability methods and criteria may not be adequate to achieve acceptable MUF (material unaccounted for) levels; (b) the limited payload of the SST leads to a very large number of fresh MOX fuel shipments; and (c) the additional security associated with plutonium under current practices could be a significant dis-incentive for utilities who otherwise may be interested in participation. The challenge here will be to incorporate the best practices from the European MOX programs with state of the art accountability techniques and creative approaches to transportation and security to provide the necessary protection for the SNM with minimum impacts. 


\subsection{Five Key Advantages}

(1) Flexibility in choosing reactor site(s) - Full MOX utllization with no reactor system modifications and ease of licensing:

No reactor systems modifications to the BWRs evaluated are necessary for full MOX core utilization. No new safety issues have been identified including severe accident conditions, and the conclusions of the existing PRA's remain valid, thereby removing schedule risk arising from licensability. Since the BWR evaluated can utilize full MOX loading through appropriate fuel design without any system level modification, there is considerable flexibility in choosing the reactor site(s).

(2) Minimal cost and schedule risks:

The cost and schedule risks associated with the described disposition capabilities are quite low. With the "Island design" BWR fuel, an initial pilot program can be implemented immediately which would quickly and cost effectively demonstrate the various elements of the disposition process such as fuel fabrication with disposition specific feed stock, safeguards implementation, transportation, storage as well as any non-technical issues such as public acceptance. These lessons could then be applied to the full MOX core disposition phase, thereby achieving well developed costs and schedules with minimal risk.

(3) Fuel and core licensing simplified:

The MOX fuel core has been designed conservatively. Peak Pu enrichment has been limited to less than $10 \%$. A defined GE/NRC licensing process and criteria for new fuel exist and BWR MOX core designs remain within those criteria, making fuel assembly design license approval easier and faster.

(4) Flexibility in transition from and return to urania core and fuel cycle:

The BWR is uniquely flexible to adapt to any variation in feed stock supply. A wide range of core design options, from full MOX core to island designs, is possible without plant system changes. The full MOX core can also be implemented in stages as existing urania fuel is discharged. For the same reason, substitution of a urania core, after the conclusion of disposition operations, can be implemented with no plant system changes and no significant reactor operating cycle interruptions. 
(5) Eariy Pu disposition committment opportunity:

The BWR "Island Design" fuel cycle provides the U.S. Government an opportunity to demonstrate a unilateral committment to the Pu Disposition process by initiating an early start fission demonstration program, thus assisting in achievement of bilateral $\mathrm{Pu}$ disposition and multilateral nonproliferation agreements. The proposed disposition process then readily provides for transparency and reciprocity with disposition in the former Soviet Union. 


\subsection{Recommendations for Near-Term Implementation}

Foremost among the near-term activities that have to be undertaken is the need to arrive at a road map for disposition of weapons plutonium with the former Soviet Union showing the plutonium quantities, plutonium forms, location, dates and methods for the disposition process. This area is outside the scope of the present study.

Nevertheless, a key factor in safeguarding the weapons plutonium in the U.S. and in the former Soviet Union is the need to proceed expeditiously with the disposition process. A number of activities have been identified to be on the critical path for the fission option. Although these activities require an early start, they are not resource or capital intensive (compared to other elements of the program); therefore, if a more attractive disposition option is found in the future, it could be initiated without having expended a great deal of resources on the fission option. The benefit of this approach, obviously, is the expeditious implementation of the disposition project.

The following recommendations are made for near-term (if not immediate) activities for using the fission option in this country:

\section{Early Demonstration of Conversion to Oxide}

Several process are available for conversion of metal to oxide. Oxide feed stock in small quantities should be fabricated with and without americium for determination of the optimum MOX fuel fabrication process.

\section{Verification of MOX Fuel Fabrication Quality}

Small quantities of MOX fuel should be produced with the disposition specific feed material interface, to confirm the quality of the as-fabricated material. This could be carried out by LANL in the TA-55 facility with input on the fuel specifications from GE, thus accelerating the critical path MOX fabrication facility design.

\section{Lead Fuel Testing}

A lead fuel testing program for full MOX core fuel should be implemented to provide the data that will be needed for fuel licensing by the NRC. Although considerable MOX fuel test data is already available, the assessment of additional benchmark data and iteration of nuclear methods is appropriate and will further reduce licensing and schedule risk. A lead fuel test program has been outlined in the report, discussing both rod tests and lead use assembly tests. The fuel for this program could be produced by LANL in the TA-55 facility. 


\section{MOX Fuel Licensing Discuselons with NRC}

Procedures exist for NRC licensing of new fuel. Nonetheless, discussions should be held as early as possible with the NRC to review their requirements and achieve mutual understanding related to licensing MOX fuel. This would help to define and design the lead fuel tests and to conduct any additional analytical evaluations that may be required.

\section{Environmental Impact Statement}

Generic and site-specific environmental impact assessments will be made prior to the decision to proceed with the fission option. Selected parallel site-specific assessments with the generic assessments will help expedite this process.

\section{Implementation of an Farly Demonstration Program}

An early demonstration program using an "island" design at one or more reactor sites is recommended. This program requires a throughput of approximately $6 \mathrm{MT}$ of MOX per year. This level of throughput is within the capabilities of the LANL TA-55 facility.

The purpose of the demonstration program is both to validate the technical basis of the fission option and to implement the administrative and non-technical aspects of the project. These aspects include the interfaces and responsibilities for various elements such as fuel fabrication, transport, storage, handling of MOX fuel at the reactor site, safeguards and site security. Public and political acceptance, for instance, could also be gauged by the support of and response to the demonstration program. a demonstration program would also provide the opportunities for comparison to other disposition options. Such an early start pilot/demonstration activity can be a quite meaningful, low risk, low cost unilateral commitment to weapons plutonium disposition. 


\section{Appendix A: Uniform Descriptive Technical Data (Existing Reactors)}

\begin{tabular}{|c|c|}
\hline lelend beedre & Recetor plant lidentfilioation \\
\hline & (Data reported for Goneric 3 Plant Caee) \\
\hline & 1. Plant retroftt and oparation sohedule (rolative to 10/104 projeot atart) \\
\hline Jut-97 & 1) time MOX buming operations start (mo) \\
\hline 3484 & 2) thermal power (MWth) - See Appendix C for epecific plants \\
\hline 1185 & 3) net electrical output (MWo) - See Appendlx C tor epectilic plants \\
\hline \multirow{3}{*}{76} & 4) capacity factor (average for 1991, 1992, and 1993) - See section 4.4 Diecusaton \\
\hline & 5) remaining plant iffe $(y)$ - See Table 1.2-1 \\
\hline & II. Freeh nox fuel secembly charectertatlos \\
\hline 83.17 & 1) reactor Pu inventory - firat core (kg PU) - TRANSITIONS FROM UAANIA CORE WITH 58 ISLAND ASSY \\
\hline 136062 & 2) reactor U inventory - first core (kg U) \\
\hline 1.434 & 3) Pu contont (kg Puhual aseembly) (ONLY 12 RODS OF 60 AAE MOX RODS) \\
\hline 0 & 4) Pu-238 fraction (kg Pu-238/kg Pu) \\
\hline 0.84 & 5) Pu-239 Iraction (kg Pu-239/kg Pu) \\
\hline 0.057 & 6) Pu-240 fraction (kg Pu-240/kg Pu) \\
\hline 0.003 & 7) Pu-241 fraction (kg Pu-241/kg Pu) \\
\hline 0 & 8) Pu-242 fraction (kg Pu-242/kg Pu) \\
\hline 303 & 9) fuel ascembly mase (kg) \\
\hline 4.56 & 10) huol assombly lonoth $(m)$ \\
\hline 0.15 & 11) fuel ascombly witth $(m)$ \\
\hline 177.54 & 12) U mass in MOX (kg U/fuel aseombly) \\
\hline 0.027 & 13) U-235 enrichment in MOX (kq U-235/kg U) \\
\hline 178.97 & 14) U mase in UO2 fuol (kg UAual assembly) \\
\hline \multirow[t]{2}{*}{ NA } & 15) U-235 onrichment in UO2 fuel (kg U-235/ kg U) \\
\hline & III. Equillibrlum oyele mox fuel rechares \\
\hline 83.17 & 1) Pu rechange (kg Pu) \\
\hline 10297 & 2) U recharpe (kgU) \\
\hline 1.434 & 3) Pu content (kg Pu/fuel aseombly) \\
\hline 0 & 4) Pu-238 fraction (kg Pu-238/kg Pu) \\
\hline 0.84 & 5) Pu-239 fraction (kg Pu-239/kg Pu) \\
\hline 0.057 & 6) Pu-240 traction ( $\mathrm{kg} P \mathrm{Pu}-240 / \mathrm{kg} \mathrm{Pu})$ \\
\hline 0.003 & 7) Pu-241 fraction (kg Pu-241/kg Pu) \\
\hline 0 & 8) Pu-242 Iraction (kg Pu-242/kg Pu) \\
\hline 177.54 & 9) U mase in MOX (kg U/uel assombly) \\
\hline 0.027 & 10) U-235 enrichment in MOX (kg U-235/kg U), \% \\
\hline 178.97 & 11) U mase in UO2 fuel (kg U/uel assembly) \\
\hline NA & 12) U-235 enrichment in UO2 fuel (kg U-235/ kg U) \\
\hline 58 & 13) number of MOX fuel assomblies \\
\hline \multirow[t]{2}{*}{11.68} & 14) cycle length (mo) - full power \\
\hline & IV. MOX fuel aseembly characteriatles at disecharge \\
\hline 2.04 & 1) Pu mass discharged per cycle in MOX fuel (kg Pu) \\
\hline- & 2) Pu mases discharged per cycle in UO2 fuel (kg Pu) \\
\hline 0.0146 & 3) Pu-238 fraction in MOX fuel (kg Pu-238/kg Pu) \\
\hline 0.458 & 4) Pu-239 fraction in MOX fuel (kg Pu-239/kg Pu) \\
\hline 0.3235 & 5) Pu-240 fraction in MOX fuel ( $\mathrm{kg}$ Pu-240/kg Pu) \\
\hline 0.1405 & 6) Pu-241 fraction in MOX fuel (kg Pu-241/kg Pu) \\
\hline 0.0634 & 7) Pu-242 fraction in MOX fuel ( $\mathrm{kg}$ Pu-242/kg Pu) \\
\hline- & (8) Pu-238 fraction in UO2 fuel (kg Pu-238/kg Pu) \\
\hline$\dot{-}$ & 9) Pu-239 fraction in uO2 fuel (kg Pu-239/kg Pu) \\
\hline
\end{tabular}


Appendix A: Uniform Deacriptive Technical Data (Continued)

\begin{tabular}{|c|c|}
\hline - & 10) Pu-240 freotion in U02 fual (kg Pu-240/kg Pu) \\
\hline - & 11) Pu-241 fraction in U02 fual (kg Pu-241/kg Pu) \\
\hline - & 12) Pu-242 frection in UO2 fud (ko Pu-242/ko Pu) \\
\hline 209200 & 13) heat generation in ons fual aceembly $(W)$ \\
\hline $1.84 E+07$ & 14) gamma radiation field (remh at surtace, midplane of fuel aseembly) \\
\hline 1540 & 16) Offective full power days (d) \\
\hline 39.2 & 16) bumup (MWdKaHM) \\
\hline \multirow{2}{*}{0.01186} & 17) fraction of Pu in hoavy motal (ko PukoHM) \\
\hline & V. Wox fud charesterietica 1 year affer dicohares \\
\hline 2.146 & 1) Pu mases in MOX fuel (ko Pu) \\
\hline$\cdot$ & 2) Pu maes in UO2 fuel (kg Pu) \\
\hline 0.0165 & 3) Pu-238 fraction in MOX fual (kg Pu-238/kg Pu) \\
\hline 0.463 & 4) Pu-239 fraction in MOX fual (kg Pu-239/kg Pu) \\
\hline 0.3234 & 5) Pu-240 fraction in MOX fual (kg Pu-240/kg Pu) \\
\hline 0.1338 & 6) Pu-241 fraction in MOX fuel (kg Pu-241/kg Pu) \\
\hline 0.0033 & 7) Pu-242 fraction in MOX tuel (kg Pu-242/kg Pu) \\
\hline - & 8) Pu-238 fraction in U02 fuel (kg Pu-238/kg Pu) \\
\hline- & 9) Pu-239 fraction in UO2 fuel (kg Pu-239/kg Pu) \\
\hline - & 10) Pu-240 fraction in UO2 fual (kg Pu-240/kg Pu) \\
\hline- & 11) Pu-241 fraction in UO2 fuel (kg Pu-241/kg Pu) \\
\hline- & 12) Pu-242 fraction in U02 fual (kg Pu-242/kg Pu) \\
\hline 1654 & 13) heat generation in one fuel assembly $(W)$ \\
\hline $1.03 E+05$ & 14) gamma radiation field (rem $\mathrm{h}$ at surface, midplane of fuel assembly) \\
\hline 1540 & 15) effective full power days (d) \\
\hline 39.2 & 16) bumup (MWd/kgHM) \\
\hline \multirow{2}{*}{0.01186} & 17) fraction of Pu in heavy metal (kg Pu/kgHM) \\
\hline & \\
\hline 2.143 & 1) Pu mases in MOX fuel (kg Pu) \\
\hline - & 2) Pu mass in $\mathrm{UO} 2 \mathrm{hual}(\mathrm{kg} \mathrm{Pu})$ \\
\hline 0.0167 & 3) Pu-238 fraction in MOX fuel (kg Pu-238/kg Pu) \\
\hline 0.4858 & 4) Pu-239 fraction in MOX fuel (kg Pu-239/kg Pu) \\
\hline 0.34 & 5) Pu-240 fraction in MOX fuel (kg Pu-240/kg Pu) \\
\hline 0.091 & 6) Pu-241 fraction in MOX fuel (kg Pu-241/kg Pu) \\
\hline 0.0865 & 7) Pu-242 fraction in MOX fuel (kg Pu-242/kg Pu) \\
\hline- & 8) Pu-238 fraction in UO2 fuel (kg Pu-238/kg Pu) \\
\hline - & 9) Pu-239 fraction in UO2 fuel (kg Pu-239kg Pu) \\
\hline- & 10) Pu-240 fraction in UO2 fual ( $\mathrm{kg} P \mathrm{Pu}-240 / \mathrm{kg} \mathrm{Pu})$ \\
\hline 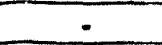 & 11) Pu-241 fraction in UO2 tuel (kg Pu-241/kg Pu) \\
\hline$\cdot$ & 12) Pu-242 fraction in UO2 fuel (kg Pu-242/kg Pu) \\
\hline 231 & 13) heat generation in one fuel assembly $(W)$ \\
\hline $1.52 E+04$ & 14) gamma radiation field (rem/h at surface, midplane of fuel assembly) \\
\hline 1540 & 15) effective full power days (d) \\
\hline 39.2 & 16) bumup (MWd/kgHM) \\
\hline \multirow{2}{*}{0.0113} & 17) fraction of Pu in heavy metal (kg Pu/kghM) \\
\hline & VII. MOX fuel charneteristics 100 yeare after cliechares \\
\hline 1.75 & 1) Pu mass in MOX fuel (kg Pu) \\
\hline$\cdot$ & 2) Pu mass in UO2 fuel (kg Pu) \\
\hline 0.0083 & 3) Pu-238 fraction in MOX fuel (kg Pu-238/kg Pu) \\
\hline 0.5386 & 4) Pu-239 fraction in MOX fuel (kg Pu-239/kg Pu) \\
\hline 0.3768 & (5) Pu-240 traction in MOX fuel (kg Pu-240/kg Pu) \\
\hline
\end{tabular}




\section{Apprendix A: Uniform Descriptive Technical Data (Continued)}

\begin{tabular}{|c|c|}
\hline 0.0013 & 6) Pu-241 fraction in MOX fuel (kg Pu-241/kg Pu) \\
\hline 0.074 & 7) Pu-242 fraction in MOX fuel ( $\mathrm{kg}$ Pu-242/kg Pu) \\
\hline- & 8) Pu-238 fraction in UO2 tual (kg Pu-238k $\mathrm{kgu}$ ) \\
\hline - & 9) Pu-239 fraction in UO2 fuel (kg Pu-239/kg Pu) \\
\hline - & 10) Pu-240 traction in U02 tual (kg Pu-240 $\mathrm{kg} \mathrm{Pu})$ \\
\hline- & 11) Pu-241 fraction in U02 tuol (kg Pu-241/kg Pu) \\
\hline- & 12) Pu-242 traction in UO2 fual (kg Pu-242/kg Pu) \\
\hline 65 & 13) heat generation in one fuel assembly $(W)$ \\
\hline $1.31 E+03$ & 14) gamma radiation tield (rem/h at surface, midplane of fuel aceombly) \\
\hline 1540 & 15) offective full power days (d) \\
\hline 39.2 & 16) bumup (MWdkgHM) \\
\hline \multirow[t]{4}{*}{0.0102} & 17) fraction of Pu in heavy metal (ko Pukg HM) \\
\hline & Vill. Pu inventory and handling at fuel febricetion feolityy (tor lidentified reactor) \\
\hline & (ret. Management and Dlaposittion of Excess Weapons Plutonium, \\
\hline & National Acadermy of Sciences, 1994, Tablo 3.1) \\
\hline C & 1) DOE attractiveness lovel of PUO2 feed material \\
\hline D & 2) DOE attractiveness level of MOX fuel pellets \\
\hline 42 & 3) average inventory of PuO2 feed material (kg Pu) \\
\hline 167 & 4) throughput of PuO2 toed matorial (kg Puy) \\
\hline 0.25 & 5) PuO2 teed material residence time or duration $(y)$ \\
\hline 4 & 6) number of manual movements of material (e.g. between gloveboxes) \\
\hline 0.8 & 7) average inventory of MOX fuel pellets (kg Pu) \\
\hline 167 & 8) throughput MOX fuel pellets (kg Pu/y) \\
\hline 0.005 & 9) MOX fuel pellet residence time or duration (y) \\
\hline 8 & 10) number of manual movements of MOX fuel pellets (e.g. between gloveboxes) \\
\hline 334 & 11) avorage inventory of MOX tuel assemblies (kg Pu) \\
\hline 167 & 12) throughput MOX fuel assemblies (kg Pu/y) \\
\hline 0.62 & 13) MOX fuel aseembly residence time or duration prior to fuel load(y) \\
\hline 8 & 14)"number of movements of MOX fuel assemblies (ASSUMES EX-REACTOR STORAQE) \\
\hline LANL & 15) staffing lovel of MOX fuel fabrication facility (number of persons) \\
\hline \multirow[t]{2}{*}{ LANL } & 16) effor at MOX fuel fabrication facility (person-hy) \\
\hline & IX. Environmental, Safoty, and Health \\
\hline $\mathbf{Y}$ & 1) requires Am removal (respond with " $y$ " or " $n$ ") \\
\hline LANL & 2) dose for fuel tab operations and intrasite transport (person-rem) \\
\hline LANL & 3) dose for reactor operations (person-rem) \\
\hline LANL & 4) Low level waste generated for fuel fab operations ( $\mathrm{m} / 3$ ) \\
\hline LANL & 5) low level waste generated for reactor operations during Pu disposition (m/3) \\
\hline \multirow[t]{2}{*}{ LANL } & 6) mixed waste generated for fuel fab operations (m3) \\
\hline & 7) mixed waste generated for reactor operations during Pu disposition (m/3) \\
\hline 348 & 8) spent fuel generated (no. of spent MOX fuel assemblles) \\
\hline LANL & 9) TRU waste generated, excluding packaging $(\mathrm{m} 3)$ \\
\hline LANL & 10) TRU waste generated, excluding packaging $(\mathrm{kg})$ \\
\hline \multirow[t]{2}{*}{4} & 11)" number of spent fuel movements to end of disposition operations (including last 10 years) \\
\hline & X. Other measures \\
\hline $\mathbf{Y}$ & 1) physical protection system meets standards in DOE Orders (respond with " $y$ " or " $n$ ") \\
\hline $\bar{Y}$ & 2) material control and accountability system moets standards in DOE Orders ( $\gamma^{\prime \prime}$ or " $\left.n "\right)$ \\
\hline $\mathbf{Y}$ & 3) is IAEA monitoring practical (i.e. nothing to prohibit monitoring) (" $y$ " or " $n$ ") \\
\hline 0 & 4) number of reactors of similar design under construction in the former republics of the USSR \\
\hline $\mathbf{0}$ & 5) number of reactors of similar design in operation in the former republics of the USSR \\
\hline & "EACH TRANSFEA IS COUNTED AS 2 FOR LOADING AND UNLOADING \\
\hline
\end{tabular}




\section{Appendix A: Uniform Descriptive Technical Data (Continued)}

\begin{tabular}{|c|c|}
\hline Full MoX & Reactor plant identification \\
\hline & (DATA REPORTED FOR GENERIC 3 REACTOR CASE) \\
\hline & 1. Plant retrofit and operation schedula (relative to $10 / 1 / 94$ project start) \\
\hline 4/1/01 & 1) time MOX buming operations start (mo) - FULL MOX BUNDLE LOADING \\
\hline 3484 & 2) thermal power (MWth) - SEE APPENDIX C FOR SPECIFIC PLANTS \\
\hline 1155 & 3) net electrical output (MWe) - SEE APPENDIX C FOR SPECIFIC PLANTS \\
\hline \multirow[t]{3}{*}{75} & 4) capacity factor (average for 1991, 1992, and 1993) - SEE DISCUSSION IN SECTION 4.4 \\
\hline & 5) remaining plant life $(y)$ - SEE TABLE 1.2-1 \\
\hline & II. Fresh MOX fuel assembly characteriatics \\
\hline 938.78 & 1) reactor Pu inventory - first core (kg Pu) - TRANSITIONS FROM URANIA CORE WITH EACH RELOAD \\
\hline 135796 & 2) reactor U inventory - first core $(\mathrm{kg} \mathrm{U})$ - TRANSITIONS FROM URANIA CORE WITH EACH RELOAD \\
\hline 5.334 & 3) Pu content (kg Pu/fuel assembly) \\
\hline 0 & 4) Pu-238 fraction (kg Pu-238/kg Pu) \\
\hline 0.94 & 5) Pu-239 fraction (kg Pu-239/kg Pu) \\
\hline 0.057 & 6) Pu-240 fraction ( $\mathrm{kg} \mathrm{Pu}-240 / \mathrm{kg} \mathrm{Pu})$ \\
\hline 0.003 & 7) Pu-241 fraction (kg Pu-241/kg Pu) \\
\hline 0 & 8) Pu-242 fraction (kg Pu-242/kg Pu) \\
\hline 303 & 9) fuel assembly mass $(\mathrm{kg})$ \\
\hline 4.55 & 10) fuel assembly length $(\mathrm{m})$ \\
\hline 0.15 & 11) fuel assembly width $(m)$ \\
\hline 173.27 & 12) U mass in MOX (kg U/fuel assembly) \\
\hline 0.0071 & 13) U-235 enrichment in MOX (kg U-235/kg U) - NATURAL URANIUM \\
\hline- & 14) U mass in UO2 fuel (kg U/fuel assembly) - (ALL MOX RODS) \\
\hline \multirow[t]{2}{*}{-} & 15) U-235 enrichment in UO2 fuel ( $\mathrm{kg} \mathrm{U}-235 / \mathrm{kg} \mathrm{U}$ ) - (ALL MOX RODS) \\
\hline & III. Equilibrium cycle MOX fuel recharge \\
\hline 938.78 & 1) Pu recharge (kg Pu) - PER REACTOR RELOAD \\
\hline 30496 & 2) U recharge (kg U) - PER REACTOA RELOAD \\
\hline 5.334 & 3) Pu content (kg Pu/fuel assembly) \\
\hline 0 & 4) Pu-238 fraction (kg Pu-238/kg Pu) \\
\hline 0.94 & 5) Pu-239 fraction (kg Pu-239/kg Pu) \\
\hline 0.057 & 6) Pu-240 fraction (kg Pu-240/kg Pu) \\
\hline 0.003 & 7) Pu-241 fraction (kg Pu-241/kg Pu) \\
\hline 0 & 8) Pu-242 fraction (kg Pu-242/kg Pu) \\
\hline 173.27 & 9) U mass in MOX (kg U/tuel assembly) \\
\hline 0.0071 & 10) U-235 enrichment in MOX (kg U-235/kg U) \\
\hline- & 11) U mass in UO2 fuel (kg U/fuel assembly) \\
\hline- & 12) U-235 enrichment in UO2 fuel ( $\mathrm{kg} \mathrm{U}-235 / \mathrm{kg} \mathrm{U})$ \\
\hline 176 & 13) number of MOX fuel assemblies \\
\hline \multirow[t]{2}{*}{11.17} & 14) cycle length (mo) - full power \\
\hline & IV. MOX fuel assembly characteristics at discharge \\
\hline 3.388 & 1) Pu nass discharged per cycle in MOX fuel (kg Pu) - PER BUNDLE IN EACH OF 176 BUNDLES \\
\hline- & 2) Pu mass discharged per cycle in UO2 fuel (kg Pu) \\
\hline 0.0099 & 3) Pu-238 fraction in MOX fuel (kg Pu-238/kg Pu) \\
\hline 0.4209 & 4) Pu-239 fraction in MOX fuel (kg Pu-239/kg Pu) \\
\hline 0.3528 & 5) Pu-240 fraction in MOX fuel ( $\mathrm{kg}$ Pu-240/kg Pu) \\
\hline 0.1508 & 6) Pu-241 fraction in MOX fuel (kg Pu-241/kg Pu) \\
\hline 0.0656 & 7) Pu-242 fraction in MOX fuel (kg Pu-242/kg Pu) \\
\hline
\end{tabular}




\section{Appendix A: Uniform Descriptive Technical Data (Continued)}

\begin{tabular}{|c|c|}
\hline- & 8) Pu-238 fraction in UO2 fuel ( $\mathrm{kg} \mathrm{Pu}-238 / \mathrm{kg} \mathrm{Pu}$ ) \\
\hline- & 9) Pu-239 fraction in UO2 fuel (kg Pu-239/kg Pu) \\
\hline- & 10) Pu-240 fraction in UO2 fuel (kg Pu-240/kg Pu) \\
\hline - & 11) Pu-241 fraction in UO2 fuel (kg Pu-241/kg Pu) \\
\hline- & 12) Pu-242 fraction in UO2 fuel (kg Pu-242/kg Pu) \\
\hline 261500 & 13) heat generation in one fuel assembly (W) \\
\hline $1.89 E+07$ & 14) gamma radiation field (rem/h at surface, midplane of fuel assembly) \\
\hline 1473 & 15) effective full power days (d) \\
\hline 37.609 & 16) bumup (MWd/kgHM) \\
\hline 0.01968 & 17) fraction of Pu in heavy metal (kg Pu/kgHM) \\
\hline & V. MOX fuel characteriatics 1 year after discharge \\
\hline 3.382 & 1) Pu mass in MOX fuel (kg Pu) - PER BUNDLE \\
\hline- & 2) Pu mass in $\mathrm{UO} 2$ fuel ( $\mathrm{kg} \mathrm{Pu}$ ) \\
\hline 0.0123 & 3) Pu-238 fraction in MOX fuel (kg Pu-238/kg Pu) \\
\hline 0.4245 & 4) Pu-239 fraction in MOX fuel (kg Pu-239/kg Pu) \\
\hline 0.3535 & 5) Pu-240 fraction in MOX fuel (kg Pu-240/kg Pu) \\
\hline 0.1439 & 6) Pu-241 fraction in MOX fuel (kg Pu-241/kg Pu) \\
\hline 0.0658 & 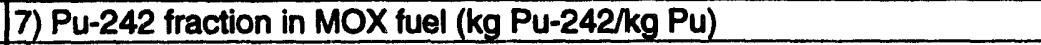 \\
\hline- & 8) Pu-238 fraction in UO2 fuel (kg Pu-238/kg Pu) \\
\hline- & 9) Pu-239 fraction in UO2 fuel (kg Pu-239/kg Pu) \\
\hline- & 10) Pu-240 fraction in UO2 fuel (kg Pu-240/kg Pu) \\
\hline - & 11) Pu-241 fraction in UO2 fuel (kg Pu-241/kg Pu) \\
\hline$\cdot$ & 12) Pu-242 fraction in UO2 fuel (kg Pu-242/kg Pu) \\
\hline 1898 & 13) heat generation in one fuel assembly (W) \\
\hline $1.30 E+05$ & 14) gamma radiation field (rem/h at surface, midplane of fuel assembly) \\
\hline 1473 & 15) effective full povier days (d) \\
\hline 37.609 & 16) burnup (MWd/kgHM) \\
\hline 0.019 .4 & 17) fraction of Pu in heavy metal ( $\mathrm{kg} \mathrm{Pu/kgHM}$ ) \\
\hline & VI. MOX fuel characteristics 10 years after discharge \\
\hline 3.213 & 1) Pu mass in MOX fuel (kg Pu) - PER BUNDLE \\
\hline- & 2) Pu mass in $\mathrm{OO} 2$ fuel ( $\mathrm{kg} \mathrm{Pu}$ ) \\
\hline 0.0128 & 3) Pu-238 fraction in MOX fuel (kg Pu-238/kg Pu) \\
\hline 0.4468 & 4) Pu-239 fraction in MOX fuel (kg Pu-239/kg Pu) \\
\hline 0.3729 & 5) Pu-240 fraction in MOX fuel ( $\mathrm{kg} \mathrm{Pu}-240 / \mathrm{kg} \mathrm{Pu}$ ) \\
\hline 0.0983 & 6) Pu-241 fraction in MOX fuel (kg Pu-24 $1 / \mathrm{kg} \mathrm{Pu})$ \\
\hline 0.0692 & 7) Pu-242 fraction in MOX fuel (kg Pu-242/kg Pu) \\
\hline- & 8) Pu-238 fraction in UO2 fuel (kg Pu-238/kg Pu) \\
\hline- & 9) Pu-239 fraction in UO2 fuel ( $\mathrm{kg} \mathrm{Pu}-239 / \mathrm{kg} \mathrm{Pu}$ ) \\
\hline- & 10) Pu-240 fraction in UO2 fuel (kg Pu-240/kg Pu) \\
\hline- & 11) Pu-241 fraction in UO2 fuel (kg Pu-241/kg Pu) \\
\hline- & 12) Pu-242 fraction in UO2 fuel (kg Pu-242/kg Pu) \\
\hline 241 & 13) heat generation in one fuel assembly (W) \\
\hline $1.78 E+04$ & 14) gamma radiation field (rem/h at surface, midplane of fuel assembly) \\
\hline 1473 & 15) effective full power days (d) \\
\hline 37.609 & 16) burnup (MWd/kgHM) \\
\hline 0.01866 & 17) fraction of Pu in heavy metal (kg Pu/kgHM) \\
\hline
\end{tabular}




\section{Appendix A: Uniform Descriptive Technical Data (Continued)}

\begin{tabular}{|c|c|}
\hline & VII. MOX fuel characteristics 100 years after diecharge \\
\hline 2.876 & 1) Pu mass in MOX fuel (kg Pu) - PER BUNDLE \\
\hline- & 2) Pu mass in $\mathrm{YO} 2$ fuel (kg Pu) \\
\hline 0.0074 & 3) Pu-238 fraction in MOX fuel (kg Pu-238/kg Pu) \\
\hline 0.4982 & 4) Pu-239 fraction in MOX fuel (kg Pu-239/kg Pu) \\
\hline 0.4156 & 5) Pu-240 fraction in MOX fuel (kg Pu-240/kg Pu) \\
\hline 0.0014 & 6) Pu-241 fraction in MOX fuel (kg Pu-241/kg Pu) \\
\hline 0.0774 & 7) $\mathrm{Pu}-242$ fraction in $\mathrm{MOX}$ fuel (kg Pu-242/kg Pu) \\
\hline- & 8) $\mathrm{Pu}-238$ fraction in UO2 fuel (kg Pu-238/kg Pu) \\
\hline- & 9) Pu-239 fraction in UO2 fuel (kg Pu-239/kg Pu) \\
\hline- & 10) Pu-240 fraction in UO2 fuel (kg Pu-240/kg Pu) \\
\hline- & 11) Pu-241 fraction in UO2 fuel (kg Pu-241/kg Pu) \\
\hline- & 12) Pu-242 fraction in UO2 fuel ( $(\mathrm{kg} \mathrm{Pu}-242 \mathrm{~kg} \mathrm{Pu}$ ) \\
\hline 94 & 13) heat generation in one fuel assembly $(W)$ \\
\hline $1.40 E+03$ & 14) gamma radiation field (rem/h at surface, midplane of fuel assembly) \\
\hline 1473 & 15) effective full power days (d) \\
\hline 37.609 & 16) bumup (MWd/kgHM) \\
\hline \multirow[t]{5}{*}{0.0167} & 17) fraction of Pu in heavy metal ( $\mathrm{kg} \mathrm{Pu} / \mathrm{kgHM}$ ) \\
\hline & \\
\hline & Vill. Pu inventory and handling at fuel fabrication facility (for identified reactor) \\
\hline & (ref. Management and Disposition of Excess Weapons Plutonium, \\
\hline & National Academy of Sciences, 1994, Table 3.1) \\
\hline$\frac{C}{D}$ & 1) DOE attractiveness level of PuO2 feed material \\
\hline$\frac{D}{360}$ & 2) DOE attractiveness level of MOX fuel pellets \\
\hline$\frac{360}{2124}$ & 3) average inventory of PuO2 feed material ( $\mathrm{kg} \mathrm{Pu}$ ) \\
\hline 2124 & 4) throughput of PuO2 feed material (kg Pu/y) \\
\hline 0.17 & 5) PuO2 feed material residence time or duration $(y)$ \\
\hline 1 & 6) number of manual movements of material (e.g. between gloveboxes) \\
\hline 10.7 & 7) average inventory of MOX fuel pellets ( $\mathrm{kg} \mathrm{Pu}$ ) \\
\hline 2124 & 8) throughput MOX fuel pellets (kg Pu/y) \\
\hline 0.005 & 9) MOX fuel pellet residence time or duration $(y)$ \\
\hline 0 & 10) number of manual movements of MOX fuel pellets (e.g. between gloveboxes) \\
\hline 4248 & 11) average inventory of MOX fuel assemblies (kg Pu) \\
\hline 2124 & 12) throughput MOX fuel assemblies (kg Pu/y) \\
\hline 0.62 & 13) MOX fuel assembly residence time or duration prior to fuel load(y) \\
\hline 8 & 14) "number of movements of MOX fuel assemblies (ASSUMES EX-REACTOR FRESH FUEL STORAGE \\
\hline 220 & 15) staffing level of MOX fuel fabrication facility (number of persons) \\
\hline \multirow[t]{3}{*}{$1.12 E+06$} & 16) effort at MOX fuel fabrication facility (person-h/y) \\
\hline & \\
\hline & IX. Environmental, Safety, and Health \\
\hline $\mathbf{Y}$ & 1) requires Am removal (respond with " $y$ " or " $n$ ") \\
\hline OPTIONAL & 1a) requires Am removal (respond with " $y$ " or " $n$ ") - COULD BE LEFT IN FUEL, SEE SECTION 8.2-4 \\
\hline 507 & 2) dose for fuel fab operations and intrasite transport (person-rem) \\
\hline 1311 & 2a) dose for fuel fab operations and intrasite transport without Am removal (person-rem) \\
\hline 31400 & 3) dose for reactor operations (person-rem) \\
\hline 110 & 3a) Additional dose for reactor operations with MOX (person-rem) \\
\hline 3384 & 4) low level waste generated for fuel fab operations $\left(\mathrm{m}^{\wedge} 3\right)$ \\
\hline 16905 & 5) low level waste generated for reactor operations during Pu disposition $\left(\mathrm{m}^{\wedge} 3\right)$ \\
\hline 47 & 6) mixed waste generated for fuel fab operations $\left(\mathrm{m}^{\wedge} 3\right)$ \\
\hline N/A & 7) mixed waste generated for reactor operations during Pu disposition $\left(\mathrm{m}^{\wedge} 3\right)$ \\
\hline
\end{tabular}




\section{Appendix A: Uniform Descriptive Technical Data (Continued)}

\begin{tabular}{|c|c|}
\hline 9328 & 8) spent fuel generated (no. of spent MOX fuel assemblies) \\
\hline 6251 & 9) TRU waste generated, excluding packaging $\left(m^{\wedge} 3\right)$ \\
\hline $6.25 E+06$ & 10) TRU waste generated, excluding packaging (kg) \\
\hline 4 & 11)"number of spent fuel movements to end of disposition operations (including last 10 years) \\
\hline & X. Other measures \\
\hline $\mathbf{Y}$ & 1) physical protection system meets standards in DOE Orders (respond with " $y^{n}$ or " $n$ ") \\
\hline $\mathbf{Y}$ & 2) material control and accountability system meets standards in DOE Orders (" $y$ " or " $n$ ") \\
\hline $\bar{Y}$ & 3) is IAEA monitoring practical (i.e. nothing to prohibit monitoring) (" $y$ " or " $n "$ ) \\
\hline 0 & 4) number of reactors of similar design under construction in the former republics of the USSR \\
\hline 0 & 5) number of reactors of similar design in operation in the former republics of the USSA \\
\hline
\end{tabular}




\section{Appendix B: Nuclear Methods And Qualification}

This section describes the methods and data used by GE to model the heterogeneous boiling water reactor core with standard urania fuel and mixed-oxide (plutonia-bearing) fuel. The methods can be categorized into four main sections: (1) the three-dimensional nuclear model, (2) lattice physics methods, (3) the thermal-hydraulic model, and (4) model qualification.

The section on the three-dimensional nuclear model discusses the PANACEA BWR core simulator code. PANACEA is a three-dimensional, coupled nuclear and thermal-hydraulic, time independent model of the BWR power distributions and thermal performance as a function of control rod position, fuel loading pattern, coolant flow, reactor pressure, and other operational and design variables. The program is capable of performing fuel cycle and thermal limits calculations. A special power and exposure iteration option is available for target exposure distributions and cycle length determinations.

The section on lattice physics methods discusses the TGBLA code. The report discusses the generation of few group neutron cross section by means of the neutron spectra calculations, the calculation of fuel rod power distributions for a fuel bundle, and the evaluation of the effects of fuel exposure. The output of TGBLA is used as input to PANACEA. The data flow between these codes in are shown in Figure B-1.

The section on the thermal-hydraulic model discusses the void-quality correlation, the bypass region calculation, the total core energy balance, and the thermal limits calculations. The thermal-hydraulic model is coupled to the neutronics model of PANACEA. Iterations are performed on power and flow until a consistent solution is obtained by PANACEA for this neutronic and thermal-hydraulic coupling.

Model qualification is presented last. Extensive operating plant tracking results are presented. Comparisons of calculations are presented for gamma scan measurements taken at the end of six operating fuel cycles. Results are also presented on comparisons of cold critical measurements taken at two plants over seven cycles of operation. This report presents the improvements made in the GE nuclear design methods. Additional information on these methods can be found in the previously reviewed and approved GE licensing topical reports. This report presents the improvements made in the GE nuclear design methods. Additional information on these methods can be found in the previously reviewed and approved GE licensing topical reports. 


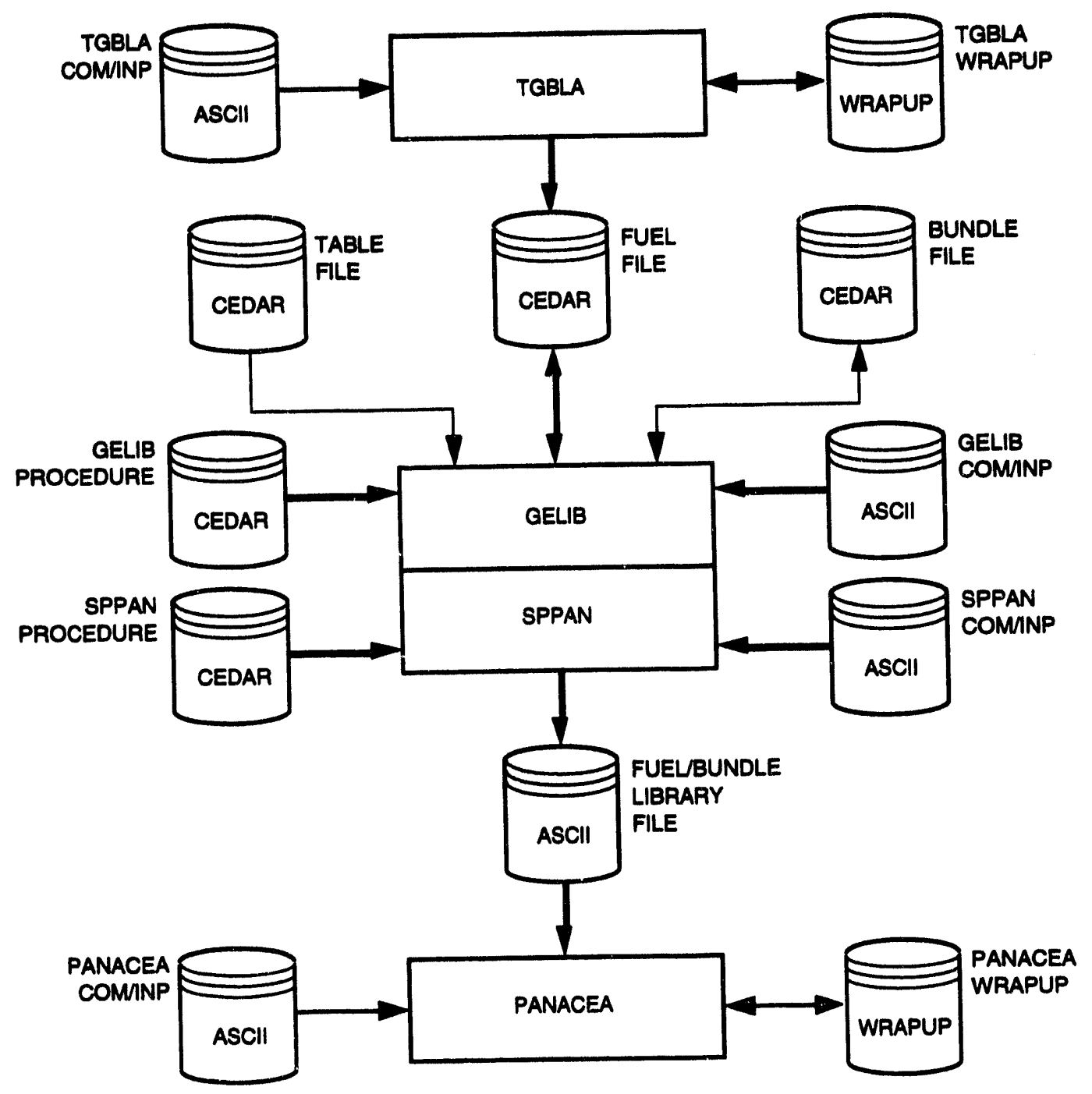

Figure B-1 Fuel/Core Design Methods Data Flow 


\section{B.1 Three-Dimensional Nuclear Model}

The nuclear model is based on coarse nodal, one-group, static diffusion theory. The mesh points are distributed approximately every two fast neutron mean free paths. Because of the short thermal neutron mean free path in a water reactor, most neutron diffusion takes place at high neutron energy. Therefore, the model is oriented toward accurate representation of fast neutron diffusion.

It is important to account for lattice fine structure in determining nuclear data for the coarsemesh global model. Separability is assumed for lattice cells consisting of one or more fuel bundles. The nuclear input preparation is performed by detailed calculations for the lattice cells or fuel bypass. Coarse-mesh diffusion parameters are fit parametrically as a function of moderator density, exposure, control, and moderator density history. (Moderator density history accounts for spatial isotopic concentrations which are affected by the neutron spectrum during burnup; the spectrum, in turn, is sensitive to moderator density.) These parametric calculations produce isotopic compositions and three-group cross-section data homogenized over the lattice cell. The methods and procedures used in the lattice model are described in Section B.2. A data reduction program (GELIB/SPPAN) processes these data into correlations and tables used in the BWR Simulator.

The nuclear model includes representation of Doppler broadening as a function of effective average fuel temperature. Xenon poisoning is considered with the spatial xenon concentration calculated for equilibrium conditions or input specified for non-equilibrium conditions. Provisions are also made to account for the reactivity effect of crud deposited on fuel rods.

Convergence of inner flux iterations is achieved through the use of successive over-relaxation. Outer void loops are converged with an under-relaxation technique. A power exposure iteration option is available. Neutron flux is converted to fission power for thermal-hydraulic coupling.

Spatial isotopic concentrations are calculated for fuel cycle analysis. Simulated in-core instrument readings can also be calculated.

\section{B.2 Detailed Lattice Physics Methods}

This section describes the process of accounting for the lattice fine structure in determining nuclear data. This data is then used as input into the three-dimensional coarse-mesh core calculations documented in Section 2.1. Due to the differences in bundle design, control state, void condition and accumulated exposure, bundle nuclear properties in a core are actually nonuniform both in transverse and axial directions. As a result, the neutron behavior in each 
axial segment of a bundle is determined not only by the nuclear properties of that segment but also by the nuclear properties of adjacent segments of that bundle and neighboring bundles. The calculation of the influence of neighboring bundles is very difficult because each bundle can potentially undergo a wide spectrum of environments, depending on its location and core operating history. However, this influence is generally small. To model the bundle, an approximation is made to account for the influence of neighboring bundles in the core simulator and to generate homogenized cross sections. This approximation is made by assuming that the bundle is located in an infinite, periodic lattice along the transverse directions and is uniform and infinitely long along the axial direction.

As a result of the preceding approximation, a bundle can be simulated as an "isolated" twodimensional heterogeneous system that may comprise fuel rods, water rods, burnable poison (gadolinia), in-channel water, channel, out-channel water, and control rod. The bundle modeling is then further divided into two stages: (1) the fuel rod cell and external region modeling by using transport theory methods, and (2) the coarse-mesh bundle modeling based on cell homogenization and diffusion theory methods.

The solution techniques begin with the generation of thermal broad-group neutron cross sections for all homogenized fuel rod cells and external regions in a bundle. In the thermal energy range, the rod-by-rod thermal spectra are calculated by a method similar to the THERMOS formalism. The major difference is that neutron leakage from rod to rod is taken into account.

The net leakage is determined by diffusion theory and is fed into the thermal spectrum calculation. Iterations between diffusion theory and thermal spectrum calculations are carried out to determine accurate, spatially dependent, thermal cross sections.

The second stop is to generate the cell and region-homogenized cross sections for the fast and epithermal broad groups. In the epithermal and fast energy range, the level-wise resonance integrals are calculated by an improved intermediate resonance approximation in which the intermediate resonance parameters are fuel-rod-temperature dependent. In addition, the fuel rod resonance escape probability is calculated according to a treatment by Mizuta.

The third step utilizes the cross sections generated in steps one and two in a two-dimensional, cross-mesh, broad-group, diffusion-theory calculation to determine the nodal flux and power distributioas in the bundle.

The fourth and final step is to perform the nuclide depletion calculation. In the depletion calculation, 100 nuclides are treated, including 25 fissile and fertile nuclides and up to 48 fission 
products plus one pseudo fission product. An improved burnup integration scheme is employed which avoids numerical problems associated with the equations encountered in poison bumup calculations.

The preceding steps are repeated for each burn step until all given burn steps are completed.

The generation of thermal cross sections and diffusion coefficients includes the condensation of thermal cross-section library from 30 groups to 16 groups, the calculation of leakage-dependent region-wise neutron spectrum, the generation of thermal leakage-dependent region-wise neutron spectrum, the generation of thermal homogenized diffusion parameters, and $P_{1}$ - blackness theory for strong absorption mediums.

The conventional library for thermal spectrum calculations is composed of 30-group thermal cross sections. A properly condensed 16-group, rather than a 30-group, thermal library, is sufficient for use to calculate the thermal spectrum correctly.

The fluxes for the group condensation of cross sections from 30 to 16 groups are calculated by using the THERMOS method for the averaged regular and the averaged gadolinia fuel rod cells. Each averaged cell is assumed to have two regions (fuel region and idding-moderator homogenized region) in the radial direction and to be infinitely long in the axial clirection.

The integral transport theory method has been widely utilized for calculating thermal neutron spectra in reactor lattices. The THERMOS computer code (based on this method) was introduced by Honeck in 1961 . The integral transport method has continued to be used along with two improvements: (1) the collision probability is calculated more accurately, and (2) the reflecting boundary condition is generalized to the albedo (or leakage-dependent) boundary condition.

\section{B.3 Thermal-Hydraulics Model}

Power-void feedback of a BWR core makes the thermal-hydraulic representation of considerable importance to the nuclear calculation. Nuclear parameters and, therefore, the power distribution are sensitive to local steam void content.

Formulas from the 1967 ASME Steam Tables are used to evaluate coolant properties as a function of reactor core pressure and inlet enthalpy. All thermal-hydraulic variables are assumed to vary linearly between nodes, where the nodal representation is the same as that for the neutron flux; therefore, the thermal-hydraulic model is consistent with the difference equations used for solution of the nuclear model. 
The flow distribution for characteristic parallel channels is determined by balancing core pressure drop. Characteristic channels are determined by combinations of total channel power, axial power shape, inlet orifice design, channel geometry (e.g., number of fuel rods, spacer design, lower and upper tie plate design), and buildup of crud on the fuel rods. The flow into individual channels is obtained by interpolation among characteristic channels of the same geometry aid orifice type as a function of radial and axial power factors and crud thickness.

The axidl enthalpy and quality distribution is determined for each channel by a nodal energy balance which considers fuel rod heat flux to the coolant, neutron moderation and gamma heating in the coolant and in the flow channel wall, heat transfer through the channel wall to the bypass region, and $\gamma,(n, \gamma)$, and $(n, \alpha)$ heating in control blades. The void distribution is given by a void-quality correlation which provides a physically oriented representation of sub-cooled and bulk boiling through a few empirical constants which describes the local flow structure.

Flow-power-void iteration is required to reach an equilibrium condition. Once this is obtained, the thermal-hydraulics model enables evaluation of the margin relative to thermal limits.

\section{B.4 Model Qualification}

The models in this report have been qualified against data obtained from operating Boiling Water Reactors. The qualification studies consist of: (1) simulation and tracking of nine operating cycles on three plants; (2) three-dimensional global power distribution comparisons with gamma scan measurements taken at the end of six cycles; and (3) cold critical measurements taken during seven cycles and two plants; and (4) burnup verification data at the end of one cycle.

In plant tracking calculations, the 3-D simulator (using cross sections generated from the lattice model) is used to simulate the behavior of a plant during operation. The reactor power, flow and pressure are input to the simulator, which then calculates the core $k_{\text {eff }}$ and power distribution as a function of cycle exposure. The operating reactor is critical; hence, the calculated $\mathbf{k}_{\text {eff }}$ is compared to 1.0. Accurate and technically well-founded simulators should calculate a $\mathbf{k}_{\text {eff }}$ close to 1.0 and the difference between $k_{\text {eff }}$ and 1.0 should not vary appreciably from plant to plant or as a function of fuel exposure. Consistency of $k_{e f f}$ bias ensures that accurate cycle length estimates will be obtained in future core designs. Tracking calculations for Hatch 1 (Cycles 1 through 3), Quad Cities 1 (Cycles 1 through 5), and Millstone 1 (Cycle 7) were performed. The overall average is 1.002 with an RMS of 0.002 , which is a quite narrow band considering the data are compiled over three plants and over nine cycles. 


\section{Appendix C: Key BWR Operational Limits Definitions}

\section{Linear Heat Generation Rate}

The Linear Heat Generation Rate (LHGR) is a measure of the heat generation rate of a fuel rod in a fuel assembly at an axial location. Limits on LHGR are specified to assure that fuel design limits will not be exceeded anywhere in the core during normal operation including anticipated operational occurrences.

Fuel design evaluations have been performed and demonstrate that the $1 \%$ plastic strain fuel design limit is not exceeded during continuous operation with LHGRs up to the uperating limit. The analysis also includes allowances for short term transient operation above the operating limit to account for anticipated operational occurrences, plus an allowance for densification power spiking.

\section{Average Planar Linear Heat Generation Rate}

The Average Planar Linear Heat Generation Rate (APHGR) is a measure of the average linear heat generation rate of all the fuel rods in a fuel assembly at an axial location. Limits on APLHGR are specified to assure that the fuel design limits will not be exceeded during anticipated operational occurrences and that the peak cladding temperature (PCT) during the postulated design basis loss of coolant accident (LOCA) will not exceed the limits specified in 10 CFR50.46.

Cycle design calculations often refer to the ratio of the highest value of APLHGR in the core (MAPLHGR, maximum APLHGR) divided by the APLHGR limit. This ratio is called MAPRAT or RAPLHGR, and must always remain bolow 1.0 throughout the cycle or the APLHGR limit will be exceeded.

\section{Minimum Critical Power Ratio}

The Minimum Critical Power Ratio (MCPR) is a measure of the operating fuel assembly power relative to the fuel assembly power that would result in the onset of boiling transition. The Safety Limit MCPR (SLMCPR) is set such that $99.9 \%$ of the fuel rods will avoid boiling transition if the limit is not violated. The operating limit MCPR (OLMCPR) is established to assure that no fuel damage results during anticipated operational occurrences. Although fuel damage would not necessarily occur if a fuel rod actual experienced boiling transition, the 
critical power at which boiling transition is calculated to occur has been adopted as a fuel design criterion.

The onset of transition boiling is a phenomena that is readily detected during the testing of various bundle designs. Based on this experimental data, correlations have been developed that are used to predict critical bundle power (i.e., the bundle power level at the onset of transition boiling) for a given set of plant parameters (e.g., pressure, mass flux, subcooling, etc.). Since plant operating conditions and bundle power levels are relatively easily monitored and determined, monitoring MCPR is a convenient way of ensuring that fuel failures due to inadequate cooling do not occur. Cycle design calculations often refer to the ratio of the OLMCPR divided by the smallest CPR calculated for the whole core. This ratio is called CPRRAT and must always remain below 1.0 throughout the cycle or the operating limit CPR will be exceeded. 


\section{Appendix D: Existing BWR Characteristics}

\section{BIG ROCK POINT NUCLEAR PLANT}

Locatlon: Chälevoix County, Michigan

4 miles NE of Charlevoix

latitude $45.3592 \mathrm{deg} \mathrm{N}$; longitude $85.1947 \mathrm{deg}$ W

Llcenses: Consumers Power Co.

Unit Information

Unit 1

Docket Number

$50-155$

Construction Permit

Operating License

Commercial Operation

License Expiration

Licensed Thermal Power [MW(t)]

Design Electrical Rating [net MW(e)]

Type of Reactor

Nuclear Steam Supply System Vendor

Cooling Water System

Type:

Source:

Source Temperature Range:

Condenser Flow Rate:

Design Condenser Temperature Rise:

Intake Structure:

Discharge Structure:

once through

Lake Michigan

38-68 ${ }^{\circ} \mathrm{F}$

49,000 gallons/minute

$20^{\circ} \mathrm{F}$

underwater crib

open discharge canal

Site Information

Total Area:

Exclusion Distance:

Low Population Zone:

Nearest City:

Site Topography:

Surrounding Area Topography:

Land Use within 5 miles:

Nearby Features:

Area of Transmission Line Corridor: Population within a 50-mile radius:

$\begin{array}{ccccc}1990 & 2000 & 2010 & 2030 & 2050 \\ 200,000 & 210,000 & 210,000 & 230,000 & 240,000\end{array}$

600 acres

0.51 mile

2.50 miles

Sault Ste. Marie, Canada;

1980 population: 81,048

gently sloping

gently sloping

commercial and industrial

The nearest town is Charlevoix 4 miles SW.

The C\&O Railroad is about 1 mile SE.

Lake Charlevoix is $\mathbf{3}$ miles $\mathrm{S}$.

D-1 


\section{BROWNS FERRY NUCLEAR POWER STATION}

Location: Limestone Country, Alabama

10 miles NW of Decatur

latitude $34.7042 \operatorname{deg} \mathrm{N}$; longitude $87.1186 \mathrm{deg}$ W

Licensee: Tennessee Valley Authority

Unit Information

Docket Number

Construction Permit

Operating License

Commercial Operation

License Expiration

Licensed Thermal Power [MW(t)]

Design Electrical Rating [net MW(e)]

Type of Reactor

Nuclear Steam Supply System Vendor

\section{Cooling Water System}

Type:

Source:

Source Temperature Range:

Condenser Flow Rate:

Design Condenser Temperature Rise:

Intake Structure:

Discharge Structure:

\section{Site Information}

Total Area:

Exclusion Distance:

Low Population Zone:

Nearest City:

Site Topography:

Surrounding Area Topography:

Land Use within 5 miles:

Nearby Features:

Area of Transmission Line Corridor: Population within a 50-mile radius:

$\begin{array}{rrr}\text { Unit 1 } & \text { Unit 2 } & \text { Unit 3 } \\ 50-259 & 50-260 & 50-296 \\ 1967 & 1967 & 1968 \\ 1973 & 1974 & 1976 \\ 1974 & 1975 & 1977 \\ 2013 & 2014 & 2016 \\ 3293 & 3293 & 3293 \\ 1065 & 1065 & 1065 \\ \text { BWR } & \text { BWR } & \text { BWR } \\ \text { GE } & \text { GE } & \text { GE }\end{array}$

once through and helper towers

Tennessee River

$40-90^{\circ} \mathrm{F}$

630,000 gallons/minute each unit

$25^{\circ} \mathrm{F}$

concrete structure in small inlet

diffuser pipes

840 acres

0.76 mile radius

7.00 miles

Huntsville;

1980 population: 142,513

flat

flat to rolling

agricultural

The nearest town is Lawngate 1 mile NE.

The Redstone Arsenal is 25 miles E.

The Southern Railroad is 6 miles S.

The Louisville and Nashville Railroad is 6 miles E. 1350 acres

$\begin{array}{ccccc}1990 & 2000 & 2010 & 2030 & 2050 \\ 760,000 & 810,000 & 850,000 & 930,000 & 1,010,000\end{array}$




\section{BRUNSWICK STEAM ELECTRIC PLANT}

Location: Brunswick Country, North Carolina 16 miles $S$ of Wilmington latitude $33.9583 \mathrm{deg} \mathrm{N}$; longitude $78.0106 \mathrm{deg} \mathrm{W}$

Licensee: $\quad$ Carolina Power and Light Co.

Unit Information

Docket Number

Construction Permit

Operating License

Commercial Operation

License Expiration

Licensed Thermal Power [MW(t)]

Design Electrical Rating [net MW(e)]

Type of Reactor

Nuclear Steam Supply System Vendor

$\begin{array}{rr}\text { Unit 1 } & \text { Unit 2 } \\ 50-325 & 50-324 \\ 1967 & 1968 \\ 1976 & 1974 \\ 1977 & 1975 \\ 2016 & 2014 \\ 2436 & 2436 \\ 821 & 821 \\ \text { BWR } & \text { BWR } \\ \text { GE } & \text { GE }\end{array}$

\section{Cooling Water System}

Type:

Source:

Source Temperature Range:

Condenser Flow Rate:

Design Condenser Temperature Rise:

Intake Structure:

Discharge Structure:

\section{Site Information}

Total Area:

Exclusion Distance:

Low Population Zone:

Nearest City:

Site Topography:

Surrounding Area Topography:

Land Use within 5 miles:

Nearby Features:

Area of Transmission Line Corridor: Population within a 50-mile radius: once through

Cape Fear River

$40-86^{\circ} \mathrm{F}$

675,000 gallons/minute each unit

$17^{\circ} \mathrm{F}$

3-mile canal from Cape Fear River

6-mile canal to Atlantic Ocean
1200 acres

0.57 mile

2.00 miles

Wilmington;

1980 population: 44,000

flat

flat

less than one-half agricultural, remainder swamps or wooded The nearest town is Southport 3 miles S.

Sunny Point Military Ocean Terminal is about 5 miles N. 3500 acres

$\begin{array}{ccccc}1990 & 2000 & 2010 & 2030 & 2050 \\ 230,000 & 250,000 & 270,000 & 300,000 & 340,000\end{array}$




\section{COOPER NUCLEAR STATION}

Location: Nemaha County, Nebraska 23 miles S of Nebraska City latitude $40.3619 \mathrm{deg} \mathrm{N}$; longitude $95.6411 \mathrm{deg}$ W

Licensee: Nebraska Public Power District

Unit Information

Docket Number

Construction Permit

Operating License

Coumercial Operation

License Expiration

Licensed Thermal Power [MW(t)]

Design Electrical Rating [net MW(e)]

Type of Reactor

Nuclear Steam Supply System Vendor

\section{Unit 1}

50-298

1968

1974

1974

2014

2381

778

BWR

GE

\section{Cooling Water System}

Type:

Source:

Source Temperature Range:

Condenser Flow Rate:

Design Condenser Temperature Rise:

Intake Structure:

Discharge Structure:

\section{Site Information}

Total Area:

Exclusion Distance:

Low Population Zone:

Nearest City:

Site Topography:

Surrounding Area Topography:

Land Use within 5 miles:

Nearby Features:

Area of Transmission Line Corridor: Population within a 50-mile radius: once through

Missouri River

$34-73^{\circ} \mathrm{F}$

631,000 gallons/minute

$18^{\circ} \mathrm{F}$

at shoreline

at shoreline

1090 acres

0.68 mile

1.00 mile radius

Lincoln;

1980 population: 171,932

flat

flat

agricultural

The nearest town is Nemaha about 1 mile S.

A railroad runs just $W$ of the site.

Indian Cave State Park is about 8 miles SSE.

6862 acres

$\begin{array}{lllll}1990 & 2000 & 2010 & 2030 & 2050\end{array}$

$\begin{array}{lllll}180,000 & 190,000 & 200,000 & 220,000 & 330,000\end{array}$ 
NEDO-32361

\section{CLINTON POWER STATION}

Location: Do Witt County, Illinois

6 miles $\mathrm{E}$ of Clinton

latitude 40.1731 deg N; longitude 88.8342 deg W

Licensee: Illinois Power Co.

Unit Information

Docket Number

Construction Permit

Operating License

Commercial Operation

License Expiration

Licensed Thermal Power [MW(t)]

Design Electrical Rating [net MW(e)]

Type of Reactor

Nuclear Steam Supply System Vendor

\section{Cooling Water System}

Type:

Source:

Source Temperature Range:

Condenser Flow Rate:

Design Condenser Temperature Rise:

Intake Structure:

Discharge Structure:

\section{Site Information}

Total Area:

Exclusion Distance:

Low Population Zone:

Nearest City:

Site Topography:

Surrounding Area Topography:

Land Use within 5 miles:

Nearby Features:

Area of Transmission Line Corridor:

Population within a 50-mile radius:

\section{Unit 1}

$50-461$

1976

1987

1987

2027

2894

933

BWR

GE

once through

Salt Creek

$32-83^{\circ} \mathrm{F}$

568,701 gallons/minute

$23^{\circ} \mathrm{F}$

concrete structure at shoreline of North Fork Salt Creek

3-mile flume discharging to Salt Creek

14,090 acres

0.60 mile radius

2.50 mile radius

Decatur;

1980 population: 93,939

flat

flat

agricultural

The nearest town is De Witt 2 miles ENE.

Weldon Springs State Park is 6 miles SW.

The Illinois Central Gulf Railroad crosses the site.

U.S. Highway I-74 is 11 miles NE.

A dam on Salt Creek near the site creates the reservoir for the cooling water system.

906 acres

$\begin{array}{ccccc}1990 & 2000 & 2010 & 2030 & 2050 \\ 730,000 & 770,000 & 790,000 & 830,000 & 870,000\end{array}$




\section{DUANE ARNOLD ENERGY CENTER}

Location: Linn County, Iowa

8 miles NW of Cedar Rapids

latitude $42.1006 \mathrm{deg} \mathrm{N}$; longitude $91.7772 \mathrm{deg}$ W

Licensee: Iowa Electric Light and Power Co.

Unit Information

Docket Number

Construction Permit

Operating License

Commercial Operation

License Expiration

Licensed Thermal Power [MW(t)]

Design Electrical Rating [net MW(e)]

Type of Reactor

Nuclear Steam Supply System Vendor
Unit 1

$50-331$

1970

1974

1975

2014

1658

538

BWR

GE

\section{Cooling Water System}

Type:

Source:

Source Temperature Range:

Condenser Flow Rate:

Design Condenser Temperature Rise:

Intake Structure:

Discharge Structure:

\section{Site Information}

Total Area:

Exclusion Distance:

Low Population Zone:

Nearest City:

Site Topography:

Surrounding Area Topography:

Land Use within 5 miles:

Nearby Features:

Area of Transmission Line Corridor: Population within a 50-mile radius: mechanical draft cooling towers

Cedar River

32-89 ${ }^{\circ} \mathrm{F}$

290,000 gallons/minute

$25^{\circ} \mathrm{F}$

structure on river shoreline

canal to shoreline

500 acres

0.27 mile

6.00 miles

Cedar Rapids;

1980 population: 110,243

flat

rolling and hilly

agricultural

The nearest town is Palo about 2 miles SW.

Several wildlife refuge areas are within 10 miles of the site.

1160 acres

$\begin{array}{lllll}1990 & 2000 & 2010 & 2030 & 2050\end{array}$

$\begin{array}{lllll}620,000 & 660,000 & 690,000 & 750,000 & 820,000\end{array}$ 
NEDO-32361

\section{DRESDEN NUCLEAR POWER STATION}

Location: Grundy County, Illinois

9 miles $\mathrm{E}$ of Morris

latitude $41.3897 \mathrm{deg} \mathrm{N}$; longitude 88.2711 deg W

Licensee: Commonwealth Edison Co.

Unit Information

Docket Number

Construction Permit

Operating License

Commercial Operation

License Expiration

Licensed Thermal Power [MW( $t)]$

Design Electrical Rating [net MW(e)]

Type of Reactor

Nuclear Steam Supply System Vendor

\section{Cooling Water System}

Type:

Source:

Source Temperature Range:

Condenser Flow Rate:

Design Condenser Temperature Rise:

Intake Structure:

Discharge Structure:

\section{Site Information}

Total Area:

Exclusion Distance:

Low Population Zone:

Nearest City:

Site Topography:

Surrounding Area Topography:

Land Use within 5 miles:

Nearby Features:

Area of Transmission Line Corridor:

Population within a 50-mile radius:

$\begin{array}{rr}\text { Unit 1 } & \text { Unit 2 } \\ 50-237 & 50-249 \\ 1966 & 1966 \\ 1969 & 1971 \\ 1970 & 1971 \\ 2010 & 2011 \\ 2527 & 2527 \\ 794 & 794 \\ \text { BWR } & \text { BWR } \\ \text { GE } & \text { GE }\end{array}$

cooling lake and spray canal

Kankakee River

$40-85^{\circ} \mathrm{F}$

471,000 gallons/minute each unit

canal from Kankakee River to a crib house

A canal carries water to a cooling lake of about 1275 acres with a hold-up time of about 3 days. The water then divides, some going to the Illinois River and some returns to the plant. Spray modules are floated in the canals.

953 acres plus 1275 acre cooling lake

0.50 mile radius

4.97 miles

Joliet; 1980 population: 77,956

flat

rolling prarie

agriculture

The nearest town is Channahon 3 miles NNE.

The General Electric Nuclear Power Plant Training

Center is $\mathrm{S}$ of the site.

A large abandoned strip mine is located in the area.

Braidwood Station nuclear plant is about 10 miles $\mathrm{S}$

La Salle County Station nuclear plant is about 27 miles

SW.

An array ammunition plant is about 7 miles $\mathrm{E}$.

2250 acres

$\begin{array}{ccccc}1990 & 2000 & 2010 & 2030 & 2050 \\ 6,820,000 & 7,050,000 & 7,200,000 & 7,450,000 & 7,710,000\end{array}$


NEDO-32361

\section{ENRICO FERMI ATOMIC POWER PLANT}

Location: Monroe County, Michigan

30 miles SW of Detroit

latitude $41.9631 \mathrm{deg} \mathrm{N}$; longitude $83.2578 \mathrm{deg} \mathrm{W}$

Licensee: Detroit Edison Co.

Unit Information

Docket Number

Construction Permit

Operating License

Commercial Operation

License Expiration

Licensed Thermal Power [MW(t)]

Design Electrical Rating [net MW(e)]

Type of Reactor

Nuclear Steam Supply System Vendor
Unit 1

$50-341$

1972

1985

1988

2025

3292

1093

BWR

GE

\section{Cooling Water System}

Type:

Source:

Source Temperature Range:

Condenser Flow Rate:

Design Condenser Temperature Rise:

Intake Structure:

Discharge Structure:

Site Information

Total Area:

Exclusion Distance:

Low Population Zone:

Nearest City:

Site Topography:

Surrounding Area Topography:

Land Use within 5 miles:

Nearby Features:

Area of Transmission Line Corridor: Population within a 50-mile radius: natural draft cooling towers

Lake Erie

34-76 ${ }^{\circ} \mathrm{F}$

836,700 gallons/minute

$18^{\circ} \mathrm{F}$

at edge of lake

to the lake via a 50-acre pond

1120 acres

0.57 mile

3.00 miles

Detroit;

1980 population: $1,203,368$

flat

flat to rolling

mostly agricultural

The town of Stony Point is adjacent to the site to the S.

Sterling State Park and General Custer Historical Site are about 5 miles $\mathrm{SW}$.

180 acres

$\begin{array}{ccccc}1990 & 2000 & 2010 & 2030 & 2050 \\ 5,370,000 & 5,630,000 & 5,840,000 & 6,230,000 & 6,650,000\end{array}$


NEDO-32361

\section{JAMES A FITZPATRICK NUCLEAR POWER PLANT}

Location: Oswago County, New York 6 miles NE of Oswago

latitude $43.5239 \mathrm{deg} \mathrm{N}$; longitude $76.3983 \mathrm{deg} \mathrm{W}$

Licensee: Power Authority of the State of New York

\section{Unit Information}

Docket Number

Construction Permit

Operating License

Commercial Operation

License Expiration

Licensed Thermal Power [MW(t)]

Design Electrical Rating [net MW(e)]

Type of Reactor

Nuclear Steam Supply System Vendor
Unit 1

50-333

1970

1974

1975

2014

2436

816

BWR

GE

\section{Cooling Water System}

Type:

Source:

Source Temperature Range:

Condenser Flow Rate:

Design Condenser Temperature Rise:

Intake Structure:

Discharge Structure:

\section{Site Information}

Total Area:

Exclusion Distance:

Low Population Zone:

Nearest City:

Site Topography:

Surrounding Area Topography:

Land Use within 5 miles:

Nearby Features:

Area of Transmission Line Corridor:

Population within a 50-mile radius: once through

Lake Ontario

$37-67^{\circ} \mathrm{F}$

352,600 gallons/minute

$32^{\circ} \mathrm{F}$

intake from the lake

discharge to the lake

702 acres

0.57 mile

3.40 miles

Syracuse;

1980 population: 170,105

flat to rolling

rolling

agricultural, industrial, residential, and recreational

The nearest town is Lakeview about 1 mile WSW.

Fort Ontario is about 5 miles SW.

Nine Mile Point Nuclear Station is about 5.0 mile W.

1000 acres

$\begin{array}{lllll}1990 & 2000 & 2010 & 2030 & 2050\end{array}$

$820,000 \quad 810,000 \quad 800,000 \quad 800,000 \quad 810,000$ 
NEDO-32361

\section{GRAND GULF NUCLEAR STATION}

Location: Claiborne County, Mississippi 25 miles $S$ of Vicksburg latitude $32.0075 \mathrm{deg} \mathrm{N}$; longitude $91.0475 \mathrm{deg} \mathrm{W}$

Licensee: System Energy Resources, Inc.

\section{Unit Information}

Docket Number

Construction Permit

Operating License

Commercial Operation

License Expiration

Licensed Thermal Power [MW(t)]

Design Electrical Rating [net MW(e)]

Type of Reactor

Nuclear Steam Supply System Vendor
Unit 1

$50-416$

1974

1984

1985

2024

3833

1250

BWR

GE

\section{Cooling Water System}

Type:

Source:

Source Temperature Range:

Condenser Flow Rate:

Design Condenser Temperature Rise:

Intake Structure:

Discharge Structure:

\section{Site Information}

Total Area:

Exclusion Distance:

Low Population Zone:

Nearest City:

Site Topography:

Surrounding Area Topography:

Land Use within 5 miles:

Nearby Features:

Area of Transmission Line Corridor:

Population within a 50-mile radius: natural draft cooling towers

Mississippi River

$32-82^{\circ} \mathrm{F}$

572,000 gallons/minute

$30^{\circ} \mathrm{F}$

a series of radial-collector wells along the shoreline discharge to river via a barge slip

2100 acres

0.43 mile radius

2.00 miles

Jackson;

1980 population: 202,895

flat to rolling

flat and rolling

wooded and recreational

The nearest town is Grand Gulf 2 miles N.

The Natchez Trace Parkway is about 6 miles SE.

The Grand Gulf Military Park is just $N$ of the site.

There are numerous hunting lodges near the site.

2300 acres

$\begin{array}{lllll}1990 & 2000 & 2010 & 2030 & 2050\end{array}$

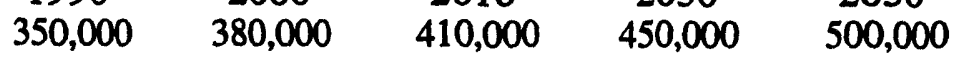




\section{EDWIN I. HATCH NUCLEAR PLANT}

Location: Appling County Georgia 11 miles $\mathbf{N}$ of Baxley latitude $31.9342 \mathrm{deg} \mathrm{N}$; longitude $82.3444 \mathrm{deg} \mathrm{W}$

Licensee: Georgia Power Co.

Unit Information

Docket Number

Construction Permit

Operating License

Commercial Operation

License Expiration

Licensed Thermal Power [MW(t)]

Design Electrical Rating [net MW(e)]

Type of Reactor

Nuclear Steam Supply System Vendor
Unit 1 Unit 2

$\begin{array}{rr}50-321 & 50-366 \\ 1969 & 1972 \\ 1974 & 1978 \\ 1975 & 1979 \\ 2014 & 2018 \\ 2436 & 2436 \\ 776 & 784 \\ \text { BWR } & \text { BWR } \\ \text { GE } & \text { GE }\end{array}$

\section{Cooling Water System}

Type:

Source:

Source Temperature Range:

Condenser Flow Rate:

Design Condenser Temperature Rise:

Intake Structure:

Discharge Structure:

Site Information

Total Area:

Exclusion Distance:

Low Population Zone:

Nearest City:

Site Topography:

Surrounding Area Topography:

Land Use within 5 miles:

Nearby Features:

Area of Transmission Line Corridor:

Population within a 50-mile radius: mechanical draft cooling towers

Altamaha River

43- $90^{\circ} \mathrm{F}$

556,000 gallons/minute each unit

$20^{\circ} \mathrm{F}$

at edge of river

120 feet from shore

2244 acres

0.78 mile

0.78 miles

Savannah;

1980 population: 141,654

flat to rolling

flat to rolling

mostly wooded

The nearest town is Cedar Crossing about 7 miles NNW.

U.S. Highway 1 is just west of the site.

4691 acres

$\begin{array}{lllll}1990 & 2000 & 2010 & 2030 & 2050\end{array}$

$\begin{array}{lllll}330,000 & 360,000 & 380,000 & 420,000 & 460,000\end{array}$ 


\section{HOPE CREEK GENERATING STATION}

Location: Salem County, New Jersey

8 miles SW of Salem

latitude $39.4678 \mathrm{deg} \mathrm{N}$; longitude $75.5381 \mathrm{deg} \mathrm{W}$

Licensee: Public Service Electric and Gas Co.

Unit Information

Docket Number

Construction Permit

Operating License

Commercial Operation

License Expiration

Licensed Thermal Power [MW( $t)]$

Design Electrical Rating [net MW(e)]

Type of Reactor

Nuclear Steam Supply System Vendor
Unit 1

$50-354$

1974

1986

1986

2026

3293

1067

BWR

GE

\section{Cooling Water System}

Type:

Source:

Source Temperature Range:

Condenser Flow Rate:

Design Condenser Temperature Rise:

Intake Structure:

Discharge Structure:

\section{Site Information}

Total Area:

Exclusion Distance:

Low Population Zone:

Nearest City:

Site Topography:

Surrounding Area Topography:

Land Use within 5 miles:

Nearby Features:

Area of Transmission Line Corridor: Population within a 50-mile radius: natural draft cooling tower

Delaware River

$34-81^{\circ} \mathrm{F}$

552,000 gallons/minute

$28^{\circ} \mathrm{F}$

at edge of river

pipe 10 feet offshore

740 acres

0.56 mile radius

5.00 miles radius

Wilmington, Delaware;

1980 population: 70,195

flat

flat

tidal marshes and grasslands

The nearest town is Port Penn about 4 miles NW in

Delaware.

The nearest railroad is 8 miles NE.

The plant is on the same site as the Salem Nuclear

Generating Station.

$\begin{array}{ccccc}\begin{array}{c}912 \text { acres } \\ 1990\end{array} & 2000 & 2010 & 2030 & 2050 \\ 4,850,000 & 4,960,000 & 5,050,000 & 5,230,000 & 5,420,000\end{array}$


NEDO-32361

\section{LA SALLE COUNTY STATION}

Location: La Salle County, Illinois 11 miles SE of Ottawa latitude $41.2439 \mathrm{deg} \mathrm{N}$; longitude $88.6708 \mathrm{deg}$ W

Licensee: Commonwealth Edison Co.

Unit Information

Docket Number

Construction Permit

Operating License

Commercial Operation

License Expiration

Licensed Thermal Power [MW( $t)]$

Design Electrical Rating [net MW(e)]

Type of Reactor

Nuclear Steam Supply System Vendor
Unit 1 Unit 2

$50-373$

1973

1982

1984

2022

3323

1078

BWR

GE
$50-374$

1973

1984

1984

2024

3323

1078

BWR

GE

\section{Cooling Water System}

Type:

Source:

Source Temperature Range:

Condenser Flow Rate:

Design Condenser Temperature Rise:

Intake Structure:

Discharge Structure:

Site Information

Total Area:

Exclusion Distance:

Low Population Zone:

Nearest City:

Site Topography:

Surrounding Area Topography:

Land Use within 5 miles:

Nearby Features:

Area of Transmission Line Corridor:

Population within a 50-mile radius: cooling pond

Illinois River

$47-85^{\circ} \mathrm{F}$

645,000 gallons/minute

$24^{\circ} \mathrm{F}$

intake from 2058 acre cooling pond, makeup from river discharge to cooling pond

3060 acres

0.32 mile

3.98 mile

Joliet;

1980 population: 77,956

flat

flat with hills along river

agricultural

The nearest town is Seneca about 5 miles NNE.

Braidwood Station (nuclear plant) is about 20 miles ENE.

Dresden Nuclear Power Station is about 22 miles NE.

2278 acres

$\begin{array}{ccccc}1990 & 2000 & 2010 & 2030 & 2050 \\ 1,160,000 & 1,220,000 & 1,260,000 & 1,310,000 & 1,370,000\end{array}$




\section{LMMERICK GENERATING STATION}

Location: Montgomery County, Pennsylvania

21 miles NW of Philadelphia latitude $40.2200 \operatorname{deg} \mathrm{N}$; longitude $75.5900 \mathrm{deg} \mathrm{W}$

Licensee: Philadelphia Electric Co.

Unit Information

Docket Number

Construction Permit

Operating License

Commercial Operation

License Expiration

Licensed Thermal Power [MW(t)]

Design Electrical Rating [net MW(e)]

Type of Reactor

Nuclear Steam Supply System Vendor
Unit 1 Unit 2

50-352 50-353

$1974 \quad 1974$

$1985 \quad 1990$

$1986 \quad 1990$

$2025 \quad 2020$

$3293 \quad 3293$

$1055 \quad 1055$

BWR BWR

GE GE

\section{Cooling Water System}

Type:

Source:

Source Temperature Range:

Condenser Flow Rate:

Design Condenser Temperature Rise:

Intake Structure:

Discharge Structure:

\section{Site Information}

Total Area:

Exclusion Distance:

Low Population Zone:

Nearest City:

Site Topography:

Surrounding Area Topography:

Land Use within 5 miles:

Nearby Features:

Area of Transmission Line Corridor:

Population within a 50-mile radius: natural draft cooling towers

Schuylkill River

42-82 ${ }^{\circ} \mathrm{F}$

450,000 gallons/minute each unit

$30^{\circ} \mathrm{F}$

intake from river

discharge to river

595 acres

0.47 mile radius

1.30 mile radius

Reading;

1980 population: 78,686

rolling

rolling

agricultural and undeveloped

The nearest town is Linfield about 1 mile SE.

Valiey Forge State Park is 10 miles SSE.

U.S. Highway I-76 is about 10 miles $S$.

$\begin{array}{ccccc}1990 & 2000 & 2010 & 2030 & 2050 \\ 6,970,000 & 7,070,000 & 7,170,000 & 7,390,000 & 7,620,000\end{array}$


NEDO-32361

\section{MIILSTONE NUCLEAR POWER STATION}

Location: New London County, Connecticut 3 miles WSW of New London latitude 41,3086 deg N; longitude $72.1681 \mathrm{deg}$ W

Licensee: Northeast Utilities

Unit Information

Docket Number

Construction Permit

Operating License

Commercial Operation

License Expiration

Licensed Thermal Power [MW(t)]

Design Electrical Rating [net MW(e)]

Type of Reactor

Nuclear Steam Supply System Vendor

$\begin{array}{rrr}\text { Unit 1 } & \text { Unit 2 } & \text { Unit 3 } \\ 50-245 & 50-336 & 50-423 \\ 1966 & 1970 & 1974 \\ 1970 & 1975 & 1986 \\ 1971 & 1975 & 1986 \\ 2010 & 2015 & 2026 \\ 2011 & 2700 & 3411 \\ 660 & 870 & 1154 \\ \text { BWR } & \text { PWR } & \text { PWR } \\ \text { GE } & \text { CE } & \text { WEST }\end{array}$

\section{Cooling Water System}

Type:

Source:

Source Temperature Range:

Condenser Flow Rate:

once through

Long Island Sound

$36-72^{\circ} \mathrm{F}$

420,000 gallons/minute for Unit 1

522,500 gallons/minute for Unit 2

$906,668 \mathrm{gallons} / \mathrm{minute}$ for Unit 3

Design Condenser Temperature Rise:

$21^{\circ} \mathrm{F}$ for Unit 1

24\% for Unit 2

$17.5^{\circ} \mathrm{F}$ for Unit 3

Intake Structure:

Discharge Structure:

on shore of Niantic Bay off Long Island Sound

discharge to Niantic Bay via holding pond

\section{Site Information}

Total Area:

500 acres

Exclusion Distance:

0.34 mile minimum

Low Population Zone:

2.40 miles radius

New Haven;

1980 population: 126,089

Site Topography:

Surrounding Area Topography:

flat

flat to rolling

Land Use within 5 miles: tural, and residential 
NEDO-32361

\section{MILLSTONE NUCLEAR POWER STATION (Continued)}

Nearby Features:

Area of Transmission Line Co ridor:

Population within a 50-mile radius:
The nearest town is Niantic 2 miles NW.

U.S. Highway I-95 is about 4 miles NNE.

Stone Ranch Military Reservation is about 6 miles NW. Harkness Memorial State Park, Bluff Point State Park, and Rocky Neck State Park are within 5 miles of the site.

The U.S. Dept. of Agriculture Plum Island facility is

$2000 \quad 2010 \quad 2030$

2050 

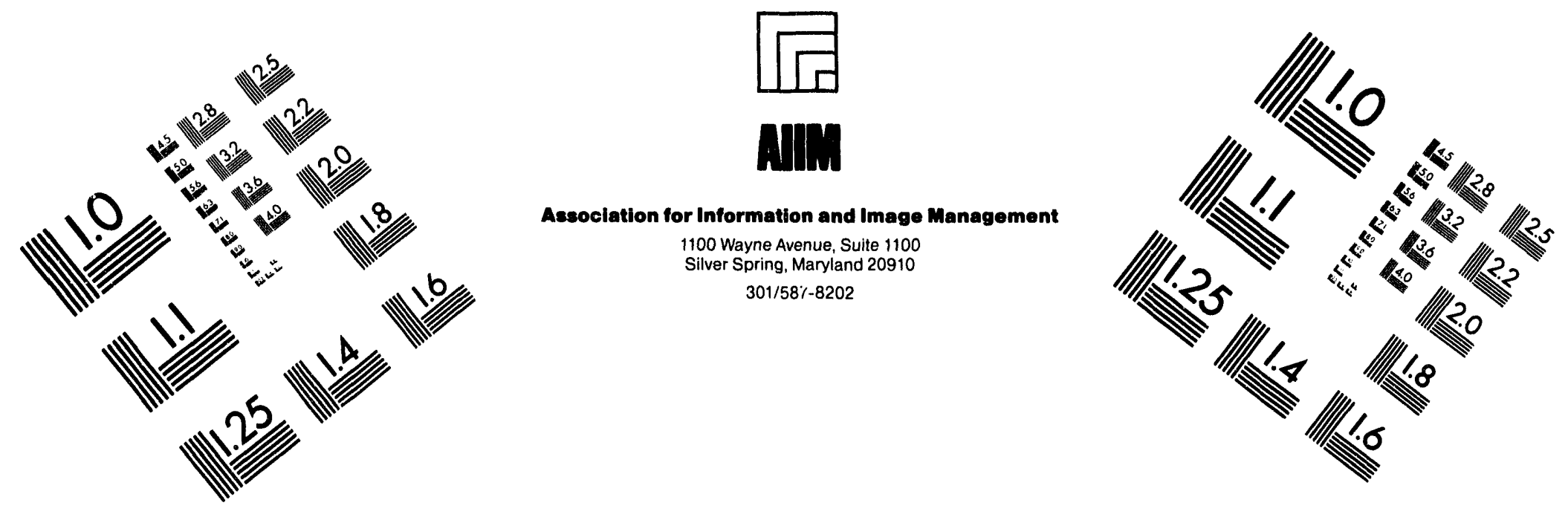

\section{Centimeter}

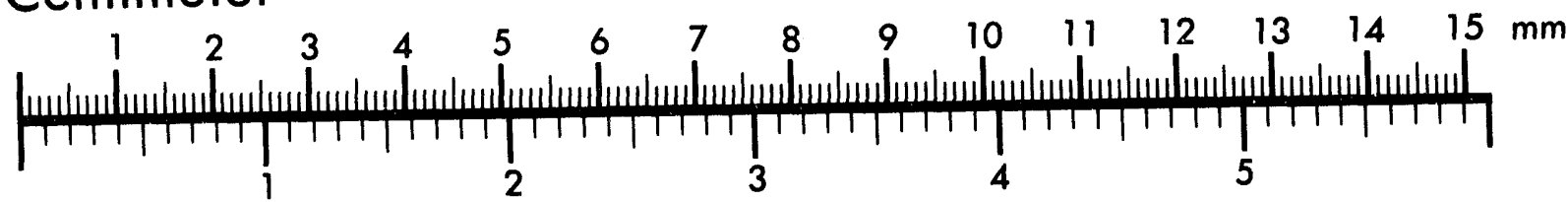

Inches
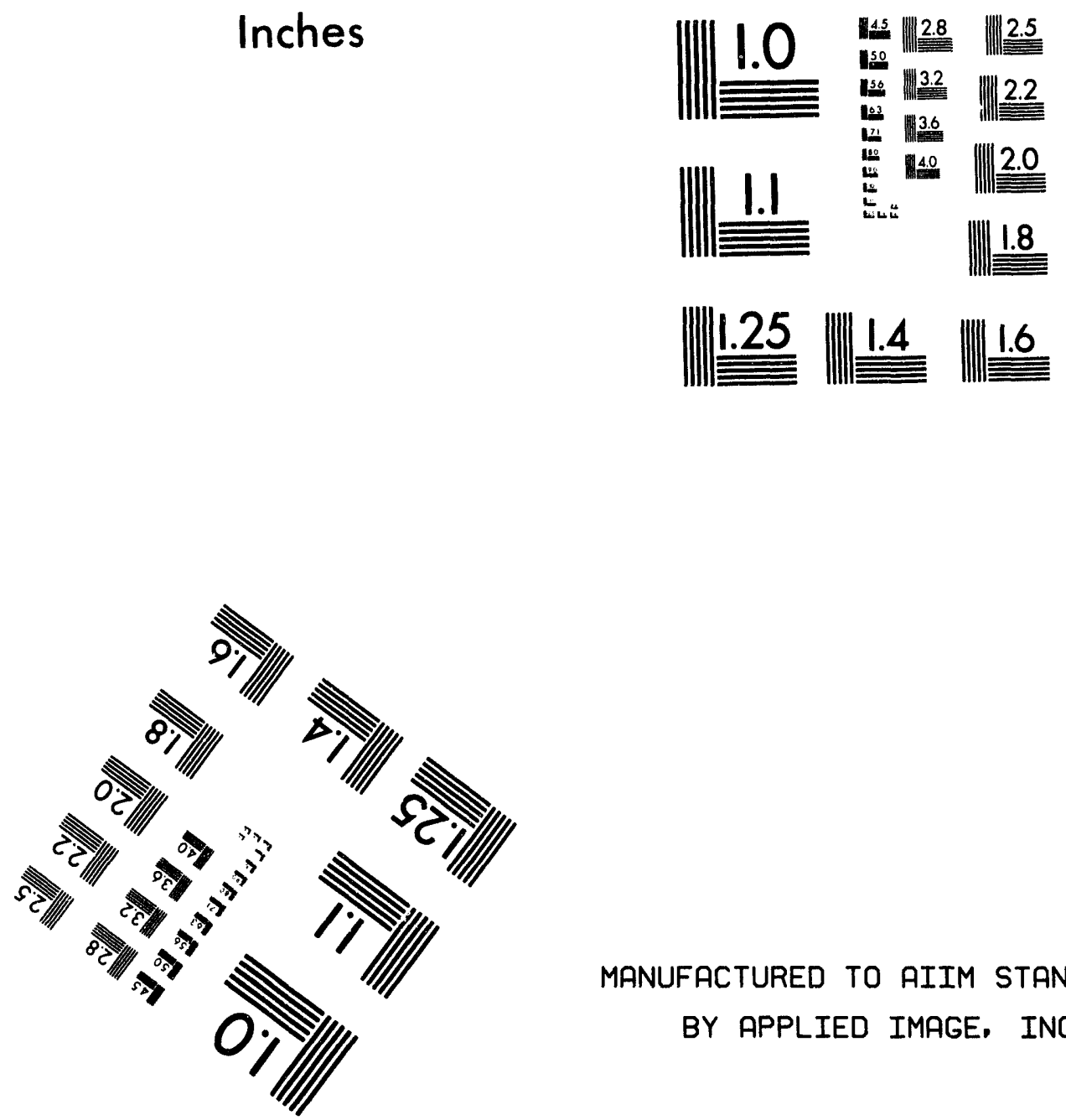

MANUFACTURED TO AIIM STANDARDS

BY APPLIED IMAGE, INC.

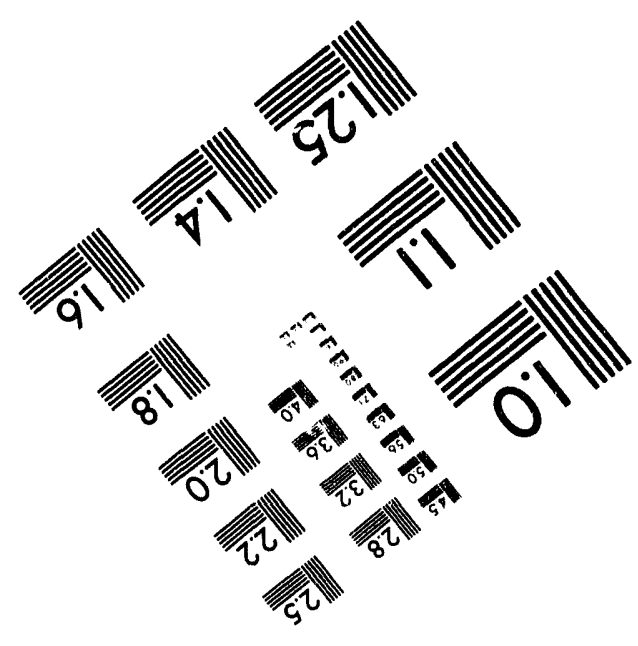



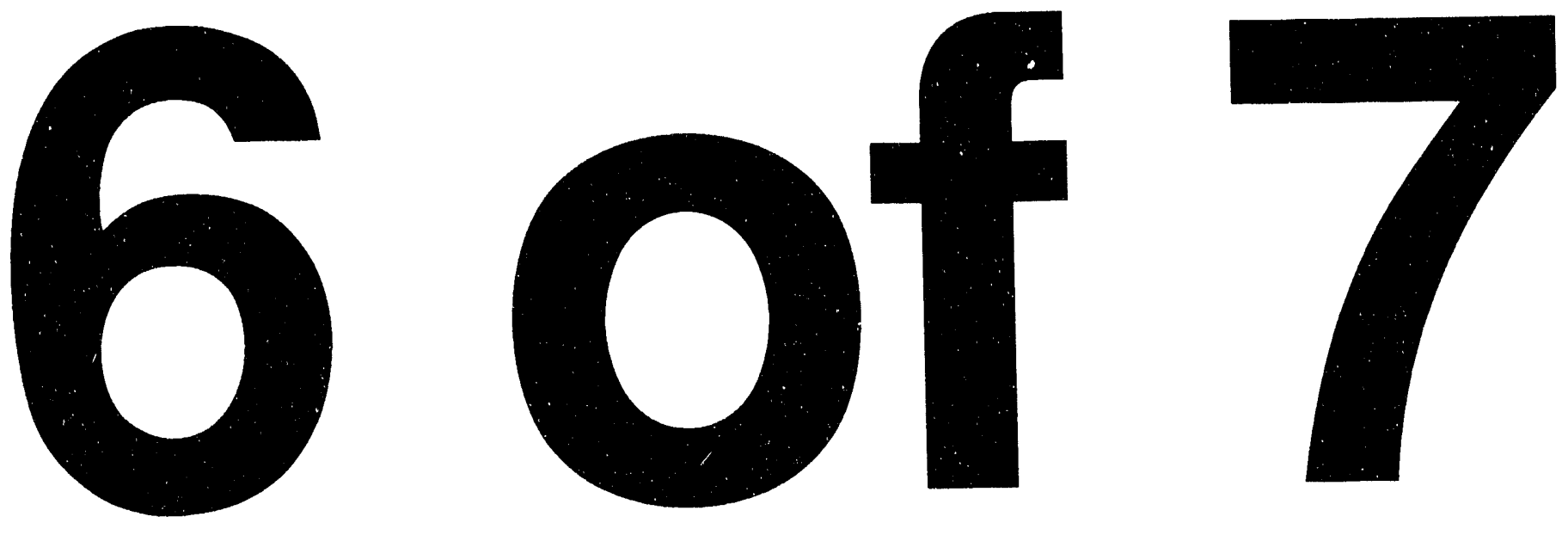
NEDO-32361

\section{MONTICELLO NUCLEAR GENERATING PLANT}

Location: Wright County, Minnesota

30 miles NW of Minneapolis

latifude $45.3333 \mathrm{deg} \mathrm{N}$; longitude $93.8483 \mathrm{deg} \mathrm{W}$

Licensee: $\quad$ Northern States Power Co.

\section{Unit Information}

Docket Number

Construction Permit

Operating License

Commercial Operation

License Expiration

Licensed Thermal Power [MW(t)]

Design Electrical Rating [net MW(e)]

Type of Reactor

Nuclear Steam Supply System Vendor

\section{Unit 1}

$50-263$

1967

1970

1971

2010

1670

545

BWR

GE

\section{Cooling Water System}

Type:

Source:

Source Temperature Range:

Condenser Flow Rate:

Design Condenser Temperature Rise: Intake Structure:

Discharge Structure:

\section{Site Information}

Total Area:

Exclusion Distance:

Low Population Zone:

Nearest City:

Site Topography:

Surrounding Area Topography:

Land Use within 5 miles:

Nearby Features:

Area of Transmission Line Corridor: Population within a 50-mile radius: once through and helper towers

Mississippi River

32-85 $\mathrm{F}$

280,000 gallons/minute

$26.8^{\circ} \mathrm{F}$

canal

canal
1325 acres

0.30 mile

1.0 mile

Minneapolis;

1980 population: 370,951

flat terraces

flat to gently sloping

agricultural and dairy farming

The nearest town is Monticello 2 miles SE.

Sherburne National Wildlife Refuge is about 9 miles $\mathrm{N}$.

Lake Maria State Park is about 6 miles WSW.

Sand Dunes State Forest and campground are 9 miles NE.

1454 acres

$\begin{array}{lllll}1990 & 2000 & 2010 & 2030 & 2050\end{array}$

$\begin{array}{lllll}2,170,000 & 2,360,000 & 2,520,000 & 2,820,000 & 3,150,000\end{array}$ 
NEDO-32361

\section{NINE MILE POINT NUCLEAR STATION}

Location: Oswago County, New York

6 miles NE of Oswago

latitude $43.5222 \mathrm{deg} \mathrm{N}$; longitude $76.4100 \mathrm{deg} \mathrm{W}$

Licensee: Niagra Mohawk Power Corp.

\section{Unit Information}

Docket Number

Construction Permit

Operating License

Commercial Operation

License Expiration

Licensed Thermal Power [MW(t)]

Design Electrical Rating [net MW(e)]

Type of Reactor

Nuclear Steam Supply System Vendor

$\begin{array}{rr}\text { Unit 1 } & \text { Unit 2 } \\ 50-220 & 50-410 \\ 1965 & 1974 \\ 1968 & 1987 \\ 1969 & 1988 \\ 2008 & 2027 \\ 1850 & 3323 \\ 620 & 1080 \\ \text { BWR } & \text { BWR } \\ \text { GE } & \text { GE }\end{array}$

\section{Cooling Water System}

Type:

Source:

Source Temperature Range:

Condenser Flow Rate:

Design Condenser Temperature Rise:

Intake Structure:

Discharge Structure:

\section{Site Information}

Total Area:

Exclusion Distance:

Low Population Zone:

Nearest City:

Site Topography:

Surrounding Area Topography:

Land Use within 5 miles:

Nearby Features:

Area of Transmission Line Corridor:

Population within a 50-mile radius:
Unit 1 - once through

Unit 2 - natural draft cooling tower

Lake Ontario

33- $77^{\circ} \mathrm{F}$

250,000 gallons/minute for Unit 1

580,000 gallons/minute for Unit 2

$32^{\circ} \mathrm{F}$ for Unit 1

$27^{\circ} \mathrm{F}$ for Unit 2

separate submerged pipelines about 1000 feet offshore diffuser pipe 555 feet long serving both units

900 acres

0.74 mile minimum

4.00 mile radius

Syracuse;

1980 population: 170,105

flat to rolling

rolling

agricultural, industrial, residential, and recreational

The nearest town is Lakeview about 1 mile WSW.

Fort Ontario is about 6 miles SW.

James A. FitzPatrick Nuclear Power Plant is 0.5 mile E.

1640 acres

$\begin{array}{ccccc}1990 & 2000 & 2010 & 2030 & 2050 \\ 820,000 & 810,000 & 790,000 & 800,000 & 810,000\end{array}$


NEDO-32361

\section{OYSTER CREEK NUCLEAR GENERATING STATION}

Location: Ocean County, New Jersey

9 miles $\mathrm{S}$ of Toms River

latitude $39.8142 \operatorname{deg} \mathrm{N}$; longitude $72.2064 \mathrm{deg} \mathrm{W}$

Licensee: GPU Nuclear Corp.

Unit Information

Docket Number

Construction Permit

Operating License

Commercial Operation

License Expiration

Licensed Thermal Power [MW(t)]

Design Electrical Rating [net MW(e)]

Type of Reactor

Nuclear Steam Supply System Vendor

\section{Unit 1}

50-219

1964

1969

1969

2009

1930

650

BWR

GE

\section{Cooling Water System}

Type:

Source:

Source Temperature Range:

Condenser Flow Rate:

Design Condenser Temperature Rise:

Intake Structure:

Discharge Structure:

\section{Site Information}

Total Area:

Exclusion Distance:

Low Population Zone:

Nearest City:

Site Topography:

Surrounding Area Topography:

Land Use within 5 miles:

Nearby Features:

Area of Transmission Line Corridor: Population within a 50-mile radius: once through

Barnegat Bay

$35-83^{\circ} \mathrm{F}$

460,000 gallons/minute

$14^{\circ} \mathrm{F}$

Forked River serves as a canal for intake and discharge to Barnegat Bay.

Forked River serves as a canal for intake and discharge to Barnegat Bay.

1416 acres

0.25 mile

2.00 miles

Atlantic City;

1980 population: 40,199

flat

rolling plains to flat lowlands

mostly undeveloped

The nearest town is Forked River about 2 miles $\mathrm{N}$.

The Garden State Parkway is 1 mile W.

There is a large influx of people seeking recreation in the summer.

322 acres

$\begin{array}{ccccc}1990 & 2000 & 2010 & 2030 & 2050 \\ 4,030,000 & 4,190,000 & 4,300,000 & 4,560,000 & 4,840,000\end{array}$




\section{PEACH BOTTOM ATOMIC POWER STATION}

Location: York County, Pennsylvania

18 miles $S$ of Lancaster latitude $39.7589 \mathrm{deg} \mathrm{N}$; longitude $76.2692 \mathrm{deg}$ W

Licensee: Philadelphia Electric Co.

\section{Unit Information}

Docket Number

Construction Permit

Operating License

Commercial Operation

License Expiration

Licensed Thermal Power [MW(t)]

Design Electrical Rating [net MW(e)]

Type of Reactor

Nuclear Steam Supply System Vendor

$\begin{array}{rr}\text { Unit 1 } & \text { Unit 2 } \\ 50-227 & 50-278 \\ 1968 & 1968 \\ 1973 & 1974 \\ 1974 & 1974 \\ 2013 & 2014 \\ 3293 & 3293 \\ 1065 & 1065 \\ \text { BWR } & \text { BWR } \\ \text { GE } & \text { GE }\end{array}$

\section{Cooling Water System}

Type:

Source:

Source Temperature Range:

Condenser Flow Rate:

Design Condenser Temperature Rise:

Intake Structure:

Discharge Structure:

\section{Site Information}

Total Area:

Exclusion Distance:

Low Population Zone:

Nearest City:

Site Topography:

Surrounding Area Topography:

Land Use within 5 miles:

Nearby Features:

Area of Transmission Line Corridor: Population within a 50-mile radius: once through with helper towers

Conowingo Pond

$34-80^{\circ} \mathrm{F}$

750,000 gallons/minute each unit

$20.8^{\circ} \mathrm{F}$

intake from Conowingo Pond through a small intake pond 5000-foot canal to Conowingo Pond

620 acres

0.51 mile minimum

1.38 miles

Lancaster;

1980 population: 54,725

rolling to hilly

rolling to hilly

agricultural and wooded

The nearest town is Slate Hill 2 miles SW.

Susquehanna State Park is about 3 miles N.

U.S. Highway I-95 is about 15 miles SE.

Conowingo Dam, about 8 miles SE on the Susquehanna

River, forms Conowingo Pond.

Unit 1 is a $40 \mathrm{MWe}$ nuclear plant on the same site and was retired from service in 1974.

Three Mile Island Nuclear Station is $\mathbf{3 5}$ miles upstream on the Susquehanna River.

1030 acres

$\begin{array}{ccccc}1990 & 2000 & 2010 & 2030 & 2050 \\ 4,660,000 & 4,850,000 & 5,010,000 & 5,280,000 & 5,570,000\end{array}$




\section{PERRY NUCLEAR POWER PLANT}

Location: Lake County, Ohio

7 miles NE of Painesville latitude $41.8008 \mathrm{deg} \mathrm{N}$; longitude $81.1442 \mathrm{deg} \mathrm{W}$

Licensee: Cleveland Electric Illuminating Co.

\section{Unit Information}

Docket Number

Construction Permit

Operating License

Commercial Operation

License Expiration

Licensed Thermal Power [MW(t)]

Design Electrical Rating [net MW(e)]

Type of Reactor

Nuclear Steam Supply System Vendor

\section{Unit 1}

$50-440$

1977

1986

1987

2026

3579

1205

BWR

GE

\section{Cooling Water System}

Type:

Source:

Source Temperature Range:

Condenser Flow Rate:

Design Condenser Temperature Rise:

Intake Structure:

Discharge Structure:

\section{Site Information}

Total Area:

Exclusion Distance:

Low Population Zone:

Nearest City:

Site Topography:

Surrounding Area Topography:

Land Use within 5 miles:

Nearby Features:

Area of Transmission Line Corridor:

Population within a 50-mile radius: natural draft cooling tower

Lake Erie

$32-79^{\circ} \mathrm{F}$

545,400 gallons/minute

$32^{\circ} \mathrm{F}$

submerged multiport structure 2550 feet offshore submerged diffuser 1650 feet offshore

1100 acres

0.55 mile radius

2.50 miles

Euclid;

1980 population: 59,999

flat

rolling

forest land, agricultural (horticulture), residential, industrial, and some recreational

The nearest town is North Perry 1 mile SW.

The Penn Central Railroad is about 3 miles $S$.

U.S. Highway I-90 is about 5 miles S.

1500 acres

$\begin{array}{ccccc}1990 & 2000 & 2010 & 2030 & 2050 \\ 2,480,000 & 2,530,000 & 2,570,000 & 2,670,000 & 2,770,000\end{array}$




\section{PILGRIM NUCLEAR POWER STATION}

Location: Plymouth County, Massachusetts

4 miles SE of Plymouth

latitude $41.9444 \mathrm{deg} \mathrm{N}$; longitude $70.5794 \mathrm{deg}$ W

Licensee: $\quad$ Boston Edison Co.

Unit Information

Docket Number

Construction Permit

Operating License

Commercial Operation

License Expiration

Licensed Thermal Power [MW(t)]

Design Electrical Rating [net MW(e)]

Type of Reactor

Nuclear Steam Supply System Vendor
Unit 1

$50-293$

1968

1972

1972

2012

1998

655

BWR

GE

\section{Cooling Water System}

Type:

Source:

Source Temperature Range:

Condenser Flow Rate:

Design Condenser Temperature Rise:

Intake Structure:

Discharge Structure:

\section{Site Information}

Total Area:

Exclusion Distance:

Low Population Zone:

Nearest City:

Site Topography:

Surrounding Area Topography:

Land Use within 5 miles:

Nearby Features:

Area of Transmission Line Corridor: Population within a 50-mile radius: once through

Cape Cod Bay

$32-83^{\circ} \mathrm{F}$

311,000 gallons/minute

$29^{\circ} \mathrm{F}$

concrete structure at edge of bay protected by a breakwater.

canal about 850 feet long

517 acres

0.33 mile

4.20 miles

Brockton;

1980 population: 95,172

flat to rolling

rolling to hilly

mostly undeveloped

The nearest town is Plymouth about 4 miles NW.

Miles Standish State Forest is about 6 miles SW.

Plymouth Rock and Plimoth Plantation historical sites are about 5 miles $W$.

174 acres

$\begin{array}{ccccc}1990 & 2000 & 2010 & 2030 & 2050 \\ 4,440,000 & 4,590,000 & 4,690,000 & 4,880,000 & 5,080,000\end{array}$




\section{QUAD-CITIES STATION}

Location: Rock Island County, Illinois 20 miles NE of Moline latitude $41.7261 \mathrm{deg} \mathrm{N}$; longitude $90.3100 \mathrm{deg}$ W

Licensee: Commonwealth Edison Co.

\section{Unit Information}

Docket Number

Construction Permit

Operating License

Commercial Operation

License Expiration

Licensed Thermal Power [MW(t)]

Design Electrical Rating [net MW(e)]

Type of Reactor

Nuclear Steam Supply System Vendor
Unit 1 Unit 2

$\begin{array}{rr}50-254 & 50-265 \\ 1967 & 1967 \\ 1972 & 1972 \\ 1973 & 1973 \\ 2012 & 2012 \\ 2511 & 2511 \\ 789 & 789 \\ \text { BWR } & \text { BWR } \\ \text { GE } & \text { GE }\end{array}$

\section{Cooling Water System}

Type:

Source:

Source Temperature Range:

Condenser Flow Rate:

Design Condenser Temperature Rise:

Intake Structure:

Discharge Structure:

\section{Site Information}

Total Area:

Exclusion Distance:

Low Population Zone:

Nearest City:

Site Topography:

Surrounding Area Topography:

Land Use within 5 miles:

Nearby Features:

Area of Transmission Line Corridor:

Population within a 50-mile radius: once through

Mississippi River

$32-85^{\circ} \mathrm{F}$

471,000 gallons/minute each unit

$24^{\circ} \mathrm{F}$

crib house at edge of river

14,000 -foot spray canal

784acres

0.50 mile

3.00 miles

Davenport, Iowa;

1980 population: 103,264

flat

flat

agricultural and small industrial park

The nearest town is Folletts 3 miles NW.

The Rock Island Railroad is 2 miles W.

Chicago, Milwaukee, and St. Paul Railroad is 1 mile E.

The Rock Island Arsenal is about 15 miles SW.

1400 acres

$\begin{array}{lllll}1990 & 2000 & 2010 & 2030 & 2050\end{array}$

$\begin{array}{lllll}740,000 & 760,000 & 780,000 & 810,000 & 850,000\end{array}$ 


\section{RIVER BEND STATION}

Location: West Feliciana County, Louisiana

24 miles NNW of Baton Rouge

latitude $30.7569 \operatorname{deg} \mathrm{N}$; longitude $91.3314 \mathrm{deg} \mathrm{W}$

Licensee: Gulf States Utility Co.

Unit Information

Docket Number

Construction Permit

Operating License

Commercial Operation

License Expiration

Licensed Thermal Power [MW(t)]

Design Electrical Rating [net MW(e)]

Type of Reactor

Nuclear Steam Supply System Vendor

\section{Unit 1}

$50-458$

1977

1985

1986

2025

2894

936

BWR

GE

\section{Cooling Water System}

Type:

Source:

Source Temperature Range:

Condenser Flow Rate:

Design Condenser Temperature Rise:

Intake Structure:

Discharge Structure:

\section{Site Information}

Total Area:

Exclusion Distance:

Low Population Zone:

Nearest City:

Site Topography:

Surrounding Area Topography:

Land Use within 5 miles:

Nearby Features:

Area of Transmission Line Corridor: Population within a 50-mile radius: mechanical draft cooling towers

Mississippi River

508,470 gallons/minute

$27^{\circ} \mathrm{F}$

at river bank

pipe extending into the river

3342 acres

0.57 mile radius

2.50 mile radius

Baton Rouge;

1980 population: 220,394

flat

flat to rolling

agricultural and forest

The nearest town is St. Francisville 3 miles NW.

Audubon Memorial State Park is about 3 miles NNE.

The Illinois Central Railroad crosses the site.

1014 acres

$\begin{array}{lllll}1990 & 2000 & 2010 & 2030 & 2050\end{array}$

$\begin{array}{lllll}800,000 & 860,000 & 920,000 & 1,010,000 & 1,110,000\end{array}$ 
NEDO-32361

\section{SUSQUEHANNA STEAM ELECTRIC STATION}

Location: Luzerne County, Pennsylvania

7 miles NE of Berwick

latitude $41.0922 \mathrm{deg} N$; longitude $76.1467 \mathrm{deg}$ W

Licensee: Pennsylvania Power and Light Co.

\section{Unit Information}

Docket Number

Construction Permit

Operating License

Commercial Operation

License Expiratior.

Licensed Thermal Power [MW(t)]

Design Electrical Rating [net MW(e)]

Type of Reactor

Nuclear Steam Supply System Vendor

\section{Unit 1 Unit 2}

50-387 50-388

$1973 \quad 1973$

$1982 \quad 1984$

$1983 \quad 1985$

$2022 \quad 2024$

$3293 \quad 3293$

$1050 \quad 1050$

BWR BWR

GE GE

\section{Cooling Water System}

Type:

Source:

Source Temperature Range:

Condenser Flow Rate:

Design Condenser Temperature Rise:

Intake Structure:

Discharge Structure:

\section{Site Information}

Total Area:

Exclusion Distance:

Low Population Zone:

Nearest City:

Site Topography:

Surrounding Area Topography:

Land Use within 5 miles:

Nearby Features:

Area of Transmission Line Corridor: Population within a 50-mile radius: natural draft cooling towers

Susquehanna River

448,000 gallons/minute each unit $14^{\circ} \mathrm{F}$

at river bank

diffuser pipe 240 feet from river bank

1075 acres

0.34 mile radius

3.00 miles

Wilkes-Barre;

1980 population: 51,551

rolling

hilly with flat river valley

wooded and agricultural

The nearest town is Beach Haven about 1 mile SW.

U.S. Highway $\mathrm{I}-80$ is 5 miles S.

The ConRail Railroad is 0.5 mile $\mathrm{E}$.

The Delaware and Hudson Railroad is 1 mile $\mathrm{E}$.

1800 acres

$\begin{array}{ccccc}1990 & 2000 & 2010 & 2030 & 2050 \\ 1,500,000 & 1,510,000 & 1,530,000 & 1,550,000 & 1,580,000\end{array}$ 


\section{VERMONT YANKEE NUCLEAR POWER STATION}

Location: Windham County, Vermont 5 miles $S$ of Brattleboro

latitude $442.7803 \mathrm{deg} \mathrm{N}$; longitude $72.5158 \mathrm{deg} \mathrm{W}$

Licensee: Vermont Yankee Nuclear Power Corp.

\section{Unit Information}

Docket Number

Construction Permit

Operating License

Commercial Operation

License Expiration

Licensed Thermal Power [MW(t)]

Design Electrical Rating [net MW(e)]

Type of Reactor

Nuclear Steam Supply System Vendor
Unit 1

$50-271$

1967

1973

1972

2013

1593

540

BWR

GE

\section{Cooling Water System}

Type:

Source:

Source Temperature Range:

Condenser Flow Rate:

Design Condenser Temperature Rise:

Intake Structure:

Discharge Structure:

\section{Site Information}

Total Area:

Exclusion Distance:

Low Population Zone:

Nearest City:

Site Topography:

Surrounding Area Topography:

Land Use within 5 miles:

Nearby Features:

Area of Transmission Line Corridor: Population within a 50-mile radius: once through and helper towers

Connecticut River

$32-74^{\circ} \mathrm{F}$

366,000 gallons/minute

$20^{\circ} \mathrm{F}$

concrete structure at edge of river

aerating structure discharging at edge of river

125 acres

0.17 mile

5.00 miles

Holyoke, Massachusetts;

1980 population: 44,678

flat

rolling to hilly

mostly wooded, some agricultural and industrial

The nearest town is Vernon about 1 mile W.

Vernon Dam is 0.7 mile downstream from the site.

Yankee Nuclear Power Station is about 20 miles WSW.

1550 acres

$\begin{array}{ccccc}1990 & 2000 & 2010 & 2030 & 2050 \\ 1,510,000 & 1,580,000 & 1,620,000 & 1,710,000 & 1,800,000\end{array}$


NEDO-32361

\section{WASHINGTON NUCLEAR PROJECT 2 (WNP-2)}

Location: Benton County, Washington

12 miles NW of Richland latitude $46.4714 \mathrm{deg} \mathrm{N}$; longitude $119.3331 \mathrm{deg}$ W

Licensee: Washington Public Power Supply System

Unit Information

Docket Number

Construction Permit

Operating License

Commercial Operation

License Expiration

Licensed Thermal Power [MW( $t)$ ]

Design Electrical Rating [net MW(e)]

Type of Reactor

Nuclear Steam Supply System Vendor
Unit 2

50-397

1973

1984

1984

2024

3323

1) 0

BV/R

GE

\section{Cooling Water System}

Type:

Source:

Source Temperature Range:

Condenser Flow Rate:

Design Condenser Temperature Rise:

Intake Structure:

Discharge Structure:

\section{Site Information}

Total Area:

Exclusion Distance:

Low Population Zone:

Nearest City:

Site Topography:

Surrounding Area Topography:

Land Use within 5 miles:

Nearby Features:

Area of Transmission Line Corridor:

Population within a 50-mile radius: mechanical draft cooling towers

Columbia River

$38-64^{\circ} \mathrm{F}$

550,000 gallons/minute

$28.7^{\circ} \mathrm{F}$

2 perforated pipe inlets supported offshore above the river bed 900 feet from pump structure on river bank buried 3-mile pipeline terminating at the river bed 175 feet from the shoreline

on Department of Energy Hanford Reservation

1.21 mile radius

3.00 miles

Spokane;

1980 population: 171,300

flat

flat

Hanford Reservation and agricultural

The nearest town is Richland 9 miles S.

The site is in the SE part of the Hanford Reservation. on Hanford Reservation

$\begin{array}{ccccc}1990 & 2000 & 2010 & 2030 & 2050 \\ 280,000 & 310,000 & 330,000 & 370,000 & 410,000\end{array}$


NEDO-32361

\section{Appendix E: Feed Material Draft Specification}

\section{$\mathrm{PuO}_{2}$ Powder, Ceramic Grade - Dry Process}

\subsection{Scope}

1.1 This specification establishes the requirements for ceramic grade plutonium dioxide powder produced from plutonium obtained during retirement of nuclear weapons.

\subsection{Applicable Documents}

2.1 The following publications form a part of this specification to the degree indicated where applicable:

ASTM B214-1964 "Sieve Analysis of Granulated Metal Powders"

ASTM 3329-1961 “Test for Apparent Density of Refractory Metals and Compounds by the Scott Volumeter"

ASTM B330-1965 "Average Particle Size of Refractory Metals and Compounds by the Fisher Sub-Sieve Sizer"

When the contents of this specification conflict with any document referenced herein, the specification takes precedence.

\subsection{General Description}

\subsection{Powder Description}

The material to be furnished in accordance with the specification shall be plutonium dioxide ready for fabrication of mixed (uranium-plutonium) oxide fuel pellets. The supplier shall furnish all required reports and information defined in this specification. The supplier shall perform and report all tests required by this specification.

\subsection{Requirements}

\subsection{Functional Criteria}

Purchaser will specify the nominal plutonium isotope compositions. The requirements of this specification are for material in the form of homogeneous powder. For this specification, 
homogeneity is defined as follows: A homogeneous lot shall consist of all material produced for a single shipment in a continuous process sequence; or it shall consist of a uniform blend of material produced in a batch-type process; and it shall consist of an amount of material produced with constant process parameters. Successful processing of this material is dependent upon the consistency of characteristics. This specification provides for a wide range of limits for certain characteristics. Once the supplier establishes a specific value for each characteristic within the wide range, he shall maintain this value within the specified narrow range. The limits of the narrow range selected must fall within the wide range. The narrow limits shall include both material and measurement variations.

\subsection{Product Specification}

All plans and procedures for obtaining (1) homogeneity, (2) samples for chemical analysis, (3) chemical composition, and (4) physical properties shall be submitted to Purchaser for concurrence.

\subsubsection{Powder Composition}

\subsubsection{Plutonium Analysis}

Plutonium minimum 85 w/o of plutonium dioxide

Isotopic Content $\quad$ Pu238, 239, 240, 241, 242 as specified

4.2.1.2 The total volatile content of the powder shall not exceed $0.05 \mathrm{wt} \%$ plutonium dioxide. The total volatile content shall be determined by weight loss on heating a one gram sample at $1000 \pm 25$ Celsius for four hours in air or by an equivalent method approved by Purchaser and agreed to by the Supplier.

4.2.1.3 The plutonium shall be in the form of plutonium dioxide produced by oxidation of plutonium metal at or below 500 Celsius.

\subsubsection{Impurities}

4.2.2.1 Total impurities, shown on Table 1, shall not exceed 2,500 ppm of plutonium.

4.2.2.2 The americium content shall not exceed $300 \mathrm{ppm}$ of plutonium.

4.2.2.3 The uranium content shall not exceed $2500 \mathrm{ppm}$ of plutonium. 
Table 1 Total Impurities

\begin{tabular}{|l|c|}
\hline Impurity & Limit PPM of Pu \\
\hline Aluminum & 400 \\
\hline Boron & 1 \\
\hline Cadmium & 1 \\
\hline Calcium & 250 \\
\hline Carbon & 500 \\
\hline Chlorine & 25 \\
\hline Chromium & 150 \\
\hline Cobalt & 75 \\
\hline Copper & 400 \\
\hline Fluorine & 130 \\
\hline Gallium & 400 \\
\hline Iron & 400 \\
\hline Lead & 400 \\
\hline Maganese & 200 \\
\hline Magnesium & 200 \\
\hline Molybdenum & 400 \\
\hline Nickel & 400 \\
\hline Nitrogen & 100 \\
\hline Silicon & 200 \\
\hline Silver & 25 \\
\hline Sodium & 400 \\
\hline Tin & 400 \\
\hline Titanium & 200 \\
\hline Thorium & 10 \\
\hline Vanadium & 400 \\
\hline Zirconium & 400 \\
\hline EDysprosium, Gadolinium, Europium, Samarium & 2 \\
\hline
\end{tabular}

4.2.2.4 The total measured impurities in Paragraph 4.2.2.1 shall be such that:

$\Sigma \mathrm{C}_{\mathrm{i}}(\mathrm{B}=)$ is equal to or less than $4.0 \mathrm{ppm}$ by weight of natural boron excluding $\mathrm{Am}-241$ where:

$\mathrm{C}_{\mathrm{i}}=$ Weight fraction in units of parts per million parts of Pu of each impurity in Paragraph 4.2.2.1, and

$(B=)=$ The natural boron equivalent of element $i$ from the Table 2 list, which is based on the neutron absorption cross sections in BNL-325 S1, and "Resonance Integral Data," ANL Newsletter No. 1, assuming a Maxwellian spectrum in the resonance region for a typical thermal reactor. The atomic weights are on the physical scale. When 
impurities are reported as less than a stated threshold of detection, the threshold value shall be used for this calculation.

\subsubsection{Physical Properties}

4.2.3.1 All material must pass through a 325-mesh U.S. Standard sieve in accordance with ASTM B214-1964, "Sieve Analysis of Granular Metal Powders".

Table 2 Boron Equivalent Data

\begin{tabular}{|l|c||c|c|}
\hline Element & $\begin{array}{c}\text { Boron Equivalent } \\
\text { ppm B/ppm of Element }\end{array}$ & Element & $\begin{array}{c}\text { Boron Equivalent } \\
\text { ppm B/ppm of Element }\end{array}$ \\
\hline Aluminum & $1.32 \times 10^{-4}$ & Magnesium & $1.42 \times 10^{-4}$ \\
\hline Boron & 1.00 & Manganese & $4.01 \times 10^{-3}$ \\
\hline Cadmium & $7.79 \times 10^{-1}$ & Molybdenum & $1.32 \times 10^{-3}$ \\
\hline Calcium & $2.87 \times 10^{-4}$ & Nickel & $1.20 \times 10^{-3}$ \\
\hline Carbon & $5.51 \times 10^{-6}$ & Nitrogen & $2.76 \times 10^{-3}$ \\
\hline Chlorine & $1.37 \times 10^{-2}$ & Samarium & 0.524 \\
\hline Chromium & $9.35 \times 10^{-4}$ & Silicon & $8.24 \times 10^{-5}$ \\
\hline Cobalt & $1.25 \times 10^{-2}$ & Silver & $2.98 \times 10^{-2}$ \\
\hline Copper & $9.45 \times 10^{-4}$ & Sodium & $3.37 \times 10^{-4}$ \\
\hline Dysprosium & $9.7 \times 10^{-2}$ & Tin & $2.06 \times 10^{-4}$ \\
\hline Europium & 0.434 & Tungsten & $7.02 \times 10^{-3}$ \\
\hline Fluorine & $3.48 \times 10^{-4}$ & Vanadium & $1.49 \times 10^{-3}$ \\
\hline Gadolinium & 4.191 & Zinc & $3.71 \times 10^{-4}$ \\
\hline Gallium & $4.000 \times 10^{-6}$ & Zirconium & $2.93 \times 10^{-5}$ \\
\hline Iron & $7.43 \times 10^{-4}$ & & \\
\hline Lead & $1.23 \times 10^{-5}$ & & \\
\hline
\end{tabular}

\subsubsection{Bulk Density}

The bulk density shall be no less than $1.0 \mathrm{gm} / \mathrm{cc}$ as determined by the Scott Volumeter per ASTM B329-1961, "Test for Apparent Density of Refractory Metals and Compounds by the Scott Volumeter". Once the Supplier has established a value for bulk density, he shall maintain this value within $\pm 0.25 \mathrm{gm} / \mathrm{cc}$ for the total amount of material supplied under the purchase contract.

\subsubsection{Surface Area}

The surface area shall be determined by the Supplier by the B.E.T. method of analysis or an equivalent method approved by Purchaser. This requirement for surface area shall not be the basis for the acceptance or rejection of material, but is requested for information only. 


\subsubsection{Particle Size}

The particle size shall be determined by ASTM B330-1965, "Average Particle Size of Refractory Metals and Compounds by the Fisher Sub-Sieve Sizer" and/or equivalent method approved by the Purchaser. This requirement for particle size shall not be the basis for the acceptance or rejection of material, but is requested for information only.

\subsection{Quality Assurance Requirements}

4.3.1 Concurrence of Purchaser is required, where indicated in this specification, of the Supplier's Quality Control Plans and Fabrication Procedures prior to the production of any material. This concurrence may involve the witnessing of tests and test equipment at the Supplier's plant. Any significant change in process operations by the Supplier is to be made known to Purchaser to permit joint performance evaluation. Such changes may be accidental or planned.

\subsubsection{Check Analysis Sample}

The Supplier shall take a sample from each lot of material sufficiently large that the Purchaser will receive a 20 gram sample. This sample is to be divided into four parts for (1) chemical analysis by Purchaser, (2) chemical analysis by Supplier, (3) chemical analysis by Referee if necessary, and (4) archive sample held by Supplier, until shipper receiver differences, if any, are settled. The sample for Purchaser shall be packaged, marked "Check Sample" and sent to Purchaser with the lot shipment. 


\subsection{Inspection}

The Supplier shall submit to the Purchaser (prior to shipment) a Certificate of Test in triplicate for each homogeneous lot of powder showing that the material conforms to this specification. The certification shall include the purchase order number, Purchaser specification designation, the results of the required tests, and a statement certifying that the material is homogeneous. The test results shall be so numbered that they can be identified with their related lot of material. The certifications shall include the results of the analyses per Paragraph 4.2, including the limit of detection for each analysis. This section shall include calculated total boron equivalent per the equation in Paragraph 4.2.2.4, if applicable.

\subsection{Packing, Marking, And Criticality Requirements}

6.1 The material shall be packed in a manner which will prevent powder loss and contamination spread during transit. Shipments must adhere to applicable United State Department of Energy and/or United States Department of Transportation shipping regulations and licenses.

6.2 The shipping containers will be decided upon jointly by the Purchaser and the Supplier such that Purchaser's license is not violated. The Purchaser must be notified and approve all pending shipments.

6.3 The external and internal surfaces of the outer container and the external surface of the inner container shall be as free from contamination as possible, and in no case shall exceed United State Department of Transportation regulation for smearable contamination.

6.4 The Supplier shall identify each container as follows:

- Outer Container:

- Container numbers, inner and outer, including permit numbers

- Purchase order numbers

- Type of material and lot number

- Metal weight of material

- Shipment class (Fissile Class I, II, or III) 
NEDO-32361

- Radiation units

- Purchaser's address (to be specified on purchase order)

- Two packing slips must be attached which include all shipping papers including two courtesy copies of AEC transfer documents, and "Certificate of Test" analysis

- Inner Container:

- Container number

- Type of material and lot number

- Gross, tare, net and metal weights 


\section{Appendix F - Plutonium Disposition Complex Deployment Capabilities}

This appendix, detailing the currently existing infrastructure at the various national laboratories, was prepared for the earlier report on plutonium disposition using the GE Advanced Boiling Water Reactor (ABWR). The appendix is included here to provide infrastructure information that is relevant to the present study, where plutonium is disposed using existing BWRs. Specifically, the information related to the possible siting of a MOX fuel fabrication facility at one of the laboratory locations applies both to the present and previous studies. 
Table F-1 Idaho National Engineering Laboratory (INEL)

\begin{tabular}{|c|c|c|}
\hline Capability Requirement & & Current Capability at INEL \\
\hline Overall Site Qualification & $\overline{\mathrm{E}}$ & $\begin{array}{l}\text { - Currently operating. Has served for } \sim 45 \text { years as DOE } \\
\text { site for part of Nuclear Materials Production Complex, } \\
\text { reactor R\&D, and fuel processing. INEL is still } \\
\text { performing these missions on a reduced scale. } \\
\text { - Occupies } \sim 890 \text { square miles } ~ 29 \text { miles west of Idaho } \\
\text { Falls, ID. } \\
\text {-Eleven Technology Areas: Test Area North (TAN), } \\
\text { Test Reactor Area (TRA), Central Facilities Area } \\
\text { (CFA), Radioactive Wiaste Management Complex } \\
\text { (RWMC), Auxiliary Reactor Area (ARA), Power Burst } \\
\text { Facility/Power Excursion Reactor Test (PBF/SPERT), } \\
\text { Idaho Chemical Processing Plant (ICPP), and Argonne } \\
\text { National Laboratory West (ANL-W), Idaho Research } \\
\text { Center (IRC), Idaho Supercomputer Center (ISC), } \\
\text { INEL Engineering. }\end{array}$ \\
\hline \multicolumn{3}{|l|}{$\begin{array}{l}\text { PuO2 Feed Material } \\
\text { Interface }\end{array}$} \\
\hline $\begin{array}{l}\text {-Pu Receiving and Storage } \\
\text { (within weapons program) }\end{array}$ & $\mathbf{E}$ & $\begin{array}{l}\text { - Management and staff experienced with SNM handling } \\
\text { including Pu. } \\
\text { SNM vault in Fuel Processing Restoration (FPR) } \\
\text { facility could receive Pu metal or oxide. }\end{array}$ \\
\hline $\begin{array}{l}\text {-Pu-to-PuO2 Conversion } \\
\text { (within weapons program) }\end{array}$ & $\mathrm{E}$ & $\begin{array}{l}\text { - Management and staff experienced with Pu processing. } \\
\text { Pu metal core fabricated and irradiated in Materials Test } \\
\text { Reactor. } \\
\text { - Hot cells in FPR facility can be used for Pu-to-PuO2 } \\
\text { conversion. }\end{array}$ \\
\hline $\begin{array}{l}\text {-PuO2 Feed Interface } \\
\text { Management }\end{array}$ & $\mathrm{E}$ & $\begin{array}{l}\text {-Experience managing } \mathrm{SNM} \text { is applicable to } \\
\text { management of interface with supplier of } \mathrm{PuO} 2 \text { feed } \\
\text { material whether or not } \mathrm{Pu} \text {-to-PuO2 conversion } \\
\text { function is located at } \mathrm{DNEL} \text {. }\end{array}$ \\
\hline MOX Fuel Fabrication & & \\
\hline $\begin{array}{l}\text {-MOX Pellet and Pin } \\
\text { Fabrication }\end{array}$ & $\mathrm{U}$ & $\begin{array}{l}\text {-Experience with Pu processing is applicable to } \\
\text { extension of capability to MOX blending, sintering, } \\
\text { and pin loading. [TBR] } \\
\text {-Fuel Processing Restoration (FPR) facility was } \\
\text { completed in } 1992 \text {, is uncontaminated, and can be } \\
\text { modified to house the MOX lines. New equipment for } \\
\text { powder blending, pellet sintering, and fuel pin loading } \\
\text { and closure is needed. } \\
\text {-FPR is six stories tall, } 160,000 \text { square feet floor space, } \\
\text { state-of-the-art radiation protection, contamination } \\
\text { control, decontamination, natural phenomena } \\
\text { protection, remote handling, } 50 / 5 \text { ton bridge crane, } \\
\text { DOE approved SNM vault. }\end{array}$ \\
\hline
\end{tabular}


NEDO-32361

Table F-1 Idaho National Engineering Laboratory (INEL) (continued)

\begin{tabular}{|c|c|}
\hline Capability Requirement & Current Capability at INEL \\
\hline \multicolumn{2}{|l|}{$\begin{array}{l}\text { MOX Fuel Fabrication } \\
\text { (Continued) }\end{array}$} \\
\hline $\begin{array}{l}\text {-BWR MOX Assembly } \\
\text { Fabrication }\end{array}$ & -FPR can be modified for MOX Assembly fabrication. \\
\hline $\begin{array}{l}\text {-Fresh MOX Assembly } \\
\text { Storage }\end{array}$ & $\begin{array}{l}\text { U -FPR can be modified for fresh MOX Assembly } \\
\text { storage. }\end{array}$ \\
\hline \multicolumn{2}{|l|}{$\begin{array}{l}\text { Spent Fuel Storage } \\
\text { Interface }\end{array}$} \\
\hline $\begin{array}{l}\text { - Spent Fuel Storage } \\
\text { (within waste disposal } \\
\text { program) }\end{array}$ & $\begin{array}{l}\text { E Environmental Impact Statement (EIS) in progress for } \\
\text { storage of commercial spent fuel (DOE Programmatic } \\
\text { Spent Nuclear Fuel Management and INEL } \\
\text { Environmental Restoration and Waste Management } \\
\text { Programs EIS). Schedule is draft Jun94. } \\
\text { - Currently available pools include Idaho Chemical } \\
\text { Processing Plant (ICPP) and naval reactor facilities. } \\
\text { - Construction of additional spent fuel storage facilities } \\
\text { planned. } \\
\text { - Manages development of storage casks. Conducted } \\
\text { Dry Rod Consolidation Technology (DRCT) project. } \\
\text { Conducted testing and analysis of metal casks for dry } \\
\text { storage. }\end{array}$ \\
\hline $\begin{array}{l}\text {-Spent Fuel Transport } \\
\text { (within waste disposal } \\
\text { program) }\end{array}$ & $\begin{array}{l}\text { E - Management and staff experienced in spent fuel } \\
\text { transport, including LWR. } \\
\text { - Manages development of transportation casks. Cask } \\
\text { Systems Development Program supports acquisition of } \\
\text { prototype casks for spent fuel transport from reactors } \\
\text { to repository. } \\
\text { - Manages transport of spent fuel, including LWR, to } \\
\text { Hot Fuel Examination Facility (HFEF) at INEL. } \\
\text { - Manages transport of spent LWR fuel assemblies from } \\
\text { reactors as part of Spent Fuel Storage Cask Testing } \\
\text { Program and other programs. }\end{array}$ \\
\hline $\begin{array}{l}\text {-Spent Fuel Interface } \\
\text { Management }\end{array}$ & $\begin{array}{l}\text {-Experience managing spent fuel operations is } \\
\text { applicable to management of interface with Nuclear } \\
\text { Waste Disposal Program whether or not spent fuel is } \\
\text { stored or transported by INEL. }\end{array}$ \\
\hline $\begin{array}{l}\text { Waste Management } \\
\text { - High Level Waste (HLW) }\end{array}$ & $\begin{array}{l}\text { E - Management and staff have recent experience in } \\
\text { designing, constructing, operating, and maintaining } \\
\text { facilities to handle, process, and store HLW. } \\
\text {-Waste treatment capabilities/facilities include handling, } \\
\text { processing, calcining, and storage. } \\
\text { - Currently stored at Idaho Chemical Processing Plant } \\
\text { (ICPP). } \\
\text { - New Waste Management Center is in planning } \\
\text { process. }\end{array}$ \\
\hline
\end{tabular}


Table F-1 Idaho National Engineering Laboratory (INEL) (continued)

\begin{tabular}{|c|c|}
\hline Capability Requirement & Current Capability at INEL \\
\hline \multicolumn{2}{|l|}{$\begin{array}{l}\text { Waste Management } \\
\text { (Continued) }\end{array}$} \\
\hline -Transuranic Waste (TRU) & $\begin{array}{l}\text { E - Management and staff have recent experience in } \\
\text { designing, constructing, operating, and maintaining } \\
\text { facilities to handle, process, and store TRU. } \\
\text { - Retrievable contact handled TRU and TRU-mixed } \\
\text { wastes currently stored at Transuranic Storage Area } \\
\text { (TSA) of Radioactive Waste Management Center } \\
\text { (RWMC). } \\
\text { - Retrievable remote handled TRU and TRU-mixed } \\
\text { waste currently stored at Intermediate-Level } \\
\text { Transuranic Storage Facility (ILTSF) of RWMC. }\end{array}$ \\
\hline -Low Level Waste (LLW) & $\begin{array}{l}\text { E - Management and staff have recent experience in } \\
\text { designing, constructing, operating, and maintaining } \\
\text { facilities to handle, process, and store LLW. } \\
\text { - LLW disposal currently at Subsurface Disposal Area } \\
\text { (SDA) of RWMC. }\end{array}$ \\
\hline -Hazardous Waste & $\begin{array}{l}\text { E - Management and staff have recent experience in } \\
\text { designing, constructing, operating, and maintaining } \\
\text { facilities to handle, process, and store hazardous } \\
\text { wastes. }\end{array}$ \\
\hline -Mixed Waste & $\begin{array}{l}\text {-Management and staff have recent experience in } \\
\text { designing, constructing, operating, and maintaining } \\
\text { facilities to handle, process, and store mixed wastes. }\end{array}$ \\
\hline $\begin{array}{l}\text { - Nuclear Waste Interface } \\
\text { Management }\end{array}$ & $\begin{array}{l}\text {-Experience managing all forms of nuclear waste is } \\
\text { applicable to management of interface with Nuclear } \\
\text { Waste Disposal Program. }\end{array}$ \\
\hline \multicolumn{2}{|l|}{ Safeguards \& Security } \\
\hline -Accountability & $\begin{array}{l}\text { - Management and staff experienced in implementing strict } \\
\text { accountability procedures for storage, handling, and transport of } \\
\text { SNM in all forms including uranium and plutonium metal, } \\
\text { oxide, components, and waste. }\end{array}$ \\
\hline -Protection & $\begin{array}{l}\text { - INEL dedicated safeguards and security contractor experience, well } \\
\text { trained and equipped to provide necessary protection and initiate } \\
\text { effective emergency responses to security infractions, is } \\
\text { applicable to providing additional personnel, fences, and guard } \\
\text { posts for Pu Disposition Complex. }\end{array}$ \\
\hline
\end{tabular}


Table F-1 Idaho National Engineering Laboratory (INEL) (continued)

\begin{tabular}{|c|c|c|}
\hline Capability Requirement & & Current Capability at INEL \\
\hline \multicolumn{3}{|l|}{$\begin{array}{l}\text { Safety and Environmental } \\
\text { Approval }\end{array}$} \\
\hline -Safety Approval & $\mathrm{U}$ & $\begin{array}{l}\text { - INEL has experience with safety approval by DOE of } \\
\text { nuclear facilities including reactors. } \\
\text {-INEL technical staff provides assistance to Office of } \\
\text { Nuclear Reactor Regulation (NRR) in instrumentation } \\
\text { and control systems, electrical and mechanical } \\
\text { components and systems, in-service and pre-service } \\
\text { inspection and testing of piping systems, equipment } \\
\text { qualification, radiological issues, operator licensing } \\
\text { examinations and training programs, standard technical } \\
\text { specifications and plant specific technical } \\
\text { specifications, license renewal activities, ALWR } \\
\text { issues, and thermalhydraulic analysis. Recent } \\
\text { emphasis has been less on operational aspects and } \\
\text { more on ALWR issues. } \\
\text {-DOE and NRC requirements are similar. Experience } \\
\text { with DOE safety approval procedures, and with } \\
\text { technical assistance to NRR, is applicable to extension } \\
\text { of capability to accommodate NRC procedures. }\end{array}$ \\
\hline -Environmental Approval & $\mathbf{E}$ & $\begin{array}{l}\text { - Management and staff experienced with environmental } \\
\text { approval requirements to obtain Record Of Decision } \\
\text { (ROD) by DOE with EPA concurrence for nuclear } \\
\text { facilities including reactors. } \\
\text { - Site data developed for ROD issued to INEL for } \\
\text { Environmental Impact Statement for Special Isotope } \\
\text { Separation Project, and other evaluations (EIS and EA } \\
\text { reports listing available upon request), is applicable to } \\
\text { Pu Disposition Complex. }\end{array}$ \\
\hline $\begin{array}{l}\text {-Federal/State/Local } \\
\text { Permitting }\end{array}$ & $\mathrm{E}$ & -INEL has experience in permitting at all levels. \\
\hline \multicolumn{3}{|l|}{ Transportation } \\
\hline -Site Access & $\mathbf{E}$ & $\begin{array}{l}\text {-Access by air via Idaho Falls, ID. } \\
\text {-Access by road includes two US highways and one } \\
\text { State highway crossing INEL boundaries. } 230 \text { miles } \\
\text { on-site roadway classified principal arterial and major } \\
\text { collector routes. } 500+\text { ton load capability on INEL } \\
\text { roads. } \\
\text {-Access by rail is Union Pacific connection at Scoville } \\
\text { Siding to government-owned spur line linking } \\
\text { developed areas within INEL. Gantry crane at Scoville } \\
\text { Siding and spur line handle up to } 160 \text { ton loads, } \sim 15^{\prime} \\
\text { high x } 10^{\prime} \text { wide for heavy equipment delivery during } \\
\text { construction. }\end{array}$ \\
\hline -Unloading \& Inspection & $\mathrm{E}$ & $\begin{array}{l}\text { - Management and staff experienced in receiving SNM } \\
\text { in all forms. }\end{array}$ \\
\hline
\end{tabular}


Table F-1 Idaho National Engineering Laboratory (INEL) (continued)

\begin{tabular}{|c|c|c|}
\hline Capability Requirement & & Current Capability at INEL \\
\hline \multicolumn{3}{|l|}{$\begin{array}{l}\text { Transportation } \\
\text { (Continued) }\end{array}$} \\
\hline -On-Site Transport & E & $\begin{array}{l}\text {-Management and staff experienced in on-site transport } \\
\text { in SNM in all forms within and between protected } \\
\text { zones. } \\
\text { - Two US highways and state roadway cross INEL } \\
\text { boundaries. } \\
\text { - Proposed BWR plant and MOX fuel fabrication facility } \\
\text { sites within } \sim 3 \text { miles of each other, with no road } \\
\text { crossings or other facilities in between. }\end{array}$ \\
\hline -Packaging for Shipment & $\mathbf{E}$ & $\begin{array}{l}\text { - Management and staff experienced in shipping SNM in } \\
\text { all forms. }\end{array}$ \\
\hline \multicolumn{3}{|l|}{ Supporting Site Assets } \\
\hline -DOE Site Office & E & $\begin{array}{l}\text {-Experienced in directing nuclear programs and large } \\
\text { projects. } 52 \text { reactor projects have been completed at } \\
\text { INEL. } \\
\text { Sponsored and participated in meeting with GE Pu } \\
\text { disposition team. }\end{array}$ \\
\hline $\begin{array}{l}\text {-Site Contractor } \\
\text { Management }\end{array}$ & E & $\begin{array}{l}\text {-Operational experience managing nuclear processing } \\
\text { and large projects including many special purpose } \\
\text { reactors. } \\
\text {-Expressed strong support for use of electric power } \\
\text { producing fission reactor for Pu disposition and } \\
\text { confidence that complex could be built and operated } \\
\text { successfully at INEL. } \\
\text { - Considers plutonium to be a national resource. }\end{array}$ \\
\hline -Work Force & & $\begin{array}{l}\text { - Technical and production oriented work force can } \\
\text { support large plutonium processing and reactor } \\
\text { construction and operation project. } \\
\text { - INEL has } \sim 12,500 \text { employees of which } \sim 4600 \text { hold } \\
\text { professional degrees. }\end{array}$ \\
\hline _Utilities & E & $\begin{array}{l}\text {-Adequate water supply and distribution system for } \\
\text { reactor makeup and complex operations. } \\
\text {-Adequate electric power provided by Idaho Power } 230 \\
\text { KV transmission line to INEL to support large } \\
\text { construction project. }\end{array}$ \\
\hline -Technology Development & E & $\begin{array}{l}\text {-Argonne National Laboratory, West is technology } \\
\text { center for DOE programs. }\end{array}$ \\
\hline -Quality Assurance & E & $\begin{array}{l}\text {-Experienced in working to DOE quality assurance } \\
\text { requirements. Also NRC and industry related (such as } \\
\text { ASME, ANS, IEEE, NQA1 \& 2, etc.) quality } \\
\text { assurance requirements. }\end{array}$ \\
\hline -Safety & $\mathrm{E}$ & $\begin{array}{l}\text { - Management and staff experienced in meeting or } \\
\text { exceeding all health and safety requirements associated } \\
\text { with nuclear programs. Long history of safe operation } \\
\text { at INEL. }\end{array}$ \\
\hline
\end{tabular}


NEDO-32361

Table F-1 Idaho National Engineering Laboratory (INEL) (continued)

\begin{tabular}{|c|c|}
\hline Capability Requirement & Current Capability at INEL \\
\hline $\begin{array}{l}\text { Supporting Site Assets } \\
\text { (continued) }\end{array}$ & \\
\hline -Environmental Protection & $\begin{array}{l}\text { E - Management and staff experienced in protection of } \\
\text { environment. Environmental restoration programs are } \\
\text { operational at INEL [TBR]. }\end{array}$ \\
\hline -Community Support & $\begin{array}{l}\text { E INEL management believes there is broad community } \\
\text { support in Idaho for nuclear activities based on long } \\
\text { history of safe operations at INEL which will facilitate } \\
\text { public acceptance of Pu Disposition Complex. } \\
\text {-Idaho Falls community leaders under "Initiative 2000" } \\
\text { will support new nuclear activities at INEL which meet } \\
\text { environmental concerns [TBR]. } \\
\text { - President of Idaho State AFL-CIO supports present } \\
\text { and future projects at INEL. } \\
\text { - Long history of support by Idaho elected } \\
\text { congressional, staie, county, and city officials. } \\
\text { Congressional delegations unanimously supported } \\
\text { defense-related projects such as Special Isotope } \\
\text { Separation Project (producing plutonium) and Complex } \\
\text { 21 at INEL. New nuclear activities WHICH DO NOT } \\
\text { INCLUDE PERMANENT STORAGE OF NUCLEAR } \\
\text { WASTE SUCH AS SPENT FUEL will be welcomed } \\
\text { [TBR]. } \\
\text {-Public interest groups include Environmental Defense } \\
\text { Institute. Is requesting large amount of information on } \\
\text { radioactive and chemical releases and accidents and } \\
\text { worker radiation records under FOlA. }\end{array}$ \\
\hline
\end{tabular}


Table F-2. Nevadla Test Site

\begin{tabular}{|c|c|}
\hline Capability Requirement & Current Capability at NTS \\
\hline Overall Site Qualification & $\begin{array}{l}\text { E Currently operating. Has served for } \sim 40 \text { years as DOE } \\
\text { site for nuclear weapons testing. Current mission is to } \\
\text { maintain readiness to resume nuclear testing if } \\
\text { required. } \\
\text {-Occupies } \sim 1350 \text { square miles } \sim 65 \text { miles northwest of } \\
\text { Las Vegas, NV. Bordered on three sides by additional } \\
4120 \text { square miles federally controlled (Nellis Air } \\
\text { Force Range). }\end{array}$ \\
\hline \multicolumn{2}{|l|}{$\begin{array}{l}\text { PuO2 Feed Material } \\
\text { Interface } \\
\text { (IF w/Nuclear Weapons } \\
\text { Complex Reconfig Program) }\end{array}$} \\
\hline $\begin{array}{l}\text {-Pu Receiving and Storage } \\
\text { (within weapons program) }\end{array}$ & $\begin{array}{l}\text { G - New capability needed for handling } \mathrm{Pu} \text { in forms for } \\
\text { processing. }\end{array}$ \\
\hline $\begin{array}{l}\text {-Pu-to-PuO2 Conversion } \\
\text { (within weapons program) }\end{array}$ & $\begin{array}{l}\text { G - New capability needed for Pu-to-PuO2 conversion } \\
\text { processing. }\end{array}$ \\
\hline $\begin{array}{l}\text {-PuO2 Feed Interface } \\
\text { Management }\end{array}$ & $\begin{array}{l}\text { U Experience managing nuclear weapons testing could be } \\
\text { extended to capability for management of interface with } \\
\mathrm{PuO} 2 \text { supplier. }\end{array}$ \\
\hline \multicolumn{2}{|l|}{ MOX Fuel Fabrication } \\
\hline $\begin{array}{l}\text {-MOX Pellet and Pin } \\
\text { Fabrication }\end{array}$ & $\begin{array}{l}\text { G - New capability needed for MOX fuel fabrication } \\
\text { processing. }\end{array}$ \\
\hline $\begin{array}{l}\text {-BWR MOX Assembly } \\
\text { Fabrication }\end{array}$ & $\begin{array}{l}\text { G } \quad \text { MOX assemblies could be fabricated in greenfield } \\
\text { facility built for MOX pellet and pin fabrication. }\end{array}$ \\
\hline $\begin{array}{l}\text {-Fresh MOX Assembly } \\
\text { Storage }\end{array}$ & $\begin{array}{l}\text {-Fresh MOX assemblies could be stored in greenfield } \\
\text { facility built for MOX pellet and pin fabrication. }\end{array}$ \\
\hline \multicolumn{2}{|l|}{$\begin{array}{l}\text { Spent Fuel Storage } \\
\text { Interface }\end{array}$} \\
\hline $\begin{array}{l}\text { Spent Fuel Storage } \\
\text { (within waste disposal } \\
\text { program) }\end{array}$ & $\begin{array}{l}\text {-New capability needed for storage of spent fuel at } \\
\text { NTS. }\end{array}$ \\
\hline $\begin{array}{l}\text {-Spent Fuel Interface } \\
\text { Management }\end{array}$ & $\begin{array}{l}\text { U - Extension of capability needed for management of } \\
\text { interface with Nuclear Waste Disposal Program } \\
\text { whether or not spent fuel is stored or transported by } \\
\text { NTS }\end{array}$ \\
\hline $\begin{array}{l}\text {-Spent Fuel Transport } \\
\text { (within waste disposal } \\
\text { program) }\end{array}$ & - New capability needed for transport of spent fuel. \\
\hline
\end{tabular}


NEDO-32361

Table F-2. Nevada Test Site

(Continued)

\begin{tabular}{|c|c|c|}
\hline Capability Requirement & & Current Capability at NTS \\
\hline \multicolumn{3}{|l|}{ Waste Management } \\
\hline -High Level Waste (HLW) & $\mathrm{E}$ & $\begin{array}{l}\text { - Management and staff experienced in handling HLW } \\
\text { [TBR]. }\end{array}$ \\
\hline -Transuranic Waste (TRU) & E & $\begin{array}{l}\text { - Management and staff experienced in handling TRU } \\
\text { [TBRI. }\end{array}$ \\
\hline -Low Level Waste (LLW) & $\mathrm{E}$ & $\begin{array}{l}\text {-Radioactive Waste Management Site in Area } 5 \text { includes } \\
92 \text { acres for surface storage and disposal of LLW. }\end{array}$ \\
\hline -Hazardous Waste & E & $\begin{array}{l}\text { - Management and staff experienced in handling } \\
\text { hazardous wastes [TBR]. }\end{array}$ \\
\hline -Mixed Waste & $\mathrm{E}$ & $\begin{array}{l}\text { - Managerent and staff experienced in handling mixed } \\
\text { wastes [1BR]. }\end{array}$ \\
\hline $\begin{array}{l}\text { - Nuclear Waste Interface } \\
\text { Management }\end{array}$ & $\mathrm{E}$ & $\begin{array}{l}\text { - Experience managing nuclear wastes is applicable to } \\
\text { management of interface with Nuclear Waste Disposal } \\
\text { Program }\end{array}$ \\
\hline \multicolumn{3}{|l|}{ Safeguards \& Security } \\
\hline -Accountability & $\mathrm{U}$ & $\begin{array}{l}\text {-Management and staff experienced in strict } \\
\text { accountability of nuclear weapons components. } \\
\text { Upgrading needed for accountability of Pu in forms for } \\
\text { processing. }\end{array}$ \\
\hline -Protection & $\mathrm{U}$ & $\begin{array}{l}\text {-NTS experience in protection of nuclear facilities is } \\
\text { applicable to providing additional personnel, fences, } \\
\text { and guard posts for Pu Disposition Complex. }\end{array}$ \\
\hline \multicolumn{3}{|l|}{$\begin{array}{l}\text { Safety and Environmental } \\
\text { Approval }\end{array}$} \\
\hline Safety Approval & $\mathrm{U}$ & $\begin{array}{l}\text { - Experienced with safety approval by DOE for weapons } \\
\text { testing related facilities. This capability will need to be } \\
\text { upgraded for DOE and NRC requirements for reactor } \\
\text { and } \mathrm{Pu} \text { processing facilities. }\end{array}$ \\
\hline -Environmental Approval & $\mathrm{U}$ & $\begin{array}{l}\text {-Management and staff have experience with } \\
\text { Environmental Assessment and Environmental Impact } \\
\text { Statement requirements. Currently preparing data for } \\
\text { EIS for upgrade alternative for Assembly/Disassembly } \\
\text { function under Nuclear Weapons Complex } \\
\text { Reconfiguration Program. This experience is } \\
\text { applicable to extension of capability needed for reactor } \\
\text { and Pu processing facilities. }\end{array}$ \\
\hline $\begin{array}{l}\text {-Federal/State/Local } \\
\text { Permitting }\end{array}$ & $\mathrm{E}$ & -Experienced in permitting at all levels. \\
\hline
\end{tabular}


Table F-2. Nevada Test Site (Continued)

\begin{tabular}{|c|c|c|}
\hline Capability Requirement & & Current Capability at NTS \\
\hline \multicolumn{3}{|l|}{ Transportation } \\
\hline- Site Access & $\mathbf{E}$ & $\begin{array}{l}\text { - Access by air via Las Vegas, NV. Desert Rock Airport } \\
\text { on NTS has } 7500^{\prime} \text { long x } 100^{\prime} \text { wide runway capable of } \\
\text { accepting jet aircraft. } \\
\text { - Access by road is four lane, divided US95 which } \\
\text { intersects I15. Mature on-site road infrastructure. } \\
\text { Adequate for construction. } \\
\text { - Access by rail via rail head at Las Vegas with Union } \\
\text { Pacific line to NTS. }\end{array}$ \\
\hline —Unloading \& Inspection & $\mathrm{U}$ & $\begin{array}{l}\text {-Management and staff experience in receiving nuclear } \\
\text { weapons components is applicable to upgrading of } \\
\text { capability needed for receiving Pu in forms for } \\
\text { processing. }\end{array}$ \\
\hline -On-Site Transport & $\mathbf{E}$ & $\begin{array}{l}\text { - Management and staff experienced in on-site transport } \\
\text { of SNM. } \\
\text {-No public roads on NTS. }\end{array}$ \\
\hline -Packaging for Shipment & $\mathrm{E}$ & $\begin{array}{l}\text { - Management and staff experienced in packaging } \\
\text { nuclear components and wastes for shipment off-site. }\end{array}$ \\
\hline \multicolumn{3}{|l|}{ Supporting Site Assets } \\
\hline -DOE Site Office & $\mathrm{U}$ & $\begin{array}{l}\text {-Experienced in directing nuclear weapons testing } \\
\text { activities. Applicable to extension of capability needed } \\
\text { for reactor and Pu processing activities. } \\
\text {-Plutonium considered to be a resource. }\end{array}$ \\
\hline $\begin{array}{l}\text { Site Contractor } \\
\text { Management }\end{array}$ & $\mathrm{U}$ & $\begin{array}{l}\text {-Operational experience managing nuclear weapons } \\
\text { testing activities. Applicable to extension of capability } \\
\text { needed for reactor and Pu processing activities. }\end{array}$ \\
\hline —Work Force & $\mathbf{U}$ & $\begin{array}{l}\text { - Trained, experienced, educated industrial base could } \\
\text { supply skilled professional, technical, craft persons. } \\
\text { Expansion needed for Pu processing and reactor } \\
\text { operation. } \\
\text {-NTS has } ~ 3500 \text { employees. }\end{array}$ \\
\hline -Utilities & $\mathrm{E}$ & $\begin{array}{l}\text { 9E6 gpd water available at NTS from } 14 \text { existing } \\
\text { wells. 2E6 gpd beyond current usage available from } \\
\text { Area } 6 \text { System for proposed Complex } 21 \text { site. } \\
\text { Additional wells can be drilled. } \\
\text {-Two independent } 138 \mathrm{kV} \text { transmissions lines } \\
\text { providing } 25-30 \mathrm{MW} \text { peak load, with } 10-15 \mathrm{MW} \text { to be } \\
\text { added within next few years. }\end{array}$ \\
\hline -Technology Development & $\mathbf{E}$ & $\begin{array}{l}\text {-Nevada Research \& Development Area (NRDA) } \\
\text { experienced with technology development for nuclear } \\
\text { rocket program. EMAD facility located in this area. }\end{array}$ \\
\hline -Quality Assurance & $\mathbf{E}$ & $\begin{array}{l}\text {-Experienced in working to DOE quality assurance } \\
\text { requirements. }\end{array}$ \\
\hline
\end{tabular}


Table F-2. Nevada Test Site

(Continued)

\begin{tabular}{|c|c|}
\hline Capability Requirement & Current Capability at NTS \\
\hline $\begin{array}{l}\text { Supporting Site Assets } \\
\text { (Continued) }\end{array}$ & \\
\hline -Safety and Health & $\begin{array}{l}\text { E - Management and staff experienced in meeting or } \\
\text { exceeding all health and safety requirements associated } \\
\text { with nuclear programs. }\end{array}$ \\
\hline -Environmental Protection & $\begin{array}{l}\text { - Management and staff experienced in protection of the } \\
\text { environment. }\end{array}$ \\
\hline -Community Support & $\begin{array}{l}\text { - Long-standing support from local communities for } \\
\text { DOE defense programs. } \\
\text { - Congressional delegation, governor, and state, } \\
\text { regional, and local officials publicly support continued } \\
\text { defense-related projects at NTS. } \\
\text { - Nevada Test Site Contractors Association supports } \\
\text { new programs at NTS. } \\
\text {-Political opposition to plutonium storage at NTS. } \\
\text { Connected with opposition to disposal of high level } \\
\text { waste at Yucca Mountain. }\end{array}$ \\
\hline
\end{tabular}


Table F-3. Oak Ridge Reservation

\begin{tabular}{|c|c|}
\hline Capability Requirement & Current Capability at ORR \\
\hline Overall Site Qualification & $\begin{array}{l}\text { - Has served for } \sim 50 \text { years as DOE site for energy R\&D } \\
\text { and weapons uranium and lithium production. Current } \\
\text { missions are energy R\&D, weapons dismantling, and } \\
\text { uranium and lithium storage, waste management, and } \\
\text { environmental restoration. } \\
\text { - Currently operating, with } ~-[T B D] \text { employees. } \\
\text { - Occupies } \sim 54 \text { square miles } \sim 17 \text { miles northwest of } \\
\text { Knoxville, TN. } \\
\text {-Three technology areas: X10 (Oak Ridge National } \\
\text { Laboratory), Y12, K25. }\end{array}$ \\
\hline Pu Feed Material In & \\
\hline $\begin{array}{l}\text {-Pu Receiving and Storage } \\
\text { (within weapons program) }\end{array}$ & $\begin{array}{l}\text { E - Management and staff experienced with SNM handling } \\
\text { including Pu. } \\
\text { - Pu could be stored in [TBD] existing facilities. }\end{array}$ \\
\hline $\begin{array}{l}\text {-Pu-to-PuO2 Conversion } \\
\text { (within weapons program) }\end{array}$ & $\begin{array}{l}\text { U/G Y12 facilities available for upgrading for Pu-to- } \\
\text { PuO2 conversion. New process equipment needed. } \\
\text {-Also greenfield available within } 10,000 \text { acres proposed } \\
\text { for Complex } 21 \text { site just West of Y12. } \\
\text { - Management and staff have lab scale Pu-to-PuO2 } \\
\text { conversion experience. [TBR] }\end{array}$ \\
\hline $\begin{array}{l}\text {-PuO2 Interface } \\
\text { Management }\end{array}$ & $\begin{array}{l}\text { - Extensive experience at ORR in } \\
\text { management/handling/transport/accountability of } \\
\text { enriched uranium, and also Pu and PuO2 [TBR], is } \\
\text { applicable to management of interface with supplier of } \\
\text { PuO2 feed material whether or not Pu-to-PuO2 } \\
\text { conversion function is located at ORR. }\end{array}$ \\
\hline MOX Fuel Fabrication & \\
\hline $\begin{array}{l}\text {-MOX Pellet and Pin } \\
\text { Fabrication }\end{array}$ & $\begin{array}{l}\text { U/G Y12 facilities available for upgrading and new } \\
\text { process equipment for MOX blending, pellet sintering, } \\
\text { and pin loading operations. } \\
\text { - Also greenfield available within proposed Complex } 21 \\
\text { site. } \\
\text { - Management and staff have pilot scale experience } \\
\text { producing MOX powder using sol-gel and gel sphere } \\
\text { processes. }\end{array}$ \\
\hline $\begin{array}{l}\text {-BWR MOX Assembly } \\
\text { Fabrication }\end{array}$ & $\begin{array}{l}\text {-Existing Y12 facilities adaptable for MOX assembly } \\
\text { fabrication. [TBR] } \\
\text { - Could also be included within MOX pellet and pin } \\
\text { fabrication facility located at either Y } 12 \text { or Complex } 21 \\
\text { site. }\end{array}$ \\
\hline $\begin{array}{l}\text {-Fresh MOX Assembly } \\
\text { Storage }\end{array}$ & $\begin{array}{l}\text { - Existing Y12 facilities adaptable for fresh MOX } \\
\text { assembly storage. [TBR] } \\
\text { - Could also be included within MOX pellet and pin } \\
\text { fabrication facility located at either Y12 or Complex } 21 \\
\text { site. }\end{array}$ \\
\hline
\end{tabular}


NEDO-32361

Table F-3. Oak Ridge Reservation (Continued)

\begin{tabular}{|c|c|c|}
\hline Capability Requirement & & Current Capability at ORR \\
\hline \multicolumn{3}{|l|}{$\begin{array}{l}\text { Spent Fuel Storage } \\
\text { Interface }\end{array}$} \\
\hline $\begin{array}{l}\text {-Spent Fuel Storage } \\
\text { (within waste disposal } \\
\text { program) }\end{array}$ & $\mathbf{E}$ & $\begin{array}{l}\text { - Facilities at ORR for temporary storage, pending } \\
\text { ultimate disposal, of spent fuel from the BWR Pu } \\
\text { Disposition Complex include [TBD] include [TBD]. }\end{array}$ \\
\hline $\begin{array}{l}\text {-Spent Fuel Transport } \\
\text { (within waste disposal } \\
\text { program) }\end{array}$ & $\mathbf{E}$ & $\begin{array}{l}\text { - Management and staff experienced in transport of spent } \\
\text { fuel. }\end{array}$ \\
\hline $\begin{array}{l}\text {-Spent Fuel Interface } \\
\text { Management }\end{array}$ & $\mathbf{E}$ & $\begin{array}{l}\text { - Experience managing spent fuel from reactors at ORR } \\
\text { is applicable to management of interface with US Spent } \\
\text { Fuel Disposal Program }\end{array}$ \\
\hline \multicolumn{3}{|l|}{ Waste Management } \\
\hline -High Level Waste (HLW) & $\mathbf{E}$ & $\begin{array}{l}\text { - Management and staff experienced in handling HLW in } \\
\text { the form of irradiated reactor components such as } \\
\text { control rods and core structural elements [TBR]. } \\
\text {-HLW is stored for future disposal at the [TBD] } \\
\text { facilities at ORNL. }\end{array}$ \\
\hline -Transuranic Waste (TRU) & $\mathbf{E}$ & $\begin{array}{l}\text {-Management and staff experienced in handling TRU. } \\
\text { - Solid transuranic waste is stored for future treatment } \\
\text { and disposal at the [TBD] facility at ORNL. Solid Pu } \\
\text { scrap from the NFS plant is also being transferred to } \\
\text { ORNL. A new facility at ORNL to treat and package } \\
\text { solid transuranic waste to specifications for ultimate } \\
\text { disposal at WIPP has been proposed to DOE. } \\
\text { - Liquid and sludge transuranic waste is stored at the } \\
\text { [TBD] storage tank facility at ORNL. A new facility at } \\
\text { ORNL to treat 3-600,000 gallons of transuranic } \\
\text { liquid/sludge to specifications for ultimate disposal at } \\
\text { WIPP has been proposed to DOE. }\end{array}$ \\
\hline - Low Level Waste (LLW) & $\mathbf{E}$ & -Management and staff experienced in handling LLW. \\
\hline -Hazardous Waste & $\mathbf{E}$ & $\begin{array}{l}\text {-Management and staff experienced in handling } \\
\text { hazardous wastes. }\end{array}$ \\
\hline -Mixed Waste & $\mathbf{E}$ & $\begin{array}{l}\text { - Management and staff are experienced in handling } \\
\text { mixed wastes. } \\
\text {-ORR has the only licensed operating Toxic Substances } \\
\text { Control Act (TSCA) incinerator in the US to handle } \\
\text { mixed waste. Currently handling liquid. Capable of } \\
\text { handling solid. }\end{array}$ \\
\hline $\begin{array}{l}\text { - Nuclear Waste Interface } \\
\text { Management }\end{array}$ & $\mathbf{E}$ & $\begin{array}{l}\text {-Experience managing all forms of nuclear waste is } \\
\text { applicable to management of interface with Nuclear } \\
\text { Waste Disposal Program. }\end{array}$ \\
\hline
\end{tabular}


Table F-3. Oak Ridge Reservation (Continued)

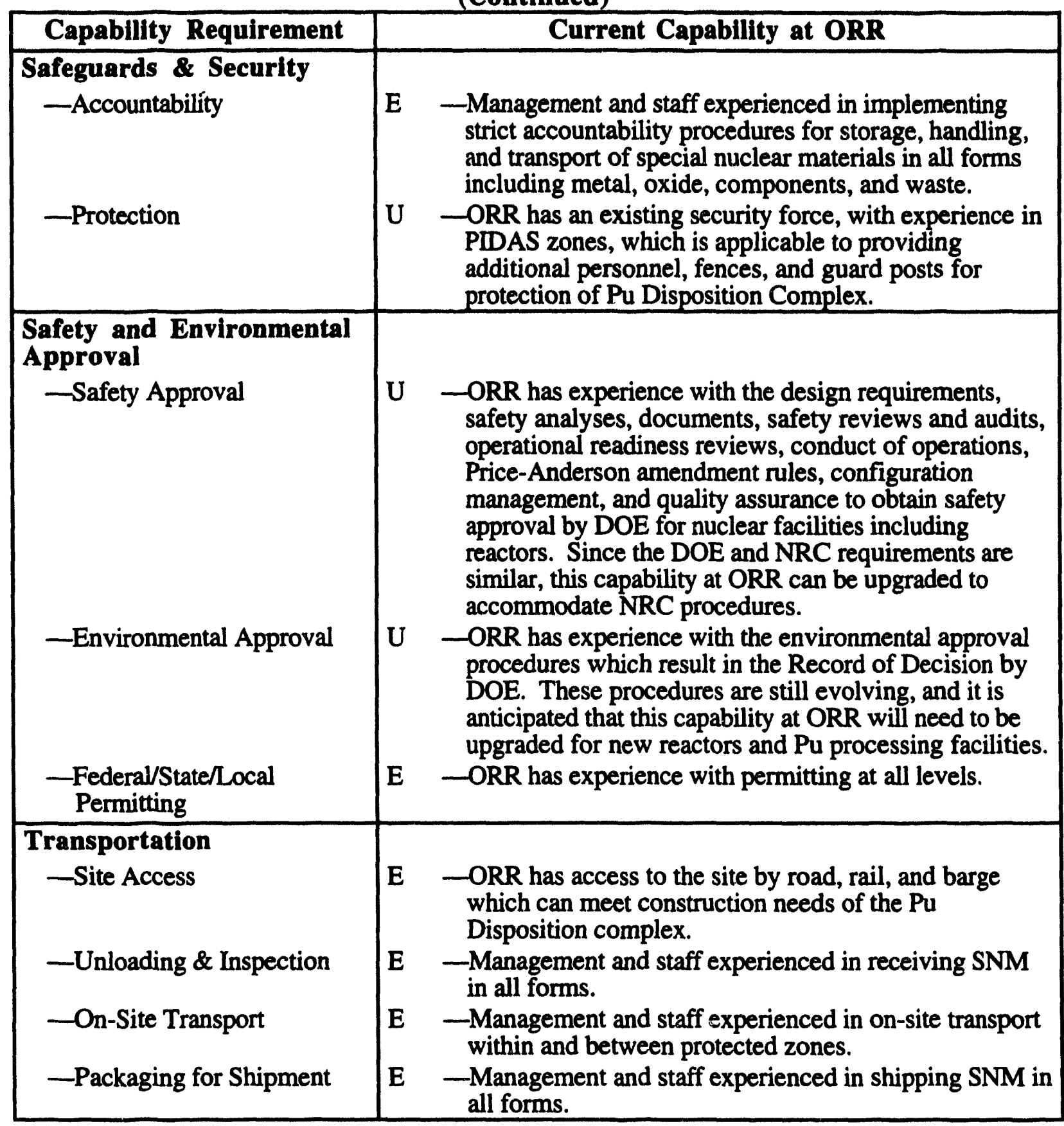


NEDO-32361

Table F-3. Oak Ridge Reservation (Continued)

\begin{tabular}{|c|c|c|}
\hline Capability Requirement & & Current Capability at ORR \\
\hline \multicolumn{3}{|l|}{ Supporting Site Assets } \\
\hline -DOE Site Office & $\mathbf{E}$ & $\begin{array}{l}\text {-Experience in directing nuclear programs and large } \\
\text { projects is applicable to Pu Disposition Complex. }\end{array}$ \\
\hline $\begin{array}{l}\text {-Site Contractor } \\
\text { Management }\end{array}$ & $\mathrm{E}$ & $\begin{array}{l}\text { Operational experience in management of plutonium } \\
\text { and tritium processing and technology and large } \\
\text { projects including reactors is applicable to } \mathrm{Pu} \\
\text { Disposition Complex. } \\
\text { Site contractor management has indicated strong } \\
\text { interest in the Pu Disposition Complex and believe } \\
\text { ORR could accommodate the program. }\end{array}$ \\
\hline -Work Force & $\mathbf{E}$ & $\begin{array}{l}\text { - Technical and production oriented work force can } \\
\text { support large plutonium and tritium construction and } \\
\text { operation project. }\end{array}$ \\
\hline -Utilities & $\mathbf{E}$ & $\begin{array}{l}\text {-Adequate water for reactor makeup and complex } \\
\text { operations. } \\
\text {-Adequate electric power and other utilities to support } \\
\text { major construction project. }\end{array}$ \\
\hline -Technology Development & $\mathbf{E}$ & $\begin{array}{l}\text { - ORNL is multipurpose research laboratory in energy } \\
\text { related areas. }\end{array}$ \\
\hline -Quality Assurance & E & $\begin{array}{l}\text {-Experienced in working to DOE quality assurance } \\
\text { requirements. }\end{array}$ \\
\hline -Safety and Health & $\mathrm{E}$ & $\begin{array}{l}\text {-Management and staff experienced in meeting or } \\
\text { exceeding all health and safety requirements associated } \\
\text { with nuclear programs. }\end{array}$ \\
\hline -Environmental Protection & $\mathrm{E}$ & $\begin{array}{l}\text { - Management and staff experienced in protection of the } \\
\text { environment. Environmental restoration programs are } \\
\text { operational at ORR. }\end{array}$ \\
\hline -Community Support & $\mathbf{E}$ & $\begin{array}{l}\text { - Indications are that state and local officials will be } \\
\text { receptive to the handling of plutonium at ORR, based } \\
\text { on recent public hearings for the Complex } 21 \text { Program } \\
\text { Environmental Impact Statement. }\end{array}$ \\
\hline
\end{tabular}


Table F-4 Pantex Plant

\begin{tabular}{|c|c|}
\hline Capability Requirement & Current Capabillity at Pantex \\
\hline Overall Site Qualification & $\begin{array}{l}\text { E - Currently operating. Has served for }>40 \text { years as DOE } \\
\text { site for assembly and disassembly of nuclear weapons. } \\
\text { Current missions are (1) fabricate chemical high } \\
\text { explosive components for nuclear weapons, (2) } \\
\text { assemble nuclear weapons for the nation's stockpile, } \\
\text { (3) maintain and evaluate nuclear weapons in the } \\
\text { stockpile, and (4) disassemble nuclear weapons being } \\
\text { retired from the stockpile. Pantex has also been } \\
\text { designated as an interim storage site for Pu pits. } \\
\text { - Occupies } \sim 16,000 \text { acres } ~ 17 \text { miles northeast of } \\
\text { Amarillo, TX. }\end{array}$ \\
\hline $\begin{array}{l}\text { PuO2 Feed Material } \\
\text { Interface } \\
\text { (IF w/Nuclear Weapons } \\
\text { Complex Reconfig Program) }\end{array}$ & \\
\hline $\begin{array}{l}\text {-Pu Receiving and Storage } \\
\text { (within weapons program) }\end{array}$ & $\begin{array}{l}\text { G Handling of Pu in metallic form for processing would } \\
\text { be new capability. }\end{array}$ \\
\hline $\begin{array}{l}\text {-Pu-to-PuO2 Conversion } \\
\text { (within weapons program) }\end{array}$ & $\begin{array}{l}\text { G - Management and staff experienced in handling } \\
\text { weapons parts containing nuclear materials. Nuclear } \\
\text { materials have not been processed in the past, and this } \\
\text { would be new capability for Pantex. }\end{array}$ \\
\hline $\begin{array}{l}\text {-PuO2 Feed Interface } \\
\text { Management }\end{array}$ & $\begin{array}{l}\text { - Experience managing nuclear weapons components } \\
\text { could be extended to capability for management of } \\
\text { interface with PuO2 supplier. }\end{array}$ \\
\hline MOX Fuel Fabrication & \\
\hline $\begin{array}{l}\text {-MOX Pellet and Pin } \\
\text { Fabrication }\end{array}$ & $\begin{array}{l}\text { G - New capability needed for Pu processing for MOX } \\
\text { fuel fabrication. } \\
\text { - Pantex has experience with process robotics, including } \\
70 \% \text { automation of glove box operations and } 100 \% \\
\text { automation of handling of the pit in the Pit Reuse } \\
\text { Program. }\end{array}$ \\
\hline $\begin{array}{l}\text {-BWR MOX Assembly } \\
\text { Fabrication }\end{array}$ & $\begin{array}{l}\text {-MOX assemblies could be fabricated in greenfield } \\
\text { facility built for MOX pellet and pin fabrication. }\end{array}$ \\
\hline $\begin{array}{l}\text {-Fresh MOX Assembly } \\
\text { Storage }\end{array}$ & $\begin{array}{l}\text {-Fresh MOX assemblies could be stored in greenfield } \\
\text { facility built for MOX pellet and pin fabrication. }\end{array}$ \\
\hline
\end{tabular}


Table F-4 Pantex Plant

(Continued)

\begin{tabular}{|c|c|}
\hline Capability Requirement & Current Capability at Pantex \\
\hline \multicolumn{2}{|l|}{$\begin{array}{l}\text { Spent Fuel Storage } \\
\text { Interface }\end{array}$} \\
\hline $\begin{array}{l}\text {-Spent Fuel Storage } \\
\text { (within waste disposal } \\
\text { program) }\end{array}$ & $\begin{array}{l}\text { G -New capability needed for storage of spent fuel at } \\
\text { Pantex. }\end{array}$ \\
\hline $\begin{array}{l}\text { Spent Fuel Transport } \\
\text { (within waste disposal } \\
\text { program) }\end{array}$ & - New capability needed for transport of spent fuel. \\
\hline $\begin{array}{l}\text { Spent Fuel Interface } \\
\text { Management }\end{array}$ & $\begin{array}{l}\text { U - Expansion of capability needed for management of } \\
\text { interface with Nuclear Waste Disposal Program } \\
\text { whether or not spent fuel is stored or transported by } \\
\text { Pantex. }\end{array}$ \\
\hline \multicolumn{2}{|l|}{ Waste Management } \\
\hline -High Level Waste (HLW) & -HLW not currently generated or disposed of on-site. \\
\hline - Transuranic Waste (TRU) & -TRU not currently generated or disposed of on-site. \\
\hline - Low Level Waste (LLW) & - LLW not currently generated or disposed of on-site. \\
\hline -Hazardous Waste & $\begin{array}{l}\text { E - Management and staff experienced in handling } \\
\text { hazardous wastes. } \\
\text { - Other than burning of high explosive materials from } \\
\text { disassembled weapons, hazardous wastes not currently } \\
\text { disposed of on-site. }\end{array}$ \\
\hline & $\begin{array}{l}\text { - Mixed wastes not currently generated or disposed of } \\
\text { on-site. }\end{array}$ \\
\hline $\begin{array}{l}\text { - Nuclear Waste Interface } \\
\text { Management }\end{array}$ & $\begin{array}{l}\text {-Experience of Manager, Amarillo Area Office in } \\
\text { Transuranic Waste Program is applicable towards } \\
\text { expansion of capability for management of interface of } \\
\text { Pu processing and reactor waste functions with } \\
\text { Nuclear Waste Disposal Program. }\end{array}$ \\
\hline \multicolumn{2}{|l|}{ Safeguards \& Security } \\
\hline -Accountability & $\begin{array}{l}\text { - Management and staff experienced in strict } \\
\text { accountability of nuclear weapons components. } \\
\text { Safeguards \& Security Directorate includes nuclear } \\
\text { material control, nuclear material accounting, and } \\
\text { enhancement for protection of SNM. Also security } \\
\text { clearance and classified document control. Expansion } \\
\text { of capability needed for accountability of Pu in forms } \\
\text { for processing. }\end{array}$ \\
\hline -Protection & $\begin{array}{l}\text {-Pantex experience in protection of nuclear weapons } \\
\text { facilities is applicable to providing additional } \\
\text { personnel, fences, and guard posts for Pu Disposition } \\
\text { Complex. } \\
\text { - Safeguards \& Security Directorate includes security } \\
\text { force program. Experienced with PIDAS zones. }\end{array}$ \\
\hline
\end{tabular}


Table F-4 Pantex Plant

(Continued)

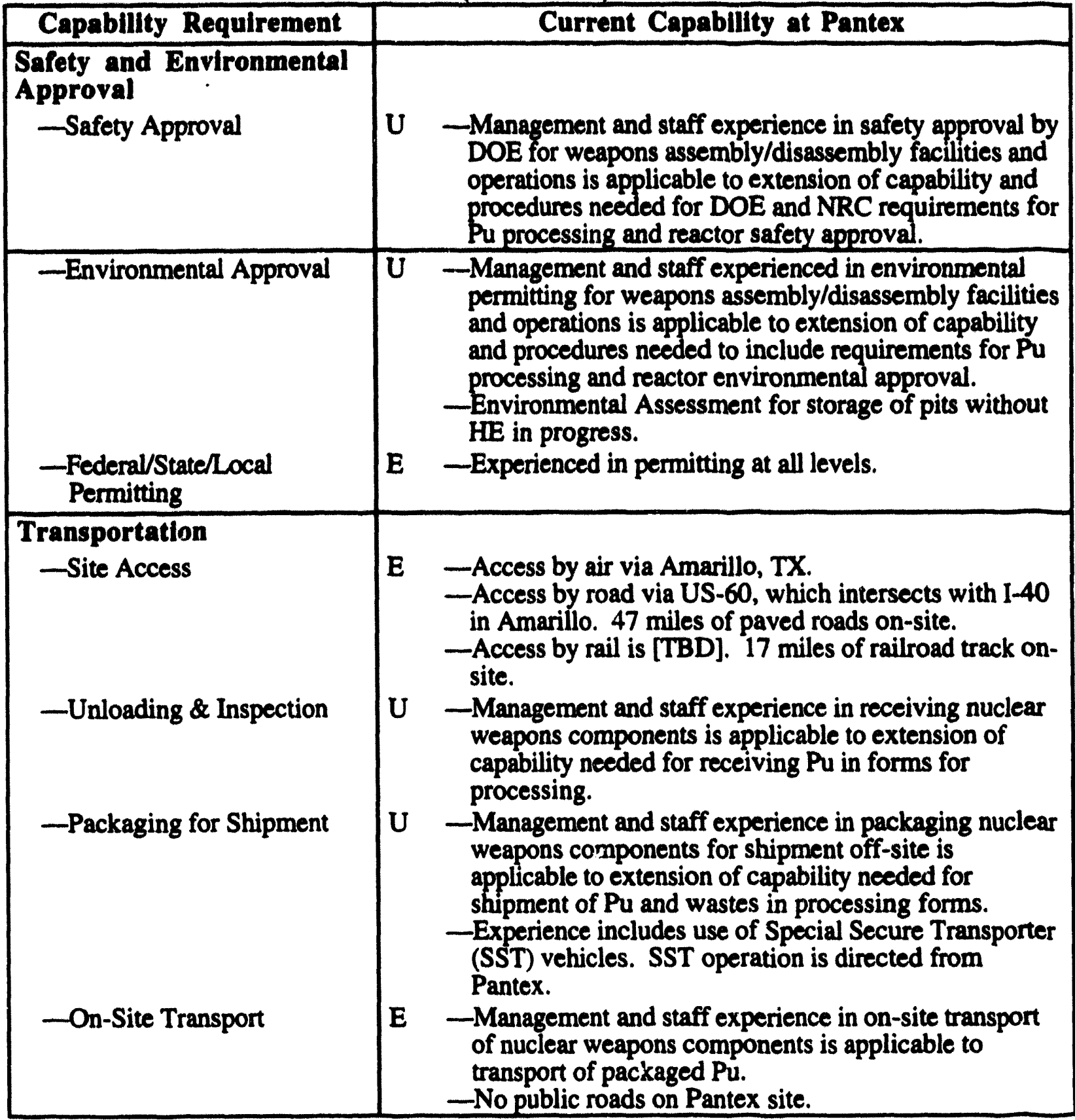


Table F-4 Pantex Plant (Continued)

\begin{tabular}{|c|c|}
\hline Capabillity Requirement & Current Capability at Pantex \\
\hline $\begin{array}{l}\text { Supporting Site Assets } \\
\text {-DOE Site Office }\end{array}$ & $\begin{array}{l}\text { E/U - Albuquerque Operations Office experience directing } \\
\text { nuclear programs and large projects is applicable to Pu } \\
\text { Disposition Complex. } \\
\text {-Amarillo Area Office experience in direction of nuclear } \\
\text { weapons assembly/disassembly related programs is } \\
\text { applicable to extension of capability needed for reactor } \\
\text { operation and Pu processing. } \\
\text {-Albuquerque Operations Office and Amarillo Area } \\
\text { Office consider plutonium to be a national resource. }\end{array}$ \\
\hline $\begin{array}{l}\text { Site Contractor } \\
\text { Management }\end{array}$ & $\begin{array}{l}\text { U Operational experience managing nuclear weapons } \\
\text { assembly/disassembly is applicable to extension of } \\
\text { capability needed for reactor and Pu processing. } \\
\text { Committed to growth by infusing new technologies } \\
\text { and broadened base of technical personnel into the } \\
\text { Pantex Plant. }\end{array}$ \\
\hline -Work Force & $\begin{array}{l}\text { U } 3000 \text { employees. } 29 \% \text { with bachelor's degree or } \\
\text { above. }>50 \% \text { of Pantex work force are technically } \\
\text { skilled employees. But not experienced with Pu } \\
\text { processing and reactor operations. }\end{array}$ \\
\hline -Utilities & $\begin{array}{l}\text { E -Adequate water available [TBR]. } \\
\text {-Adequate electric power for large construction project } \\
\text { [TBR]. }\end{array}$ \\
\hline -Technology Development & $\begin{array}{l}\text { U Advanced Technology Office (ATO) provides planning } \\
\text { and implementation of new technologies from Battelle, } \\
\text { DOE labs, industry, and universities for activities } \\
\text { related to nuclear weapons assembly and disassembly. } \\
\text { Expansion of this capability needed to support Pu } \\
\text { processing and reactor operation. } \\
\text { - Tester Design Engineering Department provides } \\
\text { design, development, modification, and maintenance of } \\
\text { automated electronic measurement systems for testing } \\
\text { of nuclear iveapons electrical circuitry. } \\
\text {-High Explosives Synthesis Facility develops new } \\
\text { chemical processes for explosives. } \\
\text { Other capabilities at Pantex applicable to technology } \\
\text { development include the Analytical Laboratory, } \\
\text { Nondestructive Evaluation (XRay) Department, Gas } \\
\text { Analysis Lab, Explosives Test Site, and others. }\end{array}$ \\
\hline -Quality Assurance & $\begin{array}{l}\text { - Experienced in working to DOE quality assurance } \\
\text { requirements. }\end{array}$ \\
\hline -Environmental Protection & $\begin{array}{l}\text { - Management and staff experienced in protection of } \\
\text { environment. Extension of capability needed for } \mathrm{Pu} \\
\text { processing and reactor operation. }\end{array}$ \\
\hline
\end{tabular}


Table F-4 Pantex Plant

(Continued)

\section{Capability Requirement Supporting Site Assets (Continued)}

-Safety and Health

- Community Support

\author{
Current Capability at Pantex
}

U - Management and staff experienced in meeting or exceeding all health and safety requirements associated with nuclear programs.

-Experience in safety for weapons assembly/disassembly facilities and operations is applicable to extension of capability needed for $\mathrm{Pu}$ processing and reactor operation such as criticality alarm and Pu detection and containment.

U - Community Relations Department activities include Information and Awareness, Community Involvement, Employee Communications, and Educational Development.

- Local community leaders in Panhandle 2000 support continuing current nuclear weapons activities at Pantex.

-Pantex is largest employer in Amarillo area.

-State political leaders concerned about long term storage of pits and potential for contamination of Ogalalla aquifer by $\mathrm{Pu}$ processing activities.

-Ranching and farming interests concerned about any adverse impact on source of water.

-University of Texas, Austin, Department of Economic Geology conducting study of geologic structure to conduct water and potential for contamination. Considers plutonium a resource.

-Public interest groups active around Pantex include Panhandle Area Neighbors and Landowners (PANAL), Serious Texans Against Nuclear Dumping (STAND), Save Texas Agricultural Resources (STAR), and Peace Farm. 


\section{Table F-5 Savannah River Site}

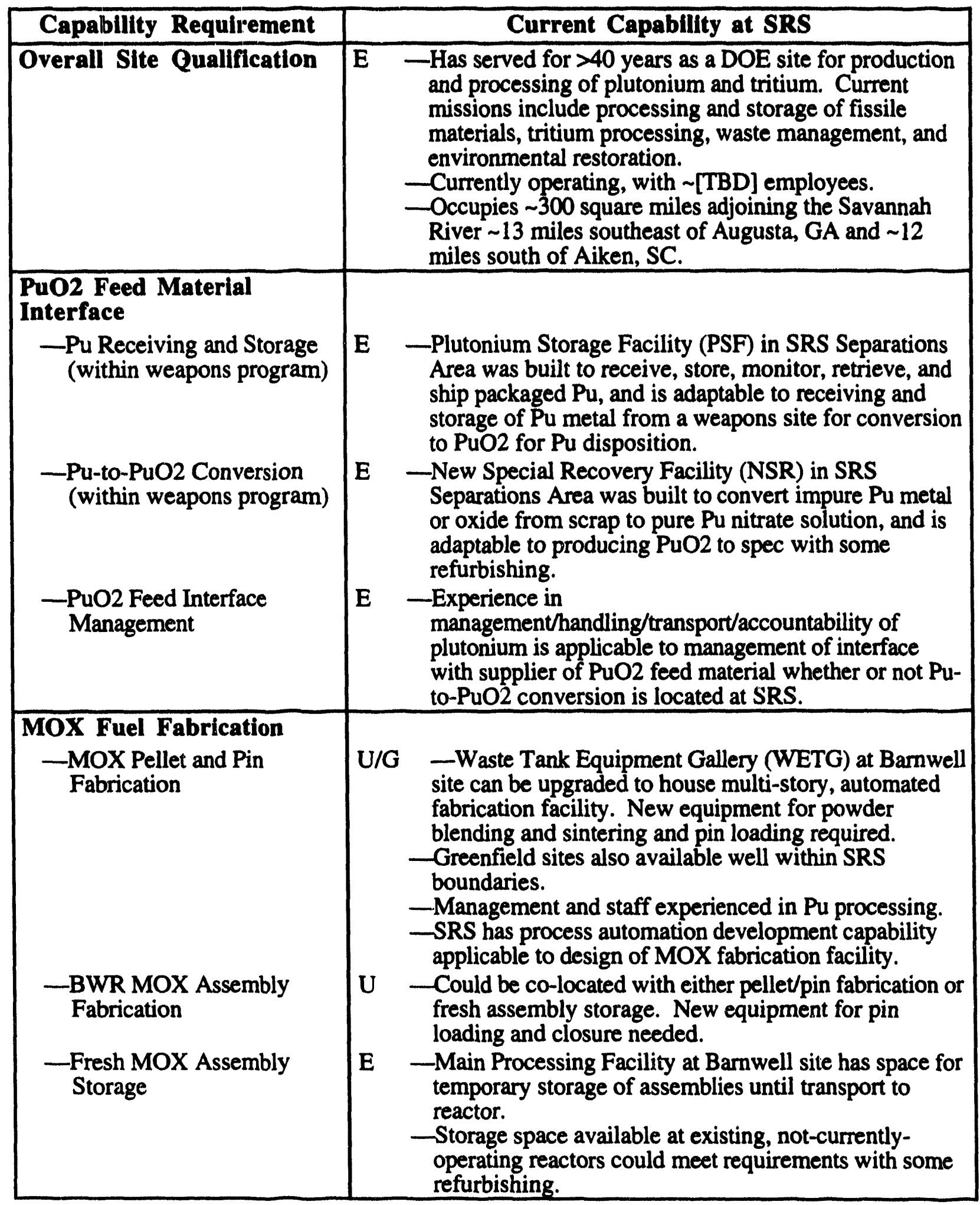


Table F-5 Savannah River Site (Continued)

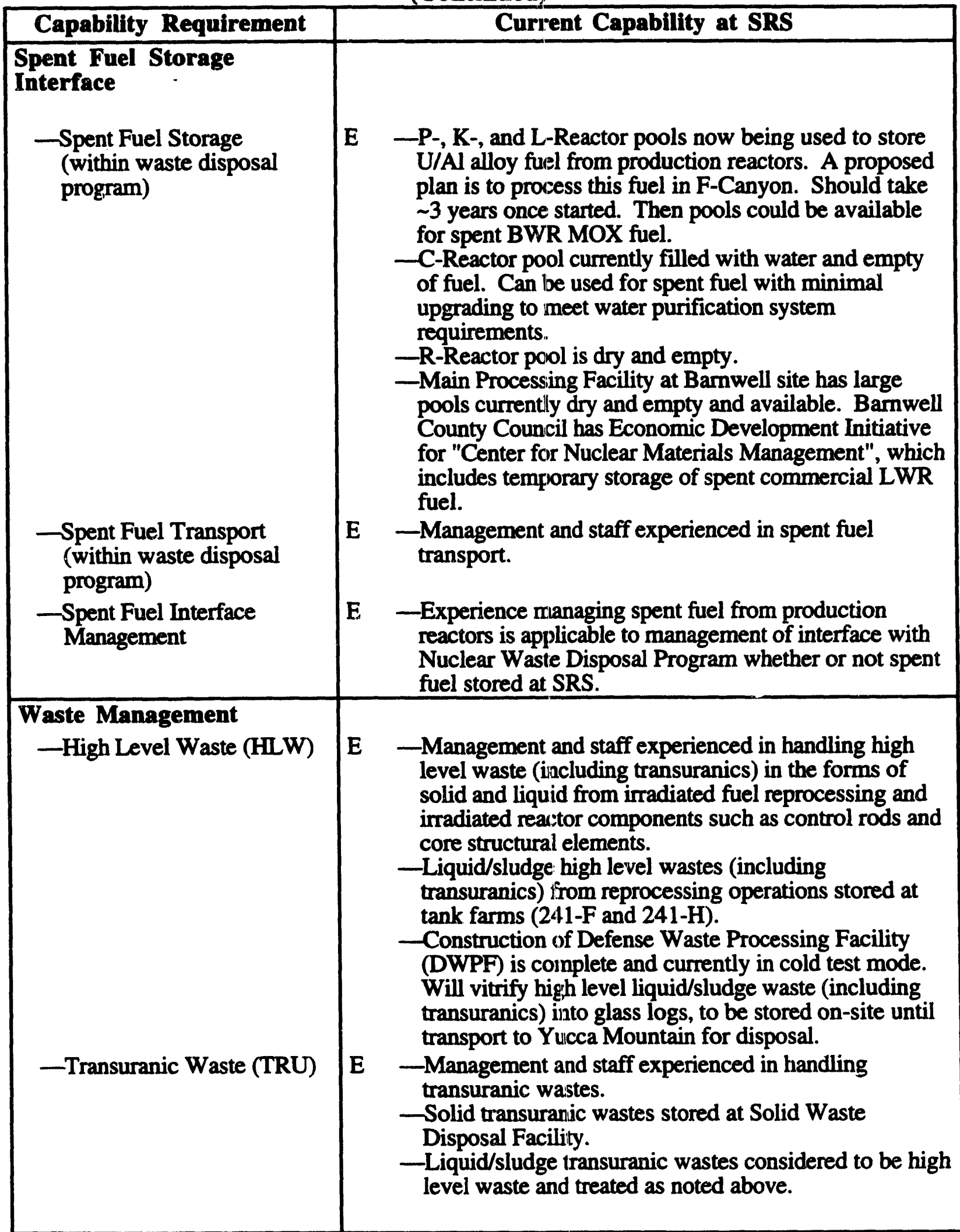


Table F-5 Savannah River Site (Continued)

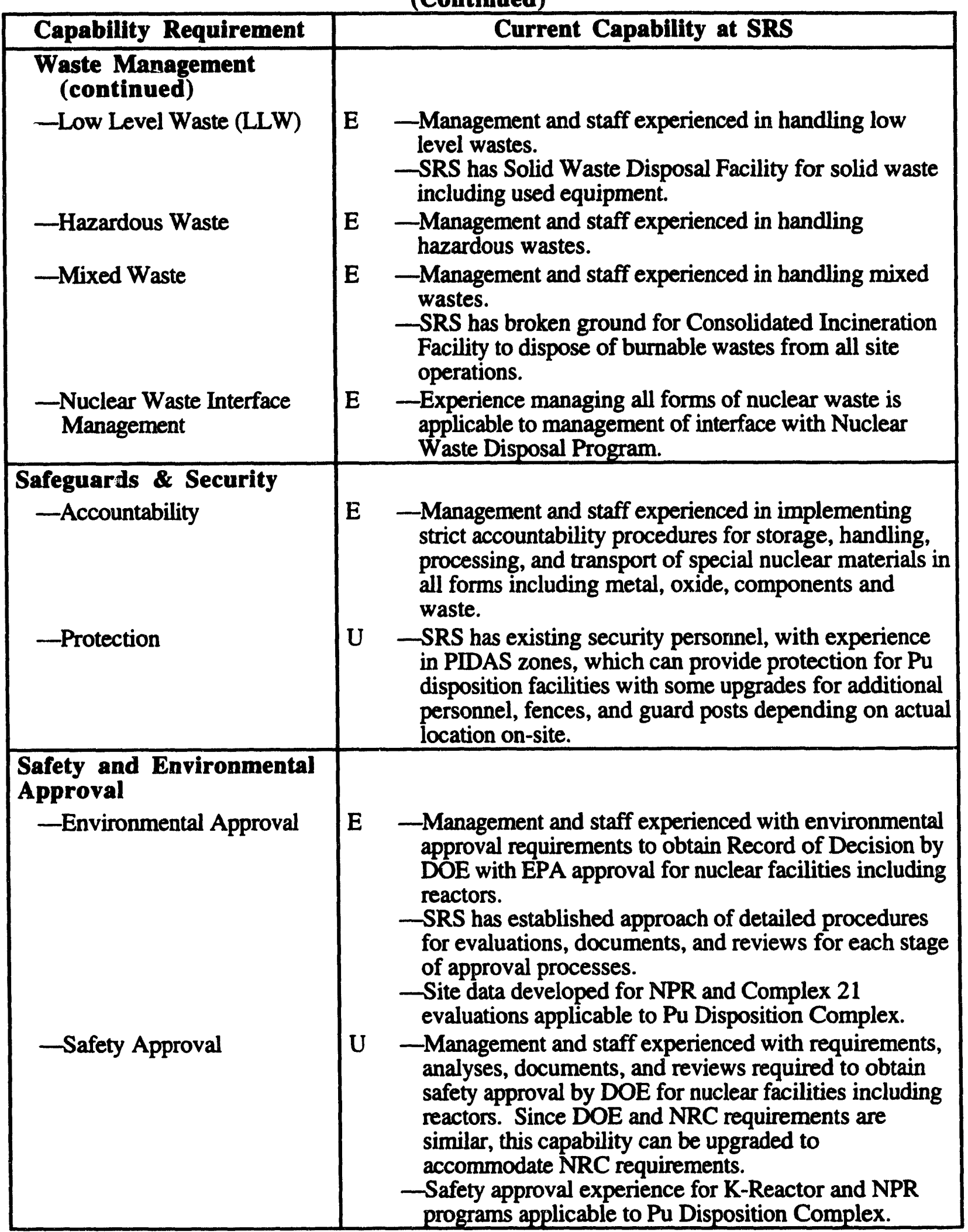


Table F-5 Savannah River Site (Continued)

\begin{tabular}{|c|c|c|}
\hline Capability Requirement & & Current Capability at SRS \\
\hline \multicolumn{3}{|l|}{$\begin{array}{l}\text { Safety and Environmental } \\
\text { Approval (Continued) }\end{array}$} \\
\hline $\begin{array}{l}\text {-Federal/State/Local } \\
\text { Permitting }\end{array}$ & $\mathbf{E}$ & -SRS has pro-active approach to permitting at all levels. \\
\hline \multicolumn{3}{|l|}{ Transportation } \\
\hline -Site Access & $\mathbf{E}$ & $\begin{array}{l}\text {-SRS has access to site by road, rail, and barge docking } \\
\text { facilities, which can meet construction needs of Pu } \\
\text { Disposition Project with minor upgrading. Savannah } \\
\text { River can be made navigable for barge transport of } \\
\text { heavy equipment. }\end{array}$ \\
\hline & $\mathbf{E}$ & $\begin{array}{l}\text { - Management and staff experienced in receiving SNM } \\
\text { in all forms. }\end{array}$ \\
\hline -On-Site Transport & $\mathbf{E}$ & $\begin{array}{l}\text { - Management and staff experienced in on-site transport } \\
\text { of SNM in all forms within and between protected } \\
\text { zones. } \\
\text { - Public road crosses SRS. }\end{array}$ \\
\hline -Packaging for Shipment & $\mathbf{E}$ & $\begin{array}{l}\text { - Management and staff experienced in shipping SNM in } \\
\text { all forms. }\end{array}$ \\
\hline $\begin{array}{l}\text { Supporting Site Assets } \\
\text {-DOE Site Office }\end{array}$ & $\mathbf{E}$ & $\begin{array}{l}\text {-Experienced in directing nuclear programs and large } \\
\text { projects. } \\
\text { - Have sponsored tours and presentations to expedite } \\
\text { independent review and evaluation of SRS capabilities } \\
\text { for Pu disposition by GE team. } \\
\text { - Have expressed interest in status of Pu Disposition } \\
\text { Study independent of site and reactor type. }\end{array}$ \\
\hline $\begin{array}{l}\text { Site Contractor } \\
\text { Management }\end{array}$ & $\mathbf{E}$ & $\begin{array}{l}\text {-Operational experience managing plutonium and tritium } \\
\text { processing and technology and large projects including } \\
\text { production reactors. } \\
\text {-Site contractor management has indicated strong } \\
\text { interest in the Pu Disposition Complex and believes } \\
\text { SRS could accommodate the program. }\end{array}$ \\
\hline -Utilities & $\mathbf{E}$ & $\begin{array}{l}\text {-Adequate water supply and distribution system for } \\
\text { reactor makeup and complex operations. Three } \\
\text { pumping stations on Savannah River can deliver 3E6 } \\
\text { gpm to SRS. } \\
\text {-Adequate electric power to support large construction } \\
\text { project. }\end{array}$ \\
\hline -Technology Development & $\mathbf{E}$ & $\begin{array}{l}\text {-Savannah River Technology Center (SRTC) at SRS } \\
\text { devoted to solving production problems and improving } \\
\text { processes of nuclear materials handling, storage, } \\
\text { processing, reactor operation, and waste management. }\end{array}$ \\
\hline -Work Force & E & $\begin{array}{l}\text { - Technical and production oriented work force can } \\
\text { support large plutonium and tritium processing and } \\
\text { reactor construction and operation project. }\end{array}$ \\
\hline
\end{tabular}


NEDO-32361

Table F-5 Savannah River Site (Continued)

\begin{tabular}{|c|c|c|}
\hline Capability Requirement & & Current Capability at SRS \\
\hline \multicolumn{3}{|l|}{$\begin{array}{l}\text { Supporting Site Assets } \\
\text { (continued) }\end{array}$} \\
\hline -Quality Assurance & $\mathrm{E}$ & $\begin{array}{l}\text {-Experienced in working to DOE quality assurance } \\
\text { requirements. }\end{array}$ \\
\hline$\longrightarrow$ Safety and Health & E & $\begin{array}{l}\text { - Management and staff experienced in meeting or } \\
\text { exceeding all health and safety requirements associated } \\
\text { with nuclear programs. }\end{array}$ \\
\hline -Environmental Protection & $\mathbf{E}$ & $\begin{array}{l}\text { - Management and staff experienced in protection of the } \\
\text { environment. Environmental restoration programs } \\
\text { operational at SRS. }\end{array}$ \\
\hline -Community Support & $\mathbf{E}$ & $\begin{array}{l}\text { SRS management believes indications are that state and } \\
\text { local officials will be receptive to new Pu processing } \\
\text { and reactor operations of Pu Disposition Complex, } \\
\text { based on response to NPR and Complex } 21 \text { proposals. } \\
\text {-SRS management has found widespread local } \\
\text { community support for operations at the site. Active } \\
\text { program of communications with community leaders } \\
\text { and public is maintained. } \\
\text {-Barnwell County Economic Development Initiative } \\
\text { includes proposal for "Center for Nuclear Materials } \\
\text { Management" which is to be a private, commercial } \\
\text { engineering laboratory working on ways to manage } \\
\text { waste. } \\
\text {-Energy Research Foundation (ERF), located in } \\
\text { Columbia SC, is leading public interest group. } \\
\text { Concerns include long term storage of nuclear materials } \\
\text { and release of tritium contaminated waste. }\end{array}$ \\
\hline
\end{tabular}




\section{Appendix G: Comparison of Urania and MOX Fuel Properties}

Fuel rod thermal-mechanical performance could be expected to be affected by the change from urania to MOX fael. A technical review of the available mixed (urania-plutonia) oxide fuel properties and performance information has been conducted to qualitatively assess differences, with respect to urania fuel, that may affect fuel rod thermal-mechanical performance relative to design and licensing criteria. The results of this review given below indicate that for the range of $\mathrm{Pu}$ enrichments considered in this study, the thermal-mechanical response of the fuel is predictable using the existing data base. As a result, MOX fuel is expected to perform as well as the standard urania fuel. The specific fuel properties and performance characteristics investigated include:

- Theoretical density

- Elastic modulus

- Creep

- Thermal expansion

- Thermal conductivity

- Enthalpy

- Melting temperature

- Radial power distribution

- Fission gas release

\section{Theoretical Density:}

Plutonia lattice parameter measurements exist to enable a quantification of the urania-plutonia theoretical density as a function of the plutonia concentration. The fuel theoretical density $(\mathrm{gm} / \mathrm{cc})$ increases with increasing plutonia concentration; the theoretical density of $10 \mathrm{wt} \%$ $\mathrm{PuO}_{2}-\mathrm{UO}_{2}$ is $\sim 0.5 \%$ greater than the theoretical density of urania. The effect of this difference on fuel rod thermal-mechanical performance is that, for the same fuel exposure accumulation and fraction of theoretical density, a slightly greater number of fissions would occur per unit volume 
of fuel thereby leading to slightly greater fuel irradiation swelling and gaseous fission product inventory. The difference, however, is relatively minor.

\section{Elastic Modulus:}

Measurements of the elastic modulus of plutonia indicate that the elastic modulus of uraniaplutonia fuel increases with increasing plutonia concentration. The elastic modulus of $10 \mathrm{wt} \%$ $\mathrm{PuO}_{2}-\mathrm{UO}_{2}$ is $-1.5 \%$ greater than the elastic modulus of urania. This difference results in a slightly stiffer pellet, and correspondingly higher stresses for a given elastic strain. This difference is relatively minor and overshadowed by the difference in fuel material creep behavior described below.

\section{Creep:}

Measurements of the temperature-dependent creep behavior of urania-plutonia compositions indicate a significant increase in the fuel creep rate relative to $\mathrm{UO}_{2}$. The creep rate of $10 \mathrm{wt} \%$ $\mathrm{PuO}_{2}-\mathrm{UO}_{2}$ at representative operating temperatures and stresses is $\sim 3$ times higher than that of Urania under the same conditions. This difference indicates a more compliant fuel pellet and correspondingly lower fuel rod cladding stresses for the same loading conditions. This difference also indicates a lower pellet-cladding interfacial pressure and correspondingly, lower pelletcladding thermal conductance and higher fuel temperatures for the same loading conditions.

\section{Thermal Expansion:}

Measurements have been performed to determine the isothermal thermal expansion coefficient of plutonia. These measurement results indicate decreased fuel thermal expansion with increasing plutonia concentration. The thermal strain at $2500^{\circ} \mathrm{F}$ for $10 \mathrm{wt} \% \mathrm{PuO}_{2}-\mathrm{UO}_{2}$ is $\sim 95 \%$ of that for urania. The effect of this difference on fuel rod thermal-mechanical performance is a slightly reduced imposed strain on the fuel rod cladding by the fuel pellet (for the same fuel temperature condition) and correspondingly reduced cladding stresses under certain loading conditions (such as a rapid power increase).

\section{Thermal Conductivity:}

Measurements of the thermal conductivity of plutonia and urania-plutonia compositions have been performed by drop calorimetry methods. The measurement results consistently show a slightly decreasing fuel thermal conductivity with increasing plutonia concentration. The thermal conductivity of $10 \mathrm{wt} \% \mathrm{PuO}_{2}-\mathrm{UO}_{2}$ at typical operating temperatures is $\sim 97 \%$ of that of 
urania. The effect of a lower fuel thermal conductivity is to increase fuel temperatures, and correspondingly fuel thermal expansion and fission gas release, for the same operating power level.

\section{Enthalpy:}

Enthalpy measurements of plutonia and urania-plutonia compositions have been performed by drop calorimetry methods. The measurement results indicate a slightly higher enthalpy for urania-plutonia than for urania at lower temperatures and a slightly lower enthalpy at higher fuel temperatures. The enthalpy of $10 \mathrm{wt} \% \mathrm{PuO}_{2}-\mathrm{UO}_{2}$ is $\sim 1 \%$ higher than that for urania at $816^{\circ} \mathrm{C}$ $\left(1500^{\circ} \mathrm{F}\right)$ and $\sim 1 \%$ lower than that for urania at $1371^{\circ} \mathrm{C}\left(2500^{\circ} \mathrm{F}\right)$. This difference is negligible.

\section{Melting Temperature:}

Extensive measurements using the thermal arrest technique have been performed by GE to determine the solidus and liquidus boundaries of urania-plutonia over the entire composition range $\left(0-100 \% \mathrm{PuO}_{2}\right)$. The measurement results indicate a decreasing melting temperature (solidus) with increasing plutonia concentration. The melting temperature of $10 \mathrm{wt} \% \mathrm{PuO}_{2}$ $\mathrm{UO}_{2}$ is $\sim 60^{\circ} \mathrm{C}$ lower than that for urania. One U.S. licensing constraint is that fuel temperatures not exceed the melting temperature during normal steady-state operation, including anticipated occurrences. Therefore, this lower fuel melting temperature will reduce, to a small extent, the operational capability of the fuel.

\section{Radial Power Distribution:}

For urania fuel, the radial power distribution across the fuel pellet is relatively flat at the start of irradiation. With continued irradiation, the progressive buildup of plutonium near the fuel pellet outer surface causes a significant peaking in the radial power distribution near the fuel surrace, with a corresponding depression of the power in the pellet interior. The effect of this timevarying change in the radial power distribution is to reduce the pellet centerline temperature for the same power level and pellet surface temperature. For urania-plutonia fuel, the presence of plutonia near the pellet surface causes an increased peaking (relative to urania) over a greater region toward the pellet surface even at the start of irradiation. This increasingly surface-peaked radial power distribution persists throughout lifetime for urania-plutonia fuel. The effect of this difference is slightly reduced fuel central temperatures, in comparison to urania fuel, for the same operating power level and fuel surface temperature. 


\section{NEDO-32361}

\section{Fisstion Gas Release:}

The release of gaseous fission products from the fuel to the fuel rod void space produces two primary effects:

- The thermal conductivity of the gaseous fission products is approximately an order of magnitude lower than the helium filler gas introduced during the fabrication of the fuel rod. Therefore, the release of gaseous fission products from the fuel pellets to the void space reduces the gas mixture thermal conductivity, reduces the conductance between the fuel pellet and the cladding, and increases fuel temperatures. The fission gas release mechanism is fuel temperature dependent, and therefore, this fuel temperature increase produces additional fission gas release.

- The release of gaseous fission products to the fuel rod void space increases the fuel rod internal pressure. The maximum fuel rod internal pressure is limited by U.S. licensing constraints. Therefore, excessive fission gas release and fuel rod internal pressure can limit the operating conditions or mechanical design of the fuel.

The available information indicates that the fission gas release behavior of urania-plutonia fuel is significantly affected by the as-fabricated fuel microstructure. For example, significant open porosity (fuel pellet pores in direct contact with the pellet surface) increases the rate of fission gas release. In addition, inhomogeneous microstructure resulting in localized islands of pure $\mathrm{PuO}_{2}$ and/or $\mathrm{UO}_{2}$ also incicases the rate of fission gas release. The preferred microstructure includes standard grain sizes $(8-10 \mu \mathrm{m})$, low open porosity $(<0.5 \%$ TD), and $100 \%$ homogeneous $(\mathrm{U}, \mathrm{Pu}) \mathrm{O}_{2}$ solid solution. With this preferred fuel microstructure, no difference in the fundamental fission gas release processes and behavior are expected relative to urania fuel.

U.S. fuel licensing criteria can be broadly categorized as either (1) thermal performance limits (e.g., fuel melting temperature limit), or (2) mechanical performance limits (e.g., fuel rod cladding stress and strain limits). The urania-plutonia fuel properties and performance assessment indicates that, relative to urania fuel, fuel thermal performance is somewhat less favorable and fuel mechanical performance is somewhat more favorable. These differences are manageable and can be accommodated either by the thermal-mechanical design of the fuel rod or specification of operating constraints. The performance area that can be influenced to minimize performance, design and licensing differences is fuel pellet fission gas riease. Application of known preferred microstructural features will eliminate the potentially significant difference. 


\section{NEDO-32361}

Based on the above evaluations, it is concluded that the criteria and limits which have been applied in the past to urania fuel can continue to be used for MOX fuel insofar as these criteria and limits involve fuel thermo-mechanical properties. More detailed evaluations will be conducted in the project phase to confirm this finding. It is possible that minor changes to the limits might be necessary to more properly take into account the slight change to fuel properties. However, these are expected to be within the margins which have been designed into the MOX fuel during this study phase. 


\section{Appendix H: Plan for Lead Fuel Testing for Verincation of MOX Fuel Performance}

\section{H.1 Introduction}

Although an extensive literature and data base are available for Mixed (uranium-plutonium) Oxide (MOX) fuel properties and its performance in LWRs, there are a number of reasons to undertake an early program of Lead Fuel Testing in the plutonium disposition project. The reasons include:

- The need to develop and demonstrate a fuel fabrication process that complements proposed processes for destruction of the pit shape. A process of hydriding followed by dehydriding has been proposed to destroy the pit shape. Currently, experimental verification of this process is under way at LANL and LLNL. Although additional work remains to be done, it is clear that any proposed MOX fabrication process should complement the work already being done in this area and still yield high quality MOX pellets.

- Although MOX pellets have been fabricated in large quantities in the past, there is little data on fabrication of MOX mixed with the Gadolinia burnable poison. There is a need to verify the fabrication parameters for this fuel.

- While nuclear analysis codes are readily available inccrporating the full complement of cross-section information needed for the design of a MOX core, it is appropriate to obtain additional benchmark data for the integrated core design codes currently in use. Additional Gd burnout profile data, especially as a function of radius within the MOX fuel rod, would aid in this study.

- In addition to the above specific factors, there is a general need to reestablish the MOX fabrication technology in this country as no large scale MOX fabrication has been carried out in more than 20 years. In this context, it is not only necessary to reestablish the old technology which could probably be accomplished very quickly but to define and incorporate automation techniques both to increase prnductivity and lower worker exposure, particularly as related to possible Am evaporation and condensation while sintering MOX pellets.

- Gamma signature has generally been used for accountability in Safeguards implementation with MOX fuel production using reprocessed plutonium. With low Am levels, this procedure will be used in conjunction with more active interrogation techniques. In addition, as stated earlier, some Am might be lost during sintering. For these reasons, it is necessary to 
document the range of $\gamma$ activities to be expected in the as-sintered pellet and its variability for a fixed Pu content.

The Lead Fuel Test program outlined below was developed by GE during this study with input received from all disciplines within GE as well as from national laboratory experts at LANL, LLNL and WHC. While cost and schedule elements are discussed at the end of this report, it is worthwhile noting here that an initial test capsule could be fabricated at a national laboratory such as LANL and placed in test in an experimental reactor such as the ATR within an year of program inception. The program envisions obtaining all the necessary confirmatory data for fuel fabrication within a year, verification of individual rod behavior within three years of start of the test program and qualification of MOX core through methods validation by about the same time.

These projections are based on pursuing an aggressive schedule, not limited by funding availability.

\section{H.2 Objectives}

In order to develop the LTA program plan, it is first necessary to state the objectives of the program. The objectives of this program can be categorized under the following four headings:

- Fuel Fabrication Validation Objectives

- Fuel Mechanical/Chemical Performance Objectives

- Fuel (Rod) Nuclear Performance Objectives

- Fuel Assembly Nuclear Performance Objectives

The objectives under each of these categories are described in more detail below:

\section{H.2.1 Fuel Fabrication Validation Objectives}

\section{- Develop MOX Processes to Complement Pit Processing}

As a first step in safeguarding the weapons plutonium, the pit which contains the plutonium in weapons has to be removed and its shape destroyed. Although a number of terhniques are available, research in this area has focused on a "chemical processing" means as a clean, safe and economical means of pit shape destruction. This process consists of hydriding and dehydriding the plutonium. In such a process, the pit shape falls apart leaving a powdery plutonium as the final state. It is therefore useful to consider MOX processing steps which 
complement this process. If the fission process is not chosen as the route to plutonium disposition, the powder Pu from this process would presumably be melted to form a desired unclassified shape.

The plutonium obtained from the hydride-dehydride process could be directly burned under a controlled oxygen partial pressure to produce plutonium oxide. This could form the starting stock for the MOX process with urania and gadolinia powders to be supplied by a commercial vendor such as GE. However, the activity of the plutonia powder and the particle size distribution thereof, may not be compatible with what is required for MOX fuel fabrication. Therefore, further studies on the milling of the plutonia powder and blending with the urania have to be conducted under controlled conditions to develop an acceptable MOX powder for sintering. In this regard, the minimum acceptable plutonia particle size in a MOX pellet is already available based on earlier studies. Initial fabrication development will therefore concern itself with the ability to meet the final fuel specifications and quality requirements in a number of respects including density, grain size and Pu distribution specifications. The urania required for these fabrication evaluations could be provided by a vendor or the laboratory could find its own source for this supply. Specifications for the MOX fuel pellet will be provided by a vendor as well as the post-sintering examination requirements.

- Demonstrate Fabricability of MOX with Gd

Although an extensive MOX fuel fabrication data base exists, there is little experience in the way of fabricating MOX fuel with Gadolinia. A major parameter of interest is the oxygen overpressure during sintering to produce acceptable final chemistry. It may be necessary to inspect and analyze fuel sintered over a range of oxygen overpressures to ascertain the correct sintering atmosphere to be used in production. It is also proposed that a range of GdPu contents be examined under this evaluation. A maximum of $10 \% \mathrm{Gd}$ (by weight fraction) is suggested as the upper limit for the investigation. The gadolinia powder could be provided by a vendor together with the specifications for this powder. Post-sintering examinations could be carried out by the laboratory based on examination specifications to be provided by a vendor. 


\section{- Efrect of Impurities on Processing and Sintering}

Americium and Gallium are expected to be present in minor or trace quantities in the initial plutonium feed. There is a need to reduce their presence as much as possible. High levels of Americium increases the $\gamma$ activity throughout the entire fuel process. The processes to reduce or eliminate americium and gallium from the initial plutonium feed material are well established but need to be integrated with the overall process flow. It has been suggested that by building the fuel fabrication factory to allow complete remote automated handling for servicing and maintenance as well as for normal operations, the level of Americium that can be present could be raised significantly. The proposed concept aims at reducing these impurities to a low enough level at the initial feedstock interface, so they do not pose any problems downstream. However, if it is desired to leave high levels of Am in the feedstock, it is necessary to quantify its behavior during pellet fabrication, particularly during sintering. It is known that Americium evaporates preferentially during sintering. Data from the initial runs will be useful in accounting for the shielding necessary to minimize worker exposure and designing cleanup systems to trap and dispose the evaporated Americium.

\section{- Validation of Fuel Pellet Quality}

A vendor could provide the fuel pellet specifications, including those for density, grain size, impurity limits and fuel chemistry. Post-sintering examinations should be conducted on sufficient pellets to validate the overall fabrication process and individual process steps to aid in fine tuning the MOX Fuel Factory Requirements, Equipment Specifications and Process Parameters. Although sintering parameters for MOX fuel are well established, a limited number of additional studies should be conducted to optimize the process, particularly with a view to reducing the waste stream and scrap recycle fraction which are the major contributors to worker exposure.

\section{H.2.2 Fuel Mechanical/Chemical Performance and Fuel Properties:}

\section{- Mechanical/Chemical Performance}

The objectives here are simply to verify the pin mechanical/chemical performance of the rod and verify its integrity.

Post-irradiation examinations will include among others: gathering fission gas release data and pin dimensional (strain) measurements as a function of fabrication/ operating variables; checking the migration of specific fission products through gamma scanning; fuel length 
change measurements; fuel-cladding interface examinations for chemical compatibility. The fabrication variables will include $\mathrm{Pu}-\mathrm{Gd}$ fraction, fuel power density and the range of allowable fuel pellet physical specifications.

- Fuel Properties

Properties of MOX fuel are readily available from previous DOE programs including the Liquid Metal Fast Reactor Development Program. However, limited additional data are needed, particularly for MOX fuel with Gadolinia poison. Material property correlation models for oxide fuel containing small fractions of rare earth materials (of which Gd is one) have been developed at GE, nevertheless, experimental confirmation of these data will be required to establish complete licensability of the fuel. Two specific properties of major interest are: fuel thermal conductivity, and Fuel Solids, Liquids temperatures.

\section{H.2.3 Fuel (Rod) Nuclear Performance Objectives}

The primary objective here is to confirm the existing Gd burnup data as a function of the radius within an individual rod. Different Pu-Gd compositions should be examined in this regard. Rods can be irradiated individually or in a cluster, in an experimental reactor such as the ATR. GE could provide the test specifications and post-irradiation examinations and conduct the associated nuclear analysis. A range of fission densities should be considered in the experiment. The results will provide the benchmark data for further calibration of nuclear codes.

\section{H.2.4 Fuel Assembly Nuclear Performance Objectives}

The results of fuel rod testing in an experimental reactor with a thermal spectrum are considered sufficient for licensability of the MOX fuel in a BWR. However, Lead Use Assembly (LUA) tests using full scale assemblies will be conducted to provide confirmatory data for the thermalmechanical performance of MOX fuel to goal exposures and for additional benchmarking of integrated nuclear analysis codes.

In conducting full assembly tests in an existing (BWR) reactor, it should be recognized that a MOX fueled assembly will be operating in a sea of urania fueled bundles and therefore the nuclear design of this full bundle cannot be expected to be identical to the MOX fuel bundle proposed as the reference. In point of fact, the test assembly has to be designed to minimize its impact on adjacent urania fueled assemblies and to operate within the limits of the criteria for which the plant was originally designed. Full scale MOX assembly tests are however recommended to provide thermo-mechanical information to goal exposures on prototype length 
fuel rods and additional nuclear data for methods validation. Full assembly tests can also be used to study the effect of different $\mathrm{Gd} / \mathrm{Pu}$ enrichments and provide data on power shaping, both axially and radially in the assembly. Post-irradiation data will be obtained on rod profilometry, length changes and fission product migration by gamma scanning, on a cycle-by-cycle basis.

\section{H.3 Preliminary Test Plan}

Based on the foregoing objectives, the following test plan is proposed:

\section{Series 1: Fuel Fabrication Verification and Fuel Properties Testing}

- MOX Fuel will be fabricated by a DOE designated latioratory based on mechanically mixed process, with Pu oxide from Pu feed stock prepared from weapons grade Pu or comparable chemistry. The process specifications from the Pu Oxide phase to MOX fuel could be arrived at jointly between GE and the fuel fabrication laboratory. The final specifications for the MOX pellet could be provided by GE. The final specifications for QA for the fuel could be specified by GE. GE could provide the initial input for sintering Gd bearing MOX fuel. Data will be obtained on Am transport during sintering.

- Range of Fuel Pellet Parameters:

- Pellets with and without purification steps to remove Gallium

- Different Pu-Gd compositions (Gd from 0 to $10 \%$ and Pu from 2 to 20\%)

- Nominal Pellet density: $96.5 \%$

- Nominal Grain Size: 10 microns

- Specific Pu-Gd combinations to be specified by GE in Phase 2.

- Post-Fabrication Tests for:

- Pellet $\gamma$ and neutron activity after fabrication

- Optical Metallography (etched and unetched)

- Fuel chemistry (O:M Ratio, impurities)

- Pu homogeneity

- Fuel density, description of porosity (by metallography) 
NEDO-32361

- Surface Roughness

- Post-fabrication examinations to be specified by the Vendor; these tests will be conducted on various batches to describe any effect of process variables on final fuel pellet characteristics

- Fuel Properties Tests:

- Thermal conductivity (by laser flash technique)

- Thermal Arrest Studies

(Above tests for various Pu-Gd compositions)

\section{- QA of Reference Fabrication Process Pellets}

Vendor to specify QA requirements; laboratory will conduct the QA audit of the fabricated pellets. Sampling plan will be based on mutually agreed parameters.

The results of this series will be documented by the laboratory with particular emphasis on process verification for production of Gd bearing and non-Gd bearing MOX fuel, and the effect of initial process step to either remove or not remove Am, gallium.

\section{Series 2: In-Reactor Rod Tests}

The in-reactor tests are aimed at developing the data and meeting the objectives described under Section H.2. The rods could be assembled by the laboratory and shipped to the test site. Individual rods or rod clusters (depending upon the test cavity size) will be irradiated in a thermal spectrum research reactor. The test site will be responsible for incoming inspection, thermal hydraulic design of the test, for obtaining the preliminary and final approvals for the test and for safety analysis of the test. The detailed test specifications could be provided by GE.

As currently envisioned, these tests would be individual capsules or a cluster of capsules. Either bare rods or capsule-type completely encapsulating the fuel rod with a gas-gap design to produce the required cladding temperature can be used. None of the tests in this series are slated to have any active monitoring devices. Indirect confirmation of the temperature will be obtained by incorporating TEDs (Thermal Expansion Devices) which have been used in the past. To the extent these tests are entirely uninstrumented and passive, the cost of these tests should be low and an aggressive schedule could be pursued. Data will be obtained as a function of exposure by removing the capsules containing the rods at regular intervals. 
Post-irradiation examination requirements could be specified by GE and could include, as noted earlier, gamma scans, fuel length changes, rod profilometry, limited fuel pellet metallography, fission gas collection, and limited microprobe examinations of the fuel-cladding interface. These examinations will be conducted at a suitable facility to be designated by DOE. GE can conduct Gd radial profile measurements in its VNC facility from shipment of samples to be made from the designated post-irradiation examination facility. Because Gd burnout occurs early in life, results on Gd radial profile in MOX fuel can be obtained with just one year of irradiation. Calibration of the nuclear codes using this data can therefore be expected early in the program.

The details of the test matrix will be worked out after meetings between the interfacing organizations. As presently envisioned, the test matrix will include at least the following parameters:

- Fuel Characteristics:

- MOX fuel with Pu preprocessed to remove Am/gallium and unpreprocessed

- MOX Rod without Gd for Comparison with $\mathrm{Gd}$ with $\mathrm{Pu}$ as variable

- Pu-Gd Composition (Up to $10 \% \mathrm{Gd}$ )

- Fuel density, grain size within allowable range

- Operating Conditions:

- Exposure

- Power Level

\section{Series 3: Lead Use Assembly Tests}

These tests will consist of full assemblies with either a partial or full complement of MOX fueled rods, with and without $\mathrm{Gd}$, to benchmark integrated nuclear analysis codes and to demonstrate rod performance to goal exposures. The matrix of fuel rod variables is expected to be similar to that for rod tests under Series 2. 


\section{NEDO-32361}

\section{H.4 Interfaces}

In order to carry out this program, a number of interfaces have to be established and the activities coordinated. A Lead Fuel Testing Interface Control Board may need to be established with specific responsibilities and assignments and charged with the conduct of the program. The required interfaces and responsibilities are:

Test Requester: Responsibilities to propose the test matrix, provide fuel cladding hardware, nuclear design, provide specifications and QA acceptance criteria for fuel, post-fuel production examination requirements, post-irradiation examination requirements, provide input for fabrication process and $\mathrm{T}-\mathrm{H}$, safety analysis as required.

Fuel Fabricator: National laboratory to be assigned by DOE. Responsible for developing MOX fuel (with and without $\mathrm{Gd}$ ) fabrication process parameters, provide input for large scale integration with automated equipment for MOX factory, for large scale integration with safeguards requirements, particularly for material accountability, for welding, inspecting and shipping completed test fuel hardware to test site, provide input to safety analysis and $\mathrm{T}-\mathrm{H}$ analysis as required, for conducting specific fuel properties measurements, and for implementing QA and inspection procedures on fabricated fuel.

Test Site (for Test Series 2): For obtaining preliminary and final test request approvals, for providing required nuclear environment to test requester for test design, for capsule T-H analysis, for safety analysis, for fabricating and assembling the test assembly except for the fuel rod(s), for post-irradiation examinations. LUA tests will be conducted at the candidate reactors chosen for disposition.

\section{H.5 Cost}

Detailed cost estimates can be generated only after some details of the test matrices, for instance relative to the number of tests, have been arrived at. Approximately 50 to 60 fuel rod tests under Series 2 are anticipated. An initial cost estimate for this program has been described in Section 6 of this report.

\section{H.6 Schedule}

Details of the schedule will be worked out during the initial phases of program inception. A preliminary schedule has been presented in Section 6. It is worthwhile pointing out that although validation of the thermo-mechanical performance to goal exposures will take several years, the only additional data needed for benchmarking nuclear methods relates to the behavior of MOX 
fuel with $\mathrm{Gd}$. This data should become available early in the program from rod tests as Gd more or less burns out during the first cycle. 


\section{Appendix I: Repository Considerations}

\section{CFRPart 961, Article VI - Criteria for Disposal}

\section{Article VI - Criteria for Disposal}

\section{A. General Requirements}

1. Criteria

(a) Except as otherwise provided in this contract, DOE shall accept hereunder only such Spent Nuclear Fuel (SNF) and/or High Level Waste (HLW), which meets the General Specifications for each fuel and waste as set forth in Appendix E, annexed hereto and made a part hereof.

(b) Purchaser shall accurately classify SNF and/or HLW prior to delivery in accordance with paragraphs B and D of Appendix E.

\section{Procedures}

(a) Purchaser shall provide to DOE a detailed description of the SNF and/or HLW to be delivered hereunder in the form and content as set forth in Appendix $F$, annexed hereto and made a part hereof. Purchaser shall promptly advise DOE of any changes in said SNF and/or HLW as soon as they become known to the purchaser.

(b) DOE's obligation for disposing of SNF under this contract also extends to other than standard fuel; however, for any SNF which has been designated by the Purchaser as other than standard fuel, as that tern is defined in appendix E, the Purchaser shall obtain delivery and procedure confirmation from DOE prior to delivery. DOE shall advise Purchaser within sixty (60) days after receipt of such confirmation request as to the technical feasibility of disposing of such fuel on the currently agreed to schedule and any schedule adjustment for each service. 


\section{Appendix I of 10CFRPart 961 - General Specifications}

\section{A. Fuel Category Identification}

1. Categories-Purchaser shall use reasonable efforts, utilizing technology equivalent to and consistent with the commercial practice, to properly classify SNF prior to delivery to DOE, as follows:

a. Standard Fuel means SNF that meets all the General Specifications therefore set forth in paragraph B below.

b. Nonstandard Fuel means SNF that does not meet one or more of the General Specifications set forth in subparagraphs 1 through 5 of paragraph B below, and which is classified as Nonstandard Fuel Classes NS-1 through NS-6, pursuant to paragraph B below.

c. Failed Fuel means SNF that meets the specifications set forth in subparagraphs 1 through 3 of paragraph B below, and which is classified as Failed Fuel Class F-1 through F-3 pursuant to subparagraph 6 of paragraph B below.

d. Fuel may have "Failed Fuel" and/or several "Nonstandard Fuel" classifications.

B. Fuel Description and Subclassification-General Specifications

1. Maximum Nominal Physical Dimensions

\begin{tabular}{|c|c|c|}
\hline & $\begin{array}{l}\text { Bolling Water } \\
\text { Reactor (BWR) }\end{array}$ & $\begin{array}{c}\text { Pressurized Water } \\
\text { Reactor (PWR) }\end{array}$ \\
\hline Overall Length & 14 feet, 11 inches & 14 feet, 10 inches \\
\hline Active Fuel Length & 12 feet, 6 inches & 12 feet, 0 inches \\
\hline Cross Section 1 & 6 inches $\times 6$ inches & 6 inches $\times 9$ inches \\
\hline
\end{tabular}

2. Nonfuel Components. Nonfuel components including, but not limited to, control spiders, burnable poison rod assemblies, control rod elements, thimble plugs, fission chambers, and primary and secondary neutron sources, that are contained within the fuel assembly, or BWR channels that are contained within the fuel assembly, or BWR channels that are an integral part of the fuel assembly, which do not require special handling, may be included as part of the spent nuclear fuel delivered for disposal pursuant to this contract. 
Note: Fuel that does not meet these specifications shall be classified as Nonstandard Fuel-Class NS-3.

3. Cooling. The minimum cooling time for fuel is five (5) years.

Note: Fuel that does not meet this specification shall be classified as Nonstandard Fuel-Class NS-3.

4. Non-LWR Fuel. Fuel from other than LWR power facilities shall be classified as Nonstandard Fuel-Class NS-4. Such fuel may be unique and require special handling, storage, and disposal facilities.

5. Consolidated Fuel Rods. Fuel which has been disassembled and stored with the fuel rods in a consolidated manner shall be classified as Nonstandard Fuel Class NS-6.

\section{Failed Fuel}

a. Visual Inspection. Assemblies shall be visually inspected for evidence of structural deformity or damage to cladding or spacers which may require special handling. Assemblies which (i) are structurally deformed or have damaged cladding to the extent that special handling may be required or (ii) for any reason cannot be handled with normal fuel handling equipment shall be classified as Failed Fuel-Class F-1.

b. Previously Encapsulated Assemblies. Assemblies encapsulated by Purchaser prior to classification hereunder shall be classified as Failed Fuel-Class F-3. Purchaser shall advise DOE of the reason for the prior encapsulation of assemblies in sufficient detail so that DOE may plan for appropriate subsequent handling.

c. Regulatory Requirements. Spent fuel assemblies shall be packaged and placed in casks so that all applicable regulatory requirements are met.

C. Summary of Fuel Classifications

1. Standard Fuel:

a. Class S-1: PWR

b. Class S-2: BWR 
2. Nonstandard Fuel:

a. Class NS-1: Physical Dimensions

b. Class NS-2: Non Fuel Components

c. Class NS-3: Short Cooled

d. Class NS-4: Non-LWR

C. Class NS-5: Consolidated Fuel Rods

3. Failed Fuel:

a. Class F-1: Visual Failure or Damage

b. Class F-2: Radioactive "Leakage"

c. Class F-3: Encapsulated

D. High-Level Radioactive Waste

The DOE shall accept high-level radioactive waste. Detailed acceptance criteria and general specifications for such waste will be issued by the DOE no later than the date on which DOE submits its license application to the Nuclear Regulatory Commission for the first disposal facility.

\section{Appendix F - Detalled Description of Purchaser's Fuel}

This information shall be provided by Purchaser for each distinct fuel type within a Shipping Lot not later than sixty (60) days prior to the schedule transportation date.

Purchaser

Contract Number/Date 1

Reactor/Facility Name

I. Drawings included in generic dossier:

1. Fuel Assembly DWG\#

2. Upper and lower end fittings DWG\#

Dossier Number:

DOE Shipping Lot Number: 
\# Assemblies Described:

BWR

PWR

Other

\section{Design Material Descriptions:}

Fuel Element:

1. Element type (rod, plate, etc.)

2. Total length: (in.)

3. Active length: (in.)

4. Cladding material (Zr, s.s., etc.)

Assembly Description:

1. Number of elements:

2. Overall dimensions (length) (cross section) (in.)

3. Overall weight:

III. Describe any distortions, cladding damage or other damage to the spent fuel, or nonfuel components within this Shipping Lot, which will require special handling procedures. (Attach additional pages if needed.)

IV. Assembly Number:

Shipping Lot \#:

1. Startup date (mo/day/yr)

2. Shutdown date (mo/day/yr)

3. Cumulative fuel exposure (MWd/MT)

4. Avg. reactor power (month)

5. Total heat output/assembly in watts, using an approved calculational method

\begin{tabular}{|l|l|l|l|l|l|}
\hline & \multicolumn{4}{|c}{ Immediation History Cycle No. } \\
\cline { 2 - 5 } & 1 & 2 & 3 & 4 & 5
\end{tabular}

Any false, fictitious or fraudulent statement may be punishable by fine or imprisonment (U.S. Code, Title 18, Section 1001).

By Purchaser:

Signature

Title

Date 


\section{BWR Spent Fuel Discharge Statiotics}

Table I1 Historically Discharged Assemblies for the GE BWR/4-6 Assembly Clase, Broken Down by Assembly Type and Discharge Year

(Reproduced from the LWR Quantities Database)

\section{LWR Quantities Database}

Historical Data through December 31, 1990

Data Broken Down By: Assembly Type, Discharge Year

Discharged Assemblies by Assembly Class: GE BWR/4-6

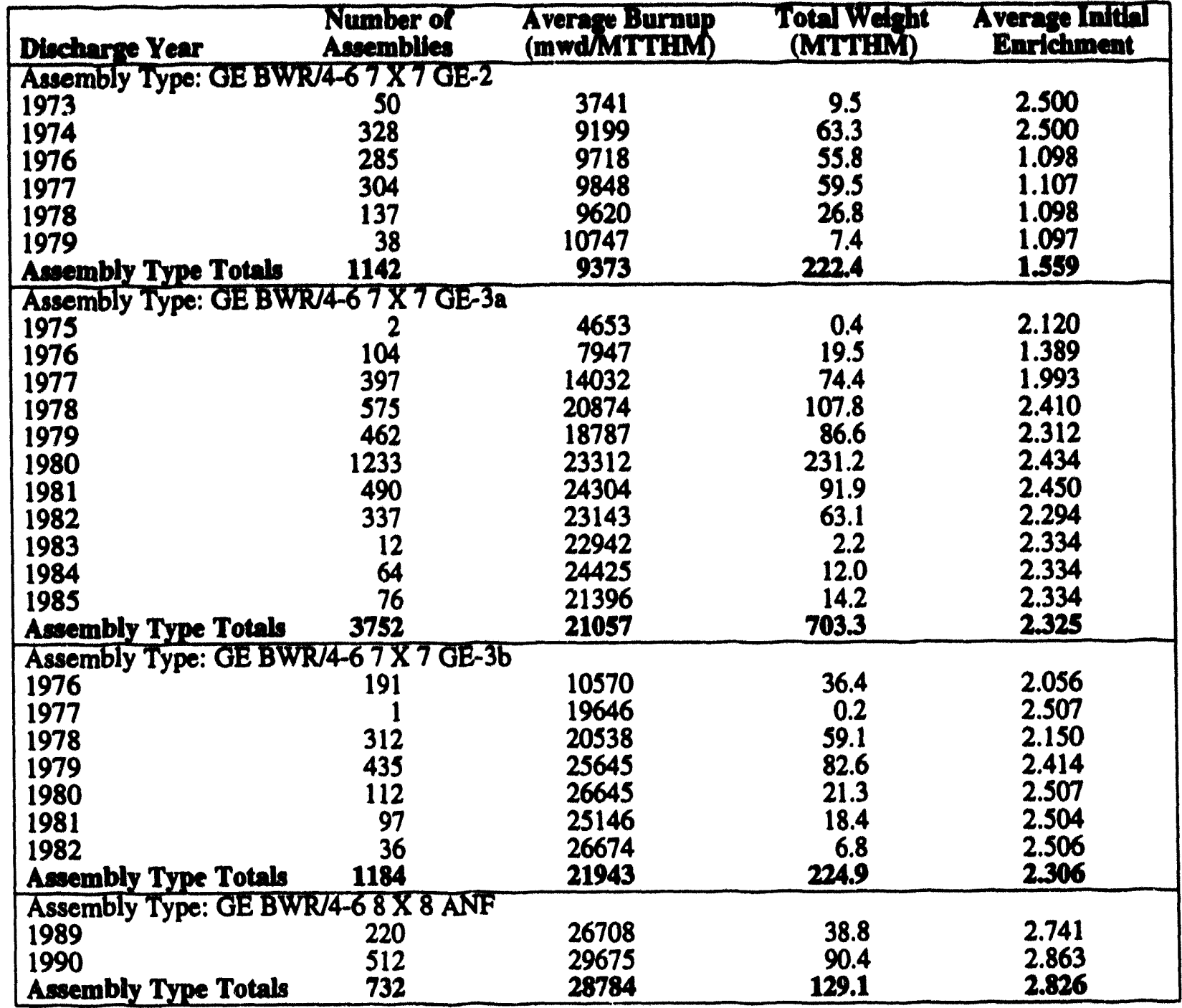

*As reported by the utilities 


\section{Table 11 (Continued)}

\section{LWR Quantities Database}

Historical Data through December 31, 1990

Data Broken Down By: Assembly Type, Discharge Year

Discharged Assemblies by Assembly Class: GE BWR/4-6

\begin{tabular}{|c|c|c|c|c|}
\hline Dlechar Year & $\begin{array}{l}\text { Number or } \\
\text { Asecenblies }\end{array}$ & $\begin{array}{l}\text { Avorag : irnup } \\
\text { (mwd/MTIM) }\end{array}$ & $\begin{array}{l}\text { Torawerist } \\
\text { (MTTEM) }\end{array}$ & $\begin{array}{l}\text { Average inita } \\
\text { Enrichment }\end{array}$ \\
\hline $\begin{array}{l}\text { Assembly 1ype: GEBW } \\
1977 \\
1978 \\
1979 \\
1980 \\
1981 \\
1982 \\
1983 \\
1984 \\
1985 \\
\text { Aesembly Type Totall }\end{array}$ & $\begin{array}{l}74-68 \times 8 \text { OE-42 } \\
112 \\
92 \\
158 \\
267 \\
392 \\
271 \\
300 \\
333 \\
19 \\
1944\end{array}$ & $\begin{array}{l}18924 \\
18801 \\
20073 \\
24241 \\
24825 \\
26187 \\
27497 \\
28652 \\
24842 \\
24993\end{array}$ & $\begin{array}{r}20.6 \\
16.9 \\
29.0 \\
49.1 \\
72.2 \\
49.9 \\
55.3 \\
61.2 \\
3.5 \\
357.6\end{array}$ & $\begin{array}{l}2.190 \\
2.238 \\
2.392 \\
2.670 \\
2.650 \\
2.735 \\
2.736 \\
2.763 \\
2.730 \\
2.631\end{array}$ \\
\hline $\begin{array}{l}\text { Assembly Type: GEBW } \\
1978 \\
1979 \\
1980 \\
1981 \\
1982 \\
1983 \\
1984 \\
1985 \\
\text { Arembly Type Totals }\end{array}$ & $\begin{array}{c}J 468 \times 8 \text { GE-46 } \\
3 \\
137 \\
621 \\
479 \\
262 \\
91 \\
146 \\
48 \\
1787 \\
\end{array}$ & $\begin{array}{l}13892 \\
17153 \\
19261 \\
22602 \\
22328 \\
29507 \\
25220 \\
16416 \\
21368\end{array}$ & $\begin{array}{r}0.6 \\
25.6 \\
116.0 \\
89.5 \\
48.9 \\
17.0 \\
27.3 \\
99.0 \\
333.7\end{array}$ & $\begin{array}{l}2.192 \\
2.177 \\
2.164 \\
2.422 \\
2.194 \\
2.685 \\
2.739 \\
2.114 \\
2.311\end{array}$ \\
\hline $\begin{array}{l}\text { Assembly Type: GE BW } \\
1980 \\
1981 \\
1982 \\
1983 \\
1984 \\
1985 \\
1986 \\
1987 \\
1988 \\
1989 \\
1990 \\
\text { Aseembly Type Totals }\end{array}$ & $\begin{array}{c}\text { J4-68X8GE-5 } \\
78 \\
33 \\
220 \\
950 \\
772 \\
630 \\
48 \\
264 \\
644 \\
343 \\
216 \\
4198 \\
\end{array}$ & $\begin{array}{r}2848 \\
20093 \\
22088 \\
27626 \\
27108 \\
19046 \\
27421 \\
12636 \\
23423 \\
27862 \\
23405 \\
23645\end{array}$ & $\begin{array}{r}14.3 \\
6.0 \\
40.0 \\
173.9 \\
141.3 \\
115.5 \\
8.8 \\
48.3 \\
117.8 \\
62.9 \\
39.5 \\
768.3\end{array}$ & $\begin{array}{l}0.762 \\
2.655 \\
2.487 \\
2.706 \\
2.678 \\
2.035 \\
2.263 \\
1.546 \\
2.256 \\
2.548 \\
2.123 \\
2.362\end{array}$ \\
\hline
\end{tabular}

*As reported by the utilities 
Table II (Continued)

LWR Quantities Database

Historical Data through December 31, 1990

Data Broken Down By: Assembly Type, Discharge Year

Discharged Assemblies by Assembly Class: GE BWR/4-6

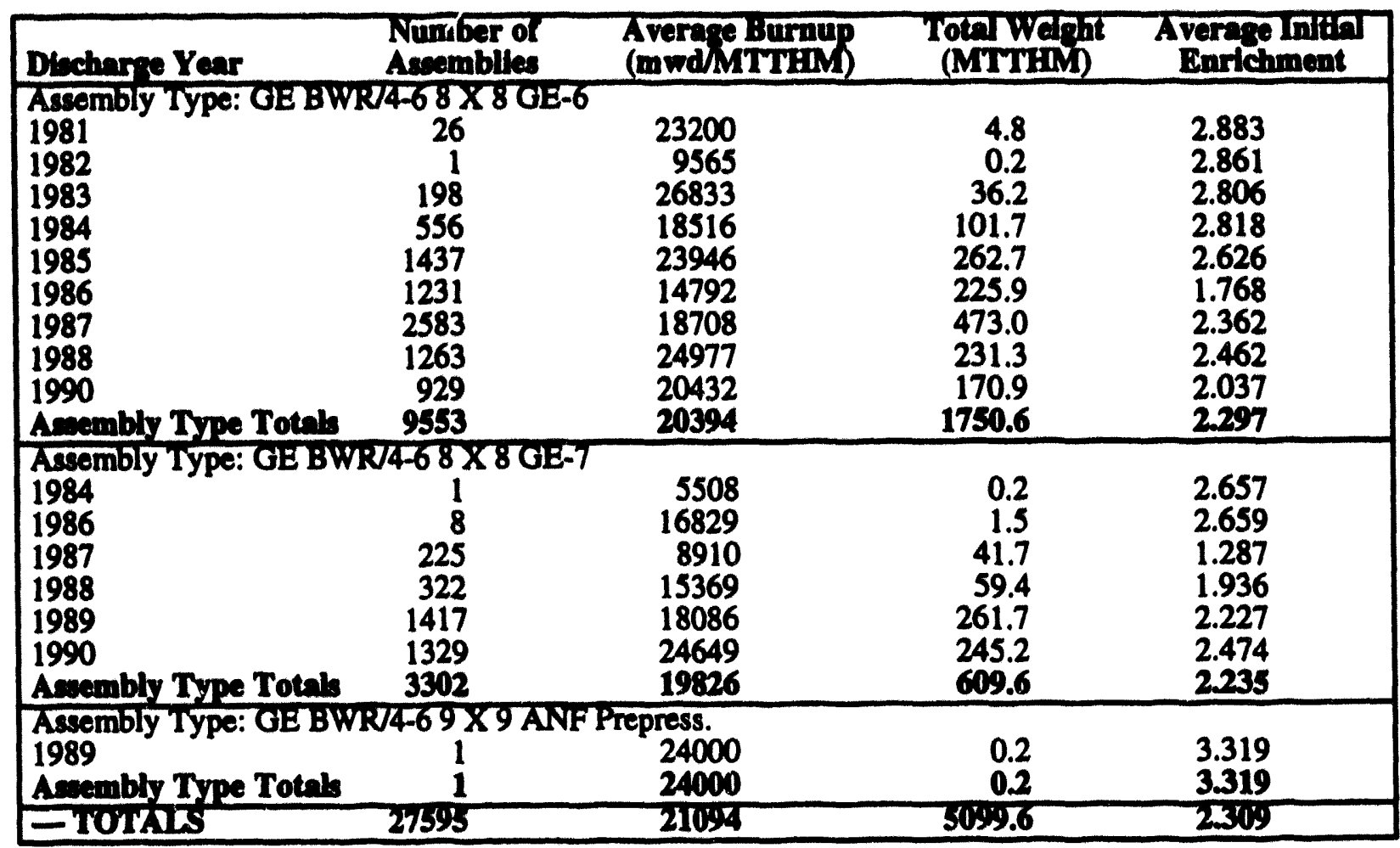

*As reported by the utilities 
Table 12 Projected Quantities of Spent Fuel From GE BWR/46 Assembly Clasa, Broken Down by Discharge Year and Burnup Bin (Reproduced From the LWR Quantities Database)

LWR Quantities Database

Projected Data: No New Orders Case with Extended Burnup

Data Broken Down By: Discharge Year and Burnup Bin

Projected Assemblies for Assembly Class: GE BWR/4-6

\begin{tabular}{|l|c|c|c|c|c|}
\hline $\begin{array}{l}\text { Discharge } \\
\text { Year }\end{array}$ & $\begin{array}{c}\text { Burnup } \\
\text { Bin }\end{array}$ & $\begin{array}{c}\text { Number of } \\
\text { Assemblies }\end{array}$ & $\begin{array}{c}\text { Average Burnup } \\
\text { (mwdMTTMM) }\end{array}$ & $\begin{array}{c}\text { Total Weight } \\
\text { (MTTMM) }\end{array}$ & $\begin{array}{c}\text { Average } \\
\text { Initial } \\
\text { Enrichment }\end{array}$ \\
\hline 1991 & $0-5000$ & 4 & 3000 & 0.7 & 0.711 \\
\hline 1991 & $15001-20000$ & 132 & 17310 & 23.5 & 1.650 \\
\hline 1991 & $20001-25000$ & 572 & 23007 & 102.1 & 2.300 \\
\hline 1991 & $25001-30000$ & 1100 & 28359 & 200.8 & 2.695 \\
\hline 1991 & $30001-35000$ & 879 & 33251 & 157.2 & 3.082 \\
\hline 1991 & $35001-40000$ & 4 & 36000 & 0.7 & 2.990 \\
\hline 1992 & $0-5000$ & 52 & 4077 & 9.2 & 0.940 \\
\hline 1992 & $10001-15000$ & 96 & 13327 & 17.0 & 1.416 \\
\hline 1992 & $15001-20000$ & 224 & 18438 & 39.5 & 1.728 \\
\hline 1992 & $20001-25000$ & 584 & 22416 & 103.7 & 2.208 \\
\hline 1992 & $25001-30000$ & 610 & 28392 & 111.2 & 2.599 \\
\hline 1992 & $30001-35000$ & 1421 & 32791 & 252.5 & 2.992 \\
\hline 1992 & $35001-40000$ & 113 & 36381 & 20.1 & 3.201 \\
\hline 1993 & $20001-25000$ & 176 & 24122 & 31.8 & 1.868 \\
\hline 1993 & $25001-30000$ & 788 & 28792 & 143.4 & 2.672 \\
\hline 1993 & $30001-35000$ & 1597 & 32959 & 286.7 & 3.060 \\
\hline 1993 & $35001-40000$ & 96 & 36000 & 16.6 & 3.310 \\
\hline 1994 & $15001-20000$ & 180 & 17600 & 31.9 & 1.950 \\
\hline 1994 & $25001-30000$ & 768 & 28610 & 138.8 & 2.806 \\
\hline 1994 & $30001-35000$ & 1449 & 32816 & 258.5 & 3.064 \\
\hline 1994 & $35001-40000$ & 575 & 37059 & 102.5 & 3.167 \\
\hline 1995 & $25001-30000$ & 264 & 29143 & 48.0 & 2.729 \\
\hline 1995 & $30001-35000$ & 2001 & 33192 & 360.5 & 3.050 \\
\hline 1995 & $35001-40000$ & 375 & 36128 & 67.2 & 3.324 \\
\hline 1996 & $25001-30000$ & 656 & 29140 & 118.9 & 2.834 \\
\hline 1996 & $30001-35000$ & 1114 & 32882 & 198.6 & 3.044 \\
\hline 1996 & $35001-40000$ & 1268 & 36340 & 224.8 & 3.213 \\
\hline 1996 & $40001-45000$ & 4 & 42000 & 0.7 & 3.140 \\
\hline 1997 & $15001-20000$ & 36 & 16000 & 6.4 & 3.200 \\
\hline 1997 & $25001-30000$ & 79 & 28206 & 14.0 & 2.845 \\
\hline 1997 & $30001-35000$ & 1717 & 33525 & 309.7 & 3.091 \\
\hline 1997 & $35001-40000$ & 1322 & 37011 & 233.9 & 3.267 \\
\hline 1998 & $25001-30000$ & 208 & 27629 & 37.7 & 3.033 \\
\hline 1998 & $30001-35000$ & 676 & 33538 & 121.7 & 3.041 \\
\hline & & & & & \\
\hline
\end{tabular}


Table 12 (Continued)

LWR Quantities Database

Projected Data: No New Orders Case with Extended Burnup

Data Broken Down By: Discharge Year and Burnup Bin

Projected Assemblies for Assembly Class: GE BWR/4-6

\begin{tabular}{|c|c|c|c|c|c|}
\hline $\begin{array}{l}\text { Discharge } \\
\text { Year }\end{array}$ & $\underset{\text { Bin }}{\substack{\text { Burnup } \\
\text { Bin }}}$ & $\begin{array}{l}\text { Number of } \\
\text { Assemblies }\end{array}$ & $\begin{array}{l}\text { Average Burnup } \\
\text { (mwd/MTTHM) }\end{array}$ & $\begin{array}{c}\text { Total Weight } \\
\text { (MTTHM) }\end{array}$ & $\begin{array}{c}\text { Average } \\
\text { Initial } \\
\text { Enrichment }\end{array}$ \\
\hline 1998 & $35001-40000$ & 1666 & 36476 & 301.7 & 3.300 \\
\hline 1998 & $40001-45000$ & 144 & 41004 & 25.6 & 3.620 \\
\hline 1999 & $15001-20000$ & 179 & 19155 & 32.4 & 2.510 \\
\hline 1999 & $25001-30000$ & 55 & 28562 & 9.7 & 3.011 \\
\hline 1999 & $30001-35000$ & 1312 & 33702 & 235.1 & 3.113 \\
\hline 1999 & $35001-40000$ & 1990 & 36638 & 354.4 & 3.209 \\
\hline 2000 & $25001-30000$ & 219 & 28024 & 39.4 & 3.062 \\
\hline 2000 & $30001-35000$ & 478 & 33399 & 86.5 & 3.228 \\
\hline 2000 & $35001-40000$ & 1835 & 36977 & 328.8 & 3.272 \\
\hline 2001 & $25001-30000$ & 198 & 29950 & 35.9 & 2.611 \\
\hline 2001 & $30001-35000$ & 385 & 33067 & 70.2 & 3.117 \\
\hline 2001 & $35001-40000$ & 1616 & 37293 & 289.7 & 3.282 \\
\hline 2001 & $40001-45000$ & 136 & 42066 & 24.3 & 3.687 \\
\hline 2002 & $25001-30000$ & 147 & 27931 & 26.7 & 3.083 \\
\hline 2002 & $30001-35000$ & 238 & 32440 & 43.2 & 3.264 \\
\hline 2002 & $35001-40000$ & 2532 & 37691 & 455.3 & 3.346 \\
\hline 2002 & $40001-45000$ & 557 & 20607 & 99.0 & 3.361 \\
\hline 2003 & $25001-30000$ & 92 & 28539 & 16.5 & 3.101 \\
\hline 2003 & $30001-35000$ & 91 & 33589 & 16.3 & 3.372 \\
\hline 2003 & $35001-40000$ & 1667 & 38031 & 298.1 & 3.385 \\
\hline 2003 & $40001-45000$ & 514 & 41087 & 91.6 & 3.356 \\
\hline 2003 & $45001-50000$ & 129 & 45039 & 22.9 & 3.874 \\
\hline 2004 & $25001-30000$ & 149 & 28315 & 26.9 & 3.077 \\
\hline 2004 & $30001-35000$ & 264 & 33177 & 48.2 & 3.185 \\
\hline 2004 & $35001-40000$ & 1721 & 38103 & 309.4 & 3.3092 \\
\hline 2004 & $40001-45000$ & 223 & 40789 & 40.0 & 3.575 \\
\hline 2005 & $25001-30000$ & 73 & 28720 & 13.1 & 3.099 \\
\hline 2005 & $30001-35000$ & 310 & 33272 & 56.2 & 3.238 \\
\hline 2005 & $35001-40000$ & 1993 & 37709 & 357.5 & 3.328 \\
\hline 2005 & $40001-45000$ & 1221 & 41297 & 215.2 & 3.509 \\
\hline 2006 & $25001-30000$ & 145 & 28271 & 26.3 & 3.104 \\
\hline 2006 & $30001-35000$ & 110 & 34122 & 20.0 & 3.544 \\
\hline 2006 & $35001-40000$ & 1439 & 38228 & 262.3 & 3.333 \\
\hline 2005 & $40001-45000$ & 283 & 42626 & 51.5 & 3.678 \\
\hline 2007 & $25001-30000$ & 73 & 28938 & 13.2 & 3.114 \\
\hline 2007 & $30001-35000$ & 185 & 32627 & 33.3 & 3.264 \\
\hline
\end{tabular}


NEDO-32361

Table 12 (Continued)

LWR Quantities Database

Projected Data: No New Orders Case with Extended Burnup

Data Broken Down By: Discharge Year and Burnup Bin

Projected Assemblies for Assembly Class: GE BWR 14-6

\begin{tabular}{|c|c|c|c|c|c|}
\hline $\begin{array}{l}\text { Discharge } \\
\text { Year }\end{array}$ & $\underset{\text { Bin }}{\text { Burnup }}$ & $\begin{array}{l}\text { Number of } \\
\text { Assemblies }\end{array}$ & $\begin{array}{l}\text { Average Burnup } \\
\text { (mwdMMTHM) }\end{array}$ & $\begin{array}{c}\text { Total } \\
\text { Weight } \\
\text { (MTTHM) }\end{array}$ & $\begin{array}{c}\text { Average } \\
\text { Initial } \\
\text { Enrichment }\end{array}$ \\
\hline 2007 & $35001-40000$ & 1618 & 37384 & 289.3 & 3.289 \\
\hline 2007 & $40001-45000$ & 1058 & 40925 & 186.5 & 3.510 \\
\hline 2008 & $25001-30000$ & 147 & 28547 & 26.7 & 3.092 \\
\hline 2008 & $30001-35000$ & 194 & 33642 & 35.3 & 3.285 \\
\hline 2008 & $35001-40000$ & 2097 & 37967 & 378.9 & 3.353 \\
\hline 2008 & $40001-45000$ & 913 & 40985 & 162.7 & 3.573 \\
\hline 2008 & $45001-50000$ & 128 & 45465 & 22.7 & 3.901 \\
\hline 2009 & $25001-30000$ & 73 & 29065 & 13.1 & 3.122 \\
\hline 2009 & $30001-35000$ & 111 & 32973 & 20.0 & 3.193 \\
\hline 2009 & $35001-40000$ & 1303 & 38147 & 233.4 & 3.297 \\
\hline 2009 & $40001-45000$ & 29 & 41310 & 5.5 & 3.359 \\
\hline 2010 & $25001-30000$ & 141 & 29221 & 25.5 & 3.164 \\
\hline 2010 & $30001-35000$ & 202 & 32951 & 36.4 & 3.281 \\
\hline 2010 & $35001-40000$ & 2020 & 38101 & 355.5 & 3.352 \\
\hline 2010 & $40001-45000$ & 1427 & 41145 & 252.7 & 3.512 \\
\hline 2010 & $45001-50000$ & 127 & 45800 & 22.6 & 3.922 \\
\hline 2011 & $25001-30000$ & 142 & 29416 & 25.7 & 3.205 \\
\hline 2011 & $30001-35000$ & 44 & 34214 & 8.0 & 3.203 \\
\hline 2011 & $35001-40000$ & 1081 & 38256 & 195.2 & 3.399 \\
\hline 2011 & $40001-45000$ & 315 & 42117 & 56.7 & 3.404 \\
\hline 2012 & $15001-20000$ & 132 & 16149 & 23.5 & 3.187 \\
\hline 2012 & $30001-35000$ & 272 & 30958 & 49.0 & 3.280 \\
\hline 2012 & $35001-40000$ & 497 & 38619 & 89.7 & 3.449 \\
\hline 2012 & $40001-45000$ & 1810 & 41647 & 324.5 & 3.561 \\
\hline 2013 & $10001-15000$ & 228 & 13349 & 41.4 & 2.971 \\
\hline 2013 & $20001-25000$ & 80 & 22262 & 14.5 & 3.048 \\
\hline 2013 & $25001-30000$ & 148 & 26255 & 26.9 & 3.082 \\
\hline 2013 & $30001-35000$ & 232 & 31593 & 42.1 & 3.170 \\
\hline 2013 & $35001-40000$ & 759 & 37903 & 138.0 & 3.403 \\
\hline 2013 & $40001-45000$ & 749 & 42575 & 134.4 & 3.494 \\
\hline 2013 & $45001-50000$ & 129 & 45128 & 22.8 & 3.596 \\
\hline 2014 & $5001-10000$ & 28 & 9457 & 5.1 & 3.217 \\
\hline 2014 & $10001-15000$ & 716 & 13694 & 128.7 & 3.285 \\
\hline 2014 & $15001-20000$ & $\overline{472}$ & 16632 & 84.0 & 3.363 \\
\hline 2014 & $20001-25000$ & 144 & 23879 & 25.6 & 3.319 \\
\hline 2014 & $25001-30000$ & 840 & 28250 & 150.8 & 3.445 \\
\hline
\end{tabular}


Table 12 (Continued)

LWR Quantities Database

Projected Data: No New Orders Case with Extended Burnup

Data Broken Down By: Discharge Year and Burnup Bin

Projected Assemblies for Assembly Class: GE BWR/4-6

\begin{tabular}{|l|c|c|c|c|c|}
\hline $\begin{array}{l}\text { Discharge } \\
\text { Year }\end{array}$ & $\begin{array}{c}\text { Burnup } \\
\text { Bin }\end{array}$ & $\begin{array}{c}\text { Number of } \\
\text { Assemblies }\end{array}$ & $\begin{array}{c}\text { Average Burnup } \\
\text { (mwd/MTTHM) }\end{array}$ & $\begin{array}{c}\text { Tota } \\
\text { Weight } \\
\text { (MTTHM) }\end{array}$ & $\begin{array}{c}\text { Average } \\
\text { Initial } \\
\text { Enrichment }\end{array}$ \\
\hline 2014 & $30001-35000$ & 324 & 32708 & 57.8 & 3.427 \\
\hline 2014 & $35001-40000$ & 881 & 38378 & 157.3 & 3.486 \\
\hline 2014 & $40001-45000$ & 985 & 42159 & 176.9 & 3.626 \\
\hline 2014 & $45001-50000$ & 200 & 47572 & 35.2 & 3.903 \\
\hline 2015 & $10001-15000$ & 188 & 14900 & 35.2 & 3.529 \\
\hline 2015 & $15001-20000$ & 176 & 16850 & 31.2 & 3.942 \\
\hline 2015 & $25001-30000$ & 256 & 29811 & 47.2 & 3.783 \\
\hline 2015 & $30001-35000$ & 151 & 31837 & 26.8 & 3.901 \\
\hline 2015 & $35001-40000$ & 111 & 37691 & 20.0 & 3.454 \\
\hline 2015 & $40001-45000$ & 1691 & 42880 & 302.4 & 3.746 \\
\hline 2015 & $45001-50000$ & 20 & 45543 & 3.6 & 3.675 \\
\hline 2016 & $5001-10000$ & 16 & 9635 & 2.9 & 3.085 \\
\hline 2016 & $10001-15000$ & 400 & 14660 & 73.6 & 3.237 \\
\hline 2016 & $15001-20000$ & 16 & 19064 & 2.9 & 3.191 \\
\hline 2016 & $25001-30000$ & 416 & 28217 & 76.6 & 3.385 \\
\hline 2016 & $30001-35000$ & 67 & 33015 & 12.2 & 3.291 \\
\hline 2016 & $35001-40000$ & 544 & 38708 & 98.8 & 3.430 \\
\hline 2016 & $40001-45000$ & 1357 & 43210 & 243.8 & 3.677 \\
\hline 2016 & $45001-50000$ & 190 & 45180 & 33.5 & 3.915 \\
\hline 2017 & $30001-35000$ & 46 & 34166 & 8.1 & 3.364 \\
\hline 2017 & $35001-40000$ & 337 & 38795 & 61.3 & 3.411 \\
\hline 2017 & $40001-45000$ & 918 & 42736 & 164.4 & 3.590 \\
\hline 2018 & $15001-20000$ & 196 & 15558 & 36.4 & 3.422 \\
\hline 2018 & $30001-35000$ & 196 & 31284 & 36.4 & 3.605 \\
\hline 2018 & $35001-40000$ & 213 & 38821 & 38.1 & 3.539 \\
\hline 2018 & $40001-45000$ & 1064 & 43255 & 192.4 & 3.626 \\
\hline 2018 & $45001-50000$ & 536 & 45218 & 94.2 & 3.843 \\
\hline 2019 & $30001-35000$ & 46 & 33844 & 8.2 & 3.343 \\
\hline 2019 & $35001-40000$ & 233 & 38041 & 42.7 & 3.349 \\
\hline 2019 & $40001-45000$ & 1053 & 42820 & 186.2 & 3.663 \\
\hline 2020 & $30001-35000$ & 47 & 33104 & 8.4 & 3.298 \\
\hline 2020 & $35001-40000$ & 314 & 37812 & 56.5 & 3.378 \\
\hline 2020 & $40001-45000$ & 1434 & 42271 & 256.6 & 3.594 \\
\hline 2020 & $45001-50000$ & 203 & 45164 & 36.1 & 3.642 \\
\hline 2021 & $30001-35000$ & 3 & 31524 & 0.6 & 2.045 \\
\hline 2021 & $35001-40000$ & 304 & 38274 & 53.6 & 3.343 \\
\hline & & & & & \\
\hline
\end{tabular}


NEDO-32361

\section{Table 12 (Continued)}

\section{LWR Quantities Database}

Projected Data: No New Orders Case with Extended Burnup

Data Broken Down By: Discharge Year and Burnup Bin

Projected Assemblies for Assembly Class: GE BWR/4-6

\begin{tabular}{|l|c|c|c|c|c|}
\hline $\begin{array}{l}\text { Discharge } \\
\text { Year }\end{array}$ & $\begin{array}{c}\text { Burnup } \\
\text { Bin }\end{array}$ & $\begin{array}{c}\text { Number of } \\
\text { Assemblies }\end{array}$ & $\begin{array}{c}\text { Average Burnup } \\
\text { (mwdMTTMM) }\end{array}$ & $\begin{array}{c}\text { Tota } \\
\text { Weight } \\
\text { (MTTMM) }\end{array}$ & $\begin{array}{c}\text { Average } \\
\text { Initial } \\
\text { Enrichment }\end{array}$ \\
\hline 2021 & $40001-45000$ & 818 & 42233 & 144.6 & 3.603 \\
\hline 2021 & $45001-50000$ & 145 & 45017 & 25.7 & 3.632 \\
\hline 2022 & $10001-15000$ & 471 & 13200 & 83.7 & 3.274 \\
\hline 2022 & $20001-25000$ & 116 & 21183 & 20.0 & 3.555 \\
\hline 2022 & $25001-30000$ & 354 & 28041 & 63.5 & 3.333 \\
\hline 2022 & $3001-35000$ & 164 & 31913 & 28.6 & 3.525 \\
\hline 2022 & $35001-40000$ & 444 & 37941 & 79.7 & 3.368 \\
\hline 2022 & $40001-45000$ & 1496 & 42142 & 267.2 & 3.591 \\
\hline 2023 & $30001-35000$ & 82 & 32669 & 14.6 & 3.246 \\
\hline 2023 & $35001-40000$ & 821 & 38459 & 148.8 & 3.468 \\
\hline 2023 & $40001-45000$ & 677 & 43486 & 120.9 & 3.742 \\
\hline 2024 & $10001-15000$ & 423 & 12745 & 76.0 & 2.945 \\
\hline 2024 & $15001-20000$ & 656 & 16928 & 114.8 & 3.263 \\
\hline 2024 & $20001-25000$ & 144 & 24913 & 25.4 & 2.831 \\
\hline 2024 & $25001-30000$ & 486 & 27659 & 86.3 & 3.378 \\
\hline 2024 & $30001-35000$ & 7 & 32928 & 1.3 & 2.857 \\
\hline 2024 & $35001-40000$ & 564 & 38382 & 98.5 & 3.255 \\
\hline 2024 & $40001-45000$ & 1111 & 41455 & 196.5 & 3.506 \\
\hline 2025 & $10001-15000$ & 452 & 13275 & 81.9 & 3.330 \\
\hline 2025 & $15001-20000$ & 292 & 17125 & 53.1 & 3.226 \\
\hline 2025 & $20001-25000$ & 124 & 22132 & 22.0 & 3.286 \\
\hline 2025 & $25001-30000$ & 553 & 27089 & 100.0 & 3.325 \\
\hline 2025 & $30001-35000$ & 511 & 33895 & 92.5 & 3.246 \\
\hline 2025 & $35001-40000$ & 1046 & 37165 & 189.2 & 3.324 \\
\hline 2025 & $40001-45000$ & 136 & 41344 & 24.8 & 3.427 \\
\hline 2026 & $10001-15000$ & 368 & 13219 & 66.5 & 3.430 \\
\hline 2026 & $15001-20000$ & 88 & 15654 & 16.3 & 3.386 \\
\hline 2026 & $20001-25000$ & 252 & 23731 & 45.0 & 3.451 \\
\hline 2026 & $25001-30000$ & 204 & 27475 & 37.9 & 3.386 \\
\hline 2026 & $30001-35000$ & 263 & 32853 & 47.2 & 3.393 \\
\hline 2026 & $35001-40000$ & 450 & 37803 & 81.8 & 3.417 \\
\hline 2026 & $40001-45000$ & 305 & 41464 & 53.0 & 3.375 \\
\hline 2027 & $10001-15000$ & 172 & 11160 & 31.8 & 3.649 \\
\hline 2027 & $15001-20000$ & 276 & 17827 & 44.8 & 3.802 \\
\hline 2027 & $20001-25000$ & 172 & 23296 & 31.8 & 3.737 \\
\hline 2027 & $30001-35000$ & 287 & 33351 & 48.8 & 3.898 \\
\hline & & & & & \\
\hline
\end{tabular}




\section{Table 12 (Continued)}

LWR Quantities Database

Projected Data: No New Orders Case with Extended Burnup

Data Broken Down By: Discharge Year and Burnup Bin

Projected Assemblies for Assembly Class: GE BWR/4-6

\begin{tabular}{|l|c|c|c|c|c|}
\hline $\begin{array}{l}\text { Discharge } \\
\text { Year }\end{array}$ & $\begin{array}{c}\text { Burnup } \\
\text { Bin }\end{array}$ & $\begin{array}{c}\text { Number of } \\
\text { Assemblies }\end{array}$ & $\begin{array}{c}\text { Average Burnup } \\
\text { (mwd/MTTMM) }\end{array}$ & $\begin{array}{c}\text { Total } \\
\text { Weight } \\
\text { (MTTMM) }\end{array}$ & $\begin{array}{c}\text { Average } \\
\text { Initial } \\
\text { Enrichment }\end{array}$ \\
\hline 2027 & $35001-40000$ & 229 & 36991 & 42.3 & 3.808 \\
\hline 2027 & $40001-45000$ & 323 & 42790 & 55.3 & 3.759 \\
\hline 2027 & $45001-50000$ & 40 & 48560 & 7.3 & 3.940 \\
\hline 2028 & $30001-35000$ & 119 & 34508 & 21.0 & 3.285 \\
\hline 2028 & $35001-40000$ & 164 & 39737 & 29.1 & 3.299 \\
\hline 2029 & $10001-15000$ & 332 & 14537 & 58.8 & 3.210 \\
\hline 2029 & $20001-25000$ & 216 & 24280 & 38.3 & 3.210 \\
\hline 2029 & $30001-35000$ & 216 & 32858 & 38.3 & 3.210 \\
\hline & & & & 17503.7 & 3.304 \\
\hline
\end{tabular}


Waste Acceptance Criteria Definition from Yucca Mountain She Charactorizetion Project Change Directive, CR No. DCP-060 dated 2/6/93

\section{Table F1.1.1 Function Description: Define Waste Acceptance Criteria}

I. Function ID Number:

1.1.1

II. Function Thile:

Define Waste Acceptance Criteria

III. Function Definition:

Determine the conditions necessary to be met by the SNF/HLW, in order for DOE to be able to accept it for disposal. (Note that the 10CFR60 definition for HLW includes SNF.)

IV. Interfaces:

A. Inputs:

$\begin{array}{llll}\text { 1.1.111 } & \text { Waste Characteristics } & \text { From: } & \text { Purchaser/Producer } \\ \text { 1.1.112 } & \text { RW-859 Data } & \text { From: } & \text { Purchaser } \\ \text { 1.1.113 } & \text { 10CFR961 } & \text { From: } & \text { Function L.1.2 }\end{array}$

B. Outputs:
1.1.101 Waste Acceptance Criteria
To:
Function 1.1.2

v. Function Requirements:

A. Constraints:

1.1.1C1 (a) High-level-waste package design in general.

(1) Packages for HLW shall be designed so that the in situ chemical, physical, and nuclear properties of the waste package and its interactions with the emplacement environment do not compromise the function of the waste packages or the performance of the underground facility or the geologic setting. 


\section{Table F1.1.1 (Continued)}

(2) The design shall include but not be limited to consideration of the following factors: solubility, oxidation/reduction reactions, corrosion, hydrating, gas generation, thermal effects, mechanical strength, mechanical stress, radiolysis, radiation damage, radionuclide retardation, leaching, fire and explosion hazards, thermal loads, and synergistic interactions.

(b) Specific criteria for HLW package design.

(1) Explosive, pyrophoric, and chemically reactive materials. The waste package shall not contain explosive or pyrophoric materials or chemically reactive materials in an amount that could compromise the ability of the underground facility to contribute to waste isolation or the ability of the geologic repository to satisfy the performance objectives.

(2) Free liquids. The waste package shall not contain free liquids in an amount that could compromise the ability of the waste packages to achieve the performance objectives relating to containment of HLW (because of chemical interactions or formation of pressurized vapor) or result in spillage and spread of contamination in the event of waste package perforation during the period through permanent closure.

(4) Unique identification. A label or other means of identification shall be provided for each waste package. The identification shall not impair the integrity of the waste package and shall be applied in such a way that the information shall be legible at least to the end of the period of retrievability. Each waste package identification shall be consistent with the waste package's permanent written records.

[10CFR60.135]

1.1.1C2 (c) Waste form criteria for HLW. High-level radioactive waste that is emplaced in the underground facility shall be designed to meet the following criteria: 


\section{Table F1.1.1 (Continued)}

(1) Solidification. All such radioactive wastes shall be in solid form and placed in sealed containers.

(2) Consolidation. Particulate waste forms shall be consolidated (for example, by incorporation into an encapsulating matrix) to limit the availability and generation of particulates.

(3) Combustibles. All combustible radioactive wastes shall be reduced to a noncombustible form unless it can be demonstrated that a fire involving the waste packages containing combustibles will not compromise the integrity of other waste packages, adversely affect any structures, systems, or components important to safety, or compromise the ability of the underground facility to contribute to waste isolation.

[10CFR60.135]

1.1.1C3 (d) Design criteria for other radioactive wastes. Design criteria for waste types other than HLW will be addressed on an individual basis if and when they are proposed for disposal in a geologic repository.

[10CFR60.135]

1.1.1C4 This requirement intentionally left blank.

1.1.1C5 This requirement intentionally left blank.

1.1.1C6 (c) The Safety Analysis Report shall include:

(5) A description of the kind, amount, and specifications of the radioactive material proposed to be received and possessed at the geologic repository operations area.

[10CFR60.21] 


\section{Table F1.1.1 (Continued)}

1.1.1C7 (b) License conditions shall include thems in the following categorles:

(1) Restrictions as to the physical and chemical form and radioisotopic content of radioactive waste.

(2) Restrictions as to size, shape, and materials and methods of construction of radioactive waste packaging.

(3) Restrictions as to the amount of waste permitted per unit volume of storage space considering the physical characteristics of both the waste and the host rock.

(4) Requirements relating to test, calibration, or inspection to assure that the foregoing restrictions are observed.

[10CFR60.43]

1.1.1C8 (b) Structures, systems, and components important to se fety:

(7) Criticality control. All systems for processing, transporting, handling, storage, retrieval, emplacement, and isolation of radioactive waste shall be designed to ensure that a nuclear criticality accident is not possible unless at least two unlikely, independent, and concurrent or sequential changes have occurred in the conditions essential to nuclear criticality saiety. Each system shall be designed for criticality safety under normal and accident conditions. The calculated effective multiplication factor (keff) must be sufficiently below unity to show at least a $5 \%$ margin, after allowance for the bias in the method of calculation and the uncertainty in the experiments used to validate the method of calculation.

[10CFR60.131]

1.1.1C9 This requirement intentionally left blank.

1.1.1C10 This requirement intentionally left blank. 


\section{Table F1.1.1 (Continued)}

\subsubsection{C11 Criteria for nuclear criticality safety:}

(a) Design for criticality safety. Spent fuel handling, packaging, transfer, and storage systems must be designed to be maintained subcritical and to ensure that, before a nuclear criticality accident is possible, at least two unlikely, independent, and concurrent or sequential changes have occurred in the conditions essential to nuclear criticality safety. The design of handling, packaging, transfer, and storage systems must include margins of safety for the nuclear criticality parameters that are commensurate with the uncertainties in the data and methods used in calculations and demonstrate safety for the handling, environment under accident conditions.

[10CFR72.124]

B. Performance:

1.1.1P1 The DOE shall accept high-level radioactive waste. Detailed acceptance criteria and general specifications for such waste will be issued by the DOE no later than the date on which DOE submits its license application to the Nuclear Regulatory Commission for the first disposal facility.

[10CFR961.11, Appondix E,D]

C. Interface:

1.1.111a Routine determinations. Prior to each shipment of licensed material, the licensee shall ensure that the package with its contents satisfies the applicable requirements of this part and of the license. The licensee shall determine that:

(a) The package is proper for the contents to be shipped;

(b) The package is in unimpaired physical condition except for superficial defects such as marks or dents; 
Table F1.1.1 (Continued)

(c) Each closure device of the packaging, including any required gasket, is property installed and secured and free of defects;

(d) Any system for containing liquid is adequately sealed and has adequate space or other specifled provision for expansion of the liquid;

(e) Any pressure relief device is operable and set in accordance with written procedures;

(f) The package has been loaded and closed in accordance with written procedures;

(g) For fissile material, any moderator or neutron absorber, if required, is present and in proper condition;

(h) Any structural part of the package which could be used to lift or tie down the package during transport is rendered inoperable for that purpose unless it satisfles the design requirements of Sec. 71.45;

(i)(1) The level of non-fixed (removable) radioactive contamination on the external surfaces of each package offered for shipment is as low as reasonably achievable. The level of non-fixed radioactive contamination may be determined by wiping an area of 300 square centimeters of the surface concerned with an absorbent material, using moderate pressure, and measuring the activity on the wiping material. Sufficient measurements must be taken in the most appropriate locations to yield a representative assessment of the non-fixed contamination levels. Except as provided under paragraph (1)(2) of this section, the amount of radioactivity measured on any single wiping material when averaged over the surface wiped, must not exceed the limits glven in Table $V$ of this part at any time during transport. Other methods of assessment of equal or greater efficiency may be used. When other methods are used, the detection efficiency of the method used must be taken into account and in no case may the non-fired contamination on the external surfaces of the package exceed ten times the limits listed in Table V. 
Tablo V Romovable Extomal Radloactive Contamination Wipo Limito

\begin{tabular}{|c|c|c|}
\hline \multirow[b]{2}{*}{ Contaminent } & \multicolumn{2}{|c|}{$\begin{array}{l}\text { Maximum Pormitalbis } \\
\text { Lmite }\end{array}$} \\
\hline & нCymm & dom/om 2 \\
\hline $\begin{array}{l}\text { Bota-gamma emitting radionuclides; all } \\
\text { radionuclides Ilves less than ten days; } \\
\text { natural uranium; natural thorium uranium- } \\
235 \text {; uranium-238; thorium-232, thorium-223, } \\
\text { and thorium-230 when contained in ores or } \\
\text { phyical concentrates }\end{array}$ & $10^{-6}$ & 22 \\
\hline All other alpha emitting radionuclides & $10^{-6}$ & 2.2 \\
\hline
\end{tabular}

(2) In the case of packages transported as exclusive use shipments by rall or highway only, the non-fixed radioactive contamination at any time during transport must not exceed ten times the levels prescribed in paragraph (1)(1) of this section. The levels at the beginning of transport must not exceed the levels prescribed in paragraph (i)(1) of this section.

(j) External radiation levels around the package and around the vehicle, $\mathrm{H}$ applicable, will not exceed the limits specified in Sec. 71.47 at any time during transportation; and

(k) Accessible package surface temperatures will not exceed the limits specified in Sec. $71.43(\mathrm{~g})$ at any time during transportation.

[10CFR71.87]

\subsubsection{1b General considerations.}

(b) The MRS must be designed to store either spent fuel or solid high-level radioactive wastes. Liquid high-level radioactive wastes may not be received or stored in an MRS. If the MRS is a water-pool type facility, the solidified waste form shall be a durable solid with demonstrable leach resistance.

[10CFR72.120] 


\section{Appendix J: Adapting Commercial MOX Fuel Fabrication Experience}

A Hazard and Operability Study (HAZOP I)' study was held to discuss the implications of processing plutonia derived from weapons or 'A' grade plutonium using a commercial MOX fabrication process such as that currently being developed for the Sellafield MOX Plant (SMP). The SMP process is based on the conversion of civil plutonia powder (arising from the reprocessing of fuel from MAGNOX and Thermal Oxide Nuclear Power Stations) to Mixed Oxide (MOX) Fuel elements. Figure J-1 indicates the main features of the proposed SMP process and this was taken as the process basis for the study.

\section{J.1 Objectives of Study}

The objectives of the meeting were to:

- Identify the key assumptions which needed to be made for the study. The assumptions related to the form of ' $A$ ' grade plutonium, its isotopic composition, etc.

- Identify the key issues (in terms of safety, operability and process) which would need to be addressed in adapting a commercial MOX fuel fabrication process to handle ' $A$ ' grade plutonia.

\section{J.2 Assumptions}

The following assumptions have been made with regard to the form and isotopic composition of the ' $A$ ' grade plutonium:

- 'A' grade plutonium contains 5 w/o Pu-240. The sensitivity of the study to plutonia, with Pu-240 content of $<5$ w/o would also be considered.

- The process will receive plutonia powder. The conversion of weapons plutonium to the oxide form will occur in a facility provided elsewhere.

- The moisture content of the plutonia powder is $1.5 \mathrm{wt} \%$.

- The plutonia powder is 5 years aged. If the age is increased the dose rates would increase due to Am growth and the heat output would increase slightly.

${ }^{1}$ HAZOP is a rigorous systematic process used principally to identify potential hazards and assess the safety of plant designs. 


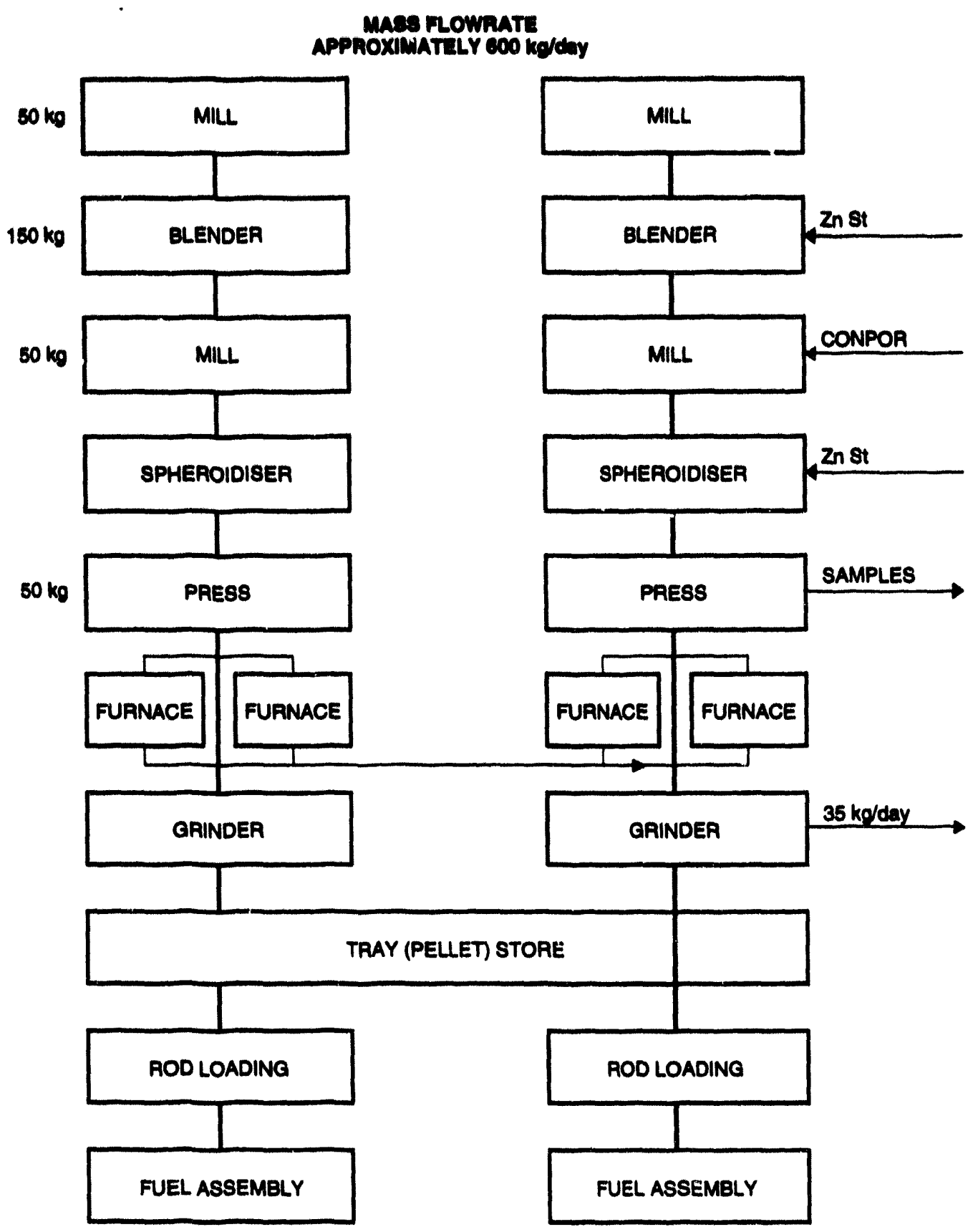

Figure J-1 SMP Process Flow Diagram 
- The HAZOP I study is based on the assumption that civil grade plutonia is replaced in terms of throughput with plutonia, derived from ' $A$ ' grade plutonium.

A comparison of these assumptions with the current SMP Reference Case is given in Table J-1.

Table J-1 Comparison of SMP Reference Fuel with Assumed 'A' Grade Fuel

\begin{tabular}{|l|l|l|}
\hline & \multicolumn{1}{|c|}{\begin{tabular}{c}
\multicolumn{1}{|c|}{ SMP Reference } \\
$\mathbf{P u O}_{2}$
\end{tabular}} & \multicolumn{1}{c|}{$\begin{array}{c}\text { Assumed 'A' Grade } \\
\text { PuO2 }\end{array}$} \\
\hline Burn Up & $45 \mathrm{Gwd} / \mathrm{teU}$ & $500 \mathrm{Mwd} / \mathrm{teU}$ \\
\hline Aging & $\begin{array}{l}5 \text { years } \\
\text { (plus 5 year cooling prior to } \\
\text { reprocessing) }\end{array}$ & 5 years \\
\hline Pu-240 Content & 10 w/o & $5 \mathrm{wt} \%$ \\
\hline Moisture & $1.5 \mathrm{w} / 0$ & $1.5 \mathrm{wt} \%$ \\
\hline
\end{tabular}

\section{J.3 HAZOP Study}

The HAZOP I study was undertaken using the key word listing given in Table J-2. The basis of the study was the proposed SMP process flow diagram shown in Figure J-1.

Table J-3 is a record of the discussions from the meeting for each of the key words listed in Table J-2.

A single action was generated against Nuclear Technology to determine the isotopic composition of typical ' $A$ ' grade plutonium. This action (Action 1.1) and its response are included in the HAZOP minutes in Table J-4.

Table J-2. Key Word List for the HAZOP I

\begin{tabular}{|ll|}
\hline EXTERNAL DOSE & SHIELDING \\
INTERNAL DOSE & LOSS OF CONTAINMENT \\
VENTILATION & EFFLUENTS \\
CRITICALITY & FIRE \\
EXPLOSION/DETONATION & IMPACT DAMAGE \\
MIXING OF FEEDS & SEISMIC \\
EXTREME WEATHER & LOSS OF SERVICES \\
INSTRUMENTATION/INTERLOCKS & MAINTAINABILITY \\
TOXICITY & CORROSION \\
DECOMMISSIONING & DOMINO \\
HUMAN FACTORS & OTHERS \\
\hline
\end{tabular}


Table J-3 HAZOP I Record Sheet

Meeting No: 1

Date: 6/12/93

\begin{tabular}{|c|c|c|}
\hline Keyword & Discussion & Action/Recommendation \\
\hline $\begin{array}{l}\text { External Dose/ } \\
\text { Shielding }\end{array}$ & $\begin{array}{l}\text { The anticipated dose rates from the ' } \mathrm{A} \text { ' } \\
\text { grade } \mathrm{PuO}_{2} \text { will be lower than those } \\
\text { being emitted from } \mathrm{PuO}_{2} \text { derived from } \\
\text { civil reactors. The response to } \mathrm{HAZOP} \\
\text { action } 1.1 \text { (see attached) provides a } \\
\text { comparison with the expected gamma } \\
\text { and neutron dose rates between civil } \\
\text { and } \mathrm{A} \text { grade } \mathrm{PuO} \mathrm{O}_{2} \text {. It is seen for } \mathrm{A} \\
\text { grade } \mathrm{PuO}_{2} \text { dose rates are lower. } \\
\text { Consequently this reduction in external } \\
\text { dose rates may enable the MOX fabri- } \\
\text { cation in the proposed plant to involve } \\
\text { more manual intervention. } \\
\text { Additionally, the required shielding } \\
\text { may be able to be reduced. }\end{array}$ & $\begin{array}{l}\text { Action } 1.1-\text { On } \\
\text { D. Winstanley (Nuclear } \\
\text { Technology. Determine the } \\
\text { isotopic composition of } \\
\text { weapons ('A') grade pluto- } \\
\text { nium that will be handled } \\
\text { within the proposed MOX } \\
\text { production facility. }\end{array}$ \\
\hline $\begin{array}{l}\text { Internal Dose/Loss } \\
\text { of Containment }\end{array}$ & $\begin{array}{l}\text { It is anticipated that ' } A \text { ' grad } \mathrm{PuO}_{2} \text { will } \\
\text { be less radiotoxic than that used in the } \\
\text { SMP process. However, the } \mathrm{PuO}_{2} \\
\text { handled will never the less be extremely } \\
\text { radiotoxic and similar precautions will } \\
\text { need to be undertaken to ensure that its } \\
\text { primary containment is maintained at } \\
\text { all times. }\end{array}$ & \\
\hline Ventilation & $\begin{array}{l}\text { The requirements as indicated above } \\
\text { are for all PuO } \mathrm{P}_{2} \text { bearing material to be } \\
\text { maintained in primary containment. In } \\
\text { some instances, containment would be } \\
\text { provided by the various ventilation } \\
\text { systems (e.g., } \mathrm{C} 5 \text { extraction on the } \\
\text { glovebox system and the } \mathrm{C} 3 / \mathrm{C} 2 \text { for } \\
\text { secondary containment. These ventila- } \\
\text { tion systems will need to be provided } \\
\text { even through the radiotoxicity of 'A' } \\
\text { grade } \mathrm{PuO}_{2} \text { is less than that for civil } \\
\text { grade } \mathrm{PuO}_{2} \text {. } \\
\text { The decay heat load of 'A' grade } \mathrm{PuO}_{2} \\
\text { will be less than that from civil grade } \\
\text { PuO } \\
\text { additional cooling or the requirement } \\
\text { for cooling to be provided by the venti- } \\
\text { lation system. }\end{array}$ & \\
\hline
\end{tabular}


Table J-3. HAZOP I Record Sheet (Continued)

\section{Meeting No: 1}

Date: 6/12/93

\begin{tabular}{|c|c|c|}
\hline Keyword & Discussion & Action/Recommendation \\
\hline Effluents & $\begin{array}{l}\text { No additional liquid/solid effluents due } \\
\text { to handling of ' } \mathrm{A} \text { ' grade } \mathrm{PuO}_{2} \text { rather } \\
\text { than civil grade } \mathrm{PuO}_{2} \text { was identified. }\end{array}$ & \\
\hline Criticality & $\begin{array}{l}\text { It was noted by Nuclear Technology } \\
\text { that the reduction of the Pu- } 240 \text { content } \\
\text { of the 'A' grade material would result } \\
\text { in an increase of the reactivity of the } \\
\text { vessels. Nuclear Technology indicated } \\
\text { that a reduction of the Pu- } 240 \text { content } \\
\text { from } 10 \text { wt\% to } 5 \text { wt\% reduces the safe } \\
\text { mass by } 5 \% \text { and it is concluded that to } \\
\text { maintain the same level of safety, the } \\
\text { proposed process vessels should be re- } \\
\text { duced by a similar fraction, through the } \\
\text { actual reduction needs to be confirmed. } \\
\text { This may have an effect on the plant } \\
\text { capacity, though the actual magnitude is } \\
\text { unclear. However, should the capacity } \\
\text { be reduced, then this may be offset by } \\
\text { how the plant is operated, controlled, or } \\
\text { by additional lines. }\end{array}$ & \\
\hline $\begin{array}{l}\text { Firel } \\
\text { Overheating }\end{array}$ & $\begin{array}{l}\text { As previously indicated (under ventila- } \\
\text { tion) the heat generation capacity of 'A' } \\
\text { grade } \mathrm{PuO}_{2} \text { is less than that for civil } \\
\text { grade } \mathrm{PuO}_{2} \text { and consequently the over- } \\
\text { heating hazard potential should be } \\
\text { reduced. }\end{array}$ & \\
\hline $\begin{array}{l}\text { Explosion/ } \\
\text { Detonation }\end{array}$ & $\begin{array}{l}\text { No additional explosion or detonation } \\
\text { hazards were identified due to the pro- } \\
\text { cessing of ' } \mathrm{A} \text { ' grade } \mathrm{PuO}_{2} \text { rather than } \\
\text { civil grade } \mathrm{PuO}_{2} \text {. }\end{array}$ & \\
\hline Impact Damage & $\begin{array}{l}\text { No additional impact scenarios were } \\
\text { identified due to the processing of ' } \mathrm{A} \text { ' } \\
\text { grade } \mathrm{PuO}_{2} \text { rather than civil } \mathrm{PuO}_{2} \text {. }\end{array}$ & \\
\hline
\end{tabular}


Table J-3 HAZOP I Record Sheet (Continued)

Meeting No: 1

Date: 6/12/93

\begin{tabular}{|l|l|l|}
\hline Keyword & \multicolumn{1}{|c|}{ Discussion } & Action/Recommendation \\
\hline Mixing of Feeds & $\begin{array}{l}\text { The potential option that the plant may } \\
\text { also process civilian PuO } \mathrm{O}_{2} \text { was identi- } \\
\text { fied. Should this occur then increased } \\
\text { dose rates compared with 'A' grade } \\
\text { PuO }{ }_{2} \text { can be expected. Consequently, } \\
\text { should civilian grade PuO }{ }_{2} \text { be handled } \\
\text { in addition to 'A' grade PuO } \\
\text { ther additional shielding and/or lower } \\
\text { cell occupancies would be required to } \\
\text { maintain whule body dose levels to } \\
\text { acceptable levels. }\end{array}$ & \\
\hline Seismic & $\begin{array}{l}\text { No additional seismic requirements } \\
\text { have been identified due to the process- } \\
\text { ing of 'A' grade plutonium rather than } \\
\text { civil plutonium. The existing provi- } \\
\text { sions would need to be maintained. }\end{array}$ & \\
\hline Extreme Weather & $\begin{array}{l}\text { No additional design requirements have } \\
\text { been identified due to the processing of } \\
\text { 'A' grade plutonium rather than civil } \\
\text { plutonium. The existing provisions } \\
\text { would need to be maintained. }\end{array}$ & \\
\hline Loss of Services & $\begin{array}{l}\text { The lower decay heat from 'A' grade } \\
\text { PuO } 2 \text { may reduce the need to provide } \\
\text { cooling following a loss of services. }\end{array}$ & \\
\hline Instrumentation/ & $\begin{array}{l}\text { The reduction in the Pu-241 (and hence } \\
\text { gamma rays) may make it more diffi- } \\
\text { Interlocks } \\
\text { This may make the provision of safety } \\
\text { protection systems, and product instru- } \\
\text { mentation more difficult to engineer. }\end{array}$ & \\
\hline Maintainability & $\begin{array}{l}\text { No additional maintainability problems } \\
\text { due to the processing of 'A' grade PuO } \\
\text { rather than civil PuO } 2 \text { were identified. } \\
\text { It was noted that the reduction in the } \\
\text { dose rates from the fissile material } \\
\text { should reduce dose update from back- } \\
\text { ground contamination levels during } \\
\text { maintenance. }\end{array}$ & \\
\hline
\end{tabular}


Table J-3 HAZOP I Record Sheet (Continued)

\section{Meeting No: 1}

Date: 6/12/93

\begin{tabular}{|c|c|c|}
\hline Keyword & Discussion & Action/Recommendation \\
\hline Toxicity & $\begin{array}{l}\text { No additional toxicity hazards due to } \\
\text { the processing of ' } \mathrm{A} \text { ' grade } \mathrm{PuO}_{2} \text { rather } \\
\text { than civil PuO } \mathrm{P}_{2} \text { were identified. } \\
\text { Although radiotoxicity of ' } \mathrm{A} \text { ' grade } \\
\mathrm{PuO}_{2} \text { is less than civil } \mathrm{PuO}_{2} \text { the } \\
\text { containment requirements would be the } \\
\text { same. }\end{array}$ & \\
\hline Corrosion & $\begin{array}{l}\text { No additional corrosion hazards due to } \\
\text { the processing of ' } \mathrm{A} \text { ' grade } \mathrm{PuO}_{2} \text { rather } \\
\text { than civil } \mathrm{PuO}_{2} \text { were identified. }\end{array}$ & \\
\hline Decommissioning & $\begin{array}{l}\text { No additional decommissioning hazards } \\
\text { due to the processing of ' } \mathrm{A} \text { ' grade } \mathrm{PuO}_{2} \\
\text { rather than civil } \mathrm{PuO}_{2} \text { were identified. } \\
\text { If the facility processed solely ' } \mathrm{A} \text { ' } \\
\text { grade } \mathrm{PuO}_{2} \text { then dose rates during } \\
\text { decommissioning would be less. }\end{array}$ & \\
\hline Domino & $\begin{array}{l}\text { It was noted that weapons grade mate- } \\
\text { rial would not be in the form of } \mathrm{PuO}_{2} \\
\text { powder required for feed to commercial } \\
\text { MOX plants and that conversion to the } \\
\text { oxide state (i.e., } \mathrm{PuO}_{2} \text { ) will need to } \\
\text { occur within an upstream plant. The } \\
\text { HAZOP study does not consider this } \\
\text { conversion plant. However, it is noted } \\
\text { that the capacity of the conversion plant } \\
\text { should match the capacity of the MOX } \\
\text { facility, i.e., the conversion plant should } \\
\text { not impose a restriction on the oper- } \\
\text { ability of the MOX plant. }\end{array}$ & \\
\hline Human Factors & $\begin{array}{l}\text { No additional human factor issues were } \\
\text { raised as the result of handling } \mathrm{PuO}_{2} \\
\text { arising from ' } \mathrm{A} \text { ' grade plutonium rather } \\
\text { than civilian } \mathrm{PuO}_{2} \text {. }\end{array}$ & \\
\hline Others & & \\
\hline
\end{tabular}


Table J-4 HAZOP Action Responses

ACTION: Determine the isotopic composition of weapons grade (' $\mathrm{A}$ ' grade) $\mathrm{PuO}_{2}$ that will be handled within the proposed MOX production facility.

It is considered that the facility will handle $\mathrm{PuO}_{2}$ that is approximately 5 years aged.

REPLY: The ratios shown below give a trade only and should not be used for specific calculations without verification that it is appropriate to do so.

'A' grade plutonium usually has a low Pu240 content ( $5 \mathrm{wt} \%)$.

Nuclear Technology have in existence a MAGNOX FISPIN run for $500 \mathrm{Mwd} / \mathrm{te}$ irradiated fuel, 5 years aged (PDEC 94) which has the following isotopic composition:

$\begin{array}{lll}\text { Pu240 4.3 wt\% } & \text { Pu236 } & \text { 9.52E-9 wt\% } \\ \text { Pu241 0.19 wt\% } & \text { Pu238 } & 3.8 \text { E-3 wt\% } \\ \text { Pu242 2.95E-3 wt\% } & \text { Pu239 } & 95.5 w t \%\end{array}$

A comparison between this composition and the SMP Shielding Design Basis (SDB) based on an arbitrary but appropriate system (i.e., plutonium surrounded by $6 \mathrm{~mm}$ steel) gives the following ratios (A Grade/SDB) for $5 \mathrm{yr}$ aged fuel.

RATIOS

$\begin{array}{llll} & \gamma & \mathrm{n} & \text { Total } \\ \text { Unshielded } & 0.05 & 0.11 & 0.05 \\ \text { Shielded } & 0.11 & 0.11 & 0.11\end{array}$

(6mm Steel)

The above ratios show that for these set of circumstances ' $A$ ' Grade plutonium give $\sim 5 \%$ of the unshielded dose rates and $10 \%$ of the shielded dose rates assumed from the reference case.

NB: Shielded dose rates are dominated by neutron dose rates. If the Pu-240 content increases then the neutron dose rates will increase. 


\section{J.4 Main Findings}

The following is a summary of the main issues raised during the study:

- Because the ' $\mathrm{A}$ ' grade plutonia contains fewer gamma and neutron emitting isotopes (see response to Action 1.1, Table J-3) the need for the fully automated processing could be relaxed which may have particular benefits for fuel assembly operations. This would not be the case if the facility had to process both civil and ' $A$ ' grade plutonium.

- Though the radiotoxicity of ' $A$ ' grade plutonium is marginally less than civil plutonium it is considered that the containment integrity of any plutonium bearing material will need to be the same to that for a civil plutonia MOX plant.

- The radiometric decay heat arising from plutonia derived from ' $A$ ' grade plutonium will be less than that from civil grade material. Consequently this may reduce the requirements to provide cooling of any plutonium bearing material holdup within the process.

- The Pu-240 content of plutonia derived from 'A' grade plutonium will be less than the corresponding Pu- 240 content of civilian grade material. This may require that the capacity of any vessel that may hold pure plutonia powder or MOX be reduced to ensure criticality safety under normal and fault conditions. Typically for plutonia with a Pu-240 content of $10 \mathrm{wt} \%$, a reduction in Pu- 240 content to $5 \mathrm{wt} \%$ reduces the safe mass by $5 \mathrm{wt} \%$.

The effects on the plant capacity because of this would need to be considered, though it is anticipated that any reduction in individual vessel capacity could be off-set by for example increasing the number of process lines etc.

- It was noted that the lower Pu-241 content of 'A' grade plutonia will result in lower external dose rates. As it is anticipated that some instrument based protection systems within the plant may, in some instances rely on the gamma rays being emitted from the plutonia (e.g., criticality instruments) then the availability of these systems could be affected.

- The capacity of any upstream facility which may be used to convert weapons grade plutonium to plutonia suitable for the commercial process should be such that it will not restrict the operation of the MOX plant. In addition the upstream plutonium conversion facility could incorporate a capability to blend the weapons grade plutonia with other material (e.g., civil plutonia or urania) such that the criticality reactivity of the material was no worse than civil plutonia. This would avoid increasing the difficulty in making a criticality safety case. 


\section{Appendix K: DOE Order Applicability Assessment}

This appendix was prepared for an earlier study on plutonium disposition using the GE Advanced Boiling Water Reactor (ABWR). It details an assessment of the applicability of the various DOE Orders to the disposition project. It is included here to provide information that is relevant to the present study, where plutonium is disposed using existing BWRs. Specifically, the information related to the safeguards provisions on the handling of Special Nuclear Materials (SNM) contained in DOE Orders are applicable to both this and the previous study.

The assessment began with a review of the Directives Checklist of DOE Orders (RL N 0000.1S) and the identification of those DOE Orders that were potentially applicable to the ABWR Complex operations and activities. Each order was reviewed for applicability and a brief justification prepared based on familiarity with the DOE Order and the ABWR Complex facility layout, activities and operation. Seventy-seven DOE Orders were determined to apply to the ABWR Complex. Orders werc considered not applicable if they simply did not apply or if the DOE Order contained no requirement statements applicable to a contractor.

A tabular summary of the Orders evaluated and the statement of applicability is provided in Table 1. This provides a quick overview of all the orders assessed and their applicability to the ABWR Complex.

An item of interest that was identified during this review is that several DOE Orders include exclusionary statements that if the reactor or facility is licensed by the NRD, then the DOE Order does not apply (i.e., 5480.6, .22, .23, .24, .26, .28 and .30). These orders are included in the assessment since it has not been determined if the DOE will actively pursue an NRC license or simply utilize the NRC expertise during the review and approval periods which could preclude the public review activities required by the formal licensing process. 
TABLE 1

DOE ORDER APPLICABILITY ASSESSMENT

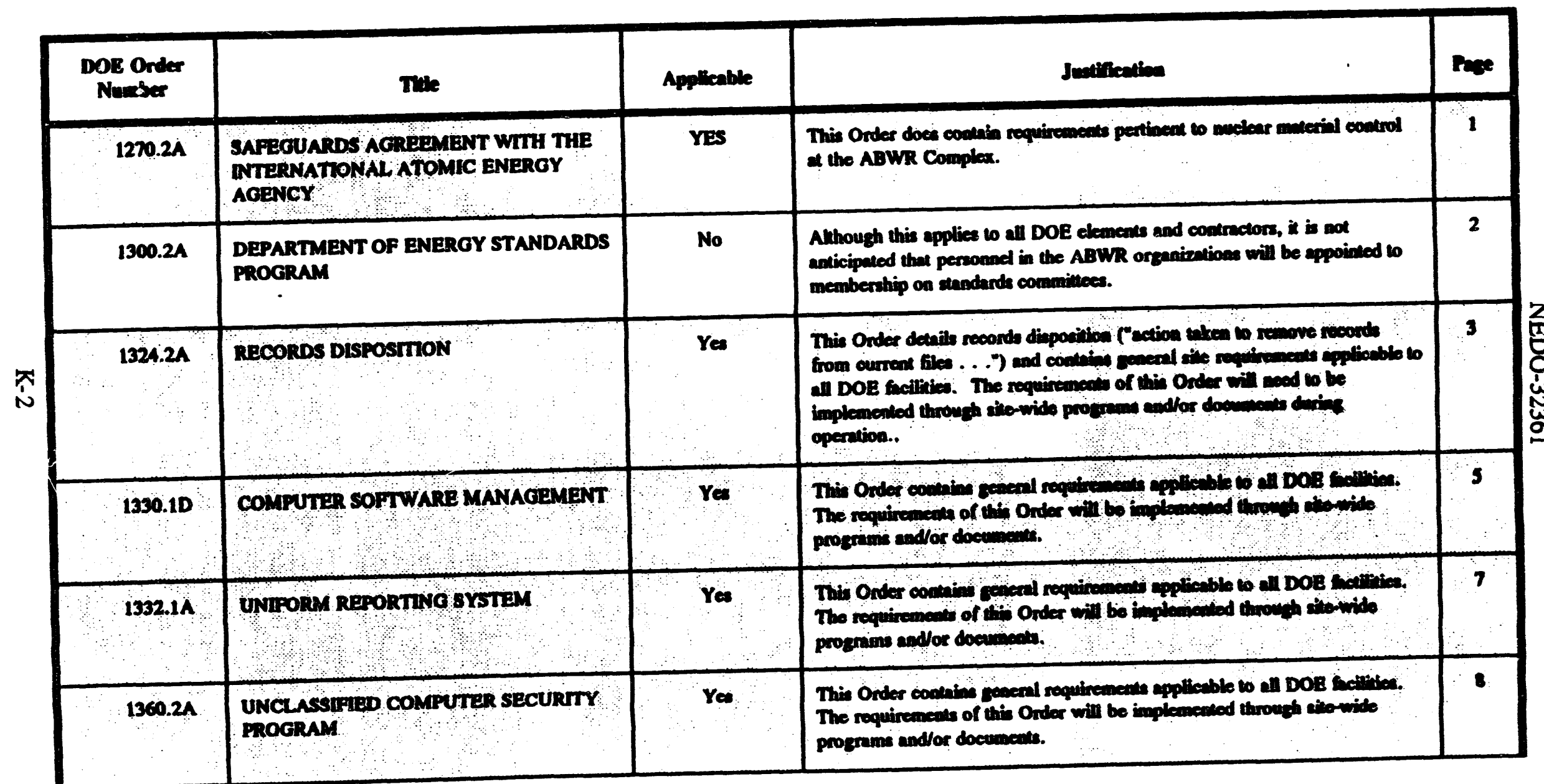

(Shaded boxes indicate applicable orders.)

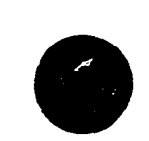



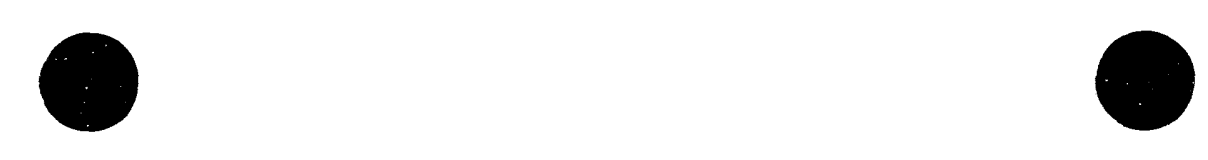

TABLE 1

DOE ORDER APPLICABILITY ASSESSMENT

\begin{tabular}{|c|c|c|c|c|}
\hline $\begin{array}{l}\text { DOE Order } \\
\text { Nomber }\end{array}$ & The & Apprientile & Juetilection & rase \\
\hline $1360.4 \mathrm{~A}$ & $\begin{array}{l}\text { SCIENTIFIC AND TECHNICAL } \\
\text { COMPUTER SOFTWARE }\end{array}$ & No & 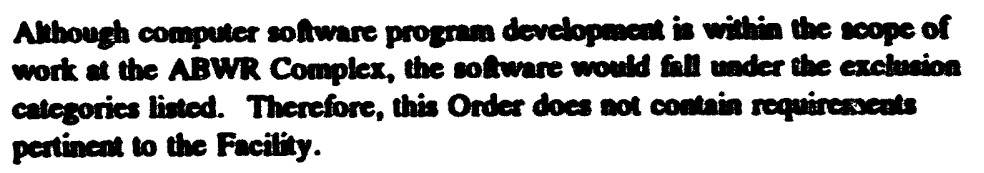 & 9 \\
\hline 1540.1 & $\begin{array}{l}\text { MATERIALS TRANSPORTATON AND } \\
\text { TRAFTC MANAGEMENT }\end{array}$ & Ye & 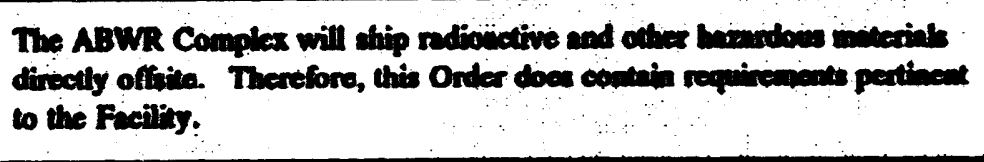 & 10 \\
\hline 1540.2 & $\begin{array}{l}\text { HAZARDOUS MATERIAL PACKAGING } \\
\text { FOR TRANSPORT - ADMINISTRATIVE } \\
\text { PROCEDURES }\end{array}$ & Yes & 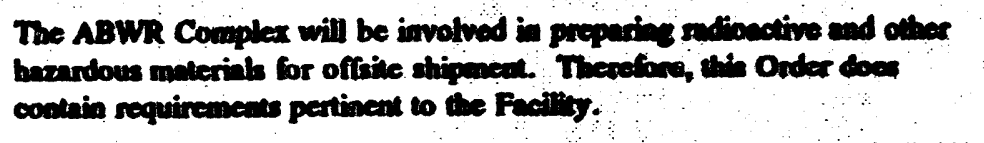 & 11 \\
\hline 1540.3 & $\begin{array}{l}\text { BASE TECHNOLOGY FOR RADIOACTIVE } \\
\text { MATERIAL TRANSPORTATION } \\
\text { PACKAGING SYSTEMS }\end{array}$ & No & 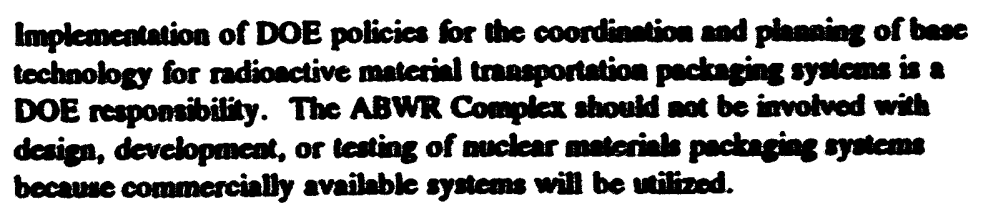 & 12 \\
\hline 1540.4 & $\begin{array}{l}\text { PHYSSICAL RROTECTON OP } \\
\text { UNCLASSIFIED IRRADIATED REACTOR } \\
\text { FUEL WN TRANSTT }\end{array}$ & Yea & 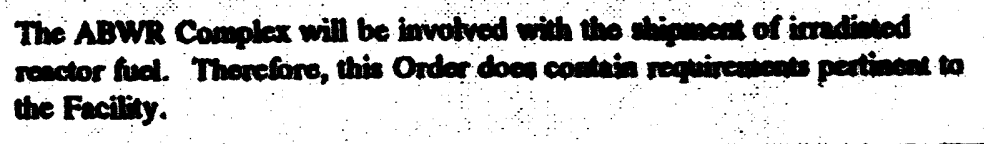 & 14 \\
\hline 3790.1B & $\begin{array}{l}\text { FEDERAL EMPLEYEE OCCUPATIONAL } \\
\text { SAFETY AND HEALTH PROGRAM }\end{array}$ & No & 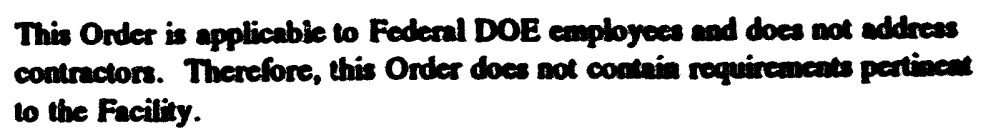 & 15 \\
\hline
\end{tabular}

(Shaded boxes indicate applicable orders.) 
TABLE 1

DOE ORDER APPLICABILITY ASSDSSMENT

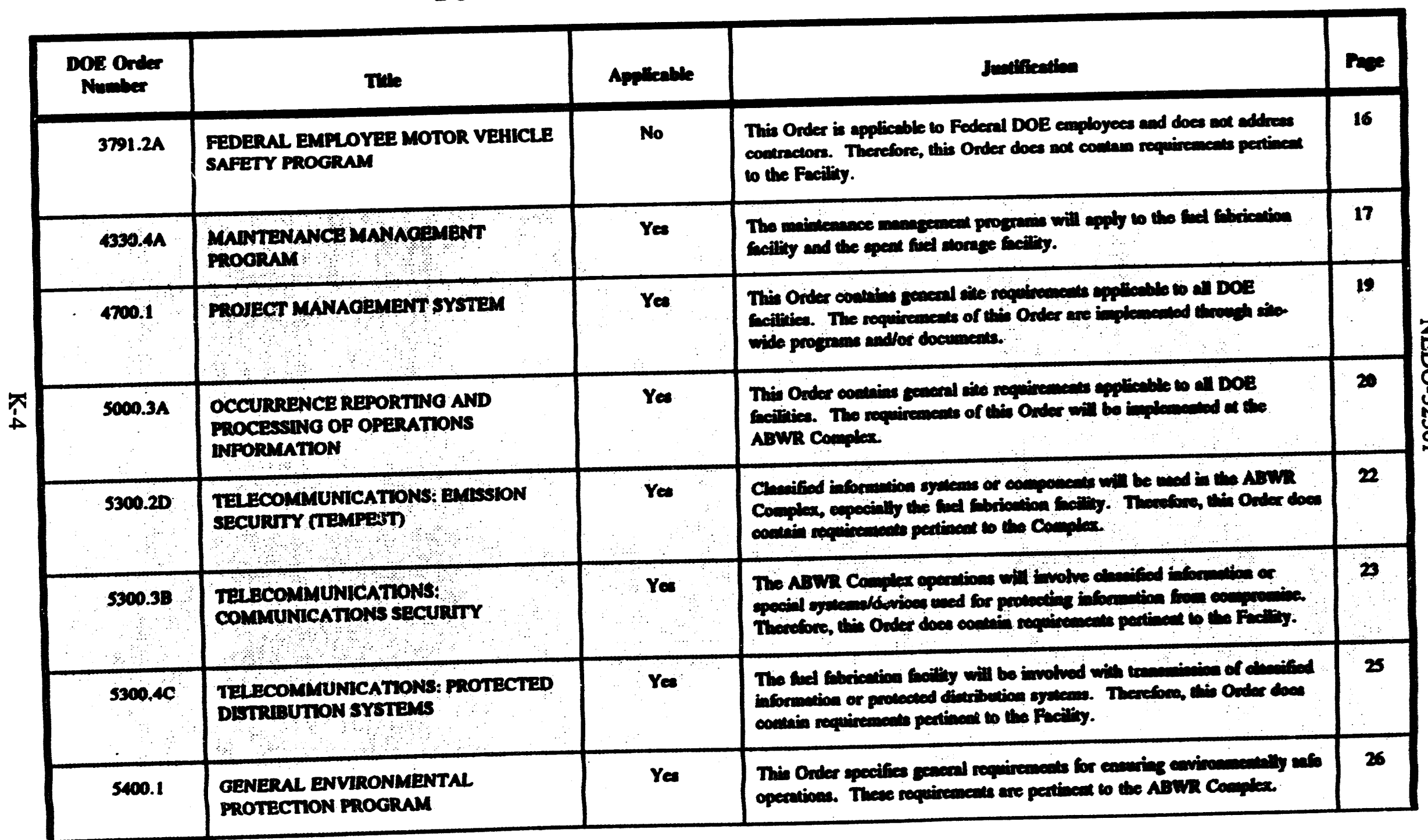

(Shaded boxes indicate applicable orders.) 

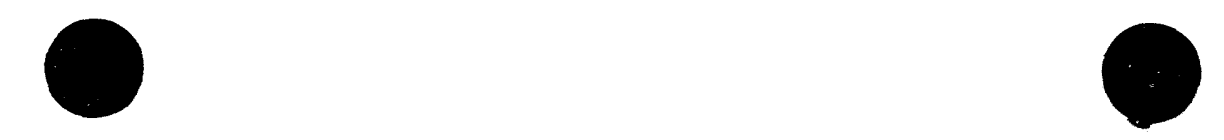

TABLE 1

DOE ORDER APPLICABILITY ASSESSMENT

\begin{tabular}{|c|c|c|c|c|}
\hline $\begin{array}{l}\text { DOB Oniver } \\
\text { Number }\end{array}$ & The & Applieatle & 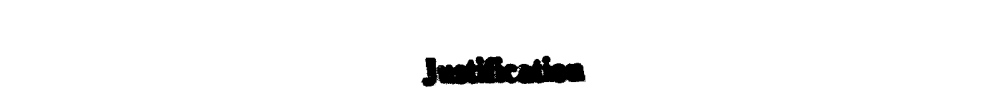 & \\
\hline $5400.2 A$ & $\begin{array}{l}\text { ENVIRONMENTAL COMPLIANCE LSSUE } \\
\text { COORDINATION }\end{array}$ & No & 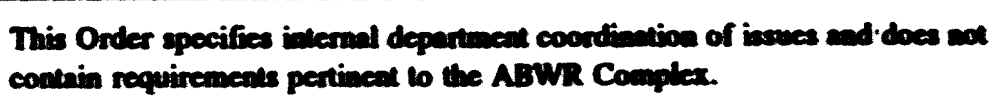 & 27 \\
\hline 5400.3 & $\begin{array}{l}\text { HAZARDOUS AND RADIOACTIVE } \\
\text { MDXED WASTE PROGRAM }\end{array}$ & No & 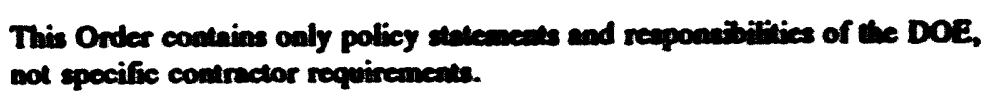 & 28 \\
\hline 5400.4 & $\begin{array}{l}\text { COMPREHENSTVE ENVIRONMENTAL } \\
\text { RESPONSE, COMPENSATION, AND } \\
\text { LABILTY ACT REQUIREMENTS. }\end{array}$ & No & 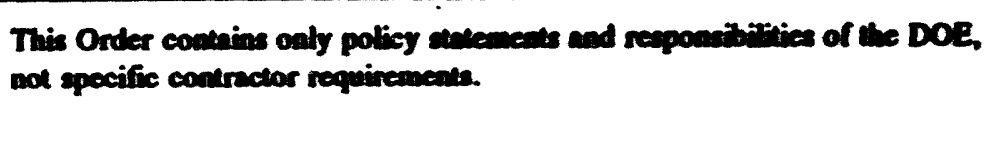 & 30 \\
\hline 5400.5 & $\begin{array}{l}\text { RADLATON PROTECTRON OP THE } \\
\text { PUBLIC AND TIE ENVTRONMENT }\end{array}$ & Yes & 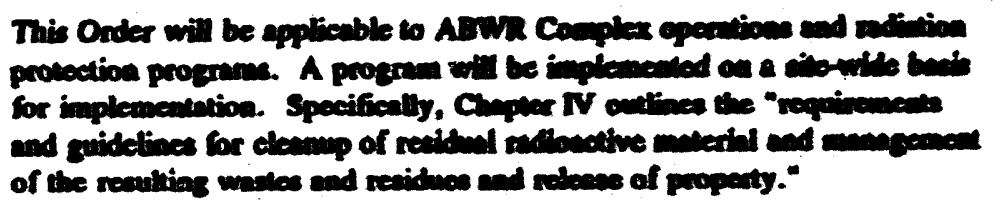 & 31 \\
\hline 540.1D & $\begin{array}{l}\text { NATTONAL ENVIRONMENTAL POLICY } \\
\text { ACT COMPLIANCE PROGRAM }\end{array}$ & No & 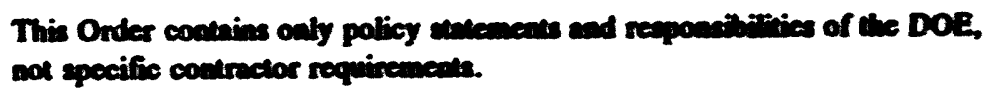 & 32 \\
\hline 5480.18 & $\begin{array}{l}\text { ENVIRONMENT, SAFETY, AND HEALTH } \\
\text { PROGRAM FOR DEPARTMENT OF } \\
\text { ENERGY OPERATIONS }\end{array}$ & No & 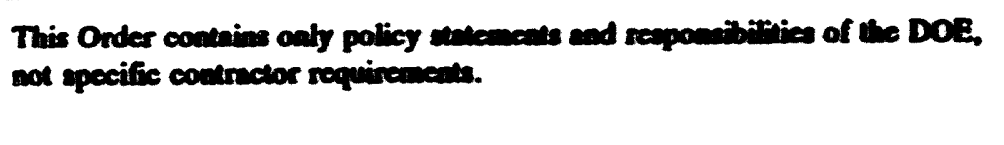 & 33 \\
\hline 5480.3 & 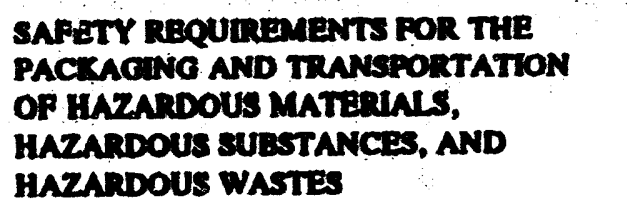 & Yes & 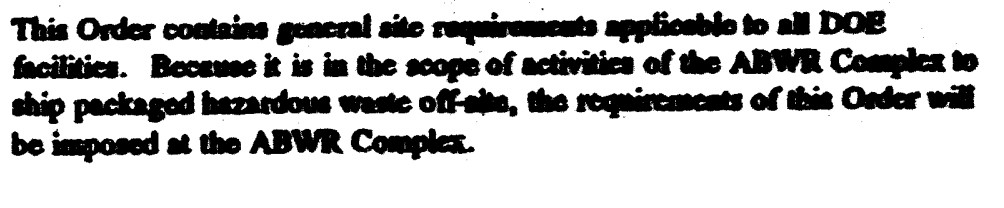 & 34 \\
\hline
\end{tabular}

(Shaded boxes indicate applicable orders.) 
TABLe 1

DOE ORDER APPLCABIUTY AGSDSSMTNT

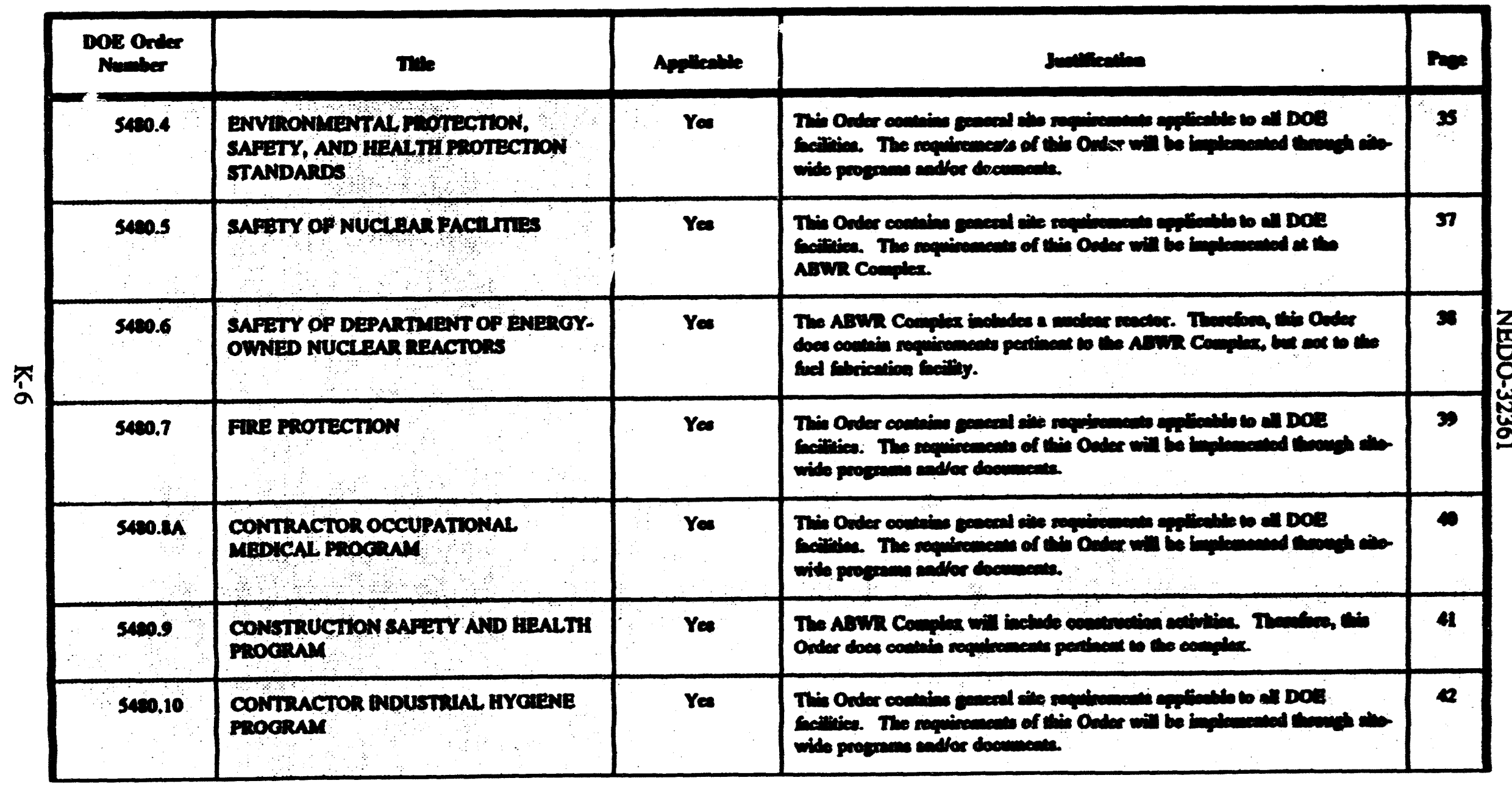

(Shaded boxes indicate applicable orders.)

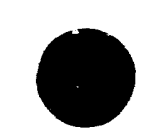


TABLE 1

DOE ORDER APPLUCABILTY ASSRSSMENT

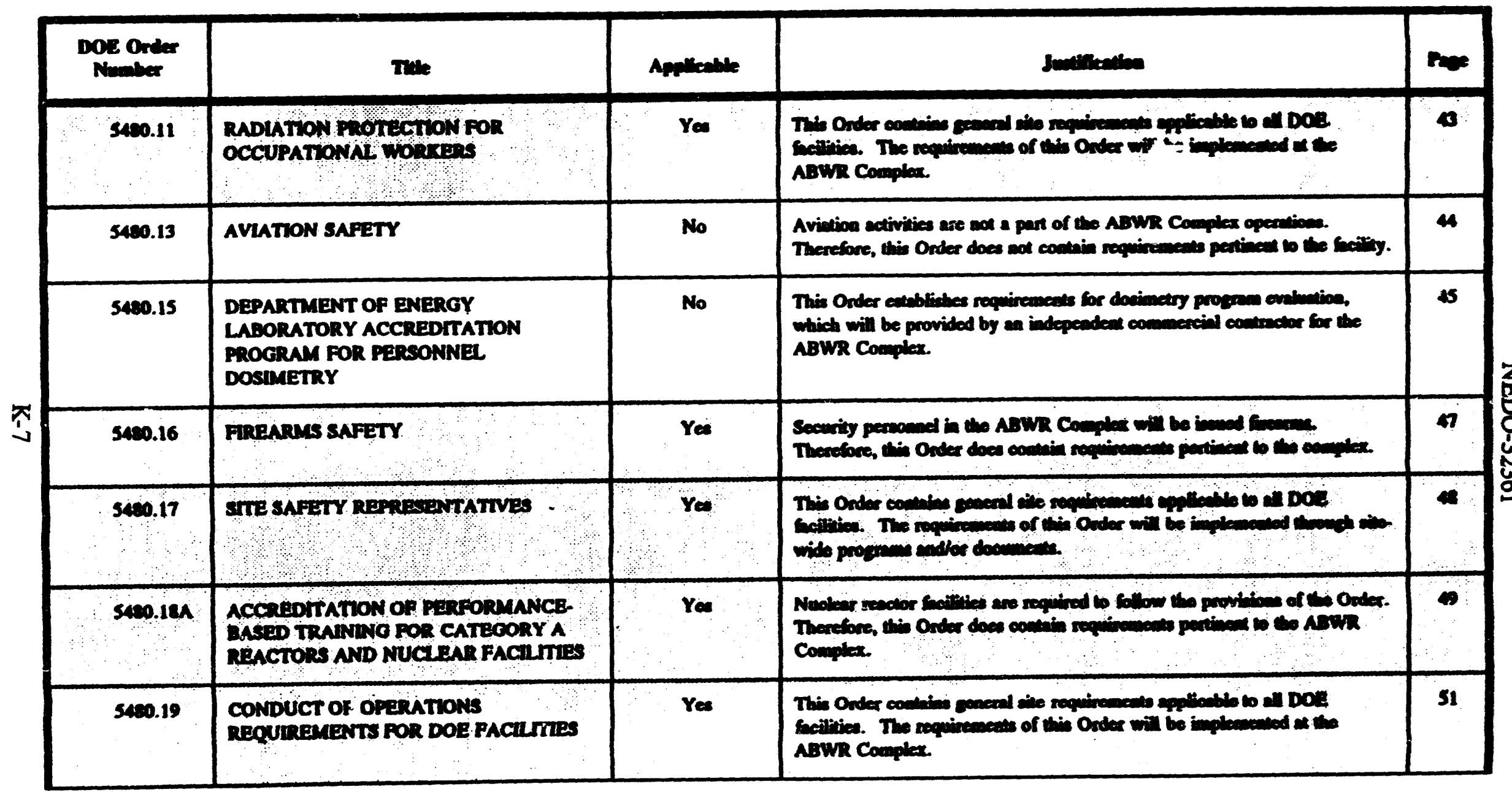

(Shaded boxes indicate applicable orders.) 

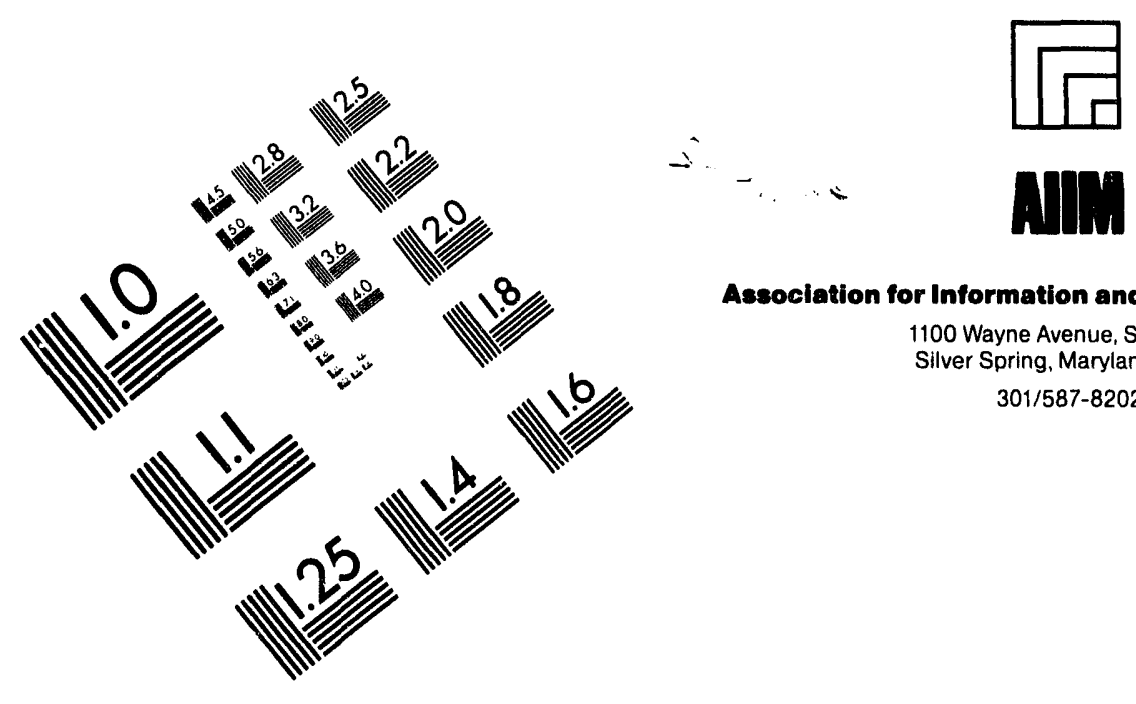

Association for Information and Image Management

1100 Wayne Avenue, Suite 1100

Silver Spring, Maryland 20910

301/587-8202

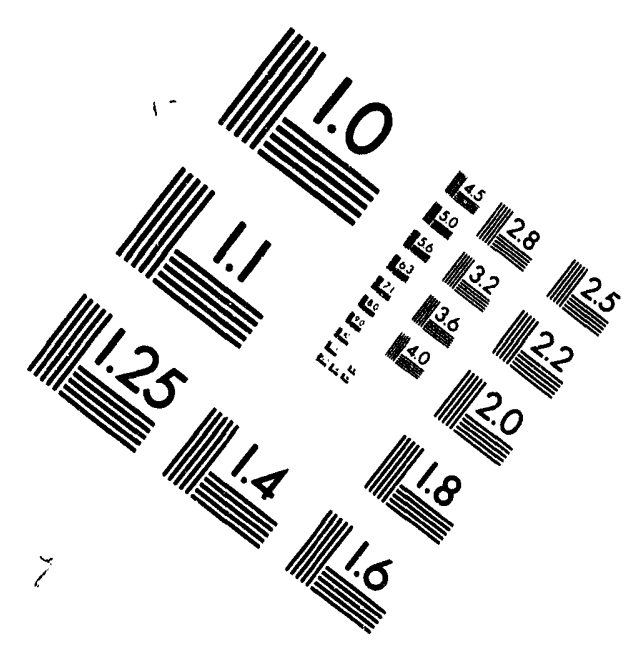

\section{Centimeter}

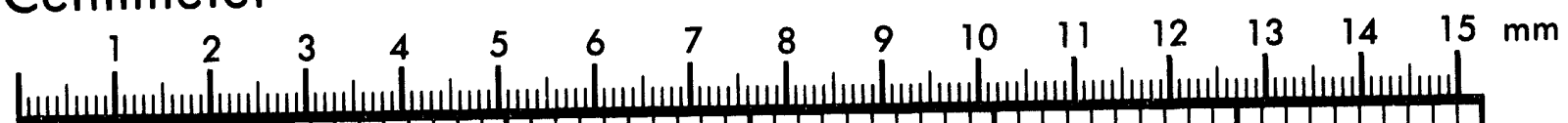

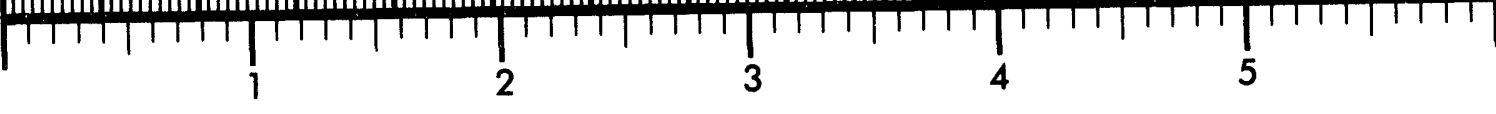
Inches
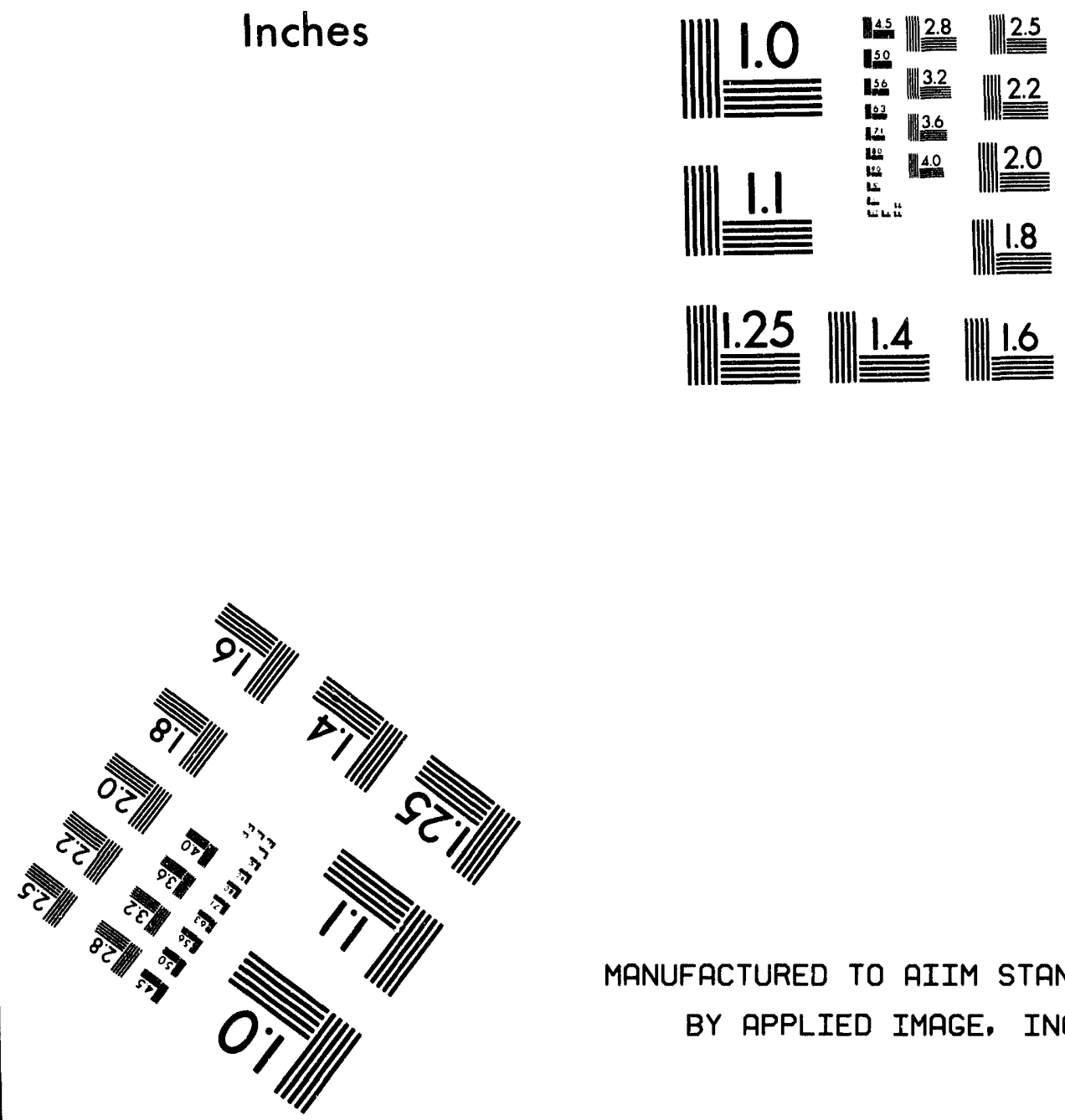

MANUFACTURED TO AIIM STANDARDS

BY APPLIED IMAGE, INC.

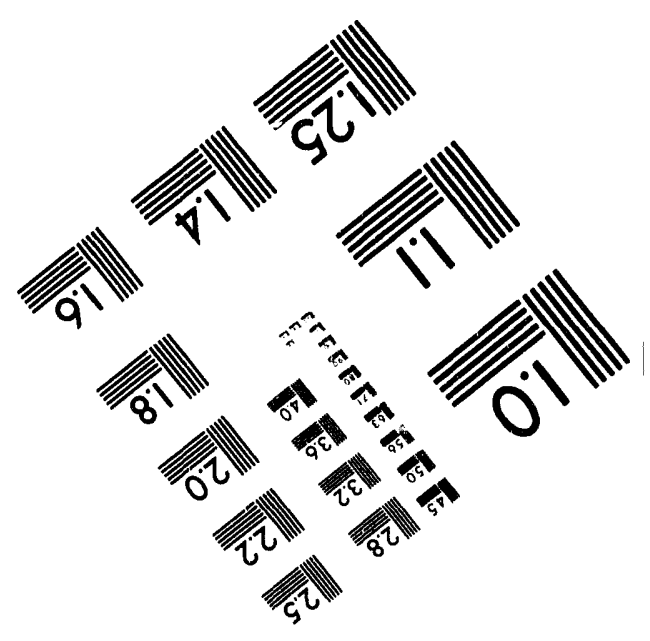



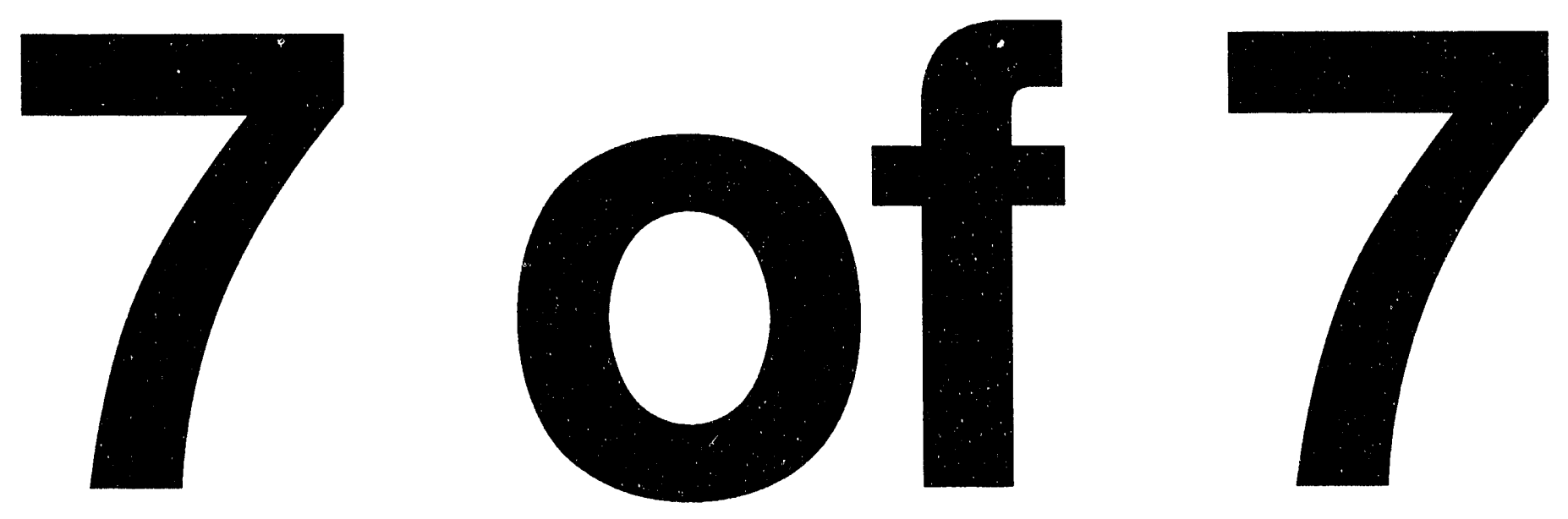
TABLE 1

DOE ORDER APPLICABILITY ASSESSMENT

\begin{tabular}{|c|c|c|c|c|}
\hline $\begin{array}{l}\text { DOZ Order } \\
\text { Nurnaber }\end{array}$ & 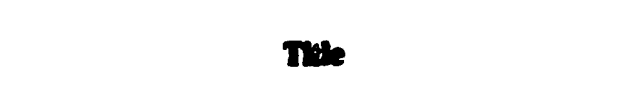 & Applicable & Juetiriention & Pase \\
\hline $\begin{array}{r}5400.20 \\
\vdots \\
\vdots \\
\vdots \\
\hdashline\end{array}$ & 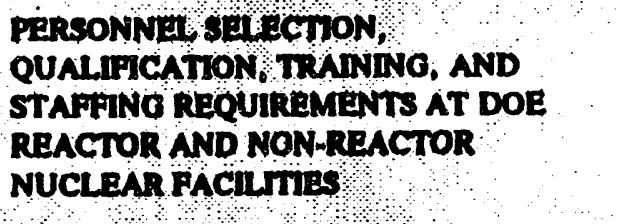 & You & $\begin{array}{l}\text { This Order contains general site requirements applicable to all DOE } \\
\text { facilities. The requirements of this Onder will be implemented at the } \\
\text { ABWR Complox. }\end{array}$ & 52 \\
\hline 5480.21 & UNREVIEWED SAFETY QUESTIONS & $Y \in$ & $\begin{array}{l}\text { This Order contains generil site requirements applicable to all DOE } \\
\text { facilities. The requirements of this Order will be implemented at the } \\
\text { ABWR Complex. }\end{array}$ & 53 \\
\hline 5480.22 & TECHNICAL SAFETY REQUIREMENTS & Yes & $\begin{array}{l}\text { This Order contains genernl sitc requirements applieable to all bOE } \\
\text { facilities. therefore, the requirements of this order will be implemented at } \\
\text { the ABWR. If the reactor is licensod by NRC, this will not apply to the } \\
\text { reactor itself, but will apply to the fuel fibrication facility and spent fuel } \\
\text { elorage facility. }\end{array}$ & 55 \\
\hline 5480.23 & NUCLEAR SAFETY ANALYSIS REPORTS & Yes & $\begin{array}{l}\text { This Order contains general site requirements applicable to all DOE } \\
\text { facilities. the requirements of thil order will be implemented at the ABWR } \\
\text { Complax. If the reactor is licensed by NRC, thit will apply to the non- } \\
\text { reactor facilitive withit the complex. }\end{array}$ & 56 \\
\hline 5490.24 & NUCLEAR CRIICALITY SAFETY & Yes & $\begin{array}{l}\text { This Onder contains general requirementi applicable to all DOE finsilitics. } \\
\text { The requiremetne of this Order will be implemented through siterwide } \\
\text { programs and/or documents. }\end{array}$ & 57 \\
\hline 5480.26 & $\begin{array}{l}\text { TRENDINO AND ANALYSIS OF } \\
\text { OPERATIONS WNORMATION USING } \\
\text { PERPORMANCE INDICATORS }\end{array}$ & Ye & $\begin{array}{l}\text { This Order contain general requiremente applicible to all DOE facilities. } \\
\text { The requiremetns of this Order will be implemented through sito-wide } \\
\text { programe and/or documents. }\end{array}$ & 58 \\
\hline
\end{tabular}

(Shaded boxes indicate applicable orders.) 
TABLE 1

DOE ORDER APPLICABILITY ASSESSMENT

\begin{tabular}{|c|c|c|c|c|}
\hline $\begin{array}{l}\text { DOE Ordex } \\
\text { Number }\end{array}$ & Tile & Applicable & Juntirication & Page \\
\hline 5480.28 & $\begin{array}{l}\text { NATURAT PHENONHNAHAZARDS } \\
\text { MTTOATON }\end{array}$ & Yo: & $\begin{array}{l}\text { This Onder contains general requirements applicable to all DOE nuclear } \\
\text { facilitien. The requiremente of this onder will be implemented at the ABWR } \\
\text { Complex. If the reactor is licensed by NRC, this will apply to the non- } \\
\text { reactor facilities within the complex. }\end{array}$ & 59 \\
\hline 5480.30 & $\begin{array}{l}\text { NUCLEARRRACTORSAFETY DESION } \\
\text { CRITERIA }\end{array}$ & Yes & $\begin{array}{l}\text { This Order containe requirements specifically applicable to the Reactor } \\
\text { Facility only. If the reactor is licensed by NRC, this Order does not apply. }\end{array}$ & 61 \\
\hline 5481.18 & $\begin{array}{l}\text { SAFETY ANALYSIS AND REVIEW } \\
\text { SYSTEM }\end{array}$ & No & This Onder has been superceeded by DOE 5480.23 for nuclear facilities. & 62 \\
\hline $5482.1 \mathrm{~B}$ & $\begin{array}{l}\text { ENVIRONMENT, SAFETY, AND HEALTH } \\
\text { APPRATSAL PROCRAM }\end{array}$ & Yes & $\begin{array}{l}\text { This Onder contains general site requirements applicable to all DOE } \\
\text { facilities. The requirements of thit Onder will be implemeated through sito } \\
\text { wide programs and/or documents. }\end{array}$ & 63 \\
\hline $5483: 1 \mathrm{~A}$ & $\begin{array}{l}\text { OCCUPATIONAL SAFETY AND HEALTH } \\
\text { PROORAM FOR DOE CONTRACTOR } \\
\text { EMPLOYEES AT COVERNMENT-OWNED } \\
\text { CONTRACTOR-OPERATED PACILITES }\end{array}$ & $\ddot{Y}$ Ye & $\begin{array}{l}\text { The ABWR Complex will likely be managed under a COCO Contract. } \\
\text { Therefore, the requirements of this onder are pertinent to the Fheility. }\end{array}$ & 64 \\
\hline 5484.1 & $\begin{array}{l}\text { BNVIRONMENTAL PROTECTION, } \\
\text { SAFETY AND HEALTH PROTECTION } \\
\text { DNPORMATION REPORTINO } \\
\text { RBQUIREMENTS }\end{array}$ & Yes: & $\begin{array}{l}\text { Thi Onder connains general site requirements applieablo to all DOE } \\
\text { facilitiew. The requinements of this Order will be implemented through tito- } \\
\text { wide programs and/or documents. }\end{array}$ & 65 \\
\hline $5500.1 \mathrm{~B}$ & EMERGENCY MANAGEMENT SYSTEM & No & $\begin{array}{l}\text { This Order outlines the policy and requirements for the Emergency } \\
\text { Management System (EMS). Requirements listed are at the DOE level. } \\
\text { Therefore, this Order does not contain requirements pertinent to the ABWR } \\
\text { Complex. }\end{array}$ & 66 \\
\hline
\end{tabular}

(Shaded boxes indicate applicable orders.) 
TABLE 1

DOE ORDER APPLICABILITY ASSESSMENT

\begin{tabular}{|c|c|c|c|c|}
\hline $\begin{array}{l}\text { DOE Order } \\
\text { Number }\end{array}$ & Thile & Applicable & Justification & Page \\
\hline 5500.28 & $\begin{array}{l}\text { EMEROENCY CATEOONIES, CLASSES, } \\
\text { AND NOTTFCATION AND REPORTING } \\
\text { REQUIREMENTS }\end{array}$ & Yoa & $\begin{array}{l}\text { This Order contains general sile requirements applicable to all DOE } \\
\text { facilities. The requirements of this Onder will be implemented at the } \\
\text { ABWR Complex. }\end{array}$ & 67 \\
\hline $5500.3 A$ & $\begin{array}{l}\text { PLANNINO AND PRBPAREDNESS FOR } \\
\text { OPERATIONAL EMEROENCIES }\end{array}$ & Yes & 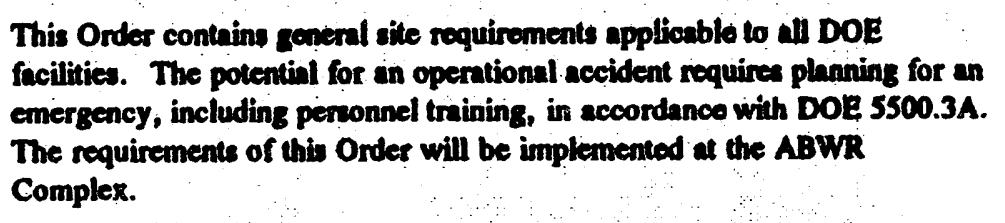 & 68 \\
\hline 5500.4 & $\begin{array}{l}\text { PUBLIC AFFAIRS POLICY AND } \\
\text { PLANNINO REQUIREMENTS FOR } \\
\text { EMERGENCIES }\end{array}$ & Yes & $\begin{array}{l}\text { This Order contains general site roquirements applioesblo to all DOE } \\
\text { facilities. The requirements of this Ordor will be implemented through sito } \\
\text { wide programs and/or documents. }\end{array}$ & 70 \\
\hline 5500.78 & $\begin{array}{l}\text { EMERGENCY OPERATING RECORDS } \\
\text { PROTECTION PROGRAM }\end{array}$ & No & $\begin{array}{l}\text { This Order contains only policy statements and responsibilities of the DOE, } \\
\text { not specific contractor requirements. }\end{array}$ & 72 \\
\hline 5500.10 & $\begin{array}{l}\text { EMEROENCY RBADWNESS ASSURANCE } \\
\text { PROGRAM }\end{array}$ & Yoo & 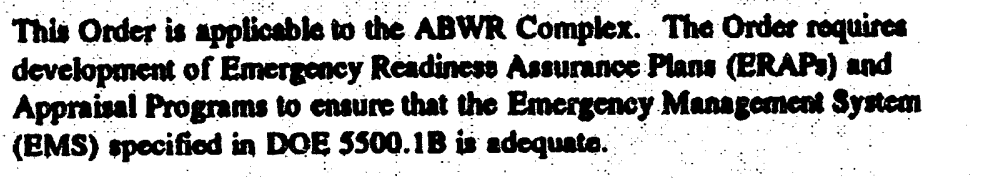 & 73 \\
\hline $5530.1 \mathrm{~A}$ & ACCIDENT RESPONSE GROUP & No & $\begin{array}{l}\text { Activities performod at the ABWR Complex will not involve nuclear } \\
\text { weapons or components thereof (assumming Pu Oxide is delivered to the } \\
\text { Fuel Fabrication Facility). Therefore, this Order does not contain } \\
\text { requirements pertinent to the ABWR Complex. }\end{array}$ & 74 \\
\hline
\end{tabular}

(Shaded boxes indicate applicable orders.) 


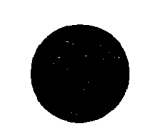

TABLE 1

DOE ORDER APPLICABILITY ASSESSMENT

\begin{tabular}{|c|c|c|c|c|}
\hline $\begin{array}{l}\text { DOE Order } \\
\text { Number }\end{array}$ & Thie & Applicable & Justirication & Page \\
\hline 5600.1 & $\begin{array}{l}\text { MANAGEMENT OF THE DEPARTMENT } \\
\text { OF ENERGY WEAPON PROGRAM AND } \\
\text { WEAPON COMPLEX }\end{array}$ & No & $\begin{array}{l}\text { The scope of the ABWR Complex operntions does not include weapons or } \\
\text { weapons components. Therefore, this Order does not contsin requirements } \\
\text { pertinent to the complex. }\end{array}$ & 75 \\
\hline 5610.1 & $\begin{array}{l}\text { PACKAGING AND TRANSPORTING OF } \\
\text { NUCLEAR EXPLOSIVES, NUCLEAR } \\
\text { COMPONENTS, AND SPECIAL } \\
\text { ASSEMBLIES }\end{array}$ & No & $\begin{array}{l}\text { The scope of the ABWR Complex operations does not include weapons or } \\
\text { weapons components. Therefore, this Order does not contain requirements } \\
\text { pertinent to the Facility. }\end{array}$ & 76 \\
\hline 5610.2 & CONTROL OF WEAPON DATA & No & $\begin{array}{l}\text { Athough fuel fabrication quantities may be classified, the ABWR Complex } \\
\text { activities do not involve weapons data. Therefore, this Order does not } \\
\text { contain requirements pertinent to the ABWR Complex. }\end{array}$ & 77 \\
\hline 5610.10 & $\begin{array}{l}\text { NUCLEAR EXPLOSIVE AND WEAPON } \\
\text { SAFETY PROGRAM }\end{array}$ & No & $\begin{array}{l}\text { The ABWR Complex is not involved in handling nuclear } \\
\text { weapons/explosives. Therefore, this Order does not contain requirements } \\
\text { pertinent to the Facility. }\end{array}$ & 78 \\
\hline 5610.11 & NUCLEAR EXPLOSIVE SAFETY & No & $\begin{array}{l}\text { The ABWR Complex is not involved in any activities dealing with nuclear } \\
\text { explosive/components. Therefore, this Onder does not contain requirements } \\
\text { pertinent to the Facility. }\end{array}$ & 79 \\
\hline 5610.13 & $\begin{array}{l}\text { JOINT DEPARTMENT OF } \\
\text { ENERGYIDEPARTMENT OF DEFENSE } \\
\text { NUCLEAR WEAPON SYSTEM SAFETY, } \\
\text { SECURTY, AND CONTROL ACTIVITIES }\end{array}$ & No & $\begin{array}{l}\text { The ABWR Complex organization is not involved with weapon system } \\
\text { safety, security, and control activities. Therefore, this order does not } \\
\text { contain requirements pertinent to the ABWR Complex. }\end{array}$ & 80 \\
\hline $5630.8 A$ & $\begin{array}{l}\text { SAFEGUARDING OF NAVAL NUCLEAR } \\
\text { PROPULSION INFORMATION }\end{array}$ & No & $\begin{array}{l}\text { The ABWR Complex operations do not involve Naval Nuclear Propulsion } \\
\text { Information. Therefore, this Order does not contain requirements pertinent } \\
\text { to the Facility. }\end{array}$ & 81 \\
\hline
\end{tabular}

(Shaded boxes indicate applicable orders.) 
TABLE 1

DOE ORDER APPLICABILITY ASSESSMENT

\begin{tabular}{|c|c|c|c|c|}
\hline $\begin{array}{l}\text { DOE Order } \\
\text { Number }\end{array}$ & Tile & Applicable & Justification & Page \\
\hline 5630.11 & $\begin{array}{l}\text { SAFEGUARDS AND SECURTY } \\
\text { PROGRAM }\end{array}$ & Ya & $\begin{array}{l}\text { Safeguards and Socurity will be an inlegenl part of the ABWR Complex } \\
\text { operations. Therefore, this Order coes contain requirements pertisent to the } \\
\text { Complex. }\end{array}$ & 82 \\
\hline$\$ 630,12 A$ & $\begin{array}{l}\text { SAFEGUARDS AND SECURTTY } \\
\text { INSPECTION AND EVALUATTON } \\
\text { PROGRAM }\end{array}$ & Yes & $\begin{array}{l}\text { This Order conthina goneral site requirements applioable to all DOE } \\
\text { facilities. The requirements of this Order will be implemented through sito- } \\
\text { wide programs and/or documents. }\end{array}$ & 83 \\
\hline $5630.13 A$ & $\begin{array}{l}\text { MASTER SAFEGUARDS AND SECURTYY } \\
\text { AGREEMENTS }\end{array}$ & Yea & $\begin{array}{l}\text { The program outlined in this Order will be implemented for the overall } \\
\text { complex. Therefore, this Order does contain requirements pentinent to the } \\
\text { ABWR Complex. }\end{array}$ & 84 \\
\hline $5630.14 A$ & $\begin{array}{l}\text { SAFEGUARDS AND SECURTY } \\
\text { PROORAM PLANNINO }\end{array}$ & Yes & $\begin{array}{l}\text { The program oullized in this Order will be implemented for the ovenall } \\
\text { complex. Therefore, this Order does contain roguirements pertinent to the } \\
\text { ABWR Complex: }\end{array}$ & 85 . \\
\hline 5630.15 & $\begin{array}{l}\text { SAFEOUARDS AND SECURTY } \\
\text { TRANINO PROGRAM }\end{array}$ & Yea & $\begin{array}{l}\text { Safegurds and Security will be an integral part of the ABWR Complex } \\
\text { operations. Therefore, this Order doet contain roquiremente pertinent to the } \\
\text { Complex. }\end{array}$ & 86 \\
\hline 5631.18 & SECURTYY EDUCATION PROCRAM & Yes & $\begin{array}{l}\text { This Order conenins general requircments applionblo to all DOE fooitities. } \\
\text { The requirements of this Order will be implemented through site-wide } \\
\text { programs and/or documents. }\end{array}$ & 88 \\
\hline $5631.2 B$ & PERSONNEL SECURTTY PROGRAM & Yea & $\begin{array}{l}\text { This Onder contains genernil requirementa applicable to all DOE ficilitice. } \\
\text { The requirements of this Order are implemented through site-wide programs } \\
\text { and/or documents. }\end{array}$ & 90 \\
\hline
\end{tabular}

(Shaded boxes indicate applicable orders.) 
TABLE 1

DOE ORDER APPLICABILITY ASSESSMENT

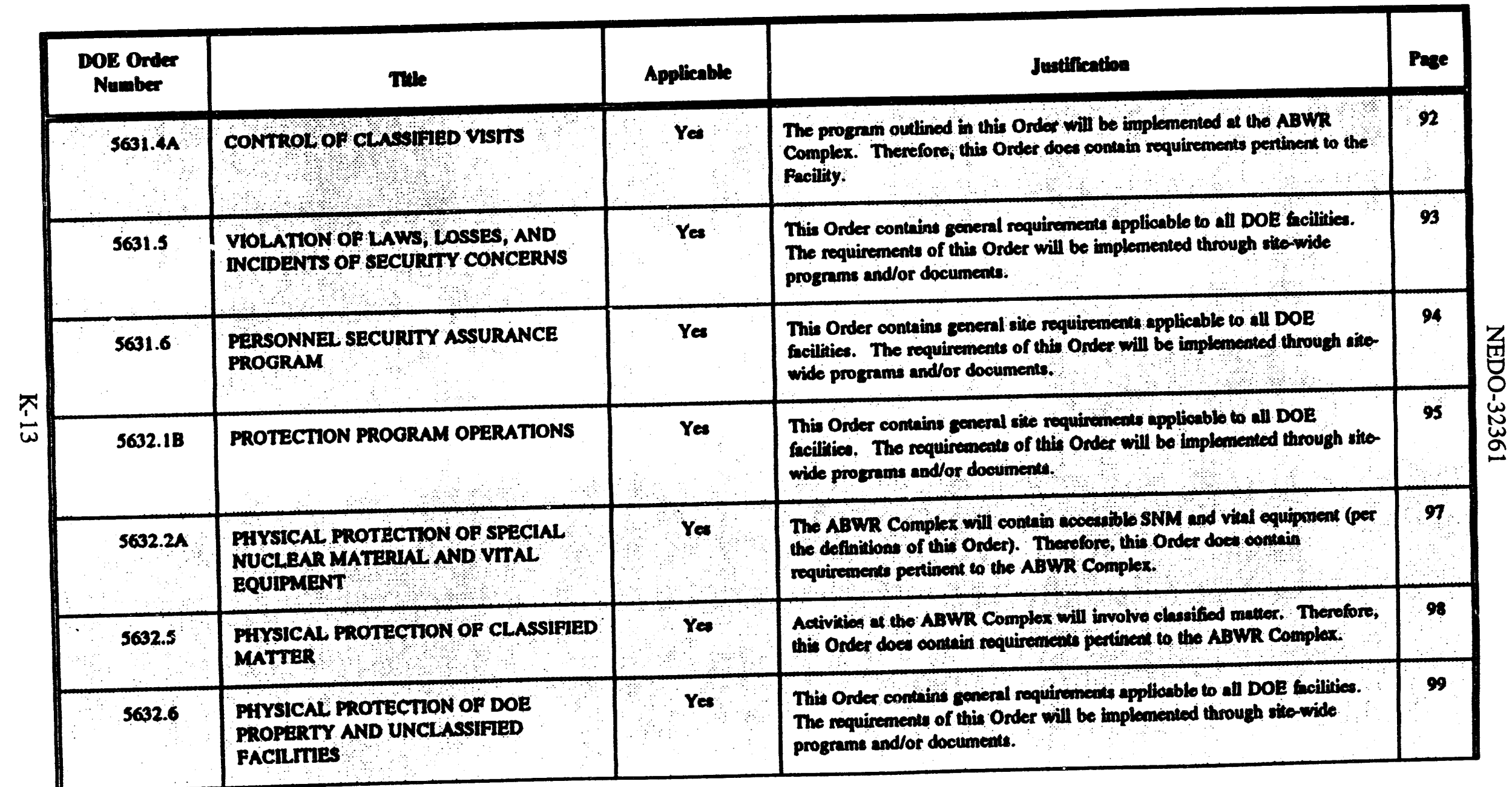

(Shaded boxes indicate applicable orders.) 
TABLE 1

DOE ORDER APPLICABILITY ASSESSMENT

\begin{tabular}{|c|c|c|c|c|}
\hline $\begin{array}{l}\text { DOP Ordex } \\
\text { Nuinber }\end{array}$ & The & Applicalle & Juetification & Page \\
\hline$\$ 632.7$ & PROTRCTUVTOROA' & Yea & 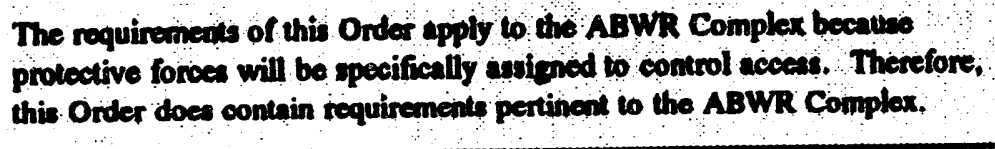 & 100 \\
\hline 5632.8 & $\begin{array}{l}\text { PROTECTON PROORAM OPERATONS: } \\
\text { SYSTPM PERFORMANCB TESTS }\end{array}$ & Yes & 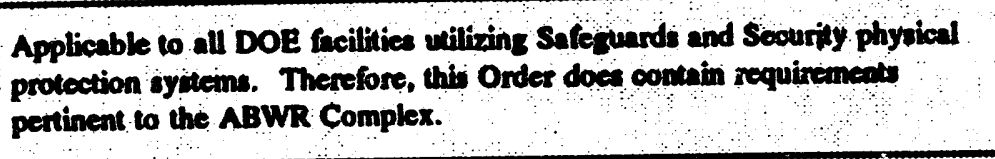 & 101 \\
\hline 5632.9 & $\begin{array}{l}\text { ISSUANCE, CONTROL, AND USE OP } \\
\text { BADOES, PACSES, AND CREDENTLAL }\end{array}$ & $Y \in$ & $\begin{array}{l}\text { The progenn oulined in this Order will be inplemented at the ABWR } \\
\text { Complex. Therefore, this Order does contin requirements pentinent to the } \\
\text { Pacility.. }\end{array}$ & 102 \\
\hline 5632.10 & $\begin{array}{l}\text { SAFEGUARDS AND SECUURTY (SES) } \\
\text { EQUIPMENT STANDARDIZATION }\end{array}$ & No & $\begin{array}{l}\text { The ABWR Complex will utilize commercially available components in the } \\
\text { Safeguards and Security systems. DOE etandards will be implemented, but } \\
\text { not developed. Therefore, this Order does not contain requirements } \\
\text { pertinent to the ABWR Complex. }\end{array}$ & 103 \\
\hline 5633.2 & 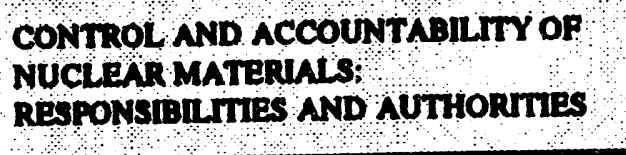 & Yo & 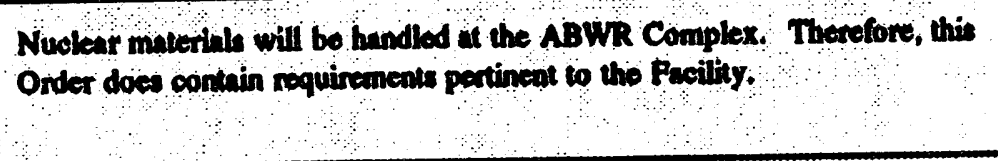 & 104 \\
\hline $\begin{array}{c}5633.3 \\
\vdots \\
\vdots\end{array}$ & $\begin{array}{l}\text { CONTROL AND ACCOUNTABILTY OF } \\
\text { NUCLEAR MATERIALS }\end{array}$ & $Y e$ & $\begin{array}{l}\text { Nucleat materials will be handled at the ABWR Complax Therefore, this } \\
\text { Order does confain requirements pertinent to the Faolity. }\end{array}$ & 105 \\
\hline $5633: 4$ & $\begin{array}{l}\text { NUCLEAR MATERIALS TRANSACTIONS: } \\
\text { DOCUMENTATION AND REPORTINO }\end{array}$ & $Y \in$ & $\begin{array}{l}\text { Nuclear muterials will be received and shipped from the ABWR Comples. } \\
\text { Therefore, this Order does contrin rogulirements pertinent to the Facility. }\end{array}$ & 106 \\
\hline
\end{tabular}

(Shaded boxes indicate applicable orders.)

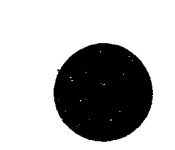


TABLE 1

DOE ORDER APPLICABILITY ASSESSMENT

\begin{tabular}{|c|c|c|c|c|}
\hline $\begin{array}{l}\text { DOE Order } \\
\text { Number }\end{array}$ & Thie & Applicable & Justification & Page \\
\hline 5633.5 & $\begin{array}{l}\text { NUCLLAR MATHALALS REPOPTINO } \\
\text { AND DATA SUBMLSSION PROCEDURES }\end{array}$ & Yes & $\begin{array}{l}\text { Nuolear melerials are rodeived, rethinod, and hipped at tho ABWR } \\
\text { Complex. Therefore, this Onder does contain requirements pertinent to the } \\
\text { complex. }\end{array}$ & 107 \\
\hline $5634.1 \mathrm{~A}$ & $\begin{array}{l}\text { FACILITY APPROVAKS, SECURTY } \\
\text { SURVEY,, AND NUCLEAR MATERHLS } \\
\text { SURVEYS }\end{array}$ & Yes & $\begin{array}{l}\text { The ABWR Complox will receive, prooced, ship and handle cleasified } \\
\text { malerial und SNM. Therefore, this Onder doet contain requirements } \\
\text { pertinent to the ABWR Complex. }\end{array}$ & 108 \\
\hline $5635.1 A$ & $\begin{array}{l}\text { CONTROL OF CLASSIFIED DOCUMENTS } \\
\text { AND INPORMATION }\end{array}$ & Yes & 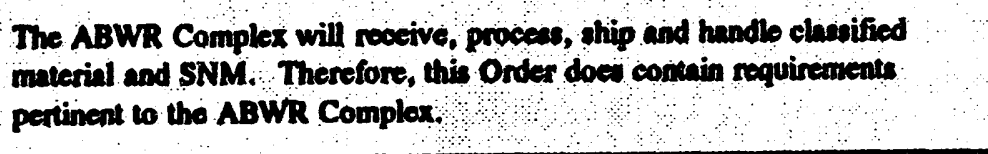 & 109 \\
\hline $5635.2 B$ & $\begin{array}{l}\text { PROTECTION OF CLASSIFIED } \\
\text { NATIONAL SECURTYY COUNCIL } \\
\text { INFORMATION }\end{array}$ & No & $\begin{array}{l}\text { It is not anticipated that NSC information will be handlod or utilized by the } \\
\text { ABWR Complex. therefore, this Order does not contain requirements } \\
\text { pertinent to the complex. }\end{array}$ & 110 \\
\hline 5635,3 & $\begin{array}{l}\text { HAND CARRYINC CLAESIFIED MATTER } \\
\text { ON AIR CAPREERS }\end{array}$ & Ye & 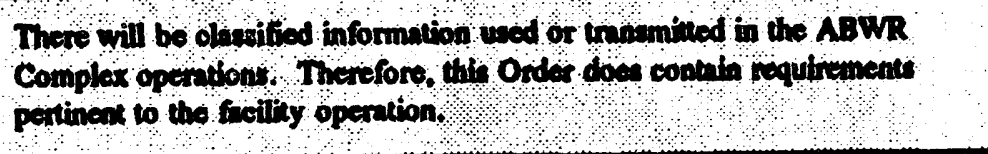 & 111 \\
\hline 5635.4 & $\begin{array}{l}\text { PROTECTION OP UNCLASSIFIED } \\
\text { CONTROLLED NUCLBAR INPORMATION }\end{array}$ & Yes & 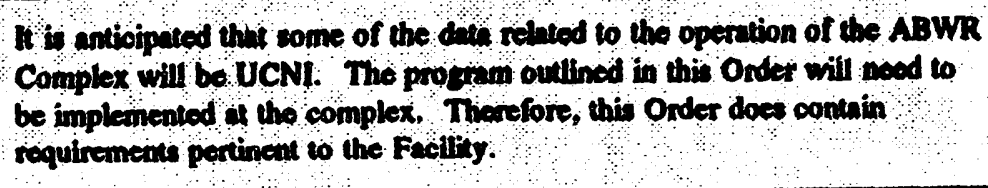 & 112 \\
\hline $5636.1 \mathrm{~A}$ & $\begin{array}{l}\text { PROHIBTIONS ON HLLCTT } \\
\text { WIRETAPPING AND EAVESDROPPING }\end{array}$ & $Y e s$ & $\begin{array}{l}\text { The progrnm oulinod in this Order will need to be implemented at the } \\
\text { ABWR Complex. Therefore, this Order does contais requirements } \\
\text { pertinent to the Facility. }\end{array}$ & 113 \\
\hline
\end{tabular}

(Shaded boxes indicate applicable orders.) 
TABLE 1

DOE ORDER APPLICABILITY ASSESSMENT

\begin{tabular}{|c|c|c|c|c|}
\hline $\begin{array}{l}\text { DOS Oniex } \\
\text { Nanber }\end{array}$ & Thle & Applieable & Justiriention & Page \\
\hline 5637.1 & $\begin{array}{l}\text { CLASSIFIED COMPUMAR SRCURTY } \\
\text { PROGRAM }\end{array}$ & Yes & 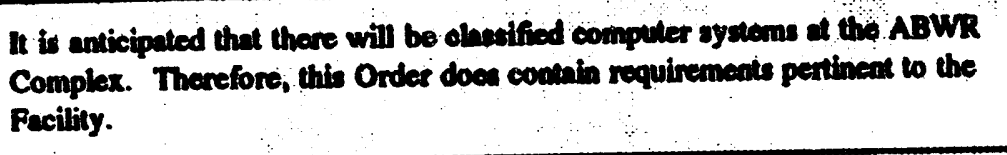 & 114 \\
\hline 5650.28 & $\begin{array}{l}\text { IDENTIFICATION OP CLASSIFIED } \\
\text { INPORUATION }\end{array}$ & Yes & 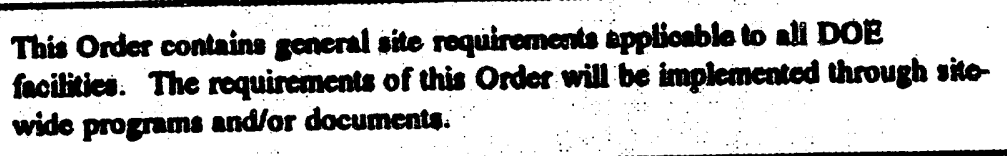 & 115 \\
\hline $5650.3 A$ & $\begin{array}{l}\text { DENTIPCATION OR UNCLASSIFIED } \\
\text { CONTROLLED NUCLEAR INFORMATION }\end{array}$ & $Y=$ & $\begin{array}{l}\text { This Order contains general sile requirements applicable to all DOE } \\
\text { facilties. The requirements of this Order will be inplemented through sito } \\
\text { wido programs and/or documents. }\end{array}$ & 116 \\
\hline 5660.1 & $\begin{array}{l}\text { MANAGBMENT OF NUCLEAR } \\
\text { MATERIALS }\end{array}$ & Yes & $\begin{array}{l}\text { The ABWR Complex will contain nuclear melerial . Thereforo, this Order } \\
\text { does contain roquiremests pertineme to the ABWR Complex. }\end{array}$ & 117 \\
\hline $5670.1 A$ & $\begin{array}{l}\text { MANAGEMENT AND CONTROL OF } \\
\text { POREIGN INTELLIGENCE }\end{array}$ & No & $\begin{array}{l}\text { This Order contains only policy statements and responsibilities of the DOE, } \\
\text { not specific contractor requirements. }\end{array}$ & 118 \\
\hline 5670.2 & $\begin{array}{l}\text { SECURITY OF FOREICN INTELLIGENCE } \\
\text { INFORMATION AND SENSITTE } \\
\text { COMPARTMENTED INFORMATION } \\
\text { FACILITIES }\end{array}$ & Nó & $\begin{array}{l}\text { This Order is applicable only if Foreign Intelligence Information is } \\
\text { requested or retained by ABWR Complex personnel. Such information is } \\
\text { not expected to be requeated or retained as a part of the ABWR Complex } \\
\text { lovel of activities. }\end{array}$ & 119 \\
\hline $5700,6 \mathrm{C}$ & QUALTTY ASSURANCE & Yes & 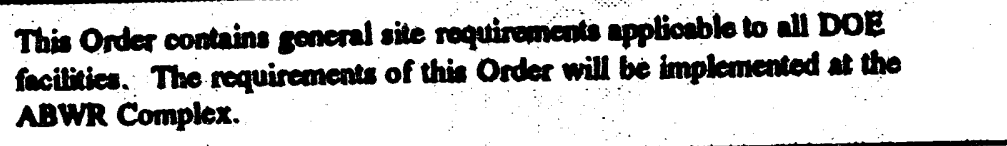 & 120 \\
\hline
\end{tabular}

(Shaded boxes indicate applicable orders.) 
TABLE 1

DOE ORDER APPLICABILITY ASSESSMENT

\begin{tabular}{|c|c|c|c|c|}
\hline $\begin{array}{l}\text { DOE Order } \\
\text { Number }\end{array}$ & Tule & Applicable & Justification & Page \\
\hline $5820.2 A$ & RADIOACTTVE WASTE MANAGEMENT & Yes & $\begin{array}{l}\text { This Order contains general site requirements applicable to all DOE } \\
\text { facilities. The ABWR Complex will contain radioactive wates and } \\
\text { therefore the requirements of this Order will be implemented at the Facility. }\end{array}$ & 121 \\
\hline $6430.1 \mathrm{~A}$ & OENERAL DESION CRITERIA & Yes & $\begin{array}{l}\text { This Order contains general site requirements applioable to all non-reactor } \\
\text { nuclear facilities. The requirements of thi Onder will be implemented } \\
\text { through programs and/or doouments for the fuel fabrication faoility. }\end{array}$ & 122 \\
\hline
\end{tabular}

(Shaded boxes indicate applicable orders.) 

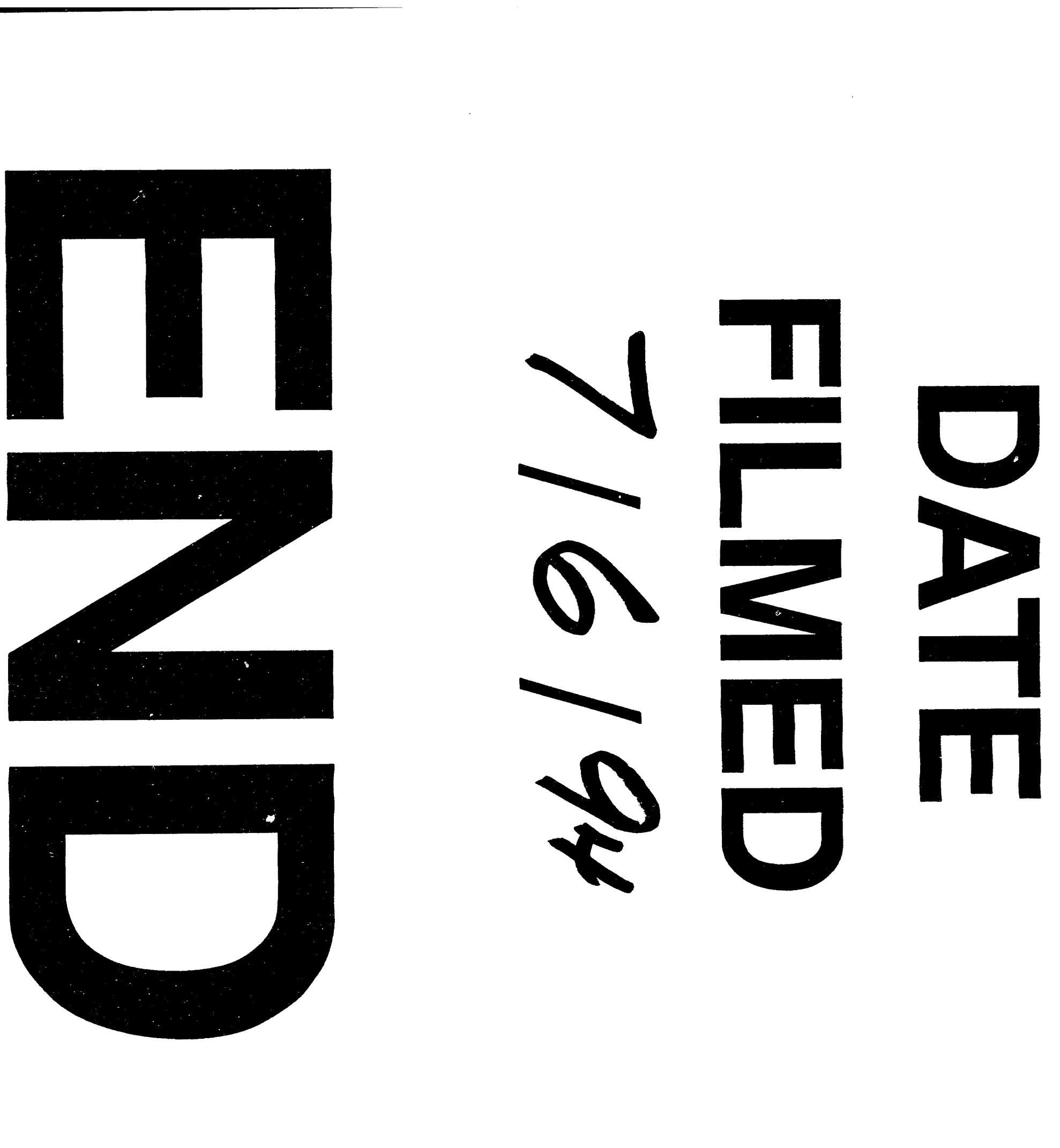

$\tau$ 